Rüdiger Hermann

\title{
Ein gemeinsamer Markt für Elektrizität in Europa
}




\section{Rüdiger Hermann}

\section{Ein gemeinsamer Markt für Elektrizität in Europa}

In der Arbeit werden vier Optionen zur Schaffung eines europäischen Strommarktes entwickelt und bewertet, in deren Mittelpunkt der Stromtransportbereich steht. Es wird ein optimales Ordnungsmodell konzipiert und aufgezeigt, wie dieses eingeführt werden kann. Die Arbeit untersucht außerdem, auf welcher Grundlage Stromtransporte durch Versorgungsgebiete abgerechnet werden können.

Rüdiger Hermann wurde 1967 in Aachen geboren und studierte Wirtschafts- und Organisationswissenschaften an der Universität der Bundeswehr in Hamburg. Seit 1994 ist er bei der Allianz Versicherungs-AG beschäftigt. 
Ein gemeinsamer Markt für Elektrizität in Europa

Rüdiger Hermann - 978-3-631-75129-9

Downloaded from PubFactory at 01/11/2019 07:57:52AM

via free access 


\title{
SCHRIFTEN ZUR \\ WIRTSCHAFTSTHEORIE UND WIRTSCHAFTSPOLITIK
}

\author{
Herausgegeben von \\ Rolf Hasse, Wolf Schäfer, Thomas Straubhaar und Klaus W. Zimmermann
}

Band 4

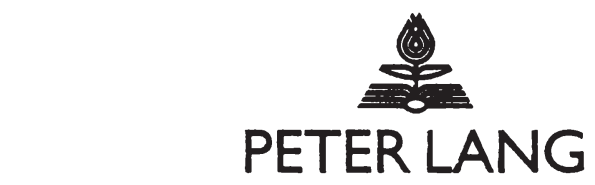

Frankfurt am Main - Berlin - Bern - New York - Paris - Wien

Rüdiger Hermann - 978-3-631-75129-9

Downloaded from PubFactory at 01/11/2019 07:57:52AM

via free access 
Rüdiger Hermann

\section{Ein gemeinsamer Markt für Elektrizität in Europa \\ Optionen einer Wettbewerbsordnung zwischen Anspruch und Wirklichkeit}

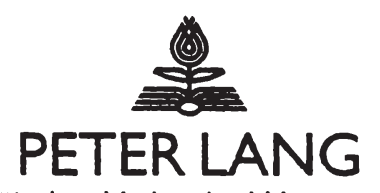

Europäischer Verlag der Wissenschaften

Rüdiger Hermann - 978-3-631-75129-9

Downloaded from PubFactory at 01/11/2019 07:57:52AM 
Die Deutsche Bibliothek - CIP-Einheitsaufnahme

Hermann, Rüdiger:

Ein gemeinsamer Markt für Elektrizität in Europa : Optionen einer Wettbewerbsordnung zwischen Anspruch und

Wirklichkeit / Rüdiger Hermann. - Frankfurt am Main ; Berlin ; Bern ; New York ; Paris ; Wien : Lang, 1997

(Schriften zur Wirtschaftstheorie und Wirtschaftspolitik ; Bd. 4)

Zugl.: Hamburg, Univ. der Bundeswehr, Diss., 1997

ISBN 3-631-32116-3

Open Access: The online version of this publication is published on www.peterlang.com and www.econstor.eu under the international Creative Commons License CC-BY 4.0. Learn more on how you can use and share this work: http://creativecommons.org/licenses/ by/4.0.

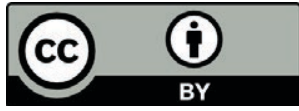

This book is available Open Access thanks to the kind support of ZBW - Leibniz-Informationszentrum Wirtschaft.

\author{
Gedruckt mit Unterstützung \\ der Universität der Bundeswehr Hamburg.
}

\author{
D 705 \\ ISSN 1433-1519 \\ ISBN 3-631-32116-3 \\ ISBN 978-3-631-75129-9 (eBook) \\ (c) Peter Lang $\mathrm{GmbH}$ \\ Europäischer Verlag der Wissenschaften \\ Frankfurt am Main 1997 \\ Alle Rechte vorbehalten.
}

Das Werk einschließlich aller seiner Teile ist urheberrechtlich geschützt. Jede Verwertung außerhalb der engen Grenzen des

Urheberrechtsgesetzes ist ohne Zustimmung des Verlages unzulässig und strafbar. Das gilt insbesondere für Vervielfältigungen, Übersetzungen, Mikroverfilmungen und die Einspeicherung und Verarbeitung in elektronischen Systemen. 


\section{INHALTSVERZEICHNIS}

Darstellungsverzeichnis

Einleitung

1 Ziele und Hemmnisse des europäischen Binnenmarktes für Elektrizität

2 Die Besonderheiten des Elektrizitätssektors

2.1 Die technischen Besonderheiten

2.2 Entwicklungsbedingte Besonderheiten

2.3 Die marktlichen Besonderheiten der Elektrizitätsversorgung

3 Wettbewerb oder Monopole für den europäischen Strommarkt?

4 Der gegenwärtige Integrationsgrad der europäischen Elektrizitätswirtschaften

4.1 Die elektrizitätswirtschaftlichen Strukturen der EU-Mitgliedsländer

4.1.1 Elektrizitätswirtschaften mit vollständig integrierten Strukturen und einem staatlichem EVU

4.1.1.1 Die Struktur der französischen Elektrizitätswirtschaft

4.1.1.2 Die Struktur der italienischen Elektrizitätswirtschaft

4.1.2 Elektrizitätswirtschaften mit mehrstufigen Strukturen und geschlossenen Versorgungsgebieten

4.1.2.1 Die Struktur der deutschen Elektrizitätsversorgung 57

4.1.2.2 Die Struktur der niederländischen Elektrizitätswirtschaft 62

4.1.3 Elektrizitätswirtschaften mit desintegrierten Strukturen und Wettbewerb in der Elektizitätsversorgung - Die Struktur der britischen Elektrizitätswirtschaft -

4.2 Stromhandel- und Austauschkapazitäten zwischen den europäischen Mitgliedsstaaten

$\begin{array}{ll}\text { 4.2.1 Organisationen für den Stromaustausch } & 75\end{array}$

$\begin{array}{lll}\text { 4.2.1.1 Die UCPTE } & 75\end{array}$

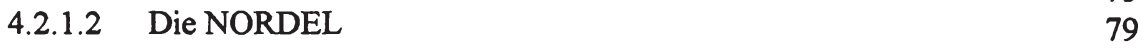

4.2.1.3 Der CENTREL-Verbund $\quad 80$

$\begin{array}{lll}\text { 4.2.2 Stromaustausch und Harmonisierungsgrad } & 81\end{array}$

4.2.2.1 Stromaustausch und Verbindungen 
5 Richtlinien und Richtlinienentwürfe zum europäischen Binnenmarkt für Elektrizität

5.1 Die Entwicklung der Richtlinien und Richtlinienentwürfe $\quad 87$

5.2 Die Vorstellungen der EU-Kommission 93

5.3 Die Vorstellungen des Europäischen Parlamentes und des Ministerrates 93

$6 \quad$ Integrationskonzepte 95

6.1 Die Weiterentwicklung des bestehenden Verbundsystems durch die europäischen EVU (1.Konzept) 95

6.1.1 Die Konzeption des Weiterentwicklungskonzeptes 95

6.1.2 Die Mittel des Konzeptes 96

6.1.3 Die Entwicklungmöglichkeiten des Konzeptes 97

6.1.4 Die Akzeptanz des Weiterentwicklungskonzeptes 101

$\begin{array}{ll}\text { 6.1.5 Der Regulierungsbedarf des Konzeptes } & 102\end{array}$

$\begin{array}{ll}\text { 6.1.6 Die Implementierung des Konzeptes } & 105\end{array}$

6.2 Das Third-Party-Access-Konzept (2.Konzept) 106

6.2.1 Die Konzeption des TPA-Konzeptes 106

$\begin{array}{ll}\text { 6.2.2 Die Mittel des Konzeptes } & 119\end{array}$

6.2.3 Die Entwicklungsmöglichkeiten des Konzeptes 120

$\begin{array}{ll}\text { 6.2.3.1 Entwicklungsszenarien } & 121\end{array}$

6.2.3.2 Zusammenhang zwischen Strompreisdisparitäten, dem Interesse am Stromhandel und den Entwicklungen im Binnenmarkt des TPA-Konzeptes

$\begin{array}{ll}\text { 6.2.4 Die Akzeptanz des Konzeptes } & 128\end{array}$

$\begin{array}{ll}\text { 6.2.5 Der Regulierungsbedarf des Konzeptes } & 130\end{array}$

6.2.6 Die Implementierung des TPA-Konzeptes 134

$\begin{array}{lll}6.3 & \text { Beurteilung der Integrationskonzepte } & 135\end{array}$

$\begin{array}{ll}\text { 6.3.1 Beurteilung der Marktkonzeptionen } & 135\end{array}$

6.3.2 Der Beitrag der beiden Integrationskonzepte zur Zielerfüllung 142

6.3.2.1 Reduzierung der Kosten der Stromversorgung 142

6.3.2.1.1 Kostenentwicklung bei Umsetzung des
Weiterentwicklungskonzeptes

6.3.2.1.2 Kostenentwicklung bei Umsetzung des TPA-Konzeptes $\quad 144$

$\begin{array}{lll}\text { 6.3.2.2 } & \text { Beurteilung der Versorgungssicherheit } & 148\end{array}$

$\begin{array}{lll}\text { 6.3.3 Ausblick: Preisentwicklung bei Umsetzung der } & \\ & \text { Integrationskonzepte }\end{array}$

7 Desintegrationskonzepte 153

$\begin{array}{lll}7.1 & \text { Vorbemerkungen } & 153\end{array}$

7.2 Das Common-Carrier-Konzept (3digennept) $)_{n n}$ - 978-3-631-75129-9 154 
7.2.1 Die Konzeption des Common-Carrier-Konzeptes 155

7.2.1.1 Gesamtmarkt und Erzeugung 155

7.2.1.2 Die Verteilung 159

7.2.1.2.1 Endabnehmerversorgung durch die Verteilerunternehmen 159

7.2.1.2.2 Wettbewerb in der Endverbraucherversorgung 162

$\begin{array}{lll}\text { 7.2.1.3 Die Netzgesellschaft } & 165\end{array}$

$\begin{array}{ll}\text { 7.2.2 Die Mittel des Common-Carrier-Konzeptes } & 169\end{array}$

$\begin{array}{lll}\text { 7.2.2.1 Der Spotmarkt } & 170\end{array}$

$\begin{array}{lll}\text { 7.2.2.2 Die Abwicklung der langfristigen Lieferverträge } & 174\end{array}$

7.2.2.3 Das Lastmanagement und die Bereitstellung der
Versorgungssicherheit

$\begin{array}{ll}\text { 7.2.3 Die Entwicklungsmöglichkeiten des Konzeptes } & 177\end{array}$

$\begin{array}{ll}\text { 7.2.4 Die Akzeptanz des Common-Carrier-Konzeptes } & 182\end{array}$

$\begin{array}{lll}7.2 .5 & \text { Der Regulierungsbedarf des Konzeptes } & 184\end{array}$

$\begin{array}{lll}\text { 7.2.6 Die Implementierung des Konzeptes } & 187\end{array}$

7.3 Das Stromhändlerkonzept (4. Konzept) 193

7.3.1 Die Konzeption des Stromhändlerkonzeptes 193

$\begin{array}{lll}\text { 7.3.1.1 Der Stromhändlerankaufsmarkt } & 195\end{array}$

$\begin{array}{lll}\text { 7.3.1.2 Der Stromhändlerabsatzmarkt } & 195\end{array}$

7.3.1.3 Der Endabnehmermarkt zwischen Verteiler und
Letztabnehmer

7.3.2 Die Mittel des Stromhändlerkonzeptes 199

7.3.2.1 Die Preisgestaltung gegenüber den Erzeugerunternehmen 199

$\begin{array}{ll}\text { 7.3.2.1.1 Einheitliche Strombezugspreise } & 200\end{array}$

7.3.2.1.2 Uneinheitliche Strombezugspreise 200

7.3.2.2 Die Preisgestaltung gegenüber den Verteilern und
Großabnehmern

$\begin{array}{lll}\text { 7.3.2.3 Die Preisgestaltung für die Koordinationsaufgaben und den } & \\ & \text { Stromtransport } & 202\end{array}$

$\begin{array}{lll}\text { 7.3.3 Die Entwicklungsmöglichkeiten } & 203\end{array}$

7.3.4 Die Akzeptanz des Stromhändlerkonzeptes 206

$\begin{array}{ll}\text { 7.3.5 Der Regulierungsbedarf des Konzeptes } & 208\end{array}$

7.3.6 Die Implementierung des Stromhändlerkonzeptes 209

$\begin{array}{ll}7.4 & \text { Beurteilung der Desintegrationskonzepte } \\ 7.4 .12\end{array}$

$\begin{array}{ll}\text { 7.4.1 Beurteilung der Marktkonzeptionen } & 212\end{array}$

7.4.2 Der Beitrag der beiden Desintegrationskonzepte zur
Zielerfüllung

7.4.2.1 Reduzierung der Kosten der Stromversorgung 220

7.4.2.1.1 Kostenentwicklung bei Umsetzung des Common-Carrier-
Konzeptes

7.4.2.1.2 Kostenentwicklung bei Umsetzung des Stromhändlerkonzeptes 224

7.4.2.2 Beurteilung der Versorgungssicherheit 
7.4.3 Ausblick: Preisentwicklung bei Umsetzung der Desintegrationskonzepte

8 Die Bedeutung der Stromtransportkapazitäten für den europäischen Binnenmarkt für Elektrizität

8.1 Stromtransportpreise 233

8.1.1 Anforderungen an Stromtransportpreise 233

8.1.2 Pauschalisierte Stromtransportpreise 236

8.1.3 Einzelfallorientierte Stromtransportpreise 239

8.1.4 Die Stromtransportpreisarten für die Ordnungskonzepte 240

8.1.4.1 Stromtransportpreise im Weiterentwicklungskonzept 240

8.1.4.2 Im TPA-Konzept 247

8.1.4.3 Im Common-Carrier-Konzept 249

8.1.4.4 Im Stromhändlerkonzept 250

8.2 Die Stromtransportkapazitätsentwicklung 251

8.2.1 Die Stromtransportkapazitätsentwicklung im
Weiterentwicklungskonzept

8.2.2 Stromtransportkapazitätsentwicklung im TPA-Konzept 254

8.2.2.1 Die Auswirkungen der Regulierung auf die Stromtransportkapazitätsentwicklung 255

8.2.2.2 Die Abhängigkeit von der Marktsituation 262

8.2.2.3 Der Einfluß von Bedarfsprognosen 270

8.2.3 Stromtransportkapazitätsentwicklung im Common-CarrierKonzept

8.2.4 Stromtransportkapazitätsentwicklung im Stromhändlerkonzept

9 Die Folgen der Stromtransportkapazitätsentwicklungen für die Ordnungskonzepte 277

$\begin{array}{lll}9.1 & \text { Für das Weiterentwicklungskonzept } & 277\end{array}$

9.2 Für das TPA-Konzept 277

9.3 Für das Common-Carrier-Konzept und das Stromhändlerkonzept

$\begin{array}{lll}10 & \text { Schlußbetrachtungen } & 287\end{array}$

$\begin{array}{ll}\text { Literaturverzeichnis } & 311\end{array}$ 


\section{DARSTELLUNGSVERZEICHNIS}

\section{Abbildungen}

1 Systematik der Ansätze

2 Stilisierter Tageslastverlauf

3 Stilisierter Jahreslastverlauf

4 Besonderheiten der Elektrizitätsbranche im Überblick

5 Koordinationsformen in Abhängigkeit ihrer Einflußvariabeln

6 Öffentliche Elektrizitätsversorgung in der Bundesrepublik Deutschland

7 Die niederländische Elektrizitätsstruktur

8 Weiterentwicklungskonzept - Szenarien im Überblick

9 Durchleitung Typ 1 (Stromtransit)

10 Durchleitung Typ 2

11 Durchleitung Typ 3

12 Durchleitung Typ 4a

13 Durchleitung Typ 4b

14 Durchleitung Typ 5

15 Stufenweise Einführung der Durchleitungsverpflichtung im TPA-Konzept 111

16 Zusammenhang zwischen den Szenarien fur das TPA-Konzept und ihren wichtigsten Einflußgrößen

17 Anbieter eines Abnehmers in Randlage

18 Entwicklung der Wettbewerbsintensität im TPA-Konzept

19 Marktmacht eines Gebiets-EVUs

20 Struktur vor der Umstrukturierung (Integrierte Unternehmen)

21 Situation nach der Umstrukturierung (Desintegrierte Unternehmen)

22 Märkte des Common-Carrier-Konzeptes

23 Endabnehmerversorgung durch Erzeuger

24 Endabnehmerversorgung durch spezielle Versorgungsunternehmen

25 Endabnehmerversorgung durch spezielle Versorgungsunternehmen bei Existenz von Verteilern

26 Gliederungskriterien für die Aufteilung der Netzgesellschaften 167

27 Konzeption des Pools im Common-Carrier-Konzept 170

28 Stromfluß im Common-Carrier-Konzept 171

29 Grenzanbieterpreisbildung im Common-Carrier-Konzept 173

30 Angebotene Stromleistungen der Anbieter in dem jeweiligen Zeitabschnitt 173

31 Einordnung der Entwicklungsszenarien

32 Ausgangspunkt: Vertikal integrierte Unternehmen 188

33 1. Implementierungsschritt: „unbundling“"

34 2. Implementierungsschritt: Abtrennung des Erzeugerbereiches 189

35 3. Implementierungsschritt: Einrichtung des Pools

36 4. Implementierungsschritt: Privatisierung und Schaffung der Verteiler 
37 5. Implementierungsschritt: Organisation der Netzgesellschaften 192

38 Marktstruktur im Stromhändlerkonzept 194

39 Angebotsabgabe an den Stromhändler 197

40 Übersicht Szenarien Stromhändlerkonzept 204

41 Ausgangspunkt: Vertikal integriete Unternehmen im Stromhändlerkonzept

209

42 1. Implementierungsschritt: „unbundling“ 210

43 2. Implementierungsschritt: Abtrennung des Erzeuger- und

Verteilerbereiches

44 3. Implementierungsschritt: Schaffung der Erzeuger- und der Verteilerunternehmen

45 Durchschnittskostenverlauf eines bestehenden und eines zu errichtenden Kraftwerks

46 Kraftwerksverteilung vor Einführung des Stromhändlerkonzeptes

226

47 Kraftwerksverteilung nach Einführung des Stromhändlerkonzeptes

48 Entwicklung der Endabnehmerpreise im Stromhändlerkonzept

49 Durchschnittspreisentwicklung bei zweigeteiltem Stromtransporttarif 237

50 Marktliche einzelfallorientierte Stromtransportpreise 241

51 Lastsituation, Stromtransportkapazitäten eines EVU 244

52 Kostensituation für das EVU in einer Hochlastphase 244

53 Kostensituation eines EVUs in einer Schwachlastphase 245

54 Angebotsabgabe bei knappen Stromtransportkapazitäten 248

55 Stromtransportkapazitätsentwicklung im Weiterentwicklungskonzept ohne Stromhandelsaktivitäten

56 Ausgangssituation eines Gebiets-EVU im TPA-Konzept 257

57 Kostensituation eines potentiellen Exporteurs 258

58 Preis eines Exporteurs für ein importierendes Unternehmen 260

59 Gesamtkosten eines Importeurs ohne Eigenerzeugung 260

60 Vergleich der Gesamtkosten bei vollständiger Eigenerzeugung und bei $\begin{array}{ll}\text { vollständigem Stromimport } & 261\end{array}$

61 Ausgangssituation 1 - Ausgelastete Stromtransportkapazitäten 263

62 Ausgangssituation 2 - Stromtransportkapazitätsknappheit 263

63 Ausgangssituation 3 - Stromtransportüberkapazitäten 264

64 Zusammenhang zwischen Widerstand und Übertragungsmenge einer elektrischen Leitung

65 Stromtransportkapazitätsentwicklung bei einem EVU mit niedrigen Erzeugungskosten und Absatzchancen

66 Überkapazitätsreduktion

67 Netznutzungsprofiländerung

68 Kapazitätsbedarfsentwicklungsprognosen und deren Auswahl für die Ausbauentscheidungen bei den EVU

69 Stromtransportpotential des Gesamtmarktes furr Durchleitungen

70 Stromtransportkapazitätsabweichungen img TPA-Konzepts-3-631-75129-9 
71 Rückgewinnung des Monopols durch ein GebietsEVU in der Situation IX

72 Grund für nicht genutzte Durchleitungskapazitäten 279

73 Stromtransportpotential für Durchleitungen 280

74 Stromtransportpotential nach Abzug der nicht nutzbaren Menge 280

75 Max. Durchleitungskapazität = beantragte Durchleitungskapazität 281

76 Kurzer Durchleitungszeitraum, hohe Durchleitungskapazität

77 Überkapazitäten > Durchleitungskapazität; langer Durchleitungszeitraum, geringe Durchleitungskapazität

78 Mittlere Durchleitungszeiträume, mittlere Durchleitungskapazitäten

282

79 Unterschiedliche Laufzeiten der Verträge und unterschiedliche Durchleitungskapazitäten

80 Mehrere Unternehmen mit gleicher Laufzeit der Verträge und abnehmenden Durchleitungskapazitäten

81 Übersicht: Stromtransportpreisarten

\section{Tabellen}

1 Zusammenhang zwischen Marktstruktur und Kosten in der Elektrizitätsversorgung

2 Informations- und Veränderungskosten nach Vollmer 42

3 Beurteilungsschema für die Ordnungsmodelle 48

4 Stromtransportpreise in der italienischen Elektrizitätswirtschaft 54

5 Abbau des Franchise-Marktes in der englischen Elektrizitätswirtschaft

6 Preisregulierung in der englischen Elektrizitätswirtschaft

7 Stromimport der EU-Staaten 1994 in GWh

8 Stromexport der EU-Staaten 1994 in TWh

9 Exportüberschuß der EU-Länder 1994 in TWh

10 Strompreisvergleich für Haushaltsabnehmer Januar 1995 ECU pro kWh

11 Strompreisvergleich für Industrieabnehmer Januar 1995 ECU pro $100 \mathrm{kWh}$

12 Steuerliche Belastung auf den Haushaltsstromverbrauch in der EU im Januar 1994 in \%

13 Verzeichnis der Verbundgesellschaften in Europa

14 Übersicht: Beurteilung Weiterentwicklungskonzept und TPA-Konzept

15 Übersicht: Beurteilung Common-Carrier und Stromhändlerkonzept

16 Übersicht: Stromtransportpreisarten

17 Marktsituationen für EVU im TPA-Konzept

18 Veränderung der Absatzchancen der EVU in Abhängigkeit von der Marktsituation 
19 Zusammenfassende Beurteilung der Integrationskonzepte 286

20 Akzeptanz der Integrationskonzepte 291

21 Übersicht: Implementierung der Integrationskonzepte 292

22 Akzeptanz des Common-Carrier-Konzeptes und des Stromhändlerkonzeptes 293

23 Implementierungsaufwand und -zeitraum der Desintegrationskonzepte 294

24 Anforderungen an Stromtransportpreise 295

25 Vergleichende Beurteilung der Ordnungskonzepte 299 


\section{ABKÜRZUNGSVERZEICHNIS}

ARE

CC

DSM

DVG

EdF

ENEL

EVU

GW

HGÜ

$\mathrm{Hz}$

IEA

kVA

$\mathrm{kV}$

$\mathrm{kWh}$

MVA

MW

NGC

REC

SEP

TPA

TWh

UCPTE

UNIPEDE

VDEW

VEEN
$=$ Arbeitsgemeinschaft regionaler

Energieversorgungsunternehmen e.V.

$=$ Common-Carrier

$=$ Demand-Side-Management

$=$ Deutsche Verbundgesellschaft

$=$ Eléctricité de France

$=$ Ente nationale per l'energia elettrica

$=$ Elektrizitätsversorgungsunternehmen

$=$ Gigawatt $=10^{6} \mathrm{~kW}$

$=$ Hochspannungsgleichstromübertragung

$=\operatorname{Hertz}(1 \mathrm{~Hz}=1$ Schwingung pro Sekunde $)$

$=$ Internationale Energie Agentur

$=$ Kilovoltampère

$=$ Kilovolt

$=$ Kilowattstunde

$=$ Megavoltampère $=1000 \mathrm{kVA}$

$=$ Megawatt $=1000 \mathrm{~kW}$

= National Grid Company

$=$ Regional Electricity Company

$=$ Samenwerkende elektriciteits-produktie betrijven (Niederländische Netzgesellschaft)

$=$ Third-Party-Access

$=$ Terrawattstunden

$=$ L'Union pour la Coordination de la Production et du Transport de 1'Electricité

(Vereinigung von EVU zur Koordination des mittel- und südeuropäischen Stromverbundnetzes)

$=$ Union Internationale des Producteurs et Distributeurs de'Energie Electrique

(weltweite Vereinigung der EVU)

= Vereinigung Deutscher Elektrizitätswerke e.V.

$=$ Verenigung van Exploitanten van Elektriziteitsbetrijven in Nederland

(Niederländische Vereinigung der EVU) 
Rüdiger Hermann - 978-3-631-75129-9

Downloaded from PubFactory at 01/11/2019 07:57:52AM

via free access 


\section{Einleitung}

„Negotiated Access“ und „Single-Buyer-System“ sind die neuesten Schlagworte in der Diskussion um die Liberalisierung des europäischen Strommarktes, des letzten vollständig durch nationale Monopole und vertikal integrierte Strukturen gekennzeichneten Wirtschaftsbereiches der EU - einer Diskussion, in der die Haltungen und Argumente so konträr sind wie die Interessenlagen der Beteiligten: Auf der einen Seite Mitgliedsländer, deren Regierungen eine Öffnung der Märkte anstreben, auf der anderen Seite Nationen, für welche die autonome Elektrizitätsversorgung einen ähnlichen Stellenwert hat wie eine eigenständige Landesverteidigung. Beeinflußt wird diese Diskussion signifikant von der gezielten Arbeit diverser Verbände. Diese Gemengelage von Interessen hat bisher jeden europäischen Veränderungsprozeß gelähmt. Die Folge: Ein gemeinsamer Markt für Elektrizität besteht bisher in der EU nicht.

Begonnen hatte die Diskussion um die Schaffung eines europäischen Strommarktes 1986, als die EU-Kommission erstmalig einen offiziellen Blick auf die Elektrizitätsstrukturen in Europa warf. Die bestehenden Monopole, Handelsbarrieren und nationalen Eigenständigkeiten widersprachen eindeutig den EU-Zielen. Ohne längere Beratungen oder Anhörungen von Verbänden äußerte die Kommission erstmalig ihre Vorstellungen von einem desintegrierten, offenen europäischen Strommarkt: Ein "Common-Carrier-System" sollte alle Verbraucher und Erzeuger verbinden und Wettbewerb um die Strombelieferung einführen. Erwartungsgemäß reagierten zuerst, schnell und hartnäckig die bestehenden europäischen EVU; in zahlreichen Veröffentlichungen legten sie ihre Haltung dar. Für sie stand fest, daß ein solches System technisch nicht realisierbar sei und das Ende der Versorgungssicherheit in Europa bedeute. Die empirische Widerlegung folgte eher zufällig, jedoch umgehend durch die Umgestaltung des britischen Elektrizitätsversorgungssystems. Trotzdem mußte die EU-Kommission von ihren Vorstellungen abrücken, nicht zuletzt deshalb, weil verschiedene nationale Regierungen die Argumentation ihrer (staatlichen) Versorgungsunternehmen übernahmen. Als Folge wurde am 22.01.1992 ein Richtlinienvorschlag von der EU-Kommission unterbreitet, der den Common-Carrier-Gedanken aufgab und eine erzwingbare Netzbenutzung durch Dritte (Third-Party-Access) bei fortbestehenden Strukturen vorsah. Doch selbst dieser Ansatz konnte in der politischen Diskussion nicht bestehen; der Richtlinienvorschlag wurde vom europäischen Parlament und vom Ministerrat abgelehnt. Weitere Beratungen und Anhörungen veranlaßten die EU-Kommission, ihren eigenen Konzeptvorschlag weiter aufzuweichen. „Negotiated Access“ - verhandelter Netzzugang - lautete die Antwort der EU-Kommission auf die Kritik: Instrument des Ende 1993 vorgelegten Richtlinienvorschlages soll ein Netzzugang von versorgungsgebietsexternen Stromlieferanten auf der Basis von frei ausgehandelten Verträgen sein. Hierdurch soll zusammen mit weiteren Mitteln ein Wettbewerb in der 
europäischen Elektrizitätsversorgung eingeführt werden, wobei dieser Wettbewerb zu einer Angleichung der Preise und einer Integration gemäß den EU-Zielen führen soll. Die Umsetzung des Negotiated Access Ansatzes scheiterte am Widerstand der französischen Regierung, die ihrerseits eine alternative Ordnungskonzeption vorschlug. Die Ordnungskonzeption betont den öffentlichen Versorgungsauftrag und weist diesen einem einzigen Elektrizitätsversorgungsunternehmen (Single-Buyer) zu. Der Single-Buyer ist verpflichtet, Großabnehmern Stromim- und Exporte zu ermöglichen, kann aber jederzeit den Stromtransport ablehnen, wenn seiner Meinung nach der öffentliche Versorgungsauftrag durch die Lieferungen eingeschränkt wird.

Unabhängig von den Kommissionsvorschlägen haben die EVU in Europa ein eigenes Konzept für die Weiterentwicklung des Binnenmarktes für Elektrizität vorgelegt. Dieses hält an den bestehenden Monopolstrukturen fest und will Effizienzgewinne durch den Ausbau von Kooperationen erreichen. Zudem sollte eine "Strombörse" aufgebaut werden, in der die EVU Strom handeln können. Als letztes ist die Übernahme des britischen Ansatzes für die EU angedacht worden. Ein derartiges auf einen zentralen Stromhändler aufbauendes Konzept weist einige Gemeinsamkeiten mit dem ursprünglichen Kommissiongedanken des CommonCarriers auf. Somit bestehen für den europäischen Strommarkt vier grundlegende Ansätze, die nach dem Grad der vertikalen Integration in zwei Kategorien eingeteilt werden können, in die Integrations- und in die Desintegrationsansätze (vgl. Abb. 1). Der verhandelte Netzzugang des letzten Richtlinienvorschlages soll hierbei als ein Sonderfall des Third-Party-Access Ansatzes angesehen werden; die zentralen Aussagen unterscheiden sich für die beiden Ansätze nicht. Auf das SingleBuyer-System als letzten ordnungspolitischen Vorschlag für die Umgestaltung der französischen Elektrizitätswirtschaft soll nur am Rande eingegangen werden, da es sich um einen einzelstaatlichen Ansatz handelt. In den Grundzügen ist der von Frankreich vorgelegten Single-Buyer-Ansatz eine weitere Modifikation des ThirdParty-Access Ansatzes.

Ziel der vorliegenden Arbeit ist es nun, die Ansätze zu Konzepten weiterzuentwickeln und darzulegen, welches Konzept geeignet ist, um die vorgegebenen politischen Ziele - Kostensenkung und ausreichende Versorgungssicherheit - zu erreichen. Gleichzeitig sollen die Auswirkungen auf den Binnenmarkt aufgezeigt werden, die sich ergeben, wenn versucht wird, die Ziele mit nicht kompatiblen Konzepten zu realisieren. Die Ausführungen werden verdeutlichen, daß ein bisher in der Diskussion vollständig vernachlässigter Teilbereich der Stromversorgung, der Stromtransport, so starke Auswirkungen auf die Konzepte hat, daß er bei zweien der vier Konzepte zu deren Scheitern führt - beim Weiterentwicklungs- und beim TPA-Konzept. Bewußt ausgeklammert werden sollen alle rechtlichen Fragestellungen und Probleme, die mit diesem Thema zusammenhängen. Es soll zudem

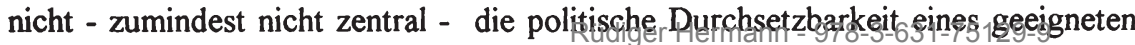


Ordnungskonzeptes diskutiert werden: die Aussagen zur Akzeptanz der Ansätze dienen lediglich als Hinweise auf potentielle Implementierungsbarrieren. Da letztlich auf dem britischen Elektrizitätsmarkt eine nicht wettbewerbskonforme Erzeugerbetriebsgröße sowie falsch gewählte Eigentümerstrukturen zu negativen Auswirkungen geführt haben, soll bei der Entwicklung der Desintegrationskonzepte ein besonderes Augenmerk der Unternehmengröße und den Eigentumsverhältnissen gelten.

Abbildung 1: Systematik der Ansätze

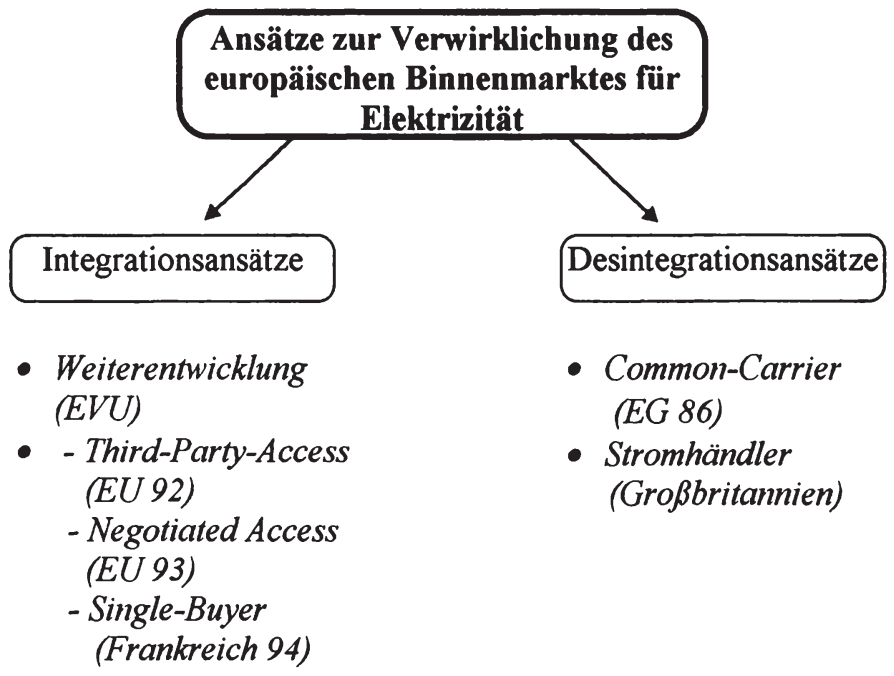

Die Literatur zu diesem Thema beschränkt sich auf eine Vielzahl von Artikeln; eine Gesamtbearbeitung dieser Fragen fehlt bisher. Die Autoren dieser Aufsätze beschäftigen sich primär mit den aktuellen Tendenzen in der politischen Willensbildung auf EU-Ebene; ferner zeigen sie potentielle Konsequenzen für die nationalen Elektrizitätswirtschaften auf. Den gegenwärtigen Stand der Integration vor Vollendung des EU-Strommarktes haben in der Bundesrepublik EISS et al ${ }^{1}$ sehr detailliert dargestellt. Einer der Autoren, PICK, beschäftige sich in seiner Dissertation ${ }^{2}$ erstmalig mit Ordnungsmodellen für den europäischen Strommarkt. KLOPFER ${ }^{3}$ überlegte, wie die bereits in einigen Staaten, insbesondere in Großbritannien,

1 EISS, H. : LUKES, R. ; PICK, H.; SCHULZ, W. : Die Ordnung des Elektrizitătsmarktes in der Europäischen Gemeinschaft, München 1990

2 PICK, H. : Ordnungsprobleme eines Europäischen Binnenmarktes für Elektrizităt, Idstein 1991

3 KLOPFER, T. : Mărkte für Strom - Internationale Erfahrungen und Übertragbarkeit auf Deutschland, München 1993 
durchgeführten Umstrukturierungen auf die Bundesrepublik Deutschland übertragen werden können. Alle Autoren grenzen weitgehend oder vollständig den Stromtransportbereich mit seinen Auswirkungen auf die Marktentwicklung in den Ordnungmodellen aus. In anderen europäischen Staaten ist bisher wenig zu diesem Thema gearbeitet und publiziert worden; die Diskussion nationaler Policy-Inputs beherrscht oftmals die elektrizitätswirtschaftliche Diskussion.

Die vorliegende Arbeit beginnt mit einer kurzen Darstellung der Ziele, die durch die Umgestaltung der europäischen Elektrizitätssektoren erreicht werden sollen und stellt dabei die von der EU-Kommission identifizierten Hemmnisse für die Verwirklichung des europäischen Strommarktes dar. Im Anschluß werden die Besonderheiten der Elektrizitätsbranche herausgearbeitet und in technische, marktliche und entwicklungsbedingte aufgeteilt. Hierbei soll überprüft werden, inwiefern die identifizierten technischen und marktlichen Besonderheiten eine Ausnahmebehandlung in der Wettbewerbspolitik rechtfertigen. Im nächsten Abschnitt sollen aus den Zielen unter Berücksichtigung der Besonderheiten Kriterien abgeleitet werden, an denen die gegenwärtige Struktur und die Ordnungsmodelle gemessen werden. Der Schwerpunkt wird dabei auf die marktlichen Ziele gelegt; die hierzu erforderlichen Kriterien werden aus der „neuen Preistheorie“ und dem Transaktionskostenansatz abgeleitet. Es folgt eine Bestandsaufnahme der gegenwärtigen Strukuren anhand von fünf ausgewählten nationalen Elektrizitätswirtschaften. Eine kurze Bewertung durch die Kriterien zeigt, daß ein immenser Handlungsbedarf besteht, den es von der EU auszufuillen gilt. Deshalb werden als nächstes die Vorstellungen der EU-Institutionen dargelegt und die daraus entstehenden Ansätze konzeptualisiert. Bei der Betrachtung der Marktentwicklungen wird auf die Szenario-Technik zurückgegriffen. Jedes Ordnungskonzept endet mit der Bewertung anhand der aufgestellten Kriterien. Dabei kristallisiert sich heraus, daß die einzelnen Ordnungsmodelle ohne eingehende Betrachtung des Stromtransportbereiches nicht abschließend bewertet werden können. Deshalb widmet sich ein eigener Abschnitt auch dem Stromtransportbereich. Es wird die Frage beantwortet, für welches Ordnungsmodell welcher Stromtransportpreis geeignet ist und wie die Stromtransportkapazitäten in den jeweiligen Ordnungsmodellen ausgebaut werden. Anschließend werden dann die Auswirkungen der Stromtransportkapazitätsentwicklungen auf die Zielerfüllung der Ordnungskonzepte aufgezeigt und die vier Ordnungskonzepte abschließend bewertet. Das Ergebnis mag in seiner Eindeutigkeit möglicherweise viele überraschen: Es bedeutet für die EU-Kommission ein „back to the roots“, nämlich zurückzukehren zu der Forderung nach einer Desintegration der nationalen Elektrizitätssektoren. 


\section{Ziele und Hemmnisse des europäischen Binnenmarktes für Elektrizităt}

Die gesellschaftspolitischen Ziele für die nationalen Elektrizitätswirtschaften stehen seit Beginn der Elektrifizierung in allen Mitgliedsstaaten fast unverändert fest. Die Elektrizitätsversorgung soll sicher und kostengünstig sein. Ferner soll in der Elektrizitätsversorgung schonend mit der Umwelt und den natürlichen Ressourcen umgegangen werden. In den einzelnen Mitgliedsstaaten werden ähnliche Konzepte benutzt, um die genannten Ziele zu erreichen: So bedeutet für alle Staaten die Sicherheit der Stromversorgung eine autarke Stromversorgung ${ }^{4}$, die den Fremdbezug von Elektrizität aus anderen Staaten ausschließt. Als weitere Maßnahme, um die Sicherheit der Stromversorgung zu steigern, setzten die Mitgliedsstaaten überwiegend heimische Primärenergieträger zur Verstromung ein. Deren Förderung ist jedoch in Europa nur zu Kosten möglich, die über dem Weltmarktpreis für diese Energieträger liegen. Bisher wird dies von den Staaten bisher hingenommen. Einem europäischen Strommarkt mit Wettbewerbsprozessen wurde die Umsetzung der Ziele nicht zugetraut; die Konsequenz dieses Sicherheitsstrebens: Es entwickelten sich in allen EU-Staaten abgeschottete Elektrizitätswirtschaften, in denen die Unternehmen mit einem (Teil-)Monopol in der Elektrizitätsversorgung ausgestattet worden sind. Einige Nationen gingen noch einen Schritt weiter; sie verstaatlichten ihre Stromversorgung.

Die so entstandenen nationalen Strukturen kollidierten Ende der achtziger Jahre mit der wirtschaftlichen Zielsetzung der EU, der Schaffung eines Raumes "ohne Binnengrenzen (...), in dem der freie Verkehr von Waren, Ressourcen, Dienstleistungen und Kapital, laut den Bestimmungen des Vertrages, gewährleistet ist "6. $\mathrm{Zu}$ diesem Konflikt vertritt die Kommission der Europäischen Union die Meinung, daß ein marktwirtschaftlich organisierter europäischer Strommarkt nicht den Zielen der sicheren und kostengünstigen nationalen Stromversorgung entgegenstehe, vielmehr durch die Einführung von Wettbewerb im Elektrizitätssektor deutliche Effizienzsteigerungen realisiert werden könnten. Deshalb fordert die Kommission eine an der europäischen Zielsetzung orientierte Umgestaltung der nationalen Elektrizitätsstrukturen. Die Umgestaltung soll die von ihr identifizierten Hemmnisse für einen europäischen Strommarkt beseitigen. Als solche Hemmnisse werden angesehen?:

4 Eine Ausnahme ist Dänemark.

5 Die VEBA AG hält diese Einstellung für „überholt“. Vgl. HARTMANN, U. : Anmerkungen zur Energiepolitik, in: EW Jg. 94 (1995), Heft 3, S. 89

6 Art. 8a des EWG-Vertrages zitiert aus : Bulletin der EG, Beilage 2/86, S. 5

7 Im folgenden entnommen aus : KOMMISSION der EUROPÄISCHEN GEMEINSCHAF-

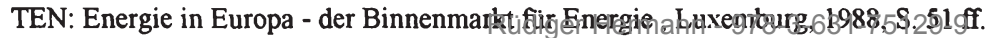


Die nationalen rechtlichen Rahmenbedingungen

Diese weichen deutlich voneinander ab und erlauben ausländischen Unternehmen zumeist nicht die Aufnahme der Elektrizitätsversorgung.

Die Stromerzeugung durch öffentliche Unternehmen

Nach Meinung der EU-Kommission beinhaltet die Idee eines offenen Binnenmarktes für Elektrizität die Stromerzeugung auf einer wirtschaftlich wettbewerbsfähigen Basis. Die bestehenden öffentlichen Unternehmen stehen dieser Zielsetzung entgegen.

Die unterschiedliche steuerliche Behandlung und der Zugang zu den Finanzmärkten

Diese führen zu Verzerrungen bei den Gesamtkosten und bestimmen die Ertragssituation einen Unternehmens wesentlich mit.

Die Verfahren für die Genehmigung von Kraftwerksneubauten

Durch die unterschiedlichen nationalen Genehmigungsverfahren können EVU ihre Investitionsprogramme nicht gleichartig durchfuihren. Damit verzerren die Genehmigungsverfahren die Bedingungen und Ausgangssituationen für den europäischen Strommarkt.

\section{Der Zugang der Stromerzeuger zur Primärenergie}

Die Kosten in der Stromerzeugung werden durch die Kosten der Primärenergieträger, die in den Kraftwerken zum Einsatz kommen, mitbestimmt. Nach Meinung der EU-Kommission bedeutet ein echter Binnenmarkt, daß Stromerzeugungsunternehmen gleiche Bedingungen für den Zugang zu den Einsatzenergien haben müssen. Damit stellen die bestehenden nationalen $\mathrm{Be}-$ schränkungen oder Auflagen Hemmnisse für den europäischen Strommarkt dar.

\section{Die Sicherheitsauflagen und Umweltschutznormen}

Diese wirken sich in unterschiedlichen Kosten aus. Sie haben Auswirkungen auf die Kosten beim Betrieb, bei der Wartung und bei der Auswahl der Produktionstechnologie von Kraftwerken. Sie sind eine Folge der nationalen und nicht der einer gemeinschaftlichen Energie- und Umweltpolitik und stehen dem Binnenmarkt entgegen.

Mit den von Sir Leon Brittan, dem seinerzeitig zuständigen Kommissar für Wettbewerbsfragen am 18.04.1991 in einer Rede vor der "Institution of Civil Engineers" in London zusammengefaßten Zielen will die Kommission die Sicherstellung der marktwirtschaftlichen Leitlinie des Binnenmarktes erreicht sehen. Die Ziele sind: 
- die Freiheit der Lieferantenwahl für Abnehmer,

- die Preisfestsetzung im freien Wettbewerb,

- die Durchleitungsmöglichkeit für Erzeuger,

- die Transparenz und die Einschränkung der staatlichen Beihilfen sowie

- die Beseitigung der Austauschhemmnisse zwischen den Mitgliedsstaaten $^{8}$.

Ein kleinster gemeinsamer Nenner zwischen der Kommissionsposition und den Vorstellungen der einzelnen Mitgliedsstaaten besteht nicht. Daher ist zunächst zu überprüfen, ob die Besonderheiten und Erfordernisse des Elektrizitätssektors zwingend Märkte mit Wettbewerb bei der Güter- und Dienstleistungbereitstellung ausschließen. Ist dies nicht der Fall, bleibt die Frage offen, welche Marktform Monopol oder Märkte mit Wettbewerb - die Stromversorgung bei ausreichendem Versorgungssicherheitsniveau zu den geringsten Kosten bereitstellen kann. Dabei geht es gleichzeitig darum festzustellen, ob Wettbewerb, wie es die EU-Kommission annimmt, zur Schaffung eines gewünschten Marktergebnisses geeignet ist.

8 Zitiert nach KOMMISSION der EUROPÄISCHEN GEMEINSCHAFTEN: EG-Nachrichten - Berichte und Informationen Nr.17 vom 29.04.1991, S.6 Vgl. dazu auch weiter die ăhnlichen Aussagen des für Energiefragen zuständigen EG-Kommissars Antonio Cardoso e Cunha auf dem XXII UNIPEDE Kongreß in Kopenhagen, zusammenfassend wiedergegeben in: BRAATZ, U.: Elektrizität ohne Grenzen - XXII UNIPEDE Kongreß in Kopenhagen, in: EW Jg. 90 (1991), Heft 16/17, S.907 und die Begründung in den Ausführungen zu Beginn der EG-Richtlinie 90/547/EWG.üdiger Hermann - 978-3-631-75129-9 
Rüdiger Hermann - 978-3-631-75129-9

Downloaded from PubFactory at 01/11/2019 07:57:52AM

via free access 


\section{Die Besonderheiten des Elektrizitätssektors}

Die „Besonderheitentheorie“ der Elektrizitätswirtschaft hat in den europäischen Staaten eine lange Tradition, deren Pflege sich eine Vielzahl von Autoren annimmt $^{9}$. Sie wurde historisch als Rechtfertigung für eine wettbewerbliche Ausnahmebehandlung in den EU-Mitgliedsstaaten herangezogen ${ }^{10}$. In der Literatur werden als Besonderheiten all jene Tatbestände bezeichnet, die eine Ausnahmeregelung für die Elektrizitätswirtschaft rechtfertigen sollen. Diese Vorgehensweise trägt nicht gerade zur Versachlichung der ordnungspolitischen Diskussion bei. Deshalb wird hier ein anderer Weg gewählt:

Definition:

Als Besonderheit der Elektrizitätswirtschaft sollen all jene Tatbestände bezeichnet werden, bei denen die Elektrizität von den traditionellen Gütern" der Preis- und Markttheorie abweicht.

Hierzu ist es zunächst erforderlich, die angesprochenen Gütereigenschaften herauszuarbeiten. Folgende Eigenschaften werden Gütern zugeordnet ${ }^{12}$.

- die Materialgebundenheit,

- die Teilbarkeit,

- der Tauschwert,

- die Wiederveräußerbarkeit.

9 Die spezifischen Merkmale des Energiemarktes werden z.B. in KOMMISSION der EUROPÄISCHEN GEMEINSCHAFTEN - Generaldirektion Energie: Energie in Europa - Der Binnenmarkt für Energie, Luxemburg 1988, S. 7 aufgezählt. Vgl. weiter van HOEK, G.A.L.: Europa im Stromverbund - Vortrag anläßlich des VDEW-Symposiums für Wirtschaftsjournalisten "Perspektiven der Stromversorgung" vom 25 bis 27.11.1991 und KOBARG, B. : Elektrizitätswirtschaft und Wettbewerb, Kiel 1973, S. 29-65

10 Vgl. z.B. RECKNAGEL, H. : Mehr Wettbewerb in der Energiewvirtschaft?, in: ET 40. Jg. (1990), Heft 4, S. 220 sowie die zahlreichen Veröffentlichungen zu diesem Thema der VDEW und der Eurelectric.

11 Trotz der Dienstleistungseigenschaften hat der Europäische Gerichtshof unterstrichen, daß es sich bei der Elektrizităt um eine Ware im Sinne des europäischen Rechtes handelt. Vgl. HANCHER, L. : Die Almelo-Entscheidung des Europäischen Gerichtshofs - Hintergrund, Bewertung, mögliche Auswirkungen, in: EW 45.Jg. (1995), S. 68-76, hier 72

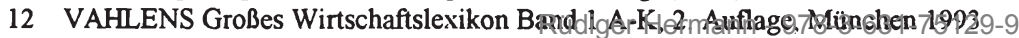


Dienstleistungseigenschaften $\sin ^{13}$ :

- die Immaterialität

- fehlende Lagerfähigkeit

- Standortgebundenheit

- fehlende Weiterveräußerbarkeit,

- erforderliche Träger sind der Mensch oder ein Objekt,

- Aufrechterhaltung eines konstanten Potentials zur Wertschöpfung, d.h. fehlender Verzehr der eingesetzten Produktionsfaktoren.

Der Verfasser unterteilt die Besonderheiten der Branche in ${ }^{14}$ :

- technische,

- entwicklungsbedingte und

- marktliche.

\subsection{Die technischen Besonderheiten}

Bei den technischen Besonderheiten wird häufig an erster Stelle die fehlende Möglichkeit genannt, elektrische Energie in großen Mengen zu speichern (fehlende Speicherfähigkeit) ${ }^{15}$. Ein vom Bundesforschungsminister finanzierter Feldversuch zeigte $^{16}$, daß Batteriespeicher in Niederspannungsnetzen sehr wohl einsetzbar sind. Bisher war die Speicherung von Gleichstrom in Batterien technisch nur bei nied-

13 VAHLENS Großes Wirtschaftslexikon Band 1 A-K, 2. Auflage, München 1993

14 In der Literatur werden zumeist ausschließlich die technischen Besonderheiten dargestellt. Entwicklungsbedingte Besonderheiten wurden bisher in der Literatur für die Elektrizitätswirtschaft nicht herausgearbeitet. Hierzu vergleiche die Einteilungen der Besonderheiten in : SCHNEIDER, R. : Elektrizităt unter wettbewerbspolitischem Aspekt, Bamberg 1966 S. 23

15 Vgl. VDEW: Der europäische Strommarkt - Dokumentation der deutschen Elektrizitătswirtschaft, Frankfurt/Main 1988, S. 17 und weiter KOBARG, G.: a.a.O., S. 57; BÖRNER, B. (Hrsg.) : Reform des Energierechts und Natur der Sache, Düsseldorf 1971 und VICKERS, J.; YARROW, G. : The British electricity experiment, in: Econimic policy - A European Forum, 12.April 1991, S. 189 und GREEN, R. ; NEWBERY, D.M. : Competition in the British Electricity Spot Market, Diskussionpapier des Centre for Economic Policy Research, Nr. 557, London 1991, S. 3

16 Diese Auffassung wird von einigen Autoren nicht uneingeschränkt geteilt. Vgl. z.B. die Zusammenfassung des Vortrags von H,-J. Haubrich in: KETTERER, H. : Stromspeicher für die

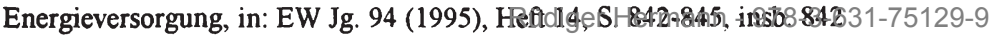


rigem Wirkungsgrad möglich. Die Kosteneinsparungen durch eine Großserienfertigung wurden bei der Wirtschaftlichkeitsanalyse berücksichtigt, die Kosten für die Entsorgung der Batterien dagegen nicht ${ }^{17}$. Die bei dem Feldversuch aufgetretenen technischen Schwächen erschienen den beteiligten Ingenieuren überwindbar $^{18}$. Trotz der technischen Realisierbarkeit wurde in der Studie festgestellt, daß der Einsatz derzeit aber nicht wirtschaftlich ist ${ }^{19}$. Schon 1950 wies Vogt in diesem Zusammenhang darauf hin, daß das Endprodukt, also die elektrische Energie an der Steckdose, nicht speicherbar ist, dagegen die Möglichkeit zur Speicherıng der Vor- und Zwischenprodukte besteht ${ }^{20}$. Unter Vorproduktspeicherung in der Elektrizitätsversorgung versteht Vogt die "Bevorratung mit Energieträgern"21. Denkbar und möglich ist sie bei fossilen, nuklearen und ausgewählten regenerativen Energieträgern. Für die Speicherung des Zwischenproduktes zieht Vogt das Beispiel der Wärmespeicher heran, wo ein Vorrat an Dampf auf Lager zur Deckung von Nachfragespitzen gehalten wurde. Heute wird diese Technik nicht mehr eingesetzt. Statt dessen werden ganze Kraftwerke im Leerlauf betrieben, um verzugslos bei Ausfall von Kraftwerken oder entstehenden Nachfragespitzen die benötigte elektrische Energie produzieren zu können ${ }^{22}$. Somit kann festgehalten werden, daß der derzeitig wirtschaftlich zu produzierende Wechsel- oder Drehstrom $^{23}$ als Endprodukt technisch nicht speicherbar ist und diese Besonderheit bei den späteren Überlegungen zur Umgestaltung des Sektors berücksichtigt werden muß.

Nach Meinung der „Vereinigung Deutscher Elektrizitätswerke (VDEW) ${ }^{24 ،}$ sind fehlende Lieferfristen für Elektrizität eine weitere Besonderheit des Elektrizitätssektors. Produktion und Verbrauch elektrischer Energie müssen gleichzeitig erfolgen. Näher betrachtet zeigt sich der folgende Zusammenhang: Großverbraucher

17 Deren Recycling ist heute bereits bis zu 95\% möglich, wozu jedoch Kosten anfallen.

18 Vgl. BACKWINKEL,R.; FREUND, H.; GRETSCH, R. ; KAUFMANN,W.; SCHMIDT,G. ; SEELE, W. ; WEISSMÜLLER,G. : Entwicklung, Errichtung und Erprobung eines Niederspannungsnetzstützpunktes mit NaS-Batterien zur Spannungsstützung, Abschlußbericht zum BMFT-Forschungsvorhaben, Essen, Februar 1991 sowie FREUND, H.: Batteriespeicher in Netzen der Energieversorgung , Brigton 1989

$19 \mathrm{Zu}$ den Nutzungsmöglichkeiten von Batteriespeicher in der Elektrizitätsversorgung vgl. BACKWINKEL, R. ; FREUND, H. ; SEELE, W. : Batteriespeicher zur Spannungsstützung in Niederspannungsnetzen, in: EW Jg.91 (1992), Heft 15, S. 975 ff.

20 Vgl. VOGT, H. : Probleme der Versorgungswirtschaft, München 1950, S. 83 ff.

21 VOGT, H. : a.a.O., S. 84

22 Mit Einschränkungen könnte man heute den Einsatz von Pumpspeicherkraftwerken als Zwischenproduktspeicherung ansehen: In Schwachlastzeiten wird Wasser in höhergelegene Becken gepumpt, um damit bei Bedarf durch Umwandlung der kinetischen Energie des Wassers Strom zu produzieren.

23 Zur Unterscheidung zwischen Wechsel- und Drehstrom siehe : MEISTER, $H$. : Elektrotechnische Grundlagen, 8. Auflage Würzburg 1986, S. $234 \mathrm{ff}$.

24 Vgl. VDEW : Der europäische Strommarkitidigara.@rnSald - 978-3-631-75129-9 
mit beeinflußbaren und/oder vorhersehbaren Strombedarfen können Stromleistungen für Zeitperioden vorbestellen. Hierdurch können sich die EVU auf die angekündigte Entnahme vorbereiten. Anders ist die Situation bei Kleinabnehmern: Die Stromentnahme aus dem Netz des einzelnen Abnehmers hängt von einer Vielzahl für das EVU nicht vorhersehbarer Faktoren ab. Die große Anzahl der Abnehmer läßt jedoch nach dem Gesetz der großen Zahl ein planbares Abnahmeprofil mit ausgesprochenen Nachfragespitzen entstehen ${ }^{25}$ (vgl. Abb. 2).

An den Nachfragespitzen müssen die EVU ihre Planung der Erzeugungs- und Transportkapazitäten ausrichten, wenn die Versorgungssicherheit für jede Nachfrage gewährleistet werden soll. Die hierdurch entstehenden Kosten können die europäischen EVU bisher über ihre Preisgestaltung an den Verbraucher weitergeben, so daß wirtschaftlich aus der Besonderheit keine Nachteil resultieren.

Als weitere technische Besonderheit wird die Leitungsgebundenheit der Elektrizitätsversorgung ${ }^{26}$ angesehen. Elektrische Energie kann nur über ein festes, in sich geschlossenes Netz zielgerichtet übertragen werden; soweit nicht futuristische Szenarien bemüht werden, ist dieses Argument stichhaltig. Gas, als ein weiterer leitungsgebundener Energieträger kann in großen Mengen auch nur über ein Leitungsnetz transportiert werden, ist jedoch einfacher zwischenzulagern und ein Absinken des Gasdrucks in den Leitungen hat geringere Auswirkungen für die Abnehmer als ein Spannungsabfall in einem Elektrizitätsnetz.

Als letzte technische Besonderheit der Elektrizitätsversorgung ist die Notwendigkeit geeigneter Energiewandler beim Verbraucher/Endabnehmer zu nennen. Die Nutzung der elektrischen Energie und ihr Wirkungsgrad sind durch die Energiewandler festgelegt ${ }^{27}$. Eine kurzfristige Substitution von Strom durch andere Energieträger wird hierdurch unmöglich ${ }^{28}$. Mittel- und langfristig ist Substitution über den Einsatz finanzieller Mittel (sog. "switching costs") theoretisch jedoch realisierbar. Da aber kein anderer Energieträger eine derartige Anwendungsvarietät ${ }^{29}$ aufweist wie die Elektrizität, spielen solche Substitutionsprozesse trotz

25 Vgl. hierzu z.B. den zweiten Bericht des VDEW-Arbeitskreises „Tarifgestaltung“: Tariflicher Bereitstellungspreis - Möglichkeiten für eine Umstellung auf eine elektrische Bemessungsgröße bei Gewerbe und Landwirtschaft, in: EW Jg. 83 (1994), Heft 25, S. 10511073 hier die Abbildungen auf Seite 1052 und 1053

26 Vgl. MIKSCH, L. : Wettbewerb als Aufgabe, Bad Godesberg 1947, S. 137

27 Vgl. MEIER,K. : Spitzenlasttarifierung - Ökonomische Effizienz und Erhaltung der Eigenwirtschaft - Eine angewandte Studie der elektrizitätswirtschaftlichen Preisbildung. Bern, Stuttgart 1983, S. $52 \mathrm{ff}$

28 Vgl. hierzu VOGELSANG, I. : Die staatliche Regulierung der amerikanischen Elektrizitätswirtschaf - Eine kritische Bestandsaufnahme, in: ZfE 1/79, S. 25

29 Vgl. GRAWE, J. : Die Rolle des Stroms zur Optimierung der Energiebedarfsdeckung, in: EW 91. Jg. (1992), Heft 18, S. 1139 
steigender Strompreise außer auf dem Wärmeenergiemarkt in den europäischen Staaten bisher kaum eine Rolle.

\section{Abbildung 2: Stilisierter Tageslastverlauf ${ }^{30}$}

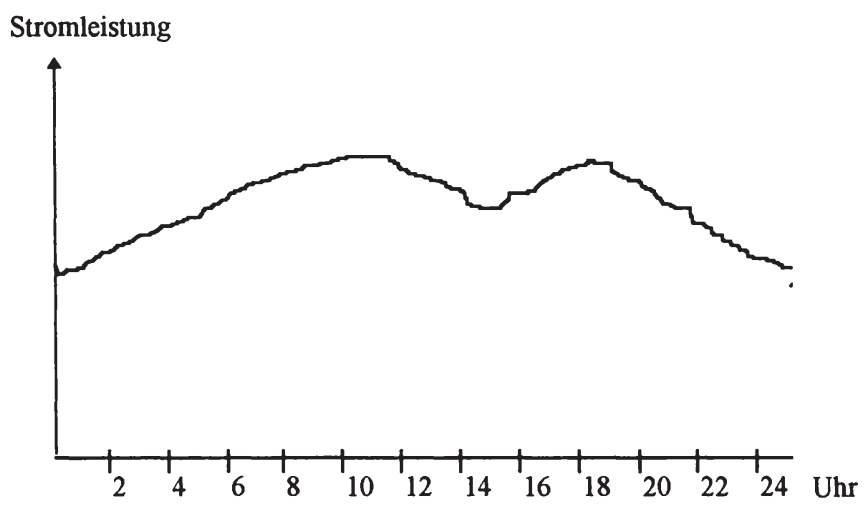

\section{Abbildung 3: Stilisierter Jahreslastverlauf}

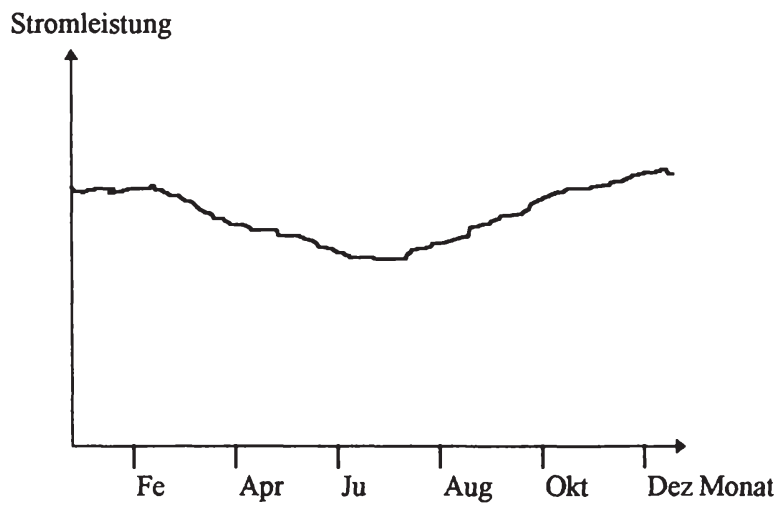

30 Tages- und Jahreslastverlauf sind stilisiert, da sie für die europäischen Länder stark abweichen. Einflußfaktoren sind das Klima, die Lebensgewohnheiten und der Grad der Elektrizitătsanwendung. 


\subsection{Entwicklungsbedingte Besonderheiten}

Als entwicklungsbedingte Besonderheiten der Elektrizitätswirtschaft sind die hohe Kapitalintensität $^{31}$, verbunden mit langen Bauzeiten ${ }^{32}$, die Existenz geschlossener Versorgungsgebiete und die Bedeutung der Versorgungssicherheit anzusehen.

Die Gründe für die hohe Kapitalintensität der europäischen Elektrizitätswirtschaften liegen in den nationalen historischen Entwicklungen. Die ersten leistungsfähigen Stromerzeugungsgeneratoren wurden Ende der siebziger Jahre des letzten Jahrhunderts gebaut ${ }^{33}$. Produktions- und Verbrauchsort waren nicht zuletzt wegen mangelnder Transportmöglichkeiten identisch. Gleichstrom niedriger Spannung wurde zur Jahrhundertwende in zunehmend größeren Blockstationen zur Versorgung räumlich eng zusammenliegender städtischer Verbraucher produziert. Die technischen Entwicklungen in der Zeit nach den Blockstationen ermöglichten den raschen Ausbau der Erzeugungskapazitäten und deren schrittweise Verbindung in Kleinnetze. Die Ausnutzung von economies of scale führte im beginnenden zwanzigsten Jahrhundert zu zunehmend größeren Erzeugungsanlagen und längeren Bauzeiten ${ }^{34}$. Gefördert wurde dieser Trend durch zwei Faktoren: Parallel zu der technischen Entwicklung der Erzeugungsanlagen wurden leistungsfähige Transformationsstationen gebaut und somit eine Stromübertragung über weitere Entfernungen ermöglicht. Gleichzeitig entdeckten in Europa viele Gebietskörperschaften die Verdienstmöglichkeiten aus der Elektrizitätsversorgung, was zu deren Zentralisierung und nachfolgender staatlicher Regulierung ${ }^{35}$ beitrug. Der Höhepunkt dieses Trends wurde nach dem zweiten Weltkrieg mit der Verstaatlichung der Elektrizitätsversorgung in Frankreich und Italien erreicht. Mit der Verstaatlichung oder der Regulierung veränderten sich die Gewinnerzielungsmöglichkeiten für die Unternehmen. Bestehende Gewinne wurden beschnitten oder auf

31 Vgl. zur Kapitalintensităt KOMMISSION der EUROPÄISCHEN GEMEINSCHAFTEN Generaldirektion Energie: Vollendung des Binnenmarktes für Gas und Elektrizităt, Brüssel 15.07.1991 und WESSEL, T. : Wirtschaftliche und rechtliche Grundlagen der Energiewirtschaft, Tagungsberichte des Energiewirtschaftlichen Instituts der Universitäı Köln, Heft 1, Köln 1951,S. 8

32 Die Bauzeiten für Kraftwerke hăngen von technischen, politischen und natürlichen Faktoren ab, die innerhalb Europas variieren. Ein Beispiel für politische Faktoren sind die unterschiedlichen Zeiten für die Genehmigungsverfahren der Kernkraftwerke in der Bundesrepublik und in Frankreich.

33 Vgl. BRUCHE, G. : Elektrizitătsversorgung und Staatsfunktion, Frankfurt/Main 1977, S.19

34 Damit verbunden sind hohe Fixkosten. Vgl. hierzu KOEPPEL, P.F.: Typische Vertrăge der Energiewirtschaft in kartellrechtlicher Betrachtung, Köln 1959, S. 13

35 Unter Regulierung soll nach SOLTWEDEL et al die direkte hoheitliche Einschrănkung der Gewerbe- und Vertragsfreiheit in bestimmten Wirtschaftsbereichen verstanden werden. Vgl. SOLTWEDEL, H. et al : Deregulierungspotentiale in der Bundesrepublik. Tübingen 1986, 
einen Prozentsatz des eingesetzten Kapitals begrenzt ${ }^{36}$. Bei staatlichen EVU wurden sie zumeist vollständig verboten. In dieser Situation nutzten die Besitzer oder Manager der EVU die technische Realisierbarkeit von Großerzeugungsanlagen. Durch den Bau von Großkraftwerken konnten sie die Kapitalintensität steigern, was bei den EVU-Besitzern, denen ein Prozentsatz auf den Kapitaleinsatz als „billiger“ Gewinn zugesagt worden war, zur Steigerung des absoluten Gewinns führte und den Managern der Staatsunternehmen ein höheres Sozialprestige bescherte $^{37}$.

Ein Sachverhalt, der direkt aus der entwicklungsbedingten hohen Kapitalintensität resultiert, ist die gute Innenfinanzierungsmöglichkeit der EVU ${ }^{38}$. Diese entsteht durch Abschreibungen und Rückstellungen. Durch die Abschreibungen fließen dem jeweiligen Unternehmen aus dem betrieblichen Umsatzprozeß finanzielle Mittel von außen zu, die einen Rückfluß für bereits investierte Mittel darstellen (Kapitalfreisetzungseffekt) ${ }^{39}$. Die im Elektrizitätssektor üblichen langen Einsatzzeiten von Kraftwerken erfordern keine direkte Reinvestition. Die vorzeitigen Rückflüsse kann das Unternehmen zu ebenfalls kapitalintensiven Investitionen nutzen, deren Rückflüsse aus Abschreibungen wieder zur Ersatzinvestion für die ersten Anlagen verwendet werden (Kapitalausweitungseffekt) ${ }^{40}$.

Die Finanzierung über Rückstellungen stellt die zweite innerbetriebliche Finanzierungsart dar. Das Unternehmen bildet Rückstellungen für Verbindlichkeiten in zuküntigen Perioden, die „, zum Stichtag dem Grunde nach, aber nicht nach Höhe und Fälligkeit feststehen. ${ }^{\text {“41 }}$ Rückstellungen entstehen im Elektrizitätssektor insbesondere für den Abriß und die Entsorgung von Kraftwerken. Durch den langen Zeitraum, in dem die Rückstellungen vor ihrer Auflösung im Unternehmen verbleiben, erhalten sie ihre Bedeutung als Finanzierungsmöglichkeit. Der Vorteil dieser beiden Innenfinanzierungsarten sind ihre niedrigen Kosten, insbesondere im Ver-

36 So z.B. in Spanien vgl. hierzu EISS e al : a.a.O., S. 612

37 Averch/Johnson-Effekt vgl. hierzu AVERCH, H. : JOHNSON, L. : Behavior of the Firm under Regulatory Contraint, in: American Economic Review, Vol. 52, 1962, S. 1053-1069 und weiter BAILEY, E.E.; COLEMANN, R.D.: The Effect of Lagged Regulation in an Averch-Johnson Model, in: Bell Journal of Economics, Vol. 2 (1971), S. 278-292

38 Vgl. hierzu : VORMBAUM, H. : Finanzierung der Betriebe, 7.Auflage, Wiesbaden 1986, S. $438 \mathrm{ff}$. und S.32 ff.

39 Vgl. hierzu: PERRIDON, L. ; STEINER, M. : Finanzwirtschaft der Unternehmung, 3. Auflage, München 1984, S. 279 f. und BERGER, T.: (Hrsg.) Finanzlexikon. München 1971 , S. 13

40 Vgl. hierzu WÖHE, G. : Grundzüge der Unternehmensfinanzierung, 5. Auflage, München 1988, S. 14 ff. u. S. 279 . PERRIDON, L. STEINER, M. : a.a.O., S. 279. Dieser Effekt wird auch als Lohmann-Ruchti-Effekt bezeichnet. Vgl. hierzu: RUCHTI, H. : Die Bedeutung der Abschreibung für den Betrieb, Berlin 1942

41 PERRIDON, L. ; STEINER, M. : a.a.O., R.ü28der Hermann - 978-3-631-75129-9 
gleich zu einer Fremdfinanzierung ${ }^{42}$. Mit Hilfe dieser Finanzierungsmittel gelang es den bestehenden europäischen EVU in Europa, kontinuierlich zu expandieren. Einer der erfolgreichsten Unternehmen hierbei ist das Rheinisch-WestfälischeElektrizitätsunternehmen (RWE).

Geschlossene Versorgungsgebiete bildeten sich bereits nach dem ersten Weltkrieg als Ergebnis der elektrizitätswirtschaftlichen Entwicklungsprozesse. Zur schnelleren Elektrifizierung der jeweiligen Landesteile und zur Vermeidung von Doppelinvestitionen führten viele europäische Länder eine Konzession für die Elektrizitätsversorgung ein. Ein Versorgungsunternehmen erhielt ein Versorgungsmonopol und wurde gleichzeitig verpflichtet, alle Verbraucher anzuschließen ${ }^{43}$. Erst in den letzten Jahren wurden verstärkt Zweifel an der Notwendigkeit geschlossener Versorgungsgebiete geäußert. Bei der Beurteilung sollte aber die Existenz geschlossener Versorgungsgebiete nicht mit integrierten Unternehmen gleichgesetzt werden: Während traditionell für Verteilerunternehmen geschlossene Versorgungsgebiete eingerichtet wurden, variiert der Integrationsgrad des Gesamtmarktes in den einzelnen Ländern beträchtlich ${ }^{44}$. In Großbritannien wird seit 1990 schrittweise versucht, die geschlossenen Versorgungsgebiete in der Stromerzeugung und Verteilung, auch für Endverbraucher, aufzulösen.

Die letzte entwicklungsbedingte Besonderheit der Elektrizitätswirtschaft ist die Bedeutung der Versorgungssicherheit. Die Versorgung mit Elektrizität wurde von den jeweiligen europäischen Regierungen für so unabdingbar erachtet, daß sie eine Sonderbehandlung der Elektrizitätswirtschaft durchsetzten oder unterstützten. Die durch geschlossene Versorgungsgebiete vor Konkurrenz geschützten EVU bekamen den angesprochenen Kontrahierungszwang auferlegt, der zusammen mit anderen staatlichen Vorgaben die Versorgungssicherheit gewährleisten sollte.

42 Vgl. zu den Kosten der unterschiedlichen Finanzierungsarten: HILSCHER, U. ; LAUBSCHER, H.-D.: Finanzierungskosten, Frankfurt/Main 1976 zitiert nach PERRIDON, L. ; STEINER, M. : a.a.O., S. 285

43 In der Bundesrepublik sind die EVU jedoch nur zum Anschluß verpflichtet, wenn der Anschluß wirtschaftlich zumutbar ist. Vgl. weiter LEDERER, P. ; BOUTTES, J.P. : Elektrizitătsmonopol kontra Wettbewerb, in: EW Jg. 90 (1991), Heft 6, S. 224-230

44 So besteht in Polen eine Trennung zwischen Erzeugung, Transport und Verteilung. Vgl. hierzu: o.V.: EG und Internationales, in: ETR42.jgr (1982) 


\subsection{Die marktlichen Besonderheiten der Elektrizitätsversorgung}

In der Fachliteratur wird seit Ende der achtziger Jahre die Diskussion geführt, ob es sich bei der Elektrizitätsversorgung um eine Dienstleistung oder um eine Gütererstellung handelt ${ }^{45}$. Ausgelöst wurde diese Diskussion durch EU-rechtlichen Bestimmungen, die sich in bezug auf Dienstleistungen und Güter unterscheiden. Für beide Argumentationslinien sprechen eine Reihe von Gründen: So bestimmt der Abnehmer über Zeit, Ort und Leistung der Inanspruchnahme bis zur maximalen Kapazität des Anbieters. Der Anbieter erstellt die Infrastruktur, welche die Versorgung erst ermöglicht; dies entspricht dem Dienstleistungscharakter. Für den Gutcharakter sprechen die physikalischen Verwendungsmöglichkeiten der Elektrizität und die eindeutige Meßbarkeit und Teilbarkeit der erbrachten Leistungseinheiten. Die Auswertung der Diskussion zeigt, daß es sich bei der Elektrizitätsversorgung um einen Sachverhalt handelt, der im Bereich der Elektrizitätsverteilung und des Elektrizitätstransportes Dienstleistungscharakter besitzt, im Bereich der Stromerzeugung aber vom Gutscharakter dominiert wird. Für die Endabnehmer ist die Elektrizität ein vollständig homogenes Gut ${ }^{46}$.

Eine weitere marktliche Besonderheit ist die kurzfristig preisunelastische Nachfrage $^{47}$; mittelfristig lassen sich dagegen Reaktionen auf Preisänderungen feststellen. Die Abnehmer werden dann bei Preissteigerungen versuchen, durch den Einsatz von effektiveren Energiewandlern ihr durch die Preissteigerung reduziertes Budget zu entlasten (vgl. Abb. 4).

Industrielle Großabnehmer haben darüber hinaus drei weitere Reaktionsmöglichkeiten:

1. Übergang zur Eigenversorgung, sofern technisch möglich,

2. Einsatz anderer Energieträger unter Inkaufnahme der bereits erwähnten switching costs und

3. die Verlagerung der Produktionsstandorte in Gebiete mit niedrigerem Strompreisniveau $^{48}$.

45 Vgl. LANGBEIN, G. : Stromimport und EG-Niederlassungsrecht - Aufriß der Problemstellungen, in: ET 37. Jg. (1987), Heft 11, S. 941 ff. . Grawe , J. stellt die Dienstleistungsaufgaben der EVU heraus. Vgl. GRAWE, J. : Energierechtliche Rahmenbedingungen für Energiedienstleistungsmärkte, in: EW Jg. 94 (1995), Heft 1 u. 2, S. 9

46 Vgl. LEFTWICH, R.H. : The Price System and Ressource Allocation. 6. Auflage, Heimsdale (Illinos) 1976, S. 28-29

$47 \mathrm{Zu}$ diesem Ergebnis kommt eine schwedische Studie mit 20.000 Kunden, die durch schweitzer Studien gestützt wird. Vgl. hierzu: MEIER, H. : UNIPEDE - Tarifkonferenz in Lausanne, in: EW Jg. 91 (1992) Heft 26, S. 1688

48 Zur Einfluß der Strompreise auf die Industrie vgl. NIJKAMP, P. : PERRELS, A. : Impacts

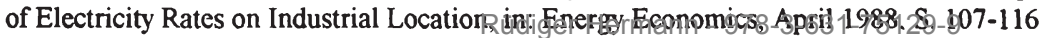




\subsection{Die Relevanz dieser Besonderheiten für die Elektrizitätswirtschaft}

Bei der Relevanz der technischen Besonderheiten ist die Leitungsgebundenheit der elektrischen Energieversorgung ein wichtiger Aspekt: Die Leitungsgebundenheit stellt zusammen mit der mangelnden Speicherfähigkeit eine Besonderheit dar, die in keiner anderen Branche zu finden ist ${ }^{49}$; sie hat primär jedoch Auswirkungen auf das Elektrizitätsnetz und nicht auf die Produktion. Des weiteren führen die technischen Zusammenhänge in einem Stromnetz dazu, daß bei einer Überlastung die gesamte Geschäftstätigkeit zusammenbrechen würde, wenn keine Überkapazitäten zur Verfügung stehen würden ${ }^{50}$. Dies würde bedeuten, daß jedwede Nachfrage nicht befriedigt wird, während in anderen Wirtschaftszweigen bei temporärer Produktionskapazitätsüberschreitung lediglich die Bedürfnisse der Nachfrager, die das Gut oder die Dienstleistung nicht mehr konsumieren können, unbefriedigt bleiben; der Verlust an Bedürfnisbefriedigung wäre damit in der Elektrizitätswirtschaft bei unzureichender Versorgungssicherheit bedeutend größer. Hieraus resultieren erhöhte Anforderungen an die Versorgungssicherheit.

\section{Abbildung 4: Besonderheiten der Elektrizitätsbranche im Überblick}

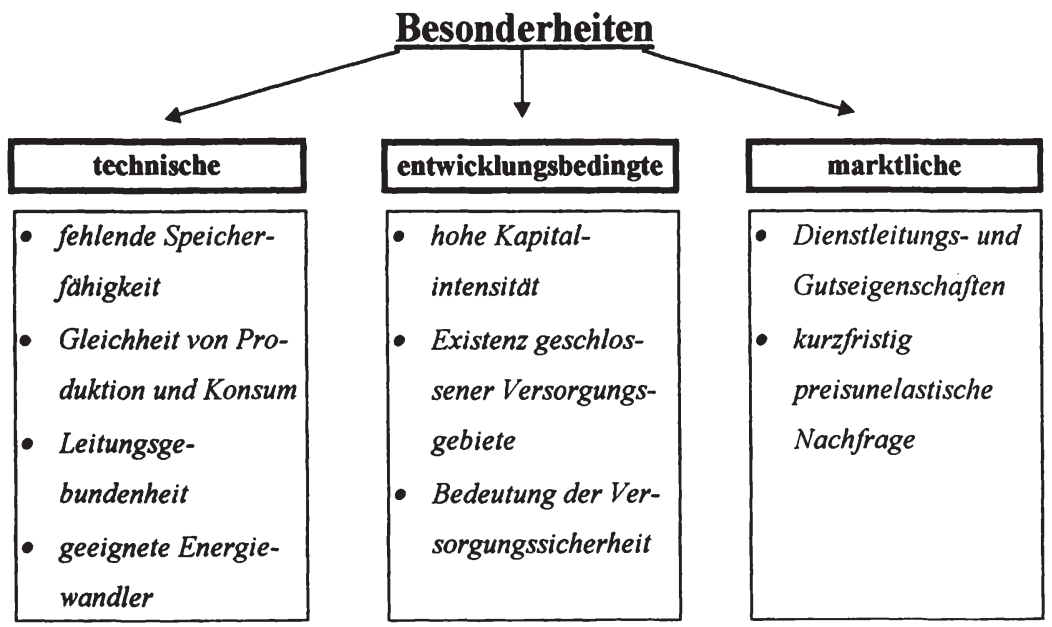

49 In der Gasversorgung ist eine begrenzte Lagerhaltung möglich.

50 Dies gilt unter der Nebenbedingung, daß keine Last abgeworfen werden kann. Die EVU sehen in der Praxis jedoch einen gezielten Lastabwurf vor und schließen hierzu auch Vertrăge mit Großabnehmern. 
Die entwicklungsbedingte Besonderheit der vollständig vertikal integrierten Versorgungsgebiete sind ein Ergebnis staatlicher Einflußnahmen unter politischen Zielsetzungen in Zeiten, in denen die europäischen Staaten ein starkes Autarkiestreben aufwiesen. Als Folge der politischen Vorgaben und der eingesetzten Regulierungsverfahren bildete sich die heutige hohe Kapitalintensität in der europäischen Elektrizitätsversorgung heraus. Ein technischer oder marktlicher Determinismus liegt jedoch nicht vor. Kleinere Staaten in der Welt und Projekte zur dezentralen Stromerzeugung in Europa verdeutlichen, daß dezentrale Stromerzeugungsanlagen funktionieren und auch unter wirtschaftlichen Gesichtspunkten Vorteile aufweisen können ${ }^{51}$. Die Erfahrungen mit der Auflösung der geschlossenen Versorgungsgebiete in Großbritannien ${ }^{52}$ zeigen zudem empirisch, daß die entwicklungsbedingte Besonderheit der vertikal integrierten Unternehmen mit geschlossenen Versorgungsgebieten in der Elektrizitätswirtschaft nicht unabänderlich ist und somit als Begründung entfällt. Lange Kapazitätsbauzeiten bei hoher Kapitalintensität zeichnen gleichfalls auch andere Industriezweige aus; somit läßt sich schlüssig daraus keine Sonderbehandlung der Elektrizitätswirtschaft ableiten.

Die angesprochenen, sich aus dem Dienstleistungscharakter der Elektrizitätsversorgung ergebenden marktlichen Besonderheiten begründen, isoliert betrachtet, keine Notwendigkeit für eine Sonderbehandlung in Wettbewerbsrecht und -politik. Eine kurzfristig preisunelastische Nachfrage gibt es bei anderen Gütern und Dienstleistungen, die zu den menschlichen Grundbedürfnissen gehören, häufiger. In fast allen Bereichen der Marktwirtschaft ist es so, daß letztendlich der Verbraucher mittels seiner Konsumentensouveränität über Leistung, Zeit und Ort der Inanspruchnahme einer Dienstleistung entscheidet und daher Kapazitäten vorgehalten werden müssen. Erst im Zusammenhang mit den technischen Eigenschaften der Elektrizität ergeben sich „echte“ Besonderheiten. $\mathrm{Zu}$ prüfen wäre, ob ein ausreichendes $\mathrm{Ma} ß$ an Versorgungssicherheit durch organisatorische oder technische Maßnahmen erreicht werden kann. Diese müssen die technische Besonderheiten derart kompensieren können, daß Wettbewerb trotz dieser Spezifika möglich wird (Second-Best-Lösung) ${ }^{53}$. Die Maßnahmen sind jedoch von dem gewählten Ordnungssystem der Elektrizitätswirtschaft abhängig und sollen daher an späterer Stelle behandelt werden.

Zusammenfassend kann festgestellt werden, daß die Besonderheiten des Elektrizitätssektors auf dem Teilmarkt der Erzeugung nicht zwingend Monopolstrukturen zur Sicherstellung der Elektrizitätsversorgung erfordern. Im Bereich

51 Vgl. KRISTOF, K. : Dezentralisierung in der Elektrizitätswirtschaft, Frankfurt/Main, New York 1992

52 Vergleiche hierzu die Darstellungen zur britischen Elektrizitătswirtschaft in Kapitel 4.1.3

53 LIPSEY, R.G. ; LANCASTER, K. : The General Theory of Second Best, in: Review of Economic Studies, Vol. 24, 1956, S. 11-32 und BOADWAY, R. : The Role of Second-Best

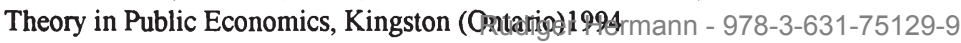


des Stromtransportes und der Stromverteilung mïssen die technischen Besonderheiten bei der Auswahl der Mittel für die Zielerfüllung berïcksichtigt werden. Sie erfordern zwangsläufig Versorgungsgebiete in denen eine Netz- oder Verteilungsgesellschaft zumindest zeitlich begrenzt den Stromtransport ${ }^{54}$ oder die Stromverteilung alleine durchführt ${ }^{55}$. Damit bleibt zu prüfen, ob in der ökonomischen Theorie Anhaltspunkte für den Ausschluß von Marktstrukturen mit Wettbewerb zu finden sind.

54 Genaugenommen besteht der Stromtransport aus einer Vielzahl von Teilbereichen wie der Laststeuerung, dem Leitungsbau, die Endnahme- und Einspeiseregelung und die Verrechnung der Leistungen sowie der Koordination der Reservehaltung.

55 Vgl. RECKNAGEL, H. : Mehr Wettbewerb ..., a.a.O., S. 220. Zu dem gleichen Ergebnis kommt auch eine Studie des Energiewirtschaftlichen Instituts der Universităt Köln. Vgl. hierzu die Zusammenfassung der wichtigsten Ergebnisse in : KLOPFER, T.; HENSING, I. ; RIECHMANN, C.; SCHULZ, W. ; WERNER, O. : Bewertung von Deregulierungsmodellen,

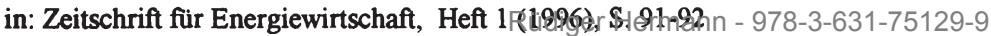




\section{Wettbewerb oder Monopole für den europăischen Strom- markt ?}

In der „klassischen“ Preistheorie erhält der Wettbewerb die Aufgabe, effiziente Strukturen zu schaffen und die maximale soziale Wohlfahrt zu erzielen. Von vielen politisch Handelnden wird Wettbewerb als Instrument zur Schaffung eines wünschenswerten Marktergebnisses verstanden. Dieser Auffassung hat die „neuere" Preistheorie widersprochen. HAYEK und andere erkannten, daß die Bedingungen, in denen der Wettbewerb der „klassischen“ Preistheorie wohlfahrtsoptimale Gleichgewichte erreicht, in der Realität nicht gegeben sind ${ }^{56}$. Statt vollständiger Markttransparenz bestehen auf den empirischen Märkten ausgeprägte Informationsdefizite. Hieraus entwickelte HAYEK einen anfangs wenig beachteten Wettbewerbsbegriff. Nach HAYEK wird Wettbewerb als Endeckungsverfahren verstanden, mit dem die Marktakteure versuchen, ihre Unwissenheit abzubauen ${ }^{57}$. Diesen Wettbewerbsbegriff baute STREIT weiter aus. Nach STREIT ${ }^{58}$ ist Wettbewerb ein Prozeß, ,in dem (1) Wissen entdeckt und verbreitet wird und (2) die Ausübung von Handlungs- und Eigentumsrechten durch tatsächliche oder potentielle Substitution von Transaktionspartnern (und Transaktionsobjekten) kontrolliert wird“. Wissen wird dabei nicht auf die alleinige Kenntnis von Preisunterschieden beschränkt, sondern um die Schaffung von Preisen durch neu aufgefundene Produkt- oder Prozeßinnovationen ${ }^{59}$ erweitert. Als Lohn für den Entdeckungsprozeß winken den aktiven Marktakteuren im Vergleich zu den Akteuren ohne Wissen höhere Gewinne. Die Initiatoren von Innovationen können gleichzeitig Entwicklungsprämien erzielen.

Beiden Wettbewerbsbegriffen gemeinsam ist die fehlende Zielrichtung des Wettbewerbs und die damit verbundene Ungewißheit des durch den Wettbewerbsprozeß erreichbaren Zustandes ${ }^{60}$. Dies schließt aus, daß Wettbewerb ein bestimmtes Marktergebnis herbeiführt. Diese Betrachtung habe, wie STREIT meint, zur Folge, daß Wettbewerb zur Erreichung von ordnungspolitischen Zielen ungeeignet sei. Er vernachlässigt hiermit jedoch den zweiten Teil seines Wettbewerbsbegriffes. Wettbewerb weist neben der Preisfindungsfunktion eine Kontrollfunktion auf. Bedin-

56 Vgl. HAYEK, F.A. von: Der Wettbewerb als Entdeckungsverfahren, in ders . Freiburger Studien, Tübingen 1969, S. 249 f.

57 Vgl. HAYEK, F.A. von: Der Wettbewerb als Entdeckungsverfahren, in ders . Freiburger Studien, Tübingen 1969, S. 249 f.

58 Vgl. STREIT, M.E. : 13 Thesen zu einer marktprozeßorientierten Wettbewerbspolitik, in: Wirtschaftswissenschaftliches Studium Heft 4 , April 1993, S. 168

59 Die Innovationsfunktion von Wettbewerb auf Mărkten betont auch TOLKSDORF, M. : Dynamischer Wettbewerb - Einführung in die Grundlagen der deutschen und internationaler Wettbewerbspolitik, Wiesbaden 1994, S. 52 ff.

60 Vgl. HAVER, R. : Versunkene Kosten - Zur Funktionsweise der unsichtbaren Hand, Freiburg 1990, S. 20 
gung für diese Kontrollfunktion ist, wie der Autor richtig ausführt, die Existenz alternativer oder potentieller Transaktionspartner oder -objekte.

Diese Sichtweise des Wettbewerbs ist von BAUMOL in seiner Theorie der „Perfectly Contestable Markets“ bereits zu Beginn der achtziger Jahre detailliert ausgefuhrt worden ${ }^{61}$. Die Theorie der „Perfectly Contestable Markets" betont die Bedeutung der potentiellen Konkurrenz für die Preisbildung. Demnach ist die Marktstruktur entgegen der „klassischen“ Preistheorie ${ }^{62}$ nicht für die Preissetzung bestimmend, sondern die Existenz von potentiellen Konkurrenten. Potentielle Konkurrenten können jedoch nur dann auf den Markt wirken, wenn Märkte vollkommen angreifbar sind, d.h. keine Marktzutritts- oder Austrittsbarrieren existieren ${ }^{63}$. STIGLER definierte, ohne auf einen konkreten Markt abzuzielen, Marktzutrittsschranken als ,a cost of producing (at some or every rate of output) which must be born by a firm which seeks to enter an industry but is not born by firms already in the industry ${ }^{664}$. Hieraus folgt, daß bei fehlenden Markzutrittsbarrieren ${ }^{65}$ oder der Existenz von potentiellen Konkurrenten ,... any industry, regardless of its market form, can (in theory) be perfectly contestable ${ }^{\text {c66 }}$.

Diese Schlußfolgerung basiert auf den von FEHL und SCHREITER ${ }^{67}$ wie folgt zusammengefaßten Annahmen:

- Der Markteintritt und Marktaustritt ist völlig frei und kostenlos.

- Allen potentiellen Konkurrenten stehen die gleichen Produktionstechniken, Produktionsverfahren und Beschaffungsmärkte zur Verfügung.

- Die Nachfrager besitzen keine Präferenzen für oder gegen einen bestimmten Anbieter, eine hohe Preiselastizität und eine hohe Reaktionsgeschwindigkeit.

- Potentielle Konkurrenten besitzen gegenüber den bestehenden Anbietern einen Aktionsvorsprung, der es ihnen ermöglicht, Vorsprungsgewinne zu erzielen und sich im Markt zu etablieren oder zumindest kurzfristige

61 BAUMOL, W.J. ; PANZAR, J.C. ; WILLIG, R.D. : Contestable Markets and the Theory of Industry Structure, erweiterte Fassung, San Diego 1988 und BAUMOL, W.J. : Contestable Markets: An Urprising in the Theory of the Industry Structure, in: American Economic Review, Vol. 72 (1982), S. 1-15

62 Vgl. BAUMOL, W.J. : Determinants ... , a.a.O., S. 507

63 Hierin sieht BAUMOL die Exklusivităt des Ansatzes der Perfectly Contestable Markets im Gegensatz zur Spieltheorie und zum Transaktionskostenansatz.

64 STIGLER, G. : The Organization of Industry, Homewood (Ill.) , 1968, S. 67

65 Vgl. hierzu weiter DEMSETZ, H. : Barriers of Entry, in: American Economic Review, Vol. 72 (1982), S. 47-57, insb. S. 47

66 BAUMOL, W.J. : Determinants of Industry Structure and Contestable Markets Theory, in: GREENAWAY, D. ; BLEANEY, M.F. : Companion to Contemporary Economic - Thought, London 1992, S. 514

67 Vgl. FEHL, U. ; SCHREITER, C. : Neuere Entwicklungen in der Preistheorie, in: WiST Heft 6, Juni 1993, S. 276 
Gewinne zu erzielen, bevor sie den Markt wieder verlassen (Hit-andrun-Aktionen ${ }^{68}$ ).

- Die Märkte sind homogen.

- Alle Marktakteure besitzen vollkommene Information.

Liegen alle diese Bedingungen auf einem Markt vor, ist in der Theorie die Marktstruktur nicht maßgebend für das Erreichen des Pareto-Optimums ${ }^{69}$ und jedes im Markt befindliche Unternehmen muß langfristig zu minimalen Kosten produzieren $^{70}$. Es stellt sich automatisch die optimale Betriebsgröße im Markt ein. In der Realität ist dies jedoch zumeist nicht der Fall; insbesondere die Annahme der vollständigen Information der Marktakteure kann bezweifelt werden ${ }^{71}$. Ferner ist der Markteinritt und -austritt gerade im Elektrizitätssektor mit seinen langen Genehmigungsverfahren und Kraftwerksbauzeiten weder frei noch kostenlos. Ähnlich stellt sich die Situation auf den Beschaffungsmärkten für die EVU in Europa dar: Durch staatliche Auflagen müssen die EVU, z.B. in der Bundesrepublik und in Großbritannien, bestehende nationale Energieträger zur Verstromung einsetzen ${ }^{72}$. Desweiteren ist die Nachfrage kurzfristig preisunelastisch und die Reaktionsgeschwindigkeit der Nachfrager wegen der switching costs gering. Wenn die Annahmen der Theorie der „Perfectly Contestable Markets“ aber nicht zutreffen, ist auch die Schlußfolgerung für den Elektrizitätsmarkt nicht anwendbar und die Ausgangsfrage muß erneut gestellt werden. Hat die Marktstruktur einen Einfluß auf das Erreichen des Pareto-Optimums und lassen sich durch Änderungen der bestehenden Marktstrukturen Kostenreduktionen erzielen?

Die Beantwortung dieser Frage wird durch die Ausführungen der ,neueren“ Preistheorie erleichtert. Die Vertreter gehen auf die Auswirkungen von Marktunvollkommenheiten, also der Abweichungen der Realität von dem Konstrukt des vollkommenen Marktes ein. Sie problematisieren insbesondere die Auswirkungen der

68 Vgl. BAUMOL, W.J. ; PANZAR, J.C. ; WILLIG, R.D. : Contestable Markets and the Theory of Industry Structure, New York 1982, S. 4

69 Im Falle des natürlichen Monopols wird ein Ramsey-optimales Marktergebnis erreicht. Vgl. BAUMOL, W.J. ; PANZAR, J.C. ; WILLIG, R.D. : Contestable Markets and the Theory of Industry Structure, New York 1982, S. 3 ff.

70 Vgl. BAUMOL, W.J. ; Determinants ... , a.a.O., S. 514

71 BAUMOL ist sich bewußt, daß die vollkommende Angreifbarkeit eines Marktes empirisch genau so selten ist, wie das Vorhandensein der vollkommenen Konkurrenz. Er sieht in seinem Ansatz vielmehr ein theoretisches Konzept, um einen in der „Klassischen“ Theorie nicht berücksichtigten Aspekt herauszuheben. Vgl. hierzu: BAUMOL, W.J. : Determinants ... a.a.O., S. 508. Gleiches gilt seiner Meinung nach für die Spieltheorie und den Transaktionskostenansatz.

72 Vergleiche die Ausfuihrungen zur britischen und zur deutschen Elektrizitätswirtschaft in den Kapiteln 4.1.3 und 4.1.2.1 . Zur Entstehung von Handelsbarrieren durch Verhandlung von Regierungen vgl. COPELAND, B.R. : Strategic Interaction Among Nations: Negotiable and Non-negotiable Trade Barriers, in: Canadian Journal of Economics Vol. 23 (1990), No.1, S. 84-108 
unvollkommenen Information bei den Marktsubjekten ${ }^{73}$. Die unvollständige Information der Marktsubjekte bewirkt, daß die Anbieter die genaue Nachfragemenge der Konsumenten nicht kennen. Für die Konsumenten sind Informationsdefizite mit der Unkenntnis über den niedrigsten Preis auf dem Markt gleichzusetzen. Die Auswirkungen der Marktunvollkommenheiten für die Marktsubjekte unterscheiden sich je nach Marktstruktur.

Bei einem Monopol ist der Preis für die Nachfrager kurzfristig ein Fixum. Deren Nachfrage nach Elektrizität ist kurzfristig preisunelastisch, für die Anbieter gegeben und schwankt im Tagesverlauf. ALCHIAN ${ }^{7}$ stellt folgende Reaktionsmöglichkeiten der Anbieter auf eine schwankende Nachfragemenge auf ${ }^{35}$.

1. Die Anbieter reagieren auf die Nachfrageänderungen mit keiner Änderung der Preise:

a) Die Anbieter können die Mengenänderungen durch eine Lagerhaltung ausgleichen (Lagerhaltung).

b) Von den Anbietern wird versucht, Informationen über die Schwankungen zu erhalten, die sie in ihre Produktionsplanungen einbeziehen (Mengenreaktion).

2. Die Anbieter reagieren auf die Nachfrageänderungen mit Preisänderungen und erzielen jeweils einen vollständigen Absatz der produzierten Menge (Preisreaktion $)^{76}$.

In der Elektrizitätswirtschaft scheidet die Lagerhaltung des Endproduktes wegen der technischen Besonderheiten aus.

In den bestehenden Monopolen der nationalen Elektrizitätswirtschaften ist der Preis in seiner Höhe vorgegeben oder nach oben begrenzt ${ }^{77}$. Damit werden die Reaktionsmöglichkeiten der Anbieter auf die Mengenreaktion beschränkt, durch die folgende Kosten bei den Anbietern entstehen:

73 Vgl. hierzu FEHL, U. ; SCHREITER, C. : Neuere Entwicklungen in der Preistheorie, in WiST Heft 6, Juni 1993, S. 279

74 ALCHIAN, A. A. : Information Costs, Pricing and Resource Unemployment, in: PHELPS, E.S. et al. : Microeconomic Foundations of Employment and Inflation Theory, London 1970 , S. $32 \mathrm{ff}$.

75 Der Ansatz ist besonders für die Elektrizitătswirtschaft mit ihrer tages- und jahreszeitlich schwankenden Nachfrage interessant.

76 CARLTON, D.W. : The Theory and Facts of How Markets Clear: Is Industrial Organisation valuable for Understanding Microeconomics?, in: SCHMALENSEE, R. : WILLIG, R.D. (Hrsg.) : Handbook of Industrial Organisation Vol. 1, Amsterdam 1989, S. 931, weist auf die damit verbundenen Kosten für die Anbieter und Nachfrager hin.

77 Vgl. hierzu die Ausführungen in Kapitel 4.Rüdiger Hermann - 978-3-631-75129-9 
- Kosten für Kraftwerke, die im Leerlauf (Heißreserve) betrieben werden müssen, um plötzlichen Nachfrageerhöhungen begegnen zu können,

- Kosten für die Vorproduktlagerung,

- Kosten für die Regelungstechnik,

- Kosten für die Informationsbeschaffung über Nachfrageschwankungen,

- Kosten für Prognoseirrtümer.

Da der Preis für Klein- und Mittelabnehmer fest ist, haben diese gleichzeitig kein Interesse, ihre Lastkurve anzugleichen und durch eine konstante Auslastung der Kraftwerke und Leitungen eine Kostenersparnis bei den EVU zu bewirken.

In einem offenen Markt ohne staatliche Regulierung sieht die Situation anders aus. Zwischen Anbietern und Nachfragern können differenzierte Liefer- und Bezugsverträge geschlossen werden, die dem einzelnen Anbieter auch eine Preisreaktion auf Nachfragemengenschwankungen ermöglicht. Von den drei Reaktionsmöglichkeiten Alchians können die Anbieter zwischen der Mengen- und der Preisreaktion ${ }^{78}$ wählen. Bei einer reinen Mengenreaktion entstehen für die Anbieter die gleichen Kosten wie in der Anbietermonopolsituation. Für die Nachfrager entstehen jedoch zusätzliche Suchkosten nach dem günstigsten Anbieter. Ohne abgestimmte Verhaltensweisen kann die Mengenreaktion der Anbieter zu einem Monopol führen, wenn die Informationsbeschaffungskosten der Nachfrager nach dem günstigsten Anbieter niedriger sind als die Nutzen aus der Informationsbeschaffung und der günstigste Anbieter seine Produktionsmenge beliebig ausdehnen kann.

Bei der Preisreaktion der Anbieter besitzen die Nachfrager zwei Handlungsalternativen. Sie können langfristig ihre Nachfragemenge reduzieren oder zeitlich verlegen, um Preisschwankungen mit Hilfe von Erfahrungszyklen auszugleichen. Diese sukzessive Nachfragemengenanpassung ist erreichbar, wenn die Nachfrager stromintensive Anwendungen in Schwachlastzeiten verlegen oder vorzeitig ihre elektrischen Geräte gegen solche austauschen, die über einen höheren Wirkungsgrad verfügen. Hierdurch entstehen ihnen jedoch folgende Kosten:

- Kosten für die Planung,

- Suchkosten für geeignete Anbieter ,

- Opportunitätskosten,

- Kapitalkosten für die Anschaffung neuer Geräte,

- Switching costs bei der Nutzung anderer Energieträger.

Ferner können die Nachfrager jegliche Nachfragemengenanpassung unterlassen, weil die Preisunterschiede der Anbieter niedriger sind als die erforderlichen Such-

78 Möglich ist auch eine Kombination aus Preis- und Mengenreaktion. Die folgenden Aussagen gelten für die Kombination sinngemäß. Rüdiger Hermann - 978-3-631-75129-9 
kosten, um den günstigsten Anbieter herauszufinden ${ }^{79}$ oder die anderen Kosten für die Nachfragemengenanpassung höher sind als der daraus erwachsene Nutzen. FEHL sieht in den unterschiedlichen Preisen von Anbietern in einem offenen Markt eine Informationsproduktion der Anbieter für die Nachfrager, die die Anbieter durch höhere Preise entlohnt bekommen. Daraus kann man schließen, daß Preisunterschiede bei unvollkommener Information Bestandteil eines Gleichgewichtes sein können.

\section{Tabelle 1: Zusammenhang zwischen Marktstruktur und Kosten in der Elek- trizitätsversorgung}

\begin{tabular}{|c|c|c|}
\hline $\begin{array}{l}\text { Markt- } \\
\text { Markblekte } \\
\text { form }\end{array}$ & $\begin{array}{l}\text { Kosten } \\
\text { für die Anbleter }\end{array}$ & $\begin{array}{l}\text { Kosten } \\
\text { für die Nachfrager }\end{array}$ \\
\hline Monopol & $\begin{array}{l}\text { - Reservehaltung } \\
\text { - Vorproduktlagerung } \\
\text { - Regelungstechnlk } \\
\text { - Informationsbeschaffung } \\
\text { - Prognoseirtümer }\end{array}$ & keine \\
\hline $\begin{array}{l}\text { offener } \\
\text { Markt }\end{array}$ & $\begin{array}{l}\text { a) bel Mengenanpassung } \\
\text { - Reservehaltung } \\
\text { - Vorprodultlagerung } \\
\text { - Regelungstechnik } \\
\text { - Informatlonsbeschaffung } \\
\text { - Prognoselrtümer } \\
\text { b) bel Prelsreaktlon } \\
\text { - Prelsblldungskosten } \\
\text { - Kosteneinsparung durch } \\
\text { ausgegllchene Lastkurve }\end{array}$ & Informationsbeschaffungskosten \\
\hline
\end{tabular}

Sofern die Nutzen für die Nachfragemengenanpassung höher sind als die Kosten, entsteht durch eine Preisanpassung der Anbieter bei den Nachfragern ein Interesse, ihre Lastkurve langfristig auszugleichen, um die höheren Preise für Spitzenlaststrom zu vermeiden. Dies führt dazu, daß die EVU weniger Kraftwerke in Heißreserve $^{80}$ betreiben müssen und niedrigere Informationsbeschaffungskosten über

79 CARLTON, D.W. : The Theory and Facts of How Markets Clear: Is Industrial Organisation valuable for Understanding Microeconomics?, in: SCHMALENSEE, R. : WILLIG, R.D. (Hrsg.) : Handbook of Industrial Organisation Vol. 1, Amsterdam 1989, S. 936

80 Jederzeit verzuglos zur Stromerzeugung eirßsëtzbagr Hermann - 978-3-631-75129-9 
Nachfrageschwankungen entstehen. Die zahlreichen Studien und Veröffentlichungen zum Demand-Side-Management ${ }^{81}$ zeigen, welches ein großes Kosteneinsparpotential hierin begründet ist.

Die Übersicht (Abb. 1) zeigt, daß letztlich die Höhe der Informationsbeschaffungskosten für die Nachfrager sowie der Saldo aus Preisbildungskosten und Einsparungen durch die ausgeglichene Lastkurve für die Auswahl der Marktstruktur entscheidend sein müssen. Die Informationskosten auf real existierenden Märkten sind jedoch stark von der vorhandenen Informationsinfrastruktur mit ihrer spezifischen Technik als Gestaltungsvariable abhängig. Sie sind keine die Marktstruktur a priori determinierende Größe. VOLLMER unterscheidet hierauf aufbauend Märkte nach der Höhe der Grenzkosten furr die Informationsbeschaffung und der Grenzkosten für die Lagerhaltung, die in der Elektrizitätswirtschaft mit den Grenzkosten der Mengenanpassung gleichgesetzt werden können (vgl. Tabelle 2).

Diese Überlegungen bedeuten für die Ausgangsfrage, daß auch die „neuere“ Preistheorie nicht eine spezielle Marktstruktur für den europäischen Elektrizitätsmarkt begründet. Welche Schlußfolgerungen können nun aus dem Transaktionskostenansatz gezogen werden?

Gegenstand des Transaktionskostenansatzes sind die Kosten des Güteraustausches auf einem Markt. Mit Hilfe dieser Kosten geht es dem Transaktionskostenansatz um die effiziente Koordination von wirtschaftlichen Leistungsbeziehungen. Damit betont der Ansatz die Koordinationsfunktion von Märkten und Organisationen ${ }^{82}$. Die Autoren lösen sich von den Annahmen der „klassischen“ Preistheorie, der vollständigen Information und der fehlenden Kosten für den Güteraustausch. Als Transaktion wird die Übertragung von Verfügungsrechten verstanden ${ }^{83}$.

Das Grundprinzip des Ansatzes kann man folgendermaßen zusammenfassen: Die Koordination von Leistungsbeziehungen verursacht Kosten. Die Akteure versuchen diese Kosten durch Organisation der Austauschbeziehungen zu minimieren. Die unterschiedlichen Organisationsformen beinhalten unterschiedliche Kosten für die verschiedenen Austauschbeziehungen. Nach Coase bieten sich als Organisationsformen Märkte und Unternehmungen $\mathrm{an}^{84}$. Für die späteren Betrachtungen

81 Vgl. MEYER, K. : Spitzenlasttarifierung, okonomische Effizienz und Erhöhung der Eigenwirtschaft - Eine angewandte Studie der elektrizitătswirtschaftlichen Preisbildung, Bern, Stuttgart 1983

82 Vgl. BAUMOL, W. J. : Determinants ..., a.a.O., S. 510

83 Diese aus den Rechtswissenschaften entnommene Definition von Austauschbeziehungen ist für den Transaktionskostenansatz sinnvoll, da hierdurch auch Mărkte für Verfügungsrechte, z.B. Optionsscheine und Benutzungsrechte, erklärt werden können.

84 Als grundlegenden Artikel hierzu: COASE, R.H. : The Nature of Firm Economic, nachgedruckt von STIGLER, G.J.; BOULDING, K.E. (Hrsg.) : Readings in Price Theory , Homewood 1984 
Tabelle 2: Informations- und Veränderungskosten nach Vollmer

Grenzkosten der Lagerhaltung

\begin{tabular}{|c|c|c|c|c|}
\hline \multirow{4}{*}{$\begin{array}{l}\text { Informa- } \\
\text { tions- } \\
\text { grenz- } \\
\text { kosten }\end{array}$} & & Null & Positiv & $\begin{array}{l}\text { prohibitiv } \\
\text { wirkend }\end{array}$ \\
\hline & Null & $\begin{array}{l}\text { vollkommen } \\
\text { flexible Preise } \\
\text { und Mengen }\end{array}$ & \begin{tabular}{|c|} 
vollkommen \\
flexible Preise; \\
verzögerte \\
Mengenanpas- \\
sung
\end{tabular} & $\begin{array}{l}\text { vollkommen } \\
\text { flexible Preise; } \\
\text { vollkommen } \\
\text { starre Mengen }\end{array}$ \\
\hline & Positiv & $\begin{array}{c}\text { verzógerte } \\
\text { Preisanpas- } \\
\text { sung; vollkom- } \\
\text { men flexible } \\
\text { Mengen }\end{array}$ & $\begin{array}{c}\text { verzogerte } \\
\text { Preis- und } \\
\text { Mengenanpas- } \\
\text { sung }\end{array}$ & \begin{tabular}{|c|} 
verzögerte \\
Preisanpas- \\
sung; \\
vollkommen \\
starre Mengen
\end{tabular} \\
\hline & $\begin{array}{l}\text { prohibitiv } \\
\text { wirkend }\end{array}$ & $\begin{array}{c}\text { vollkommen } \\
\text { starre Preise ; } \\
\text { vollkommen } \\
\text { flexible } \\
\text { Mengen }\end{array}$ & $\begin{array}{c}\text { vollkommen } \\
\text { starre Preise ; } \\
\text { verzögerte } \\
\text { Mengenan- } \\
\text { passung }\end{array}$ & $\begin{array}{l}\text { vollkommen } \\
\text { starre Preise } \\
\text { und Mengen }\end{array}$ \\
\hline
\end{tabular}

Quelle: VOLLMER, F.U. : Informations- und Veränderungskosten in makroökonomischen Transmissionsmodellen, Frankfurt/M. , Bern, New York 1986, S. $52^{85}$

sind zwei Fragestellungen, zu denen sich die Autoren des Ansatzes äußern, bedeutend:

- Welche Transaktionskostenarten gibt es und wie entstehen sie?

- Welche Koordinationsform wirtschaftlicher Leistungsbeziehungen ist unter welchen Bedingungen effizient?

Bezüglich der Transaktionskosten unterscheidet man gewöhnlich fünf Gruppen:

- Suchkosten,

- Informationskosten,

- Verhandlungskosten,

- Durchsetzungskosten,

- Opportunitätskosten.

85 Diese Einteilung wird bei der Beurteilung von Ordnungsmodellen für den europäischen Strommarkt interessant, um die Auswirkungen einer Stromhandelsinfrastruktur auf die Informationskosten einschätzen zu können. Rüdiger Hermann - 978-3-631-75129-9 
Transaktionsprobleme werden als Vertragsprobleme angesehen ${ }^{86}$. Als Ursache furr die durch die Vertragsprobleme entstehenden Kosten wird in der Theorie das $\mathrm{Zu}$ sammenwirken von ,human and environmental factors" angesehen.

Die human factors werden in die auf SIMON ${ }^{87}$ zurückgehende begrenzte Rationalität und das opportunistische Verhalten von Menschen unterteilt. Unter opportunistischem Verhalten wird das Bestreben der Menschen verstanden, bei ihrem Handeln günstige Situationen und Konstellationen egoistisch auszunutzen, auch unter Einsatz von List und Tücke. Das Verhalten beinhaltet ferner die unvollständige und verzerrte Wiedergabe von Informationen an andere Akteure und setzt unvollständige Informationslagen bei den Akteuren voraus.

Als „environmental factors“ werden die Unsicherheit und die Marktstruktur angesehen. Die Unsicherheit resultiert aus der Umweltdynamik und der Umweltkomplexität. Beide Faktoren erschweren den Marktteilnehmern die Beurteilung der Folgen eigener Verhaltensweisen, die Wirkungen der Verhaltensweisen der anderen Marktakteure und die Auswirkungen veränderter Rahmenbedingungen.

Durch die Existenz der begrenzten Rationalität, des opportunistischen Verhaltens und der Unsicherheit wird eine Situation des „Information Impactness" geschaffen, welche sich durch Informationsasymmetrien ${ }^{88}$ bei den Akteuren auszeichnet und zu deren Abbau Kosten erforderlich werden ${ }^{89}$.

Der letzte von WILLIAMSON in seinen „Organizational Failures Framework“90 aufgefuihrte Faktor für die Entstehung von Transaktionskosten ist die Marktstruktur. Er betont jedoch, daß es nicht die Marktstruktur an sich ist, die die Transaktionskosten determiniert, sondern identifiziert die Wettbewerbsintensität in der jeweiligen Markstruktur als den Einflußfaktor der Transaktionskosten. Die Frage, ob ein Markt oder eine Unternehmung zur Lösung der Koordinationsfunktion geeigneter sei, wird von den Autoren des Transaktionskostenansatzes von den Merkmalen der zu betrachtenden Transaktion abhängig gemacht. Hierbei wird zwischen

- Spezifität,

- Unsicherheit und

- Häufigkeit

unterschieden.

86 Vgl. KAAS, K.P. ; FISCHER, M. : Der Transaktionskostenansatz in: WISU 8-9/93, S. 686

87 Vgl. SIMON, H.A. : Administrative Behavior, New York 1961, S. 24

88 Vgl. uur Entstehung von Informationsasymmetrien KOOPMANN, T. : Three Essays of the State of Economic Science; New York 1957, S. 147

89 Vgl. KAAS, K.P. : Marketing als Bewältigung von Informations- und Unsicherheitsproblemen im Markt, in: Die Betriebswirtschaft, 50. Jg. (1990), S. $540 \mathrm{ff}$.

90 Vgl. WILLIAMSON, O.E. : Markets and Hierachies. Analysis and Antitrust Implications, New York 1975, S. 20 ff. . Eine gute Zusammenfassung in ders.: Transaction Cost Economics , in SCHMALENSEE, R. ; WILLIG, R.D. (Hrsg.): Handbook of Industrial Organisation, Vol. 1, Amsterdam 1989, S. 135-182 diger Hermann - 978-3-631-75129-9 
Die Spezifität als Transaktionsmerkmal stellt auf die zur Transaktion erforderlichen Investitionen ab. Hierbei können zwischen der Standort-, Sachkapital- und Humankapitalspezifität unterschieden werden. Hinzugefügt werden müssen spezifische Investitionen für einen einzelnen Abnehmer ${ }^{91}$ und die aus erforderlichen immatriellen Kapital resultierenden Kosten ${ }^{92}$. Bei steigender Spezifität erhöhen sich die gegenseitigen Abhängigkeiten bei den Transaktionspartnern. Die Kosten für die Begrenzung der daraus erwachsenden Risiken steigen mit zunehmender Abhängigkeit. Durch die Wahl einer unternehmensinternen Organisation der Koordinationsaufgaben können diese Kosten und die Risiken der Spezifität für den Endprodukthersteller reduziert werden ${ }^{93}$. Im Falle einer Transaktion mit niedriger Spezifität sind die Risiken für den Anbieter gering und der Markt als Organisationsform reduziert die Kosten.

Unsicherheit als zweites Transaktionsmerkmal bezieht sich auf die Informationsgewinnung und -auswertung. Unsicherheit führt dazu, daß der einzelne Akteur bei Vertragsabschluß das genaue Ergebnis der Übertragung der Verfügungsrechte nicht kennt. Um Abweichungen von seinen Vorstellungen zu begrenzen, sind Kosten erforderlich ${ }^{94}$.

Als letztes Transaktionsmerkmal ist die Häufigkeit der Leistungserstellung zu nennen. Je schneller Transaktionen wiederholt werden, desto höher können die Kosteneinsparungen durch eine Standardisierung der Transaktionen sein.

Die Abbildung 5 zeigt in Anlehung an die Ausführungen von WILLIAMSON ${ }^{95}$ eine Einschätzung über die jeweilige effiziente Koordinationsform bei Vorliegen der Transaktionsmerkmale.

Die bilaterale Koordination sieht bei rechtlicher Selbständigkeit der Transaktionspartner eine Koordination über glaubhafte Zusicherungen als Absicherungsmaßnahmen vor. Bei der trilateralen Koordination werden zur Lösung von Konflikten und zur Klärung von Lücken in Verträgen ein unabhängiger Dritter, z.B. eine Schiedsstelle, herangezogen. Wie die Graphik zeigt, stellt der Transaktionskosten-

91 Diese Kosten sind insbesondere für Zulieferfirmen, z.B. in der Automobilindustrie, relevant.

92 Z.B. die für das Erreichen eines Bekanntheitsgrades erforderlichen Marketingaufwendungen.

93 Vgl. BAUMOL, W.J. : Determinants ..., a.a.O., S. 510 f.

94 Als Beispiel kann hier der Bau eines Hauses angeführt werden. Bei Vertragsabschluß bestehen Plăne, die jedoch nicht restlos eindeutig sind oder unterschiedlich interpretiert werden können. Der Bauherr muß deshalb wăhrend der Bauphase durch Kontrollen gewăhrleisten, daß das endgültige Ergebnis mit seinen Vorstellungen übereinstimmt. Hierdurch entstehen Kosten für die Fahrt zur Baustelle, für Verhandlungen mit Handwerken etc.

95 Vgl. WILLIAMSON, O.E. : Die ökonomischen Institutionen des Kapitalismus, Tübingen 1990, S. $89 \mathrm{ff}$. 
ansatz die Koordinationsform als eine Variable des Entscheidungsprozesses der Akteure dar. Unternehmen werden damit als Alternativen zu Märkten gesehen.

\section{Abbildung 5: Koordinationsformen in Abhängigkeit ihrer Einflußvariabeln}

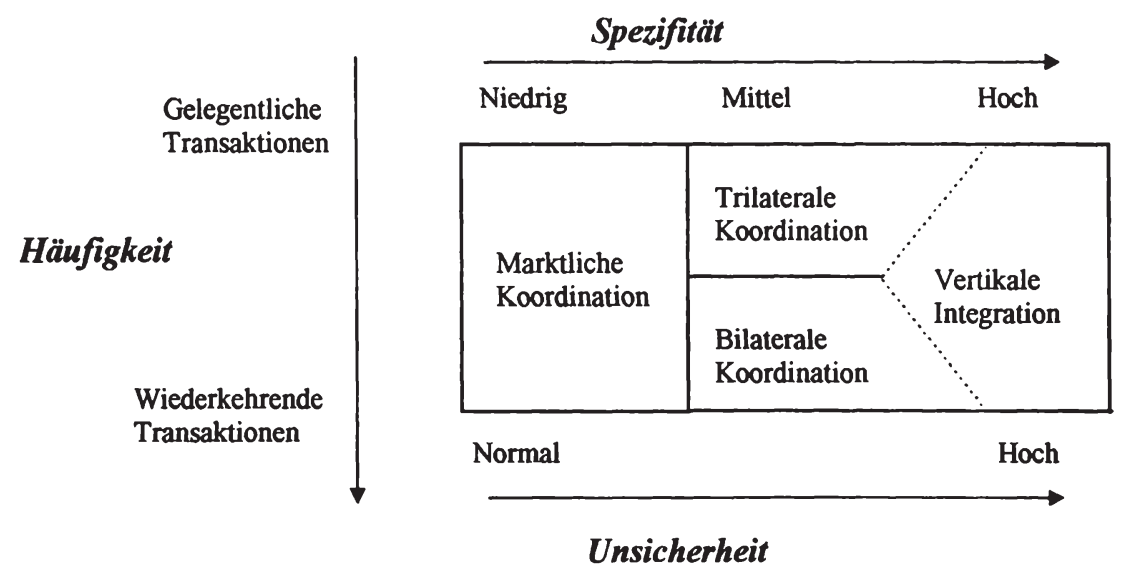

Quelle: In Anlehnung an WILLIAMSON nach KAAS, K.P. ; FISCHER, M. : Der Transaktionskostenansatz in: WISU 8-9/93 S. 686

Wenn Unternehmen als Alternative zu Märkten gesehen werden, impliziert dies die Frage nach abprüfbaren Kriterien für die effiziente Koordinationsform ${ }^{97}$, die über die allgemeinen Ausführungen zu den Transaktionsmerkmalen hinausgehen ${ }^{98}$. Diese existieren bisher nicht. Trotzdem soll der Elektrizitätssektor in das Schema von Williamson eingestuft werden. Zuvor muß jedoch folgendes festgehalten werden:

Für die gesamte Wertschöpfungskette der Elektrizitätsversorgung ergeben die Alternativen zur Koordination von Coase ${ }^{99}$ - Unternehmen oder Märkte - keinen Sinn. Die vollständige Koordination der Leistungsbeziehungen in einem Unter-

96 Vom Verfasser leicht verändert.

97 BORCHERT, GROSSEKETTLER wăhlen einen gănzlich anderen Ansatz. Für sie ist die vertikale Integration eine Sonderform des Unternehmenswachstums. Vgl. BORCHERT, M. ; GROSSEKETTLER, H. : Preis- und Wettbewerbstheorie: Marktprozesse als analytisches Problem und ordnungspolistische Gestaltungsaufgabe, Stuttgart 1985, S. 105

98 Für die Elektrizitătswirtschaft ist besonders die Bestimmung des optimalen vertikalen Integrationsgrades interessant.

99 Vgl. COASE, R.H. : The Nature of Firm Economic, nachgedruckt von STIGLER, G.J. ; BOULDING, K.E. : Readings in Price TheöryigHomewoed 199378-3-631-75129-9 
nehmen wäre gleichzusetzen mit der Eigenversorgung jedes Abnehmers. Dies ist insbesondere für Haushaltsabnehmer in städtischen Ballungszentren weder praktikabel noch ökonomisch effizient. Deshalb bestehen die beiden Alternativen ausschließlich für die vorgelagerten Wertschöpfungsstufen und für die Großverbraucherstromversorgung. Die folgende Einstufung der Elektrizitätsversorgung bezieht sich deshalb ausschließlich auf diese Wertschöpfungsstufen.

Die Spezifität als erste Eigenschaft ist im Elektrizitätssektor gering. Der einzelne Großabnehmer investiert nicht speziell für einen Lieferanten, da es sich bei der Elektrizität um ein vollkommen homogenes Gut handelt. Die gleiche Situation liegt bei den Anbietern, also den Kraftwerksbetreibern vor; beim Bau und Betrieb eines Kraftwerks werden keine Investitionen für einen einzelnen Abnehmer getätigt. Die im Kraftwerk produzierte Elektrizität wird in ein Leitungsnetz eingespeist, aus dem die Abnehmer den Strom entnehmen. Im Stromtransport- und Verteilungsbereich beschränken sich die anbieter- und abnehmerspezifischen Investitionen auf die Anschlußleitungen zwischen Abnehmer und Anbieter sowie dem Netz, also den letzten Metern zu einem Einspeiser oder Abnehmer. Ansonsten ist auch in diesem Wertschöpfungsbereich der Elektrizitätsversorgung die Spezifität gering.

Unsicherheit bei den Elektrizitätsliefer- und Bezugsverträgen besteht für die Anbieter in der Höhe der tatsächlichen Abnahmemenge. Begrenzt wird diese Unsicherheit durch bestehende Erfahrungen über den Lastverlauf und zeitlich differenzierte Abnahmeverträge mit Großabnehmern. Für die Abnehmer besteht die Unsicherheit in der Ungewissenheit über die Versorgungssicherheit bestehend aus Versorgungsquantität und -qualität. Wird die Versorgungssicherheit über separate Verträge von einem anderen Anbieter bereitgestellt, besteht keine Unsicherheit für die Stromabnehmer.

Die Transaktionen werden im Elektrizitätssektor kontinuierlich getätigt. Lediglich die Höhe der durch die Transaktion gelieferten Leistungsmenge variiert.

Demzufolge ist für die Elektrizitätsversorgung die marktliche Koordinationsform in der Theorie des Transaktionskostenansatzes effizient. Dies bedeutet gleichzeitig, $\mathrm{da} ß$ die bestehende Koordinationsform der vertikalen Integration für die angesprochenen Wertschöpfungsstufen Effizienzverluste beinhaltet, durch deren Abbau Kosten reduziert werden können.

Die Versorgungssicherheit muß, obwohl sie Bestandteil der Unsicherheit ist, unabhängig von der Koordinationsform wegen der gesellschaftspolitischen Zielsetzung gewährleistet werden. Darüber hinaus ist die Eigenerstellung der Versorgungssicherheit bei Kleinabnehmern nicht effizient. Sie ist abhängig von der kon- 
kreten Ausgestaltung der Rahmenbedingungen sowie von technischen und organisatorischen Maßnahmen ${ }^{100}$. Somit kann folgendes festgehalten werden:

Die Besonderheiten des Elektrizitätssektors erfordern ebensowenig wie die Schlußfolgeringen aus den theoretischen Überlegungen zur Preistheorie und zum Transaktionskostenansatz zwingend vertikal integrierte Monopole in geschlossenen Versorgungsgebieten. Nötig sind jedoch wegen der Leitungsgebundenheit der Elektrizitätsversorgung Gebiete, in denen ein Unternehmen (zeitlich begrenzt) den Stromtransport oder die Stromverteilung durchführt. Im Bereich der Stromerzeugung sind marktliche Strukturen mit Wettbewerb Monopolen vorzuziehen. Sie reduzieren die Regulierungskosten wegen der kontrollierenden Funktion des Wettbewerbs. Die im nächsten Kapitel folgende Bestandsaufnahme der nationalen Elektrizitätswirtschaften in Europa wird zudem zeigen, daß empirisch die Umgestaltung einer vollständig integrierten monopolisierten Elektrizitätswirtschaft gelungen ist. Damit ist die Forderung der EU-Kommission nach einer marktlichen Umgestaltung der nationalen Elektrizitätswirtschaften zielkonform.

Welche Schlußfolgerungen können nun aus den theoretischen Ausführungen für die Gestaltung einer Marktordnung gezogen werden, die die Ziele des Strommarktes verwirklicht?

$\Rightarrow$ Es gibt keine Hinweise darauf, daß vertikal integrierte Monopole die Elektrizitätsversorgung in Europa zu niedrigeren Kosten bereitstellen können als marktwirtschaftliche Strukuren mit Wettbewerb.

$\Rightarrow$ Damit Wettbewerb in einem europäischen Strommarkt kostenmindernd wirkt, müssen folgende Bedingungen und Faktoren erfüllt sein:

- Die vertikale Integration der Elektrizitätsstrukturen muß aufgehoben werden.

- Eine Stromhandelsinfrastruktur muß geschaffen werden.

- Es müssen Teilmärkte entstehen.

- Es müssen Mitbewerber oder potentielle Konkurrenten existieren.

- Innovationen sollten vorhanden sein.

- Es dürfen keine oder nur geringe Marktzutritts- oder Austrittsbarrieren vorhanden sein.

- Informationen über Angebots- und Nachfragepreismengenkombinationen müssen von den Marktsubjekten beschaffbar sein.

- Die einzelnen Marktsubjekte müssen über eine begrenzte Marktmacht verfügen.

- Die Transaktionen müssen eine niedrige Spezifität bei begrenzter Unsicherheit aufweisen.

$100 \mathrm{Vgl}$. hierzu die Ausführungen zur Sicherstellung der Versorgungssicherheit in den

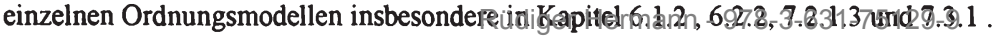


Das folgende Schema faßt die Bedingungen und begünstigenden Faktoren zu Kriterien zusammen, mit Hilfe derer die vorgeschlagenen und vorzuschlagenden Ordnungsmodelle für den europäischen Strommarkt bewertet werden sollen. Die vollständige Erfüllung dieser Kriterien bedeutet gleichzeitig, das das jeweilige Ordnungsmodell das Ziel des europäischen Strommarktes, eine kostengünstige und sichere Elektrizitätsversorgung, verwirklicht.

Tabelle 3: Beurteilungsschema für die Ordnungsmodelle

Kriterien zur Beurteilung der Ordnungsmodelle

Vertlkale Integratlon aufgelöst ?

Stromhandelsinfrastruktur geschaffen ?

Tellmärkte geschaffen ?

Marktzutrittsbarrieren reduzlert ?

Wettbewerbsintensität gesteigert?

Marktmacht einzelner Anbleter u.Nachfrager begrenzt ?

Reduzlerung der Gesamtkosten der Elektrizitätsversorgung ?

Versorgungssicherheit gewährleistet? 


\section{Der gegenwärtige Integrationsgrad der europäischen Elektrizitătswirtschaften}

Die von der EU-Kommission vorgesehene Verwirklichung kann nur dann erfolgreich sein, wenn die vorhandenen stromwirtschaftlichen Gegebenheiten und Organisationsformen ausreichend berücksichtigt werden. Deshalb werden nun die elektrizitätswirtschaftlichen Strukturen der einzelnen Mitgliedsstaaten typisiert dargestellt. Im Anschluß werden die bestehenden europäischen Zusammenschlüsse und Organisationen der Stromwirtschaft mit ihren Aufgaben erläutert. Mit Hilfe dieser Ausführungen soll dann ein angepaßter Implementierungsprozeß für das zu entwickelnde Ordnungskonzept umrissen und der Implementierungsaufwand sowie die Akzeptanz des jeweiligen Ordnungskonzeptes in den Mitgliedsstaaten eingeschätzt werden.

\subsection{Die elektrizitätswirtschaftlichen Strukturen der EU- Mitgliedsländer}

\subsubsection{Elektrizitätswirtschaften mit vollständig integrierten Strukturen und einem staatlichem EVU}

Hauptmerkmal dieser Gruppe ist pro Mitgliedsland ein einziges, staatliches EVU. Dieses übernimmt alle Teilbereiche der elektrizitätswirtschaftlichen Wertschöpfungskette. Zudem verfügt das EVU über das ausschließliche Recht, Elektrizitätsleitungen zu errichten und Endabnehmer zu versorgen. Ausgenommen von dieser Versorgungspflicht sind lediglich Eigenversorger. Der staatliche Besitz der Unternehmen ist gleichzusetzen mit einer vollständigen staatlichen Kontrolle unter Betonung des „öffenlichen Interesses an der Elektrizitätsversorgung ${ }^{“ 101}$. Sowohl die Tarife als auch die Strompreise unterliegen dem staatlichem Diktat und werden als energiepolitisches Instrument benutzt. Gewinne dürfen die Unternehmen nicht erzielen. Die EVU sind alleinig zum Stromhandel mit Nachbarstaaten autorisiert. Durchleitungs- oder Transitmöglichkeiten bestehen maximal für industrielle Eigenversorger zwischen ihren Produktionsstätten. In diese Kategorie der Elektrizitätswirtschaften können die französische, die griechische, die luxemburgische und die italienische eingeordnet werden.

101 Vgl. MATHIS, C. : Vorschlăge für eine Neuorientierung der Elektrizitătsivirtschaft in

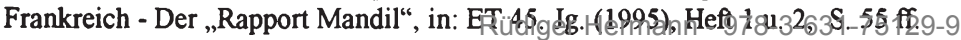




\subsubsection{Die Struktur der französischen Elektrizitätswirtschaft}

Zentrales Unternehmen der französichen Elektrizitätswirtschaft ist die Eléctricité de France (EdF). Sie wurde am 08.04.1946 durch das Nationalisierungsgesetz als "etablissement public a caractère industriel et commercial" geschaffen. Die EdF als das französische EVU ist mit einem staatlich verliehenen Monopol im Bereich der Stromerzeugung, des Stromtransportes und der Elektrizitätsverteilung ausgestattet worden. Nur sie darf Strom über die Grenzen Frankreichs im- oder exportieren. Ferner ist ausschließlich die EdF autorisiert, die Kernenergie zur Stromerzeugung einzusetzen. Ausgenommen von der Verstaatlichung wurden im Bereich der Stromerzeugung private Unternehmen, deren durchschnittliche Jahreserzeugung in den Jahren 1942 und 1943 unter 12 Mio kWh lag sowie Anlagen zur Eigenerzeugung bei stromintensiven Industrien ${ }^{102}$. Weitere Ausnahmen sind die als unbedeutend eingestuften Erzeugungsanlagen mit einer Gesamtkapazität unter $8000 \mathrm{kVA}^{103}$ und die Anlagen der französischen Kommunen zur Müllverbrennung mit den daraus erzeugten Elektrizitätsmengen. Im Bereich der Elektrizitätsverteilung sind von der Verstaatlichung gemischtwirtschaftliche Gesellschaften, an denen der Staat die Mehrheit der Eigentumsanteile hält und analoge Regiebetriebe, die sich aus den kommunalen Körperschaften konstituiert haben, sowie Verbrauchergenossenschaften ${ }^{104}$ ausgenommen worden. In bezug auf den Gesamtmarkt spielen die nicht verstaatlichten EVU keine Rolle.

Die Organisation der EdF ist in dem Nationalisierungsgesetz Art. 20-22 in den Grundzügen festgelegt worden. Demnach soll die EdF eine zentralistische, stark hierarchisch geprägte Organisationsstruktur aufweisen: Die Unternehmensführung unterliegt einem ursprünglich mit 18 Mitgliedern besetzten Verwaltungsrat ${ }^{105}$. Nach mehrfachen Änderungen in der Zahl besteht dieser Rat heute aus 15 Mitgliedern. ${ }^{106}$ Unterhalb der Unternehmensführung folgt die Direktionsebene. Die wichtigste Direktion ist die Direktion "de la production et du transport". Sie ist weiter in Abteilungen unterteilt. Die Abteilung für die hydraulische Produktion gliedert sich weiter in neun Arbeitseinheiten auf, die die Wasserkraftwerke der EdF betreiben. Die Abteilung fur die thermische Produktion unterteilt sich ebenfalls in neun Arbeitseinheiten.

102 Bis zu einer Leistung von 850 kVA. Vgl. Art. 8 des Nationalisierungsgesctzes. Gleichzeitig wurde in dem Gesetz die Entschädigung der Enteigneten geregelt. Vgl. weiter MARTIN, J.M. : Die französische Energiewirtschaft - Entwicklung und Perspektiven. in: ZfE Heft 3, 1990, S. 228

103 Einheit für die elektrische Scheinleistung einer Anlage.

104 Vgl. FIQUET, A. : a.a.O., S. 555

$105 \mathrm{Vgl}$. MÜLLENDORF, R. : Die französische Elektrizitätswirtschaft und der europăische Stromhandel, in: GLÜCKAUF 125 (1989), Nr. 21/22, S. 1351

106 Dekret vom 17.12.1953 
Sieben regionale Unterabteilungen der Stromtransportabteilung betreiben und unterhalten den ihnen zugewiesenen Abschnitt des französischen Stromtransportnetzes. Zusätzlich ist ein Bereich geschaffen worden, der den überregionalen Stromtransport koordiniert und verwaltet ${ }^{107}$.

Die Direktion für die Versorgung industrieller Großabnehmer ist neben ihrer Kernaufgabe auch für den Stromaustausch mit anderen europäischen Ländern und den Ankauf von elektrischer Energie von Eigenerzeugern zuständig.

Im Verteilungsbereich der EdF ist eine gemischte Direktion geschaffen worden, die neben dem Betrieb des Mittel- und Niederspannungsnetzes und der Versorgung der Endabnehmer auch Aufgaben für die französische Gasgesellschaft übernimmt. Die Direktion unterteilt sich in 18 regionale Abteilungen mit 99 Versorgungszentren. Unterhalb der Versorgungszentren existieren die Unterabteilungen, von denen es in Frankreich über 300 gibt und die sich wiederum in ca. 900 Bezirksverwaltungen untergliedern.

Die Tarifstruktur der EdF ist traditionell durch Kundengruppentarife geprägt.

Haushalten und Kleinabnehmern wird der sogenannte "tarif bleue" in Rechnung gestellt, der für Kunden mit einer Leistung kleiner als 36 kVA gilt. Die Preise weisen einen nach Leistung und Wahlmöglichkeit differierenden Grundpreis und einen variablen von der Tageszeit abhängigen Preis pro in Anspruch genommener kWh auf.

Sonderabnehmern wird der sogenannte "tarif jaune" berechnet. Er gilt für Abnehmer zwischen $36 \mathrm{kVA}$ und $250 \mathrm{kVA}$ und differenziert nach Verbrauch, Jahreszeit, Tageszeit und Auslastungsgrad der Angebotsseite. Er weist ebenso wie der "tarif bleue" eine Grundbetrag und einen Arbeitspreis auf.

Für Großabnehmer wurde bereits in den fünfziger Jahren der sogenannte "tarif verte" eingeführt. Großabnehmer ab einer Leistungsnachfrage von $1 \mathrm{MW}$ können diesen weiter unterteilten Tarif benutzen. Die Struktur ähnelt dem gelben Tarif. Die Intervalle der Berechnungsgrundlage des Arbeitspreises sind jedoch kleiner.

Die staatlichen Unternehmen in Frankreich unterliegen einer umfassenden Regulierung. Ziel der staatlichen Regulierung ist die Überwachung der Unternehmen in Hinblick auf die Ziele des Allgemeinwohles. Die Strategie zur Sicherstellung der staatlichen Ziele in den öffentlichen Unternehmen wird in Frankreich "planification" genannt. Sie setzt sich aus drei Bereichen zusammen:

a) Die „controle“

Als "controle“ wird eine Aufsichtsform des Verwaltungsrechts mit zahlreichen Eingriffsmöglichkeiten in die Unternehmensaktivitäten bezeichnet. Die „controle“ im Bereich der Geschäftsführung der EdF wird durch das Finanzministerium und

$107 \mathrm{Vgl}$. hierzu die Beschreibung in : EdF: entreprise nationale industrielle et comerciale, La

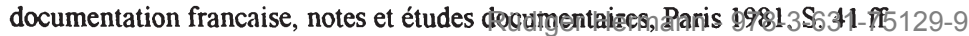


das Ministerium für Industrie und Forschung durchgeführt. Jeweils ein Vertreter der beiden Ministerien sind im Führungsgremium der EdF vertreten.

b) Der Fünf-Jahres-Plan

Ein weiteres Steuerungsinstrument des Staates für die EdF ist der französische Fünfjahresplan, der größere Kapazitätserweiterungen oder Ersatzinvestitionen der EdF festlegt. Die EdF arbeitet bei der Erstellung des Planes ihre Vorschläge aus und ergänzt diese um die Planungen der anderen in der Elektrizitätswirtschaft tätigen Unternehmen. Die Vorschläge werden anschließend von einer Arbeitsgruppe für die Energiepolitik überprüft und münden in den "rapport general du Plan"(Bericht des Gesamtplanes) ${ }^{108}$, der vom Parlament genehmigt werden muß. Zusätzlich muß die Regierung ihre Zustimmung für Investitionen in die Kernenergie geben.

\section{c) Verträge}

Ein weiterer Bereich der „planification“ sind die zwischen dem Staat und den öffentlichen Unternehmen geschlossenen Verträge. In dem derzeit gültigen Vertrag sind die allgemeinen Ziele der französischen Energiepolitik festgelegt und für den Elektrizitätsbereich näher präzisiert ${ }^{109}$. Als Besonderheit wurde die Maximierung des Stromexportes für die EdF in den Vertrag aufgenommen.

Eine weitere Form der staatlichen Kontrolle ist die Überwachung der EdF-Aktivitäten durch das französische Parlament. Darüber hinaus kann der Staat über die Vergabe von Konzessionen auf die Elektrizitätswirtschaft einwirken. Eine Konzession des Staates ist für die Errichtung einer Anlage zur Stromerzeugung oder Verteilung in Frankreich eine unabdingbare Voraussetzung. Mit dem Inanspruchnehmen der Konzession muß sich der Konzessionsnehmer zu technischen und wirtschaftlichen Bedingungen, die in den sogenannten "cashier des charges" festgelegt sind, verpflichten. Bestandteil der "cashier des charges“ sind Preisobergrenzen für den produzierten Strom und Abgaben an die französischen Gebietskörperschaften sowie technische Standards und die Übernahme der Versorgungspflicht für die Endabnehmer. Kernkraftwerke unterliegen einem gesonderten Genehmigungsverfahren.

Die Konzessionen haben eine bestimmte Laufzeit, nach deren Ende die errichtete Anlage an den Konzessionsgeber, also den Staat, fällt.

Der Neubau von Kapazitäten durch von der EdF unabhängige Erzeuger ist heute ausschließlich nach dem Gesetz vom 02.08.1949 möglich. Demnach benötigt der potentielle Erzeuger eine Genehmigung zur Errichtung einer Stromerzeugungsan-

108 Vgl. GINOCCHIO, R. : Législation de l'éctricité, Paris 1977, S. 79 f.

$109 \mathrm{Vgl}$. zu den Zielen der französischen Energiepolitik VILIAN, M. : La politique de l'energie en France, de la seconde guerre mondial a l'horizon 1985, Paris 1969, S. 101 und GILSBACH, A. : Die Konzeption der französisohen Energiepplitik \&Mïnchem 1965,9S. $61 \mathrm{f}$. 
lage. Die Genehmigung kann jedoch nur dann ausgesprochen werden, wenn die beabsichtigte Anlage der Deckung des Eigenbedarfes dient und eine Bestätigung der EdF vorgelegt wird, die nachweist, daß die beabsichtigte Anlage nicht den Planungen und Interessen der EdF zuwiderläuft. Ferner muß sich die EdF bereit erklären, die überschüssige Elektrizitätsmenge aufzukaufen.

Ein Schwerpunkt der staatlichen Einflußnahme auf die Elektrizitätswirtschaft bestand traditionell in der Preisgestaltung. Nach zahlreichen unbefriedigenden Erfahrungen mit einer auf Indexbindungen oder Höchstpreisen basierenden Preisregulierung wurde die grundsätzliche Freiheit der Preisbildung der EdF mit dem 01.12.1986 erlassen. Der Staat behält sich jedoch das Recht vor, durch Dekrete die Strompreise zu begrenzen, um hohe Inflationsraten, konjunkturelle Krisen und Notsituationen zu bekämpfen. So erfolgte am 29.07.1988 ein erstes Dekret, das die Entwicklung der Strompreise an die Gestehungskosten bindet. Die Gestehungskosten setzen sich aus den Kosten für Neuinvestitionen, den Betriebskosten der Anlagen und den Brennstoffkosten zusammen. Gemäß Artikel 4 des Dekretes wird die Entwicklung der Strompreise durch den Wirtschaftsminister jährlich neu geregelt. Die Preise für den An- und Verkauf elektrischer Energie durch die nicht nationalisierten Elektizitätserzeuger oder Verteilerunternehmen sind im Dekret vom 20.05.1955 gesetzlich geregelt.

\subsubsection{Die Struktur der italienischen Elektrizitätswirtschaft}

Prägendes Unternehmen der italienischen Elektrizitätswirtschaft ist die „Ente nationale per l'energia elettrica" (ENEL). Sie ist als voll integriertes EVU im Besitz von Erzeugungs-, Transport- und Verteilungskapazitäten ${ }^{110}$. Innerhalb der überwiegenden Anzahl der Versorgungsgebiete besitzt die ENEL das Verteilungsmonopol, welches mit der Versorgungspflicht gesetzlich verbunden ist. Die lokalen neben der ENEL bestehenden Verteilerunternehmen werden ausschließlich von der ENEL beliefert. Zusätzlich besitzt die ENEL das Import- und Exportmonopol für elektrische Energie über die italienischen Grenzen ${ }^{111}$. Die Durchleitungsmöglichkeiten durch das Netz der ENEL sind nach dem Gesetz zur Nationalisierung der Elektrizitätswirtschaft aus dem Jahre 1962 auf Lieferungen von Industrieeinspeisern oder Staatsunternehmen an eigene Unternehmen begrenzt worden. Seit dem Beginn der achtziger Jahre kann zusätzliche Energie aus regenerativen Energieträgern und Gemeinschaftskraftwerken durch das Transportnetz der ENEL geführt werden. Die Preise für die Durchleitung werden mit Hilfe einen fiktiven

$110 \mathrm{Vgl}$. ENTE NAZIONALE PER L'ENERGIA ELETTRICA: I Prezzi del l'energia elettrica in Italia - in vista delmercato mico europeo, Rom 1991

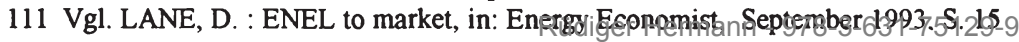


Übertragungsweges berechnet. Erster Transportpreisbestandteil ist der Transportpreis pro $5000 \mathrm{kWh}$. Dieser berechnet sich pro $100 \mathrm{~km}$ der jeweiligen Spannungsebene. Zweiter Bestandteil sind die angenommenen Verluste pro $100 \mathrm{~km}$ Leitungslänge. Dritter Preisbestandteil ist der Preis für die Transformation ebenfalls pro $5000 \mathrm{kWh}$ und den dabei anfallenden Verlusten. Die Verluste muß das den Transport beantragende Unternehmen ausgleichen oder bei der ENEL bezahlen.

Tabelle 4: Stromtransportpreise in der italienischen Elektrizitätswirtschaft

\begin{tabular}{|l|l|c|}
\hline Leitungen auf der & Transportpreis (Lit/kWh) & angenom. Verluste(\%) \\
\hline \hline $380 \mathrm{kV}$ Ebene & $0,9^{*} 5.000 / \mathrm{h}$ pro $100 \mathrm{~km}$ & 1 \\
\hline $220 \mathrm{kV}$ Ebene & $1,4^{*} 5.000 / \mathrm{h}$ pro $100 \mathrm{~km}$ & 2 \\
\hline $130 \mathrm{kV}$ Ebene & $3,2^{*} 5.000 / \mathrm{h}$ pro $100 \mathrm{~km}$ & 3 \\
\hline & & \\
\hline & & angenom. Verluste(\%) \\
\hline Transformation & Preis (Lit/kWh) & 1 \\
\hline Hoch-/Hochspannung & $1,0^{*} 5.000 / \mathrm{h}$ & 1 \\
\hline Hoch-/Mittelspannung & $1,0^{*} 5.000 / \mathrm{h}$ & \\
\hline
\end{tabular}

Quelle: VIK Dokumentation Nr. 200 Mai 1991 " Durchleitung als Wettbewerbsinstrument in der Stromversorgung" Essen 1991, S. 17

Intern unterteilt sich die ENEL in drei Organe - den Präsidenten, dem Verwaltungsrat und das Prüferkollegium. Unterhalb dieser drei Organe ist die ENEL in zwei Sparten - die Generaldirektion und die Spezialdienste - unterteilt. Hierarchisch unterstellt sind der Generaldirektion die Zentraldirektion und die nach geographischen Gesichtspunkten unterteilten Abteilungen. Die Abteilungen untergliedern sich weiter in Distrikte, Teildistrikte und Zonen ${ }^{112}$.

Die ENEL war bis 1992 eine Körperschaft des öffentlichen Rechtes ${ }^{113}$, welche der staatlichen Kontrolle unterlag. Das Betriebsvermögen der ENEL gehörte dem italienischen Staat sowie den Regionen und den Kommunen. Die ENEL handelte geschäftlich jedoch als privatrechtliche juristische Person. 1992 wurde nach Parlamentswahlen ein Regierungsbeschluß getroffen, die Staatskonzerne auf ihre Privatisierungsmöglichkeiten zu überprüfen. Die Staatsunternehmen, unter ihnen auch

112 Vgl. Art. 11 des ENEL Statuts

113 Vgl. D'ERNO, V. : Die italienische Energiewirtschaft - Entwicklung und Aussicht, in: ZfE $1 / 90$, S. 19 
die ENEL, hatten eine Grundsatzerklärung zur Privatisierung ihres jeweiligen Unternehmens abzugeben. Ausgegangen wurde von einer Umwandlung der Staatsbetriebe in selbständige Aktiengesellschaften. Die ENEL sprach sich für ihre Privatisierung unter der Bedingung aus, daß der Staat vorher die Verbindlichkeiten in Höhe von 43 Mrd. DM übernähme ${ }^{114}$. Im Dekret Nr. 333 vom 11.07.1992 wurde die Umwandlung der ENEL in eine Aktiengesellschaft verkündet, wodurch die ENEL in Zukunt dem italienischen Privatrecht unterliegt ${ }^{115}$. Die Aktien der ENEL befinden sich zur Zeit noch in Staatsbesitz ${ }^{116}$. Mit der praktische Umsetzung der Privatisierungsüberlegungen konnte frühestens 1994 begonnen werden ${ }^{117}$, da das italienische Recht eine Privatisierung erst nach zwei geprüften Jahresabschlüssen mit Gewinnen vorsieht. Bei dem Verkauf der Aktien strebt die italienische Regierung eine weitgehende Streuung der Aktien $a^{118}$. Aktiv unterstützt wurden die Privatisierungsabsichten durch die öffentliche Meinung in Italien. Bisher ist es zu dem Verkauf jedoch noch nicht gekommen.

Neben der ENEL bestehen 40 eigenbetriebliche und 102 kommunale EVU. Die 40 eigenbetrieblichen Unternehmen sind gemäß dem königlichen Dekret vom 15.10.1925 $5^{119}$ organisiert. Die anderen $102 \mathrm{EVU}$ werden von ihren Gemeinden ohne rechtliche und organisatorische Trennung betrieben ${ }^{120}$. Außerdem existieren 497 Klein-EVU in Privateigentum. Sie wurden 1962 von der Nationalisierung ausgenommen, weil ihre Erzeugungsmenge zwischen 1959 und 1962 weniger als 15 Mio kWh jährlich betrug. Die lokalen EVU, die gemäß Art. 4 Nr. 5 des Gesetzes vom 06.12.1962 eine Ausnahme bei der durchgeführten Verstaatlichung bilden, verbleiben in kommunalem Besitz ${ }^{121}$. Veränderungen bei den Klein- EVU sind nicht geplant. Sie bleiben weiter im Privatbesitz in den Rechtsformen der GmbH, der AG, der Genossenschaft und der Personengesellschaft bestehen ${ }^{122}$.

114 Die für ein Unternehmen in dieser Größe überzogenen Verbindlichkeiten resultieren teilweise aus staatlichen Interventionen, z.B. den regionalen Förderprogrammen.

115 Vgl. LANE, D. : a.a.O., S. 15

116 Vgl. LAMBRECHT, F. : Transmission and Distribution in Liberalized Power Markets, in: ET 45. Jg. (1995), Heft 1 u. 2, S. 105

117 Die Privatisierungspläne gingen von einem Beginn im Oktober 1994 aus. Der Zeitplan konnte jedoch nicht eingehalten werden, so daß mit dem Verkauf der Masse der Aktien im Laufe des Jahres 1995/96 gerrechnet werden muß.

118 Vgl. LANE, D. : a.a.O., S. 15

119 Trotz der zu dieser Zeit in Italien regierenden Faschisten unter Mussolini wurden die Gesetze und Dekrete noch im Namen des italienischen Königs herausgegeben. Vgl. hierzu DTV-Atlas zur Weltgeschichte, Band 2, 8. Auflage München 1983, S. 159

120 Vgl. EISS, H. ; LUKES, R. ; PICK, H. ; SCHULZ, W. : a.a.O. , S. 541

121 Vgl. EISS, H. ; LUKES, R. ; PICK, H. ; SCHULZ,W. : a.a.O., S. 551

122 Vgl. BACKHAUS, L. : Verstaatlichung von Wirtschaftsunternehmen, Köln. Berlin, Bonn, München 1978, S. $30 \mathrm{f}$. 
$\mathrm{Zu}$ den Eigenerzeugern gehören in Italien im wesentlichen Unternehmen der chemischen und metallurgischen Industrie. Eigenerzeuger nach dem Gesetz vom 06.12.1962 Art.4 Nr.6 ist, wer einen Eigenverbrauchsanteil von mindestens 70\% aufweist ${ }^{123}$ und seiner geringen Bedeutung wegen nicht verstaatlicht wurde.

In den italienischen Stromtarifen werden Haushalts-, Industrie-, Kleinverbraucher-, Selbsterzeuger, Weiterverteiler und Beleuchtungskunden unterschieden ${ }^{124}$. Alle Tarife werden öffentlich bekannt gemacht und gelten für das gesamte Land. Die Tarife weisen zumeist eine zweigeteilte Struktur auf.

Die ENEL unterliegt, obwohl sie traditionell selbständig handelte, der staatlichen Regulierung.

Mit der Kontrolle ist heute noch das CIPE (Comitao Interministeriale per la Programmazione Economico) und der Minister für Industrie, Handel und Kunsthandwerk $^{125}$ beauftragt. Das CIPE besteht aus dem Ministerpräsidenten und verschiedenen Staatsministern. Das Industrieministerium ist Genehmigungs- und Überwachungsbehörde. Dessen Preisaufsicht beurteilt und kontrolliert die realen tarifären Abnahmebedingungen auf ihre Vereinbarkeit mit den Richtlinien der CIPE. Das Industrieministerium überwacht ferner die Höchstpreise. Hiervon sind insbesondere die Verbraucher- und Sonderabnehmertarife und der Strombezugspreis der ENEL von nicht nationalisierten Unternehmen sowie die Tarife für die Durchleitung elektrischer Energie betroffen. Weitere Aufsicht wird in Zusammenhang mit speziellen Subventionsprogrammen von den zuständigen Ministerien auf die Elektrizitätsunternehmen durchgeführt. Anläßlich der Privatisierungspläne wurde der Ersatz der Höchstpreisregulierung durch eine Bindung der Elektrizitätspreise an die Entwicklung der Verbraucherpreise vorgeschlagen. Der Vorschlag wurde bisher jedoch nicht umgesetzt.

Ein besonderes Problem für die italienische Elektrizitätswirtschaft stellt die steigende Elektrizitätsnachfrage dar. Die bestehenden Kraftwerken sind zu einem Teil veraltet und die Gesamterzeugung kann in den bestehenden Kraftwerke nicht erhöht werden. So müssen bis zum Jahr 2000 neue Kraftwerke gebaut werden. In der öffentlichen Diskussion sind die Kraftwerkstypen und ihre Standorte umstritten. So versucht die ENEL kurzfristig über erhöhte Stromimporte die gestiegene Nachfrage zu befriedigen. Am 21.12.1988 wurde durch das CIPE ein Notprogramm genehmigt, welches den Bau kleinerer Gasturbinenkraftwerke und die Erweiterung der bestehenden thermischen Kraftwerke vorsieht ${ }^{126}$. Die Präferenz für Gaskraftwerke in dem Notprogramm resultierte aus der öffentlichen Meinung, die Kraft-

$123 \mathrm{Vgl}$. ENEL-Bericht 1985

124 Vgl. ENTE NAZIONALE PER L'ENERGIA ELETTRICA: a.a.O.

125 Vgl. EISS H. , LUKES, R. ; PICK, H. ; SCHULZ, W. : a.a.O., S. 548

126 Vgl. D'ERNO, V. : a.a.O., S. 27

Rüdiger Hermann - 978-3-631-75129-9 
werksstandorte anderer konventioneller Energieträger aus Umweltschutzgründen ablehnt. Massiver Protest der Öffentlichkeit richtete sich gegen den Ausbau und die Nutzung der Kernkraft, so daß diese zur zukünftigen Bedarfsdeckung in Italien nicht herangezogen werden kann.

\subsubsection{Elektrizitätswirtschaften mit mehrstufigen Strukturen und geschlossenen Versorgungsgebieten}

Die Elektrizitätswirtschaften dieser Kategorie weisen unterschiedliche Strukturen und eine Vielzahl von Unternehmen auf. Gemeinsam verfügen sie über geschlossene Versorgungsgebiete in der Endabnehmerversorgung und integrierte mehrstufige Organisationsformen. Die Anzahl der Versorgungsstufen variiert dagegen. Die Integration wird zumeist über Verträge realisiert. Die Besitzverhältnisse und die Eigentumsanteile an den EVU weichen in den Mitgliedsstaaten dieser Gruppe deutlich voneinander $a b$. Zumeist ist eine nationale Gebietskörperschaft an den EVU beteiligt. Wettbewerb in der Endabnehmerversorgung besteht in diesen Elektrizitätswirtschaften nicht. Wettbewerb um die Belieferung von Verteilerunternehmen durch Erzeuger ist beschränkt nur in Spanien vorhanden ${ }^{127}$. Weitere Unterschiede bestehen hinsichtlich der rechtlichen Rahmenbedingungen ihrer Organisationsformen und ihrer staatlichen Regulierung. In diese Gruppe können die Elektrizitätswirtschaften Belgiens, der Bundesrepublik, Dänemarks, Spaniens, Irlands, Portugals und der Niederlande eingeordnet werden.

\subsubsection{Die Struktur der deutschen Elektrizitätsversorgung}

Innerhalb der öffentlichen Elektrizitätsversorgung ${ }^{128}$ der Bundesrepublik Deutschland lassen sich drei vertikal untereinander verknüpfte Marktstufen unterscheiden.

a) Die Interregionalstufe,

b) Die Regionalstufe,

c) Die lokale Stromversorgung ${ }^{129}$.

127 EISS et al : a.a.O., S. $635 \mathrm{f}$.

128 Es gibt darüber hinaus in der Bundesrepublik die industrielle Kraftwirtschaft und die Elektrizitătserzeugung der Deutschen Bundesbahn. Beide sind jedoch nicht Bestandteil ordnungspolitischer Veränderungen. 
a) Die Interregionalstufe

Auf der Interregionalstufe existieren acht Unternehmen, die sich zur Deutschen Verbundgesellschaft (DVG) zusammengeschlossen haben ${ }^{130}$. Die EVU besitzen ein miteinander verbundenes Hochspannungsnetz der 380- und 220-kV-Ebene zum überregionalen Stromtransport. Ziel des Zusammenschlusses ist die Koordination des überregionalen Stromaustausches und der abgestimmte Ausbau und Betrieb der unternehmenseigenen Netze sowie der Erzeugungsanlagen. Das deutsche Verbundsystem der DVG befindet sich auf der Stufe des Elektrizitätsverbundsystems, d.h. es werden Absprachen über die gemeinsame Nutzung von Hochspannungsleitungen, den Import- und Export von elektrischer Energie sowie über Investitionsvorhaben getroffen. Die einzelnen Unternehmen besitzen die vollständige Eigenständigkeit und betreiben ihr Netz eigenverantwortlich.

Die Unternehmen der Interregionalebene unterscheiden sich hinsichtlich des vertikalen Integrationsgrades. Einzelnen EVU wie das RWE oder die VEW sind auf allen Marktstufen von der Stromerzeugung bis zur lokalen Verteilung tätig. Andere Unternehmen der Interregionalstufe beliefern vorwiegend oder ausschließlich Unternehmen der Regionalebene oder kommunale Verteilerunternehmen, nicht jedoch Letztabnehmer ${ }^{131}$.

Seit 1950 werden in der Bundesrepublik Deutschland Durchleitungen über das Versorgungsgebiet eines anderen EVU der Interregionalstufe hinweg vorgenommen. Erforderlich wurden diese Durchleitungen durch territorial nicht zusammenhängende Versorgungsgebiete einzelner EVU und der Wiederaufnahme der Lieferbeziehungen zu den Alpenländern.

\section{b) Die Regionalstufe}

Die Versorgung auf der Regionalebene wird von Unternehmen durchgeführt, die über Mittel- und Niederspannungsnetze zum regionalen Transport elektrischer Energie verfügen. Die Unternehmen haben sich zur Arbeitsgemeinschaft regionaler Energieversorgungsunternehmen e.V. (ARE) zusammengeschlossen ${ }^{132}$. Die Arbeitsgebiete der regionalen EVU erstrecken sich, mit Ausnahme der Stadtstaaten, über sämtliche Bundesländer. Sie beliefern überall dort die Endverbraucher oder kommunalen Verteilerunternehmen, wo die Unternehmen der Interregionalebene keine Endverbraucherversorgung durchführen ${ }^{133}$.

129 Vgl. WAGNER, G. : Abbau regionaler Strompreisdisparităten, Studie der Bundesanstalt für Landeskunde und Raumordnung, Bonn 1985, S. 63

130 Nach dem Zusammenschluß des Badenwerkes mit der Energieversorgung-Schwaben reduziert sich die Zahl auf acht Unternehmen.

131 Vgl. GRONER, H. : Die Ordnung der deutschen Elektrizitătswirtschaft. Baden-Baden 1975, S. 93

$132 \mathrm{Vgl}$. ARBEITSGEMEINSCHAFT REGIONALER ENERGIEVERSORGUNGSUNTERNEHMEN - ARE-e.V. : Tătigkeitsbericht 1986-1987, Hannover 1988, S. 2 ff. . Vgl. dazu auch die selbstgestellten Aufgaben der ARE im Tătigkeitsbericht S. 228 f. .

133 Vgl. WAGNER, G. : a.a.O., S. 64 
c) Die lokale Stromversorgung

Die unterste vertikale Stufe der Elektrizitätsversorgung wird von den Unternehmen der lokalen Stromversorgung gebildet. Sie befinden sich zumeist in Gemeindebesitz und betreiben ein Niederspannungsnetz zur Belieferung der Endverbraucher mit elektrischer Energie. Häufig verfuggen diese Unternehmen über keine eigenen Erzeugungskapazitäten. Sie beziehen ihren Strom von den Unternehmen der Regional- und Interregionalebene.

Gebietsschutzverträge teilen das Bundesgebiet in abgegrenzte Versorgungsgebiete auf, in denen ein EVU das ausschließliche Versorgungsmonopol besitzt. Die Gebietsschutzverträge lassen sich in Konzessionsverträge und die unterschiedlichen Formen der Demarkationsverträge unterteilen. Sie bilden die Voraussetzung zur Ausschaltung des Wettbewerbs auf dem deutschen Strommarkt ${ }^{134}$.

Konzessionsverträge werden zwischen einer Gebietskörperschaft und einem EVU geschlossen. Die Gemeinde oder die Stadt räumt dem EVU das ausschließliche Recht ein, den kommunalen Verkehrsraum und kommunale Grundstücke zur Verlegung von Stromleitungen zu benutzen ${ }^{135}$. Als Gegenleistung zahlt das Unternehmen Abgaben an die Kommune. Die Laufzeit dieser Verträge ist heute auf 20 Jahre beschränkt worden ${ }^{136}$.

Der lückenlose Schutz der EVU vor Wettbewerb wird durch das Systems von Demarkationsverträgen erzielt. Man unterscheidet zwei Typen von Demarkationsverträgen - die sogenannten A- und B-Verträg $\mathrm{e}^{137}$. Bei den A-Verträgen handelt es sich um vertikale Stromliefer- und -bezugsabsprachen über die Art und Tiefe des Stromaustausches. Dabei wird weiter nach dem Abgrenzungskriterium der Versorgungsgebiete in die geographische Demarkationsabrede und die Abnehmerdemarkation unterschieden. Bei der geographischen Demarkation verpflichtet sich das strombeziehende EVU, den gelieferten Strom ausschließlich für die Verteilung im eigenen Versorgungsgebiet zu verwenden, d.h. einen Verkauf in andere Versorgungsgebiete oder den Verkauf an andere EVU zu unterlassen. Das stromliefernde EVU verpflichtet sich, keine Endverbraucher im Versorgungsgebiet des strombeziehenden EVU zu beliefern. Bei der Abnehmerdemarkation, die über Grenzmen-

134 Vgl. BUDERATH, J. : Die Ordnungspolitik in der deutschen Elektrizitătswirtschaft, Bad Homburg 1962, S. 6 ff.

135 Zur Entwicklung der Konzessionsverträge in Deutschland vgl. HÜFFER, U. ; TETTINGER, J. : Rechtsfragen beim Versorgerwechsel nach Ablauf von Konzessions-vertrăgen, Stuttgart, München, Hannover, Berlin 1992, S. 25 ff.

136 Vgl. WAGNER, G. : a.a.O., S. 66. Die Konzessionsabgaben sind in der Bundesrepublik Deutschland in die energiepolitische Diskussion gelangt. Von verschiedenen Kreisen ist eine Reform gefordert worden. Vgl. hierzu BRAUN, D. : Reform des Konzessionsabgabewesens, in: ET 41. Jg. (1991), S. 456 ff. und CRONENBERG, M. : Reform der Konzessionsabgaben, in: ET 41. Jg. (1991), Heft 7, S. 450 ff.

137 Vgl. WAGNER, G. : a.a.O., S. 67 vgl. auch weiter SCHIFFER, H.W.: Stabile Preise in Sicht, in: ET 6/86, S. $484 \mathrm{ff}$. 
genabkommen realisiert werden, behält sich das stromliefernde EVU das Recht vor, Abnehmer einer bestimmten Größe im Versorgungsgebiet des strombeziehenden EVUs unmittelbar zu versorgen ${ }^{138}$. In den sogenannten B-Verträgen verpflichtet sich eine Gemeinde, die Versorgung von Endabnehmern in ihrem Gemeindegebiet zu unterlassen. Zwischen den Unternehmen der Interregionalebene sind weitere Verträge zur Einschränkung des Wettbewerbs getroffen worden. Durch wechselseitige Verträge haben die Unternehmen die Bundesrepublik in "Interessengebiete" aufgeteilt und Aufnahmekriterien zur DVG aufgestellt.

Nach den Eigentumsverhältnissen werden die EVU in Unternehmen der öffentlichen Hand, in gemischtwirtschaftliche und in private Unternehmen unterteilt ${ }^{139}$. Als Unternehmen der öffentlichen Hand werden solche mit einem Anteil der öffentlichen Hand von mehr als $95 \%$ bezeichnet. Private Unternehmen weisen einen Anteil privater Kapitalgeber von mehr als $75 \%$ auf. Unternehmen mit Eigentumsverhältnissen zwischen diesen beiden Fällen werden den gemischtwirtschaftlichen zugerechnet.

\section{Abbildung 6: Öffentliche Elektrizitätsversorgung in der Bundesrepublik Deutschland}

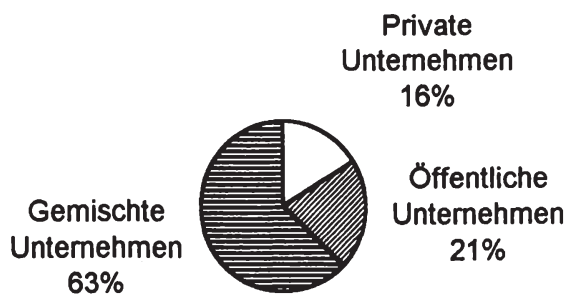

Quelle : VDEW 1994

In der deutschen Elektrizitätswirtschaft werden die Preise und die Vertragsgestaltung zwischen EVU und Verbrauchern nach den Abnehmergruppen unterschieden. 
Man differenziert zwischen Sonder- und Tarifabnehmern ${ }^{140}$. Sonderabnehmer untergliedern sich weiter in vier Kategorien ${ }^{141}$ :

a) Weiterverteilende EVU,

b) Industrielle Großabnehmer mit einem Anschluß an das Hochspannungsnetz,

c) Mittelständische Industrie- und Gewerbebetriebe mit Anschluß an das Mittelspannungsnetz,

d) Nachtstromspeicherheizungs- und Wärmepumpenbesitzer .

Tarifabnehmer sind alle Abnehmer, die keine Sonderabnehmer sind, insbesondere Haushaltsabnehmer und Kleingewerbetreibende. Während Sonderabnehmer die Lieferbedingungen und -preise mit ihrem zuständigen EVU frei aushandeln können, sind die Tarifabnehmer an standardisierte Verträge und Tarife gebunden. Die Stromtarife und Preise für Tarifkunden sind veröffentlichungspflichtig.

Im Rahmen der staatlichen Regulierung ${ }^{142}$ muß der Marktzutritt eines EVUs vom Wirtschaftsminister genehmigt werden. Die Genehmigung kann nur verweigert werden, wenn die Aufnahme der Versorgung negative Auswirkungen auf die Sicherheit und Preisgünstigkeit der Stromversorgung hat. Die deutsche Regulierung sieht ferner eine Preisaufsicht vor. Nach der Bundestarifordnung für Elektrizität müssen Tarifänderungen der Preisbehörde vorgelegt werden. Die Anhebung der festgelegten Höchstpreise bedarf der Genehmigung des Wirtschaftsministeriums und wird nur erteilt, wenn das beantragende EVU die Anhebung des Preises mit der Kosten- und Erlösentwicklung stichhaltig begründen kann. Die Genehmigung ist befristet und kann widerrufen werden.

140 Vgl. SCHIFFER, H.-W. : Preisbildung in der Energiewirtschaft, in: ET 41. Jg. (1991), Heft 12, S. 817 und SCHIFFER, H.-W. : Energiemarkt Bundesrepublik Deutschland, Köln 1988, S. $124 \mathrm{ff}$.

141 Vgl. EISS, H. et al : a.a.O., S. 224

142 Zum aktuellen Stand der Regulierung in der deutschen Elektrizitätsıvirtschaft vergleiche LEWINGTON, I. ; WEISHEIMER, M. : Zur Regulierung in der deutschen Elektrizitătswirtschaft - Eine Analyse anhand neuer ökonomischer Ansätze und praktischer Erfahrungen, in:

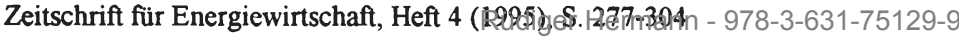




\subsubsection{Die Struktur der niederländischen Elektrizitätswirtschaft}

\section{Abbildung 7: Die niederländische Elektrizitätsstruktur}

\section{Erzeugung Stromtransport $\quad$ Verteilung}

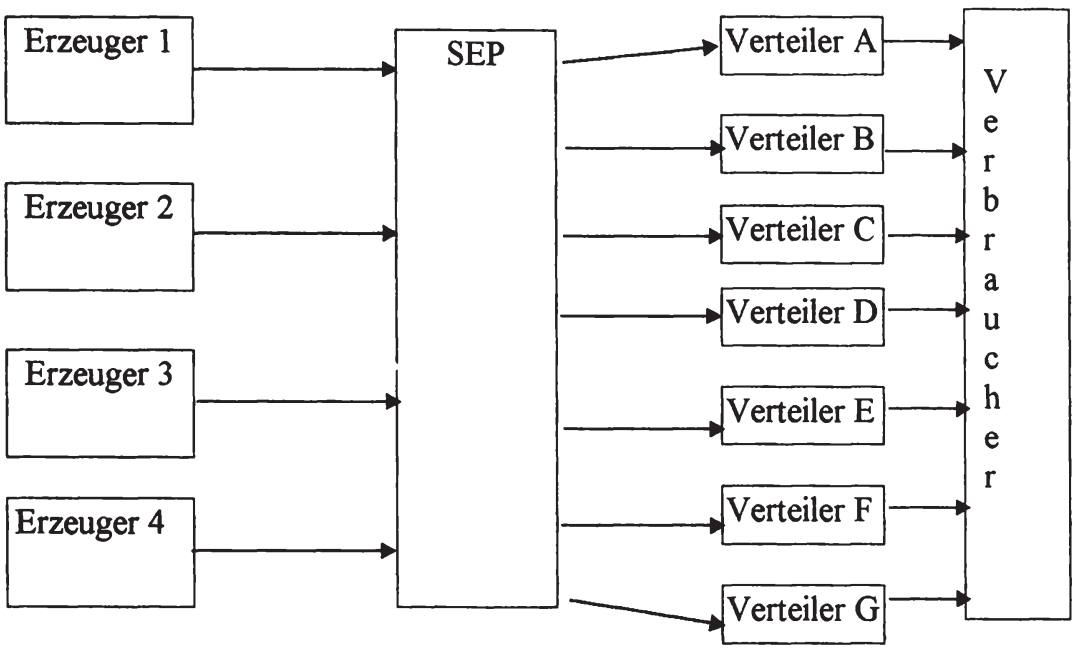

Die niederländische Elektrizitätswirtschaft weist eine dreigeteilte Struktur auf ${ }^{43}$.

In der Stromerzeugung sind zur Zeit vier Unternehmen tätig. Die Erzeuger sind regional konzentriert und befinden sich überwiegend in kommunalem Besitz ${ }^{144}$. Der Transport der elektrischen Energie über das 380/220-kV-Netz wird von der $\mathrm{SEP}^{145}$ als Eigentümerin des Netzes ${ }^{146}$ durchgeführt. Anteilseigner können satzungsgemäß öffentliche Gebietskörperschaften oder Rechtspersönlichkeit besitzende, privatrechtliche Körperschaften werden, die in der Elektrizitätsversorgung tätig sind ${ }^{147}$. Hierbei handelt es sich um die vier großen Erzeugungsunternehmen.

143 Vgl. DEREGULIERUNGSKOMMISSION : a.a.O., S. 5

144 Vgl. PRIECKAERTS, J.M. :a.a.O., S. 384

145 NV Samenwerkende elektriciteits-produktie betrijven, gegründet 1949

146 Vgl. GRÜNDLING, F-J. ; PICK H. : Die europăische Energiewirtschaft vor dem Binnenmarkt, in:ZfE 1/90 S. 1-15, hier S. 11

147 Năhere Ausführungen siehe SCHMITZ, E. : Die Anwendung der Wettbewerbsregeln des

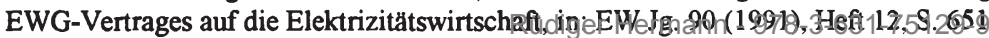


Über den Stromtransport hinaus hat die SEP die Aufgabe, einen gemeinsamen nationalen Stromplan zu erstellen und die aus dem Verbundbetrieb der "I'Union pour la Coordination de la Production et du Transport de l'Electricité" (UCPTE) erwachsenden Aufgaben zu erfüllen. Dadurch ist sie gleichzeitig auch für den Stromaußenhandel der Niederlande zuständig ${ }^{148}$. Ein weiteres wesentliches Tätigkeitsfeld der SEP ist der Einkauf der zur Verstromung benötigten Primärenergieträger auf den vorgelagerten Marktstufen. Ferner trägt sie durch koordinierende und beratende Tätigkeiten zur Optimierung der nationalen Elektrizitätsversorgung bei ${ }^{149}$. In der Stromverteilung und der Endabnehmerversorgung sind kommunale Unternehmen mit einem geschlossenen Versorgungsgebiet tätig. Die Verteilerunternehmen sind zum Anschluß jedes Verbrauchers in ihrem Versorgungsgebiet verpflichtet, es sei denn der Anschluß ist dem Verteilerunternehmen aus wirtschaftlichen Gründen nicht zuzumuten ${ }^{150}$.

Die faktische Integration der niederländischen Elektrizitätswirtschaft wird über Verträge erreicht. Die Verträge werden zwischen Erzeuger und Verteilern, die auch die niederländischen Großabnehmer beliefern, geschlossen. Bei den Verträgen zwischen Erzeugern und Verteilern verpflichtet sich der Erzeuger keinen Abnehmer (Großabnehmer) im Verteilungsgebiet des Verteilungsunternehmens mit Strom zu beliefern. Die weitere Integration in bezug auf die Großabnehmer erfolgt in der niederländischen Elektrizitätsversorgung indirekt. Der einzelne Großabnehmer hat drei Alternativen:

a) den Bezug der benötigten elektrischen Energie von seinem Verteilerunternehmen,

b) den Strombezug aus dem Ausland über die SEP,

c) die Eigenerzeugung.

Verbleiben die industriellen Großabnehmer bei ihrem Verteilungsunternehmen, wird ihnen durch die von der VEEN ${ }^{151}$ vorgeschlagenen und von ihren Mitgliedern angewandten allgemeinen Geschäftsbedingungen ein Eigenerzeugungs- und ein Weiterveräußerungsverbot auferlegt ${ }^{152}$. Der zusätzliche Strombezug von einem Dritten ist ebenfalls ausgeschlossen. Für die zweite Alternative, dem Strombezug aus dem Ausland, sind ausreichende Leitungskapazitäten der SEP erforderlich.

148 Dieses Ausschließlichkeitsrecht beim Stromaußenhandel ist der Anlaß eines umfassenden Rechtsstreits vor dem Europäischen Gerichtshof gewesen. Vgl. hierzu: HANCHER, L. : Die Almelo-Entscheidung des Europäischen Gerichtshofs - Hintergrund, Bewertung, mögliche Auswirkungen, in: EW, 45.Jg. (1995), Heft 1/2, S. 68-76, hier S. 68

149 Vgl. PRIECKAERTS, J.M. : a.a.O., S. 385

150 Art. 12 Abs. 4 Elektriciteitswet 1989

151 Verenigung van Exploitanten van Elektriziteitsbedrijven in Nederland (Zusammenschluß der niederländischen EVU)

152 Vgl. SCHMITZ, E. : a.a.O., S. 651 Rüdiger Hermann - 978-3-631-75129-9 
Zudem hat die SEP ein Anrecht auf ein „angemessenes Durchleitungsentgelt“. Deshalb ist bisher von den seit 1989 bestehenden Möglichkeiten zum Stromimport für Großabnehmer kein Gebrauch gemacht worden. Ein direkter Wettbewerb der Erzeuger um die Großabnehmer findet nicht statt.

In den niederländischen Stromtarifen wird zwischen Kleinverbraucher und Sonderabnehmer unterschieden. Kleinverbraucher sind alle privaten Haushalte und gewerbliche Kunden der EVU. Die Tarife weisen eine zweigeteilte Struktur mit einem Fixkostenanteil für den Anschluß und die Zählermiete sowie einem variablen Anteil für die verbrauchte $\mathrm{kWh}$ auf. Niederländische Sonderabnehmer werden in drei Unterkategorien unterteilt: Großabnehmer, Mittelspannungsgroßabnehmer und Hochspannungsgroßabnehmer. Großabnehmer sind Verbraucher ab einer Abnahmeleistung von $50 \mathrm{kVA}$ und einer jährlichen Stromentnahme von mehr als $100.000 \mathrm{kWh}$. Mittelspannungsgroßabnehmer als zweite Unterkategorie verfügen über eine Anschlußleistung kleiner als $5 \mathrm{MW}$ und werden von den EVU mit einer eigenen Tarifstrukur bedient. Völlig frei ist die Preis- und Tarifgestaltung in den Niederlanden für sogenannte Giganten, das sind Hochspannungsabnehmer mit einer Anschlußleistung größer als $5 \mathrm{MW}^{153}$.

Durch das Elektrizitätsgesetz von 1989 reduzierten sich die regionalen Strompreisdisparitäten und es kam zu Kostensenkungen ${ }^{154}$, die die Preissenkungen durch die Verbilligung der Primärenergieträger unterstützten. In den niederländischen Strompreisen sind keine Konzessionsabgaben enthalten, da diese in den Niederlanden nicht erhoben werden. Einige EVU müssen jedoch spezielle Umweltabgaben an die Kommunen bezahlen, die auf den Strompreis verteuernd wirken.

Die niederländische Regulierung umfaßt folgende Instrumente:

\section{a) Der nationale Elektrizitätsplan}

Die SEP erstellt jährlich einen nationalen Elektrizitätsplan, der die Einzelplanungen der Verteiler- und Erzeugerunternehmen berücksichtigt und die Vorhaben der SEP wiedergibt ${ }^{155}$. Der Plan ist vom Wirtschaftsminister genehmigen zu lassen.

b) Die Genehmigungspflicht von Anlagen zur Elektrizitätserzeugung und -verteilung

Zum Bau und Betrieb von Anlagen zur öffentlichen Elektrizitätserzeugung über 5 MW ist eine Konzession erforderlich ${ }^{156}$. Ausgenommen wurden die Anlagen mit

153 Vgl. EISS et al : a.a.O. S. $436 \mathrm{ff}$.

154 Vgl. ZIJ, G. : Geplanter Markt oder Monopol mit Wettbewerb? - Eine Bilanz der niederlăndischen Losung, in: ET 43. Jg. (1993), Heft 10, S. 674

$155 \mathrm{Vgl}$. Art. 12 und 44 Elektriciteitswet

156 Vgl. Art. 2 Elektriciteitswet 1989 
einer Leistung kleiner als 5 MW und dezentrale Erzeugungsanlagen. Die dezentralen Erzeugungsanlagen unterliegen jedoch einer Zustimmungspflicht der SEP, wenn die an einem Standort errichteten Anlagen eines Unternehmens zusammen mehr als $25 \mathrm{MW}$ überschreiten ${ }^{157}$. Die Konzession an ein Unternehmen kann vom Wirtschaftsminister aufgehoben werden, wenn das Unternehmen kein Kraftwerk betreibt ${ }^{158}$.

\section{c) Die Preisregulierung}

In dem Elektrizitätsgesetz ${ }^{159}$ ist der SEP die Lieferung von Elektrizität an Konzessionäre ohne die vorherige Preisgenehmigung untersagt worden. Gleichzeitig wurden einheitliche Tarife für die Stromlieferungen der Konzessionäre an die SEP festgelegt ${ }^{160}$. Für die Verteilerunternehmen sind Höchstpreise zur Belieferung ihrer Abnehmer auf Vorschlag der VEEN vom Wirtschaftsminister erlassen worden. Dieser kann die Tarife für die Stromlieferungen an die SEP und die Höchstpreisvorschläge für die Verteilungsunternehmen der VEEN ablehnen, wenn sie dem Ziel einer ausreichenden nationalen Elektrizitätsversorgung widersprechen oder $\mathrm{Ab}$ nehmergruppen andere quersubventionieren sollen oder müssen ${ }^{161}$. Unter besonderen Voraussetzungen kann einzelnen Verteilerunternehmen eine Freistellungen von den Höchstpreisen erteilt werden ${ }^{162}$.

\subsubsection{Elektrizitätswirtschaften mit desintegrierten Strukturen und Wettbewerb in der Elektizitätsversorgung - Die Struktur der britischen Elektrizitătswirtschaft}

Bisher einziges Mitgliedsland mit einer desintegrieten Struktur in der Elektrizitätsversorgung ist Großbritannien. Die desintegrierte Struktur beschränkt sich auf England und Wales. In Schottland und Nordirland existieren geschlossene Versorgungsgebiete mit jeweils einem mit einem Monopol in der Elektrizitätsversorgung ausgestatteten Unternehmen. Teilweise sind die schottischen EVU bereits organisatorisch und rechtlich entbündelt worden ${ }^{163}$.

157 Vgl. Art. 40 Abs. 1 Elektriciteitswet 1989

158 Vgl. Art. 6 Elektriciteitswet 1989

159 Vgl. Art. 25 Abs. 1 Elektriciteitswet 1989

$160 \mathrm{Vgl}$. Art. 25 Abs. 2 Elektriciteitswet 1989. Grundlage der traditionellen Preisregulierung ist das Prijzenwet vom 24.03.1961, welches mehrfach modifiziert wurde.

161 Vgl. Art. 28 Abs. 1 Elektriciteitswet 1989

162 Vgl. Art. 33 Elektriciteitswet 1989

163 Vgl. BOULDING, P.G. : Die Preisentwicklung in der britischen Elektrizitätswirtschaft, in: Zeitschrift für Energiewirtschaft, Heft 4 (1995) S. 2826-394, hier/8-32581-75129-9 
"A vertically integrated, administered generation and transmission structure was replaced over-night (on March 1990) by one that ist de-integrated and markt-based ..."164 Durch die Umstrukturierung ist eine vollständig desintegrierte Marktstruktur mit zwei Marktstufen geschaffen worden. Die erste Marktstufe ist der Großhandelsmarkt; die Endabnehmerversorgung stellt die zweite Marktstufe dar.

Durch die Desintegration sind getrennte Unternehmen in

- der Elektrizitätserzeugung,

- dem Elektrizitätstransport,

- der Elektrizitätsverteilung,

- der Endversorgung von Abnehmern

entstanden.

Zur Koordination des Gesamtsystems wurde ein Pool installiert, der die Laststeuerung und weitere Aufgaben übernimmt ${ }^{165}$.

Im Stromerzeugungssektor wurde das vor der Umstrukturierung bestehende Unternehmen in drei getrennte Unternehmen aufgeteilt ${ }^{166}$.

- National Power,

- PowerGen,

- Nuclear Electric ${ }^{167}$.

Neben den so entstandenen Erzeugern produzieren die Industrie, hauptsächlich zur Eigenversorgung, und die ebenfalls neugeschaffenen regionalen Verteilerunternehmen elektrische Energie. Zusätzlich treten die EdF und die beiden schottischen EVU sowie neu auf den Markt eintretende, unabhängige, private Erzeuger in den Wettbewerb ein.

164 VICKERS, J. ; YARROW, G. : a.a.O., S. 188. Eine umfassende Darstellung der Privatisierung ihres historischen Vorlaufes erbringt HENNEY, A. : A Study of the Electricity Industry in England and Wales, London 1994

165 Ursache für die Trennung ist die Annahme, daß ausschließlich im Stromtransportbereich ein natürliches Monopol besteht.Vgl. BARTELS, R. ; COHEN, R. ; HOEHN, T. : Das Elektrizitătssystem in Großbritannien: Erste Erfahrungen und Perspektiven. in: Zeitschrift für Energiewirtschaft, Heft 1 (1991), S. 27 und VICKERS, J. ; YARROW, G. : a.a.O., S. 190

166 Aus diesem Grund kommen Vickers und Yarrow zu dem Schluß, daß die Reform nicht weit genug gegangen sei. Vgl. VICKERS, J. ; YARROW, G. : a.a.O., S. 204

167 Vgl. ESSER, C. : Umstrukturierung und Privatisierung der Elektrizitätsivirtschaft in Eng-

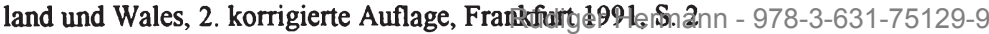


Mit der Elektrizitätsïbertragung ist die National Grid Company (NGC) betraut. Sie ist als Holdinggesellschaft organisiert. Eigentümer sind die neu entstandenen, regionalen Verteilungsgesellschaften ${ }^{168}$. Die NGC gibt zur Koordination des Elektrizitätsmarktes Standards und technische Anforderungen an Betrieb, Instandhaltung und Entwicklung der Stromversorgung heraus. Sie sind für die das Übertragungsnetz inanspruchnehmenden Unternehmen und Eigenerzeuger verbindlich. Weitere Aufgaben der Netzgesellschaft sind ${ }^{169}$.

- der Betrieb , die Instandhaltung und der Ausbau der Hochspannungsnetze,

- die Abwicklung von Stromimporten und -exporten über die Kupplungsstellen zu anderen Netzen,

- die zentrale Lastverteilung, die Sicherstellung der Versorgungssicherheit und -qualität,

- der Betrieb der beiden Pumpspeicherkraftwerke in Dinorwig und Festiniog ${ }^{170}$ sowie

- die Übernahme der Poolverwaltung und die anschließende Abrechnung der Poolgeschäfte.

Auf der Verteilungsstufe sind zwölf Regional Electricity Companies (RECs) geschaffen geworden ${ }^{171}$. Die RECs betreiben die regionalen Mittel- und Niederspannungsnetze $(132-240 \mathrm{kV})$ zwischen den Hochspannungsnetzausstiegspunkten und den Anschlüssen der Endverbraucher und müssen Normen und Standards zum Anschluß von Endverbrauchern an ihre Netz aufstellen und veröffentlichen. Sie haben die Möglichkeit, einen gewissen Anteil an Elektrizität entweder selbst zu erzeugen oder aus Beteiligungskraftwerken zu beziehen.

In der Endabnehmerversorgung sind in erster Linie die Verteilerunternehmen tätig. Die Unternehmen sind zur vollständigen, organisatorischen Trennung ihres Verteilungsgeschäftes von der Endverbraucherversorgung verpflichtet. Als Verteiler müssen die RECs von ihren Endversorgungsgeschäftsbereichen die gleichen $\mathrm{Ge}-$ bühren verlangen wie von anderen Endversorgern. Weitere Endversorger sind die Erzeugerunternehmen. Zusätzlich können andere bisher nicht in der Elektrizitätsversorgung tätige Unternehmen Lizenzen, sogenannte Second-Tier-Lizenzen , erwerben.

168 Vgl. WEYMANN-JONES, T. G. : Electricity Privatisation, Aldershot, Brookfield, Hong Kong, Singapore, Sydney 1990, S. 26

169 Vgl. ESSER, C. : a.a.O., S. 5

170 Vgl. GREEN, R. ; NEWBERY, D.M. : Competition ..., a.a.O., S. 3

171 Vgl. NATIONAL WESTMINSTER BANK : Privatising Electricity in the United Kingdom Problems In Store, Quarterly Review, Londoni NovH 1988. 
Die erste Marktstufe ist in England und Wales die Großhandelsebene. Auf ihr beziehen die Endversorger und Großabnehmer ihre benötigte elektrische Energie von den Erzeugern. Zusätzlich muß auf der Großhandelsstufe die Abstimmung der Verträge mit den Kapazitäten der Netzgesellschaft und der Verteilerunternehmen durchgeführt werden. Der Großhandelsmarkt im neuen Elektrizitätsversorgungssystem unterteilt sich in einen Spotmarkt und einen Markt für Optionsverträge; wobei der Pool das verbindende Element ist.

Teilnehmer des Spotmarktes sind alle mit Lizenzen ausgestattete Erzeuger- und Verteilerunternehmen, die französische Elektrizitätsgesellschaft EdF und die Besitzer von Endversorgungslizenzen. Darüber hinaus steht der Spotmarkt auch Endabnehmern mit einer Mindestgröße von $10 \mathrm{MW}$ offen. Die Erzeuger müssen, um am Spotmarkt teilnehmen zu können, Mitglieder des Pools sein.

Neben den Stromlieferverträgen des Spotmarktes können die Unternehmen Optionsverträge abschließen ${ }^{172}$. Die Verträge sind inhaltlich unabhängig von den tatsächlichen technisch-physikalischen Zusammenhängen und dienen der Stabilisierung der Preisschwankungen des Spotmarktes sowie der Abnahmekapazitätssicherung. Für die Erzeuger sind die Optionsverträge eine Strategie zur Reduzierung ihres langfristigen unternehmerischen Risikos, da die Auslastung von Kapazitäten langfristig und nicht wie im Pool nur einen Tag im voraus geplant werden muß. Bei den Optionsverträgen unterscheidet man zwischen einseitigen und zweiseitigen Verträgen ${ }^{173}$. Einseitige Verträge können zwischen Erzeugern und Endversorgern abgeschlossen werden. Das Endversorgungsunternehmen zahlt dem die Option gebenden Erzeuger eine Optionsgebühr, um als Gegenleistung die Differenz zwischen Pool-Preis und dem Vertrag zugrunde liegenden Preis, vom Erzeuger erstattet zu bekommen, wenn der Poolpreis über dem Vertragspreis liegt. Neben dem Preis ist in dem Optionsvertrag die Elektrizitätsmenge festgehalten, für die die Option gelten soll. Dabei gilt zwischen dem zugrunde gelegten Elektrizitätspreis und der Optionsgebühr folgende Beziehung: Je niedriger der Bezugspreis ist, desto höher ist die Optionsgebühr. Einseitige Optionsverträge kommen am häufigsten zum Abschluß. Zweiseitige Verträge wurden in der Anfangszeit ausschließlich von der EdF auf dem Großhandelsmarkt angeboten. Sie sehen Zahlungen des Erzeuger bei Überschreiten des Poolpreises über den oberen Bezugspreis vor. Bei Unterschreiten des Pool-Preises unter den zweiten Bezugspreis muß der Endversorger dem Erzeuger die Differenz bezahlen. Eine Optionsgebühr fält zumeist nicht an. Liegt der Poolpreis zwischen den beiden Bezugspreisen, so sind von den Vertragspartnern keine Zahlungen zu tätigen.

172 Vgl. VICKERS, J. ; YARROW, G. : a.a.O., S. 208 f.

173 Vgl. HOLMES, A. : HOLMES, A. : Twisting by the Pool, in: Energy Economist, Juli 1991, Heft 17, , S. 9 
Weitere Variationen der Optionsverträge können sich hinsichtlich der Vertragsdauer, der Unterbrechbarkeit und der Bezugszeitpunkte ergeben.

Das Poolsystem sieht die zentrale Koordination aller Kraftwerke vor. Alle Stromlieferungen, auch die durch Optionsverträge abgesicherten, werden über die vom Poolverwalter zu erstellende „merit order" gesteuert. Die Teilnehmer verpflichten sich, die Regeln des Pools und der Geschäftsabwicklung anzuerkennen ${ }^{174}$. Mit der Führung des Elektrizitätspools ist die NGC beauftragt worden, die den Pool als getrennten Geschäftsbereich mit eigener Buchführung führen muß. Ihr werden von den Poolmitgliedern die erforderlichen Informationen zur Verfügung gestellt. Für die Deckung der entstehenden Kosten erhält die Netzgesellschaft von den Poolmitgliedern eine Verwaltungsgebühr ${ }^{175}$. Die Koordination im Pool erfolgt folgendermaßen: Täglich um 10.00 Uhr übermitteln alle Erzeuger mit einer Erzeugungskapazität über $100 \mathrm{MW}$ Nettoengpaßleistung der Poolverwaltung folgende Informationen $^{176}$ :

\section{a) Zustand der Kraftwerke}

Der Zustand der Kraftwerke kann mit in Betrieb, kurzfristig anfahrbar und außer Betrieb angegeben werden.

b) Verfügbarkeit der Kraftwerke für jede halbe Stunde des folgenden Tages

c) Die Angebotspreis-Mengen-Kombinationen

Die Erzeugerunternehmen können der Poolgesellschaft mehrere Preis-MengenKombinationen angeben, zu denen sie bereit sind, elektrische Energie an den Pool zu liefern. Theoretisch sind diese Preis-Mengen-Kombinationen Teil der Angebotsfunktion des Erzeugerunternehmens. In der Praxis kann von dem Unternehmen aber auch versucht werden, den späteren Preis über die Preis-Mengen-Kombinationen zu manipulieren.

d) Preise für Reservehaltungsdienstleistungen der Erzeugerunternehmen.

Erzeuger geben an, zu welchem Preis sie bereit sind, Kraftwerke in Reservebereitschaft zu halten, um bei kurzfristigen, unvorhergesehenen Bedarfsspitzen Elektrizität zu produzieren ${ }^{177}$.

174 Vgl. VICKERS, J. ; YARROW, G. : a.a.O., S. 195

175 Vgl. ESSER, C. : a.a.O., S. 10

176 Vgl. ESSER, C. : a.a.O., S. 10 f. und BARTELS et al : a.a.O., S. 31

177 Das Angebot der Erzeuger zum Reservebetrieb von Kraftwerken kann mit der Sekundärregelung im UCPTE-Verbund verglichen werden. Der Unterschied besteht darin, daB die integrierten EVU des UCPTE-Verbundes eine gewisse Kraftwerkskapazităt kostenlos in Reserve zu halten haben und das in Ansprochenehmenden Unemehmen aasschligeßlich die 
e) Preise für die temporär befristete Produktion über die angebotenen PreisMengen-Kombinationen.

Die letzte Preisinformation ist mit den Preisen für die Inanspruchnahme der Primärregelung in Verbundnetzen gleichzusetzen ${ }^{178}$. Über die Nutzung der zusätzlichen Hilfs- und Service-Leistungen entscheidet der Grid Operator ${ }^{179}$, sie werden getrennt von den Stromlieferungen abgerechnet. Nachdem die Netzgesellschaft diese Informationen von den Lizenzinhabern erhalten hat, erstellt sie für jede Periode des folgenden Tages eine „merit order". Hierzu bedient sie sich eines speziellen EDV-/ Software-Systems. In der „merit order" werden die Angebote nach den Preisen hierarchisch geordnet. Zusätzlich werden die bereitgestellten Mengen vermerkt. Der „merit order“ wird dann die prognostizierte Nachfrage für die Perioden des nächsten Tages gegenübergestellt. Durch den Vergleich wird der Grenzanbieter bestimmt. Das ist jener Elektrizitätserzeuger, der gerade noch benötigt wird, um die Nachfrage zu decken. Die verbleibenden teureren Anbieter werden unter normalen Umständen, d.h. wenn die tatsächliche Nachfrage die prognostizierte nicht überschreitet, nicht zur Elektrizitätsversorgung herangezogen. Der Preis des Grenzanbieters, der sogenannte „System Marginal Price (SMP) ${ }^{\text {“180 }}$, dient als eine Grundlage der Preisbildung für den Elektrizitätspool. Die Erzeuger erhalten für ihre gelieferte Elektrizität den sogenannten „Pool-Input-Price (PIP)“. Dieser besteht aus SMP, erhöht um einen Kapazitätspreis. Der Kapazitätspreis setzt sich aus dem Wert einer ausgefallenen Leistungseinheit, reduziert um die durch den Ausfall reduzierten Grenzkosten und der Eintrittswahrscheinlichkeit des Ausfalls, zusammen. Letztendlich ist der Kapazitätspreis ein Teil der Gesamtkosten der Versorgungssicherheit. Die Differenz zwischen PIP und dem Angebot des Erzeugerunternehmens ist der Gewinn. Demnach erhält der Grenzanbieter definitionsgemäß keinen Gewinn.

Der Preis (Pool-Output-Price, POP), den Verteilerunternehmen, "Second Tier"Versorger und die Endversorger als Poolmitglieder in Rechnung gestellt bekommen, berechnet sich nach folgender Formel:

$$
\text { POP = PIP + Uplift }
$$

Der „Uplift“ soll die Kosten der Netzgesellschaft decken, die durch

- die Reservehaltung und Bereitstellung,

- der Kraftwerksverfügbarkeit,

- dem Prognoseirrtum,

Stromleistung bei Aushilfslieferungen bezahlt. Der Preis enthălt jedoch kein Kapazitătselement.

178 Vgl. die Aussagen zur Primărregelung innerhalb des UCPTE-Verbundes im Kapitel 4.2.1.1 .

179 Vgl. LAMPRECHT, F. : Wettbewerb und Regulierung in der britischen Elektrizitătswirtschaft, in: ET 42. Jg. (1992), Heft 11, S. 777

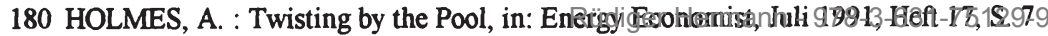


- etwaiger Übertragungsbeschränkungen,

- Qualitäts- und Sicherheitsdienstleistungen,

- kurzfristige Einsätze

entstehen ${ }^{181}$.

Darüber hinaus muß die Differenz zwischen POP und PIP die Kosten für den Stromtransport der NGC decken.

Die finanzielle Abrechnung der Lieferverträge durch den Pool erfolgt innerhalb der nächsten 28 Tage $^{182}$ durch die Netzgesellschaft. Sie übernimmt gleichzeitig eine Vermittlerrolle bei der Festlegung der Geschäftsverbindungen und erhält die zur Abrechnung erforderlichen Informationen von den Poolmitgliedern ${ }^{183}$.

Der Endabnehmermarkt des englisch-walisischen Elektrizitätsmarktes kann in zwei Teilmärkte aufgegliedert werden:

1. dem Franchise-Markt,

2. dem Nicht-Franchise-Markt.

Im Franchise-Markt unterliegen die Endabnehmer der Versorgungspflicht der RECs und können ihre Versorger nicht wählen. Der Franchise-Markt soll stufenweise bis 1998 abgebaut und dann aufgelöst werden.

Tabelle 5: Abbau des Franchise-Marktes in der englischen Elektrizitätswirtschaft

\begin{tabular}{|l|l|}
\hline Kunden mit Spitzenlast & frei ab \\
\hline mehr als 1 MW & 31.03 .1990 \\
\hline mehr als 0,1 MW & 31.03 .1994 \\
\hline alle Kunden & 31.03 .1998 \\
\hline
\end{tabular}

Quelle: BARTELS, , R. ; COHEN, R. ; HOEHN, T. : Das Elektrizitätswirtschaftssystem in Großbritannien - Erste Erfahrungen und Perspektiven, in: ZfE $1 / 91$, S. 29

181 Vgl. ESSER, C. : a.a.O., S. 13

182 Vgl. BARTELS et al : a.a.O., S. 31

183 Zur Entwicklung der Pool-Preise, des Uplifts etc. siehe BARTELS et al : a.a.O., S. 32 ff. und die Abb. im Anhang von ESSER, C. : a.a:Qidiger Hermann - 978-3-631-75129-9 
Im Nicht-Franchise-Markt unterliegen die Endabnehmer nicht der Versorgungspflicht der RECs. Sie müssen einen Liefervertrag mit einem Versorgungsunternehmen abschließen ${ }^{184}$.

Die aus den staatlichen EVU hervorgegangenen Unternehmen sind bis auf die Nuclear Electric privatisiert worden ${ }^{185}$. Zur Finanzierung der Kernkraftwerke wurde von der Regierung den RECs Abnahmeverpflichtungen und den anderen Erzeugern eine Abgabe auferlegt.

Tarifstrukturen bestehen derzeit ausschließlich für den Franchise-Markt. In diesem Teilmarkt der Endversorgungsmarktes werden überwiegend zweigliedrige Tarife angewandt. Für die Kunden der Endversorgungsunternehmen im Nicht-FranchiseMarkt bestehen keine festen Tarife, doch entwickelten die Versorgungsunternehmen standardisierte Angebote, um die Transaktionskosten bei den Abnehmern mit niedrigen Abnahmenmengen zu reduzieren.

Mit der Umstrukturierung der britischen Elektrizitätswirtschaft mußte ein neues Regulierungsinstrumentarium ${ }^{186}$ geschaffen werden, mit dem die britische Regierung glaubte, die energiepolitischen Ziele für die Elektrizitätswirtschaft erreichen zu können. Insbesondere die Umsetzung der Primärenergieträgerpolitik mußte ermöglicht werden.

So wurde zur Gesamtüberwachung des neu geschaffenen Elektrizitätsversorgungssystems die Position des Generaldirektors für die Elektrizitätsversorgung (Director-General of Electricity Supply - DGES) geschaffen. Die Hauptaufgaben des DGES liegen in der sicherstellenden Überwachung der Versorgungssicherheit. Die Befriedigung jeder gerechtfertigten Elektrizitätsnachfrage hat der Generaldirektor als politische Vorgabe auferlegt bekommen. Darüber hinaus ist er für die Förderung des Wettbewerbs innerhalb der Elektrizitätswirtschaft Großbritanniens zuständig. Des weiteren soll er die Innovationsraten und damit verbunden auch die Effizienzsteigerungen innerhalb des Stromerzeugungs-, Stromtransport- und Stromverteilungsbereiches überwachen.

184 Standardtarife existierten dabei bisher nicht. Es ist jedoch davon auszugehen, daß bei zunehmendem Marktzutritt von Kleinabnehmern die einzelnen Versorger standardisierte Verträge und Tarife herausbringen werden. Vgl. LANG, G. : Die Strompreisentwicklung in England und Wales seit der Privatisierung, in: EW Jg. 91 (1992), Heft 21, S. 1385

$185 \mathrm{Zu}$ den Privatisierungsplänen der britischen Regierung siehe DEPARTMENT of ENERGY: Electricity Privatisation, London 1988, so wurden am $10.03199160 \%$ von PowerGen und National Power an der Borse zum Verkauf angeboten. Vgl. 0.V. : That curious UK electricity pool price, in: Energy Economist, Februar 1991, S. 9

186 Zur Regulierung der Elektrizitătswirtschaft allgemein siehe HELM, D.R. ; YARROW, G. : The Regulation of Utilities, Oxford 1988 und CREW, M. ; KLEINDORFER, P. : The Economics of Public Utility Regulation, Londödig e86-1ermann - 978-3-631-75129-9 
Neben dieser Aufsichtsinstitution erfolgt die Regulierung innerhalb der Elektrizitätswirtschaft durch Lizenzen. Dabei sind die Lizenzen für die einzelnen Elektrizitätsbereiche mit unterschiedlichen Bedingungen verknüpft.

Es gibt vier verschiedenen Arten von Lizenzen:

a) Erzeugungslizenzen,

b) Übertragungslizenzen,

c) Öffentliche Stromversorgungslizenzen (Public Electricity Supply),

d) Second-Tier Lizenzen.

Die verschiedenen Lizenzen können generell an alle in der Elektrizitätsbranche tätigen Unternehmen vergeben werden. Den Lizenznehmern ist es verboten, verschiedene Geschäftsbereiche gegenseitig zu subventionieren. Um dies zu verhindern, sind die Lizenznehmer zu einer getrennten Buchführung für jede Sparte verpflichtet ${ }^{187}$. Des weiteren ist den Lizenzinhabern eine diskriminierende Behandlung von Kunden mit gleichen Kriterien verboten. Die Übertragungslizenz wurde ausschließlich an die National Grid Company vergeben, während die RECs für die nächsten 35 Jahre mit den Verteilungslizenzen betraut wurden. Damit werden ihnen im Bereich des lokalen und regionalen Stromtransportes weiterhin Monopolrechte eingeräumt. Die Second-Tier- und Erzeugungslizenzen stehen allen Unternehmen zu einem Ausgabepreis von 100 Pfund offen. Des weiteren sollen durch die mit den Lizenzen verbundenen Auflagen die Versorgungssicherheit im neuen Elektrizitätsversorgungssystem gefördert werden. Ein Erzeuger verpflichtet sich mit dem Erwerb einer Lizenz zu einem festgelegten Maß an Brennstoffbevorratung, um Lieferengpässe in der Primärenergieträgerzufuhr überbrücken zu können. Darüber hinaus sind die Verteilerunternehmen zur Einhaltung der von der Regulierungsinstitution aufgestellten Sicherheitsstandards gezwungen. Hierdurch soll erreicht werden, daß die RECs ihre Kosten nicht zu Lasten der Versorgungssicherheit senken ${ }^{188}$.

Die dritte Komponente des britischen Regulierungssystems ist die Preisregulierung. Wegen der wettbewerblichen Konzeption des Erzeugungs- und Endversorgungsbereiches ist sie auf die Übertragungs- und Verteilungsgebühren beschränkt worden. Bei dem angewandten Verfahren handelt es sich um eine Höchstpreisregulierung, die an den jährlichen Preissteigerungen angelehnt wird. Die Höchstpreisregulierung wird nach dem Preisindexbindungsverfahren (Retail Price Index) durchgefuhrrt ${ }^{189}$.

187 Diese Forderung wurde von der EG-Kommission in ihren Richtlinienvorschläge auch für den EG-Binnenmarkt für Elektrizität gefordert, wogegen Vertreter der Elektrizitătswirtschaften anführen, daß dies nicht möglich wäre.

188 Vgl. ESSER, C. : a.a.O., S. 22

189 Vgl. VICKERS, J. ; YARROW, G. : a.a.他:Bigl93Hermann - 978-3-631-75129-9 
Tabelle 6 : Preisregulierung in der englischen Elektrizitätswirtschaft

\begin{tabular}{|l|l|l|}
\hline Regulierter Preis & Formel & Anmerkungen \\
\hline $\begin{array}{l}\text { Übertragung (NGC) } \\
\text { Einnahmen/max } K W\end{array}$ & RPI - X & $\mathrm{X}=0$ bis 31.03 .93 \\
\hline $\begin{array}{l}\text { Verteilung (RECs) } \\
\text { Einnahmen/kWh }\end{array}$ & RPI + X & $\begin{array}{l}\mathrm{X} \text { liegt zwischen 0 \% und } 2.5 \% \text { für die } \\
\text { verschiedenen RECs bis } 1995\end{array}$ \\
\hline $\begin{array}{l}\text { Lieferung durch RECs } \\
\text { Einnahmen/kWh }\end{array}$ & RPI - X+Y & $\mathrm{X}=0$ bis 31.03 .93 \\
\hline
\end{tabular}

$\mathrm{X}$ von der Regulierungsbehörde festzulegender Wert

Y steht für Kosten, die nicht der Kontrolle der RECs unterliegen

RPI steht für Retail Price Index (der Verbraucherpreisindex)

Quelle: BARTELS, , R. ; COHEN, R. ; HOEHN, T. : Das Elektrizitätswirtschaftssystem in Großbritannien - Erste Erfahrungen und Perspektiven, in: ZfE $1 / 91$, S. 29

Weitere Beschränkungen in ihren unternehmerischen Handlungsspielräumen sind den beiden größten Stromerzeugern, der National Power und der PowerGen, den zwölf RECs und der Stromtransportgesellschaft auferlegt worden. So kann die Netzgesellschaft über den aus den beiden Pumpspeicherkraftwerken in Spitzenzeiten hergestellten Strom keine elektrische Energie kaufen oder verkaufen. Den RECs wurde eine Beteiligung an Erzeugungsanlagen bis zu einem Gesamtumfang von $15 \%$ des Eigenbedarfes vorgeschrieben, um zu verhindern, daß durch eine Ausweitung der Eigenerzeugung der RECs eine Reintegration des Elektrizitätsmarktes stattfindet.

Aus den gleichen Gründen ist der Marktanteil, den die beiden Großerzeuger als Second-Tier- Versorger erreichen dürfen, in den meisten Versorgungsgebieten auf $15 \%$ des Gesamtumsatzes beschränkt worden. Bis 1998 kann dieser Prozentsatz in einigen Gebieten $25 \%$ betragen.

Die gesamte britische Regulierung unterliegt den gleichen Problemen, die andere Länder mit den verschiedenen Regulierungsformen und Instrumentarien ebenfalls kennen. WEYMANN-JONES faßt diese wie folgt zusammen: " In particulary, optimal regulatory policy is viewed as a game between a principal (the regulator) and 
an agent (the utility) whose objectives differ, and in which the agent has a monopoly over the information needed by the regulator"190.

\subsection{Stromhandel- und Austauschkapazitäten zwischen den euro păischen Mitgliedsstaaten}

\subsubsection{Organisationen für den Stromaustausch}

\subsubsection{Die UCPTE}

In den 20er Jahren wurden die ersten Verbindungen zwischen den mitteleuropäischen Stromnetzen durch die Gründung der CIGRE ${ }^{191}$ hergestellt. Der weitere Ausbau der Verbindungen diente der Vermaschung der Netze und fand nach geographischen Gesichtspunkten statt. Nach Ende des zweiten Weltkrieges wurde der Wiederaufbau der europäischen Netzverbindungen angegangen. Dazu wurde aufgrund einer Empfehlung der OECD und der nationalen Elektrizitätswirtschaften 1951 die UCPTE (Union pour la coordination de la production et du transport de l'électricité) gegründet ${ }^{192} . \mathrm{Zu}$ den Gründungsmitgliedern zählten die Niederlande, Belgien, Luxemburg, Frankreich, die Bundesrepublik Deutschland, die Schweiz, Italien und Österreich. Später schlossen sich Spanien, Portugal, Jugoslawien und Griechenland $a^{193}$, während die EU-Länder Dänemark und Großbritannien nicht beitraten. Es gehören also auch Nicht-EU-Länder zur UCPTE. In der UCPTE sind die EVU zusammengeschlossen, die über ein eigenes geschlossenes Versorgungsgebiet und ein Übertragungsnetz verfügen. Die Unternehmen unterliegen einer mehr oder weniger starken behördlichen Aufsicht ${ }^{194}$. Technisch realisiert wurde der Zusammenschluß auf der Hochspannungsebene bis

190 WEYMANN-JONES, T.G. : Electricity Privatisation, Aldershot-Brookfield. Hong Kong, Singapore, Sydney 1990, S. 2

191 CIGRE ( Conference Internationale des Grande Reseaux Electrique a Haute Tension) vgl. SCHWICKARDI, G. : a.a.O., Bd. 2, S. 69

192 Vgl. VDEW: Der europäische Strommarkt, Frankfurt/Main 1988, S. 23

193 Vgl. STRAUSS, L. ; KARAS, P. : Die Bedeutung der Gleichstromnetzkupplung EtzenrichtHradec für die Stromnetze West- und Osteuropas, in: EW Jg. 92 (1993), Heft 12, S. 713

194 Vgl. BÖRNER, B. : Frankreichstrom nack Degtschland, 2 ana. O,9853-631-75129-9 
1958. 1993 umfaßte die gesamte installierte Kraftwerkskapazität $387.000 \mathrm{MW}^{195}$. Ca. $10 \%$ der erzeugten Stromleistung wurde über eine nationale Grenze ausgetauscht. Insgesamt existierten 125 grenzüberschreitende Hochspannungskupplungen der 220- und 380- kV Ebene mit einer Kapazität von 75.000 MVA, was ca. $15 \%$ der installierten Kraftwerksleistung entspricht ${ }^{196}$. Das Leitungsnetz umfaßte $1990150.000 \mathrm{~km}$, wogegen 1985 noch $130.000 \mathrm{~km}$ Leitungen betrieben wur$\operatorname{den}^{197}$. Die beachtliche Übertragungskapazität wurde bisher nur sekundär zum Stromhandel benutzt.

Die UCPTE sah ihre ersten Ziele in ${ }^{198}$.

- der Förderung und Beschleunigung des Wiederaufbaus der nationalen Netze und des Stromverbundes nach dem zweiten Weltkrieg, durch den weite Teile der Elektrizitätsinfrastruktur in Europa zerstört worden waren.

- vorbeugenden Maßnahmen gegen temporären Wassermangel zur Elektrizitätserzeugung aufgrund klimatischer Schwankungen in den Alpen.

- Der bereits vor dem Krieg gegründete Verbund zwischen Wasserkraftwerken in den Alpen und Kohlekraftwerken im Ruhrgebiet sollte wiederaufgebaut und intensiviert werden.

- der Erhöhung der Versorgungssicherheit und -qualität. Schwerpunkte bei der Versorgungssicherheit waren und sind die Ausfallzeiten in den Netzen. Die Frequenzstabilisierung ist das vorherrschende Ziel der Versorgungsqualitätssteigerung.

Heute ist die Förderung einer sicheren, wirtschaftlichen und umweltverträglichen Versorgung oberstes Ziel der UCPTE. Verwirklicht werden soll dieses Ziel durch eine verbesserte Reservehaltung und Aushilfe bei Kraftwerksausfällen in den Mitgliedsstaaten ${ }^{199}$. Die Aushilfslieferungen können durch schwankende Wasserstände, erforderliches, geplantes oder unvorhergesehenes Abschalten von Kraftwerken,

$195 \mathrm{Vgl}$. STRAUSS, L. : Osteuropa bereitet Anbindung ans Westnetz vor. in: INFORMATIONSZENTRALE DER ELEKTRIZITÄTSWIRTSCHAFT e.V. (Hrsg.): StromThemen 10. Jg. ,Nr. 5 , Frankfurt 1993, S. 2

196 Vgl. UCPTE: Betrieb des westeuropaischen Verbundnetzes, Arnheim (Niederlande) 1990, S. 10

197 Vgl. DVG Bericht 1990, Heidelberg 1990

198 Vgl. HOEK, van G.A.L. : Europa im Stromverbund, Vortrag anläßlich des VDEW-Symposiums für Wissenschafts-Journalisten „Perspektiven der Stromerzeugung „vom 25. bis 27.11.1991 abgedruckt in: Strom der Zeit 1991, Frankfurt/M. 1991, S. 1-9

199 Vgl. SCHWICKARDI, G. : a.a.O., Bd. 2, S.699diger Hermann - 978-3-631-75129-9 
Streiks und durch Belastungsspitzen entstehen ${ }^{200}$. Die Aushilfe ist in der UCPTE automatisiert worden und tritt ein, ohne daß weitere Absprachen zwischen Lieferanten und Beziehern erforderlich sind. Die Wirtschaftlichkeit der Stromerzeugung soll durch die Optimierung des Stromaustausches gefördert werden. Dies beinhaltet für die UCPTE-Mitglieder die Möglichkeit zum kurzfristigen Stromaustausch ohne Garantieleistungen sowie langfristig mit Garantieleistungen ${ }^{201}$. Des weiteren sollen große Gemeinschaftskraftwerke mehreren EVU eine weitere Ausnutzung der economies of scale mit den damit verbundenen Kostensenkungen ermöglichen ${ }^{202}$. Zudem ist gemäß den Aussagen der UCPTE ein Spotmarkt für kurzfristige Elektrizitätslieferungen eingerichtet worden.

Die Voraussetzungen für die Teilnahme am Stromverbund sind:

- Kraftwerke und Leitungen werden eigenverantwortlich und selbständig gebaut.

- Die eigenen Kraftwerke eines Mitglieds-EVU müssen zusammen mit Gemeinschafts- oder Vertragskraftwerken den Bedarf des EVU dekken ${ }^{203}$.

- Jeder Partner muß über die notwendige Infrastruktur verfügen.

- Die Infrastruktur wird dem Verbund freiwillig und kostenlos zur Verfügung gestellt ${ }^{204}$.

Betrachtet man die Ziele und Aufgaben der UCPTE und deren technische sowie organisatorische Umsetzung, kristallisiert sich die zentrale Bedeutung des Stromtransportnetzes furr das Funktionieren des Systems heraus. Technisch handelt es sich bei dem UCPTE-Verbundnetz um ein Drehstromnetz, welches von seinen

200 Ein Beispiel hierfür sind die Aushilfslieferungen der UCPTE-Mitglieder an die französische EdF im Jahre 1990.

201 Garantieleistung bedeutet in diesem Zusammenhang die Sicherstellung der Stromlieferung auch bei Engpässen des liefernden Unternehmens.

202 Vgl. BOLLE, F.: Wettbewerb und Kooperation in der Elektrizitătswirtschaft, Schriftenreihe des Energiewirtschaftlichen Institutes der Universităt Köln, Nr. 39, Köln 1990, S. 21 sieht für die amerikanische Elektrizitătsversorgung eine Ausschöpfung der economies of scale in den 70er Jahren. Selbst wenn man einen gewissen time-lag in der Entwicklung zwischen der amerikanischen und den europäischen Elektrizitätswirtschaften annimmt, so kann davon ausgegangen werden, daß die Ausnutzung der economies of scale auch in Europa an ihre Grenzen gestoßen ist.

$203 \mathrm{Vgl}$. MICHAELIS, H. : Gesamtwirtschaftliche Aspekte, Ein EWG-Binnenmarkt für Elektrizităt, in: ET, 37. Jg. (1987), Heft 16/17, S. 1030

$204 \mathrm{Vgl}$. LICHTENBERG, H. : Der europăische Stromverbund, in: LUKES, R. (Hrsg.): Ein EWG-Binnenmarkt für Elektrizităt - Realităt oder Utopie, Köln, Berlin, Bonn, München 1988, S. 92 und weiter MANSFELD, L. : Gebietsmonopole für Elektrizitătsversorger, in: technologie und management 1/89, [süß̊ger Hermann - 978-3-631-75129-9 
Netzkupplungen her nicht für große Stromtransporte konzipiert ist. Das Netz wird parallel betrieben und ist mit einer automatischen Leistungsregelung sowie Einrichtungen zum Netzschutz versehen. Die Netzregelung und damit auch die Störungsaushilfe und Frequenzhaltung werden durch zwei Mechanismen - die Primär- und die Sekundärregelung - sichergestellt ${ }^{205}$. Für die Primärregelung ist es erforderlich, daß jedes Kraftwerk eine gewisse Marge zwischen 2 und 5\% seiner maximalen Auslastung nicht ausfährt. Gleiches gilt für die Übertragungskapazitäten, die ebenfalls eine Reservemarge aufweisen müssen. Bei Ausfall eines Kraftwerks oder einer unvorhergesehenen Lastspitze sinkt die Frequenz im gesamten Verbundnetzes ab. Daraufhin wird die Produktion in den Kraftwerken gesteuert erhöht, bis die Sollfrequenz erreicht ist. Technisch übernimmt ein in jedem Kraftwerk installierter Primärregler, der bei Frequenzabweichungen von $20 \mathrm{mHz}$ die Leistungsabgabe erhöht, diese Aufgabe. Die Mitgliedstaaten des UCPTE-Verbundes haben als Vorgaben für ihre an der Primärregelung teilnehmenden Kraftwerke festgelegt, daß sie in fün Sekunden ihre Einspeisung um 1,25\% und innerhalb von 30 Sekunden um $2,5 \%$ erhöhen können müssen ${ }^{206}$. Zur Feinabstimmung der Frequenz im Netz dient die sogenannte Sekundärregelung. Hierbei setzt jedes EVU für sein Netz einen zentralen Regler ein, der mit den jeweiligen Regelblöcken der Kraftwerke verbunden ist ${ }^{207}$. Reicht die Primärregelung nicht aus, um die Frequenz zu halten, werden in Reserve gehaltene Laufwasserkraftwerke oder andere in Heißreserve betriebene Kraftwerke eingesetzt, um eine Entlastung des Netzes zu bewirken. Gleichzeitig ist das EVU mit dem Ausfall oder der unvorhergesehenen Lastspitze dazu verpflichtet, alles zu unternehmen, um die benötigte elektrische Energiemenge selber bereitzustellen. Die Verrechnung der Stromlieferungen wird später auf der Basis der gemessenen Lastflüsse ${ }^{208}$ vorgenommen. Hierzu produziert das EVU zu einem festgelegten Zeitraum über den eigenen Bedarf hinaus und liefert an die ehemaligen Lieferanten, wodurch die Lastflüsse umgekehrt werden.

Stromlieferungen in Form des Stromhandels nehmen in der UCPTE-Hierachie eine geringere Bedeutung ein als die Aushilfslieferungen ${ }^{209}$ und werden über ein Einsatzprogramm realisiert. Jeder UCPTE-Partner meldet einer Zentralstelle in

205 Vgl. VDEW: Der europäische Strommarkt, a.a.O., S. 23. Zur Veranschaulichung vgl. das Zahlenbeispiel in LICHTENBERG, H. : a.a.O., S. 94

$206 \mathrm{Vgl}$. STRASSBURG, W. : Stromverbund für Gesamteuropa?, in: Stromthemen Extra, Nr. 54, S. 4

207 Vgl. HEUK, K. : Vorlesungsskript für den Studienzweig Elektrotechnik. Eine gute Übersicht über die Zusammenhänge in der Netzsteuerung gibt das Schaubild in: STOTZ, J. : Anbindung des Höchstspannungsnetzes der neuen Bundeslănder an den westeuropăischen Verbund, in: EW Jg. 93 (1994), Heft 13, S. 718

$208 \mathrm{Zu}$ den Lastflüssen im UCPTE-Netz vergleiche UCPTE-Jahresbericht 1993, Wien 1994, S. 41

209 Vgl. HOEK, van G.A.L. : Vortrag .., a.a.O., S. 5 und UCPTE: Betrieb des westeuropäischen Verbundsystems, Anheim 1990, S. 8 ff Rüdiger Hermann - 978-3-631-75129-9 
Laufenburg/Schweiz die beabsichtigten Geschäfte. Der Tag ist dabei in halbstündige Perioden eingeteilt. Die Stelle in Laufenburg erstellt dann für jede Periode eines Tages ein Gesamtregelprogramm. Die Preise für die Lieferungen und die Aushilfe, sofern die Aushilfe pagatorisch beglichen werden soll, werden bilateral ohne Beteiligung der UCPTE zwischen den EVU ausgehandelt. In Zukunft ist beabsichtigt, ein Informationssystem ${ }^{210}$ ("Strombörse") einzurichten, um damit einen vergrößerten Spotmarkt zu installieren. Des weiteren erhofft die UCPTE, die Vertragsabwicklung und die Preisgestaltung durch das Informationssystem zu vereinfachen.

Ein weiterer Ausbau des UCPTE-Verbundes nach Osten und nach Süden (Nordafrika) ist in Vorbereitung. So soll durch eine Drehstromverbindung über die Straße von Gibraltar das Stromnetz Marokkos mit dem spanischen verbunden werden. Marokko ist seit 1992 mit den Ländern Algerien und Tunesien elektrizitätswirtschaftlich verbunden ${ }^{211}$.

\subsubsection{Die NORDEL}

Das skandinavische Gegenstück zur UCPTE ist die NORDEL. Sie arbeitet mit der mitteleuropäischen Organisation zusammen. In der NORDEL haben sich die EVUs Dänemarks (skandinavischer Teil), Finnlands, Norwegens und Schwedens zusammengeschlossenen. Assoziiert ist das isländische EVU, zu dem jedoch keine Verbindungsleitungen bestehen ${ }^{212} .1992$ wurden 347 TWh in der NORDEL (ohne Island) erzeugt ${ }^{213}$. Der Anteil der thermischen Kraftwerke betrug $199015 \%$, der atomaren Kraftwerke $24 \%$ und $61 \%$ der erzeugten Stromleistung wurden in Wasserkraftwerken produziert ${ }^{214}$. Hierin spiegelt sich die Dominanz der Wasserkraft mit ihrem Nutzungspotential in der Elektrizitätserzeugung Skandinaviens wieder $^{215}$.

Von der Zielsetzung unterschied sich die NORDEL bis zur Liberalisierung des norwegischen Elektrizitätsmarktes nicht von der UCPTE. In Norwegen findet zur

210 Welches gemäß der UCPTE-Philosophie dezentral strukturiert sein soll.

211 Vgl. DVG: Bericht 1992, a.a.O., S. 14. Auch international nehmen die Zusammenschlüsse zu Stromverbunden zu. So schließen sich z.B. die Elektrizitătswirtschaften Bahrains, Kuwaits, Katars und Saudi-Arabiens zusammen. Vgl. Nachrichten in: ET, 43. Jg. (1993), Heft 7, S. 486

212 Vgl. DVG: Die Stromversorgung in Deutschland, Heidelberg 1994, S. 16

213 Vgl. BIERHOFF, R. : Ausblick ..., a.a.O. , S. 747 und für 1991 DVG: Daten aus der Verbundwirtschaft der Bundesrepublik Deutschland, Heidelberg 1993

214 Der Anteil der Wasserkraftnutzung in Mitteleuropa ist wesentlich niedriger. Vgl. hierzu die Werte für die UCPTE-Mitgliedsländer in: UCPTE: Jahresbericht 1993, a.a.O., S. 74 ff.

215 NORDEL: Jahresstatistik 1990 
Zeit eine Umgestaltung der Elektrizitätswirtschaft statt. Durch die damit verbundenen Auswirkungen auf die Zusammenarbeit in der NORDEL hat diese am 10.02.1993 eine neue Satzung verabschiedet. Nach dieser Satzung verlagern sich die Schwerpunkte der NORDEL-Arbeit auf beratende und empfehlende Maßnahmen sowie der technischen Koordinierung von Elektrizitätsströmen und der Sammlung von Informationen ${ }^{216}$.

Kurzfristige Stromlieferungen werden innerhalb der NORDEL nach dem Mittelwert der Grenzkosten entlohnt. Dies führt dazu, daß die Erträge für Stromlieferungen innerhalb der NORDEL für die Elektrizitätswirtschaften mit einem Stromüberschuß $\beta^{217}$ wenig lukrativ sind. Aus diesem Grund sind die EVU mit einem großen Wasserkraftpotential an einer weiteren Verknüpfung der Verbundnetze in Europa und einer Öffnung der Märkte für den Stromhandel interessiert. Ihr Ziel ist es, den mitteleuropäischen Strommarkt beliefern zu können.

\subsubsection{Der CENTREL-Verbund}

Einige Staaten des ehemaligen Ostblocks haben ihr Interesse bekundet, UCPTEMitglieder zu werden. Hierzu gründeten die Verbundunternehmen Polens, Tschechiens, Ungarns und der Slowakischen Republik 1992 als organisatorisches Gremium die CENTREL ${ }^{218}$. Der Beitritt wird jedoch von der UCPTE kritisch bewertet, da die EVU weder die erforderliche Infrastruktur mitbringen, noch einen ausreichenden Versorgungsqualitätsstandard aufweisen ${ }^{219}$. Erfahrungen haben gezeigt, daß Frequenzabweichungen im alten osteuropäischen Verbundnetz vielfach $0,5-1,0 \mathrm{~Hz}$ oder $1-2 \%$ von der Nennfrequenz betragen haben. Dies hätte zur Folge, daß die UCPTE-EVU Aushilfsleistung zur Frequenzaufrechterhaltung bei einem denkbaren Parallelbetrieb dem Ostnetz bereitstellen müßten ${ }^{220}$. Für Aushilfslieferungen in dem dann erforderlichen Umfang bestehen weder die erforderlichen Leitungs- noch die Kraftwerkskapazitäten im jetzigen UCPTE-Netz. Darüber hinaus sehen die UCPTE-Mitglieder keine ökonomische Notwendigkeit, ihren ausreichenden und funktionierenden Stromverbund nach Osten auszudehnen $^{221}$. Trotzdem haben die UCPTE-Mitglieder die Aufnahme der CEN-

216 Vgl. HAGENMEYER, E. : Stromversorgung in Europa, in: atomwirtschaft, Juli 1993, S. 509. Zur Umgestaltung der norwegischen Elektrizitätswirtschaft siehe WIEDSWANG, G. : Die Liberalisierung des norwegischen Energiemarktes - Konsequenzen für den internationalen Stromaustausch, in: ET 43. Jg. (1993), Heft 10, S. $678 \mathrm{ff}$.

217 Hierzu gehort in erster Linie Norwegen mit seinen großen Wasserkraftpotentialen.

218 Vgl. BIERHOFF, R. : Ausblick ..., a.a.O. , S. 748

219 HOLZER, J. : a.a.O., S. 149

220 Dies entspricht der Leistung von 10 modernen Kernkraftwerken oder ca. 20 Steinkohlekraftwerken.

221 Vgl. HOEK, van G.A.L. : Vortrag ..., a.a.OR, \&d.6j ff. Hermann - 978-3-631-75129-9 
TREL-Länder nicht grundsätzlich abgelehnt. Sie sehen jedoch in dem erfolgreichen Betrieb des CENTREL-Gebietes als Inselnetz die wesentliche Voraussetzung für die langfristige Aufnahme ${ }^{222}$. Bis zum Zusammmenschluß soll der Stromaustausch und die gegenseitige Unterstützung über die bestehenden oder noch zu errichtenden Gleichstromverbindungen intensiviert werden ${ }^{223}$. So arbeiten die westeuropäischen EVU zunehmend mit den Unternehmen des ehemaligen Ostblocks zusammen. Die EdF, das Bayernwerk und die PreussenElektra haben eine Zusammenarbeit bei der Entwicklung der osteuropäischen Stromwirtschaften vereinbart. Dazu zählen Projekte in Rußland, der Tschechischen und der Slowakischen Republik $^{224}$. Bei einem EU-Beitritt der CENTREL-Länder werden die aufgezeigten Probleme und unterschiedlichen technischen Standards zu einer erschwerten elektrizitätswirtschaftlichen Integration führen.

\subsubsection{Stromaustausch und Harmonisierungsgrad}

\subsubsection{Stromaustausch und Verbindungen}

Der Stromaustausch zwischen den Staaten befindet sich auf einem, im Vergleich zu anderen Wirtschaftssektoren, niedrigen Niveau. Außer in Italien und Dänemark ${ }^{225}$ hat nur in den Niederlanden der grenzüberschreitende Stromhandel einen Anteil von über $10 \%$ an der Nettostromerzeugung. Bei den meisten europäischen Staaten liegt der Anteil unter 5\% der Nettostromerzeugung. Eine Ausnahme ist Luxemburg, das durch seine Lage und Größe keine ausreichenden eigenen Erzeugungsanlagen errichtet hat und daher prozentual hohe Stromflüsse über die Grenzen aufweist. Damit fiel der grenzüberschreitende Handel mit elektrischer Enegie ein Jahr nach Vollendung des Binnenmarktes gering aus.

222 Die Vorbehalte gegen einen zügigen Anschluß des CENTREL-Netzes an das UCPTE-Netz durch deren Mitglieder wird deutlich, wenn man die Frequenzabweichungen im osteuropäischen Verbundnetz und im UCPTE-Netz betrachtet. Vgl. hierzu Tabelle 4 in MÜLLER, L. : Network Ost, in: ENERGIE SPEKTRUM, April 93, S. 30. Ein Probebetrieb wurde am 29. und. 30.09.93 durchgefuihrt. Vgl. DVG: Jahresbericht 1993. Heidelberg 1994, S. 5

223 Vgl. BIERHOFF, R. : Ausblick ..., a.a.O. , S. 747

$224 \mathrm{Vgl}$. Nachrichten, in: ENERGIE SPEKTRUM, Juli 92, S. 76 und RADZIO, H. : Die deutsche Stromwirtschaft blickt nach Osten, aber auch nach Westen, in: ET 43. Jg. (1993), Heft 10, S. 654

225 Zur Entwicklung der dänischen Stromimporte in den achtziger Jahren vgl.TUROWSKI, R. :

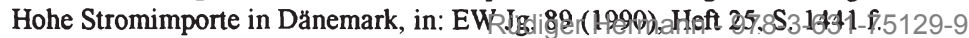


Tabelle 7: Stromimport der EU-Staaten 1994 in GWh

\begin{tabular}{|l|r|l|r|}
\hline Belgien & 9,05 & Irland & - \\
\hline Dänemark & 1,78 & Italien & 38,57 \\
\hline Bundesrepublik Deutschland & 35,65 & Luxemburg & 5,02 \\
\hline Griechenland & 0,82 & Niederlande & 10,71 \\
\hline Spanien & 5,10 & Portugal & 2,26 \\
\hline Frankreich & 3,38 & Großbritannien & 16,89 \\
\hline
\end{tabular}

Quelle: EUROSTAT: Energie : Monatliche Statistik 4/95

Tabelle 8: Stromexport der EU-Staaten 1994 in TWh

\begin{tabular}{|l|r|l|r|}
\hline Belgien & 5,07 & Irland & \\
\hline Dänemark & 6,62 & Italien & 1,08 \\
\hline Bundesrepublik Deutschland & 33,08 & Luxemburg & 0,56 \\
\hline Griechenland & 0,43 & Niederlande & 0,29 \\
\hline Spanien & 3,25 & Portugal & 1,37 \\
\hline Frankreich & 66,88 & Großbritannien & - \\
\hline
\end{tabular}

Quelle: EUROSTAT: Energie : Monatliche Statistik 4/95

Stromlieferungen dienen in erster Linie als Aushilfslieferungen und zum Ausgleich jahreszeitlicher Schwankungen in der Elektrizitätserzeugung einiger Länder. und werden gemäß der UCPTE-Satzung primär durch Kompensationsgeschäfte ausgeglichen (vgl. Tabelle 9).

Bereits in den 50er Jahren wurden erste lokale Verbindungsleitungen zwischen der UCPTE und den RGW-Staaten aufgebaut. Diese bestehen zwischen Österreich und Ungarn, Griechenland und Bulgarien, dem ehemaligen Jugoslawien und Ungarn, Rumänien und Bulgarien. Die Leitungen haben jedoch nur regionale Bedeutung ${ }^{226}$. Die Technik der Gleichstromübertragung wurde gewählt, da die Netzsteuerung und die Frequenzstabilität in den Netzen zu stark abwichen. Heute wird die Hochspannungs-Gleichstromübertragung (HGÜ) auch genutzt, weil sie bei größeren

226 Vgl. STRASSBURG, W. : Stromverbund fur Gesamteuropa, in: IZE: StromThemen, 8. Jg. Frankfurt 1991, S. 5 
Übertragungsentfernungen Kostenvorteile gegenüber Drehstromverbindungen aufweist. Neben den angesprochenen Verbindungen bestehen weitere Gleichstromverbindungen zwischen der UCPTE, der NORDEL und dem Netz Großbritanniens ${ }^{227}$, welches keinem Verbundsystem angeschlossen ist.

\section{Tabelle 9: Exportüberschuß der EU-Länder 1994 in TWh}

\begin{tabular}{|l|r|l|r|}
\hline Belgien & $-3,98$ & Irland & \\
\hline Dănemark & 4,84 & Italien & $-37,49$ \\
\hline Bundesrepublik Deutschland & $-2,57$ & Luxemburg & $-4,46$ \\
\hline Griechenland & $-0,39$ & Niederlande & $-10,42$ \\
\hline Spanien & $-1,85$ & Portugal & $-0,89$ \\
\hline Frankreich & 63,5 & Großbritannien & $-16,89$ \\
\hline
\end{tabular}

Quelle: Eigene Berechnung aus EUROSTAT: Energie : Monatliche Statistik 4/95

1995 wurde eine Verbindung zwischen der UCPTE und der NORDEL in Betrieb genommen. Die HGÜ-Leitung mit einer Kapazität von 500 MW verbindet Lübeck und Arrie (Schweden) ${ }^{228}$. Der Hauptzweck dieser Verbindung ist die verbesserte Nutzung der bisher nicht genutzten skandinavischen Wasserkraftpotentiale ${ }^{229}$. Eine weitere HGÜ-Leitung ist zwischen der dänischen ELKRAFT und der VEAG in den neuen Bundesländern beschlossen, wodurch mittels eines $42 \mathrm{~km}$ langen Seekabels bei einer Übertragungskapazität von $600 \mathrm{MW}$ das UCPTE-Netz weiter mit dem NORDEL-Netz verbunden wird. Mit der Inbetriebnahme wird 1995 gerechnet $^{230}$. Hinter diesen technischen Investitionen steht die Förderung eines Wirtschaftsraumes Ostsee und die Erschließung der Wasserkraftpotentiale Nordeuropas. In der Prüfungsphase sind Kabelverbindungen zwischen den Niederlanden und Norwegen sowie zwischen Schweden und Polen ${ }^{231}$. Darüber hinaus ist Korsika mit Italien über eine Gleichstromverbindung an das italienische Stromnetz angeschlos-

$227 \mathrm{Vgl}$. die genauen Errichtungsdaten der einzelnen Gleichstromleitungen in: BRINKMANN, K.: Einfuhrung in die elektrische Energiewirtschaft, Braunschweig 1971, S. 56 ff.

$228 \mathrm{Vgl}$. HAGENMEYER, E. : Stromversorgung in Europa, in: atomwirtschaft, Juli 1993, S. 510

229 Schătzungen gehen davon aus, daß bisher ca $6 \mathrm{Mrd}$. kWh ungenutzt blieben. Vgl. DVG: Bericht 1992, a.a.O., S. 12

230 Vgl. HAGENMEYER, E. : Stromversorgung..., a.a.O., S. 510 und STOTZ, J.: Anbindung des Höchstspannungsnetzes der neuen Bundesländer an den westeuropäischen Verbund, in: Elektrizitătswirtschaft, Jg. 93 (1994), Heft 13, S. 715-722, hier S. 722

231 Vgl. BIERHOFF, R. : Ausblick ... , a.a.O.RSdZ7G H Hermann - 978-3-631-75129-9 
sen worden ${ }^{232}$. Griechenland soll über eine Gleichstromverbindung $1997 \mathrm{mit}$ der italienischen Republik verbunden werden ${ }^{233}$.

Abgesehen von den lokalen Gleichstromleitungen ist die UCPTE mit dem osteuropäischen Verbundnetz seit 1983 über eine HGÜ-Verbindung in Dürnrohr (Österreich) verbunden. Im August 1992 kam eine 600 MW HGÜ-Kupplung zwischen Bayern und der Tschechischen Republik hinzu, die die Übertragungskapazität zu den osteuropäischen Elektrizitätsnetzen verdoppelte ${ }^{234}$. Am Ende dieser Entwicklung könnte ein europäisches „Supernetz“ stehen, das bis zum Ural reichen könnte ${ }^{235}$.

\subsubsection{Harmonisierungsgrad}

Ein ähnliches Bild wie das Stromaustauschvolumen zeigt die Harmonisierung der Rahmenbedingungen der Elektrizitätsversorgung. Die Elektrizitätsversorgungsstruktur ist in jedem Land unterschiedlich. Zu einer Rechtsangleichung ist es bisher nicht gekommen. Eine Aufnahme der Elektrizitätserzeugung oder Verteilung ist in keinem EU-Mitgliedsland für Unternehmen aus anderen Ländern möglich. In den Staaten mit nationalisierten Unternehmen ist diese sogar für Unternehmen des eigenen Landes untersagt. Darüber hinaus existieren die unterschiedlichsten Regulierungsinstrumente und Verfahren, die zumeist bei einer Investitionsaufsicht beginnen und bei den verschiedenen Formen der Preisaufsicht enden. Zudem sind die nationalen Elektrizitätspreise, insbesondere für die Industrie, häufig durch politische Zielsetzungen geprägt und variieren in Europa beträchtlich ${ }^{236}$ (Tabelle 10 und 11).

Ein Ziel des EU-Binnenmarktes ist die Steuerharmonisierung als Teil der Angleichung der allgemeinen Rahmenbedingungen. Die folgenden Daten zeigen die unterschiedlichen Steuersätze in den Mitgliedsstaaten ${ }^{237}$ (Tabelle 12).

232 Vgl. SCHWICKARDI, G. : a.a.O., Bd. 2, S. 70 . $\mathrm{Zu}$ den technischen Bedingungen und den Einsatzmöglichkeiten der Hochspannungsgleichstromübertragung (HGÜ) vergleiche HAPPOLT, H. ; OEDING, P. : Elektrische Kraftwerke und Netze, 5. Auflage, Berlin 1978, S. $607 \mathrm{ff}$.

233 Vgl. HAGENMEYER, E. : Stromversorgung..., a.a.O., S. 511

234 Vgl. DVG: Bericht 92 , a.a.O., S. 12

235 Vgl. HAGENMEYER, E. : Elektrizitătswirtschaft an der Jahrhundertwende - europăischer Wettbewerb kontra lokale Partnerschaft?, in: EW Jg. 94 (1995), Heft 20, S. 1267

236 Zur Problematik der internationalen Strompreisvergleiche siehe LANG, G. : Spielball der Wechselkurse? Strompreise im internationalen Vergleich, in: ENERGIE, Jg. 39, Nr. 4, April 1987, S. $47 \mathrm{ff}$.

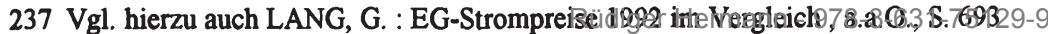


Tabelle 10: Strompreisvergleich für Haushaltsabnehmer Januar 1995 ECU pro $100 \mathrm{kWh}$

Abnahme: $3500 \mathrm{kWh}$ darunter Nachtstrom 1300, bei $4-9 \mathrm{~kW}$ bestellte Leistung alle Steuern eingeschlossen

\begin{tabular}{|l|r|l|r|}
\hline Belgien & 15,00 & Irland & 8,25 \\
\hline Dänemark & 14,78 & Italien & 19,82 \\
\hline $\begin{array}{l}\text { Bundesrepublik } \\
\text { Deutschland* }\end{array}$ & 15,87 & Luxemburg & 11,37 \\
\hline Griechenland & 7,64 & Niederlande* & 8,45 \\
\hline Spanien & 12,25 & Portugal & 13,22 \\
\hline Frankreich* & 12,96 & Großbritannien* & 10,21 \\
\hline
\end{tabular}

* Mittelwert aus mehreren Angaben fur Regionen oder Stadte

Quelle: EUROSTAT: Statistik kurzgefaßt - Energie und Industrie 1995/8, Luxemburg 1995

Tabelle 11: Strompreisvergleich für Industrieabnehmer Januar 1995 ECU pro $100 \mathrm{kWh}$

Abnahme: $24 \mathrm{GWh} / \mathrm{Jahr}$, Inanspruchnahme 6000 Stunden ohne Umsatzsteuer

\begin{tabular}{|l|r|l|r|}
\hline Belgien & 7,35 & Irland & 5,88 \\
\hline Dänemark & 4,90 & Italien & 7,88 \\
\hline $\begin{array}{l}\text { Bundesrepublik } \\
\text { Deutschland* }\end{array}$ & 9,67 & Luxemburg* & 5,87 \\
\hline Griechenland & 5,67 & Niederlande & 5,62 \\
\hline Spanien & 6,85 & Portugal* & 9,83 \\
\hline Frankreich* & 6,50 & Großbritannien* $^{*}$ & 5,33 \\
\hline
\end{tabular}

- Mittelwert aus mehreren Angaben fur Regionen oder Stadte

Quelle: EUROSTAT: Statistik kurzgefaßt - Energie und Industrie 1995/8, Luxemburg 1995 
Tabelle 12: Steuerliche Belastung auf den Haushaltsstromverbrauch in der EU im Januar 1994 in \%

Abnahme: $1200 \mathrm{kWh} / \mathrm{Jahr}$

\begin{tabular}{|l|r|l|r|}
\hline Belgien & 17,8 & Irland & 11,1 \\
\hline Dänemark & 49,9 & Italien & 20,5 \\
\hline Bundesrepublik Deutschland & 20,4 & Luxemburg & 5,7 \\
\hline Griechenland & 15,3 & Niederlande & 14,9 \\
\hline Spanien & 13,1 & Portugal & 5,1 \\
\hline Frankreich & 22,6 & Großbritannien & 0 \\
\hline
\end{tabular}

Quelle: EUROSTAT: Energiepreise 1973-1994

Weitere eigenstaatliche Tendenzen sind bei der Umwelt- und Ressourcenschonung zu erkennen. Jedes europäische Mitgliedsland hat ein eigenes anzustrebenes Niveau definiert und eigene Maßnahmen zum Umweltschutz durchgeführt.

Vergleicht man abschließend die für den Strommarkt aufgestellten Ziele der EUKommission, so kann festgehalten werden, daß die Freiheit der Lieferantenwahl, außer für Großabnehmer und Stromversorger in England und Wales, nirgendwo innerhalb der EU verwirklicht wurde. Eine Preisfestsetzung im freien Wettbewerb findet für alle Abnehmergruppen nicht statt ${ }^{238}$. Eine Durchleitungsmöglichkeit für Erzeuger besteht bisher nur in den Niederlanden und in Großbritannien. Die staatlichen Beihilfen für die Elektrizitätswirtschaft sind weder transparent noch einheitlich und die bestehenden Austausch- und Handelshemmnisse bestehen weiter. Daraus folgt, daß die bisherigen Strukturen zu keinem europäischen Binnenmarkt für Elektrizität geführt haben und der wirtschaftliche Integrationsgrad des Stromsektors hinter dem anderer Wirtschaftszweige hinterherhinkt. Hieraus erwächst in Handlungsbedarf, den es auszufullen gilt.

238 In Großbritannien ist die Preissteigerung an dër Entwicklung desTPreisindekes5gelonden. 


\section{Richtlinien und Richtlinienentwürfe zum europăischen Binnenmarkt für Elektrizităt}

Die EU-Kommission stellte in der Vergangenheit mehrere Richtlinienentwürfe zur Verwirklichung des europäischen Binnenmarktes für Elektrizität auf. Während die Richtlinienentwürfe zur Preistransparenz ${ }^{239}$ und zum Elektrizitätstransit über Hochspannungsnetze in Kraft getreten sind, steht die Verabschiedung der Richtlinienvorschläge, die mit Strukturveränderungen verbunden sind, durch das Europäische Parlament und dem Ministerrat noch aus.

\subsection{Die Entwicklung der Richtlinien und Richtlinienentwürfe}

Nachdem die Ziele für den EU-Binnenmarkt im allgemeinen und im speziellen für den EU-Strommarkt bereits in den achtziger Jahren aufgestellt wurden, erschien 1986 ein Weiß-buch, in dem die Vorstellungen und Maßnahmen zur Verwirklichung des EU-Binnenmarktes bis zum 01.01.1993 skizziert wurden. Die Maßnahmen für den Energiesektor sind in diesem Weißbuch nicht ausgefürt worden. Daher erschien 1988 ein Sonderbericht der EU-Kommission "Der Binnenmarkt für Energie", in dem zum ersten Mal der Begriff des "Common Carrier" für die leitungsgebundenen Energieträger eingeführt wurde, ohne jedoch den Begriff oder die zugrunde gelegte Ordnungskonzeption für den EU-Strommarkt zu erläutern ${ }^{240}$. Die konkreten Vorstellungen der EU-Kommission mündeten in mehreren Richtlinienvorschlägen, die 1990 vorgelegt wurden. Die erste verabschiedete Richtlinie sieht die Veröffentlichung von Strompreisen für Großabnehmer durch das Statistische Amt der Europäischen Gemeinschaft vor. Die Preise wurden bis dahin nicht veroffentlicht ${ }^{241}$.

Am 29.10.90 erließ der EU-Ministerrat die sogenannte "Elektrizitätstransitrichtlinie" (90/547 EWG), die die Umsetzung der Vorstellungen der EU-Mitgliedsstaaten hinsichtlich der Benutzungsmöglichkeiten der europäischen Stromverbundnetze zum Stromaustausch über die Grenzen von einzelnen Mitgliedsstaaten regelt. Die Richtlinie weist die Mitgliedsstaaten im Art. 1 an, die notwendigen Bestimmungen zu erlassen, um den Elektrizitätstransit zwischen den Hochspannungsnetzen zu begünstigen. Unter Elektrizitätstransit wird gemäß Art. 2

239 Vgl. LANG, G. : Die EG-Richtlinie zur Strompreistransparenz, in: EW Jg. 89 (1990), Heft 24, S. 1357

$240 \mathrm{Vgl}$. KOMMISSION DER EUROPÄISCHEN GEMEINSCHAFT - Generaldirektion Energie : Energie in Europa - Der Binnenmarkt für Energie, Luxemburg 1988, S. 52

241 Vgl. LANG, G. : Die EG-Richtlinie zur Strompreistransparenz, in: EW Jg. 89 (1990), Heft 24, S. 1357 
Abs.1 jede Beförderung von Elektrizität verstanden, die "die folgenden Voraussetzungen erfüllt:

a) Die Beförderung wird von der (den) in jedem einzelnen Mitgliedsstaat für ein großes Hochspannungsnetz zuständigen Gesellschaft(en) durchgefürt, das im Gebiet eines Mitgliedsstaats gelegen und am Verbund der europäischen Hochspannungsnetze beteiligt ist; Verteilungsnetze sind ausgenommen;

b) das Erzeugungsnetz bzw. das Endabnahmenetz liegt im Hoheitsgebiet der Gemeinschaft;

c) bei der Beförderung wird mindestens eine innergemeinschaftliche Grenze überschritten."242

Die Bestimmungen gelten für die in Tabelle 13 aufgeführten Unternehmen ${ }^{243}$ :

Die folgenden Artikel der Richtlinie legen fest, daß Vertragspartner die nationalen Netzgesellschaften sein müssen und die Verträge keine Diskriminierungen und mißbräuchliche Vorschriften enthalten dürfen. Ferner dürfen die Verträge die Versorgungssicherheit und Dienstleistungsbereitstellung nicht beeinträchtigen oder gefährden (Art. 3 Abs.2).

Art. 3 Abs. 3 regelt die Anzeigepflicht der Verträge und Vertragsverhandlungen bei der EU-Kommission. Darüber hinaus legt die Richtlinie fest, welcher Kreis die bei der EU-Kommission eingerichtete Schlichtungsstelle anrufen kann. Nach der Entscheidung der Schlichtungsstelle kann die EU-Kommission das im Gemeinschaftsrecht vorgesehene Verfahren zur Durchsetzung der Entscheidung einleiten.

Die Kommission sah durch die sogenannte Transitrichtlinie nicht die Verwirklichung des europäischen Strommarkt sichergestellt, da die Richtlinie keinen Wettbewerb um Endverbraucher einfürte. Ihrer Meinung nach leistet die Richtlinie nur einen bescheidenen Beitrag zur Integration der nationalen Elektrizitätswirtschaften. Deshalb wurden von der Kommission weitere Überlegungen angestellt, wie die aufgestellten Ziele verwirklicht werden können. Die Überlegungen mündeten in den später heftig diskutierten Richtlinienentwurf der EU-Kommission vom 22.01.1992. Der Entwurf sieht für die Elektrizitätswirtschaft eine phasenweise Einführung von Veränderungen vor ${ }^{244}$, die im folgenden aufgezeigt wird:

In der ersten Phase soll der Elektrizitätstransit und die Transparenz der von den individuellen Endverbrauchern zu zahlenden Preisen erhöht werden.

242 Richtlinie des Rates vom 29.10.1990 über den Transit von Elektrizitătslieferungen über große Netze (90/547/EWG).

243 Hierbei sind seit der Richtlinie Änderungen eingetreten durch Fusionen, Umbenennungen und dem Anschluß des ostdeutschen Stromnetzes an das Gemeinschaftsgebiet.

$244 \mathrm{Vgl}$. KOMMISSION DER EUROPÄISCHEN GEMEINSCHAFT: Bulletin der EG 1/2, Luxemburg 1992 S. 47 
Tabelle 13: Verzeichnis der Verbundgesellschaften in Europa

\begin{tabular}{|l|l|l|}
\hline \multicolumn{3}{|c|}{ Verzeichnis der Gesellschaften und Netze der Gemeinschaft, } \\
die unter die EU-Richtlinie fallen
\end{tabular}

Quelle: Richtlinie des Rates vom 29.10.1990

In der zweiten Phase soll die Konkurrenz auf den europäischen Elektrizitätsmärkten durch Öffnung der Märkte für die Stromerzeugung und für die Errichtung von Hochspannungsleitungen belebt werden. Die Maßnahmen sollen unterstützt werden durch eine erhöhte Kostentransparenz bei den Elektrizitätsversorgungsunternehmen (EVU), um einen fairen, nicht diskriminierenden Wettbewerb zu erreichen. Gleichzeitig sollen in dieser Phase die integrierten Unternehmen organisatorisch und in der Rechnungslegung ,entbündelt“245 und ein beschränkter Zugang für Dritte zu den Netzen der EVU eingeführt werden. Der Zugang soll zunächst auf die Großabnehmer mit einer Abnahmemenge von jährlich über $100 \mathrm{GWh}$ (rd. 25

245 Im Sinne von einer Aufteilung der Unternehmen in Sparten mit getrennter Rechnungslegung 
MW Anschlußleistung) und Verteilerunternehmen mit einem Anteil von mehr als drei Prozent an der nationalen Stromverteilung beschränkt werden ${ }^{246}$.

Die dritte Phase der vorgeschlagenen Richtlinie sieht die weitergehende Öffnung der Netze für Dritte (Third-Party-Access) vor.

Der erste Zugang für Dritte sollte nach den Vorstellungen der EU-Kommission ab 1993, die weitere Ausdehnung ab $1996^{247}$ und der Abschluß der Phasen bis 1998 erzielt werden ${ }^{248}$. Der Erstellung der Phasen und der Richtlinienentwürfen lagen als Leitlinien die Vermeidung von Überregulierungen, die Einhaltung der Subsidiarität, des politischen Dialogs sowie des schrittweisen Vorgehens zugrunde ${ }^{249}$.

Als Reaktion auf den Kommissionsvorschlag bestätigte der EU-Rat die Bedeutung der Vollendung des Energiebinnenmarktes für die Integration der Märkte. Er betonte, daß die Erstellung von Richtlinien an den Grundsätzen der schrittweise Realisierung, der Transparenz und der Nichtdiskriminierung orientiert werden sollte ${ }^{250}$. Darüber hinaus ersucht der Rat die EU-Kommission, ihre Vorschläge zur Einfuihrung eines größeren Wettbewerbs zu prüfen und insbesondere die unterschiedliche Lage in den einzelnen Mitgliedsstaaten und die Besonderheiten des Elektrizitätsmarktes zu berücksichtigen ${ }^{251}$.

Die Wirtschaftsministerkonferenz vom 25. und 26.03.1992 hat sich in einem Beschluß grundsätzlich für mehr Wettbewerb ausgesprochen, empfiehlt jedoch wegen fehlender Praxiserfahrungen mit Wettbewerbsmodellen in der Elektrizitätswirtschaft ein behutsames Vorgehen. In den Vorschlägen der EU-Kommission sieht die Konferenz die Gefahr einer Überregulierung und äußerte Bedenken gegen den Netzzugang Dritter in Verbindung mit der geplanten Entbündelung ${ }^{252}$. Die europäischen EVU kritisierten den Vorschlag vehement. Sie sehen in dem Vorschlag einen Eingriff in ihre Eigentumsrechte und eine Gefährdung der Versorgungssicherheit. Ferner müßte vor einer Öffnung der Märkte erst die Harmonisierung der Rahmenbedingungen durchgeführt werden, damit bestehende Wettbewerbsverzerrungen nicht einzelne EVU benachteiligen.

246 Diese scheinbar niedrige Zugangsschwelle würde in der Bundesrepublik den Ausschluß jedes regionalen und lokalen Verteilerunternehmens von der Durchleitung bedeuten.

247 Vgl. ET-Redaktion : Kompromißsuche hallt an, in: ET 42. Jg. (1992), Heft 8, S. 500

248 Vgl. o.V. : Liberalisierung der Energiemărkte umstritten, in: ET 42. Jg. (1992), Heft 7, S. 491

249 Vgl. KOMMISSION DER EUROPÄISCHEN GEMEINSCHAFT: Bulletin der EG 1/2, Luxemburg 1992, S. 47. Die aufgestellten Zeitplăne sind durch die nicht zeitgerechte Umsetzung der Richtlinie bereits hinfallig geworden.

250 Vgl. KOMMISSION DER EUROPÄISCHEN GEMEINSCHAFT: Bulletin der EG 11, Luxemburg 1992, S. $46 \mathrm{f}$.

251 Vgl. KOMMISSION DER EUROPÄISCHEN GEMEINSCHAFT: Bulletin der EG 11, Luxemburg 1992, S. 47

252 Vgl. BÖWING, H. ; DEHMER, R. ; DIENES, K. : EG und Internationales, in: ET 42. Jg. (1992), Heft 7 , S. 482 
Trotz der heftigen Diskussion um die Vorschläge und der massiven Kritik ${ }^{253}$ von seiten der EVU hielt die EU-Kommission an ihren Vorschlägen fest. Im folgenden Zeitraum entstand eine lebhafte politische Diskussion zwischen Kommission, Ministerrat und Europäischem Parlament über die Struktur des zukünftigen europäischen Elektrizitätsmarktes. Sowohl im Ministerrat als auch im europäischen Parlament entstanden zwei gegensätzliche Grundhaltungen. In beiden Institutionen sahen Vertreter des Wettbewerbsgedankens und der Öffnung der Märkte in der Liberalisierung und dem Abbau der zwischenstaatlichen Stromhandelshemmnisse das geeignete Mittel um die Integration voranzutreiben. Andere Gruppen in beiden Institutionen forderten mit Hinweis auf das Subsidiaritätsprinzip die Beibehaltung der nationalen Eigenständigkeiten. Vertreter dieser Gruppen weisen den Regierungen der Mitgliedsländer das alleinige Recht zu, über das Ausmaß des Wettbewerbs in der Stromversorgung zu entscheiden ${ }^{254}$. Die Auseinandersetzung um die vorgeschlagene EU-Richtlinie mündete in der Ablehnung des Kommissionsvorschlages durch Ministerrat und Parlament. Das europäische Parlament forderte die Betonung des öffentlichen Dienstleistungscharakters, der mit dem Schlagwort des „service public“ zusammengefaßt wurde. Darüber hinaus forderte das Europäische Palament vor jeder Umstrukturierung eine umfassende Harmonisierung der Rahmenbedingungen, insbesondere hinsichtlich der Umweltschutzauflagen und der Steuergesetze. Im Gegensatz zur Haltung der EU-Kommission soll nach der Meinung des Europäischen Parlamentes ein Zugang zum Netz eines EVU nicht erzwingbar sein und aufgrund freier Verhandlung zwischen den betroffenen Unternehmen erfolgen. Staaten mit zentralistischer Elektrizitätsstruktur sollen von der Liberalisierung ausgenommen werden, wenn sie benötigte Kraftwerkskapazitäten ausschreiben. Durchleitungsanträge sollen von den Unternehmen dieser Länder bei einer Einschränkung des öffentlichen Versorgungsauftrags abgelehnt werden können. Übereinstimmung herrscht zwischen dem Europäischen Parlament und der Kommission in bezug auf die Abschaffung der Ausschließlichkeitsrechte für den Bau von Stromerzeugungsanlagen. Damit zeigen das europäische Parlament und der Ministerat, daß sie keine klaren Zielvorstellungen und Konzepte für die Schaffung eines europäischen Strommarktes haben.

Als Folge der Stellungnahme des Europäischen Parlamentes am 17.11.1993 modifizierte die EU-Kommission ihren Vorschlag. Sie sieht nun nicht mehr einen erzwingbaren Netzzugang vor, sondern will einen ,verhandelten Netzzugang Dritter“ (NTPA) erreichen ${ }^{255}$. Neben der Abschaffung der Ausschließlichkeitsrechte hielt

253 Vgl. z.B. GRAWE, J. : Der Kommissionsentwurf für eine EG-Richtlinie "Elektrizităt" aus der Sicht der deutschen Elektrizitătswirtschaft, in: EW Jg. 91 (1992), Heft 14. S. 880 ff. und SCHAVOIR, R. : Common Carriage: Geordneter Rückzug? , in: ET 40. Jg. (1990), Heft 3, S. 109

$254 \mathrm{Vgl}$. HELFRISCH, F. : EU-Binnenmarkt für Strom aus Verbrauchersicht, in: ET 44. Jg. (1994), Heft 1/2, S. 27 f.

255 Vgl. 0.V. : EU und Internationales: Die Europăische Kommission zur Vereinbarkeit des

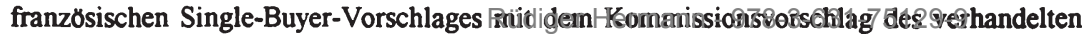


die Kommission an der schrittweisen Einführung von Wettbewerb fest. Verändert wurden desweiteren die Vorschläge für den Verteilungsbereich. Eine Beschränkung des Wettbewerbs im Verteilungsbereich soll in den Staaten möglich sein, die die Endverbraucherversorgung als Staatsaufgabe definieren oder definiert haben ${ }^{256}$. Hierin gibt die Kommission den Forderungen des Europäischen Parlamentes nach Anerkennung des „service public"-Auftrages der EVU, insbesondere in den romanischen Staaten, nach. Dies reichte der französischen Regierung jedoch nicht. Frankreich schlug vor, das System des Alleinverkäufers (Single-Buyer) ${ }^{257}$ als eine zweite Alternative zum verhandelten Netzzugang in Europa zuzulassen. Der französische Vorschlag räumt dem Single-Buyer das alleinige Reicht ein, über alle Teilbereiche der Elektrizitätsversorgung autonom zu entscheiden. Beantragte Durchleitungen und Stromlieferungen kann der Single-Buyer ablehnen, wenn seiner Meinung nach der öffentliche Versorgungsauftrag eingeschränkt oder gefährdet wird. 1995 ließ die EU-Kommission prüfen, ob das Single-Buyer System neben dem verhandelten Netzzugang in Europa existieren kann, ohne daß Verzerrungen im innereuropäischen Stromhandel eintreten. Die bis Anfang 1996 vorgelegten Studien verneinen dies. Aus diesem Grund lehnte die EU-Kommission den französischen Vorschlag ab. Am 11.02.96 brachte die französische Seite einen Kompromißvorschlag bei einer Sitzung auf Expertenebene ein. Dieser Vorschlag will die Verpflichtung zur Liberalisierung des europäischen Elektrizitätsmarktes auf das Marktsegment der Großabnehmer mit Absatzmengen über $100 \mathrm{GWh} / \mathrm{Jahr}$ pro Abnehmer begrenzen. Der Komprißvorschlag wurde insbesondere von deutscher Seite als nicht ausreichend abgelehnt ${ }^{258}$. Die Auseinandersetzungen zwischen den einzelnen EU-Institutionen über die geeigneten Maßnahmen zur Integration der europäischen Elektriztitätswirtschaften sind zur Zeit noch nicht abgeschlossen. Im folgenden sollen nun die hinter den Vorschlägen stehenden Konzepte kurz umrissen werden.

Netzzuganges, in: ET 45. Jg. (1995) Heft 5, S. 324 und KOMMISSION DER EUROPÄISCHEN GEMEINSCHAFT: Energy in Europe, 1994 Annual Energy Review, Luxemburg 1995, S. $145 \mathrm{f}$.

256 Vgl. BIERHOFF, R. : Ausblick auf die künftige elektrizitătswirtschaftliche Zusammenarbeit, in: EW Jg. 93 (1994), Heft 13, S. 744 f. und STRASSBURG, W. : Energie-, Umwelt- und Wettbewerbspolitik im Widerstreit., in: ET 44. Jg. (1994), Heft 9, S. 593 ff.

257 Vgl. MICHAELIS, H. : Der Weg zu einem europäischen Binnenmarkt für Energie, in: Energiewirtschaftliche Tagesfragen, 46. Jg. (1996), Heft 4, S. 214-217

258 Vgl. PRITZSCHE, K.; MEIER, A. : EU-Liberalierung - jüngste Entwicklungen, in: Ener-

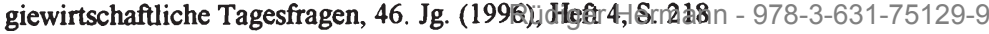




\subsection{Die Vorstellungen der EU-Kommission}

Faßt man die Elemente der bisherigen Äußerungen und Richtlinienvorschläge der EU-Kommission zusammen, so können vier wesentliche Bestandteile herausgestellt werden $^{259}$ :

- Aufhebung der Ausschließlichkeitsrechte im Kraftwerks- und Leitungsbau,

- (Rechnungslegungsmäßige) Trennung von Erzeugung, Transport und Verteilung (Unbundling),

- Benutzung der Netze durch Dritte (Third-Party-Access),

- Zuweisung der Übertragungsnetze zu Netzbetreibern.

Die einzelnen Elemente können jedoch nicht, wie von Vertretern der Elektrizitätswirtschaft und der nationalen Regierungen praktiziert, getrennt bewertet werden, sondern müssen in ihrem Zusammenwirken betrachtet werden. Daher werden die auf den Richtlinienvorschlag von 1992 zurückgehenden Vorstellungen der EUKommission in dem zweiten Ordnungsmodell (TPA-Konzept) konkretisiert und anschließend anhand der aufgestellten Kriterien bewertet.

1993 weisen Veröffentlichungen und Äußerungen von Mitgliedern der EU-Kommission daraufhin, daß die EU-Kommission die Umsetzung des britischen Modells für ganz Europa befürworten würde. Die Umsetzung eines an die britische Struktur angelehnten Ordnungsmodells für den europäischen Binnenmarkt soll in dem vierten Konzept (Stromhändler) analysiert und bewertet werden.

Die urspüngliche Zielsetzung der EU-Kommission mit einer weitgehenden Einführung von Teilmärkten bei vollständiger Desintegration wird im dritten Konzept (Common-Carrier) entworfen und anhand der Kriterien überprüft.

\subsection{Die Vorstellungen des Europăischen Parlamentes und des Ministerrates}

Die Einwände des Ministerrates, der französischen Regierung und des Europäischen Parlamentes lassen auf von der EU-Kommission abweichende Ziele schließen. Die wiederholte Betonung des „service-public“-Auftrages der EVU in Europa und des Subsidiaritätsprinzips ergibt folgendes Bild:

259 Vgl. KLOPFER, T. : Mărkte für Strom - Internationale Erfahrungen und Übertragbarkeit auf Deutschland, München 1993 , S. 53 Rüdiger Hermann - 978-3-631-75129-9 
- Die nationalen Regierungen sind ebenso wie die Vertreter im Europäischen Parlament nicht bereit, die bisherigen nationalen Strukturen in den jeweiligen Mitgliedsstaaten aufzugeben.

- Sie lehnen eine grundlegende Einführung von Wettbewerbsmodellen ab.

- Sie wollen den Status quo um eine Ausdehnung des zwischenstaatlichen Stromaustausches auf freiwilliger Basis modifizieren.

Für die europäische Integration und die Vollendung des europäischen Strommarktes hätte dies zur Folge, daß die bisherigen vertikal integrierten Strukturen zumindest in der Mehrzahl der Mitgliedsländer beibehalten werden würden. Ob die Länder mit nicht vollständig zentralistischen Strukturen eine wettbewerbliche Öffnung ihrer Märkte zulassen würden, wenn die Mehrzahl der Mitgliedsländer hiervon Abstand nimmt, bleibt zu bezweifeln. Ein Elektrizitätshandel jedoch würde immer auf freiwilliger Basis und ohne Beteiligung von Verbrauchern stattfinden. Beantragte Durchleitungen können mit Hinweis auf eventuelle Einschränkungen des öffentlichen Versorgungsauftrages jederzeit abgelehnt werden. Diese Vorstellungen decken sich weitgehend mit denen der europäischen EVU und sollen in dem ersten Ordnungsmodell (Weiterentwicklungskonzept) eingehend dargestellt und hinsichtlich ihres Zielerreichungsgrades anhand der aufgestellten Kriterien bewertet werden. 


\section{Integrationskonzepte}

\subsection{Die Weiterentwicklung des bestehenden Verbundsystems durch die europăischen EVU (1.Konzept)}

\subsubsection{Die Konzeption des Weiterentwicklungskonzeptes}

Kerngedanke des Weiterentwicklungskonzeptes ist der freiwillige Ausbau des bestehenden Verbundsystems, um die Ziele des UCPTE-Verbundes ${ }^{260}$ mit niedrigeren Kosten zu erreichen.

Das Weiterentwicklungskonzept sieht folgendes vor:

- den Ausbau des bisherigen Verbundbetriebes,

- die Intensivierung des bestehenden Stromhandels innerhalb der Rahmenbedingungen vor Inkraftreten der EU-Richtlinien zur Vollendung des EU-Binnenmarktes für Elektrizität ${ }^{261}$,

- die weitere Steigerung der Versorgungssicherheit.

Diese Maßnahmen und Ziele sollen auf die folgenden, weitgehend auf die UCPTE zurückgehenden Richtlinien aufbauen:

- Eigenverantwortlichkeit der EVU in der Stromversorgung,

- Freiwilligkeit ${ }^{262}$ in der Durchführung von Maßnahmen und bei der Teilnahme am Stromhandel,

- weiterbestehende nationale Besonderheiten,

- Rücksichtnahme auf historisch gewachsene nationale Strukturen ${ }^{263}$.

Zur Verwirklichung des Konzeptes sind einige Vorbedingungen erforderlich: Die UCPTE und die NORDEL müssen ihre Infrastruktur und ihre Verbundnetze für den Binnenmarkt mit seinen Zielen zur Verfügung stellen, d.h. auch Nicht-EUMitglieder müssen für den weiteren Ausbau des Verbundbetriebes gewonnen werden. Darüber hinaus muß der Wille der EVU vorhanden sein, an den Maßnahmen teilzunehmen und den Verbund in die beabsichtigte Richtung weiterzuentwickeln. Insbesondere gilt dies für die beabsichtigten Kosteneinsparungen durch Effizienzerhöhungen. Für den europäischen Elektrizitätsmarktes bedeutet das Konzept die Beibehaltung der geschlossenen Versorgungsgebiete, innerhalb derer ein EVU das Versorgungsmonopol für alle Endverbraucher besitzt. Es findet keine weitere In-

260 Vergleiche zu den Zielen des Binnenmarktes Kapitel 1.

261 Vgl. RIEMER, H.W. : EG-Binnenmarkt - Technische Aspekte der Herausforderung der deutschen Elektrizitătswirtschaft, in: EW Jg. 91 (1992), Heft 17, S. 1083

262 Vgl. RIEMER, H.W. : a.a.O., S. 1080

263 Hierdurch wird der Vorschlag des EuropdiscictioneParlamentes-veilgehend ưngesetet. 
tegration oder Desintegration statt. Teilnehmer am freiwilligen Stromhandel sind die Unternehmen der überregionalen Verbundebene. Die bestehenden Vorteile des Verbundbetriebes, insbesondere die geringeren Kosten des hohen Versorgungssicherheitsniveaus durch partnerschaftliche Reservehaltung, werden auch weiterhin genutzt werden können. Durch die Konzeption vorgegeben können drei Arten von Stromhandelsgeschäften entstehen bzw. bereits bestehende verstärkt werden:

a) Kurzfristige Stromlieferungen auf Basis von Kompensationsgeschäften zum Ausgleich außergewöhnlicher Betriebssituationen. Die angesprochenen Geschäfte sind weniger dem Stromhandel zuzuordnen als den Maßnahmen zur Erhöhung der Versorgungssicherheit.

b) Stromlieferungen zwischen benachbarten EVU und deren monetäre Bezahlung. Die Lieferungen können sowohl kurzfristigen als auch langfristigen Charakter haben, da das UCPTE-Reglement langfristige Stromlieferverträge als Teil der Eigenverantwortlichkeit akzeptiert.

c) Stromlieferungen zwischen nicht benachbarten EVU und deren Transport über das Hochspannungsnetz eines dritten Verbundunternehmens.

\subsubsection{Die Mittel des Konzeptes}

Die Mittel zum Ausbau des bisherigen Verbundbetriebes in Richtung einer Verbundwirtschaft ${ }^{264}$ sind:

- der gemeinsame Kraftwerksbau ${ }^{265}$,

- die schrittweise Ausdehnung der gemeinsamen Nutzung bestehender Kraftwerke,

- die gemeinschaftliche Brennstoffbeschaffung und -verwaltung,

- die Einrichtung einer Strombörse,

- die Verbesserung der europäischen Regelungstechnik zum Ausbau der Versorgungssicherheit und zur Abwicklung der Stromhandelsgeschäfte.

Die Strombörse besteht aus einem Informationssystem, in dem die beteiligten EVU überschüssige Stromerzeugungskapazitäten anbieten. Alle Teilnehmer haben dann die Möglichkeit, bilaterale Verträge über Stromlieferungen abzuschließen. Ermög-

264 Zur Erlăuterung der Betriebsarten vgl. PICK, H. : Die Ordnung ..., a.a.O., S. 72. In den USA wird die Verbundwirtshaft als tight energy pool bezeichnet, vgl. FEDERAL ENERGY REGULATORY COMMISSION: The Transmission Task Forces Report to the Commission, Electricity Transmission: Realities, Theory and Policy Alternatives, London 1989, S. $17 \mathrm{ff}$. mit den Angaben über die Anzahl und dem Stromabsatzvolumen der Unternehmen.

265 Erste Beispiele für diesen Trend ist die Beteiligung deutscher EVU an dem Atomkraftwerk

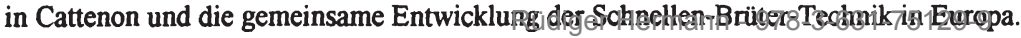


licht werden soll mit der Strombörse die kurzfristige Optimierung zwischen Eigenerzeugung der EVU und Fremdbezug. Gemäß Schmitt kommt diese Wirtschaftlichkeit "allen (Verbrauchern) zugute" ${ }^{\text {266 }}$. Darüber hinaus wird die Strombörse als Informations- und Preisbildungsmittel für die Stromlieferungen des zweiten und dritten Typs benötigt. Die Preise werden bei der bereits teilweise betriebenen Strombörse jedoch nicht nach dem Auktionsverfahren bestimmt, sondern durch die angesprochenen bilateralen Verträge ${ }^{267}$. Zur $\mathrm{Ab}$ - und Verrechnung der Stromlieferungen ist eine Messung der Lastströme innerhalb der nationalen sowie zwischen den nationalen Netzen notwendig, um ungewollte Stromflüsse zu minimieren. Die auf Lieferverträgen beruhenden Lastflüsse müssen auf der Grundlage der Messungen monetär abgerechnet werden. Die nicht auf Lieferverträgen beruhenden ungewollten Lastflüsse können durch geeignetes Vorstellen der Kupplungsregler zwischen den nationalen Verbundnetzen in späteren Zeitperioden ausgeglichen werden. Solange die ungewollten Lastflüsse auf einem niedrigen Niveau verbleiben, werden die EVU keine Bedenken gegen das bewährte Verfahren haben. Denkbar ist aber auch eine monetäre Entlohnung unbeabsichtigter Lastflüsse, die jedoch wenig Sinn gibt. Der Empfänger der Stromflüsse hat keine niedrigeren Kosten durch die Lieferung zu erwarten. Den eingesparten Brennstoffkosten steht ein erhöhter Regelungsaufwand entgegen.

Konzeptionell ungelöst bleibt die Frage, wie die Nutzung der Verbundinfrastruktur geregelt wird. Bisher stellten die nationalen EVU ihre Infrastruktur dem Verbund kostenlos zur Verfugung, da die Inanspruchnahme durch andere begrenzt war und der Gesamtnutzen der Verbundteilnahme die Kosten bei weitem überstieg. Anders könnte dies werden, wenn einige EVU sich am Stromhandel weniger beteiligten, andere dagegen sehr. Sind die wenig Beteiligten gleichzeitig die Haupttransitpartner der am Stromhandel Teilnehmenden, ist fraglich, ob die kostenlose Infrastrukturbereitstellung bestehen bleibt. Eine Lösung wäre die genaue Messung der Stromflüsse und einer darauf aufbauenden Infrastrukturabgabe der Benutzer. Eine weitere Lösungsmöglichkeit könnte das Abführen eines Teiles der Ersparnisse des Stromhandels in einen Fond sein und die anschließende Ausschüttung nach der prozentual zur Verfügung gestellten Infrastruktur.

\subsubsection{Die Entwicklungmöglichkeiten des Konzeptes}

Aufbauend auf den Voraussetzungen, Bedingungen und Mitteln des Weiterentwicklungskonzeptes lassen sich vier Entwicklungsszenarien darstellen.

266 SCHMITT, F.J. : Europåische Stromversorgung auf neuen Wegen, in: Glückauf 127 (1991), Nr. 5/6, S. 223

267 Vgl. SCHMITT, F.J. : a.a.O., S. 223 Rüdiger Hermann - 978-3-631-75129-9 
Szenario 1 : Stagnation des Stromhandels

Szenario 2 : Ausbau des Stromhandels auf niedrigem Niveau

Szenario 3 : Intensivierung des Stromhandels

Szenario 4 : Entwicklung zum Energiemaklersystem

Zwischen den Szenarien, dem Stromhandelsvolumen und der Bereitschaft zum Stromhandel besteht folgender Zusammenhang ${ }^{268}$.

\section{Abbildung 8: Weiterentwicklungskonzept - Szenarien im Überblick}

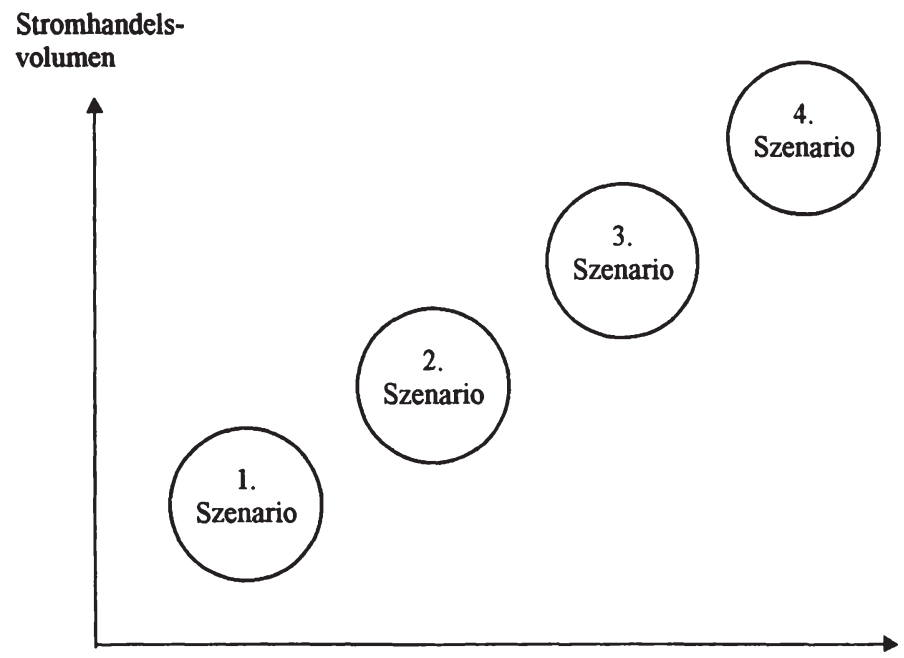

Bereitschaft zum

Stromhandel

Szenario 1: Stagnation des Stromhandels und die Beschränkung der Weiterentwicklung auf die Versorgungssicherheit, Versorgungsqualität und Reservehaltung

268 Auf die Bereitschaft zum Stromhandel ist auch vom jeweiligen Unternehmertyp abhăngig. Zur Typologisierung von Unternehmern vgl. BORCHERT, M. ; GROSSEKETTLER, H. : Preis- und Wettbewerbstheorie: Marktprozesse als analytisches Problem und ordnungspolitische Gestaltungsaufgabe, Stuttgart 1985, S. \$19diger Hermann - 978-3-631-75129-9 
Faktoren die das Eintreten des Szenarios fördern :

- geringe Strompreisdisparitäten,

- fehlender öffentlicher und/oder politischer Druck zu Strompreissenkungen,

- Betonung der nationalen Eigenständigkeit in der Elektrizitätsversorgung,

- eine reine Höchstpreisregulierung.

Durch gemeinsame Absprachen und die Entwicklung von Technologien zur Verbesserung der Versorgungssicherheit können positive Effekte durch den EUBinnenmarkt für den Verbraucher entstehen. Ebenso kann durch den Ausbau der bestehenden Kupplungskapazitäten zu den Ländern der EU, die bisher nur gering an die Verbundnetze angeschlossen sind, z.B. Griechenland, Großbritannien und Irland, die Versorgungssicherheit und Reservehaltung dieser Länder verbessert und gleichzeitig Kosten eingespart werden. Eine weitere Kooperation ist bei diesem Szenario im Bereich der Spannungshaltung denkbar, um das bisherige Stabilitätsniveau weiter zu erhöhen.

Szenario 2 : Ausbau des Stromhandels auf einem insgesamt niedrigen Niveau

Geringe Strompreisdisparitäten und das Interesse einiger weniger EVU am Stromhandel fördern das Eintreten des zweiten Szenarios. Gründe für das Interesse dieser Elektrizitätswirtschaften können kurzfristige Probleme bei der Strombedarfsdeckung sein.

Die Probleme können aus

- dem Ausfall von Kraftwerken,

- einem starken Nachfrageanstieg,

- der mittelfristigen Wasserknappheit durch trockene Sommer

resultieren.

An einem Eintreten dieses Szenarios hat Italien ein besonderes Interesse. 
Szenario 3 : Intensivierung des Stromhandels

Ein intensiver Stromhandel auf dem europäischen Strommarkt des Weiterentwicklungskonzeptes kann durch ein hohes Interesse der EVU am Stromhandel zustande kommen. Das Interesse der EVU kann durch folgende Sachverhalte entstehen:

- Massive Überkapazitäten in einer Elektrizitätswirtschaft und eine Öffentlichkeit, die Quersubventionen und hohe Preise durch die Überkapazitäten zunehmend weniger toleriert,

- Unzureichende Kraftwerkskapazitäten in Elektrizitätswirtschaften bei fehlenden Zubaumöglichkeiten,

- Die Androhung stromintensiver Industriezweige zur Eigenerzeugung überzugeben oder ihre Standorte zu verlagern; insbesondere dann, wenn die betroffenen EVU keine Quersubventionmöglichkeiten haben,

- Eine politische Beeinflussung der EVU zu Stromim-/exporten.

Szenario 4 : Entwicklung zum Energiemaklersystem

In den USA haben sich Energiemaklersysteme auf freiwiliger Basis entwickelt, die auf der Grundlage kurzfristiger Grenzkosten Effizienzsteigerungen bewirkten ${ }^{269}$. Anders als bei der im Aufbau befindlichen Strombörse werden bei den amerikanischen Energiemaklersystemen innerhalb kurzer Zeitperioden Liefer- und BezugsEVU durch ein Computerprogramm ermittelt und automatisch zugewiesen. Restriktionen können gleichzeitig erfaßt und mit einbezogen werden. Das Eintreten des Szenarios für den europäischen Elektrizitätsmarkt begünstigende Faktoren sind:

- hohe Strompreisdisparitäten,

- freie Leitungskapazitäten,

- eine funktionierende Teilnahmeregulierung,

- die hohe Bereitschaft zur Teilnahme am Stromhandel von seiten der $\mathrm{EVU}^{270}$,

- Überkapazitäten in Ländern mit niedrigen Erzeugungspreisniveau.

Welche Entwicklung bei der Umsetzung des Konzeptes tatsächlich eintreten würde, hängt fast vollständig von der Akzeptanz des Konzeptes und der Bereit-

269 Vgl. BARKER, J.M. Jr. : Electric Energy Brokering: An Explanation and Status Report, in: Public Utility Fortnightly, Februar 1982, S. 28 ff.

270 Die Sachverhalte, die das Interesse am Stromhandel fordern, sind identisch mit denen des Szenarios 3. 
schaft zum Stromhandel bei den beteiligten EVU der Verbundebene ab, nicht jedoch von den gesetzten Rahmenbedingungen oder den Markteffekten. Eine direkte Einflußnahme der europäischen Institutionen auf das Marktgeschehen besteht nicht.

\subsubsection{Die Akzeptanz des Weiterentwicklungskonzeptes}

Die Akzeptanz des Konzeptes bei den EVU fur das von ihnen entwickelte Konzept ist selbstverständlich ${ }^{271}$. Die bestehenden Vorteile des Verbundbetriebes werden auf freiwilliger Basis ausgebaut und das einzelne EVU hat die Möglichkeit zu wählen, welche Maßnahmen es durchführt. Kurzfristige Aushilfslieferungen ohne Abnehmerverluste können von den EVU genutzt werden, um eine kostenintensive, kurzfristige Steigerung der eigenen Erzeugungskapazitäten zu vermeiden. Je nach staatlicher Regulierungspraxis innerhalb der einzelnen Länder variiert jedoch die Bereitschaft zur langfristigen Teilnahme am Stromhandel: In den Ländern, die ihren EVU eine prozentual feste Verzinsung des eingesetzten Kapitals garantieren und die über ein hohes Strompreisniveau verfügen, wird die Bereitschaft zu langfristigen Strombezugsverträgen gering sein. Der Grund besteht darin, daß die Stromimporte das eingesetzte Kapital und somit auch den Gewinn des EVU reduzieren. In Mitgliedsländern mit gleicher Regulierungspraxis und niedrigen Erzeugerpreisen wird die langfristig gewinnsteigernde Wirkung der Exporte zur Teilnahme am Stromhandel führen. Die Versorgungsunternehmen in Staaten, die keine Gewinne in der Elektrizitätsversorgung erlauben, müßten scheinbar indifferent sein oder zur Ausnutzung der Einsparpotentiale durch den Stromhandel bereit sein. Die Realität widerspricht dieser These jedoch: Die französische EdF als ein EVU aus dieser Gruppe, die wegen ihrer niedrigen Strompreise theoretisch als Anbieter auftreten könnte, hat die Bedeutung einer unabhängigen nationalen Elektrizitätsversorgung immer wieder betont. Dies verwundert um so stärker, weil die französische Importabhängigkeit bei Primärenergieträgern hoch ist ${ }^{272}$.

Die industriellen Großverbraucher werden dieses Konzept, wie sie in zahlreichen Veröffentlichungen zum Ausdruck gebracht haben ${ }^{273}$, ablehnen. Sie bekommen keine Möglichkeit, ihren Belieferer zu wählen und Strompreisdisparitäten in Europa auszunutzen. Die Machtstellung des EVU bei Strombelieferungsverhandlungen ist unverändert.

271 Vgl. VDEW: Für europäische Kooperation - gegen Zwangsregulierung durch Brüssel, Stellungsnahme vom 15.07.1991, Frankfurt/M. 1991 S. 3 ff.

272 Ein Grund hierfür könnte nach Meinung des Verfassers die Ablehnung jeder Marktöffnung oder Verănderung durch die EVU-Manager sein.

$273 \mathrm{Vgl}$. hierzu stellvertretend für die europäischen stromintensiven Industriezweige die Veroffentlichungen der VIK, z.B. : VIK : „Durchleitung als Wettbewerbsinstrument in der Stromversorgung", Essen 1990 
Die Haushaltsabnehmer werden dieses Konzept mehrheitlich befürworten, garantiert es doch weiterhin eine gesicherte Elektrizitätsversorgung auf hohem Niveau. Werden gleichzeitig Kosteneinsparungen durch die Angleichung der Rahmenbedingungen innerhalb der EU an den Verbraucher wenigstens teilweise weitergegeben, wird die Akzeptanz steigen ${ }^{274}$. Außerdem ist es bisher den EVU gelungen, weite Teile der Bevölkerung mit ihrem Argumentationsmuster für eine integrierte Elektrizitätsversorgung und die geschlossenen Versorgungsgebiete zu gewinnen. Die Einbeziehung von kommunalen Vertretern innerhalb der Versorgungsunternehmen erleichtert dies.

\subsubsection{Der Regulierungsbedarf des Konzeptes}

Der Regulierungsbedarf des Konzeptes verteilt sich auf folgende Bereiche:

a) Verhinderung von Monopolrenten

b) Verteilung der Einsparungen

c) Sicherung der Teilnahme am Stromhandel
-> Mittel: Preisregulierung

-> Mittel: Aufteilungsregeln zwischen EVU und

Verbrauchergruppen

-> Mittel: Aufsicht über die Unternehmensführung

\section{$\mathrm{Zu}$ a) Verhinderung von Monopolrenten}

Eine Regulierung mit dem Ziel, unangemessene Monopolrenten zu verhindern, ist beim Weiterentwicklungskonzept erforderlich. Eine derartige Regulierung hat in den meisten EU-Mitgliedsländer Tradition. Die einzelnen Regulierungsmaßnahmen und -ansätze innerhalb der EU sind nicht angeglichen, so daß konsequenterweise eine Harmonisierung der europäischen Preisregulierungsvorschriften erforderlich wird.

274 Diese These kann man mit dem Verhalten der amerikanischen Verbraucher in den siebziger Jahren begründen. In diesem Zeitraum sanken die Strompreise und es kamen keine Forderungen nach verstärktem Wettbewerb auf. Erst als Ende der Siebziger, Anfang der achtziger Jahre die Strompreise anstiegen, forderten die Verbraucherverbände die Einführung wettbewerblicher Prozesse in der Elektrizitătswirtschaffier Hermann - 978-3-631-75129-9 
$Z u$ b) Verteilung der Einsparungen zwischen EVU und Verbrauchern

Zielsetzung des Weiterentwicklungs-Konzeptes ist es, Kosteneinsparungen in der Elektrizitätsversorgung zu erzielen. Daraus erwächst die Frage, wie Kosteneinsparungen aufgeteilt werden sollen. Eine einfache Lösung wäre die freie Verfügung der EVU über die Kosteneinsparungen. Dies würde positive Anreize zur Entwicklung des Stromhandels bewirken. Eingeschränkt würden die Anreize durch die nationalen Regulierungsbestimmungen, die den EVU keine Ausdehnung des Gewinnes bei Kosteneinsparungen aus dem Stromgeschäft zubilligen. Daher muß, um positive Effekte zu erzielen, den EVU ein Teil der Kosteneinsparungen als Gewinn überlassen werden ${ }^{275}$. Der Rest muß an die Verbraucher weitergegeben werden. Verbraucherverbände sprachen sich für die vollständige Weitergabe der Kosteneinsparungen an die Verbraucher aus. Dann ist eine Aufsicht über die Teilnahme am Stromhandel erforderlich, da keine inhärenten Anreize für die EVU zur Teilnahme bestehen.

\section{Zu c) Sicherung der Teilnahme am Stromhandel}

Das Verhältnis aus relativen Grenzkosten in der Stromerzeugung und dem Umfang überschüssiger Erzeugungskapazitäten bestimmt die Regulierungserfordernisse zur Teilnahme am Stromhandel. Hierbei sind folgende Kombinationen zu unterscheiden:

1. Niedrige Grenzkosten ohne Überkapazitäten

EVU mit im europäischen Vergleich niedrigen Grenzkosten und ohne überschüssige Erzeugungskapazitäten können nicht andere EVU beliefern und sind an einem Bezug zur Kostensenkung nur bei außergewöhnlichen Betriebssituationen interessiert. Über das bisherige Regulierungsniveau hinausgehende Maßnahmen sind nicht erforderlich.

2. Niedriges Grenzkostenniveau und hohe Überkapazitäten

Ein Unternehmen mit niedrigen Grenzkosten und hohen Überkapazitäten ${ }^{276}$ ist bestrebt, die überschüssige Elektrizität abzusetzen. Dies hat für das EVU den Vorteil zusätzlicher Deckungsbeiträge bei unverändertem Eigenkapitaleinsatz. Gleichzeitig kann ein Stromabsatz mit hohen Deckungsbeiträgen bei staatlichen Investitionsaufsichtsbehörden als Argument für weitere Investitionen eingesetzt werden. Eine staatliche Aufsicht muß in diesem Fall überprüfen, ob die aus dem Stromexport gewonnenen Deckungsbeiträge auch wirklich genutzt werden, um den Verbrauchern Preisreduzierungen zu gewähren.

275 In den USA können EVU zwischen 25 und 50\% der Kosteneinsparungen als Gewinne einbehalten.Vgl. hierzu BOLLE, F. : Wettbewerb und Kooperation..., a.a.O., S. 19

276 Ein Beispiel wăren hohe Überkapazităten in Grund- und Mittellastkraftwerken mit ihren niedrigen Grenzkosten bis zur Kapazitătsgrentzear Hermann - 978-3-631-75129-9 
3. Mittleres Grenzkostenniveau ohne Überkapazitäten

Versorgungsunternehmen der Verbundebene mit mittleren Grenzkosten und ohne überschüssige Erzeugungskapazitäten sind potentielle Kunden für EVU mit niedrigen Grenzkosten und freien Kapazitäten bei temporärem Anstieg der Nachfrage. Das EVU ist indifferent gegenüber den Stromlieferungen, da der Strombezug investitionsneutral wirkt. Eine staatliche Aufsicht muß daher die Ausnutzung von Kosteneinsparungspotentialen durch Strombezug überprüfen.

4. Mittleres Grenzkostenniveau und Überkapazitäten

Ein Unternehmen mit mittleren Grenzkosten und überschüssigen Kapazitäten ${ }^{277}$ wird in Zeiten geringer Nachfrage auf der Verbundebene seine Kapazitäten nicht zur Erzielung von Deckungsbeiträgen einsetzen können. In Zeiten hoher Nachfrage können Strommengen abgesetzt werden, wenn die Nachfrage der EVU mit hohen Grenzkosten dem Angebot der EVU mit niedrigen Grenzkosten übersteigt ${ }^{278}$. In Zeiten niedriger europäischer Stromnachfrage, wird das EVU aber nicht die Angebote günstiger Anbieter nutzen, da es zuerst die eigenen Kapazitäten einsetzen will, um einen Deckungsbeitrag zu erzielen. Eine Regulierung sollte in dieser Situation in der Marktteilnahmekontrolle bestehen. Es muß sichergestellt werden, daß das EVU seine überschüssigen Kapazitäten zu einem realistischen Preis anbietet und keine Verhandlungen über Stromlieferungen ablehnt.

\section{Hohes Grenzkostenniveau ohne Überkapazitäten}

Ein EVU mit hohen Grenzkosten ohne überschüssige Erzeugungskapazitäten ist ein potentieller Strombezieher. Eine staatliche Aufsicht sollte ein besonderes Augenmerk auf die Ursachen der hohen Grenzkosten legen und überprüfen, ob das Unternehmen alle Möglichkeiten zur Kostenreduzierung, also auch durch Fremdbezug, ausgenutzt hat.

\section{Hohes Grenzkostenniveau und Überkapazitäten}

Unternehmen mit hohen Grenzkosten und überschüssigen Kapazitäten werden keine Absatzchancen haben, können eventuell aber durch preisgünstigeren $\mathrm{Zu}$ kauf absolute Kosteneinsparungen erzielen. Die Motivation hierzu wird jedoch gering sein, da die Einsparungen durch Fremdbezug die Ineffizienz der Versorgung durch das EVU offenbaren. Die staatliche Regulierung unterscheidet sich nicht von dem Fall ohne überschüssige Kapazitäten.

277 Denkbar sind ehemals als Mittellastkraftwerke eingesetzte ăltere Kraftwerke, die noch zur Spitzenbedarfsdeckung eingesetzt werden.

278 Es wurden bei diesen Überlegungen keine Restriktionen durch die Stromtransportkapazităten vorausgesetzt. 
Letztlich bedeuten die Regulierungseingriffe, daß die EVU auf der Grundlage von Informationen der Strombörse nachweisen müssen, daß sie kostenreduzierende Angebote angenommen oder angeboten haben. Diese regulierenden Eingriffe widersprechen jedoch dem Prinzip der Freiwilligkeit, das der Konzeption zugrunde gelegt worden ist.

\subsubsection{Die Implementierung des Konzeptes}

Eine Implementierung des Konzeptes erscheint einfach und ohne hohe Kosten möglich. Die erforderliche Regelungs- und Steuerungstechnik ist zum Großteil bereits vorhanden oder kann erweitert werden. Gleiches gilt für den Aufbau der Strombörse. Die Installationen eines reinen Informationssystems, an dem die beteiligten EVU "on-line" angeschlossen sind, stellt im Zeitalter der digitalen Datenverarbeitung und Übertragungstechnik kein Problem dar und kann durch die Kosteneinsparungen unproblematisch finanziert werden ${ }^{279}$. Defizite hinsichtlich der erforderlichen Infrastruktur bestehen in Griechenland, Irland und in Großbritannien, dreier Mitgliedsstaaten, die bisher nicht oder nur indirekt an das UCPTENetz angeschlossen sind. In Großbritannien müssen die bestehenden Gleichstromverbindungen durch Drehstromverbindungen ausgestauscht werden und eine Regelungstechnik nach UCPTE-Standard eingerichtet werden. Ferner muß sich Großbritannien der gemeinsamen Frequenzsteuerung anschließen. Griechenland ist bisher nur über das Netz des ehemaligen Jugoslawien an das UCPTE-Netz angeschlossen. Seit Ausbruch des Krieges ist diese Verbindung nicht mehr sicher und genügt nicht den Anforderungen. Als Folge wurde Griechenland nicht mit dem UCPTE-Netz parallelgeschaltet ${ }^{280}$. Da eine Normalisierung derzeit nicht absehbar ist, muß Griechenland über eine leistungsfähige Verbindung an das UCPTE-Netz angeschlossen werden, über die dann auch der Stromaustausch zu den Mitgliedsstaaten durchgeführt werden kann. Irland kann bisher nicht am europäischen Stromaustausch teilnehmen, weil die Verbindungen fehlen.

Eine Voraussetzung für positive Auswirkungen des Weiterentwicklungskonzeptes im Sinne der aufgestellten Ziele ist eine Bewußtseinsänderung beim Management der EVU. Diese zu initiieren wird schwieriger werden als die Schaffung der organisatorischen und technischen Voraussetzungen des Konzeptes.

Wenn diese Voraussetzungen für eine Intensivierung des Stromhandels geschaffen wurden und bei Eintreten des dritten oder vierten Entwicklungsszenarios das Han-

279 Beim FEB sind 1987 laufende Kosten in Höhe von 433000 \$ angefallen, denen Ersparnisse in Höhe von 40 Mio.\$ gegenüberstanden. Vgl. hierzu BOLLE, F. : Wettbewerb und Kooperation, a.a.O., S. 63.

280 Dies führte zu Netzbeeintrăchtigungen. Vgl. hierzu: UCPTE: Jahresbericht 1993 , Wien 1994, S. 45 
delsvolumen steigt, wird das Bedürfnis seitens der EVU steigen, die Transaktionskosten zu senken und von der Börse zu einem Energiemaklersystem überzugehen. Die EVU könnten zuerst selbst entscheiden, ob sie weiter an der Strombörse teilnehmen oder ihre Handelsaktivitäten durch den Energiemakler automatisch abwickeln lassen wollen. Ebenso ist ein gleichzeitiges Operieren in beiden Handelsinstitutionen mit unterschiedlichen Angeboten möglich.

Die Schaffung der technischen Einrichtungen und die Umgestaltung der Organisationen zum Ausbau des bestehenden Verbundes in der EU müßte nach maximal zwei Jahren abgeschlossen sein.

\subsection{Das TPA-Konzept (2.Konzept)}

\subsubsection{Die Konzeption des TPA-Konzeptes}

„Third-Party-Access“(TPA) ist das in der Fachliteratur meist diskutierte Schlagwort für den europäischen Strommarkt ${ }^{281}$. Einer der Gründe für die Popularität des TPAs ist die scheinbar einfache dahinterstehende Konzeption, welche rechtlich jedoch Probleme aufwirft ${ }^{282}$. „Third-Party-Access“ bedeutet, daß ein EVU, welches über ein Stromnetz verfügt, dieses anderen für Durchleitungen zur Verfügung stellen muß.

Grundlage des TPA-Konzeptes sind die bestehenden Strukturen der nationalen Elektrizitätswirtschaften innerhalb der EU-Staaten. Die UCPTE und die NORDEL werden als zwischenstaatliche, elektrizitätswirtschaftliche Institutionen akzeptiert. Aufbauend auf dem gegenwärtigen System soll durch Einführung einer Durchleitungsverpflichtung für EVU mit einem geschlossenen Versorgungsgebiet Wettbewerb in der Elektrizitätswirtschaft eingeführt werden ${ }^{283}$. Dabei ist die „Durchleitung" einer der entscheidenden Begriffe.

281 Vgl. RIEMER, H.W. : a.a.O., S. 1086

282 Vgl. BAUER, J.F. : Die Europäische Gemeinschaft und das Recht der leitungsgebundenen Energie, Baden-Baden 1993. Im folgenden sollen die rechtlichen Probleme ausgeklammert werden, da sie Gegenstand umfangreicher Diskussionen der Juristen sind und für die ökomomische Betrachtung der Ordnungskonzepte hinsichtlich der Effizienz und gesamtgesellschaftlichen Wohlfahrt sowie ihrer Bewertung hinsichtlich des Zielerfüllungsbeitrages nicht ausschlaggebend sein dürften.

283 Vgl. RIEMER, H. W. : a.a.O., S. 1080 Rüdiger Hermann - 978-3-631-75129-9 
Durchleitung wird folgendermaßen definiert:

Unter einer Durchleitung wird im elektrizitätswirtschaftlichen Sprachgebrauch der ökonomische Transport ${ }^{28}$ elektrischer Energie durch das Leitungsnetz eines $E V U$, welches die elektrische Energie nicht produziert hat, verstanden ${ }^{285}$.

Unter dem Oberbegriff werden eine Vielzahl von Tatbeständen zusammengefaßt, die in fünf Typen ${ }^{286}$ eingeteilt werden können:

\section{Durchleitung Typ 1 (Stromtransit)}

Ein EVU A speist in das Netz eines EVU B Strom ein. Ein weiteres EVU C entnimmt den Strom an einer anderen Netzgrenze.

\section{Abbildung 9: Durchleitung Typ 1 (Stromtransit)}

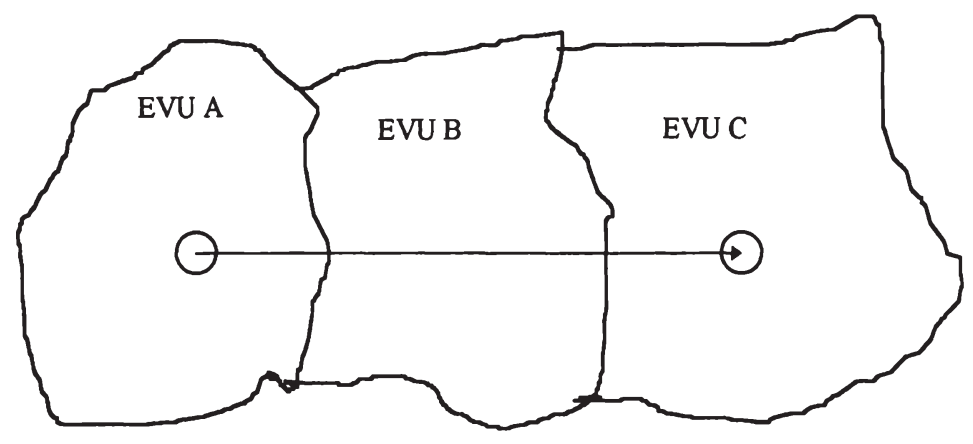

Dieser Fall wird in bezug auf das EVU B im folgenden auch als Stromtransit bezeichnet.

284 Dem okonomischen Stromtransport stehen keine physikalischen Lastflüsse gegenüber.

285 Gröner bezeichnet als Durchleitung ausschließlich den Transport durch das Hoch- und Mittelspannungsnetz eines EVU. Vgl. GRÖNER, H. : Wettbewerb in der Elektrizitătswirtschaft, in: Energiewirtschaft, Heft 2 (1979), S. 112-117, hier S. 116

$286 \mathrm{Vgl}$. SCHWEPPE, F.-C. : Economic Impediments to Power Transfer, in: KELLY, K. (Hrsg.): Non-Technical Impediments to Poüreingerantsfen, Coolurabla 307hio-19877, \$.976 ff. 


\section{Durchleitung Typ 2}

Ein EVU A speist Strom in das Netz eines EVU B ein. Ein Abnehmer im Versorgungsgebiet von B entnimmt die Elektrizität aus dem Netz von B. Der gleiche Durchleitungstyp liegt vor, wenn ein Erzeuger im Netz von EVU B an das EVU A Strom liefern will.

\section{Abbildung 10: Durchleitung Typ 2}
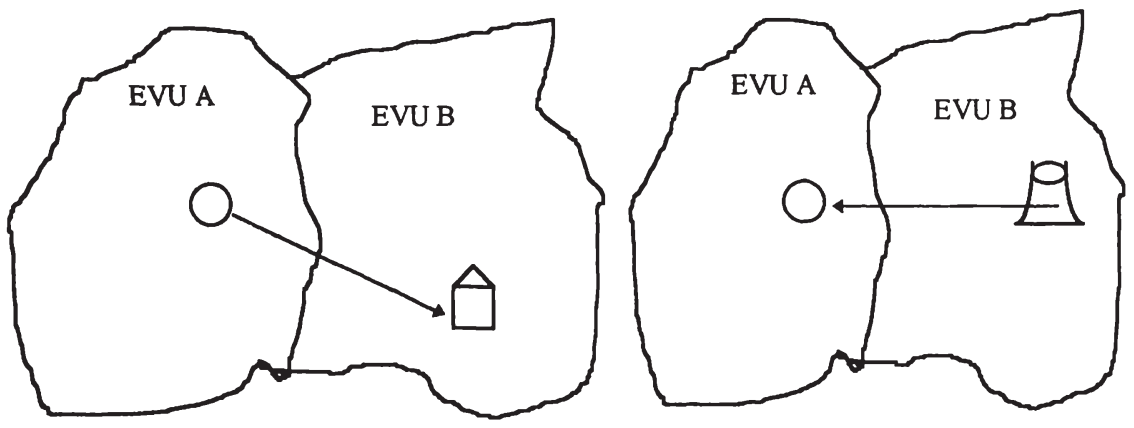

\section{Durchleitung Typ 3}

Ein Erzeuger, der nicht Netzbetreiber ist, speist in das Netz seines Gebiets-EVUs Strom ein, der von einem Abnehmer des gleichen Gebiets-EVU entnommen wird.

\section{Abbildung 11: Durchleitung Typ 3}

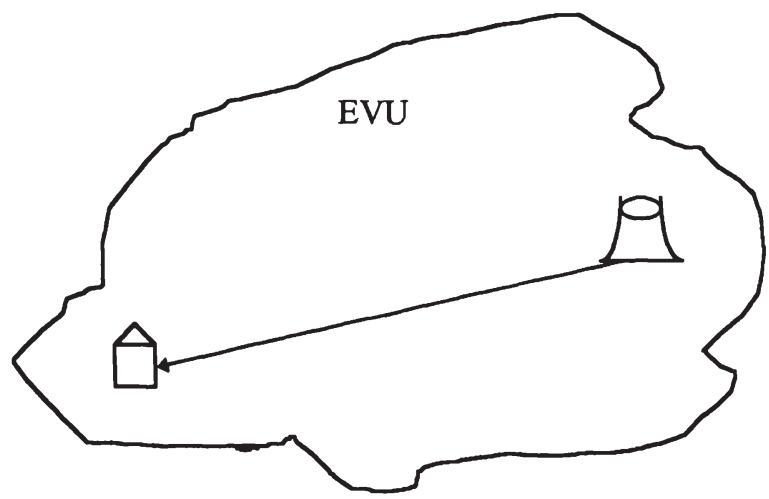


Neben diesen generellen Fällen der Durchleitung, ist die Kombination der einzelnen Arten denkbar.

\section{Durchleitung Typ 4}

EVU A liefert elektrische Energie über das Leitungsnetz des Gebiets-EVUs B zu einem Abnehmer innerhalb des Versorgungsgebietes des EVU C. Gleicher Fall liegt dann vor, wenn ein Erzeuger innerhalb des Versorgungsgebietes des EVU C über das Netz des EVUs B zu dem EVU A liefert.

\section{Abbildung 12: Durchleitung Typ 4a}

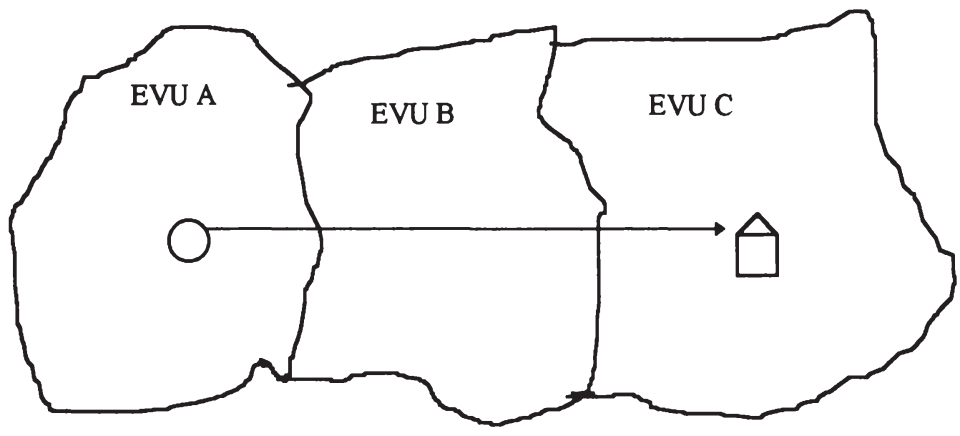

\section{Abbildung 13:Durchleitung Typ 4b}

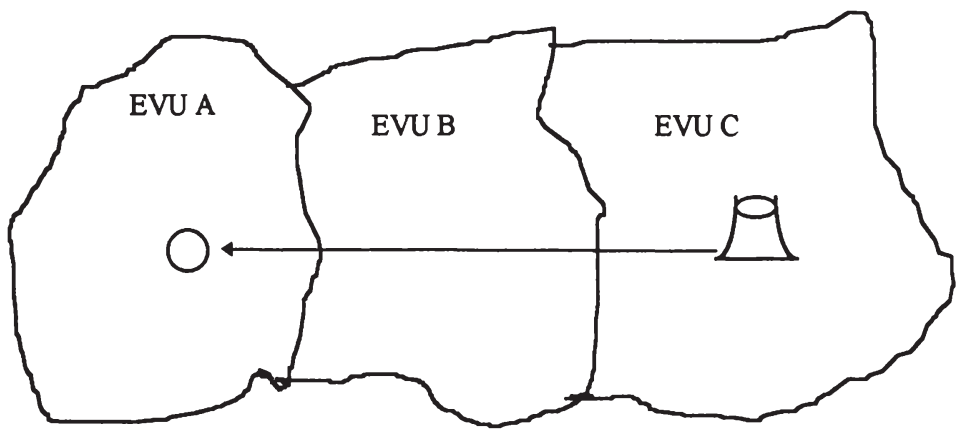




\section{Durchleitung Typ 5}

Ein Erzeuger innerhalb des Versorgungsgebietes des EVU A liefert Strom zu einem Abnehmer innerhalb des Versorgungsgebietes von EVU C über das Leitungsnetz von EVU B.

\section{Abbildung 14: Durchleitung Typ 5}

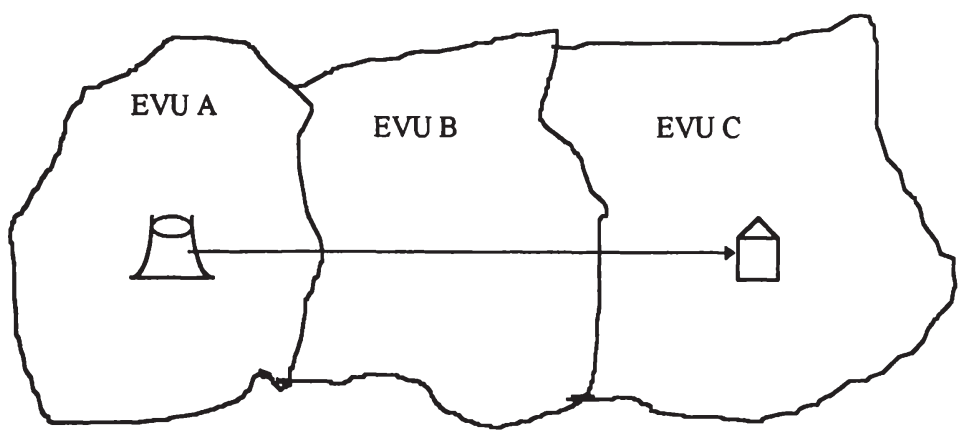

Für das EVU B handelt es sich bei diesem Durchleitungstyp um einen reinen Stromtransit.

Das TPA-Konzept sieht nun die Einführung der Durchleitungsverpflichtung in drei Stufen vor:

In der ersten Stufe soll die Durchleitungsverpflichtung für die EVU der Verbundebene zum ersten Male zum Einsatz kommen. Hierdurch soll Wettbewerb zwischen den EVU um die günstigsten Strombeschaffungsmöglichkeiten eingeführt werden. Es werden ausschließlich Durchleitungen des Typs 1 zustande kommen können. Potentielle Stromhandelsgeschäfte der ersten Stufe des Konzeptes können kurzfristige Aushilfslieferungen und langfristige Handelsaktivitäten sein. Problematisch wirken sich bei den langfristigen Geschäften die Netzkapazitäten des Durchleiters aus ${ }^{287}$.

287 Die Handelsmöglichkeiten zwischen benachbarten EVU sind in diesen Überlegungen nicht eingeschlossen. Für EVU, die Stromlieferungen mit benachbarten EVU anstreben, unterscheidet sich das TPA-Konzept hinsichtlich der Stromhandelsgeschäftsoptionen nicht von

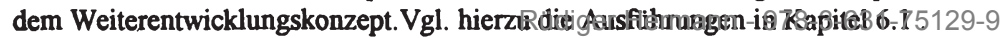


Die zweite Stufe räumt industriellen Großabnehmern und Verteilerunternehmen ein Durchleitungsrecht ein. Das Durchleitungsrecht ist erforderlich, damit Großabnehmer und Verteiler ab einer bestimmten Stromabnahmemenge zwischen ihrem Gebiets-EVU und einem anderen Stromhandelsunternehmen wählen können. Hierdurch soll wettbewerbliches Verhalten um Letztabnehmer initiiert werden. Bei einem externen Strombezug durch diese Abnehmer muß das betroffene GebietsEVU bei ausreichenden Netzkapazitäten die Stromdurchleitung abwickeln.

Die dritte Stufe sieht vor, allen Endverbrauchern die Möglichkeit zu eröffnen, den Stromlieferanten frei zu wählen. Alle Abnehmer können jedoch auch weiter zu alten Vertragskonditionen bei ihrem Gebiets-EVU verbleiben.

\section{Abbildung 15: Stufenweise Einführung der Durchleitungsverpflichtung im TPA-Konzept}

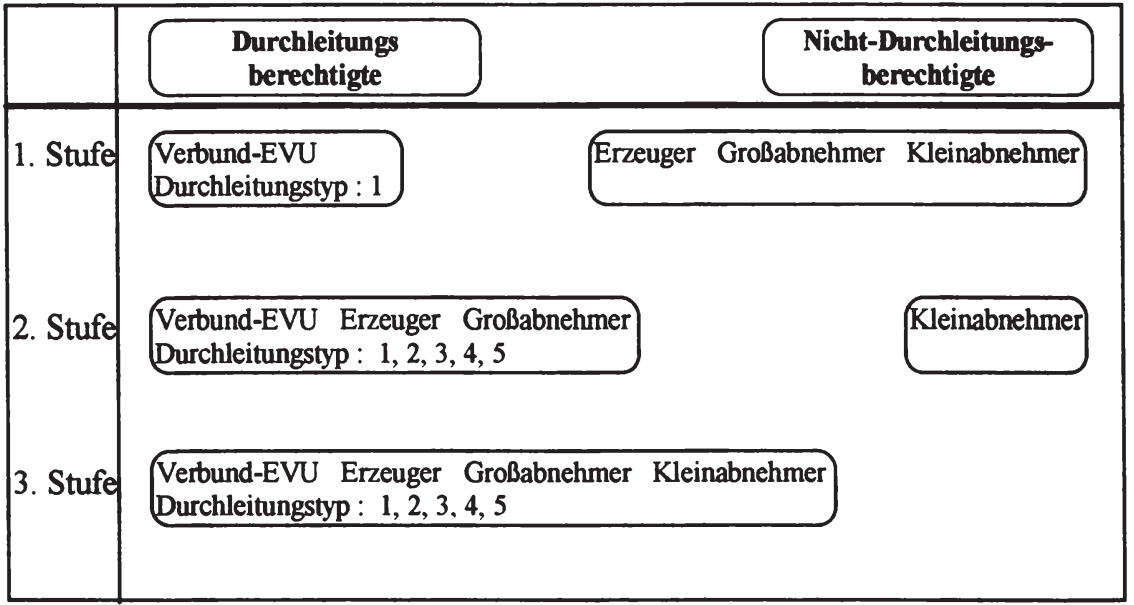

Der hinter dem Konzept stehende Grundgedanke besteht darin, daß es sich bei dem Leitungsnetz eines EVU mit geschlossenem Versorgungsgebiet um eine Art Infrastruktur handelt, deren Potential allen Interessierten offen stehen soll. Das Versorgungsnetz innerhalb eines Versorgungsgebietes bleibt in der Hand eines EVU, welches weiterhin zum Anschluß der Kleinverbraucher verpflichtet ist (Versorgungspflicht). Das Betriebsmonopol des EVUs mit dem Leitungsnetz besteht fort.

Mit der Einführung des Konzeptes sind mehrere Forderungen verknüpft, aus denen die Regeln im TPA-Konzept abgeleitet werden: 
a) Das durchleitende EVU darf durch die Durchleitung nicht in seinem Netzbetrieb und seiner Versorgungsleistung unzumutbar beeinträchtigt werden. Konkret bedeutet dies, daß das EVU überschüssige Leitungskapazitäten besitzen muß, die den Durchleitungsbegehrenden technisch und organisatorisch zur Verfügung gestellt werden können .

b) Das Durchleitungsentgelt muß alle Kosten des durchleitenden Unternehmens für die zu erbringenden Leistungen abdecken, da sonst die Rentabilität des Netzes sinken würde. Eine Unterdeckung wäre ferner eine wettbewerbsverzerrende Subvention des Durchleiters zugunsten des EVU, das den Stromtransport benötigte.

Eiss et $\mathrm{a}^{288}$ und darauf bezugnehmend Pick ${ }^{289}$ sind der Meinung, daß die Durchleitungsverpflichtung zu dauerhaften und tragfähigen institutionellen Regeln führt, "wenn das Erbringen von Durchleitungsleistungen für den Netzbetreiber zu einer regulären wirtschaftlichen Aktivität wird." ${ }^{290}$.

Folgende Voraussetzungen sind für die Verwirklichung des Konzeptes notwendig:

a) Der Wille der EVU zur Teilnahme am Stromhandel und damit zum wettbewerblichen Verhalten ist erforderlich. Zudem müssen die EVU bereit sein, Durchleitungen abzuwickeln ${ }^{291}$.

b) Einige " Pionier"- Verbraucher müssen zu Beginn der zweiten und dritten Stufe des Konzeptes bereit sein, Strom nicht von ihrem Gebiets-EVU zu beziehen. Sie zeigen damit risikoaversen Abnehmern, daß die Inanspruchnahme der Durchleitung nicht zum Zusammenbruch der eigenen Versorgung führt und wirken somit erhöhend auf den Wettbewerbsdruck.

Generelle Voraussetzung zur erfolgreichen Implementierung des Konzeptes ist eine geeignete Regelungs- und Meßtechnik, die alle anfallenden Lastflüsse über die Grenzen der jeweiligen Versorgungsgebiete hinaus erfaßt und beeinflussen kann. Für ungewollte Lastflüsse muß zudem der bestehende Abrechnungsmodus des UCPTE-Netzes auf das TPA-Konzept angepaßt werden. Des weiteren muß eine Durchleitung für die sie beantragende Unternehmen durchsetzbar sein. Den Unternehmen, die potentiell zur Durchleitung gezwungen werden sollen, muß die Möglichkeit genommen werden, über atarifäre Handelshemmnisse die Durchleitung unattraktiv werden zu lassen.

288 Vgl. EISS, H. ; LUKES, R. ; PICK, H. ; SCHULZ, W. : Die Ordnung des Elektrizitätsmarktes ...., a.a.O., S. 72 ff.

289 Vgl. PICK, H. : a.a.O., S. 86

290 Vgl. PICK, H. : a.a.O., S. 86

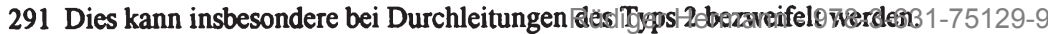


Ungeklärt sind bei der Konzeption eine Reihe wesentlicher Punkte:

a) Die Vertragsgestaltung,

b) die Situation bei Rückkehr der Verbraucher zum Gebiets-EVU,

c) das Durchleitungsentgelt ${ }^{292}$,

d) die Versorgungspflicht und die Versorgungssicherheit ,

e) die Auswahl der Durchleitungsanträge.

\section{Zu a) Die Vertragsgestaltung}

Zwischen dem Strom liefernden und dem -beziehenden Unternehmen oder Abnehmern müssen Absprachen über den Umfang und die Dauer der angestrebten Stromlieferung getroffen werden. Gleichzeitig sind die Sicherheit und die Qualität der Lieferung und deren Abnahme festzulegen. Zwischen den Vertragspartnern und den durchleitenden EVU muß ein Vertrag abgeschlossen werden, der das Verhalten des Durchleiters bei Ausfall der Lieferung (Übernahme der Reservehaltung) und bei Schwankungen in der Lieferspannung und -frequenz sowie die Bezahlung der Übertragungsverluste regelt. Bisher existieren keine direkten Vertragsentwürfe für Stromlieferungen im Rahmen des TPA-Konzeptes. Doch lassen sich in der europäischen Praxis genügend Vorbilder für derartige Verträge finden ${ }^{293}$.

\section{Zu b) Die Situation bei Rückkehr der Verbraucher zum Gebiets-EVU}

In der zweiten und dritten Stufe des Konzeptes können Abnehmer wählen, von welchem Stromerzeuger sie ihre benötigte Energie beziehen. Nach Ablauf der ersten externen Lieferverträge kann es zu folgenden Situationen kommen:

- Der Abnehmer wünscht die Wiederaufnahme der Versorgung durch das Gebiets-EVU, weil er keinen Lieferanten für den Anschlußzeitraum nach der Belieferung durch ein externes EVU findet. Es stellt sich dann die Frage, ob das Gebiets-EVU gezwungen werden kann, die Versorgung des Abnehmers zu übernehmen. Mit der Frage verbunden sind die Bedingungen, zu denen die Versorgung durch das Gebiets-EVU wieder aufgenommen werden muß. Ohne die Übernahme der Versorgung durch das Gebiets-EVU müßte der Abnehmer zur Eigenerzeugung übergehen. Der Weg der Eigenerzeugung ist jedoch nicht für alle Abnehmer aus räumlichen oder rechtlichen Gründen möglich ${ }^{294}$. Deshalb sieht ein Vorschlag ${ }^{295}$ eine Ankündigungspflicht von sechs Monaten für die Un-

292 Vgl. hierzu die detaillierte Darstellung im Kapitel 8.1.4.2 zu den Stromtransportpreisarten für das TPA-Konzept.

293 Letztlich basiert jede Stromlieferung der EdF an einen britischen Abnehmer auf einen solchen Durchleitungsvertrag.

294 Ein Hindernis kann z.B. die in den meisten Lăndern der EU existierende Genehmigungspflicht für Energieerzeugungsanlagen und die hiermit verbundenen Auflagen darstellen.

295 Vgl. GRAWE, J. : Der Kommissionsentwurf für eine EG-Richtlinic "Elektrizită" aus der

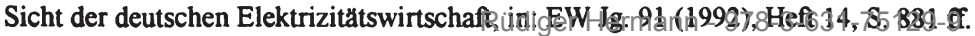


ternehmen vor, die aus der Versorgung des Gebiets-EVUs ausgeschieden sind und eine Rückkehr beabsichtigen. Hiergegen wehren sich die EVUs zu Recht. Die Kapazitätsbauzeiten und ökonomischen Planungszeiträume liegen in der Elektrizitätswirtschaft über sechs Monaten. Eine erneute Versorgungspflicht seitens der EVU nach einer derart kurzen Ankündigungsperiode würde dazu führen, daß die EVU in ihren Kapazitätsberechnungen eine Reserve für Abnehmer einplanen müßten, die nicht mehr von ihnen mit elektrischer Energie versorgt werden. Die Kosten hierfür würden die Preise des EVU steigen lassen, da für die verbleibenden Abnehmer weiterhin die Kostenüberwälzung gültig ist. Gleichzeitig sinkt das Wettbewerbspotential des EVU. Sinnvoller wäre ein höherer Ankündigungszeitansatz, z.B. 18 Monate, und höhere Preise im ersten Jahr nach der Versorgungswiederaufnahme. In 18 Monaten können die Unternehmen ihre Kapazitäten erweitern und notfalls kleinere Kraftwerke auf Öloder Gasbasis in Betrieb nehmen. Die hierfür anfallenden höheren Kosten sollten von dem betroffenen Abnehmer übernommen werden. Durch diese Maßnahmen würde die Versorgungssicherheit der Abnehmer garantiert. Für Abnehmer, die aus den Versorgungsverträgen mit ihrem Gebiets-EVU ausscheiden wollen, resultiert daraus bei risikoaversem Verhalten eine Mindestlaufzeit der Stromlieferverträge von 18 Monaten. Dem Abnehmer verbleibt vor und während der Ankündigungsperiode die Möglichkeit, mit dem Gebiets-EVU als Marktteilnehmer über den Stromliefervertrag zu verhandeln. Risikofreudigere Abnehmer können auch kürzere Verträge abschließen, wenn sie davon überzeugt sind, kurzfristig ausreichende Angebote auf dem Strommarkt zu bekommen. Erleichtert werden kann dieses Verhalten durch Eigenerzeugungspotentiale eines Abnehmers.

- Das Gebiets-EVU lehnt die weitere Durchleitung für bestehende Lieferverträge zwischen einem Abnehmer in seinem Versorgungsgebiet und einen externen Lieferanten mit Hinweis auf die erforderliche eigene Nutzung der Transportkapazitäten $a b^{296}$. In diesem Fall befindet sich der Abnehmer in einem Dilemma. Ein Erzwingen der weiteren Durchleitung ist wegen der mit dem Konzept verbundenen Regeln nicht möglich. Daher bleibt dem Verbraucher nicht anderes übrig, als bei seinem ehemaligen Gebiets-EVU um die Wiederaufnahme der Versorgung zu bitten. Bei etwaigen Preisverhandlungen befindet er sich in einer äußerst schlechten Position. Im Grenzfall kann das EVU je nach konkreter Ausgestaltung der rechtlichen Rahmenbedingungen des Konzeptes eine Wiederaufnahme aus technischen Gründen ablehnen. Der Verbraucher ist dann gezwungen, zur Eigenerzeugung überzugehen. 
$Z u$ c) Die Versorgungspflicht und die Versorgungssicherheit

Bei den Aussagen zum TPA ist die Verantwortung für die Versorgungssicherheit bisher nicht angesprochen worden. Es stellt sich daher die Frage, wer für die Bereitstellung der Versorgungssicherheit zuständig sein soll und welche Auswirkungen dies auf ihre tatsächliche Entwicklung hat. Bevor auf die verschiedenen Alternativen eingegangen wird, sollen zunächst die Eigenschaften der Versorgungssicherheit analysiert werden.

Versorgungssicherheit im engeren Sinne bedeutet die ununterbrochene Strombezugsmöglichkeit bei gegebenem Qualitätsniveau seitens des Abnehmers. Eine Unterbrechung kann durch unterschiedliche Faktoren begründet sein: In einem vollkommen abgeschlossenen Versorgungsgebiet eines EVU, also ohne Verbindungsleitungen zu anderen, kann die Versorgung zusammenbrechen, wenn die temporäre Stromabnahme größer ist als die temporäre Stromerzeugung. Gründe können kurzfristige, unvorhergesehene Lastspitzen oder der Ausfall von Erzeugungsanlagen sein. Als weitere Ursache ist die Unterbrechung der Leitung zwischen Kraftwerk und Abnehmer denkbar ${ }^{297}$. Um die Versorgungssicherheit zu erhöhen, betreiben die EVU innerhalb ihrer Versorgungsgebiete eine Vielzahl unterschiedlicher Kraftwerke. Hiermit werden die Auswirkungen eines einzelnen Kraftwerksausfalls begrenzt. Gleichzeitig werden Kraftwerkskapazitäten oberhalb der prognostizierten Leistung vorgehalten, um einer kurzfristigen Erhöhung der Nachfrage durch eine schnelle Produktionsausweitung begegnen zu können. Eine Versorgungsunterbrechung durch Leitungszusammenbruch versuchen die EVU durch überdimensionierte Maschennetze auszuschließen. Bei Ausfall einer Leitung kann die Versorgung über eine Vielzahl anderer Leitungen aufrecht erhalten werden. Durch diese Maßnahmen zur Gewährleistung der Versorgungssicherheit entstehen den EVU Kosten. Sie können gesenkt werden, wenn sich EVU wie im UCPTE oder NORDEL-Verbund zusammenschließen und ihre Netze im Parallelbetrieb fahren. Der Ausfall eines einzigen Kraftwerks hat dann prozentual geringere Auswirkungen auf das Gesamtnetz. Gleichzeitig ist der absolute Bedarf an "Heißreserve" geringer. Die Verknüpfung der Netze erlaubt den Transport über weite Strecken auch bei Ausfall von Kupplungsstellen.

Pick spricht "ohne einer empirischen Überprüfung vorzugreifen"298, von einem natürlichen Monopol bei der Erstellung von Versorgungssicherheit, da die gemeinsam erstellte Versorgungssicherheit kostengünstiger ist als die Summe der einzel erstellten. Weiterhin stellt er richtig fest, daß jeder am Netz angeschlossene von der Bereitstellung der Versorgungssicherheit profitiert, egal ob oder inwieweit er

297 Es wurde von einem Strahlennetz ausgegangen. Bei Versorgung eines Großabnehmers direkt aus dem Ring- oder Maschennetz eines EVU ist diese Unterbrechung weniger wahrscheinlich. Zum Aufbau von Netzen siehe: HARPOLT, H. ; OEDING, G. : Elektrische Kraftwerke und Netze, 5. Auflage, Berlin 1978, S. 312 und FREUND, H. ; ROSENOW, V. ; WOLFF, H.-P. : Ein erweitertes (n-1) - Planungsprinzip für vermaschte Hochspannungsnetze, in: EW Jg. 90 (1991), Heft 13, S. 747

298 Vgl. PICK, H. : a.a.O., S. 110

Rüdiger Hermann - 978-3-631-75129-9

Downloaded from PubFactory at 01/11/2019 07:57:52AM 
selbst dazu beiträgt. Zusammen mit der Nichtrivalität in der Nutzung geht er davon aus, daß es sich bei der Versorgungssicherheit um ein öffentliches Gut handelt. Öffentliche Güter ${ }^{299}$ beeinhalten die Tendenz der Nutzer zum Trittbrettfahrerverhalten ${ }^{300}$, d.h. der einzelne ist versucht, keinen Beitrag zu leisten, aber von der Gesamterstellung zu profitieren. Im bisherigen europäischen Verbundnetz war dies kein Problem. Alle EVU haben ihren Beitrag zur Reservehaltung erbracht und Maßnahmen zur Spannungs- und Frequenzhaltung durchgeführt. Ein Anreiz, diese Leistungen nicht zu erbringen, existierte nicht, da die EVU innerhalb ihres Versorgungsgebietes die Kosten voll weitergeben konnten und staatliche Regulierungsstellen keine Einwände äußerten. Mit Einführung der Durchleitungsverpflichtung ändert sich der Zusammenhang. Ein Unternehmen, welches Endverbraucher im Versorgungsgebiet eines anderen beliefern will, stärkt seine Wettbewerbssituation, wenn es die Versorgungssicherheitsbereitstellung reduziert, dadurch Kosten einspart und somit zu niedrigeren Preisen anbieten kann. Bei Ausfall der Lieferung hofft das Unternehmen, daß das Gebiets-EVU die Lieferung übernehmen wird und es dann die Kosten tragen muß, die wesentlich niedriger sind als die Kosten zur Prävention. Dies heißt, daß der Anreiz zum Trittbrettfahrerverhalten durch Einführung des TPA-Konzeptes steigt. Verhalten sich alle EVU oder Erzeuger gleich, sinkt das Versorgungssicherheitsniveau beträchtlich.

Scheinbar am einfachsten wäre es, wenn der Lieferant fïr die Versorgungssicherheit zuständig wäre. Bei Ausfall seiner Einspeisung trennt der Durchleiter den Abnehmer von seinem Netz ab. Hierdurch würde theoretisch das Trittbrettfahrer-Verhalten ausgeschlossen. Einspeisung und Entnahme wären im Einklang. Praktisch ist dies jedoch wenig sinnvoll: Der Durchleiter bekommt den Ausfall der Einspeisung zu demselben Zeitpunkt signalisiert, wie die negativen Auswirkungen eintreffen, da sich elektrische Energie annähernd mit Lichtgeschwindigkeit ausbreitet. Das anschließende sofortige Abschalten des Abnehmers würde zwar die Ursache des $\mathrm{Zu}$ sammenbruchs oder der Störung abstellen, die Beeinträchtigung der Versorgung kurzfristig aber nicht verhindern können. Hieraus folgt, daß der Durchleiter, um eigene Schäden zu begrenzen, gezwungen ist, bei einer Einspeisung mit ungewissem Sicherheitsniveau, eine Reservehaltung vorzunehmen. Relativierend muß darauf hingewiesen werden, daß der soeben skizzierte Fall eines vollständigen, unangekündigten Ausfalles eines bedeutenden Einspeisers eine Seltenheit in der elektrizitätswirtschaftlichen Störungspraxis darstellt. Kraftwerksausfälle kündigen sich vorher an und die erforderliche Leistung kann von anderen Kraftwerken bezogen werden. Außerdem wird es sich in den ersten Jahren nach der Einführung des Konzeptes bei den Einspeisern um EVU handeln, die selbst zahlreiche Möglichkeiten haben, den Ausfall einzelner Erzeugungsanlagen zu kompensieren. Gleiches gilt für

299 Vgl. z.B. eine Definition in BASSELER, U. ; HEINRICH, J. ; KOCH, W. : Grundlagen und Probleme der Volkswirtschaft, 13. Auflage, Köln 1991, S. 45

300 Vgl. BASSELER, U. ; HEINRICH, J. ; KOCH,dWer ala.6ıąn40678-3-631-75129-9 
den Leitungsausfall: Durch die Vermaschung der Netze und die Existenz mehrerer Kupplungsstellen zwischen zwei Versorgungsgebieten kann der Ausfall einer Leitung durch Umschalten aufgefangen werden, solange das Gesamtnetz nicht an der Kapazitätsgrenze betrieben wird. Dies ist aus betriebstechnischen Gründen außer in Spitzenlastzeiten eines Jahres, nicht zu erwarten. Erst wenn es sich bei den Einspeisern um reine Stromerzeuger mit teilweise nur einem Kraftwerk handelt, die keine Verträge zur Übernahme der Versorgungssicherheit mit anderen EVUoder Erzeugern geschlossen haben, ist die Aufrechterhaltung der Versorgungssicherheit durch die Durchleitung realistisch gefährdet. Dem kann begegnet werden, wenn die Verantwortung für die Versorgungssicherheit einem Marktteilnehmer zugewiesen wird, der gegebenenfalls Schadensersatz leisten muß. Im Gegenzug erhält er ein Entgelt für die Bereitstellung der Versorgungssicherheit ${ }^{301}$. Pick schlägt dazu die Zuweisung der Versorgungssicherheit auf das Gebiets-EVU vor, also auf den Durchleiter, welcher hierfür ein angemessenes Entgelt erhalten soll. Für den Endverbraucher besteht der Vorteil in der ununterbrochenen, weiter bestehenden Versorgungssicherheit in einer Hand. Technische und organisatorische Auflagen zur Teilnahme am Konzept sowie hohe Schadensersatzleistungsansprüche wären eine Möglichkeit, die Inanspruchnahme der Leistung zu begrenzen. Aus der Zuweisung der Versorgungsverantwortung zum Gebiets-EVU resultiert die Frage nach einem angemessenen Entgeltes ${ }^{302}$. Ein weiteres von Pick nicht berücksichtigtes Problem der Versorgungssicherheit ist der Kapazitätszubau. Ein EVU, welches bisher seine Kapazitätsplanung an der Gesamtnachfrage in seinem Versorgungsgebiet orientiert hatte, verliert durch die Durchleitungsverpflichtung einen bestimmten Prozentsatz an Abnehmern und damit an Erzeugungskapazitätsauslastung, für die es weiterhin die Versorgungssicherheit zu erbringen hat. In der ersten Zeit wird dies kein Problem sein. Die vorher genutzten Anlagen zur Erzeugung stehen weiterhin zur Verfügung. Von einem über das selbst benötigte Maß hinausgehenden Zubau an Kapazitäten wird das EVU absehen. Zur Aufrechterhaltung der Versorgungssicherheit müßte es aber einige Anlagen zur Versorgungssicherheitsbereitstellung für den Konkurrenten bauen. Dazu könnte es aber nur bereit sein, wenn die Gewinne aus der Bereitstellung der Versorgungssicherheit den entgangenen Gewinnen durch Wegfall der Abnehmer überwiegen. Dies scheint jedoch unwahrscheinlich, da die Versorgungssicherheitsbereitstellung nur einen kleinen Teil der Elektrizitätsversorgung ausmacht.

Als letzte Alternative bliebe die Eigenverantwortlichkeit des Abnehmers für das gewünschte Versorgungssicherheitsniveau. Ein Abnehmer kann sein gewünschtes Versorgungssicherheitsniveau entweder direkt bei seinem Einspeiser anfordern und vertraglich ausgestalten oder selber geeignete Maßnahmen, wie den Einsatz von Notstromaggregaten und die Vorbereitung eines Lastabwurfes, treffen. Des wei-

301 Dies entspricht einer Erstellung eines öffentlichen Gutes durch ein Unternehmen und die Finanzierung über Gebühren.

302 Auf diese Problematik wird in Kapitel 8. Rrithereeingegangen. 978-3-631-75129-9 
teren kann er die Versorgungssicherheit zu einem vertraglich festgesetzten Preis von seinem alten Gebietsversorger beziehen. Eine weitere Möglichkeit ist der $\mathrm{Be}-$ zug von einem unbeteiligten Dritten, z.B. einem anderen EVU oder einem unabhängigen Erzeuger. So entstünde ein Markt für Versorgungsicherheitsleistungen, der Entgeltprobleme erübrigen würde. Gleichzeitig sollten die Schadensersatzansprüche bei Versagen des Systems gesetzlich näher geregelt werden, um bei Eintritt lange Verhandlungen zu verkürzen und weitere Anreize zur Vermeidung zu geben. Hierdurch könnte gleichzeitig ein neuer Versicherungszweig entstehen.

\section{Zu e) Die Auswahl der Durchleitungsanträge}

Offen ist in der Konzeption, nach welchen Kriterien ein durchleitendendes EVU seine Antragssteller auswählen soll, wenn die Durchleitungsnachfrage größer ist als das verfügbare Angebot. Zur Lösung des Problems kommen zwei Ansätze in Frage:

Am scheinbar einfachsten ist die Zuweisung der Durchleitungskapazität durch das durchleitende EVU. Bei reguliertem, festgelegtem und pauschalisiertem Durchleitungsentgelt pro $\mathrm{kWh}$ hat der Durchleiter scheinbar keine Präferenz für einen bestimmten Antragssteller. Der Durchleiter wird jedoch zuerst versuchen, die vorhandenen Überkapazitäten an andere EVU zu vergeben, die Kunden außerhalb des eigenen Versorgungsgebietes beliefern wollen (Durchleitung Typ 1), um so die Kapazität für Durchleitungen in das eigene Versorgungsgebiet $\mathrm{zu}$ verringern (Durchleitungstypen 2-5). Bei Antrag auf Durchleitung zu einem Abnehmer innerhalb des Versorgungsgebietes wird der Durchleiter die Auswahl nach der Höhe der entfallenden Deckungsbeiträge vergeben, d.h. das Unternehmen, bei dem der kleinste Deckungsbeitragswegfall zu erwarten ist, bekommt den Zuschlag. Gleichzeitig wird der Durchleiter bestrebt sein, die überschüssigen Übertragungskapazitäten an eine möglichst geringe Anzahl von Erzeugern zu vergeben, um die Transaktionskosten gering zu halten. Die Transaktionskosten müssen mit den erforderlichen zusätzlichen Reserveleistungskosten abgewogen werden, da die Auswirkungen des Ausfalls eines großen Einspeisers relativ größer sind als bei kleinen.

Ein anderes Vergabeverfahren ist die Versteigerung der Durchleitungskapazitäten. ${ }^{303}$ Nachteilig ist bei Übernachfrage, daß damit der Durchleiter unverdienterweise einen Anteil an den Effizienzgewinnen anderer Erzeuger erhält. Vorteilhaft ist dagegen der Anreiz zum Leitungsausbau für einen Durchleiter, der sich einer Übernachfrage nach Durchleitungskapazitäten für Durchleitungen des Typs 1 gegenüber sieht. Durch dieses Verfahren wird die Spezialisierung der EVU gefördert

303 Zur Preisbildung bei Versteigerungen siehe BORCHERT, M. ; GROSSEKETTLER, H. Preis- und Wettbewerbstheorie: Marktprozesse als analytisches Problem und ordnungspolitische Gestaltungsaufgabe, Stuttgart 1985, S. 16 figer Hermann - 978-3-631-75129-9 
und die Verlagerung der Geschäftsaktivitäten eingeleitet. Die Versteigerung ist unabhängig von der gewählten Durchleitungsgebührenform denkbar. Sie kann bei pauschalisierten Durchleitungsgebühren um eine einmalige Gebühr stattfinden oder um den Durchleitungspreis pro Übertragungseinheit. Für das Funktionieren des Konzeptes wirkt sich die Versteigerung negativ aus. Die einzelnen Vertragspartner eines Stromliefergeschäftes müssen bei dem Vertragsabschluß prüfen, ob für den gewünschten Durchleitungsumfang ausreichende Übertragungskapazitäten vorhanden sind. Ferner besitzen sie keine Kenntnisse über den Durchleitungspreis. Dies erschwert den Vertragsabschluß beträchtlich, d.h. die Abnehmer erhalten einen Anreiz, bei ihrem Gebiets-EVU zu verbleiben.

\subsubsection{Die Mittel des Konzeptes}

Im Gegensatz zum Weiterentwicklungskonzept, wo sowohl der verstärkte Ausbau der Versorgungssicherheit als auch die Strombörse als Mittel zur Verwirklichung der Zielvorgaben des EU-Strommarktes eingesetzt werden sollen, kommt das TPA-Konzept mit der Durchleitungsverpflichtung aus. Der sich bildende Markt soll auf den Rahmenbedingungen aufbauend über die Mittel zur Geschäftsabwicklung entscheiden.

Die Durchleitungsverpflichtung muß, um erfolgreich wirken zu können, folgendes einschließen:

a) Regeln, wann eine Durchleitung von einem Gebiets-EVU durchgefürt werden muß.

Optimal wäre es, wenn die Regeln so präzise sind, daß erzwungene Durchleitungen auf seltene Fälle begrenzt bleiben. Aus den Forderungen an das Konzept können zwei Regeln abgeleitet werden:

- Eine Durchleitung kann nur bei ausreichenden Durchleitungskapazitäten eines Gebiets-EVU erzwungen werden.

- Das EVU darf durch die Abwicklung der Durchleitung nicht in seinem Netzbetrieb (Qualität, Sicherheit etc.) gestört werden.

b) Die Durchsetzbarkeit mit den dazu erforderlichen Verfahren und Institutionen.

In der zweiten und dritten Konzeptstufe ist mit einer Durchleitung der Typen zwei bis fünf mit einem Umsatzverlust für ein Gebiets-EVU zu rechnen. Daher wird das 
Gebiets-EVU bestrebt sein, die Durchleitung abzulehnen ${ }^{3 / 4}$. Die Entscheidung, ob eine beantragte Durchleitung zu Recht verweigert wurde, kann entweder von einer juristischen Institution getroffen werden oder von einer speziell eingerichteten, unabhängigen Institution, welche mit Fachleuten besetzt ist. Gegen die juristische Entscheidung sprechen lange Verfahren, hauptsächlich wegen des erforderlichen Einholens von Gutachten. Eine unabhängige Institution wird gegen den Vorwurf der fehlenden Kompetenz oder der Voreingenommenheit durch die Besetzung zu kämpfen haben. Erforderlich ist jedoch eine durchsetzungsfähige Institution, die Entscheidungen möglichst schnell und sachlich fundiert triff. Eine weitere Aufgabe der Institution müßte es sein, nach der Anzeige eines Unternehmens, die unbillige Erschwernis einer Durchleitung zu überprüfen und Sanktionen zu verhängen. Erste Sanktionsmöglichkeiten wären Geldstrafen, über Schadensersatzansprüche der Durchleitungsbegehrenden hinaus. Die härteste Maßnahme könnte im Entzug des Versorgungsmonopols/-rechtes bestehen. Es kann zu Recht bezweifelt werden, daß letztere Maßnahme bei staatlichen Unternehmen durchsetzbar ist.

\subsubsection{Die Entwicklungsmöglichkeiten des Konzeptes}

Die Entwicklungsmöglichkeiten nach der Vollendung der dritten Stufe des TPAKonzeptes hängen letzlich von folgenden Faktoren $\mathrm{ab}^{305}$.

a) dem Interesse der EVU, der Großabnehmer und der Kleinabnehmer (ab der dritten Phase) am Stromhandel und

b) den Durchleitungsverhinderungsmöglichkeiten der EVU ${ }^{306}$.

Die beiden Faktoren bestimmen das Stromhandelsvolumen, sofern ausreichende Stromtransportkapazitäten in Europa vorhanden $\operatorname{sind}^{307}$. Wichtigste Einflußvariablen auf den ersten Faktor sind die bestehenden Erzeugungs- und Abgabepreisdisparitäten.

304 Vorausgesetzt wurde, daß der Umsatz/Gewinn aus der integierten Versorgung höher ist als der aus dem Durchleitungsentgelt entstehende.

305 Die denkbaren Entwicklungen in der ersten und zweiten Phase des Konzeptes sollen an dieser Stelle nicht behandelt werden. Die Marktentwicklungen in der ersten Stufe unterscheiden sich faktisch nicht von dem Weiterentwicklungsmodell. Die zweite Phase des Konzeptes wird als Marktsegment der dritten Phase bei den folgenden Betrachtungen eingeschlossen.

306 Wie EVU Durchleitungen verhindern können, wird ausführlich in Kapitel 8.2.2 dargestellt.

307 Diese Restriktion wird in Kapitel 8.2 năher änalygsiertlermann - 978-3-631-75129-9 


\subsubsection{Entwicklungsszenarien}

Es können folgende Szenarien für die Entwicklung des europäischen Strommarktes bei der Verwirklichung des TPA-Konzeptes aufgestellt werden ${ }^{308}$ (vgl. Abb. 16):

Szenario I : Stagnation des Stromhandels

Szenario II : Stromhandel auf niedrigem Niveau

Szenario III : Intensiver Stromhandel

Szenario I : Stagnation des Stromhandels

Zum stagnierenden Stromhandelsvolumen kommt es dann, wenn die EVU, die eine schlechte Wettbewerbssituation auf dem europäischen Elektrizitätsmarkt haben, erfolgreich Durchleitungsbegehren der Typen zwei bis fünf ablehnen können. Können zudem die EVU erzwungene Durchleitungen langfristig erschweren, unrentabel werden oder sogar durch gezielte Maßnahmen scheitern lassen, so führt dies zum Erliegen jeder verdrängenden Durchleitung. Das Eintreten des Szenarios wird begünstigt durch eine mangelnde oder mangelhafte Regulierung, geringe Stromtransportüberkapazitäten und niedrige Strompreisdisparitäten in Europa. Die Strompreisdisparitäten in Europa bestimmen maßgeblich das Interesse der Verbraucher am Stromhandel.

Szenario II : Stromhandel auf niedrigem Niveau

Das Eintreten dieses Szenarios kann durch vier Faktoren ausgelöst werden:

- regionale Strompreisdisparitäten,

- divergierende Durchleitungsverhinderungsmöglichkeiten in Europa,

- uneinheitliche Regulierung,

- regionale Stromtransportkapazitätsengpässe.

308 Die zweidimensionale graphische Darstellungsweise wird mangels dreidimensionaler Darstellungsmoglichkeiten gewăhlt. 
Abbildung 16: Zusammenhang zwischen den Szenarien für das TPA-Konzept und ihren wichtigsten Einflußgrößen

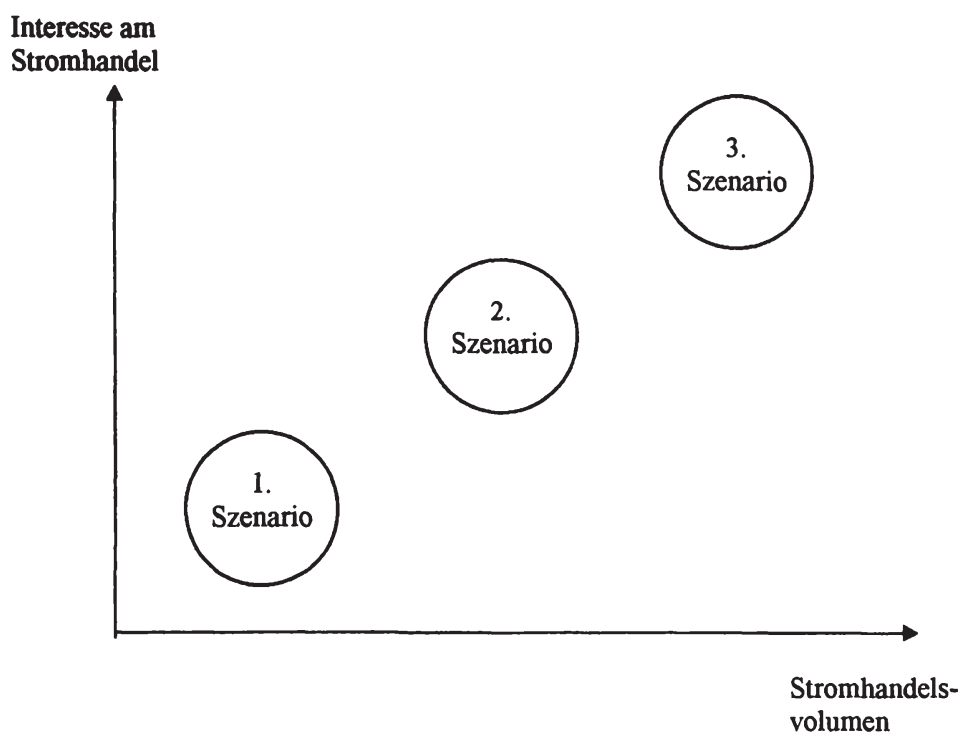

Durchleitungs-

verhinderungs-

moglichkeit

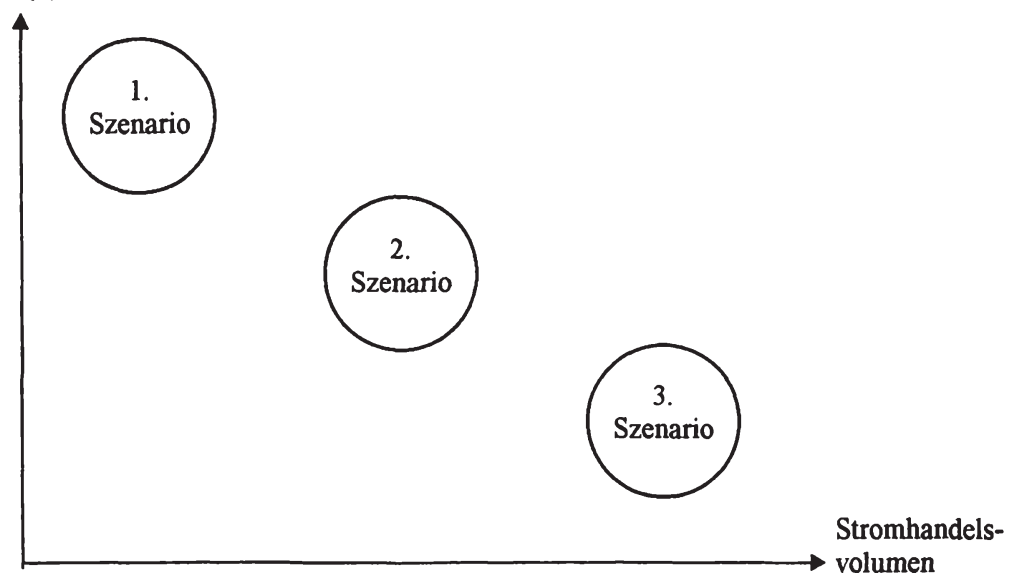

Die vier Faktoren können in beliebiger Kombination und mit unterschiedlichem Ausprägungsgrad zu dem mit dem Szenario verbundenen niedrigen Stromhandelsvolumen führen. Bei besonders ungünstiger Konstellation dieser Faktoren ist eine 
vollständige Einstellung der Stromhandelsaktivitäten innerhalb der EU oder in einigen Regionen anzunehmen.

Szenario III : Intensiver Stromhandel

Ein intensiver Stromhandel kann entstehen, wenn das Interesse der Marktteilnehmer am Stromhandel hoch ist. Hohe Preisdisparitäten in Europa und hohe Stromtransportüberkapazitäten sind hierfür ebenso eine Voraussetzung wie die europaweite erfolgreiche Regulierung der Netzverfügungsrechte, damit Durchleitungsbegehren nicht von den netzbetreibenden EVU abgelehnt werden können. Zusätzlich ist von seiten der Verbraucher die Bereitschaft erforderlich, neue Lieferanten zu akzeptieren und somit das scheinbar erhöhte anfängliche Risiko der niedrigeren Versorgungssicherheit zu tragen. Die EVU werden sich bei Eintreten des Szenario langfristig spezialisieren. Ergebnis des Prozesses werden reine Stromtransporteure oder Erzeuger sein.

\subsubsection{Zusammenhang zwischen Strompreisdisparitäten, dem Interesse am Stromhandel und den Entwicklungen im Binnenmarkt des TPA- Konzeptes}

Für die Endabnehmer bedeuten Strompreisdisparitäten in Europa die Möglichkeit, durch Auswahl ihres Lieferanten Preissenkungen erzielen zu können. Deshalb ist das Interesse am Stromhandel und an Durchleitungen bei Abnehmern umso höher, je bedeutender die Preisunterschiede in Europa sind. Zur näheren Verdeutlichung der Zusammenhänge soll an dieser Stelle der Begriff des Anbieters modifiziert werden und zwar wie folgt:

Als Anbieter soll ein Stromerzeuger definiert werden, der Strom in das Versorgungsgebiet eines Gebiets-EVUs zu niedrigeren oder gleichen Preisen wie das Gebiets-EVU liefern kann, d.h. dessen Stromerzeugungskostenunterschied zum Gebiets-EVU höher ist als die Stromtransportkosten ${ }^{309}$.

Für die Abnehmer in einem Versorgungsgebiet sind ausschließlich die so definierten Anbieter interessant, da es sich bei der Elektrizität um ein weitgehend homogenes Gut handelt. Ihre Entscheidung für oder gegen den Strombezug vom GebietsEVU hängt in der Anfangsphase bei bestehenden alternativen Anbietern von den 
subjektiv erwarteten Transaktionskosten ab. Die Abnehmer müssen die erforderlichen Informations-, Such- und Vertragskosten sowie die, mit einer Durchleitung verbundenen langfristigen Risiken, den Preisdifferenzen gegenüberstellen ${ }^{310}$. Mit zunehmenden Erfahrungswerten werden die subjektiven Einschätzungen über die erforderlichen Transaktionskosten durch objektive Erfahrungen ersetzt. Nur wenn die Summe aus Abgabepreis und Transaktionskosten niedriger ist als der Preis des Gebiets-EVU, werden die Abnehmer aktiv am Stromhandel teilnehmen ${ }^{311}$.

Anders als die Verbraucher haben die EVU verschiedene Handlungsalternativen und stehen einer ungleich komplizierteren Marktsituation gegenüber. Aus diesem Grund soll im folgenden das Interesse der EVU bei verschiedenen Erzeugungspreisdisparitätenkonstellationen ${ }^{312}$ analysiert und Rückschlüsse auf die Marktentwicklung gezogen werden. Hierbei sollen nicht absolute Preisabstände herangezogen werden sondern die relativen Abstände. Es wird unterstellt, daß die EVU keine Durchleitungsverhinderungsmöglichkeiten haben. Zur methodischen Vereinfachung werden drei Kategorien von Stromerzeugungspreisen innerhalb des Binnenmarktes gebildet:

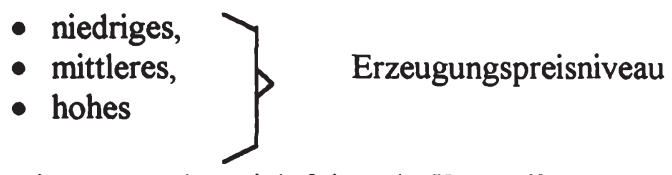

Hieraus ergeben sich folgende Konstellationen:

\section{EVU mit niedrigem Erzeugungspreisniveau}

Der einfachste zu analysierende Fall liegt bei einem EVU mit niedrigem Preisniveau und ausgelasteten Erzeugungskapazitäten vor. Selbst wenn es ein Absatzpotential bei benachbarten Gebiets-EVU erkennt, kann es dieses nicht nutzen. Die Preisgestaltung oder die Anreize zur Preisgestaltung sind unverändert, so daß kein Grund für sinkende oder steigende Preise erkennbar ist. Besitzt das EVU Überkapazitäten und ein geeignetes Absatzpotential, so wird es versuchen, darüber die eigene Ertragslage zu verbessern, d.h. die Durchleitung eines anderen EVU zu wünschen oder notfalls zu erzwingen suchen. Das Interesse der EVU am Stromhandel ist dementsprechend groß.

Offen ist noch die Frage, ob die EVU mit niedrigem Erzeugungspreisniveau Kapazitäten für den Gebiets-externen Absatz errichten werden. Für die Frage sind folgende Faktoren relevant:

310 Vergleiche hierzu die Aussagen in Kapitel 3.

311 Die Transaktionskosten bei einem Bezug von dem Gebiets-EVU sind für den Abnehmer in der Regel gleich Null. Sie weisen zumindest keinen relativen Anstieg auf.

312 Auf die Gründe für unterschiedliche Erzeugungspreise soll in der vorliegenden Arbeit nicht eingegangen werden. Vergleiche hierzu LANG, G. : Das Strompreisniveau in den EG-Ländern 1991, in : ET 41. Jg. (1991), Heft 10, \$.6afife Hermann - 978-3-631-75129-9 
- das Absatzpotential,

- die Preisabstände auf den relevanten Märkten zu den Mitanbieter,

- ausreichende Stromtransportkapazitäten,

- Stromtransportkosten,

- die erwarteten Reaktionen der Konkurrenten, insbesondere der GebietsEVU,

- die erzielbaren Gewinne.

Während die ersten beiden Faktoren ausreichend prognostizierbar sind, gefährden die Stromtransportkapazitäten, -kosten und Reaktionen der Konkurrenten ${ }^{313}$ den Absatz des EVU mit niederigem Erzeugungspreisniveau, wenn die Erzeugungskapazitäten eines Gebiets-EVUs verdrängt werden sollen. Deshalb kann an dieser Stelle festgehalten werden, daß die Stromtransportkapazität und die Durchleitungsverhinderungsmöglichkeiten bei ausreichenden Absatzmärkten, Preisunterschieden und Gewinnerzielungschancen für den Aufbau von Erzeugungskapazitäten zum Export bei einem EVU mit niedrigen Erzeugungspreisen den Ausschlag geben werden ${ }^{314}$.

\section{EVU mit mittlerem Erzeugungspreisniveau}

Für ein EVU mit mittlerem Preisniveau ist es entscheidend, zwischen wievielen Anbietern die Abnehmer in seinem Versorgungsgebiet wählen können. Andererseits können für das Angebot des Gebiets-EVU auch Abnehmer in anderen Versorgungsgebieten bestehen.

- Fehlende Anbieter

Existieren keine Anbieter oder wenige Anbieter mit einem kleinen begrenzten Angebot für das Versorgungsgebiet des Gebiets-EVU, so wird die Durchleitungsoption von wenigen Abnehmern genutzt werden können. Da die Nachfrage nach Lieferanten höher ist als das Angebot im Versorgungsgebiet, steht das EVU unter keinem direkten Druck, seine Preise zu senken. Will es jedoch alle Abnehmer weiter beliefern ${ }^{315}$, können niedrigere Preise für die Durchleitungsberechtigten eingeräumt werden. Andererseits können die Preise für den gleichen Kreis auch steigen, wenn die Kapazitäten des Anbieters erschöpft sind und das Gebiets-EVU die entfallenden Deckungsbeiträge den verbleibenden Kunden aufbürdet. Begrenzt wird die Preissteigerungsmöglichkeit durch potentielle neue Anbieter und der Möglichkeit zur Eigenerzeugung von seiten der Abnehmer.

313 Diese drei Faktoren kőnnen auch als Durchleitungsverhinderungsmøglichkeiten bezeichnet werden.

314 Diese zentrale Aussage wird im Kapitel 9 bedeutend werden.

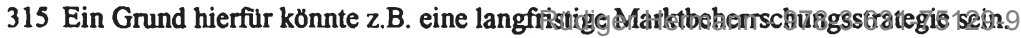


Des weiteren besteht die Gefahr, daß stromintensive Industriezweige abwandern.

- Zahlreiche Anbieter

Bei zahlreichen Anbietern für das Versorgungsgebiet steigt die Inanspruchnahme der Durchleitungsmöglichkeit. Das EVU wird gezwungen sein, seine Preise zu senken, um so die Verbraucher weiter zu beliefern und Umsatz- bzw. Gewinneinbußen zu vermindern. Gleichzeitig wird das Gebiets-EVU versuchen, die Durchleitungen zu verhindern.

- Nachfrager

Ein weiterer Fall stellt sich dar, wenn sich in der Nähe ein Gebiets-EVU mit hohem Preisniveau befindet. Dann ist die Lieferung an das externe EVU oder an Verbraucher innerhalb des Versorgungsgebietes des EVUs mit hohem Preisniveau denkbar. Gleichzeitig werden Großabnehmer des externen EVU Belieferungsmöglichkeiten erkunden, um in den Genuß von günstigerem Strom zu kommen. Hat das Gebiets-EVU mit mittlerem Preisniveau ausreichende Überkapazitäten, so wird es eine Belieferung sowohl des EVUs als auch der Großabnehmer anstreben.

- Anbieter und Nachfrager

Liegen beide Situationen vor, d.h. in der Nähe zu einem potentiellen Abnehmer in einem Versorgungsgebiet eines EVUs liegen einer oder mehrere Lieferanten, so wird das EVU bemüht sein, die Abwanderung der eigenen Abnehmer zu verhindern. Dies kann durch Preisermäßigungen erreicht werden, die ausgeglichen werden können durch die eigene Belieferung an Dritte oder durch die Ablehnung der Durchleitung. Die Ablehnung der Durchleitung kann das Gebiets-EVU mit mittlerem Preisniveau erwirken, indem es seine begrenzten Leitungsüberkapazitäten zum verstärkten Export aus seinem Versorgungsgebiet nutzt. Hierdurch schafft es die zulässige Bedingung für die Ablehnung eines Durchleitungsersuchens. Dies hätte zur Folge, daß die Preise im Versorgungsgebiet nicht steigen und zusätzliche Gewinne aus dem Export erwirtschaftet werden. Denkbar ist jedoch auch, daß das Gebiets-EVU nach der Ausschaltung der Durchleitungsmöglichkeit die Preise aufgrund seiner wiedererlangten Marktmacht anhebt.

\section{EVU mit hohen Preisen/Kosten}

Einfacher in der Analyse stellt sich die Situation eines EVU mit hohen Kosten/Preisen dar. Sind keine potentiellen Anbieter in der Nähe, so ist mit keiner Inanspruchnahme der Durchleitung und unveränderten Preisen zu rechnen. Denkbar sind jedoch auch sinkende Preise, wenn Großabnehmer glaubhaft mit der

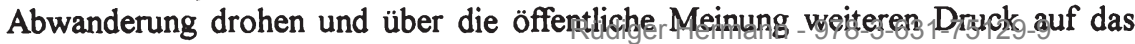


EVU ausüben. Bestehen Stromerzeugungsüberkapazitäten, kann das EVU durch den Absatz an externe Abnehmer Deckungsbeiträge erzielen, die preisreduzierend im Versorgungsgebiet wirken können. Die Preise für den Stromexport müssen jedoch unter den hohen Preisen für die Gebietsabnehmer liegen, damit der Strombezug für externe Abnehmer interessant werden kann. Zu bezweifeln ist jedoch, ob die Abnehmer, die der Anschlußpflicht unterliegen, bereit sind, Stromexporte zu niedrigeren Preisen als ihren eigenen Strombezugspreis zu akzeptieren.

Existieren Anbieter für das Versorgungsgebiet mit hohem Preisniveau, so werden die Großverbraucher versuchen, über Durchleitungsgesuche preisgünstige Strombezugsquellen zu erschließen. Nachdem die freien Übertragungskapazitäten erschöpft sind, müssen die verbleibenden Abnehmer mit weiteren Preissteigerungen rechnen, da das Unternehmen erneut ein Monopol für die Versorgung der Abnehmer besitzt. Bestehen zahlreiche Anbieter, bei ausreichenden Übertragungskapazitäten, würde dies für das EVU eine starke Inanspruchnahme der Durchleitungsoption bedeuten. Um nicht alle Großabnehmer zu verlieren, muß das EVU in diesem Fall dem berechtigten Verbraucherkreis massive Preisnachlässe gewähren. Ohne eine funktionierende Regulierungskontrolle würden die verbleibenden Kleinverbraucher die fehlenden Deckungsbeiträge übernehmen müssen. Dem entgegenwirken könnten massive Proteste der Verbraucherorganisationen in der betroffenen Region. In dieser Konstellation wird das EVU gezwungen sein, Effizienzsteigerungen einzuleiten.

Diese Überlegungen zeigen, daß alle EVU bis auf jene mit einem relativ niedrigen Stromerzeugungspreisniveau kein Interesse haben, Durchleitungen abzuwickeln und somit langfristig Kunden zu verlieren. Den EVU mit einem Interesse am Stromhandel steht meistens ein EVU entgegen, daß die Durchleitung in sein Versorgungsgebiet verhindern will ${ }^{136}$. Deshalb wird das Bestreben dieser EVU sein, Durchleitungen präventiv zu verhindern oder erzwungene Durchleitungen scheitern zu lassen. Deshalb scheidet zwangsläufig das dritte Szenario (intensiver Stromhandel) für den europäischen Binnenmarkt des TPA-Konzeptes aus. In welche Richtung sich der europäische Elektrizitätsmarkt bei Umsetzung des TPA-Konzeptes entwickelt, hängt daher ausschließlich von den Durchleitungsverhinderungsmöglichkeiten der EVU ab. Diese werden, wie die Ausführungen zu den Stromtransportkapazitäten zeigen werden, entscheidend von den Netzverfügungsrechten abhängen.

316 Eine Ausnahme liegt dann vor, wenn ein EVU den Strombedarf in seinem Versorgungsgebiet nicht ohne Import decken kann. 


\subsubsection{Die Akzeptanz des Konzeptes}

Die Akzeptanz des TPA-Konzeptes ist bei den beteiligten Gruppen sehr unterschiedlich und variiert teilweise je nach eingeführter Stufe.

Die EU-Kommission stieß mit ihren Vorschlägen für eine wettbewerbliche Öffnung der nationalen Elektrizitätswirtschaften sowohl bei diversen Verbänden als auch bei den EU-Institutionen auf Ablehnung. Deshalb sprach sie sich anfänglich für die Einfuihrung einer Durchleitungsverpflichtung aus $\mathrm{s}^{317}$. Hierdurch wollte sie zumindest die Öffnung der nationalen Märkte für Stromimporte erreichen. Mittlerweile mußte sie von der Forderung nach einer Durchleitungsverpflichtung abstandnehmen. Trotzdem hält die Kommission die Zwangsverpflichtung immer noch für ein geeignetes Mittel, um eine wettbewerbliche Öffnung der nationalen Elektrizitätsmärkte zu erreichen.

Die einzelnen Mitgliedsländer sind in ihrer Haltung zur Einführung eines auf dem TPA-Gedanken basierenden Konzeptes unterschiedlich; die Akzeptanz hängt überwiegend von der Durchsetzbarkeit der nationalen politischen Ziele ab. Das TPA-Konzept besitzt eine hohe Attraktivität für die Länder mit niedrigen Elektrizitätspreisen und Überkapazitäten, z.B. für die französiche Republik. Die nationalen Regierungen dieser Länder müßten das TPA-Konzept aus wirtschaftspolitischen Gründen befürworten, um die Auslastung der bestehenden Überkapazitäten zu erreichen und zusätzliche Deckungsbeiträge für die nationale Elektrizitätsversorgung zu erzielen. Die Abwanderung von Goßabnehmern brauchen die EVU dieser Länder nicht zu befürchten. Merkwürdigerweise lehnt aber Frankreich ein auf dem TPA-Gedanken aufbauendes Konzept ab. Begründet wird dies mit

- „Unsicherheit bezüglich der Rentabilität von Investitionen,

- Unverträglichkeit mit der Versorgungspflicht und Sicherheit der langfristigen Versorgung,

- Unmöglichkeit der Gleichbehandlung aller Kunden mit ähnlichen Verbrauchseigenschaften,

- Quersubventionen zugunsten weniger Großabnehmer auf Kosten der verbleibenden Kunden ${ }^{\star 318}$.

Anders stellt sich die Akzeptanz des Konzeptes in Ländern mit relativ hohen Strompreisen und/oder subventionierten einheimischen Energieträgern dar. Die Regierungen dieser Länder werden das Konzept ablehnen, da es zu einer kurz- und

317 Vgl. o.V. : EG und Internationales - Anhörung zu Strom- und Gas-Richtlinienvorschlăgen, in: ET 42. Jg. (1992), Heft 12, S. 850

318 MATHIS, C. : Vorschläge für eine Neuorientierung der Elektrizitătswirtschaft in Frankreich

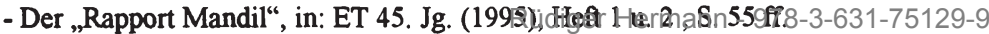


mittelfristigen Verteuerung der Elektrizitätsversorgung bei Klein- und Haushaltsabnehmern führt. Zudem besitzt das Konzept für Großabnehmer die Möglichkeit, energiepolitische Auflagen im Stromerzeugungsbereich zu umgehen. So könnte die deutsche Steinkohleförderung durch Stromimporte größeren Umfangs ausgehebelt werden. Die Ziele der Arbeitsplatzerhaltung und der Sicherung eines Anteils heimischer Energieträger am Gesamtverbrauch könnten nicht mehr gewährleistet werden. Italienische Regierungen ${ }^{319}$ werden sich eher für die Einführung eines Ordnungsmodells im EU-Rahmen aussprechen, das Stromimporte verstärkt ermöglicht und die Bezugspreise sinken läßt.

Das Bundeswirtschaftsministerium lehnt die Einführung des Netzzuganges ab, weil es darin eine weitgehend neue Regulierungsform sieht. Des weiteren wird seitens des BMWi befürchtet, die Umsetzung des Richtlinienentwurfes erfordere ein dem britischen Modell angelehntes System mit einer umfassenden staatlichen Kontroll $^{320}$. In einer eigenen Anfrage des Verfassers an das Bundesministerium für Wirtschaft wurden die einzelnen Richtlinienvorschläge der EU-Kommission als unausgereift und widersprüchlich bezeichnet ${ }^{321}$.

Die europäischen EVU haben sich fast geschlossen gegen das TPA-Konzept gestellt $^{322}$. Sie sehen in dem erzwingbaren Zutritt von Mitbewerbern einen der Teilenteignung ähnlichen Eingriff in ihre Verfügungsrechte. Zudem weisen sie auf die Besonderheiten der Branche hin, die ihrer Meinung nach einen derartig gestalteten Wettbewerb ausschließen. Sie fordern vor Einführung von Wettbewerb die Harmonisierung der Rahmenbedingungen innerhalb der $\mathrm{EU}^{323}$. Hinter dieser Forderung kann der Gedanke stehen, daß die angesprochene Harmonisierung hinsichtlich der Subventionen, Umweltschutzauflagen und Genehmigungsverfahren, wenn überhaupt, dann nicht in absehbarer Zeit durchführbar ist; bis zur Harmonisierung und der dann folgenden Einführung partiellen Wettbewerbs könnten dann weiterhin Monopolrenten von den Unternehmen erzielt werden. Gleichzeitig gewinnen die EVU Zeit, Vorkehrungen zur Abwehr der Durchleitungsgesuche zu treffen ${ }^{324}$.

319 Vgl. hierzu die Ausführungen zur italienischen Elektrizitătswirtschaft, insbesondere zu den Stromerzeugungsengpässen.

$320 \mathrm{Vgl}$. die Äußerungen des MinR M. Cronenberg, in: o.V. : Energieıvirtschaft - BMWi für Liberalisierung des Ordnungsrahmens, in: ET 42. Jg. (1992) Heft12, S. 850

$321 \mathrm{Vgl}$. Anfrage des Verfassers an das BMWi vom 11.11.1993 . Prinzipiell erachtet das BMWi das Durchleitungssystem als ein „wirksames Instrument“ zur Förderung von Wettbewerbsgeschehen.

322 Z.B. RIEMER, H.W. : EG-Binnenmarkt - Technische Aspekte der Herausforderung der deutschen Elektrizitătswirtschaft, in: EW 91. Jg. (1992), Heft 17, S. 1075 ff. und für die EdF: LEDERER, P. ; BOUTTES, J.P. : a.a.O., S. 225 ff.

323 Vgl. 0.V. : Feilschen um mehr Wettbewerb, in: ENERGIE SPEKTRUM, Januar 94, S. 8

324 Eine solche Maßnahme wăre z.B. die Verringerung der Netzkupplungskapazităten und der Leitungskapazităten. 
Seitens der EVU wird weiter angeführt, daß eine Einführung von Wettbewerb nicht erforderlich ${ }^{325}$ sei, da bereits ein hohes Maß an vergleichendem Wettbewerb bestehe. Darüber hinaus befürchten die EVU durch Einführung des TPA-Konzeptes Nachteile für die langfristige Versorgungssicherheit, die optimale Kraftwerksparkzusammensetzung und den Ausbau des Leitungsnetzes ${ }^{326}$. Weitere negative Effekte sind in ihren Augen

- steigende Preise für Kleinverbraucher,

- eine zunehmende Unternehmenskonzentration,

- die Nicht-Einführung der Kraft-Wärme-Kopplung,

- eine weitere Umweltverschmutzung und

- fehlende Anreize zur rationellen Energieanwendung.

Zudem wird die Grundlage der bisherigen europäischen Zusammenarbeit in der Elektrizitätswirtschaft, die gegenseitige Partnerschaft, ihrer Meinung nach erschwert. In den Vorschlägen zur Einführung des TPA-Konzeptes sehen sie keine Liberalisierung des europäischen Strommarktes sondern eine weitere Regulierung ${ }^{327}$. Ferner befürchten die EVU durch die Zustimmung zur Einführung der ersten Stufe des Konzeptes, die Tür für die weiteren Konzeptstufen aufzustoßen. Gerade die zweite Stufe ist für sie kritisch. Während die Verbund-EVU bei der Durchleitungsmöglichkeit den Markt in der ersten Stufe wegen der geringen Teilnehmerzahl selbst mitsteuern können, löst die Durchleitungsoption für Großabnehmer in der zweiten Stufe erstmalig das Versorgungsmonopol der EVU auf. Es kommt bei Inanspruchnahme zu einem Verbraucherverlust für einige EVU. Können die entgangenen Deckungsbeiträge nicht auf die anderen Abnehmer verteilt werden, weil Regulierungsinstitutionen ihre Aufgabe erfolgreich durchführen, kann es zu Verlusten kommen. Für europäische EVU wäre das eine gänzlich neue Situation. Weniger gefurchtet wird von den Gebiets-EVU die dritte Stufe des Konzeptes. Der Masse der Kleinverbraucher wird nicht zugetraut, daß sie aus den Verträgen ausscheiden.

Neben den Verbundunternehmen lehnen auch die EVU unterhalb dieser Ebene das Konzept ab. Sie haben ähnlich wie Großabnehmer in der ersten Stufe keine veränderten Strombezugsmöglichkeiten; erst in der zweiten Stufe können sie ihren Lieferanten frei wählen. Gleichzeitig müssen sie jedoch die Abwanderung ihrer Großverbraucher $\mathrm{zu}$ anderen Erzeugern oder zu den Verbund-EVU aus folgendem Grund befürchten: Die Verbund-EVU können Großabnehmer direkt aus dem Hochspannungsnetz beliefern und benötigen keinerlei Infrastruktur des Verteilungsunternehmens. Deshalb haben die Verbundunternehmen einen Kostenvor-

325 Vgl. z.B. die zahlreichen Veroffentlichungen der VDEW zu diesem Thema.

326 Vgl. GRAWE, J. . Der Kommissionsentwurf ... , a.a.O., S. 882

327 Vgl. MAGERL, H. : Elektrizitătswirtschaftliche Tagesfragen, in: EW Jg. 92 (1993), Heft 14, S. 869 
teil bei der Großverbraucherbelieferung gegenüber den Verteilungsunternehmen. Darüber hinaus besteht für die Klein- und Mittelverteilerunternehmen in Europa die Gefahr, durch einen einsetzenden Konzentrationsprozeß vom Markt verdrängt zu werden. Aus diesen Gründen werden die Verteilungsunternehmen die Gefahren höher einschätzen als die möglichen Gewinne durch die Verwirklichung des TPAKonzeptes. Deshalb ist die Akzeptanz des Konzeptes bei den regionalen Verteilerunternehmen gering ${ }^{328}$.

Die industriellen Großabnehmer befürworten die Einführung des TPA-Konzepte $^{329}$. Ihnen geht es in erster Linie nicht um die Einführung von tatsächlichem Wettbewerb, sondern um die Verringerung der Strompreise. Diesen Effekt erhoffen sich die Interessenverbände durch die potentielle Wahlmöglichkeit, d.h. durch eine Steigerung ihrer Marktmacht. Bei dem Verbleib der Großabnehmer bei ihrem EVU zu niedrigeren Preisen haben sie gleichzeitig den Vorteil der unverändert hohen Versorgungssicherheit ohne Such- und Transaktionskosten.

Die kleinen und mittleren Endverbraucher werden tendenziell das Konzept ablehnen, sofern sie überhaupt von geplanten Veränderungen Kenntnis erlangen. Sie werden die einseitige Bevorteilung von Großabnehmern zu ihren Lasten befürchten. Die in der dritten Stufe tatsächlich bestehende Möglichkeit zum Wechsel des Lieferanten werden sie wegen des erforderlichen Aufwandes und der vermeintlich reduzierten Versorgungssicherheit negativ bewerten. An eventuelle, auch für Kleinabnehmer mögliche Strompreissenkungen durch Einführung des Konzeptes werden sie nicht glauben.

\subsubsection{Der Regulierungsbedarf des Konzeptes}

In der ersten Konzeptstufe des TPA-Konzeptes erhalten ausschließlich VerbundEVU Durchleitungsrechte. Der Regulierungsbedarf unterscheidet sich auf dieser Stufe nicht von dem des Weiterentwicklungsmodells.

In der zweiten Konzeptstufe müssen folgende Teilbereiche von der Regulierung erfaßt werden:

328 Einige der Unternehmen verăndern jedoch bereits ihre Position. Vgl. o.V. : Für ungeteilten Strom-Wettbewerb, in: ET 44. Jg (1994), Heft 4, S. 256

329 Vgl. VIK: „Durchleitung als Wettbewerbsinistrumentếm,ma. O978-3-631-75129-9 
a) Verhinderung von Monopolrenten

b) Mißbrauchskontrolle über die Netzverfügungsrechte

c) Verhinderung atarifärer Handelshemmnisse

e) Verhinderung von Quersubventionierung
-> Mittel: Preisregulierung

-> Mittel: Netzbetreiberkontrolle

-> Mittel: Netzbetriebskontrolle -> Mittel: Preisregulierung

\section{$\mathrm{Zu}$ a) Verhinderung von Monopolrenten}

Die bereits klassische Preisregulierung ist bei Einführung des TPA-Konzeptes weiterhin erforderlich, um Monopolrenten der EVU zu verhindern. Besonders gefährdet durch die Marktstellung der EVU sind die in der zweiten Stufe noch der Anschlußpflicht unterliegenden Klein- und Mittelabnehmer. Darüber hinaus müssen die Regulierungsinstitutionen kontrollieren, ob ein Gebiets-EVU seine Strompreise für die Durchleitungsberechtigten heraufsetzt. Die Preisanhebung für Durchleitungsberechtigte können die EVU dann am Markt durchsetzen, wenn sie wissen, daß kein Anbieter für ihre Abnehmer existiert oder keine freien Übertragungskapazitäten vorhanden sind. Bei ausreichenden Übertragungskapazitäten wird die auf dem Markt erzielbare Preisobergrenze durch die Summe aus Erzeugungspreis und Durchleitungsgebühren des günstigsten externen Anbieter für die nicht vertraglich gebundene Abnehmer festgelegt. Deshalb ist in diesem Fall eine Regulierung der Übertragungskapazitäten und der Durchleitungspreise erforderlich, da das GebietsEVU als Netzmonopolist hierüber die Preise des Anbieters außerhalb des Versorgungsgebietes steuern kann.

\section{Zu b) Mißbrauchskontrolle über die Netzverfügungsrechte}

Die Netzbetreiberkontrolle hat in der zweiten Konzeptstufe die Aufgabe, die Abwicklung der berechtigten Durchleitungen sicherzustellen und den Ablehnungsbestrebungen der Gebiets-EVU entgegenzuwirken. Die Konzeption des TPA-Konzeptes enthält als gerechtfertigte Ablehnungsgründe mangelnde freie Übertragungskapazitäten und die unzumutbare Belastung des Verbund-EVU durch die Durchleitungen. Daraus folgt, daß eine wie auch immer geartete Regulierung die Kosten und Auslastungsgrade des Leitungsnetzes kennen oder sich in vertretbarer Zeit Einblicke verschaffen können muß. Für die Regulierungsinstitutionen am einfachsten ist die Kontrolle der freien Kupplungskapazitäten zu den benachbarten Versorgungsgebieten. Schwerer zu kontrollieren sind die Ablehnungen durch mangelnde Transportkapaziäten innerhalb des Versorgungsgebietes, da diese vielfälti- 
gen Einflüssen ausgesetzt sind ${ }^{330}$. Eine in diesem Bereich erfolgreiche Regulierung ist nur bei hohen Aufwendungen für die Informationsgewinnung möglich.

\section{$\mathrm{Zu} \mathrm{c)} \mathrm{Verhinderung} \mathrm{atarifärer} \mathrm{Handelshemmnisse}$}

Gebiets-EVU können versuchen, Durchleitungen durch atarifäre Handelshemmnisse, wie „Anschlußkriterien“ und Netzanschlußspezifikationen in bezug auf die Versorgungssicherheit, zu diskriminieren. Die Aufgabe der Regulierungsinstitutionen ist es, auf Antrag diese Anschluß- und Durchleitungskriterien zu überprüfen, ungerechtfertigte $\mathrm{zu}$ untersagen und zu sanktionieren.

\section{Zu d) Verhinderung von Quersubventionierung}

Die Gefahr der Quersubventionierung besteht immer dann, wenn für einzelne Abnehmergruppen die Wettbewerbsintensität hoch ist und andere Abnehmer weiterhin dem Versorgungsmonopol des Gebiets-EVUs unterliegen; eine derartige Situation liegt zwingend während der zweiten Implementierungsphase vor. Gebiets-EVU können die Preise für ihre Großabnehmer senken, um damit diese vom gebietsexternen Strombezug abhalten. Gleichzeitig können sie die Preise für die Kleinabnehmer anheben. Die Aufgabe der Preisregulierung ist es nun, die Kosten für die jeweilige Abnehmergruppe zu bestimmen und zu überprüfen, ob eine Quersubventionierung vorliegt. Die bereits traditionelle Diskussion ${ }^{331}$ über Quersubventionen zeigt, daß es bisher den nationalen Regulierungsinstitutionen nicht gelungen ist, Quersubventionen zu verhindern, deshalb ist zu bezweifeln, daß sich diese Situation im TPA-Konzept ändern wird. Auf jeden Fall steigen die Regulierungsaufwendungen durch die beabsichtigte staatliche Verhinderung von Quersubventionen weiter an.

In der dritten Konzeptstufe dehnen sich die Regulierungsbereiche nicht aus. Durch die Zugangsberechtigung einer Vielzahl von Verbrauchern steigt jedoch der Regulierungsaufwand im Bereich der Monopolmachtbegrenzung, der Mißbrauchskontrolle und der Verhinderung atarifärer Handelshemmnisse. Bei real existierenden freien Wahlmöglichkeiten auch für die Masse der Kleinabnehmer kann die Regulierung, die sich mit der Verhinderung von Quersubventionen beschäftigt, eingestellt werden. Es ist jedoch ein Trugschluß zu glauben, die Preisregulierung könne eingestellt werden, weil die Kleinabnehmer in dieser Konzeptphase aus dem Versorgungsmonopol entlassen werden. Relevant ist der Preis des externen Anbieters beim Abnehmer. Je höher die Stromtransportkosten des externen Lieferanten sind, desto weniger besteht für die Abnehmer die Chance, einen Anbieter zu finden, dessen Preis unter dem des Gebiets-EVU liegt. In einer solchen Situation verfügt das

$330 \mathrm{Vgl}$. hierzu die Ausführungen in Kapitel 8.2.2 zu den Durchleitungsabwehrpotentialen der EVU.

331 Vgl. RIEMER, H.-W. : a.a.O., S. 232 ff. Rüdiger Hermann - 978-3-631-75129-9 
Gebiets-EVU über ein begrenztes Monopol, welches weiterhin durch die Preisregulierung überwacht werden muß.

In bezug auf die Regulierungsinstitutionen für die drei Konzeptstufen kann folgendes festgehalten werden: Eine einzige europäische Regulierungsinstitution, die allen Regulierungserfordernissen auf jeder Konzeptstufe Rechnung trägt, ist nicht denkbar. Eine solche Institution müßte vollständige Information über den Betrieb der Netze, die Preisbildung und die Durchleitungsabwehrmaßnahmen der EVU besitzen. Die Preisregulierung hat in den Mitgliedsländern eine lange Tradition, weshalb auf die bestehenden Regulierungsbehörden zurückgegriffen werden kann. Die zusätzliche Netzbetreiber- und Netzbetriebskontrolle muß entweder von den bestehenden nationalen Regulierungsbehörden mit übernommen oder von einer zusätzlichen Institution erbracht werden. Denkbar ist eine spezielle Kommission, die auf Antrag Durchleitungsablehnungen von Gebiets-EVU überprüft. Diese Überprüfung im nachhinein hat den Nachteil, daß sie ausschließlich Strafen für die ungerechtfertigte Ablehnung aussprechen kann. Damit von den Strafen eine präventive Wirkung ausgeht, müßten sie so hoch angesetzt sein, daß jedes EVU von einer ungerechtfertigten Ablehnung Abstand nimmt. Dies funktioniert aber nur, wenn die EVU überzeugt sind, daß die Regulierungsinstitution eine ungerechtfertigte Ablehnung so sicher nachweisen kann, daß sie einer rechtlichen Prüfung standhält. Dies ist jedoch zu bezweifeln, da die dazu erforderlichen Informationen nur von dem EVU, welches überprüft werden soll, kommen können. Deshalb ist eine kontinuierliche Netzbetreiber- und Netzbetriebskontrolle zieladäquater, die alle relevanten Informationen kontinuierlich erhebt und darauf aufbauend Durchleitungsablehnungen bewerten kann.

\subsubsection{Die Implementierung des TPA-Konzeptes}

Das Hauptmittel des TPA-Konzeptes zur beabsichtigten Verwirklichung seiner Ziele weist einen großen Vorteil auf - die einfache Einfürung. Die Durchleitungsverpflichtung ist rechtlich zwar umstritten, davon abgesehen jedoch einfach anzuordnen. Alles weitere soll sich daraus im Marktgeschehen entwickeln.

Begleitend müssen die erforderlichen Regulierungsinstitutionen geschaffen werden. Bei Einführung der ersten Stufe des Konzeptes ist der Regulierungsaufwand noch gering und es können Erfahrungen gesammelt werden, die bei der weiteren Einführung der Konzeptphasen umgesetzt werden.

Der Implementierungsaufwand ist äußerst niedrig. Durch die stufenweise Ausdehnung des Marktzutritts auf weitere Marktteilnehmer werden große Umwandlungen vermieden, die zu hohen Übergangsverlusten führen könnten. Die vorgesehenen Ankündigungsfristen für das Ausscheiden aus der Versorgung und für die Rück- 
kehr zum Gebiets-EVU lassen den betroffenen Unternehmen ausreichend Zeit, sich der veränderten Situation anzupassen.

Die möglicherweise eintretenden Verluste aus nicht ausgelasteten Erzeugungsanlagen einiger EVU sind bei erfolgreicher Regulierung marktwirtschaftliche Normalität und daher im Rahmen des Selektionsprozesses wünschenswert.

Der erforderliche Implementierungszeitraum ist beim TPA-Konzept im Vergleich zu den noch zu betrachtenden Desintegrationskonzepten gering. Die erste Stufe, die Durchleitungsverpflichtung für Verbund-EVU, ist bereits umgesetzt worden, weshalb konzeptionell mit der zweiten Stufe sofort begonnen werden könnte. Die dritte Stufe könnte dann in zwei bis vier Jahren folgen.

\subsection{Beurteilung der Integrationskonzepte}

\subsubsection{Beurteilung der Marktkonzeptionen}

Beide Integrationskonzepte sehen weder eine rechtliche noch eine organisatorische Trennung der Stromerzeugung, des Stromtransportes, der Stromverteilung und der Endabnehmerversorgung vor ${ }^{332}$. Damit werden die bestehenden vertikal integrierten Unternehmensstrukturen nicht aufgelöst. Im TPA-Konzept bewirkt die Durchleitungsverpflichtung lediglich eine gedankliche Trennung des Netzes von der Stromerzeugung und -verteilung, welche jedoch durch die Bedingungen - ausreichende Stromtransportkapazitäten, keine Einschränkung des Netzbetriebes durch die Durchleitung - wieder aufgehoben wird. Das Stromtransportnetz erhält in beiden Integrationskonzepten keine reine Infrastrukturaufgabe für den europäischen Strommarkt, sondern wird als Bestandteil der Elektrizitätsversorgung des jeweiligen Gebiets-EVU angesehen. Hierdurch unterliegt das Stromtransportnetz weiterhin der ausschließlichen Verfügung des Gebiets-EVUs mit allen Nachteilen für den europäischen Strommarkt.

Im Weiterentwicklungskonzept dient die bestehende Infrastruktur zum bilateralen Stromaustausch ausschließlich der Versorgungssicherheitsbereitstellung im UCPTE-Netz. Installationen und Institutionen, deren primäre Aufgabe der Stromhandel ist, existieren nicht. Die Errichtung einer Infrastruktur für den Stromhandel sieht das Konzept nicht vor. Mit einem freiwilligen Ausbau durch die EVU ist nicht

332 Die wichtigsten Punkte einer Marktanalyse für Energiemărkte zeigt Gröner auf. Hierzu gehört seiner Meinung nach der vertikale Integrationsgrad. Vgl. GRÖNER, H. : Energiepolitische Konzeptionen zwischen Wettbewerb und Lenkung, in: Energiewirtschaft, Heft 2 (1978), S. 91-98, hier S. 98 
rechnen, da deren Interesse am Stromhandel gering ist. Eine Ausnahme bilden die EVU, die über keine ausreichenden Erzeugungsanlagen verfügen und zur Deckung des Bedarfes auf den Stromimport ausweichen müssen. Sie allein sind jedoch nicht in der Lage, die erforderliche Infrastruktur für den Stromhandel zu erstellen.

Ähnlich ist die Situation bei Verwirklichung des TPA-Konzeptes: Vorgesehen ist die Errichtung einer Stromhandelsinfrastruktur in Europa nicht. EVU, die ein starkes Interesse an Stromlieferungen zu anderen EVUs oder Abnehmern außerhalb des eigenen Versorgungsgebietes haben, werden bereit sein, die Investitionen für den eigenen Stromtransport zu tätigen. Die EVUs, in deren Gebiete gegen ihren Willen Strom geliefert werden soll, werden hierfür keine Stromtransportkapazitäten oder technische Anlagen zur Regelung errichten. EVU, die keinen Strom liefern und deren Abnehmer keinen Strom von anderen Lieferanten beziehen wollen, könnten Kapazitäten für einen Stromtransit anderer EVU aufbauen. Dies hängt jedoch von den Erträgen aus dem Stromtransit $\mathrm{ab}^{333}$. Darüber hinaus sieht das Konzept keinerlei Institutionen und Instrumente vor, die eine Marktorganisation schaffen. Jeder Verbraucher muß die für ihn erforderlichen Informationen beschaffen und Verträge über die Lieferung und Versorgungssicherheitsbereitstellung getrennt abschließen. Somit wird in beiden Konzepten keine Stromhandelsinfrastruktur in Europa errichtet, die Stromlieferungen zwischen den Marktteilnehmern dienen könnte.

Die fehlende Auflösung der vertikalen Integration der nationalen Elektrizitätswirtschaften und das Fehlen einer Stromhandelsinfrastruktur bewirken, daß weiterhin keine Teilmärkte in Europa durch die Einführung der Konzepte geschaffen werden, auf denen Wettbewerbsprozesse kostensenkend wirken können.

Im Weiterentwicklungskonzept ist die Marktteilnehmeranzahl auf die bestehenden EVU der Verbundebene begrenzt. Ein Marktzutritt neuer EVU oder von Stromabnehmern ist nicht möglich. Bei Umsetzung des TPA-Konzeptes wird durch die Durchleitungsverpflichtung der Gebiets-EVU ein Zugang für andere Anbieter zu den Abnehmern eines Gebiets-EVUs theoretisch geschaffen. Die jeweiligen $n a-$ tionalen Marktzutrittsbarrieren ${ }^{334}$ ändern sich durch Einführung des TPA-Konzeptes jedoch nicht ${ }^{335}$. Ferner verhindern die in den Mitgliedstaaten existierenden Ausschließlichkeitsrechte beim Leitungsbau, daß Anbieter oder reine Betreibergesellschaften eine Alternative zum Leitungsnetz des Gebiets-EVU schaffen können. Aus den Netzverfügungsrechten der Gebiets-EVU entstehen folgende Marktzutrittsbarrieren:

333 Vgl. hierzu die Ausführungen in Kapitel 9.

$334 \mathrm{Vgl}$. hierzu die Ausführungen in Kapitel 4.

335 Sie sollen nicht Gegenstand der weiteren Batrabhtangen sein. - 978-3-631-75129-9 
- die Gefahr langfristiger Durchleitungseinschränkung ${ }^{336}$,

- die reduzierte Versorgungssicherheit für die Durchleitungen durch mangelnde Netzzugriffsrechte, die ein potentieller Exporteur nicht ausgleichen kann,

- Transaktionskosten der externen Anbieter, um Anteile an der Netzkapazität der Gebiets-EVU zu erhalten,

- höhere Netzbenutzungskosten des Exporteurs im Vergleich zum Gebiets-EVU.

Die bereits bestehenden Erzeugungskapazitäten wirken für die existierenden EVU mit einem eigenen Versorgungsgebiet in Europa nicht als Marktzutrittsbarriere. Lediglich für etwaige Neuanbieter bilden sie eine Hürde. Damit kann für das TPAKonzept festgehalten werden: Die bestehenden Marktzutrittsbarrieren in den Versorgungsgebieten werden durch das Konzept zumindest in der Anfangsphase verringert, ohne jedoch vollständig abgebaut zu werden. Die langfristigen Marktzutrittsbarrieren hängen von den Gestaltungsmöglichkeiten der Gebiets-EVU beim Durchleitungsentgelt und bei den Durchleitungsverhinderungsmöglichkeiten ab.

Im Weiterentwicklungskonzept bleibt sowohl die potentielle als auch die tatsächliche Wettbewerbs- und Konkurrenzsituation auf den nationalen Elektrizitätsmärkten unverändert. Ein möglicher Wettbewerb zwischen den Unternehmen ist maximal auf der Verbundebene denkbar. Eine Konkurrenzsituation kann jedoch nur dann entstehen, wenn mehrere Verbundunternehmen als Anbieter mit niedrigen Strompreisen auftreten und auf eine begrenzte Anzahl von Nachfragern treffen. Dies ist jedoch wegen der Größe der Versorgungsgebiete, die über die Stromtransportkosten Strombezugspreisunterschiede ausgleichen, und den begrenzten Stromtransportkapazitäten in Europa unwahrscheinlich. Aus diesem Grund wird die Wettbewerbsintensität auf dem europäischen Strommarkt der Verbundunternehmen gering sein. Die ausschließlich freiwillige Teilnahme am Stromhandel kann ferner keine Wettbewerbsprozesse initiieren, die die Monopolrenten der EVU beschneiden könnten. Die Beschränkung des Wettbewerbs auf Aushilfs- und Koordinierungslieferungen sowie des Stromhandels zwischen den Verbund-EVU verhindert des weiteren, daß Endverbraucher oder regionale Verteiler ihren Lieferanten europaweit suchen können. Dieses sichert den EVU die Kostenüberwälzungsmöglichkeiten bei fehlendem unternehmerischen Risiko.

Im TPA-Konzept ist die Wettbewerbsintensität abhängig von der jeweiligen Konzeptstufe. In der ersten Konzeptstufe steigt die Anzahl der Wettbewerber auf den Elektrizitätsmärkten durch die Einführung des TPA-Konzeptes nicht. Diese Situation ändert die Durchleitungsberechtigung für Großabnehmer und Verteilungsunternehmen in der zweiten Konzeptstufe. Als Anbieter stehen die EVU in Konkurrenz zueinander um die durchleitungsberechtigten Abnehmer. Begrenzt wird die 
Anzahl der Anbieter durch die bestehenden Gebietskupplungskapazitäten. So haben Abnehmer am Rande eines Versorgungsgebietes eine deutlich höhere Anzahl von konkurrierenden Anbietern. Neben dem direkt angrenzenden EVU kommen weitere EVU hinzu, für die das benachbarte EVU den Stromtransit durchführen würde. Das Strom transportierende EVU hätte kein Interesse, die Durchleitung durch sein Versorgungsgebiet bei ausreichenden Kapazitäten zu verhindern, wenn es durch das Stromtransportentgelt zusätzliche Deckungsbeiträge erzielen kann.

\section{Abbildung 17: Anbieter eines Abnehmers in Randlage}

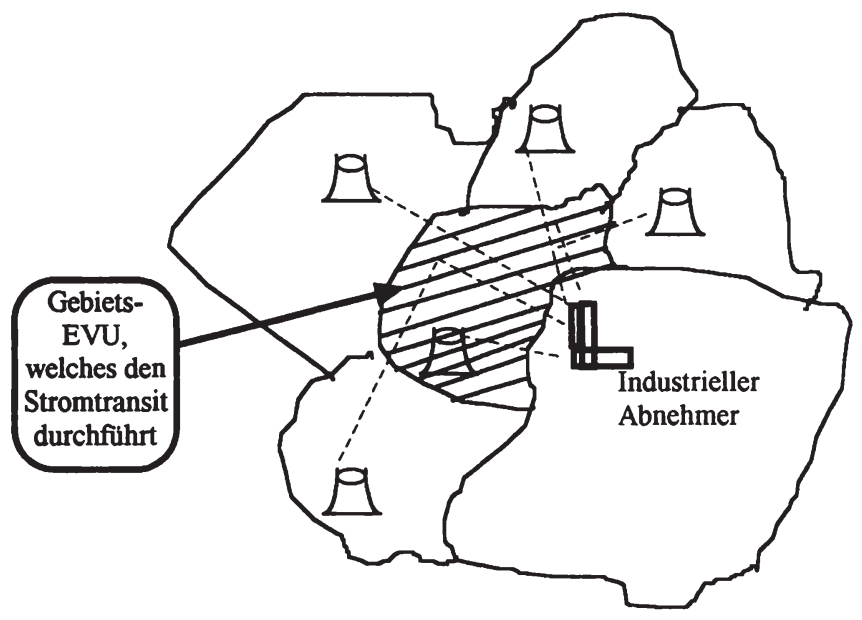

In der dritten Konzeptstufe erhöht sich lediglich die Anzahl der Abnehmer, während die Anzahl der Konkurrenten auf der Anbieterseite konstant bleibt.

In welcher Konzeptstufe entstehen durch die Einfürung des TPA-Konzeptes Wettbewerbsprozesse, die Kostensenkungen bewirken können?

In der ersten Konzeptstufe erfolgen Stromlieferungen an andere EVU ausschließlich auf freiwilliger Basis. Theoretisch treten bei einer Nachfrage eines EVU mehrere andere Gebiets-EVU um die Belieferung in Wettbewerb; in der Praxis ist dies jedoch nicht der Fall, da bei den bestehenden europäischen Erzeugungspreisdisparitäten und den bestehenden Versorgungsgebietsgrößen maximal ein EVU als Lieferant für ein nachfragendes EVU in Frage kommt ${ }^{337}$. Die vorgesehenen erzwingba- 
ren Durchleitungen durch das Gebiet eines anderen EVUs kann es theoretisch nur bei dem ersten Durchleitungstyp (Stromtransit) geben. Die Art der Durchleitung läßt jedoch bei ausreichenden Stromtransportkapazitäten kein Interesse an einer Durchleitungsablehnung bei einem EVU entstehen. Damit entsteht in der ersten Konzeptstufe des TPA-Konzeptes kein Wettbewerb zwischen den EVU. In der zweiten Konzeptphase verändert sich die Wettbewerbssituation für die durchleitungsberechtigten Großabnehmer und Verteilerunternehmen. Um ihre Nachfrage konkurriert das Gebiets-EVU mit anderen EVU. Die Wettbewerbsintensität hängt dabei von folgenden Faktoren ab:

- den Erzeugungspreisdisparitäten ,

- den Erzeugerüberkapazitäten,

- den Stromtransportkapazitäten und ihrer Verteilung innerhalb der Gemeinschaft,

- den Durchleitungsverhinderungsmöglichkeiten der Gebiets-EVU,

- dem Durchleitungsentgelt.

Betrachtet man die bestehenden europäischen Überkapazitäten, so kommt man zu dem Schluß, daß für eine flächendeckende Verdrängung eines Gebiets-EVUs in der Belieferung von Großabnehmern und Verteilerunternehmen keine ausreichenden Erzeugungsüberkapazitäten vorhanden sind. Lediglich einzelne Verbraucher oder kleinere Verteilerunternehmen können in der zweiten Stufe des TPA-Konzeptes den Lieferanten wechseln; hierdurch begrenzt sich die reale Erzeugungskapazität und die Wettbewerbsintensität kurz- bis mittelfristig. Die Wettbewerbsintensität kann steigen, wenn Erzeugungsunternehmen oder Gebiets-EVU mit einer günstigen Kostensituation und geeignetem Absatzpotential ihre Stromproduktion ausdehnen, neue Anbieter in den Markt drängen oder die Nachfrage eines GebietsEVUs sinkt und dadurch zusätzliche Überkapazitäten entstehen. Bei dem derzeitigen Alter der europäischen Kraftwerke ${ }^{338}$ und den Strombedarfsvorhersagen ist mit dem letzten Fall nicht zu rechnen. Ferner führt der Marktzutritt von Neuanbietern wegen den bestehenden Planungs- und Genehmigungszeiträume ${ }^{339}$ sowie der Bauzeiten von Kraftwerken ${ }^{340}$ in der zweiten Konzeptstufe nicht zu einer Erhöhung der Wettbewerbsintensität ${ }^{341}$.

Die Wettbewerbsintensität nach Beginn der dritten Stufe des TPA-Konzeptes unterscheidet sich für den Teilmarkt der Großabnehmer und Verteiler nicht von dem der zweiten Konzeptstufe. Lediglich können wegen des längeren Zeitraumes neue Anbieter auf den Markt eintreten, wodurch die Wettbewerbsintensität erhöht wird.

338 Für die Kernenergie siehe o.V. : Jahrbuch der Atomwirtschaft, Düsseldorf 1993

339 Diese sind je Mitgliedsstaat unterschiedlich und variieren zwischen sechs Monaten und mehreren Jahren. Vgl. hierzu die Ausführungen zu den Genehmigungsverfahren in den einzelnen Staaten in : EISS et al. : Die Ordnung a.a.O.

340 Für die Kernenergie siehe o.V. : Jahrbuch der Atomwirtschaft, Düsseldorf 1993

341 Dies wird durch die Entwicklungen seit deriblinsetzung dernTransit-Richtlinje bescatigt. 
Wie entwickelt sich nun die Wettbewerbsintensität fur das Marktsegment der Kleinabnehmer? Kein EVU in Europa verfügt über Überkapazitäten, die innerhalb eines bestehenden Versorgungsgebietes die gesamte Stromerzeugung eines $\mathrm{Ge}-$ biets-EVU ersetzen könnte. Zudem existieren die dafür erforderlichen Stromtransportkapazitäten in Europa nicht. Des weiteren ist die Kleinabnehmerbelieferung im Vergleich zur Belieferung von wenigen Großabnehmern für den Exporteur wegen der damit verbundenen Transaktionkosten uninteressant. Großabnehmer sind in ihrem Nachfrageverhalten flexibler als Kleinabnehmer; sie sind häufig dazu bereit, mit dem EVU Verträge zu schließen, die einen Lastabwurf in Spitzenlastzeiten vorsehen, und weisen zumeist eine ausgeglichene Lastkurve auf. Aus diesen Gründen muß das liefernde Unternehmen für Großabnehmer geringere Versorgungssicherheitsaufwendungen tätigen als bei vielen Kleinabnehmern bei gleicher Abnahmemenge. Darüber hinaus wird es für die Vertragsparteien einfacher sein, eine Durchleitung größeren Umfanges für einen Abnehmer zu beantragen und durchzusetzen als für eine Vielzahl unterschiedlicher Kleinabnehmer. Aus diesen Gründen wird die Wettbewerbsintensität für das Marktsegment der Kleinabnehmer auch nach Einführung der Durchleitungsrechte in der dritten Stufe unverändert bleiben. Erst wenn die in dem EU-Richtlinienvorschlag eingebrachte Aufhebung der Ausschließlichkeitsrechte zum Betrieb von Leitungen und Erzeugungsanlagen durchgesetzt würde ${ }^{342}$, könnte die Wettbewerbsintensität für die Kleinabnehmer steigen.

Negative Auswirkungen auf die Wettbewerbsintensität können durch die Haltung der EVU zur Einführung von Wettbewerb entstehen. Wenn die EVU geschlossen die Einführung von Wettbewerb auf ihren, bisher durch Monopole geschützten Märkten ablehnen, weil langfristig auch Unternehmen mit einer günstigen Kostensituation Nachteile durch den Wettbewerb erwarten, kann es frühzeitig zu einem abgestimmten Verhalten kommen ${ }^{343}$. Dies würde trotz der potentiellen Konkurrenz jegliches wettbewerbliches Verhalten ausschließen.

Somit kann festgehalten werden, daß durch die Einführung des TPA-Konzeptes auch bei Ausklammern der Durchleitungsverhinderungsmöglichkeiten lediglich eine zeitlich befristete niedrige Wettbewerbsintensität um europäische Großabnehmer und Verteilerunternehmen geschaffen wird, welche Ausgangspunkt für Kostenreduzierungen und Preissenkungen sein könnte. Die geforderte hohe Wettbewerbsintensität wird durch das Konzept nicht erreicht.

Die Marktmacht der einzelnen EVU auf den nationalen Elektrizitätsmärkten wird im Weiterentwicklungskonzept keine Veränderungen aufweisen, da keinerlei Marktmacht beeinflussende Faktoren - wie der Abbau der Marktzutrittsbarrieren oder die Aufhebung nationaler Sonderstellungen für die Elektrizitätswirtschaft verändert werden. Die Marktmacht einzelner EVU auf dem europäischen Strom-

342 Vgl. hierzu die Ausfuihrungen in Kapitel 5.

343 Die Wechselwirkungen zwischen dem Verhalten der EVU und den zur Verfügung stehenden Kapazităten im Transportbereich werden in Kapijel 8.2.21analysient8-3-631-75129-9 
markt der Verbundunternehmen ${ }^{344}$ ist dagegen gering. Durch die Kostenüberwälzungsmöglichkeit und die staatlichen Auflagen für eine eigenständige Elektrizitätsversorgung können potentielle Lieferanten nicht auf die nationalen Elektrizitätsmärkte oder die Bezugsentscheidungen potentieller Bezieher einwirken. Jedes Unternehmen besitzt die Möglichkeit, keinen Stromhandel durchzuführen.

\section{Abbildung 18: Entwicklung der Wettbewerbsintensität im TPA-Konzept}

Wettbewerbsintensităt

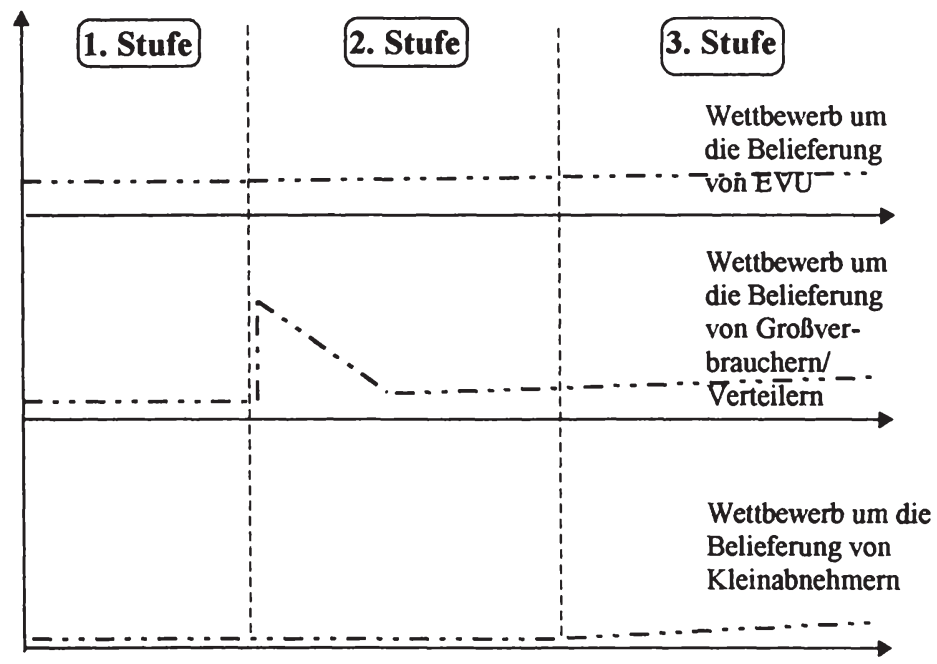

Zeit

Im TPA-Konzept wird die Marktmacht der Gebiets-EVU ab der zweiten Konzeptstufe eingeschränkt. Die Auflösung des vollständigen Monopols in der Strombelieferung von Abnehmern führt dazu, daß einzelne externe Anbieter einige Abnehmer aus der Masse der Kunden des Gebiets-EVU abziehen. Nach Abschluß der Stromlieferverträge zwischen Exporteuren und ehemaligen Abnehmern des Gebiets-EVUs sind die Durchleitungskapazitäten des Gebiets-EVUs vergeben oder die Überkapazitäten der Exporteure ausgelastet. Das Gebiets-EVU verfügt wieder über einen festen Stamm von Abnehmern. Die absolute Zahl ist zurückgegangen. Für die verbleibenden Abnehmer hat das Gebiets-EVU seine ursprüngliche Marktmacht wiedererlangt. 


\section{Abbildung 19: Marktmacht eines Gebiets-EVUs}

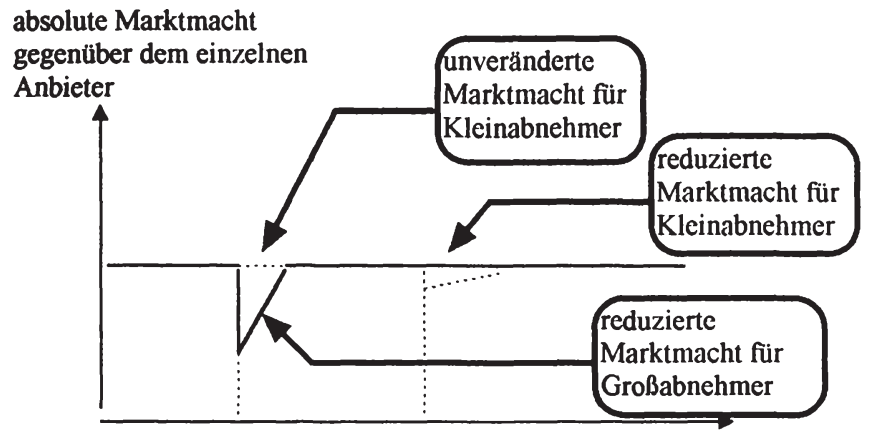

1. Konzeptstufe 2. Konzeptstufe 3. Konzeptsstufe t

Das Auslaufen von Lieferverträgen zwischen Exporteuren und Abnehmern verändert die Marktmacht des Gebiets-EVUs nicht. Wird der Vertrag nicht verlängert, muß der Abnehmer um die Wiederaufnahme der Belieferung durch das GebietsEVU bitten. Ein anderer Abnehmer hat dann die Chance, die freien Durchleitungskapazitäten für eine externe Belieferung zu nutzen. Für das Gebiets-EVU werden lediglich die Vertragspartner ausgetauscht. Somit kann festgehalten werden, daß das TPA-Konzept die Marktmacht der Gebiets-EVU nur kurzfristig verringert.

\subsubsection{Der Beitrag der beiden Integrationskonzepte zur Zielerfüllung}

\subsubsection{Reduzierung der Kosten der Stromversorgung}

\subsection{Kostenentwicklung bei Umsetzung des Weiterentwicklungskonzeptes}

Im Stromerzeugungsbereich sind kurzfristig, wie die Ausführungen zum Weiterentwicklungskonzept gezeigt haben, nur geringe Kostenreduzierungen durch verstärkte Aushilfslieferungen denkbar. Langfristig könnten theoretisch Kosteneinsparungen durch gemeinsame europäische Kraftwerksbauten oder der gemeinsamen Kraftwerksnutzung durch mehrere EVU sowie durch langfristige Lieferverträge erzielt werden. Am Ende der Entwicklung könnte eine gemeinsame „merit order" den Kraftwerkseinsatz in Europa steuern. Das Eintreten dieses im dritten und vierten Entwicklungsszenarios beschriebenen Zustandes kann jedoch bezwei-

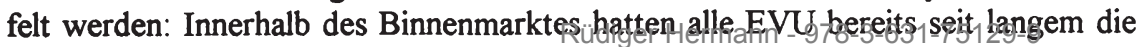


Möglichkeit, langfristige Stromlieferverträge abzuschließen und Kraftwerke gemeinsam zu bauen oder zu nutzen. Die bisher durchgeführten Gemeinschaftsprojekte weisen jedoch nur einen geringen Umfang auf ${ }^{45}$. Durch das Weiterentwicklungskonzept werden keine Rahmenbedingungen geändert, die in der Zukunft ein geändertes Verhalten der EVU bewirken könnten. Des weiteren ändert die Implementierung des Weiterentwicklungskonzeptes nicht die bestehenden nationalen Vorgaben an die Elektrizitätsversorgung, die zumeist die nationale Eigenversorgung mit elektrischer Energie vorsehen. Ferner entsteht durch das Konzept keinerlei Druck, ineffiziente Kraftwerke stillzulegen und durch Innovationen Kosten im Erzeugungsbereich zu reduzieren. Deshalb ist nicht mit einer Ausdehnung des Stromhandels und mit langfristigen Kosteneinsparungen im Erzeugungsbereich zu rechnen.

Im Stromtransportbereich wird die Zunahme der Aushilfslieferungen und die verstärkte gemeinsame Versorgungssicherheitsbereitstellung die Stromtransportkosten ansteigen lassen.

Der Verteilerbereich ist vom Weiterentwicklungskonzept nicht betroffen. Dagegen steigen die Transaktionskosten durch Verwircklichung des Weiterentwicklungskonzeptes in folgenden Bereichen an:

\section{a) Bilaterale Verträge}

Bei Abschluß, Abwicklung und Verrechnung von bilateral im Weiterentwicklungskonzept abgeschlossenen Stromlieferverträgen entstehen Transaktionskosten. Diese müssen um die Transaktionskosten zur Verrechnung der ungewollten Lastflüsse erhöht werden.

b) Zusätzliche Regelungstechnik

Zur Abwicklung und Verrechnung der Verträge ist eine umfangreichere Meß- und Regelungstechnik erforderlich als sie bisher in einigen Staaten der EU besteht. Durch deren Errichtung für die Lieferungen im Weiterentwicklungskonzept entstehen Transaktionskosten.

c) Verhalten bei Grenzfällen/Stromausfällen

Die Vertragspartner müssen Absprachen treffen, wie sie sich bei Ausfall einer Lieferung oder bei außergewöhnlichen Betriebssituationen verhalten. Durch den erforderlichen Abstimmungsprozeß und die vertragliche Vereinbarung entstehen weitere Transaktionskosten. 
d) Versorgungssicherheitsbereitstellung

Welcher Vertragspartner stellt welche Versorgungssicherheitsleistung zur Verfügung? Wie werden bei Eintritt des Reservefalles entstehende Kosten verrechnet? Zur Klärung und Festlegung dieser Fragen sind Aufwendungen erforderlich, die die Transaktionskosten weiter erhöhen.

Somit kann festgehalten werden, daß durch das Weiterentwicklungskonzept die Transaktionskosten zweifelsohne ansteigen werden.

Die Ausführungen zum Regulierungsbedarf des Konzeptes zeigen, daß ein Anstieg bei den Regulierungsaufwendungen durch die Implementierung des Konzeptes ausgelöst wird. Zusätzlich zu den bereits bestehenden Regulierungsbereichen, insbesondere der Preisaufsicht, muß die Stromhandelsteilnahmekontrolle und die Kontrolle über die Weitergabe von Kosteneinsparungen eingeführt werden. Besonders die gestiegenen Anforderungen an die Regulierung wirken dabei kostenerhöhend.

Betrachtet man nun abschließend die Gesamtkostenentwicklung nach Einführung des Weiterentwicklungskonzeptes, so stehen den kurzfristig gestiegenen Transaktionskosten und den zusätzlichen Regulierungsaufwendungen die geringen Kosteneinsparungen durch eine Intensivierung der gemeinsamen Versorgungssicherheitsbereitstellung in Europa gegenüber. Die Gesamtkostenbilanz ist somit kurzfristig eindeutig negativ.

Langfristig könnte das kurzfristige Ergebnis durch einen zunehmenden Stromhandel, die Errichtung von zusätzlichen Gemeinschaftskraftwerken und durch die gemeinsame Nutzung bestehender Kraftwerke verändert werden. Doch haben die Ausführungen zu der Stromerzeugungskostenentwicklung gezeigt, daß hiermit nicht gerechnet werden kann; damit führt das Weiterentwicklungskonzept auch langfristig zu steigenden Kosten.

\subsection{Kostenentwicklung bei Umsetzung des TPA-Konzeptes}

Die Kosten in der Stromverteilung und der Endabnehmerversorgung werden vom TPA-Konzept nicht betroffen. Verändern können sich lediglich die Stromerzeugungs- und die Stromtransportkosten sowie die Kosten für die Bereitstellung der Versorgungssicherheit.

In der ersten Stufe des TPA-Konzeptes bei Einführung der Durchleitungsverpflichtung für die Verbund-EVU besteht folgendes Kostensenkungspotential :

$$
\Delta \mathrm{K}_{\mathrm{GM}}=\sum \mathrm{Kv}_{\mathrm{s}}-\left(\sum \mathrm{Kv}_{\mathrm{L}}+\sum \mathrm{K}_{\mathrm{T}}\right)
$$




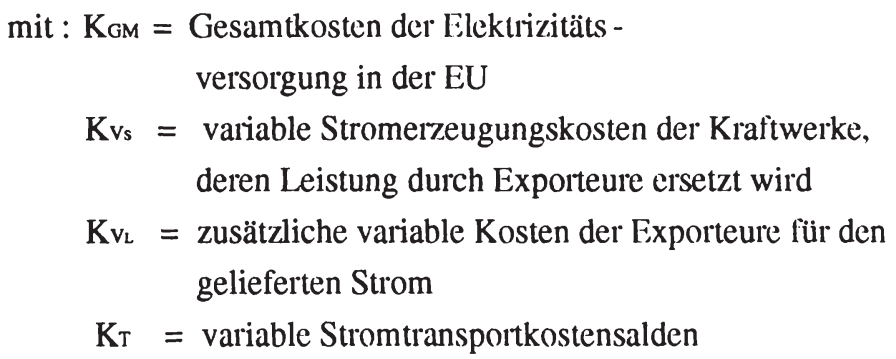

Diese Formel besagt, daß das Einsparpotential des TPA-Konzeptes auf der ersten Implementierungsstufe durch die Differenz der variablen Stromerzeugungskosten und der erforderlichen Stromtransportkosten festgelegt wird. Die absolute Höhe der entstehenden Stromtransportkosten muß um die verminderten gebietsinternen Stromtransportkosten der Importeure gekürzt werden. Es sind sogar Konstellationen denkbar, in denen dieser Saldo negativ wird, das heißt, die Stromtransportkosten durch einen speziellen Stromimport sind geringer als die Stromtransportkosten bei vollständiger Eigenversorgung eines Gebiets-EVUs.

Auf die Fixkosten der Stromerzeugung haben die Lieferungen in der ersten Stufe keinen Einfluß. Der Grund hierfür liegt in der Kostenüberwälzungsmöglichkeit des Gebiets-EVU auf seine der Versorgungspflicht unterliegenden Endverbraucher. Das Gebiets-EVU wird in dem kurzen Zeitraum der ersten Implementierungsstufe seine nicht ausgelasteten Erzeugungskapazitäten nicht stillegen, da die Stillegung Kapital vernichtet. Langfristig wird die nicht mehr benötigte Kapazitätsmenge genutzt, um ansonsten erforderliche Kraftwerksersatzinvestitionen zu verschieben.

In der zweiten Konzeptstufe ist eine Reduzierung der Fixkosten an eine erfolgreiche Regulierung gebunden. Besteht für die Gebiets-EVU, die Abnehmer verlieren, die Möglichkeit, Fixkosten auf die Kleinabnehmer zu verlagern, reduzieren sich die Fixkosten nicht. Das Kosteneinsparpotential ist ohne eine erfolgreiche Regulierung mit dem der ersten Stufe identisch, auch wenn die Nutznießer der Einsparungen nicht mehr die EVU, sondern teilweise die Großabnehmer und Verteilerunternehmen sind. Bei erfolgreicher Regulierung lautet die Gleichung:

$$
\Delta \mathrm{KGM}_{\mathrm{GM}}=\sum \mathrm{Kv}_{\mathrm{s}}-\left(\sum \mathrm{Kv}_{\mathrm{L}}+\sum \mathrm{K}_{\mathrm{T}}\right)+\Delta \mathrm{K}_{\mathrm{FoM}}
$$




$$
\begin{aligned}
\text { mit }: \mathrm{KGM}^{=} & \text {Gesamtkosten der Elektrizitäts }- \\
& \text { versorgung in der EU } \\
\mathrm{Kv}_{\mathbf{s}}= & \text { variable Stromerzeugungskosten der Kraftwerke. } \\
& \text { deren Leistung durch Exporteure ersetzt wird } \\
\mathrm{Kv}_{\mathbf{L}}= & \text { zusätzliche variable Kosten der Exporteure für den } \\
& \text { gelieferten Strom } \\
\mathrm{KT}_{\mathrm{T}}= & \text { variable Stromtransportkostensalden } \\
\Delta \mathrm{KF}_{\mathrm{F}}= & \text { Fixkostensenkung bei den Gebiets - EVU (Importeure), } \\
& \text { die Abnehmer verlieren, minus Fixkostensteigerung bei } \\
& \text { Erzeugern, die an Externe liefern (Exporteure) }
\end{aligned}
$$

In der dritten Konzeptstufe besteht die Kostenüberwälzungsmöglichkeit der EVU auf die Kleinabnehmer theoretisch nicht. Deshalb könnten ineffiziente Stromerzeugungsanlagen endgültig stillgelegt werden. Diese Selektion wird jedoch durch die Stromtransportkosten begrenzt. Erst wenn die Erzeugungskosten eines Kraftwerks des Gebiets-EVUs über den Lieferkosten ${ }^{346}$ eines Exporteurs liegen, wird das Kraftwerk keinen Strom absetzen können. Voraussetzung für die Selektion ist die Existenz ausreichender Lieferkapazitäten bei Anbietern außerhalb des Versorgungsgebietes und ausreichende Stromtransportkapazitäten des Gebiets-EVUs. Erst wenn diese Situation vorliegt, muß ein Gebiets-EVU, dessen Erzeugungskapazitäten durch externe Anbieter verdrängt worden sind, seine Transportkapazitäten umorientieren, d.h., an die veränderten Lastflüsse anpassen. Die dazu erforderlichen Investitionen erhöhen die Fixkosten ${ }^{347}$. Dies bedeutet, daß sich das Potential für langfristige Kostenreduktionen durch die langfristigen Veränderungen der Stromtransportfixkosten vermindert.

$$
\Delta \mathrm{K}_{\mathrm{GM}}=\sum \mathrm{Kv}_{\mathrm{s}}-\left(\sum \mathrm{Kv}_{\mathrm{L}}+\sum \mathrm{K}_{\mathrm{T}}+\Delta \sum \mathrm{K}_{\mathrm{FT}}\right)+\Delta \mathrm{K}_{\mathrm{FOM}}
$$

346 Die Lieferkosten bestehen aus dem Preis der gelieferten kWh multipliziert mit der bezogenen Leistung und den erforderlichen Stromtransportkosten.

347 Das hierdurch auch eine Moglichkeit fur das Gebiets-EVU besteht, Durchleitungen zu diskriminieren, wird in Kapitel 8.2.2 ausfuhrlichidangestellt:nann - 978-3-631-75129-9 


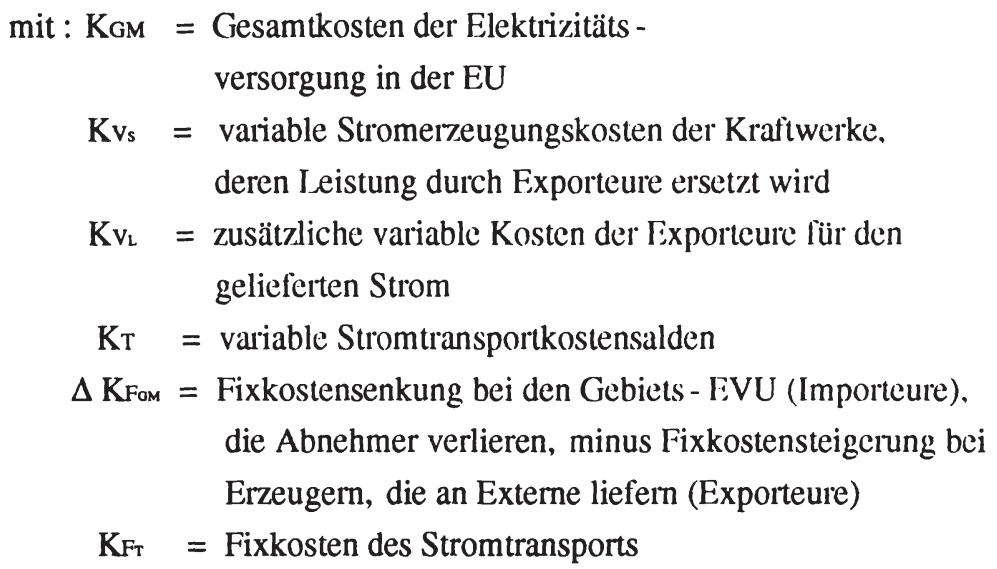

Die Veränderung der Fixkosten der Stromerzeugung entspricht der Fixkostensenkung bei den Gebiets-EVU, die Abnehmer verlieren, verringert um die Fixkostensteigerungen der Erzeuger, die Strom an Abnehmer in anderen Versorgungsgebieten liefern.

Allgemein fallen im TPA-Konzept alle Transaktionskostenarten zusätzlich an. Anbieter und Nachfrager werden die Kosten in unterschiedlicher Höhe zu tragen haben. Hierbei muß zwischen den Abnehmergruppen unterschieden werden: Kleinabnehmer werden in erster Linie Such- und Informationskosten aufbringen müssen, die Kosten für die Durchleitungsbeantragung und Durchsetzung werden aber den Anbietern überlassen. Bei Großabnehmern kann vermutet werden, daß sie bereit sein werden, diese Transaktionskosten zusätzlich zu tragen, um niedrigere Strombezugspreise realisieren zu können. Bei hoher Wettbewerbsintensität zwischen den Erzeugern werden die Lieferanten diese Kosten übernehmen müssen.

Die aus den „enviromental factors“ resultierenden Transaktionskosten betreffen alle Markteilnehmer bis auf die jeweiligen Gebiets-EVU, da diese die Marktstruktur maßgeblich über ihre Netzverfügungsrechte und Quersubventionierungsmöglichkeiten bestimmen. Für das Gebiets-EVU besteht die Unsicherheit im Bereich der Unternehmensumwelt ausschließlich aus dem Verhalten der Regulierungsinstitutionen.

Die aus der Häufigkeit einer Transaktion resultierenden Kosteneinsparungen können nur erzielt werden, wenn Durchleitungen standardisiert und weitgehend automatisch beantragt, abgewickelt und verrechnet werden können. Die dazu erforderlichen Instrumente sind im Konzept nicht geplant. Sie müßten erst von interessierten Marktteilnehmern geschaffen werden. Hierdurch fallen zusätzliche Transaktionskosten an. 
Die Ausführungen zum Regulierungsbedarf ${ }^{48}$ zeigen, daß dieser im Vergleich zur bestehenden Struktur durch die Einführung des TPA-Konzeptes steigen wird. $\mathrm{Zu}$ sätzlich zu der in den meisten europäischen Staaten vorherrschenden Preisaufsicht muß die Netzbetreiber- und Netzbetriebskontrolle eingeführt werden. Hierdurch werden weder die Regulierungsaufwendungen minimiert, noch werden die Regulierungskosten abgesenkt. Damit wirkt das TPA-Konzept dem Kostenziel für den EU-Strommarkt entgegen ${ }^{349}$.

Den aufgezeigten geringen Kosteneinsparpotentialen in Europa stehen bei der Einführung des TPA-Konzeptes Kostensteigerungen für die Versorgungssicherheitsbereitstellung für den Stromtransport und für die Regulierung sowie zusätzliche Transaktionskosten gegenüber. Die Kosteneinsparung auf der Erzeugungsseite hängt entscheidend von den Durchleitungsverhinderungsmöglichkeiten und ausreichenden Stromtransportkapazitäten ab. Zusätzlich ist der Preis für die Stromtransportleistung ein entscheidender Faktor ${ }^{350}$. Gelingt es den Gebiets-EVU, Durchleitungen in ihr Versorgungsgebiet zu verhindern, so fehlen die Einsparungen im Erzeugungsbereich. Gelingt es den Gebiets-EVU nicht, die Durchleitungen zu verhindern, hängt der Kostensaldo vom Preiswettbewerb zwischen den Anbietern ab. Damit ist eine abschließende Bewertung ohne Betrachtung der Durchleitungsverhinderungsmöglichkeiten und der Stromtransportpreise nicht möglich ${ }^{351}$.

\subsubsection{Beurteilung der Versorgungssicherheit}

Die bestehenden Rahmenbedingungen und nationalen Auflagen sowie die organisatorischen und technischen Maßnahmen der UCPTE haben in den meisten Mitgliedsstaaten zu einem hohen Versorgungssicherheitsniveau geführt. Defizite bei einzelnen südeuropäischen Mitgliedsstaaten sind zumeist auf nationale Besonderheiten oder eine ungünstige geographische Ausgangsposition ${ }^{352}$ zurückzuführen. Dieses bestehende Versorgungssicherheitsniveau der Stromversorgung in den Mitgliedsstaaten wird durch die Umsetzung des Weiterentwicklungskonzeptes nicht beeinträchtigt. Im Gegenteil, das erklärte Ziel der bestehenden EVU ist der Ausbau der Versorgungssicherheit durch die gemeinsame Reservehaltung. Das Ziel ist jedoch nicht unkritisch zu sehen: Innerhalb der UCPTE ist ein Niveau erreicht worden, das wesentlich über dem des ehemaligen Ost-Netzes liegt. Bei den europäischen Verbrauchern ist das Bedürfnis nach einer erhöhten Versorgungssicher-

348 Siehe Kapitel 6.2.5.

$349 \mathrm{Zu}$ den Zielen vergleiche Kapitel 1.

350 Vgl. hierzu die Ausführungen im Kapitel 8.1 und 8.2.2 .

351 Diese wird in Kapitel 8 unter Berücksichtigung der beiden Faktoren erbracht.

352 Z.B. eine Insellage.

Rüdiger Hermann - 978-3-631-75129-9 
heit, bis auf wenige Ausnahmen an den Randlagen, nicht erkennbar. Mit den Großabnehmern sind spezielle Verträge zur Spannungshaltung und zum gezielten Lastabwurf bei einer Übernachfrage abgeschlossen worden. Ein weiterer Anstieg der Versorgungssicherheit scheint daher nicht nötig. Des weiteren steigen die Grenzkosten der Versorgungssicherheitsbereitstellung nach Ausschöpfung aller Vorteile durch die gemeinsame europäische Bereitstellung wieder steil an. Anstatt die Versorgungssicherheit in den Mitgliedsstaaten mit hohem Niveau weiter auszubauen, wäre es den EVU bei Verwirklichung des Konzeptes möglich, für das bestehende Niveau die Kosten durch die Reduzierung der Reservekapazitäten zu senken und damit das zweite Ziel des europäischen Strommarktes, die Kostensenkung, zu fördern.

Bei Verwirklichung des TPA-Konzeptes besteht, wie die Ausführungen zu den Mitteln des Konzeptes gezeigt haben, die Gefahr, daß die Unternehmen ihre Aufwendung zur Bereitstellung der Versorgungssicherheit reduzieren, um hierdurch Kosten zu sparen und die politisch Handelnden bei Ausfall der Stromversorgung von den scheinbar negativen Auswirkungen des Konzeptes zu überzeugen. Keine der drei vorgeschlagenen Lösungsmöglichkeiten ${ }^{353}$ ist problemlos; in jedem Fall ist die Bereitstellung der Versorgungssicherheit im Vergleich zu dem derzeitigen Verfahren im TPA-Konzept mit höheren Kosten verbunden. In diesem Bereich fördert die Umsetzung des TPA-Konzeptes nicht die Ziele der kostengünstigen und sicheren Stromversorgung in Europa. Die Versorgungssicherheit kann jedoch durch die aufgezeigten Verfahren gewährleistet werden.

\subsubsection{Ausblick : Preisentwicklung bei Umsetzung der Integrationskonzepte}

Die Preisfestsetzung für Abnehmer der Verbund-EVU unterliegt im Weiterentwicklungskonzept weiterhin dem "öffentlich" überwachten Diktat der Verbundunternehmen. Ein preisfestsetzender Wettbewerb um Endverbraucher findet nicht statt. Die gegebene Kostenbindung der Elektrizitätspreise an die "begründeten" Kosten der Versorgung bleibt bestehen. Aus diesem Grund kann von einem Preiswettbewerb bei Verwirklichung des Weiterentwicklungskonzeptes nicht gesprochen werden.

Wegen der beschriebenen geringen Kosteneinsparpotentiale durch die Implementierung des Weiterentwicklungskonzeptes können Preissenkungen durch gesunkene Kosten ebenfalls ausgeschlossen werden. Denkbare zukünttige Preissenkungen im nationalen Bereich werden nicht durch einen Preiswettbewerb bewirkt werden,

353 Zuweisung der Versorgungssicherheitsbereitstellung auf das Gebiets-EVU. Eigenverantwortlichkeit für die Versorgungssicherheit bei dem Lieferanten und externer Bezug als

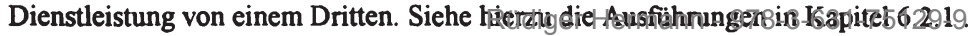


sondern können z.B. in sinkenden Rohstoffpreisen begründet sein. Hierdurch wird das Ziel des Binnenmarktes für Elektrizität, relativ sinkende Kosten und Preise zu erreichen, nicht erfullt.

Im TPA-Konzept kann ein Preiswettbewerb nach den Ausführungen zur Wettbewerbsintensität und zur Marktmacht ausschließlich temporär für Großabnehmer und Verteilerunternehmen stattfinden. Die gesamten Marktprozesse für eine begrenzte Anzahl von Abnehmern dieses Abnehmersegmentes hängen jedoch von den Durchleitungsverhinderungsmöglichkeiten und den Stromtransportgebühren ab. Deshalb kann entgegen den Aussagen einiger Autoren ${ }^{354}$ ohne die Betrachtung dieser beiden Faktoren keine Aussage über die Kosten- und Preisentwicklung im TPA-Konzept getroffen werden. Für die anderen Abnehmergruppen kann es zu keinem Preiswettbewerb und damit zu keiner Kosten- und Preisreduzierung kommen. Im Gegenteil: Die Masse der Kleinverbraucher wird sich auf Strompreissteigerungen einstellen müssen, wenn es den Regulierungsinstitutionen nicht gelingt, eine Quersubventionierung von Großabnehmern zu verhindern ${ }^{355}$. Ebenso können Preissteigerungen für die Kleinabnehmer entstehen, wenn die EVU die entgangenen Deckungsbeiträge von den Verbrauchern holen.

354 Vgl. z.B. : RIEMER, H.-W. : a.a.O., S. 1084 ff. sowie das Interview mit Dr. Dietmar Kuhnt in o.V.: Energiewirtschaft und Umweltschutz im EG-Binnenmarkt, ET 42. Jg. (1992), Heft 6, S. $346 \mathrm{ff}$.

$355 \mathrm{Ob}$ es sich hierbei um den Aufbau oder den Abbau von Quersubventionen handelt, soll nicht Gegenstand der weiteren Betrachtungen seiReüdiger Hermann - 978-3-631-75129-9 
Tabelle 14: Übersicht: Beurteilung Weiterentwicklungskonzept und TPAKonzept

\begin{tabular}{|c|c|c|}
\hline Kriterlen zur Beurteilung der Ordnungsmodelle & $\begin{array}{l}\text { erft } \\
\text { Weiterentw.- } \\
\text { konzept }\end{array}$ & IPA-Konzept \\
\hline Vertikale Integration aufgelöst? & Nein & Nein \\
\hline Stromhandelsinfrastruktur geschaffen? & Nein & Nein \\
\hline Teilmärkte geschaffen ? & Nein & Nein \\
\hline Marktzutrtttsbarieren reduziert? & Nein & Ja \\
\hline Wettbewerbsintensitöt gestelgert? & Nein & $\begin{array}{l}\text { Kene doschlos-s. } \\
\text { sende Aussogoe } \\
\text { modech }\end{array}$ \\
\hline Marktmacht einzelner Anbleter U.Nachfrager begrenzt ? & Nein & $\begin{array}{l}\text { Kone coschllos- } \\
\text { sende Aussogee } \\
\text { moolich }\end{array}$ \\
\hline Reduzlerung der Gesamtkosten der Elekttrztö̈tsversorgung? & Nein & $\begin{array}{l}\text { Kene abschlles- } \\
\text { sende Aussoge } \\
\text { mogilch }\end{array}$ \\
\hline Versorgungssicherheit gewährleistet? & $\mathrm{Ja}$ & Ja \\
\hline Ausbllick Prelsentwicklung: & $\begin{array}{l}\text { Unveränderte } \\
\text { Strompreise in } \\
\text { Europo }\end{array}$ & $\begin{array}{l}\text { Kenene abschlos. } \\
\text { sencoe Aussogoge } \\
\text { móglich }\end{array}$ \\
\hline
\end{tabular}


Rüdiger Hermann - 978-3-631-75129-9

Downloaded from PubFactory at 01/11/2019 07:57:52AM

via free access 


\section{Desintegrationskonzepte}

\subsection{Vorbemerkungen}

Die Idee zur Auflösung der integrierten EVU und Umwandlung in verschiedene Marktstufen wurde im europäischen Raum erstmals von GRÖNER ${ }^{356}$ als Konzept für die deutsche Elektrizitätswirtschaft entwickelt. GRÖNER versuchte durch sein Konzept, Wettbewerb in der Elektrizitätswirtschaft zu eröffnen. Er konzentrierte sich auf den Wettbewerb in der Stromerzeugung und skizzierte darauf aufbauend ein Bild eines desintegrierten Gesamtsektors. Der Netzgesellschaft wies er den überregionalen Stromtransport als Aufgabenbereich zu. Die erforderlichen Verträge sollten direkt zwischen Erzeugern und Verbrauchern zustande kommen. Die Steuerung des Gesamtsystems hat Gröner mit dem Hinweis, die Koordination werde der Marktmechanismus übernehmen, offen gelassen. Die einzige europäische Anwendung eines ähnlichen Konzeptes stellt das englisch-walisische Elektrizitätssystem dar.

Erste Ansätze zu einem desintegrierten Konzept für den europäischen Binnenmarkt kamen von EISS et al ${ }^{357}$ und PICK ${ }^{358}$. Sie klammerten jedoch wesentliche Elemente wie $z . B$. die Koordination ${ }^{359}$, den Umstrukturierungsproze $\beta^{360}$, die Betriebsgrößen und die Zuordnung der Versorgungssicherheit auf ein verantwortliches Unternehmen oder eine Institution aus. Ausgelassen wurde des weiteren, wie eine kostengünstige Durchmischung der Abnehmer durch Gestaltung der Versorgungsgebie$\mathrm{te}^{361}$ erreicht werden kann.

In den folgenden Ausführungen sollen als Erzeuger Unternehmen bezeichnet werden, die ausschließlich Strom produzieren und auf der noch zu beschreibenden Großhandelsstufe elektrische Energie anbieten; die Erzeugung endet an der Hochspannungskupplung des Kraftwerks zum Transportnetz. Der Transporteur, auch als Netzgesellschaft bezeichnet, unterhält und betreibt das Hochspannungsnetz zur interregionalen und überregionalen Stromübertragung. Es handelt sich innerhalb der EU überwiegend um das $380-\mathrm{kV}$ - und $220-\mathrm{kV}-\mathrm{Netz}$; in einigen ländlichen Gebieten werden vereinzelt zum überregionalen Stromtransport 110$\mathrm{kV}$-Leitungen verwandt. Die Verteilerunternehmen transportieren den Strom auf der lokalen Ebene zu den einzelnen Verbrauchern ${ }^{362}$. Kennzeichen von Vertei-

356 Vgl. GRONER, H. : Die Ordnung des ... a.a.O., S. $438 \mathrm{ff}$.

357 Vgl. EISS et al : Die Ordnung ..., a.a.O., S. 78 ff.

$358 \mathrm{Vgl}$. PICK, H. : Ordnungsrahmen ... , a.a.O.

359 Eigene Aussage von PICK, H. : Ordnungsrahmen ..., a.a.O., S. 129

$360 \mathrm{Vgl}$. hierzu die Ausführungen zur Implementierung der beiden Integrationskonzepte in den Kapiteln 6.1.6 und 6.2.6.

361 D.h., die Kundenzusammenstellung je Versorger.

362 Sehr viele Autoren unterscheiden Stromtransport und Stromverteilung über die Span-

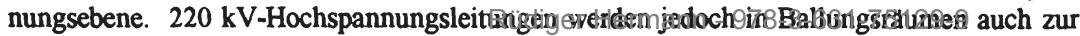


lerunternehmen ist der physikalische Stromtransport von der Hochspannungsübergabestation des Hochspannungsnetzbetreibers bis zum Anschluß des Letztabnehmers $^{363}$.

Überlegungen des Verfassers der vorliegenden Arbeit, ein desintegriertes Konzept ohne einen Pool oder einer anderen Institution zur Laststeuerung anzustreben, blieben erfolgslos. Die Besonderheiten der Elektrizitätsbranche, insbesondere die fehlende Speicherfähigkeit und die erforderliche Identität von einer schwankenden Entnahme mit der Stromproduktion stellten unüberwindliche Hindernisse dar. Ferner verhinderte die kurzfristig nicht steuerbare Entnahme im Kleinverbraucherbereich eine derartige Konzeption. Deshalb werden bei dem im Anschluß dargestellten ersten Desintegrationskonzept (Common-Carrier-Konzept) der Erzeugerpool und beim zweiten Konzept (Stromhändler-Konzept) die Netzgesellschaft als Koordinationsinstrument vorgesehen. Übernimmt die Netzgesellschaft die Aufgaben des Pools, so resultiert daraus ein Stromhandel der Netzgesellschaft ${ }^{364}$. Durch die Schaffung mehrerer Unternehmen für die Wertschöpfungskette der Elektrizitätsversorgung unterscheiden sich die beiden Konzepte von den zuvor dargestellten Integrationskonzepten.

\subsection{Das Common-Carrier-Konzept (3. Konzept)}

Ein Common-Carrier ist im Sinne des folgenden Konzeptes eine Netzgesellschaft, die für andere Strom durch ihr Netz transportiert. Die Netzgesellschaft selber handelt nicht mit elektrischer Energie.

stădtischen Verteilung eingesetzt, so z.B. in Paris. Die Spannungsstufe ist somit kein Kriterium für den Stromtransport. Eine geographische Festlegung, z.B. nach der Entfernung zwischen Erzeugung und Verbrauchsort, berücksichtigt nicht die Abnahmeverhältnisse. Beispielsweise kann eine betragsmäßig gleiche Entfernung im Ruhrgebiet als regionaler Stromtransport bezeichnet werden, im französichen Zentralmassiv aber der lokalen Verteilung zugeordnet werden. Aus diesen Gründen sollte die Zweckbestimmung als alleiniges Unterscheidungsmerkmal herangezogen werden.

363 Großabnehmer haben in der Regel einen direkten Anschluß an das Hochspannungsnetz. Sie benotigen daher weder die technische Infrastruktur noch die sonstigen Serviceleistungen des Verteilerunternehmens.

364 Vgl. auch PICK, H. : Ordnungsrahmen ... , a.a.O., S. 132 und EISS, H. ; LUKES, R. ; PICK, H.; SCHULZ, W. : Die Ordnung ... ,ailal.ge, I.186mann - 978-3-631-75129-9 


\subsubsection{Die Konzeption des Common-Carrier-Konzeptes}

\subsubsection{Gesamtmarkt und Erzeugung}

Ausgangspunkt des Konzeptes ist die Desintegration und die Einführung von Wettbewerb auf der Erzeugerseite der Elektrizitätsversorgung. Die gesamte Elektrizitätsversorgung wird in die Elektrizitätserzeugung, den -transport, die -verteilung und die Endabnehmerversorgung aufgegliedert. Damit wird der Wertschöpfungsprozeß vollständig integrierter EVU in einzelne Stufen unterteilt.

\section{Abbildung 20: Struktur vor der Umstrukturierung (Integrierte Unternehmen)}

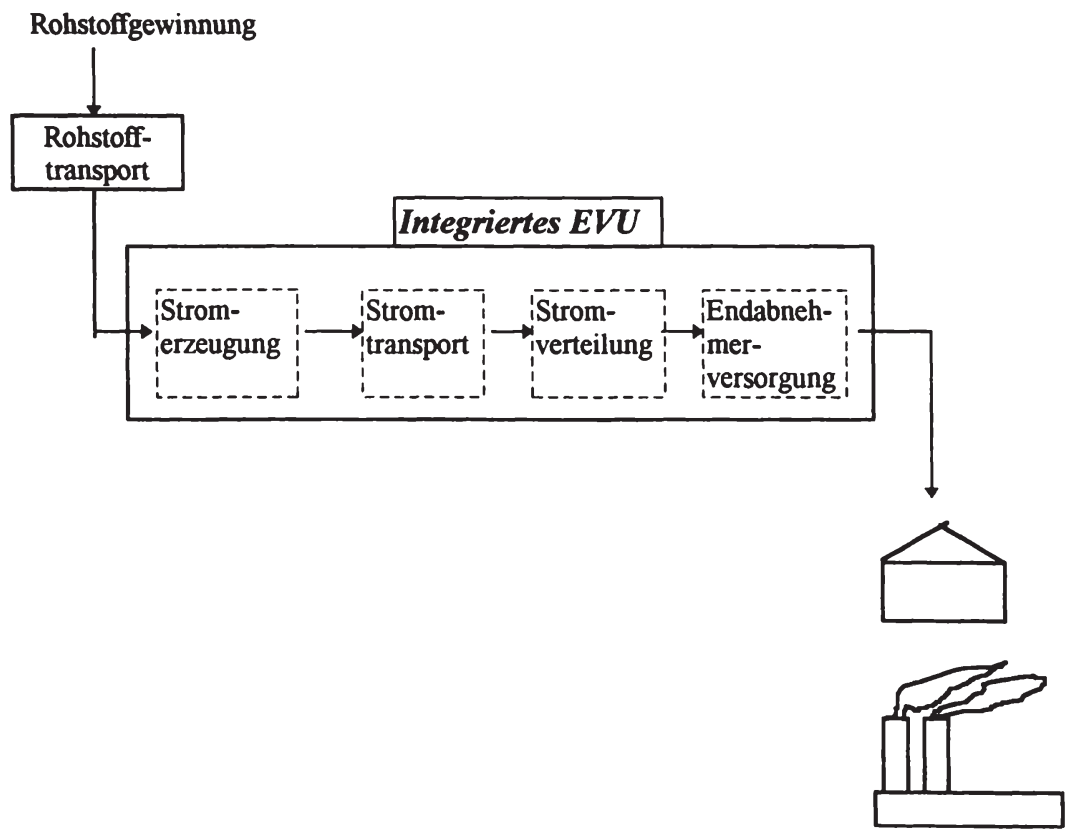




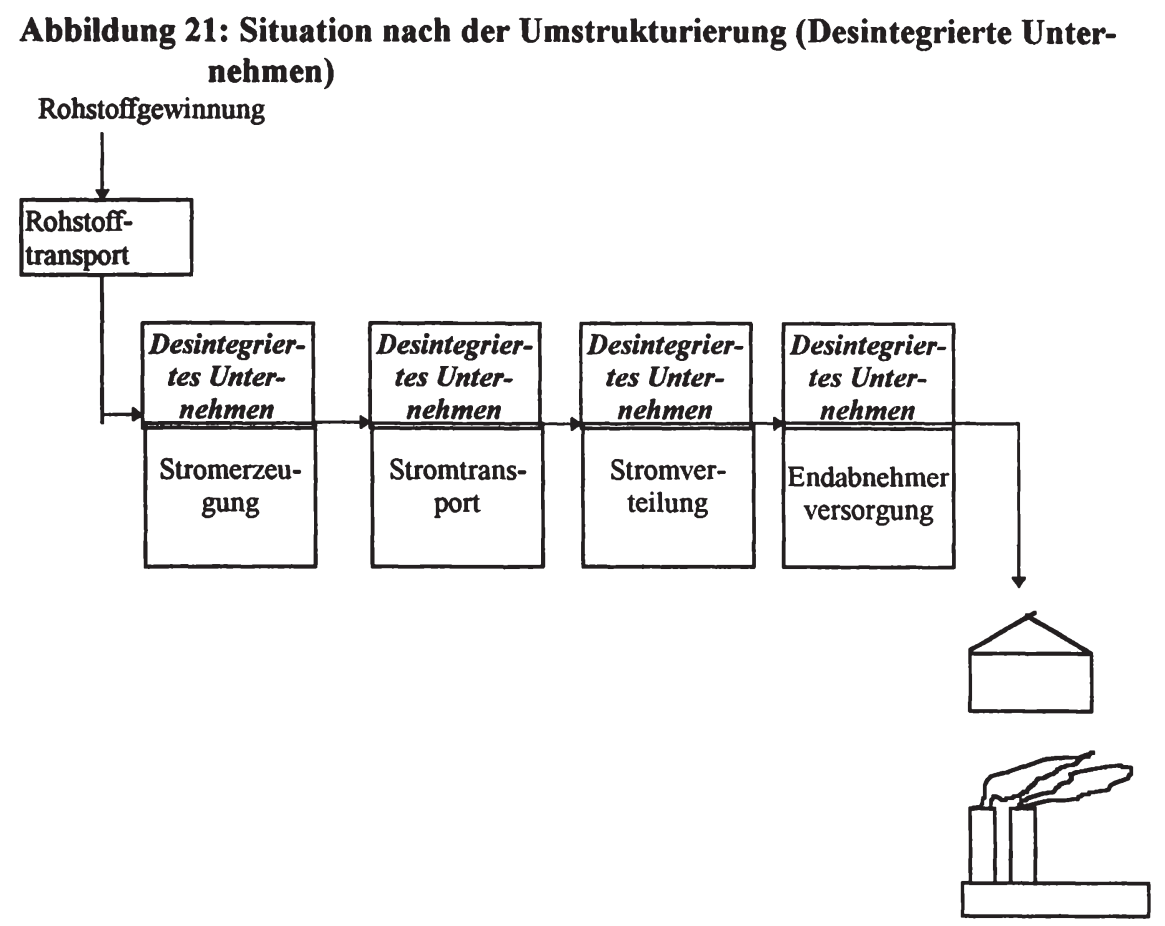

Auf jeder Stufe werden mehrere Unternehmen eingerichtet, die einen Anteil aus dem Gesamtwertschöpfungsprozeß übernehmen. Daraus folgt, daß in den Ländern, in denen die bestehende Elektrizitätsstruktur von der beabsichtigten abweicht, eine Aufteilung der existierenden Unternehmen erforderlich ist.

Nach der Aufspaltung der integrierten Unternehmen entstehen innerhalb des Binnenmarktes vier Teilmärkte:

a) der Elektrizitätsgroßhande ${ }^{365}$ zwischen Erzeugern und Verteilern sowie den Großverbrauchern,

b) der Markt für Stromtransportleistungen,

c) der Markt für Aushilfslieferungen (Reservehaltung und Zusatzstrom) ${ }^{366}$,

d) der Verbrauchermarkt zwischen Verteiler und Endabnehmer ${ }^{367}$.

365 Der Begriff wurde vom Verfasser so gewählt, weil er den Sachverhalt analog zu den anderen Sektoren treffend bezeichnet.

$366 \mathrm{Vgl}$. GRÖNER, H. : Grundzüge einer Wettbewerbsordnung für die Elektrizitătswirtschaft, in: RÖPER, B. : Wettbewerbsprobleme der Elektrizitătsversorgung - Der Begriff Monopolgrad, Berlin 1972, S. 47-69, hier S. 55 ff.

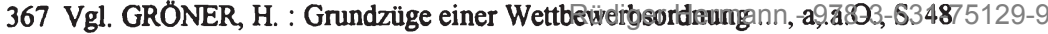


Abbildung 22: Märkte des Common-Carrier-Konzeptes

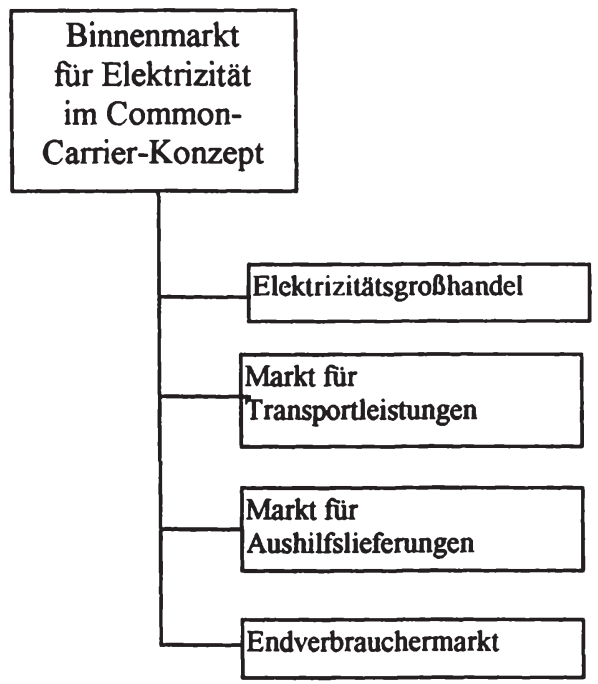

Der Elektrizitätsgroßhandel und der Markt für Aushilfslieferungen sollen über einen Pool in Zusammenarbeit mit der Netzgesellschaft abgewickelt werden. Die aus der Aufspaltung oder Umstrukturierung entstehenden Erzeuger sollen ausschließlich Strom produzieren ${ }^{368}$ und Handelsbeziehungen zum Verteiler oder Großabnehmer aufnehmen. Gleichzeitig sollten der Gebietsschutz aufgehoben und die bestehenden Ausschließlichkeitsrechte für die Stromerzeuger abgeschaff werden; in der Folge können dann weitere unabhängige Erzeuger entstehen.

Es stellt sich die Frage, welche Eigentumsverhältnisse und welche Betriebsgrößen die weiterbestehenden oder neugeschaffenen Erzeugerunternehmen aufweisen sollten.

\section{Eigentumsverhältnisse}

Die eine Elektrizitätsproduktion aufnehmenden unabhängigen Unternehmen sollten vollständig in privaten Händen liegen. Sie sind sowohl als Eigentümergesellschaften als auch als Kapitalgesellschaften denkbar. Öffentliche Gebietskörperschaften können sich als Aktionäre an den Erzeugern beteiligen, doch sollte ihr

368 Hiermit ist nicht gemeint, daß die Erzeuger nicht auch Fernwărme erzeugen können. Sie sollen lediglich in keinen anderen Teilbereiche der Wertschöpfungskette in der Elektrizitătsversorgung tătig werden. 
Anteil so niedrig sein, daß eine Beeinflussung der Unternehmensaktivitäten ausgeschlossen werden kann.

Neben den privaten unabhängigen Erzeugern können die aus der Desintegration resultierenden Unternehmen in Privateigentum überführt werden. Denkbar sind auch hier Unternehmen, an denen die jeweiligen Mitgliedsstaaten der EU beteiligt sind, die aber als eigenständige Unternehmen keinen Einflußnahmen nationaler Regierungen unterliegen. Hierdurch könnte verhindert werden, daß einzelne Regierungen direkt oder indirekt durch Subventionen den marktwirtschaftlichen Selektionsprozeß verfälschen.

\section{Betriebsgrößen}

Ein besonderes Problem bei der Umstrukturierung der bestehenden Märkte sind die Betriebsgrößen der Erzeugerunternehmen. Langfristig sollte sich die optimale Betriebsgröße durch den Marktprozeß bilden ${ }^{369}$. Trotzdem werden die integrierten Unternehmen bei Einführung des Konzeptes aufgeteilt werden müssen, wozu Vorstellungen in bezug auf eine günstige Ausgangslage für die erwarteten Marktprozesse erforderlich sind.

Theoretisch denkbar ist als kleinste Betriebsgröße die eines einzelnen Kraftwerks. Die Obergrenze wird durch die EU-Kartellrichtlinien festgelegt. Demnach wird die oberste Betriebsgröße durch die (theoretische) Annahme einer marktbeherrschenden Stellung festgelegt; diese ist jedoch abhängig von den Betriebsgrößen der anderen Marktteilnehmer. Gleichzeitig muß berücksichtigt werden, daß eine marktbeherrschende Stellung nicht auf den gesamten europäischen Elektrizitätsmarkt bezogen werden darf, sondern auf die relevanten geographisch-elektrizitätswirtschaftlichen Teilmärkte. Ein Erzeugerunternehmen mit beispielsweise 30\% der gesamten Erzeugungskapazität des Marktes hat, bezogen auf den Gesamtmarkt keine beherrschende Stellung, wenn mindestens ein anderes Unternehmen eine ähnliche Größe aufweist und weitere Anbieter geringerer Größe existieren. Konzentriert sich der Anteil auf eine europäische Region, so kann über die Stromtransportkosten ein regionales Monopol entstehen. Hieraus folgt für die Gestaltung der Betriebsgrößen bei der Umstrukturierung im Erzeugerbereich: Tendenziell sollten kleinere Betriebsgrößen gewählt werden, um zu verhindern, daß regionale Monopole entstehen. Der Verfasser schlägt Betriebsgrößen in dicht besiedelten Gebieten bis zu $10 \mathrm{GW}$ Spitzenleistung und in Gebieten mit einer niedrigen Abnehmerdichte bis zu $5 \mathrm{GW}$ vor $^{370}$. $5 \mathrm{GW}$ entsprechen einer Erzeugung von neun durchschnittlichen modernen Braun- oder Steinkohlekraftwerken oder drei bis fünf Kernkraft-

369 Hiervon geht Hagenmeyer aus. Vgl. HAGENMEYER, E. : Die Elektrizitătswirtschaft an der Jahrhundertwende ..., a.a.O., S. 1271

370 Das einfachste Kriterium zur Unterscheidung zwischen Gebieten mit hoher Abnahmedichte und mit niedriger Abnehmerdichte ist die Einwohnerzahl pro Quadratkilometer. Als niedrig sollte eine Abnehmerdichte unter 50 Einwohner pro Quadratkilometer angesehen werden und als dicht eine Abnehmerdichte von über50d/Abnehmen pro Qoäratdibmeter29-9 
werken $^{371}$. Eine Begrenzung unterhalb der $5 \mathrm{GW}$-Grenze hält der Verfasser nicht für sinnvoll, da die vorhandenen Kostendegressionsverläufe bei den bestehenden konventionellen Kraftwerken sonst nicht ausgenutzt werden können.

Werden bei der Aufteilung Abweichungen von der maximalen Betriebsgröße zugelassen, ist zu prüfen, ob diese in Hinblick auf die beabsichtigten Ziele sinnvoll sind. Dies ist regelmäßig immer dann zu bezweifeln, wenn ein Unternehmen durch die Abweichung von der Konzeption einen Wettbewerbsvorteil erlangt.

In Ländern mit bereits bestehenden eigenständigen Erzeugern ${ }^{372}$ sollte überprüft werden, ob diese der Konzeption, insbesondere hinsichtlich der Unabhängigkeit von staatlichen Stellen und den bestehenden Kapitalverflechtungen, entsprechen.

\subsubsection{Die Verteilung}

Die Aufgabe der Verteilerunternehmen ist der Transport elektrischer Energie von der Netzkupplung der Transportgesellschaft bis zum Endverbraucher. Eine weitere Aufgabe kann der Zwischenhandel mit Elektrizität sein; hieraus können zwei unterschiedliche Verteilerkonzeptionen entwickelt werden:

- Verteilerunternehmen übernehmen die Endabnehmerversorgung.

- Die Stromverteilung wird von der Endverbraucherversorgung getrennt.

(Wettbewerb in der Endverbraucherversorgung)

\subsection{Endabnehmerversorgung durch die Verteilerunternehmen}

Die Verteilerunternehmen erhalten die Versorgungspflicht für alle Endverbraucher ihres Netzes mit Ausnahme der Großabnehmer ${ }^{373}$. Als Gegenleistung erhalten sie ein räumlich und sachlich begrenztes Versorgungsmonopol. Gegenüber den Erzeugern treten sie als Nachfrager auf, die Strombezugsverträge abschließen und die bezogene Energie an den Endverbraucher zu bestimmten Preisen und Tarifen abgeben.

371 Vgl. INFORMATIONSZENTRALE DER ELEKTRIZITÄTSWIRTSCHAFT e.V. (Hrsg.) : StromBASISWISSEN : Stromerzeugung in Wärmekraftwerken, Nr. 105. S. 1-4 , Frankfurt/M. 1988 S. 4

372 Z. B. Großbritannien und Italien. Die in anderen Staaten bestehenden Erzeuger sind entweder direkter Bestandteil eines vertikal integrierten EVUs oder durch vertragliche Bindungen in ihrer Abnehmerwahl langfristig und ausschließlich gebunden.

373 Diese Konzeption ist auch in Australien gewăhlt worden. Vgl. SCHOLL, R. : Reform der Elektrizitătsindustrie in Australien - Das Beispiel der State Electricity Commission of Vic-

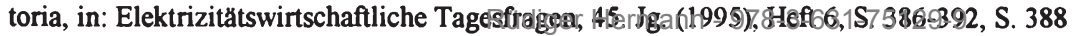


Ähnlich wie bei den Erzeugerunternehmen stellt sich auch bei den in vielen Mitgliedsländern bestehenden Verteilerunternehmen ${ }^{374}$ die Frage nach den Eigentumsverhältnissen und der Betriebsgröße.

\section{Eigentumsstrukturen}

Als Eigentümer der Verteilerunternehmen kommen Genossenschaften, öffentliche lokale Gebietskörperschaften und private Kapitalgesellschaften in Betracht. GRÖNER $^{375}$ ist der Meinung, daß sowohl genossenschaftliche Organisationen als auch Unternehmen in kommunalem Besitz als Eigentümer von Verteilerunternehmen geeignet sind. Bei kommunalen Unternehmen schlägt er weitergehend die Einführung eines Ausschreibungswettbewerbes um den Betrieb des Verteilungsnetzes vor. Zur Steigerung der Akzeptanz des Konzeptes können in diesem Bereich die nationalen Besonderheiten berücksichtigt werden. Berücksichtigt werden muß jedoch, daß genossenschaftliches Eigentum nur bei geringen Betriebsgrößen von den Eigentümer in der Realität kontrollierbar ist.

\section{Betriebsgrößen}

GRÖNER $^{376}$ schlägt bei der Schaffung der Verteilerunternehmen Betriebsgrößen mit einer Absatzobergrenze vor, die der Produktion eines Kraftwerks entspricht, ohne eine Leistungsgröße anzugeben. Mit einer solchen Betriebsgrößenobergrenze wäre die Nachfragemacht der Verteiler auf der Großhandelsebene begrenzt $\mathrm{t}^{377}$. Die Regulierung der Verteilungsmonopole würde durch die niedrige Betriebsgröße erleichtert werden, weil kleinere Unternehmen schlechtere Möglichkeiten haben, ihre tatsächliche Ertragslage zu verschleiern. Eventuell auch bei kleineren Unternehmen anfallende Monopolrenten würden, wenn überhaupt, nur langfristig ausreichen, um Konzentrationsprozesse durch Unternehmensaufkäufe einzuleiten.

Nach der Meinung des Verfassers sollte die maximale Betriebsgröße eines Verteilerunternehmen durch seine Spitzenleistungsabnahme ${ }^{378}$ festgelegt werden. Die Spitzenleistungsabnahme eines Verteilerunternehmens nach der Aufteilung, sollte nicht höher sein als die Spitzenleistungsproduktion eines Erzeugers, um die Nachfragemacht des Monopolunternehmens zu begrenzen, also bei ca. $5 \mathrm{GW}$.

Welche Betriebsgrößen zwischen diesen beiden Polen für die Verteilergesellschaften gewählt werden, ist fur die Marktprozesse auf der Großhandelsebene unbedeu-

374 Z.B. in Großbritannien, der Bundesrepublik und Frankreich.

375 Vgl. GRÖNER, H. : Die Ordnung des ... a.a.O., S. 438

376 Vgl. GRÖNER, H. : Die Ordnung des ... a.a.O., S. 438

377 Außerdem wird die tatsăchliche Betriebsgröße hierdurch nicht festgelegt, da Kraftwerksgrößen je nach Technologie und Marktstruktur sowie der historischen Entwicklung variieren.

378 Die Spitzenleistungsabnahme ist die höchste Leistungsentnahme aus dem Netz des Stromtransporteurs in einem Jahr. 
tend. Die Betriebsgrößen der Verteiler haben jedoch Auswirkungen auf deren Effizienz ${ }^{379}$. Denkbar wären darüber hinaus unterschiedliche Betriebsgrößen der Verteilerunternehmen, bei denen nationale Besonderheiten berücksichtigt werden könnten.

Für den Verteilungsbereich haben die bestehenden EVU mehrfach auf die Gefahren einer mit dem Konzept verbundenen fehlenden Abnehmerdurchmischung hingewiesen, insbesondere, wenn Großabnehmer aus Versorgungsgebieten „herausgebrochen" werden. Fraglich ist, ob die Abnehmerdurchmischung überhaupt erforderlich ist ${ }^{380}$. Begründet wird die Forderung nach einer Durchmischung der Abnehmer mit den Vorteilen im Erzeugerbereich, die aus den reduzierten Lastschwankungen entstehen ${ }^{381}$. Bei kleinen Betriebsgrößen der Verteiler und nicht dem Anschlußzwang unterliegenden Großverbrauchern wird die Durchmischung nicht im geschlossenen Versorgungsgebiet des Verteilers erreicht, sondern erst im Transportnetz. Für den Erzeugerbereich besteht hierin kein Unterschied. Trotzdem weist die Durchmischung Vorteile auf: Die Entnahme der Verteiler aus dem Transportnetz ist kontinuierlich, wodurch die Abrechnung vereinfacht wird und die technischen Installationen geringer dimensioniert werden müssen. Zudem wird die Lastkoordination dezentral von den Verteilerunternehmen vorgenommen, wodurch Transaktionskosten bei der Koordination durch den Großhandelsmarkt eingespart werden können. Die Abnehmerdurchmischung weist jedoch auch die Gefahr der Quersubventionierung zwischen den Abnehmergruppen auf; diese Gefahr entsteht bei einem Versorgungsgebiet mit jeweils einem Verteilungsunternehmen pro Abnehmergruppe nicht. Ferner fehlt die Abnehmerdurchmischung wie sie von der deutschen Elektrizitätswirtschaft verstanden wird in ländlichen, nicht oder gering industrialisierten Gebieten bei lokalen Verteilerunternehmen bereits heute. Eine Bewertung, ob durchmischte oder nach Abnehmergruppen getrennte Versorgungsgebiete im Verteilerbereich besser sind, ist ohne eine weitergehende Analyse nicht möglich ${ }^{382}$. Durch eine mangelnde oder suboptimale Durchmischung werden die Stromlieferungen und die Versorgungssicherheit nicht eingeschränkt. Aus diesem Grund sollte bei Zielkonflikten zwischen der marktadäquaten Betriebsgröße und der Abnehmerdurchmischung der Betriebsgröße Vorrang eingeräumt werden.

379 Hierzu liegen bisher keine empirischen Untersuchungen vor.

380 Die Deregulierungskommission verneint dies. Vgl. hierzu DEREGULIERUNGSKOMMISSION : a.a.O., S. 5 ff.

381 Vgl. VDEW: Der europăische Strommarkt, a.a.O., S. 21

382 Dies sollte an dieser Stelle nicht GegenstandddereUntensuohungseinß-631-75129-9 
7.2.1.2.2 Wettbewerb in der Endverbraucherversorgung

Eine andere Konzeption der Verteilerseite wird erforderlich, wenn langfristig Wettbewerb um jeden Endverbraucher eingeführt werden soll. Wettbewerb um jeden Letztabnehmer schließt die Existenz geschlossener Versorgungsgebiete im Endverbraucherbereich aus; der Endverbraucher muß die Möglichkeit besitzen, das Leitungsnetz des Verteilerunternehmens gegen ein Entgelt zu benutzen.

Der Wettbewerb um die Endabnehmer kann durch ein einstufiges Elektrizitätsversorgungssystem ohne Verteilerunternehmen eingefüht werden:

\section{Abbildung 23: Endabnehmerversorgung durch Erzeuger}

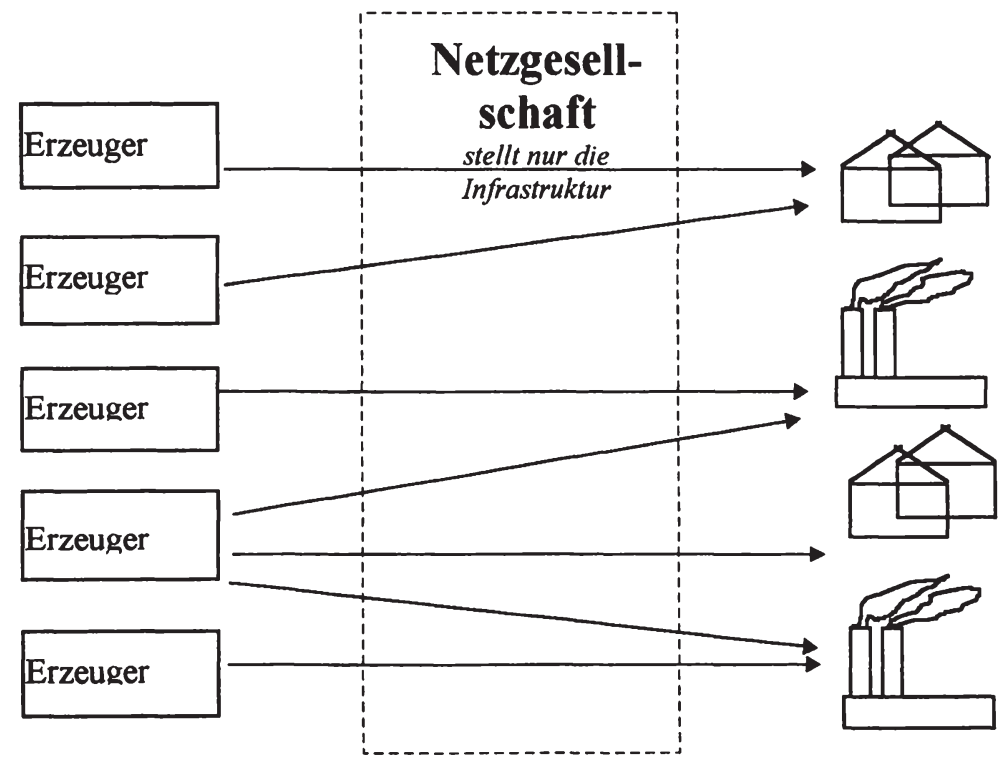

Die Netzgesellschaft übernimmt neben dem überregionalen und regionalen Stromtransport die Verteilung und kann somit die einzelnen Erzeuger direkt mit den Endverbrauchern verbinden. Die Verträge kommen zwischen Endverbraucher und Erzeuger zustande. Der Vorteil des Systems ist die gute konzeptionelle Überschaubarkeit und die wenigen Handelsstufen. Es weist jedoch auch Nachteile auf: 
a) Die einzelnen Erzeuger müssen eine Vielzahl von Verträgen mit kleinen Abnehmern schließen; ohne eine Standardisierung steigen die Transaktionskosten beträchtlich.

b) Die Erzeuger müssen bei den Vertragsabschlüssen auf eine geeignete Durchmischung der Abnehmer achten; die Informationen über das kumulierte Abnahmeverhalten verschiedener Abnehmer ist aber erst nach einer Testphase zu erhalten.

c) Von seiten der Endverbraucher, insbesondere der Masse der Klein- und Haushaltsabnehmer, müssen ebenfalls hohe Suchkosten aufgewendet werden, um den für sie günstigsten Anbieter herauszufinden. Zudem muß die erforderliche Infrastrukturbereitstellung mit der Netzgesellschaft für jeden Einzelfall geprüft werden.

Möglich ist auch die Einfuihrung von Versorgungsunternehmen (vgl. Abb. 25) ohne und mit der Existenz von Verteilerunternehmen. Als Versorger sollen Unternehmen bezeichnet werden, die als alleinige Vertragspartner der Endverbraucher für diese die benötigten Stromleistungen und die zur Lieferungen erforderlichen Leistungen entweder selbst erstellen oder auf dem europäischen Strommarkt beschaffen. Dem Geschäft muß daher kein tatsächlicher Stromfluß zugrunde liegen.

Darüber hinaus übernimmt der Versorger die Vertragsgestaltung, bündelt die Last und achtet auf eine Durchmischung. Bei einer Konzeption mit Verteilerunternehmen ist eine Bedingung für das Funktionieren des Systems die Durchleitungsverpflichtung für Verteiler.

Als Versorgungsunternehmen kommen die Erzeugungsunternehmen sowie die Verteilergesellschaften und unabhängige Anbieter in Frage. Während die Übernahme der Versorgung durch die Erzeuger wettbewerblich unproblematisch ist, entsteht bei den Verteilerunternehmen eine dem TPA-Konzept ähnliche Konstellation: Das einzelne Unternehmen wird bestrebt sein, die an das eigene Verteilungsnetz angeschlossenen Endabnehmer zu beliefern und den Absatz anderer Versorger zu behindern. Dies wäre ein Schritt in Richtung einer Reintegration. Vermieden werden kann diese Situation, wenn die Verteilergesellschaft bei Aufnahme des Versorgungsgeschäftes die Auflage bekommt, mit keinem Endabnehmer des eigenen Versorgungsgebietes Stromlieferverträge abzuschließen. Es kann jedoch bezweifelt werden, ob Verteiler dann noch ein Interesse daran haben, als Versorger tätig zu werden. Denkbar sind auch unabhängige, bisher nicht in der Elektrizitätsversorgung tätige Unternehmen, die auf dem geschaffenen Markt agieren. 


\section{Abbildung 24: Endabnehmerversorgung durch spezielle Versorgungsunter- nehmen}

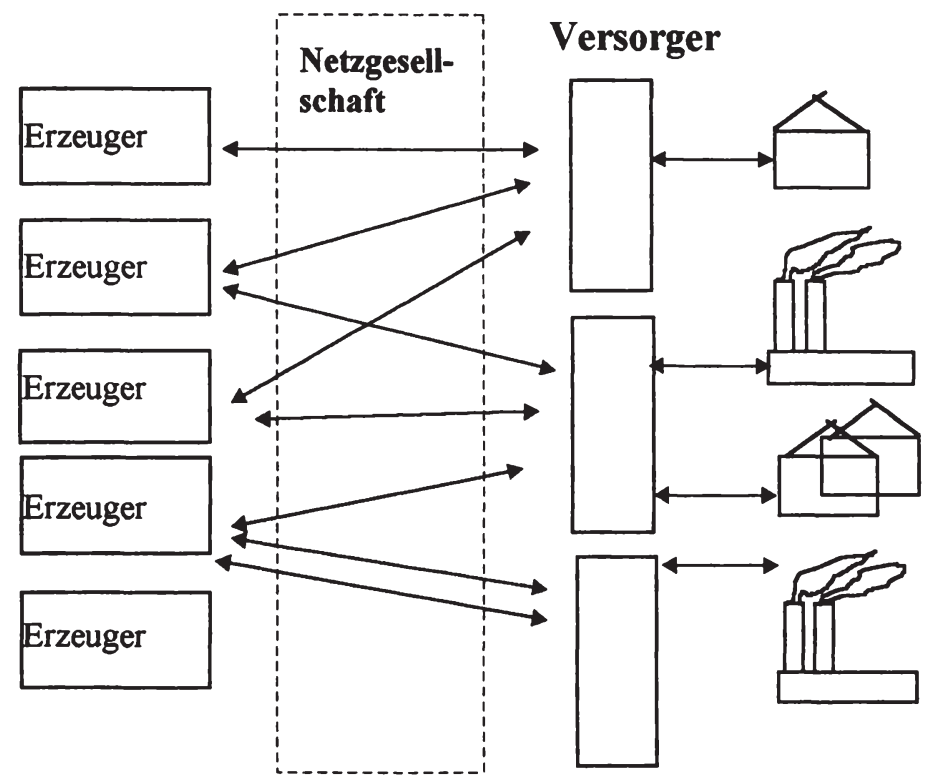

Ein weiteres Problemfeld der Desintegration ist die Versorgungspflicht. Wie aus der europäischen Diskussion geschlossen werden kann, ist die Aufhebung der Versorgungspflicht für die Kleinabnehmer nicht durchsetzbar und politisch nicht gewollt. Deshalb müssen Versorgungsgebiete geschaffen werden, in denen ein Unternehmen die Anschlußpflicht übernimmt. Hierfür wird das Unternehmen eine Kompensation verlangen. Für deren Festlegung und Kontrolle ist eine mit beträchtlichen Aufwand verbundene Regulierung erforderlich.

Unter Abwägung der Zusammenhänge schlägt der Verfasser die Endabnehmerversorgung für Kleinabnehmer durch die Verteilerunternehmen vor. Die in diesem Marktsegment mit einem Versorgungsmonopol ausgestatteten Verteilerunternehmen sollen die Anschlußpflicht für alle Kleinabnehmer auferlegt bekommen. Dieser primäre Verzicht auf die Einführung eines Wettbewerbs in der Endverbraucherbelieferung reduziert die Transaktions- und Regulierungskosten und vereinfacht die Implementierungsphasen des Common-Carrier-Konzeptes. Darüber hinaus wird die Akzeptanz des Konzeptes durch den Verzicht erhöht. Nach Beendigung der Implementierung des Konzeptes und dessen Bewährung kann dann über die Ein- 
führung des Wettbewerbs in der Endverbraucherversorgung für Kleinabnehmer nachgedacht werden.

Abbildung 25: Endabnehmerversorgung durch spezielle Versorgungsunternehmen bei Existenz von Verteilern

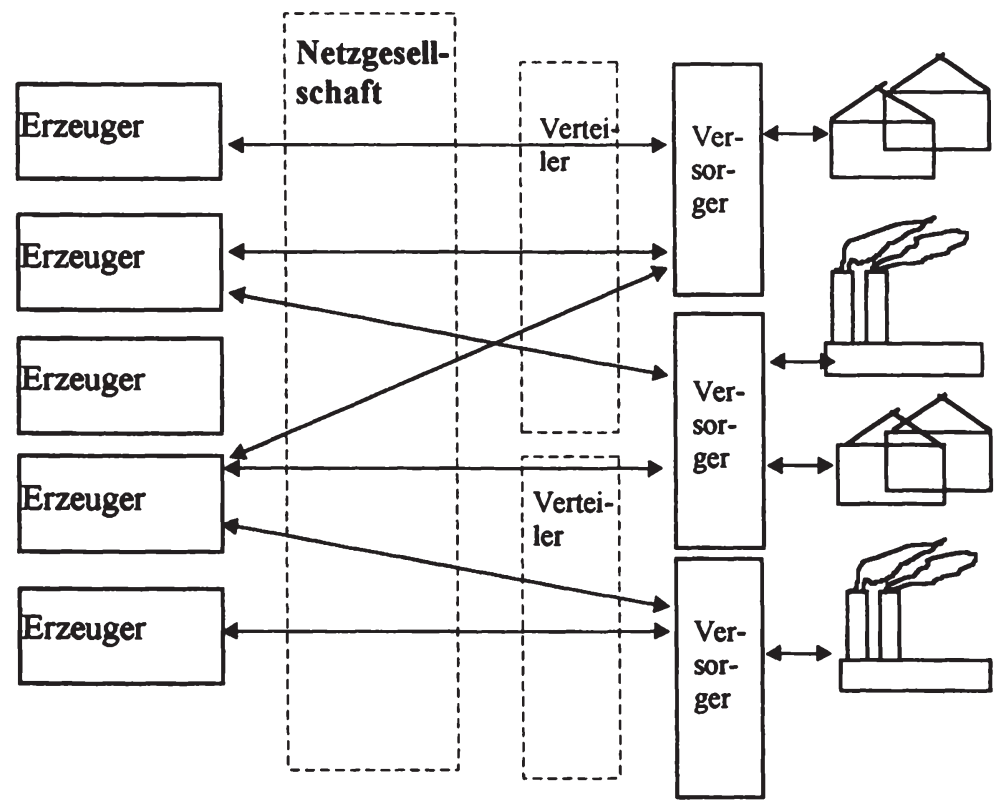

\subsubsection{Die Netzgesellschaft}

Aufgabe der Netzgesellschaft ist es, Stromerzeuger und -verteiler bzw. Großabnehmer physikalisch miteinander $\mathrm{zu}$ verbinden ${ }^{383}$. $\mathrm{Zu}$ diesem Zweck unterhält und betreibt sie ein 380- und 220- kV Leitungsnetz für den regionalen und überregionalen Stromtransport und ist nach EISS et $a^{384}$ als reiner Dienstleister tätig. Außer

383 Die detaillierten Aufgaben der Betriebsführung eines Stromnetzes zeigt die Graphik auf S. 11 in DVG: Jahresbericht 1993, Heidelberg 1994, S. 11

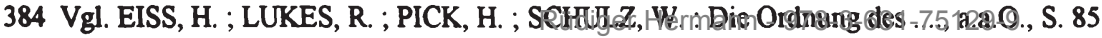


zur Reservehaltung für den Netzbetrieb ${ }^{385}$ sollte sich die Netzgesellschaft nicht an der Stromerzeugung und -verteilung beteiligen. Die Stromerzeugung der Netzgesellschaft kann den Betrieb von Spitzenlastkraftwerken wie z.B. Pumpspeicherkraftwerke umfassen, mit denen in außergewöhnlichen Betriebssituationen das Netz stabilisiert werden kann. Die Netzgesellschaft kann diese Reservehaltungskapazitäten jedoch auch von einem anderen Anbieter bereitstellen lassen. Sie sollte jedoch immer die technischen Bedingungen zum Anschluß von Kraftwerken und Verteilern für das Leitungsnetz festlegen ${ }^{386}$. Ebenso muß sie beim Vertragsabschluß zwischen Erzeugern und Abnehmern beteiligt werden. Ihre vorhandenen Kapazitäten bilden für die Vertragspartner eine Restriktion. Des weiteren sollte die Netzgesellschaft für die Bereitstellung der erforderlichen Infrastruktur zum Stromhandel über die Grenzen der EU zuständig sein. Für die erbrachten Stromtransportleistungen erhält die Netzgesellschaft von den Nutzern ein angemessenes Entgelt ${ }^{387}$.

Ähnlich wie bei den Verteilerunternehmen stellen sich auch im Stromtransportbereich die Fragen, welche Anzahl an Netzgesellschaften mit welcher Betriebsgröße in Europa geschaffen werden und welche Eigentümer die so entstandenen Unternehmen aufweisen sollen.

\section{Anzahl und Betriebsgrößen}

\section{a) Europaweite Netzgesellschaft}

Denkbar ist die Schaffung einer einzigen EU-weiten Netzgesellschaft, die die beschriebenen Aufgaben in allen Mitgliedsländern übernimmt und als Zusammenschluß der nationalen Stromtransporteure entsteht. Die Vorteile einer solchen Gesellschaft bestehen in der Vereinheitlichung des Netzbetriebes und der Anschlußnormung sowie den positiven Auswirkungen für die Integration der nationalen Elektrizitätswirtschaften zu einer einzigen europäischen. Eine einzige Netzgesellschaft kann am schnellsten die bestehenden Engstellen für den überregionalen Stromaustausch, die aus den alten Versorgungsgebietsgrenzen resultieren, abbauen und die nationalen Stromtransportnetze zu einem europäischen zusammenfügen. Durch diese Konzeption wird der Infrastrukturcharakter des Stromtransportnetzes betont.

Der Nachteil einer einzigen Netzgesellschaft liegt in ihrer Größe und den damit verbundenen Problemen für ein effizientes Management, zumal bei einer Institution dieser Größe, die Bürokratisierung immer negative Auswirkungen auf die Effizienz hat. Des weiteren fällt der vergleichende Wettbewerb in dem weiterhin mit einem

385 Vgl. zu den Aufgaben der Betriebsführung bei Stromnetzen DVG: Jahresbericht 1993, Heidelberg 1994, S. 11

386 Vgl. PICK, H. : Ordnungsmodelle ..., a.a.O., S. 159

387 Zur Stromtransportentgeltproblematik vgl. Kapitele8Hermann - 978-3-631-75129-9 
Monopol ausgestatteten Stromtransportbereich weg, der Aufschlüsse über Effizienzverluste beim Betrieb zulassen würde. Zudem ist zu erwarten, daß eine einzige europäische Netzgesellschaft auf eine geringere Akzeptanz treffen würde als mehrere kleinere Netzgesellschaften.

\section{b). Mehrere regionale Netzgesellschaften}

Die Schaffung mehrerer Netzgesellschaften hat den Vorteil, daß regionale und nationale Besonderheiten hinsichtlich der Laststruktur und des vorhandenen Netzes besser berücksichtigt werden können. Des weiteren wird die Kontrolle der Unternehmen durch die geringeren Betriebsgrößen erleichtert und gleichzeitig die Akzeptanz erhöht. Nachteilig wirken sich die erforderlichen Aufwendungen für Abrechnungen zwischen den Netzgesellschaften und der Koordinationsbedarf für übergreifende Stromlieferungen aus.

Eine Abschätzung, ob die erforderliche Koordination zwischen den Netzgesellschaften günstiger innerhalb eines Unternehmens geleistet werden kann, müßte durch eine genaue Analyse erbracht werden, die aber in das Problem der fehlenden empirischen Erhebungsmöglichkeiten hineinläuft. In Anbetracht der Probleme in großen Monopolgesellschaften neigt der Verfasser dazu, dezentrale Strukturen vorzuziehen. Dies impliziert die Frage nach der Aufteilung des Gemeinschaftsgebietes auf die Netzgesellschaften.

Abbildung 26: Gliederungskriterien für die Aufteilung der Netzgesellschaften

\section{Mögliche Gliederungskriterien bei mehreren Netzgesellschaften}

1. Geographische Kriterien

2. Elektrizitätswirtschaftliche Kennzahlen

3. Bestehende Versorgungsgebiete

\section{Geographische Kriterien}

Geographisch zusammenhängende Teile der EU könnten innerhalb einer Netzgesellschaft zusammengefaßt werden. Während diese Aufgliederung auf der Iberischen Halbinsel und in Skandinavien einfach umzusetzen ist, wird die Grenzziehung in Mitteleuropa schwieriger, da keine klaren geographischen Einheiten existieren. Zudem berücksichtigen geographische Kriterien nicht die elektrizitäts- 
wirtschaftlichen Gegebenheiten wie die Abnehmerdichte und Abnehmerstruktur sowie die Verteilung der Erzeuger.

\section{Elektrizitätswirtschaftliche Kennzahlen}

Als geeignete elektrizitätswirtschaftliche Größen können hierzu die Abnehmerzahl und die Abnahmestruktur herangezogen werden. Diese Gliederungskriterien haben den Vorteil, daß gleiche Marktgrößen mit Unternehmen gleicher wirtschaftlicher Bedeutung geschaffen werden, zwischen denen ein vergleichender Wettbewerb am ehesten möglich wäre. Die erforderliche Regulierung kann standardisiert und damit vereinfacht werden. Die Folge dieser Aufteilung ist eine unterschiedliche geographische Netzgebietsgröße: In dicht besiedelten oder/und industrialisierten Gebieten Europas entstehen geographisch kleine Netzgesellschaftsbereiche, während in ländlichen, dünn besiedelten Regionen eine Netzgesellschaft für ein geographisch großes Gebiet zuständig wäre.

\section{Bestehende Versorgungsgebiete}

Als weiteres Gliederungskriterium kommen die bestehenden nationalen Versorgungsgebiete in Betracht. Dies hätte zur Folge, daß die geographische Ausdehnung, die Leitungslänge und die Abnahmestruktur zwischen den Netzgesellschaften stark variieren würde. Nachteilig ist der geringe Integrationsbeitrag einer solchen Netzbereichsgliederung zu sehen, der bestehende Engpässe im Stromtransport innerhalb der EU nicht zwangsläufig aufhebt, und die beabsichtigte Integration des europäischen Strommarktes nach elektrizitätswirtschaftlichen Kriterien damit ausgeschließen würde.

Welche Gliederungskriterien sollten nun zur Aufteilung des EU-Gebietes herangezogen werden? Für das Common-Carrier-Konzept ist das erste und das dritte Gliederungskriterium ungeeignet, da die beiden Kriterien unterschiedliche Betriebsgrößen bei den Netzgesellschaften schaffen, ohne sicherzustellen, ob die geschaffenen Unternehmen ihre Aufgaben im Common-Carrier-Konzept und fur die Integration des Gesamtmarktes erfüllen können. Damit verbleiben die elektrizitätswirtschaftlichen Kennzahlen als geeignete Gliederungskriterien. Trotzdem ist damit die Anzahl noch nicht festgelegt: Die bereits angesprochenen Nachteile großer Netzgesellschaften, wie die negativen Folgen von Bürokratisierung, mangelnde Kontrolle und die Gefahr der Ausnutzung des Monopols lassen drei oder vier europäische Netzgesellschaften als nicht zielkonform erscheinen. Im Gegensatz dazu entstehen bei kleinen Netzgesellschaften mit wenigen angeschlossenen Erzeugern und einer geringen Abnehmerzahl hohe Kosten für das Lastmanagement und die Reservehaltung. Hieraus resultieren bezogen auf die gesamte EU Wohlfahrtsverluste. Der Verfasser schlägt daher für die Netzgesellschaft eine Mindestgröße von $15 \mathrm{GW}$ Spitzenanschlußleistung seitens der Erzeuger bei mindestens drei Kraftwerkstypen und den damit verbundenen Primärenergieträgern sowie eines_Minglesțkraftoverks- 
anzahl von 20 vor. $15 \mathrm{GW}$ entsprechen 11-12 modernen Kernkraftwerken oder 21 Kohlekraftwerken $^{388}$. Die Anzahl der Kraftwerke ist erforderlich, um den Ausfall eines einzelnen Kraftwerkes durch Produktionsausdehnung der anderen Kraftwerke und einen geordneten Lastabwurf ausgleichen zu können und gleichzeitig ein flexibles Lastmanagement zu ermöglichen.

Schwerer als die Mindestgröße ist die oberste Betriebsgröße für die Netzgesellschaften festzulegen. Der Verfasser schlägt hierfür max. acht europäische Netzgesellschaften vor, damit eine Regulierung der Netzgesellschaften möglich ist und Effizienzverluste aus „Mammut"-Organisationen begrenzt werden.

\section{Eigentumsform}

Als Eigentumsform für die Netzgesellschaft kommen die gleichen Alternativen wie für die Verteilergesellschaften in Betracht:

- privates Eigentum in Form einer Kapitalgesellschaft,

- staatlicher Besitz,

- öffentliche Unternehmen, bei denen der Staat alleiniger Anteilseigner ist $^{389}$ oder die Anteilsmehrheit hält,

- genossenschaftiche Unternehmen der Verteiler und/oder Erzeugerunternehmen ${ }^{390}$.

Dem Verfasser erscheint eine Kapitalgesellschaft, deren Aktien zu einem Drittel den Erzeugern, zu einem Drittel dem Verteiler und zu einem Drittel der öffentlichen Hand gehören, geeignet, die Aufgabenerfüllung und den Interessenausgleich zu gewährleisten.

\subsubsection{Die Mittel des Common-Carrier-Konzeptes}

Zentrales Mittel des Common-Carrier-Konzeptes, um die Desintegration zu ermöglichen und Märkte entstehen zu lassen, ist der Aufbau eines Pools. Der Pool übernimmt zwischen den Erzeugern und der Netzgesellschaft das Lastmanagement und die Bereitstellung eines Teiles der Versorgungssicherheit des Gesamtversor-

$388 \mathrm{Vgl}$. zu den aktuellen Betriebsgroßen in der Stromerzeugung BÖRNER, B. : Frankreichstrom nach Deutschland? Baden-Baden 1990, S. 60

389 Diese Eigentumsform wurde in Victoria, Australien, für die geschaffene Netzgesellschaft gewăhlt. Vgl. hierzu SCHOLL, R. : a.a.O., S. 386

390 Zur Umwandlung staatlichen Eigentums in privates bei Mărkten mit unvollstăndigen Informationen siehe DEMOUGIN, D. ; SINN, H.-W. : Privatisation, Risk-taking and the Com-

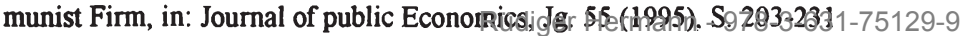


gungssystems. Ziel des Pools ist es, die Gesamtkosten der Elektrizitätsversorgung zu minimieren und die erforderliche Koordination zu übernehmen. Mitglieder sollten Erzeuger ab einer Mindestgröße ${ }^{391}$ werden. Als weitere Mitglieder des Pools kommen die Verteilerunternehmen und Großabnehmer mit direkter Zugangsmöglichkeit zu den Erzeugern in Betracht. An der Laststeuerung sollten nur die großen Einspeiser, z.B. ab einer Größe von 100 MW, teilnehmen.

\section{Abbildung 27: Konzeption des Pools im Common-Carrier-Konzept}

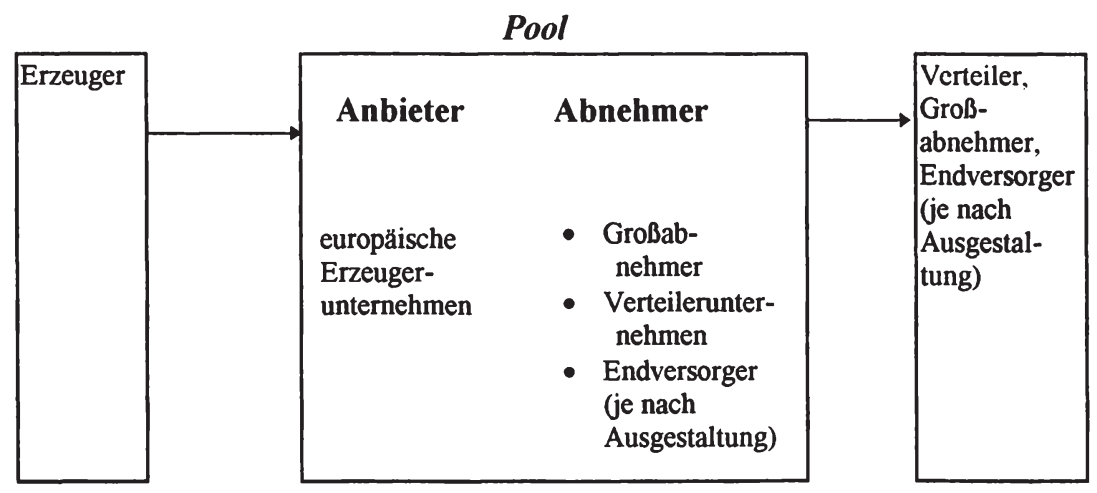

Den Transaktionen entspricht kein tatsächlicher Stromfluß3392 (vgl. Abb. 28).

Die Arbeit des Pools gliedert sich in drei Tätigkeitsfelder:

a) Der Spotmarkt für kurzfristige Lieferverträge,

b) Die Abwicklung der langfristigen Lieferverträge,

c) Das Lastmanagement und die Bereitstellung der Versorgungssicherheit.

\subsubsection{Der Spotmarkt}

Der Spotmarkt innerhalb des Erzeugerpools funktioniert ähnlich wie eine Börse ${ }^{393}$. Die Erzeuger mit freien Kapazitäten melden an die Poolleitung Angebotspreise und 
dazugehörige Stromleistungen, die Verfügbarkeit ihrer Kraftwerkskapazitäten und einen Preis für eine eventuelle Reservehaltung. Hinzu kommt die Zustandsmeldung der einzelnen Kraftwerke ${ }^{394}$. Die Meldungen und die Preis-Mengen-Kombinationen der Erzeuger müssen zu einer bestimmten Zeit am Vortag beim Pool abgegeben werden ${ }^{395}$. Bei der Zustandsmeldung geben die Erzeuger an, welche Kraftwerke "in Betrieb", "außer Betrieb" oder "kurzfristig anfahrbar" sind.

Die Verteilerumternehmen geben ihre kurzfristigen Strombedarfe bei der Poolgesellschaft auf. Wie soll nun die Zuordnung zwischen Erzeugern und Nachfragern durchgeführt werden, und mit welchen Preisen müssen die Stromlieferungen bezahlt werden?

\section{Abbildung 28: Stromfluß im Common-Carrier-Konzept}

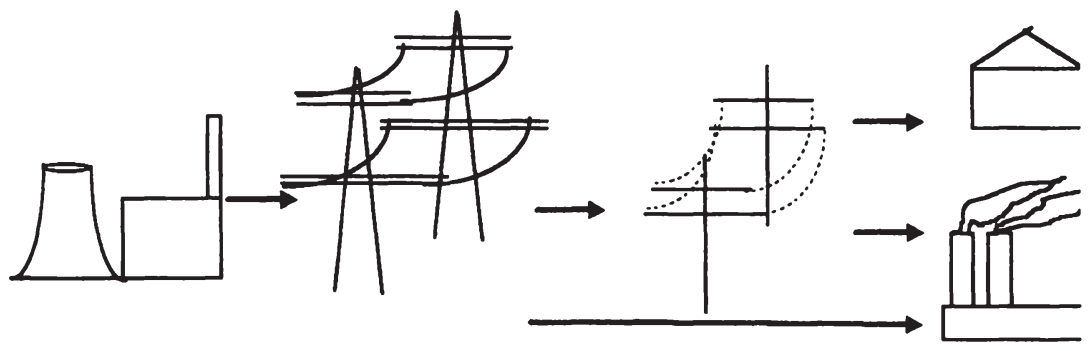

Kraftwerk

Hochspannungsnetz Mittelspannungsnetz

Verbrauch

Eine Zuordnung der Erzeuger zu den Nachfragern nach Kriterien ist nicht durchführbar, da ein preis- und mengendifferenziertes Angebot einer rein mengendifferenzierten Nachfrage gegenüber steht. Eine in dieser Situation zielkonforme $\mathrm{Zu}$ ordnungsregel ist dem Verfasser nicht bekannt. Deshalb muß für den Spotmarkt eine andere Lösung gefunden werden ${ }^{396}$ :

Erzeuger werden nicht direkt bestimmten Nachfragern zugeordnet. Der Pool kauft die angezeigte Nachfrage zu jedem Zeitpunkt von den Erzeugern und gibt die

393 Auf die Anforderungen an Güter, damit diese auf Börsen gehandelt werden kőnnen, geht BORCHERT, M. ; GROSSEKETTLER, H. : a.a.O., S. 14 f . ein. Siehe auch weiter FRIEDMAN, D. ; OSTROY, J. : Competitivity an Theoretical Investigation , in: The Economic Journal, Vol. 95 (1995), S. 22-53 ,insbesondere die Ausführungen 7u der SingleShot-Preisbildung.

394 Vgl. PICK, H. : Ordnungsmodelle ..., a.a.O., S. 153

395 Die Angebotspreise werden in Form von Preis-Mengen-Relationen abgegeben. Diese werden auf einer individuellen Angebotskurve des Erzeugers beruhen.

396 Die folgenden Ausfiuhrungen sind an das britische Verfahren angelehnt. Siehe hierzu auch die Ausführungen in Kapitel 4.1.3. 
Mengen an die Nachfrager weiter. Damit besitzt der Pool ein Ankaufsmonopol für kurzfristige Elektrizitätslieferungen. Der Pool stellt dann eine sogenannte „merit order" für den nächsten Tag auf. Hierzu ordnet er die Erzeuger gemäß ihrer Preisangebote in eine Hierarchie ein und vermerkt gleichzeitig die angebotenen Mengen. Bei einer Nachfrage der Verteiler oder der Großabnehmer wird zuerst das günstigste Angebot eingesetzt. In der Regel reichen die angebotenen Mengen des günstigsten Anbieters zur Nachfragedeckung nicht aus, so daß die in der hierarchischen Ordnung folgenden Anbieter eingesetzt werden. Das Verfahren wird solange angewandt, bis der Anbieter bestimmt ist, der die letzte verbleibende Nachfrage befriedigt. Er wird als Grenzanbieter bezeichnet ${ }^{397}$.

Bei der Zuordnung muß die Poolleitung gleichzeitig die Leitungskapazitäten der Netzgesellschaft berücksichtigen. Sie können beim Einsatz der Kraftwerke nach der „merit order" eine Restriktion darstellen.

Die Entlohnung der Erzeuger erfolgt im britischen System einheitlich nach dem Preis des Grenzanbieters, d.h. des Angebotspreises des letzten in Anspruch genommenen Erzeugers. Die Differenz zwischen dem individuell gebotenen Preis und dem Preis des Grenzanbieters ist der zusätzliche Gewinn des Erzeugers. Denkbar ist jedoch auch eine an der amerikanischen "Split the Savings"-Regel orientierte Preisfestsetzung: Die Differenz zwischen Grenzanbieterpreis und Angebotspreis des in Anspruch genommenen Erzeugers wird zwischen dem Erzeuger und dem Verteiler oder Großabnehmer aufgeteilt. Beachtet werden muß, daß mit der Aufteilungsregel im Vergleich zur Grenzanbieter-Preisfestsetzung geringere Anreize zu Effizienzsteigerungen verbunden sind; gleichzeitig sinkt die Attraktivität des Erzeugermarktes furr "newcomer".

Zur Absicherung des Spotmarktpreises und zur Schaffung einer größeren Planungssicherheit können Erzeuger und Verteiler Optionsverträge miteinander abschließen. Diese unterscheidet man in Anlehnung an die britischen Vorbilder in einseitige und zweiseitige Optionsverträge.

Bei einseitigen Optionsverträgen wird dem Verteiler als Optionsnehmer von dem optiongebenden Erzeuger die Übernahme der Differenz zwischen Spotmarktpreis und Optionspreis garantiert, sofern dieser positiv ist; hierfur erhält er ein Optionsentgelt. Für das Verteilerunternehmen besteht der Vorteil in einer oberen Begrenzung der Preisschwankungen. Andererseits kann auch der Verteiler dem Erzeuger einen Mindestpreis garantieren. Der zweiseitige Optionsvertrag bewirkt bei Unterschreiten eines Mindestpreises auf dem Spotmarkt die Zahlung der Differenz zwischen Marktpreis und Erzeugeroptionspreis durch den Verteiler und bei Überschreiten einer Obergrenze die Bezahlung der Differenz zwischen Marktpreis und Verteileroptionspreis durch den Erzeuger. Ein Optionsentgelt entfällt.

397 Vgl. hierzu auch die Ausführungen zu dem Pool der englisch-walisischen Elektrizitătsıvirtschaft in Kapitel 4.1.3. 
Abbildung 29: Grenzanbieterpreisbildung im Common-Carrier-Konzept

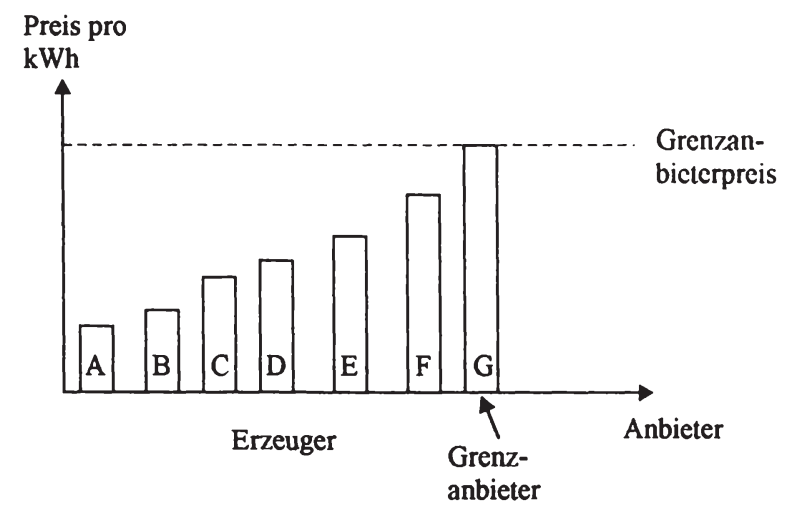

Abbildung 30: Angebotene Stromleistungen der Anbieter in dem jeweiligen Zeitabschnitt

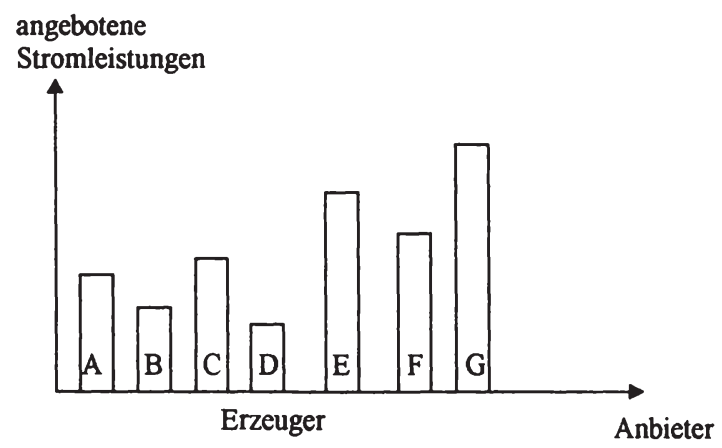

Noch offen ist die Finanzierung des Spotmarktes innerhalb des Pools; hierzu sind drei Varianten denkbar:

a) Differenz aus Pool-Input und Pool-Output-Preis Zur Finanzierung des Spotmarktes wird der Pool-Output-Preis höher als der PoolInput-Preis angesetzt. Die Differenz dient der Kostendeckung des Pools. 
b) Entgelt nach der Leistungsinanspruchnahme

Eine andere Möglichkeit wäre die Aufteilung der Kosten nach der Inanspruchnahme der Leistungen. Die Leistung des Pools besteht in dem Erstellen der ,merit order", der Zuweisung der Anbieter zu den Nachfragern, der Inrechnungstellung und der Reservehaltung sowie dem Lastmanagement. Da den Transaktionen keine tatsächlichen Stromflüsse entsprechen (siehe Abbildung 28: Stromfluß), ist die Leistungsinanspruchnahme der Poolmitglieder unabhängig von der gehandelten Stromleistung. Erst durch die Übernahme der Reserveleistungsbereitstellung oder des Reseveleistungsmanagements wird die Leistungsinanspruchnahme abhängig von der gehandelten Stromleistung. Hieraus kann ein zweigeteiltes Poolentgelt entwickelt werden: Der einzelne Erzeuger oder Abnehmer auf dem Großhandelsmarkt bezahlt dem Pool eine pauschalisierte Gebühr pro abgewickeltem Stromhandelsgeschäft; für die Reservehaltung bezahlt der Nachfrager ein an die Stromleistung gebundenes Entgelt.

c) Verwendung der Angebots-Grenzanbieter-Preisdifferenz zur Finanzierung Eine andere Alternative ist die Abzweigung eines Teils der Differenz zwischen dem Grenzanbieterpreis und den Angebotspreisen der anderen Erzeuger zur Poolfinanzierung. Dies ist auch bei Einführung der „Split the Savings“-Regel denkbar.

Der Verfasser spricht sich für eine Preisfestsetzung nach dem Grenzanbieterpreissetzungsverfahren und die Finanzierung des Pools durch eine Pool-InputPool-Output-Preisdifferenz aus. Hierdurch werden schnell hohe Anreize zu Effizienzsteigerungen geschaffen und Neuanbieter zum Markteintritt bewegt. Durch die Kombination in der Erhebung werden die Transaktionskosten minimiert.

\subsubsection{Die Abwicklung der langfristigen Lieferverträge}

Neben kurzfristigen Angeboten und Nachfragen, zu deren Absicherung Optionsverträge abgeschlossen werden können, werden alle Marktteilnehmer bestrebt sein, wenigstens einen Teil ihres Angebotes oder ihrer erwarteten Nachfrage durch langfristige Verträge abzusichern. Langfristige Verträge benötigen keine Börse, die schnell die erforderliche Koordinationsaufgaben übernimmt; weshalb langfristige Verträge ohne den Pool zustande kommen können. Denkbar ist, daß sich spezielle Makler bilden, die Erzeuger und Verteiler/Großabnehmer zusammenführen.

Die langfristigen Stromlieferverträge müssen in die Gesamtmarktkoordination eingebunden werden. Hierzu ist eine Institution erforderlich, die langfristige Lieferverträge erfaßt und mit der Netzgesellschaft abstimmt. Diese Aufgabe kann am einfachsten vom Pool übernommen werden. Die Abwicklung der langfristigen Lieferungen sollte für die Netzgesellschaft Vorrang Yor den Spotmarktgeschägthen ha- 
ben, wenn Leitungsengpässe entstehen. Ansonsten würde der Markt für langfristige Stromverträge zusammenbrechen. Für die Netzgesellschaft haben die langfristigen Lieferverträge den Vorteil der erhöhten Planungssicherheit und könnten deshalb mit niedrigeren Transportgebühren belegt werden.

\subsubsection{Das Lastmanagement und die Bereitstellung der Versorgungssicherheit}

Die Frequenz- und Spannungshaltung beim Lastmanagement erreicht der Pool über eine geeignete Regelungs- und Steuerungstechnik, die den Zugriff auf die an das Lastmanagement angeschlossenen Kraftwerke ermöglicht. So ist es denkbar, daß die Erzeuger einen Teil ihrer Kapazitäten dem Pool zum Lastmanagement gegen Entgelt zur Verfügung stellen.

Für die Versorgungssicherheit muß der Pool Kraftwerke in Reserve halten, die bei Ausfall von Kraftwerken oder Leitungen die ausgefallene Last übernehmen können. Die Reservehaltung wird durch drei Instrumente realisiert:

a) Die Poolleitung kann über die prognostizierte Elektrizitätsbedarfsmenge hinaus Kapazitäten bei Erzeugern nachfragen und diesen dafür ein Entgelt bezahlen, welches einen festen Kapazitätsanteil und einen variablen Anteil nach der Höhe der tatsächlichen Inanspruchnahme beinhaltet. Der variable Anteil sollte in erster Linie die Brennstoffkosten bei Einsatz des Kraftwerkes zur Reservehaltung abdecken. Potentielle Anbieter der Reservehaltungskapazitäten sind Erzeuger, die in der $\mathrm{Zu}$ ordnung nach der "merit order" mit ihren Angeboten über denen des Grenzanbieters liegen.

b) Ein weiteres Instrument der Reservehaltung und des Lastmanagements ist das Angebot einiger Erzeuger, Strom über ihre angebotene Leistung hinaus für den Spotmarkt zu produzieren ${ }^{398}$. In extremen Spitzennachfragesituationen kann die Poolleitung von den Erzeugern eine Ausweitung der Stromproduktion anfordern. Die Kosten für diese Maßnahme werden durch die gleichzeitige Angebotsabgabe für diese Leistung mit dem Angebot für den Spotmarkt minimiert.

c) Die Poolgesellschaft überprüft ständig die Anzahl der abgegebenen Angebote und die Entwicklung der Erzeugungskapazitäten, um zu verhindern, daß langfristig die angebotenen Mengen auf dem Spotmarkt die nachgefragten Mengen unterschreiten. Bei einer Verknappung des Angebotes erhöht sie die Preise für Reservehaltungskapazitäten. Hierdurch wird die Rentabilität von nicht kontinuierlich zur 
Strombedarfsdeckung eingesetzten Kraftwerke gesteigert; es entsteht ein Anreiz diese Kraftwerke zu errichten.

Die Kosten des Pool für das Lastmanagement und die Bereitstellung der Versorgungssicherheit können über eine prozentual erhöhte Stromtransportgebühr erhoben werden. Eine solche Gebühr entspricht dem öffentlichen Gutscharakter dieser Dienstleitungen.

Abschließend stellt sich die Frage, wer die Fïhrung des Pools übernehmen soll. Denkbar ist die Führung durch die Netzgesellschaft oder ein genossenschaftlicher Betrieb des Pools durch die Poolmitglieder.

Die Vorteile der Geschäftsführung durch die Netzgesellschaft liegen in der einfachen Koordination zwischen Lastmanagement, Vertragsgestaltung und Bereitstellung der Übertragungskapazitäten sowie der vereinfachten Überwachungsmöglichkeiten der Netzanschlußkriterien. Negativ wirkt nach Meinung des Verfassers die Gefahr einer indirekten organisierten Reintegration des Elektrizitätsmarktes unter der Führung der Netzgesellschaft und deren Neigung zur Bürokratisierung durch die Aufgabenerweiterung. Des weiteren wird eine Regulierung der Netzgesellschaft erschwert, da diese bei der Gemeinkostenzuordnung zwischen Netzgesellschaft und Pool Wahlmöglichkeiten ausnutzen könnte. Deshalb erscheint dem Verfasser, insbesondere in Hinblick auf die Begrenzung der Marktmacht der Netzgesellschaft als Monopolist, die genossenschaftliche Geschäftsführung des Pools besser geeignet, die Ziele der Umstrukturierung zu fördern.

Offen ist die Frage, ob die Gebiete der Netzgesellschaften identisch mit den Gebieten, in denen ein Pool die Koordination des Gesamtmarktes übernimmt, sein sollen oder müssen. Zwingend erforderlich ist dies nicht: Eine Netzgesellschaft kann auch für zwei Poole die Stromtransportfunktion übernehmen. Dies hätte den Vorteil, daß die Netzgesellschaft ausreichend Kupplungskapazitäten zwischen den beiden Pool-Gebieten errichtet, die zu Lieferungen zwischen den beiden Pools genutzt werden können. Andererseits vereinfacht insbesondere bei der Führung des Pools durch die Netzgesellschaft, die Identität aus Netzgesellschaftsgebiet und Pool-Gebiet die Betriebsführung des Netzes und die Verrechnung der Stromtransportkosten. Der Verfasser schlägt folgendes vor: Wenn wenige große Netzgesellschaften eingerichtet werden, sollten die Netzgesellschaften mit einer hohen Abnehmerdichte und einer großen Anzahl Pool-berechtigter Erzeuger, Verteiler und Abnehmer für mehrere Strompoole den Stromtransport übernehmen. Bei Netzgesellschaftsgrößen, die sich an dem Vorschlag der minimalen Betriebsgröße einer Netzgesellschaft orientieren, sollte je Netzgesellschaft ein Pool eingerichtet werden. 


\subsubsection{Die Entwicklungsmöglichkeiten des Konzeptes}

Entscheidenden Einfluß auf die Entwicklung des europäischen Strommarktes nach der Einfuihrung des Common-Carrier-Konzeptes hat die tatsächlich eintretende Wettbewerbsintensität. Diese hängt von einer Vielzahl von Faktoren ab, nicht zuletzt von dem Wunsch der Marktteilnehmer, durch ihr individuelles Verhalten Wettbewerbsprozesse zu initiieren. Die Summe der Faktoren, die auf das Wettbewerbsgeschehen Einfluß nehmen, ist schwer abzuschätzen; aus diesem Grund sollen in dem folgenden Abschnitt funf unterschiedliche Szenarien entwickelt und die Faktoren aufgezeigt werden, die ihr Eintreten nach der Implementierung begünstigen.

\section{Abbildung 31: Einordnung der Entwicklungsszenarien}

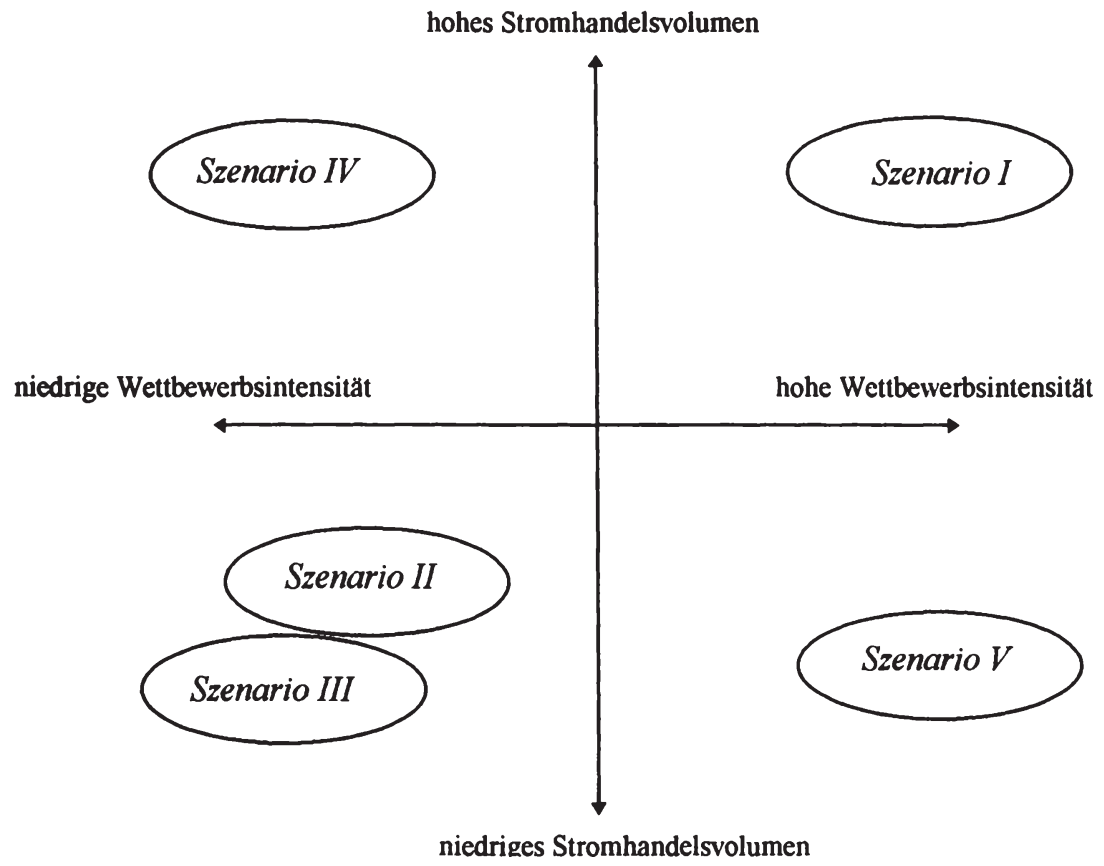


Szenario I : Hohes Stromhandelsvolumen und intensive Wettbewerbsaktivitäten

Das erste Szenario geht von einem hohen Handelsvolumen auf dem Spotmarkt und dem Markt für langfristige Stromlieferverträge sowie intensiven Wettbewerbsaktivitäten der Marktteilnehmer aus. Begünstigende Faktoren des ersten Szenarios sind:

a) Eine geeignete Erzeuger-Betriebsgröße und geographische Verteilung der Erzeuger

Konzentrieren sich die Erzeugungskapazitäten eines Anbieters mit hohem Marktanteil auf eine Region, d.h. er erreicht innerhalb dieser Region einen Marktanteil von über $50 \%$, so erlangt das Unternehmen eine marktbeherrschende Stellung, die negative Auswirkungen auf den regionalen oder lokalen Wettbewerb zeigen wird. Ist die gleiche Kapazität auf mehrere Netzgesellschaften verteilt, so beeinträchtigt die absolute Höhe der Kapazitäten das Wettbewerbsgeschehen auf dem gesamten europäischen Strommarkt nicht.

b) Neue, unabhängige Anbieter

Für das Szenario sind unabhängige, neu auf den Markt drängende Erzeuger wichtig; sie üben einen starken Wettbewerbsdruck auf die bestehenden Erzeuger aus und erzwingen damit Effizienzsteigerungen. Voraussetzung fur die Aufnahme der Elektrizitätserzeugung durch neue Anbieter sind niedrige Markteintrittsbarrieren und gute Finanzierungsmöglichkeiten.

c) Überkapazitäten in der Erzeugung Anfängliche Überkapazitäten im Erzeugungssektor begünstigen die Entwicklung zu einem hohen Wettbewerbsniveau. Die Erzeuger müssen in dieser Situation bestrebt sein, ihre Kapazitäten auszulasten und werden somit eher zu einem Preiswettbewerb neigen als bei fehlenden Überkapazitäten. Gleichzeitig sollten sich die Verteilergesellschaften marktkonform verhalten, d.h. keine Präferenz für oder gegen einen bestimmten Anbieter entwickeln, sondern das beste Angebot hinsichtlich Preis und Qualität auswählen ${ }^{399}$.

d) Eine zielkonform wirtschaftende Netzgesellschaft

Für die Netzgesellschaft gilt: Ausreichende Leitungskapazitäten und niedrige Stromtransportgebühren sowie eine funktionierende Organisation beeinflussen das Wettbewerbsverhalten der Marktteilnehmer positiv.

399 Die Gefahr besteht darin, daß die aus den ehemals integrierten EVU hervorgegangenen Unternehmen ihre traditionellen Bindungen wieder aufnelımen werden. Dadurch würden erneut, wenn auch in anderer organisatorischer Forga, wertikahintegrierce Stukturengentstehen. 
e) Strompreisdisparitäten

Ein weiterer begünstigender Faktor für das Eintreffen des Szenarios sind große Strompreisdisparitäten in der Ausgangssituation vor der Implementierung. Sie steigern das Interesse der entstehenden Marktteilnehmer auf allen Teilmärkten an günstigen Strombezugsquellen. Insbesondere von den Großabnehmern im industriellen Bereich wird bei den bestehenden Strompreisdisparitäten in Europa ein Wettbewerbsdruck ausgehen.

\section{f) Funktionsfähiger Pool}

Ein hohes Stromhandelsvolumen und eine hohe Wettbewerbsintensität kann sich nur einstellen, wenn der Pool die ihm zugewiesenen Aufgaben erfüllt und keine Marktteilnehmer diskriminiert werden. Zugleich müssen die mit der Poolinanspruchnahme anfallenden Transaktionkosten niedrig sein, damit die Preisdisparitäten zwischen den einzelnen Anbietern nicht durch hohe Transaktionskosten ausgeglichen werden. Dies hätte zur Folge, daß der Spotmarkt von den Marktteilnehmern zur Nachfragedeckung nicht herangezogen werden würde.

Szenario II : Geringe Wettbewerbsintensität und ein geringes Stromhandelsvolu men

Das zweite Szenario sieht eine geringe Wettbewerbsintensität und ein geringes Handelsvolumen auf dem Spotmarkt vor. Große Betriebsgrößen bei Verteilern und Erzeugern, bei gleichzeitiger regionaler Konzentration, begünstigen das Eintreffen des Szenarios. Im Extremfall stehen sich regional ein Erzeuger und ein Verteilungsunternehmen als alleinige Geschäftspartner gegenüber, so daß über eine langfristige Vertragsgestaltung der Wettbewerb mit anderen Erzeugern ausgeschlossen werden kann. Folgende Faktoren begünstigen das Eintreten des Szenarios II:

\section{a) Hohe Stromtransportpreise}

Hohe Stromtransportgebühren gleichen Strompreisdisparitäten aus und reduzieren die Wettbewerbsintensität. Somit wirken die Stromtransportpreise als Marktzutrittsbarrieren.

\section{b) Regional unzureichende Stromtransportkapazitäten fïr den Stromhandel} Regional ausreichende Stromtransportkapazitäten sind erforderlich, damit mehrere Konkurrenten auf einem regionalen Markt vorhanden sein können.

\section{c) Mangelnde Aufgabenerfüllung durch die Netzgesellschaft /Pool}

Übernimmt die Netzgesellschaft oder der Pool die ihnen übertragenen Aufgaben

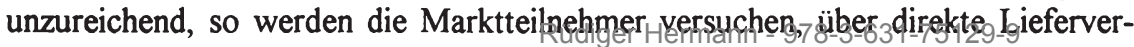


träge mit einer langen Laufzeit, die Abhängigkeit vom Pool zu verringern. Die Abhängigkeit von der Netzgesellschaft kann im Common-Carrier-Konzept von den Marktteilnehmern nicht reduziert werden ${ }^{400}$.

Szenario III : Kein Wettbewerb und kein Stromhandel

Das dritte Szenario geht von keinem wettbewerblichen Verhalten der Marktteilnehmer und der langfristigen Einstellung der Spotmarktaktivitäten aus.

Begünstigt wird das Eintreten des dritten Szenarios durch ein vollständig risikoaverses Verhalten der Marktteilnehmer. Sichern sie ihre Lieferkapazitäten und Abnahmemengen durch langfristige Verträge $a b$, bestehen bei Auslaufen der Verträge keine Wahlmöglichkeiten für die Vertragspartner. Letztlich wird hierdurch ein Wettbewerb langfristig ausgeschlossen. Gründe für das risikoaverse Verhalten von Abnehmern können mangelnde Erzeugungskapazitäten oder eine Verknappung der Kapazitäten sein. Eine solche Verknappung kann bei einem schnell steigenden Strombedarf oder bei nicht bedarfsgerechten Neubauten von Kraftwerken entstehen. Des weiteren können langfristig fehlende Strompreisunterschiede zwischen den Erzeugern das Interesse der Verteiler zur Selektion des Angebotes reduzieren. Hohe Poolkosten können ferner das Interesse der Verteiler an kurzfristigen Lieferverträgen und damit auch die Wettbewerbsintensität sinken lassen. Den gleichen Effekt haben hohe Stromtransportkosten.

Szenario IV : Hohes Stromhandelsvolumen und niedrige Wettbewerbsintensität

Das vierte Szenario geht von einem hohen Stromhandelsvolumen bei niedriger Wettbewerbsintensität aus. Diese Situation kann auf dem europäischen Strommarkt des Common-Carrier-Konzepts eintreten, wenn die meisten Stromhandelsverträge langfristig abgeschlossen werden und der Spotmarkt ausschließlich für die Bedarfsdeckung kurzfristiger Bedarfszuwächse oder für Aushilfslieferungen herangezogen wird. Überwiegend langfristige Stromhandelsverträge können dann zustande kommen, wenn risikoaverse Nachfrager auf der Großhandelsebene versuchen, Preisschwankungen durch ausschließlich langfristige Verträge auszuschließen. Ein anderer Grund für den Abschluß langfristiger Stromlieferverträge kann die Erwartung einer Angebotsverknappung durch die Nachfrager sein, die durch die abgeschlossenen Verträge ihr Preisniveau über einen planbaren Zeitraum stabil halten wollen.

400 Ausnahme ist die Eigenerzeugung, die aber für die überwiegende Mehrheit der Abnehmer nicht in Frage kommt. 
Szenario $V$ : Hohe Wettbewerbsintensität und niedriges Stromhandelsvolumen

Eine hohe Wettbewerbsintensität bei niedrigem Stromhandelsvolumen kann durch eine stark schrumpfende Nachfrage auf dem Endverbrauchermarkt - z.B. in Folge einer Rezession oder einer Energiesteuer - herbeigeführt werden. Sie führt dazu, daß die Verteiler ihre Nachfrage reduzieren. Die Konsequenz: Die überwiegend durch langfristige Stromlieferverträge abgedeckte Elektrizitätmenge wird an den Endverbraucher geliefert. Die Nachfrage auf dem Spotmarkt sinkt und das Angebot stagniert. Als Folge sinkt der Preis auf dem Spotmarkt. Die Wettbewerbsintensität ist nun abhängig von dem Verhalten der Anbieter. Versuchen sie durch niedrigere Preisangebote zu den Anbieter zu gehören, die in den nächsten Perioden zur Bedarfsdeckung herangezogen werden, steigt die Wettbewerbsintensität. Scheiden Anbieter aus dem Elektrizitätsmarkt aus, ist das Verhältnis aus Anbieter und Nachfrage entscheidend. Zur Verdeutlichung ein Beispiel:

Ausgangssituation: $\quad 100$ Nachfrager mit einen Bedarf von $1 \mathrm{MW}$ über eine Stunde Anzahl der Anbieter 10

Anbieter pro kWh: 0,01

Nach dem Nachfragerückgang:

a)

100 Nachfrager mit einem Bedarf von 0,6 MW über eine Stunde

Anzahl der Anbieter : 10

Anbieter pro $\mathrm{kWh}: 0,016 \Rightarrow$ gestiegene

Wettbewerbsintensität

b)

100 Nachfrager mit einemBedarf von $0,6 \mathrm{MW}$

über eine Stunde

Anzahl der Anbieter : 5

Anbieter pro kWh: 0,0083 => gesunkene

Wettbewerbsintensität

Diese Überlegungen zu den Szenarien zeigen, daß die konkrete Ausgestaltung der Konzeption und die Ausgangssituation einen bedeutenden Einfluß auf die spätere Entwicklung und damit auf die langfristige Zielerreichung hat. 


\subsubsection{Die Akzeptanz des Common-Carrier-Konzeptes}

Die Akzeptanz des Common-Carrier-Konzeptes wird bei den zu betrachtenden Gruppen unterschiedlich sein, nicht zuletzt deshalb, weil sie unterschiedlich von den Umstrukturierungen des Konzeptes betroffen sein werden.

Die EU-Kommission hat sich in ihren ersten Veröffentlichungen ${ }^{401}$ eindeutig für eine desintegrierte europäische Elektrizitätsstruktur ausgesprochen, welche Wettbewerb in dem letzten, durch staatliche Monopole geprägten Bereich der europäischen Volkswirtschaften einführt. Bis heute sind von der EU-Kommission keine konkreten Vorstellungen hinsichtlich der Umsetzung des Desintegrationsgedankens ${ }^{402}$ geäußert worden. Sie hat, wegen des Widerstandes der EVU, des Europäischen Parlament und einiger nationaler Regierungen einen Richtlinienvorschlag in Richtung TPA-Konzept vorgestellt, ohne jedoch ihre ursprünglichen Ziele aufzugeben ${ }^{403}$. Die mit dem Common-Carrier-Konzept verbundene Einführung von Wettbewerb auf der Erzeugerseite, die Marktöffnung für neue Anbieter und die Angleichung der Rahmenbedingungen in der Elektrizitätswirtschaft bewertet sie nach wie vor positiv ${ }^{404}$.

Die Mehrheit der nationalen Regierungen lehnt eine Desintegration ab. Als primärer Grund werden die mit der Desintegration verbundenen Umstrukturierungen genannt, deren Kosten ihrer Meinung nach ebenso wie die langfristigen Auswirkungen und Risiken nicht absehbar sind. Für einige nationale Regierungen bedeutet die mit der wettbewerblichen Öffnung der Märkte verbundene Aufgabe einer nationalen Elektrizitätspolitik einen weiteren Verlust an Eigenständigkeit in einem bedeutenden Wirtschaftssektor. Sie befürchten, ihre Regulierungs- und Steuerungsmöglichkeiten zu verlieren und diesen Wirtschaftssektor den Marktprozessen und der EU-Politik überlassen zu müssen. In beide scheinen sie kein Vertrauen zu setzen. Ein Beispiel hierfür ist Frankreich: Traditionell durch zentralistische Strukturen und eine aktive Industriepolitik mit staatlichen Einflußnahmen geprägt, fällt es den Franzosen schwer, ihre national hergestellte Unabhängigkeit ${ }^{405}$ auf dem Elektrizitätssektor abzugeben.

401 Vgl. KOMMISSION DER EUROPÄISCHEN GEMEINSCHAFT- Generaldirektion Energie: Energie in Europa - Der Binnenmarkt für Energie, Luxemburg 1988, S. $15 \mathrm{f}$.

402 Vgl. KOMMISSION DER EUROPÄISCHEN GEMEINSCHAFT - Generaldirektion Energie: a.a.O., S. 7

403 Vgl. o.V. : EG und Internationales, in: ET, 42. Jg. (1992), Heft 8, S. 558

404 HAUBRICH, H.-J. : Neue Ordnungsrahmen und Systemführungskonzepte in der Elektrizitătswirtschaft, in: ET 43. Jg. (1993) Heft 10, S. 662

$405 \mathrm{Vgl}$. z.B. die französischen Kernenergieprogramme und hierzu: BERGBACKER, F.; WESSEL, T. : Die Energiewirtschaft im gemeinsamen Markt, Baden-Baden 1963, S. $129 \mathrm{ff}$. und KRAMER, H. : Nuklearpolitik in Westeuropa und die Forschungsaufgaben der Euratom, Koln 1975, S. 78 ff. 
Die im europäischen Vergleich höchste Akzeptanz wird das Konzept in den Niederlanden und in Großbritannien aufweisen. Beide Länder haben in den letzten Jahren Erfahrungen mit Umstrukturierungen in der Elektrizitätswirtschaft sammeln können und werden daher die Auswirkungen der Veränderungen auf die Versorgungssicherheit realistischer einschätzen als andere Länder. Zudem weicht die elektrizitätswirtschaftliche Struktur der beiden Staaten im Vergleich zu den anderen Staaten am geringsten von den geforderten Strukturen des Common-CarrierKonzeptes ab. Weitere Vorbehalte der nationalen Regierungen bestehen hinsichtlich einer Privatisierung der staatlichen Unternehmen in der Elektrizitätswirtschaft. Sie befürchten den in Großbritannien beobachtbaren Arbeitsstellenabbau und die Reduzierung ihres Einflusses.

Die massivste Ablehnung einer Desintegration kommt von den europäischen $E V U^{406}$. Sie lehnen eine Aufteilung in einzelne dezentralisierte Bereiche ab, weil sie damit Machtverluste hinnehmen müssen. Weitere Gründe für ihre Ablehnung sind der Verlust ihrer Gebietsmonopole und ihrer Sonderrechte hinsichtlich Finanzierung und Forschungsbeihilfen. Eine besonders starke Ablehnung wird das Konzept durch die staatlichen Unternehmen erfahren. Diese Unternehmen waren bisher nicht zu marktkonformen Verhaltensweisen gezwungen und befürchten deshalb, ihre gewohnten Arbeits- und Funktionsweisen nicht weiterführen zu können und im entstehenden Wettbewerb über eine schlechte Ausgangsposition zu verfügen. Des weiteren lehnen Sie die geplante Privatisierung ab. Geringer werden die Bedenken gegen die Privatisierung bei den EVU ausfallen, die bisher Diversifikationsstrategien verfolgt haben und erfolgreich auf anderen Wettbewerbsmärkten operieren ${ }^{407}$. Durch folgende Argumente begründen die EVU ihre Ablehnung einer Desintegration:

a) Die Desintegration hat negative Auswirkungen auf die Versorgungssicherheit.

b) Die Desintegration führt zu verstärkten Quersubventionierungen ${ }^{408}$.

c) Die Besonderheiten der Elektrizitätsbranche lassen Wettbewerb nicht zu.

406 Z.B. DVG: Stellungnahme der deutschen Elektrizitătswirtschaft zur Mitteilung der Kommission an Rat und Parlament: Die Ausweitung der innergemeinschaftlichen Stromlieferungen, ein grundlegender Beitrag zur Vollendung des Binnenmarktes für Energie, KOM (89) 336 endgültig vom 14.07.89, Heidelberg 06.08.1989

407 Z.B. die deutsche RWE, deren neueste Diversifizierungsfeld der Tclekommunikationsdienst ist.

408 Diese sollen zu Lasten der Kleinverbraucher gehen. Vgl. hierzu VDEW: Für europăische

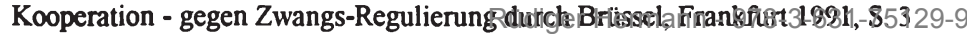


Das funktionierende desintegrierte englische System wird auch heute noch als nationale Besonderheit abgetan, dessen langfristige Folgen erst abgewartet werden müssen.

Die stromintensive Industrie wird allgemein die Einführung marktwirtschaftlicher Strukturen in einem bisher verstaatlichten und mit einem Monopol ausgestatteten vorgelagerten Wertschöpfungsprozeß begrüßen, nicht zuletzt deshalb, weil sie Effizienzsteigerungen und sinkende Preise mit der Umstrukturierung verbindet. Trotzdem werden die einzelnen nationalen Industrien eine geteilte Meinung zur Einführung des Konzeptes haben: Die Industrieunternehmen, die bisher durch staatliche Interventionen niedrige Strompreise als Standortvorteil nutzen konnten, werden die Umstrukturierung ablehnen. Anders dagegen ist die Haltung der Unternehmen in Staaten, die wegen hoher nationaler Auflagen ${ }^{409}$ hohe Strompreise bezahlen müssen. Für sie bedeutet eine Angleichung der rechtlichen Rahmenbedingungen eine relative Verbesserung der Wettbewerbsfähigkeit in Europa ${ }^{410}$.

Die Mehrheit der Klein- und Haushaltsabnehmer wird das Common-Carrier-Konzept ablehnen, weil es für sie die Auflösung der gesicherten Versorgungsgebiete bedeutet. Des weiteren sieht die angesprochene Gruppe mit der Einführung des Konzeptes eine weitere Abgabe nationaler Kompetenzen an eine fremd erscheinende EU-Bürokratie verbunden. Dies läuft dem aktuellen Trend zu einer stärkeren Betonung der nationalen Eigenständigkeiten innerhalb der EU zuwider. Ferner wird eine eigenständige Energiepolitik von der Bevölkerung als ein ureigenstes nationales Interesse verstanden. Diese Haltung basiert auf den Erfahrungen mit den Erdölkrisen der siebziger Jahre. Die bestehenden Energie- und Rohstoffabhängigkeiten fast aller europäischer Staaten von Nicht-EU-Ländern werden dabei allerdings übersehen.

\subsubsection{Der Regulierungsbedarf des Konzeptes}

Eines der Hauptbestandteile des Common-Carrier-Konzeptes ist die Einführung von Wettbewerb auf der Erzeugerseite und auf Teilen des Endabnehmermarktes. Trotzdem erfordert das Konzept eine Überwachung ${ }^{411}$, damit nicht durch individuelles Verhalten einzelner Marktteilnehmer Wettbewerb ausgeschlossen wird und das in der Erzeugung aufgelöste Monopol nicht durch ein anderes ersetzt wird.

409 Z.B. durch Umweltschutzauflagen, staatliche Anordnungen zum Einsatz heimischer Energietrăger oder zur Förderung der Kernenergie wie in Großbritannien.

410 Auf dem Weltmarkt verändert sich die Position dieser Unternehmen nur im Verhăltnis zu europäischen Anbietern.

411 Vgl. SCHWEPPE, F. : Spotpricing in ElectricityigBostenin1289, S91 1923-631-75129-9 
Träger der Regulierung können wie in den anderen Konzepten auch unabhängige Regulierungsbehörden ${ }^{411}$, bestehende europäische Behörden oder nationale Regulierungsinsitutionen der Mitgliedsstaaten sein.

\section{a) Investitionskontrolle im Erzeugerbereich}

Im Erzeugerbereich scheint dem Verfasser bei ausreichenden Kapazitäten keine Regulierung erforderlich. Lediglich der Betrieb der Kraftwerke und die Art der Investitionen bedarf der Überwachung, damit verhindert wird, daß Erzeugerunternehmen über verminderte Sicherheitsstandards und Umweltschutzauflagen einen Wettbewerbsvorteil erhalten. Problematisch erscheint diese Art der Überwachung nicht, da sie bereits in weiten Teilen der europäischen Wirtschaft üblich ist.

Ferner müssen Investitionen im Verhältnis zu den erwarteten Nachfrageentwicklungen überprüft werden. Bestehen ausreichende Kapazitätsinvestitionen, muß keine Intervention der Regulierungsinstitutionen erfolgen. Erst bei absehbarer Unterdeckung der Nachfrage sollte sie tätig werden. Eine erste Maßnahme, um absehbare Unterdeckungen zu beheben, ist die Veröffentlichung oder das Ausschreiben der fehlenden Kapazitäten; wirkt dies nicht, kann als letzte Maßnahme die fehlende Kapazität von staatlichen Unternehmen, dem Pool oder der Netzgesellschaft als weiterer Bewerber bereitgestellt werden und anschließend, nach Information über die Ertragssituation, der Verkauf angestrebt werden. Hierdurch könnten gleichzeitig weitere unabhängige Anbieter geschaffen werden.

In Großbritannien wurde zur Erzeugungskapazitätsregulierung und -kontrolle die Lizenzerteilung von Neuanlagen eingeführt ${ }^{412}$. Die erhobene Lizenzgebühr wird zur Finanzierung der Regulierung genutzt ${ }^{413}$. Beachtet werden sollte jedoch, daß hohe Lizenzgebühren als Marktzutrittsbarrieren wirken, die den gewünschten Marktzugang für Neuanbieter im Erzeugungssektor behindern und damit potentiell die Wettbewerbsintensität reduzieren ${ }^{414}$.

\section{b) Anschlußkriterienüberwachung}

Des weiteren bedarf der Marktzugang, d.h. der Anschluß an das Netz des Stromtransporteurs und die Teilnahme am Pool, technischer und organisatorischer Bedingungen, deren Einhaltung überprüft werden muß. Diese Aufgabe kann von der Netzgesellschaft am kostengünstigsten übernommen werden. Bei einer ebenso

411 Vgl. SCHOLL, R. : Reform der Elektrizitătsindustrie in Australien ..., a.a.O. , S. 388

412 Vgl. LAMBRECHT, F. : Wettbewerb und Regulierung..., a.a.O.. S. 777 f.

413 Vgl. ESSER, C. : Umstrukturierung und Privatisierung ..., a.a.O., S. 3 f.

414 In Großbritannien ist dies mit einer Lizenzgebühr von 100 Pfund nicht der Fall. Siehe hierzu Kapitel 4.1.3. 
denkbaren Überwachung der Anschlußkriterien durch den Pool besteht die Gefahr, daß neue Wettbewerber diskriminiert werden, insbesondere dann, wenn es sich um einen reinen Erzeugerpool handelt.

\section{c) Kartell- und Marktaufsicht}

Unternehmen in homogenen Märkten erfahren mit zunehmender Marktentwicklung, daß sie durch Absprachen oder Fusionen höhere Gewinne erzielen können als in Märkten mit einer hohen Wettbewerbsintensität. Deshalb werden die Unternehmen versuchen, durch Kartelle ein „Kollektivmonopol“415 zu bilden. Für die europäische Kartellaufsicht folgt daraus: Sie muß Unternehmenskonzentrationen und Verhaltensweisen beobachten und ab einer festgelegten Unternehmensgröße die Genehmigung für Unternehmenszusammenschlüsse und -beteiligungen geben oder versagen. Des weiteren sollten die Kartellbehörden kontrollieren, daß die Erzeuger nicht durch abgestimmtes Verhalten beim Bietwettbewerb den Poolpreis manipulieren.

\section{d) Kontrolle der monopolisierten Marktstufen}

Im Bereich der Elektrizitätsverteilung erhalten die neu geschaffenen Verteilerunternehmen ein Versorgungsmonopol für kleinere und mittlere Abnehmer. Dieses Versorgungsmonopol bedarf der Überwachung und Preisregulierung, um eine ungebührliche Ausnutzung der Marktstellung zu verhindern ${ }^{416}$. Bei genossenschaftlichem Eigentum der Verteilerunternehmen durch die angeschlossenen Endabnehmer könnte diese Regulierung entfallen.

Zusätzlich ist eine Überwachung der finanziellen Ausstattung und der Geschäftsführung nötig, um frühzeitig Unternehmensinsolvenzen zu vermeiden, die die Versorgungssicherheit für die Abnehmer gefährden und umfangreiche Maßnahmen zur Aufrechterhaltung der Versorgung erfordern.

Das Monopol der Netzgesellschaft muß ebenso wie das der Verteilerunternehmen reguliert werden. Die Preisregulierung ist hierbei mit der Kontrolle der Stromtransportgebührenerhebung identisch ${ }^{417}$.

415 Vgl. hierzu BORCHERT, M. ; GROSSEKETTLER, H. : a.a.O.. S.101

416 Zur Regulierung nationaler Monopolbereiche siehe JOSKOW. P. : ROSE, N. : The Effects of Economic Regulation, in: SCHMALENSEE. R. ; WILLIG, R.D. : Handbook of Industrial Organisation Vol. 2, Amsterdam 1989, 1453 ff. . Die Auswirkungen einer unzureichenden Preisregulierung im Verteilerbereich sind in Großbritannien beobachtbar.: Vgl. hierzu LOTTERMANN, T. : Die Preisentwicklungen im britischen Elektrizitătssystem. in: Elektrizitătswirtschaft, Jg. 94 (1995), Heft 3, S. 93-98, hier S. 95

417 Vgl. PICK, H. : Ordnungsmodelle ... , a.a.ORi\$lidj28 Hermann - 978-3-631-75129-9 
Finanziert werden könnte der Regulierungsaufwand über die angesprochenen Lizenzen oder über einen sog. "uplift"418 des Pools. Dies hätte den Vorteil, keine zusätzlichen Erhebungsinstrumentarien zu erfordern. Eine weitere Option ist die Finanzierung über einen Stromtransportentgeltaufschlag. Nachteilhaft wirkt dabei, $\mathrm{da} ß$ der Regulierungsaufwand ausschließlich von Unternehmen bezahlt würde, die einen Absatz erzielen, obwohl die meisten Regulierungsaufwendungen davon unabhängig sind. Als letzte Finanzierungsalternative bleibt die Übernahme der Kosten durch die EU oder die einzelnen Länder und deren Deckung durch die allgemeinen Steuern. Der Verfasser zieht eine Mischfinanzierung der Regulierung vor: Die Überwachung der Anschlußkriterien solite die Netzgesellschaft übernehmen, die die dadurch entstehenden Kosten in die Stromtransportentgelte einrechnet. Die anderen Regulierungsaufwendungen sollten durch das allgemeine Steueraufkommen und die Lizenzgebühren ${ }^{419}$ für die Unternehmen finanziert werden.

\subsubsection{Die Implementierung des Konzeptes}

Die Implementierung eines Ordnungsmodells, welche die gesamte europäische Elektrizitätswirtschaft neu strukturiert, kann nur durch einen schrittweisen Prozeß durchgeführt werden ${ }^{420}$. Dabei sollten die vorgelagerten Implementierungsschritte zum einen die Voraussetzungen für die nächste Stufe schaffen, in sich abgegrenzt durchführbar sein und die weiteren Schritte organisatorisch vorbereiten. Am Ende des Prozesses steht die konzeptionelle Ausgangssituation für die bereits aufgezeigten Entwicklungen.

\section{Implementierungsschritt: „unbundling “}

Als erster Implementierungsschritt ist das sogenannte "unbundling", die organisatorische Trennung der integrierten EVU in die Unternehmensbereiche Stromerzeugung, Stromtransport und Stromverteilung zu sehen ${ }^{421}$. Die organisatorische Trennung kann sich in der ersten Phase des Implementierungsschrittes auf das Rechnungswesen beschränken und im folgenden durch die betriebliche Aufteilung der Bereiche weitergefürt werden. Am Ende des ersten Implementierungsschrittes

418 ESSER, C. : a.a.O., S. 13

419 Bei der Lizenzgebührenhöhe sollte berücksichtigt werden. daß hierdurch nicht zusătzliche Marktzutrittsbarrieren für Neuanbieter geschaffen werden, die den Zielen des Konzeptes entgegen wirken.

420 Dies wird auch von Büchner und Gröner als der beste Weg zur Systemöffnung verstanden. Vgl. hierzu LAMPRECHT, F. : Transmission ..., a.a.O. , S. 105 und GRONER. H. : Grundzüge einer Wettbewerbsordnung ..., a.a.O., S. 67

421 Dieser Schritt ist bereits in dem Richtlinienentwurf der Kommission zum TPA-Konzept enthalten. 
sollte die Einführung von Verrechnungspreisen zwischen den Unternehmensbereichen stehen.

Legende für die folgenden Graphiken:

Rechtlich und organisatorisch sclbständiges Unternehemen

Unternehmen mit zwei organisatorisch $u$. buchhalterisch getrennten Sparten
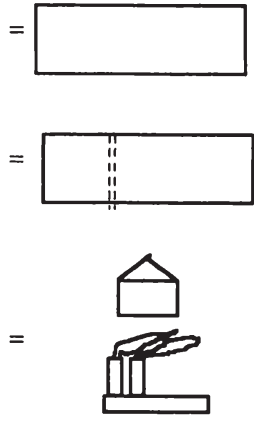

Verbraucher (Haushalts- und Industrie-)

$=$

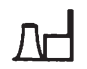

Hochspannungsnetz

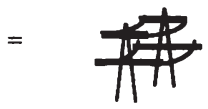

Verteilungsnetz

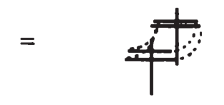

Leistungsbereitstellung erfolgt zu Verrechnungspreisen

= ニニジ

Leistungsbereitstellung erfolgt zu Marktpreisen

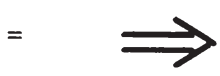

Unternehmensinterne Leistungsbereitstellung

$=$

\section{Abbildung 32 : Ausgangspunkt: Vertikal integrierte Unternehmen}

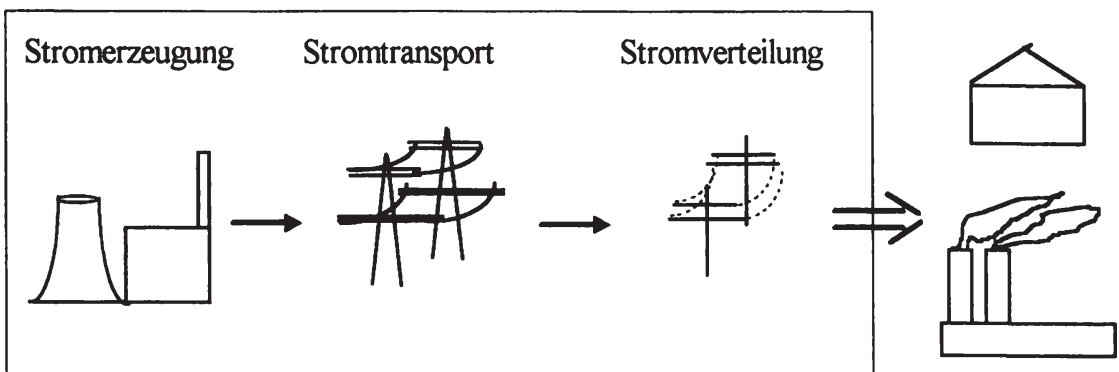


Abbildung 33 : 1. Implementierungsschritt: „unbundling“

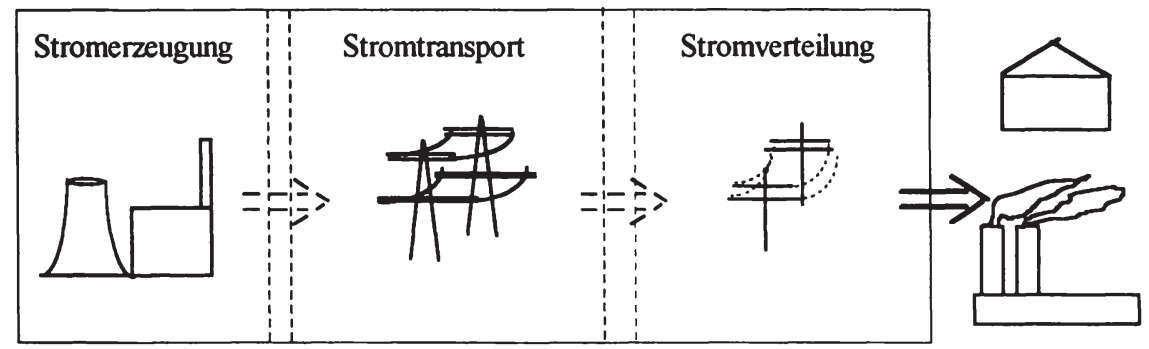

2. Implementierungsschritt: Abtrenmung des Erzeugerbereiches

In dem zweiten Implementierungsschritt sollte der Erzeugerbereich unter Beibehaltung der Betriebsgrößen und des Eigentümers aus den integrierten EVU herausgelöst werden. Die Lieferungen an die verbleibenden Unternehmensteile werden anfangs auf der Grundlage der ehemaligen Verrechnungspreise abgewickelt werden, im folgenden zunehmend durch Marktpreise. Transport- und Verteilungsunternehmen sind für das Lastmanagement zuständig. Das Transportunternehmen erstellt jeweils eine "merit order“. Am Ende des zweiten Implementierungsschrittes sollten Erzeugerunternehmen nach den vorher aufgestellten Kriterien entstehen. Denkbar ist, daß Erzeuger Kraftwerke oder Erzeugungsstandorte in Europa zugewiesen bekommen. Des weiteren sollte als Vorstufe zum Pool in diesem Implementierungsschritt parallel zu den Umstrukturierungen im Erzeugerbereich das Lastmanagement der Transportgesellschaft organisatorisch von den anderem Netzbetrieb getrennt werden.

\section{Abbildung 34 : 2. Implementierungsschritt: Abtrennung des Erzeugerbereiches}

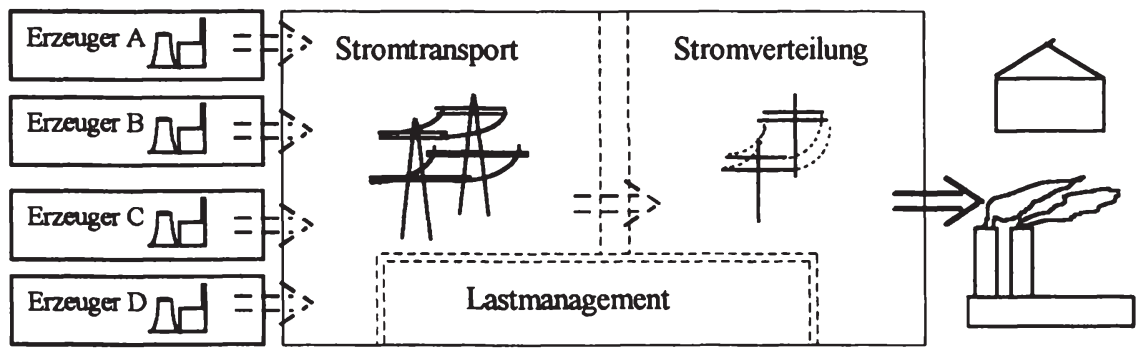




\section{Implementierungsschritt: Einrichtung des Pools}

Der dritte Implementierungsschritt wird durch die Schaffung des Erzeugerpools geprägt sein. Aus der organisatorisch vorbereiteten Trennung des Lastmanagements vom Transport- und Verteilungsbereich wird sich der Pool zuerst als Erzeugerpool konstituieren können. Zur Vorbereitung der späteren Privatisierung kann in diesem Implementierungsschritt der Verteilerbereich organisatorisch aufgeteilt und von der Netzgesellschaft abgetrennt sowie Verrechnungspreise eingeführt werden. Gleichzeitig könnten Durchmischungsanalysen und Lastsimulationen durchgeführt werden, damit empirisches Datenmaterial verfügbar wird, welches der Pool für die späteren Stromhandelsaktivitäten der Marktteilnehmer nutzen kann.

Am Ende dieses Implementierungsschrittes wird die endgültige Poolkonzeption umgesetzt werden können, indem die Verteilerbereiche der Unternehmen Poolzugang erhalten.

\section{Abbildung 35: 3. Implementierungsschritt: Einrichtung des Pools}

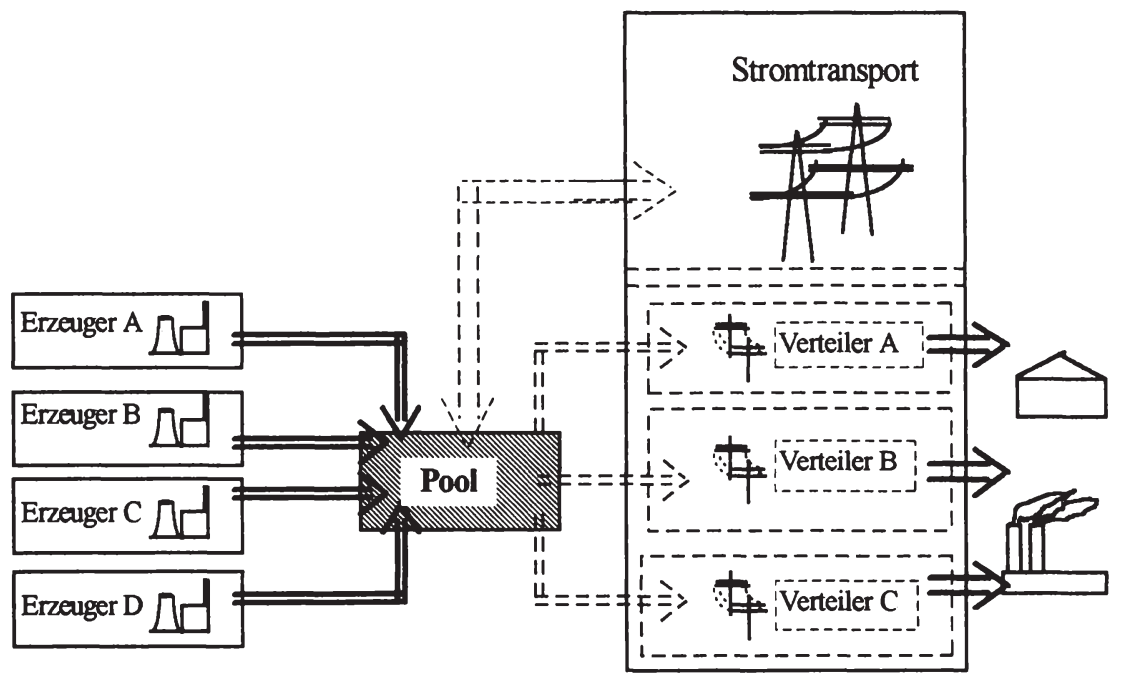

\section{Implementierungsschritt: Privatisierung und Schaffung der Verteiler}

$\mathrm{Zu}$ Beginn des vierten Implementierungsschrittes werden die bisher organisatorisch abgetrennten Verteilungskapazitäten in ein neu zu schaffendes Unternehmen eingefügt. Ferner werden im vierten Implementierungsschritt die Eigentumsstrukturen umgewandelt. Die Privatisierung der Erzeuger und Verteiler kann beispielsweise durch die Umwandlung in eine der deutschen Aktiengesellschaft ähnliche europäische Rechtsform erreicht werden. Die Anteile ehemals staatlicher Unternehmen

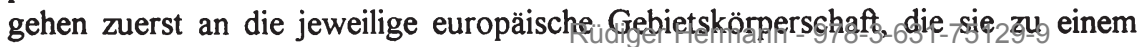


bestimmten Zeitpunkt an der Börse verkauft. Hierdurch fließen Gelder in die jeweiligen Haushaltskassen, wodurch als positiver Nebeneffekt deren Verschuldungssituation verbessert oder zumindest der finanzpolitische Spielraum vergrößert werden kann.

Problematisch erscheint dem Verfasser der Übergang der Verteilerunternehmen in genossenschaftliches Eigentum. Die Umwandlung der Verteilergesellschaften in eine Genossenschaft wird durch den erforderlichen Kapitalbedarf der Endabnehmer erschwert. Ob die Endabnehmer bereit sein würden, für einen Anteil an einem Elektrizitätsverteilungsunternehmen eine Anschaffungssumme auszugeben, kann bezweifelt werden. Daher schlägt der Verfasser vor, ausschließlich staatliche Monopolunternehmen in genossenschaftliches Eigentum zu überführen. Dies hätte zudem den Vorteil, daß die einzelnen Eigentumsformen in einen vergleichenden Wettbewerb treten, der mittel- und langfristig Aussagen über deren Effizienz zuläßt. Die nicht aus staatlichem Eigentum entstehenden Verteilerunternehmen können die Rechtsform der AG einnehmen.

\section{Abbildung 36 : 4. Implementierungsschritt: Privatisierung und Schaffung der Verteiler}

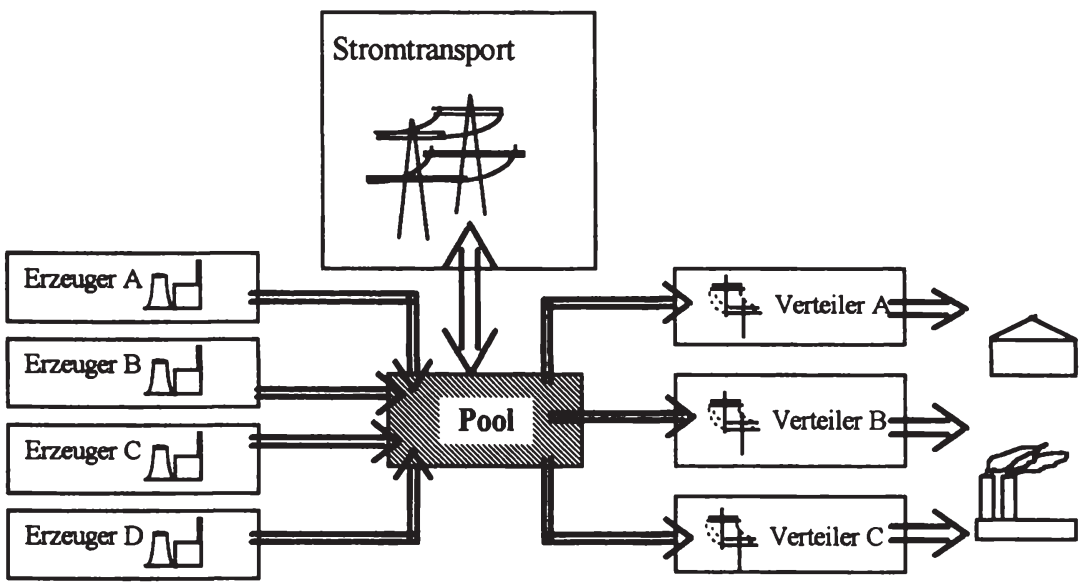

5. Implementierungsschritt: Organisation der Netzgesellschaften

In dem fünten Implementierungsschritt werden die Netzgesellschaften aufgeteilt, regionalisiert und den verschiedenen Pools zugewiesen. 


\section{Abbildung 37: 5. Implementierungsschritt: Organisation der Netzgesellschaften}

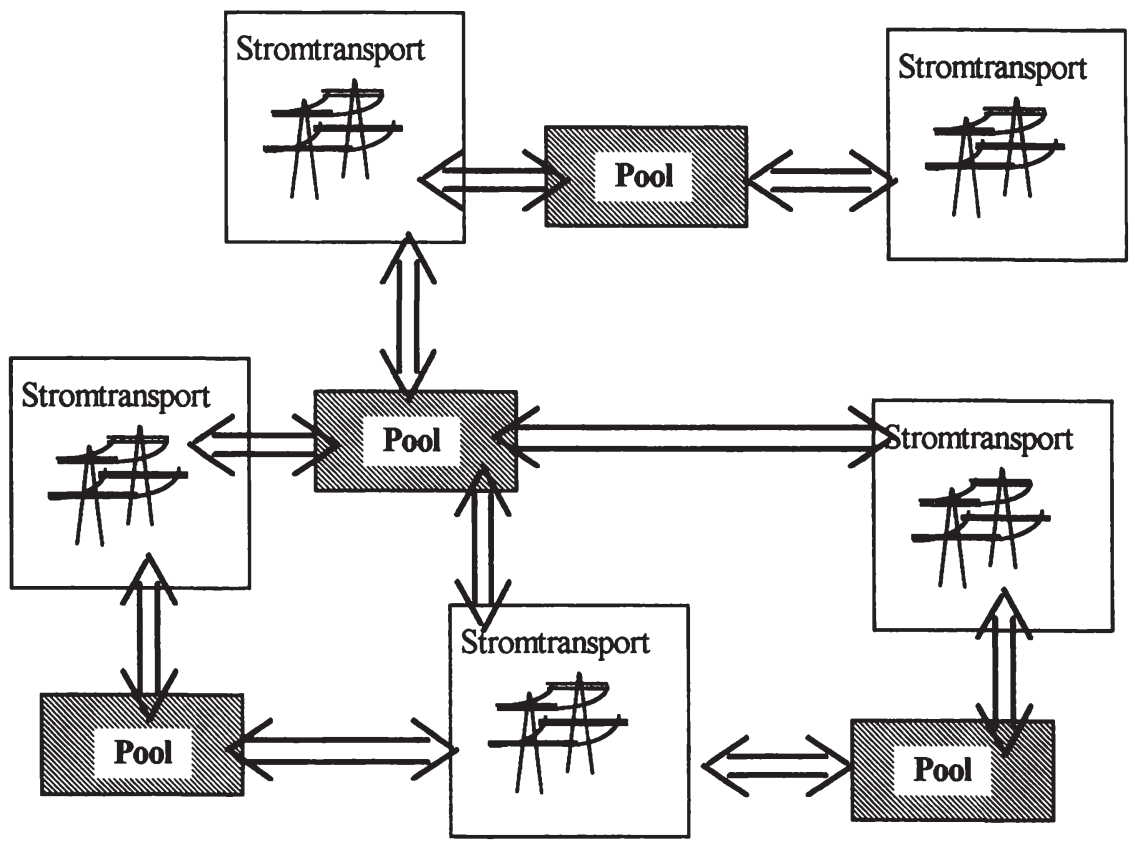

Begleitend zu den jeweiligen Implementierungsschritten sollten die Regulierungskonzepte eingefürt und die erforderlichen Institutionen ihre Arbeit aufnehmen. Der gesamte Implementierungsproze $ß$ sollte zudem von einem unabhängigen Expertenteam überwacht und ausgewertet werden, damit Erfahrungen der ersten Implementierungsphasen in die folgenden einfließen können.

Durch die aufgezeigte schrittweise Einführung des Konzeptes können Übergangsverluste und Einschränkungen bei der Versorgungssicherheit durch die Umstrukturierungen vermieden werden. Nachteilig wirkt jedoch die Dauer des Implementierungsprozesses. Die gewünschten Strukturen können nur dann ohne negative Auswirkungen eingenommen werden, wenn die einzelnen Implementierungsphasen sich über einen ausreichenden Zeitraum erstrecken. Der Verfasser schätzt die Mindestdauer der ersten ebenso wie die der zweiten Implementationsphase auf ein Jahr. Für die dritte Phase geht er wegen der höheren Komplexität und des Koordinierungsaufwandes von einer zweijährigen Mindestzeit aus. Der vierte und fünfte Implementierungsschritt kann teilweise parallel oder überlappend ablaufen. Deshalb schätzt der Verfasser den Zeitbedarf beider Schritte auf ein bis zwei Jahre. 
Somit kann für den Gesamtprozeß ein Zeitbedarf von ca. sechs bis acht Jahren errechnet werden. In Anbetracht des Umfangs und der Bedeutung der Umstrukturierungen sowie der Höhe der angestrebten Einsparungen scheint der Implementierungsaufwand und der Zeitraum gerechtfertigt.

Der Implementierungsprozeß berücksichtigt die bestehenden unterschiedlichen Strukturen in Europa. Sowohl die bereits desintegrierte Elektrizitätswirtschaft Großbritanniens als auch die vollständig integrierte Frankreichs werden schrittweise in die neue Struktur überführt.

\subsection{Das Stromhändlerkonzept (4. Konzept)}

Als Stromhändler soll im folgenden ein Unternehmen bezeichnet werden, daß Strom von Erzeugungsunternehmen kauft und an Verteilungsunternehmen oder Großabnehmer wieder verkauft. Der Stromhändler betreibt ein Stromtransportnetz und übernimmt die Koordination zwischen Stromangebot und Stromnachfrage.

\subsubsection{Die Konzeption des Stromhändlerkonzeptes}

Die Grundkonzeption des als letztes darzustellenden Konzeptes unterscheidet sich nicht von dem vorherigen Modell. Ausgangspunkt ist die Desintegration der bestehenden europäischen EVU. Durch Aufspaltung sollen Erzeugerunternehmen, Transportgesellschaften und Verteiler entstehen. Netzgesellschaft und Erzeugerunternehmen werden vollständig voneinander getrennt. Die Trennung muß gesetzlich festgeschrieben werden, um die beabsichtigten, später zu betrachtende Prozesse auch langfristig sicherzustellen.

Zentrale Organisation des Stromhändlerkonzeptes ist eine Netzgesellschaft, die folgende Aufgaben übernehmen soll:

- das Lastmanagement,

- die Einsatzsteuerung der Kraftwerke,

- die Bereitstellung der Versorgungssicherheitsleistung bis zur Verteilerebene,

- die Überwachung der technischen und organisatorischen Anschlußbedingungen an das Stromhändlernetz,

- die Messung der Lastflüsse ,

- den Stromtransport,

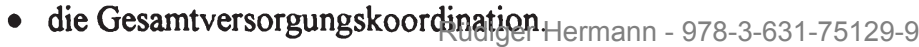


Damit unterscheiden sich die Aufgaben der Netzgesellschaft signifikant von denen eines reinen Stromtransporteurs im Common-Carrier-Konzept.

Das Stromhändlerkonzept sieht drei Marktstufen ${ }^{422}$ vor:

a) Der Stromhändlerankaufmarkt,

b) Der Stromhändlerabsatzmarkt,

c) Der Endabnehmermarkt zwischen Verteiler und Letztabnehmer.

\section{Abbildung 38: Marktstruktur im Stromhändlerkonzept}

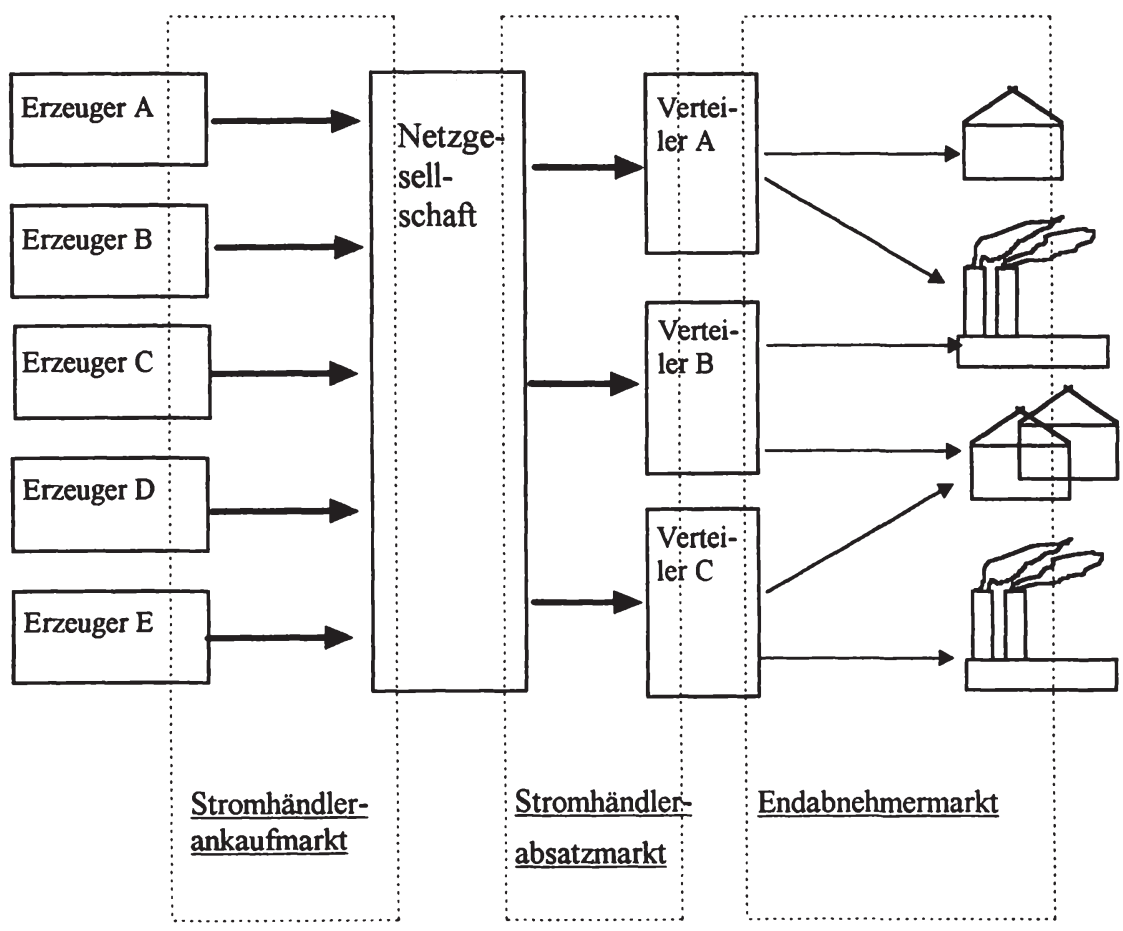

422 Diese in der Literatur nicht verwandten Begriffe hat der Verfasser ausgewăhlt, um die Marktposition des Stromhändlers herauszustellen und das Ordnungsmodell auch terminologisch von den anderen abzugrenzen. Rüdiger Hermann - 978-3-631-75129-9 


\subsubsection{Der Stromhändlerankaufmarkt}

Auf der Anbieterseite des Stromhändlerankaufmarkts sollen die aus der Desintegration entstandenen Erzeugerunternehmen in Wettbewerb zueinander treten. Einziger Nachfrager dieser Marktstufe ist die Netzgesellschaft, die die gesamte von den Endverbrauchern benötigte Stromleistung aufkauft ${ }^{423}$.

Die Anforderungen des Stromhändlerkonzeptes an die Betriebsgröße der Erzeugerunternehmen sind gering. Die existierenden Erzeuger stehen stets der mit einem Monopol ausgestatteten Netzgesellschaft im Markt gegenüber. Die konzeptkonforme Erzeugerbetriebsgröße wird überschritten, wenn ein einzelner Erzeuger ein Stromerzeugungspotential auf dem Stromhändlerbeschaffungsmarkt erlangt, mit dem er die Bezugspreise der Netzgesellschaft beeinflussen kann. Die Erzeuger sollten nach der Abspaltung von den bestehenden integrierten EVU privatisiert werden, um die daraus resultierenden Anreize für den Wettbewerb nutzen zu können.

\subsubsection{Der Stromhändlerabsatzmarkt}

Alleiniger Anbieter auf dem Stromhändlerabsatzmarkt ist die Netzgellschaft. Nachfrager sind die Großabnehmer ab einer festzulegenden Größe und die aus der Desintegration hervorgegangenen Verteilerunternehmen.

Aufgabe der Verteiler ist der lokale Stromtransport und der Zwischenhandel zwischen Netzgesellschaft und Endabnehmern. Zwischen den Verteilerunternehmen wird kein Wettbewerb eingeführt. Der traditionell viel zitierte vergleichende Wettbewerb soll Aufschlüsse über die Effizienz der Unternehmen erbringen ${ }^{424}$. Für die Sicherheit der Verteilungseinrichtungen ist das jeweilige Verteilerunternehmen zuständig. Hinsichtlich der Organisation des Verteilerbereichs gelten prinzipiell die gleichen Aussagen wie für das Common-Carrier-Konzept. Bei der Schaffung der Verteilerunternehmen muß jedoch weniger auf die Durchmischung geachtet

423 Zuzüglich ihrer eigenen und den Netzverlusten der Verteilergesellschaften.

424 Vgl. hierzu: INSTITUT FÜR BILANZANALYSEN: Die Elektrizitătswirtschaft in der Bundesrepublik Deutschland, Würzburg 1979, S. 4 ; GRAWE, J. : Ein gemeinsamer Strommarkt in der EG aus der Sicht der Energieversorgungsunternehmen, in : LUKES, R. (Hrsg.): Ein EWG- Binnenmarkt für Elektrizităt: Realităt oder Utopie; Vortrăge und Diskussionsberichte anläßlich einer wissenschaftlichen Tagung in Münster/W. am 29./30. Oktober 1987, Köln, Berlin, Bonn, München 1988, S. 165-175, S. 51 ff. Und MANSFELD, L. : Gebietsmonopole für Elektrizitătsversorger?, in : technologie \& management. Heft 1, 1989 , S. $29 \mathrm{ff}$. 
werden, da alle Endabnehmer indirekt bei der Netzgesellschaft, die das Lastmanagement übernimmt, angeschlossen sind.

Eigentümer der Verteilerunternehmen können die bereits beim Common-CarrierKonzept angesprochenen Gruppen sein.

Großabnehmer sollten in diesem Ordnungsmodell direkt an die Netzgesellschaft angeschlossen werden, um den für sie unnötigen Zwischenhandel der Verteiler zu umgehen.

\subsubsection{Der Endabnehmermarkt zwischen Verteiler und Letztabnehmer}

Die Endabnehmermarktstufe verändert sich prinzipiell durch die Einführung des Konzeptes nicht. Den Verteilerunternehmen obliegt weiterhin die Versorgungsund Anschlußpflicht für die Letztabnehmer. Ausgeschlossen hiervon sind lediglich Großabnehmer, die Zugang zum Stromhändlerabsatzmarkt haben. Die Konzeption zu dieser Marktstufe ist mit der des Common-Carrier-Konzeptes identisch.

Offen sind nun die Koordination im europäischen Elektrizitätsmarkt des Stromhändlerkonzeptes und die Ausgestaltung des Stromhändlers.

Die Koordination zwischen den einzelnen Marktstufen übernimmt die Netzgesellschaft als Stromhändler. An sie geben die Erzeuger ihre Angebote ab. Verteiler und Großabnehmer zeigen ihren Strombedarf an. Für die Gesamtkoordination des Marktes ist eine dreigeteilte Angebots- und Nachfrageanzeige bei der Netzgesellschaft sinnvoll ${ }^{425}$.

Der erste Teil der Order oder des Angebotes sollte die langfristig absehbaren Erzeugungskapazitäten und Nachfrageprognosen beinhalten. Seitens der Erzeuger kann das langfristige Angebot die Kraftwerkskapazitätsplanung umfassen, bei der Leerzeiten, Routinereparaturzeiten und jahreszeitliche Schwankungen bei Wasserkraftwerken erfaßt werden. Der Verfasser ist der Meinung, daß diese Planung der EVU für ein Jahr im voraus erstellt werden und nur absehbare Veränderungen berücksichtigen sollte.

425 Einen anderen Periodenentwurf wählen Hanselmann et al. Sie zeigen auf, wie der hier als kurzfristig bezeichnete Kraftwerkseinsatz praktisch ablaufen kann. Vgl. HANSELMANN, M. ; ALBIGER, J. ; KALTSCHMITT, M. : Mittelfristige Kraftwerkseinsatzplanung - Problemstellung und Lösungsansătze, in: Elektrizitătswirtschaft Jg. 95 (1996), Heft 4, S. 204209. Eine ab dem 01.06.96 in Finnland geplante Strombörse soll den Handel mit Stromlieferungen in 168 Stunden und spăter umfassen. Vgl. o.V. : Finnland: Start der

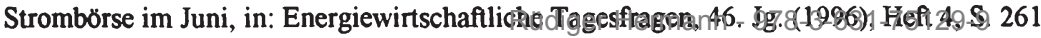


Der zweite Teil der täglichen Order und Angebote kann den mittelfristigen Bedarf und das Angebot wiedergeben. Im Gegensatz zur langfristigen Planung können hier bereits Erkenntnisse über die Markt- und Produktionslage berücksichtigt werden. Beispielsweise kann der plötzlich auftretende Defekt einer bestimmten Baugruppe eines Kraftwerkes Überprüfungen an den Kraftwerken gleichen Typs erforderlich machen. Diese Angebotsminderung kann dann von der Netzgesellschaft berücksichtigt werden. Durch die Order der Verteiler können mittelfristige Nachfrageschwankungen in die Planung eingehen ${ }^{426}$. Ein denkbarer Ansatz wäre der Zeitraum zwischen einem und sieben Tagen.

Das kurzfristige Angebot/Order sollte sich auf die für den nächsten Tag erwartete Situation beschränken. Sie wird Grundlage der von der Netzgesellschaft zu erstellenden „merit order". Mit Hilfe der „merit order" bestimmt die Netzgesellschaft die Preise für die Erzeuger ${ }^{427}$. Darüber hinaus vergibt die Netzgesellschaft Reservehaltungsaufträge an Erzeuger, deren Kapazitäten voraussichtlich nicht zur Strombedarfsdeckung verwendet werden.

\section{Abbildung 39: Angebotsabgabe an den Stromhändler}

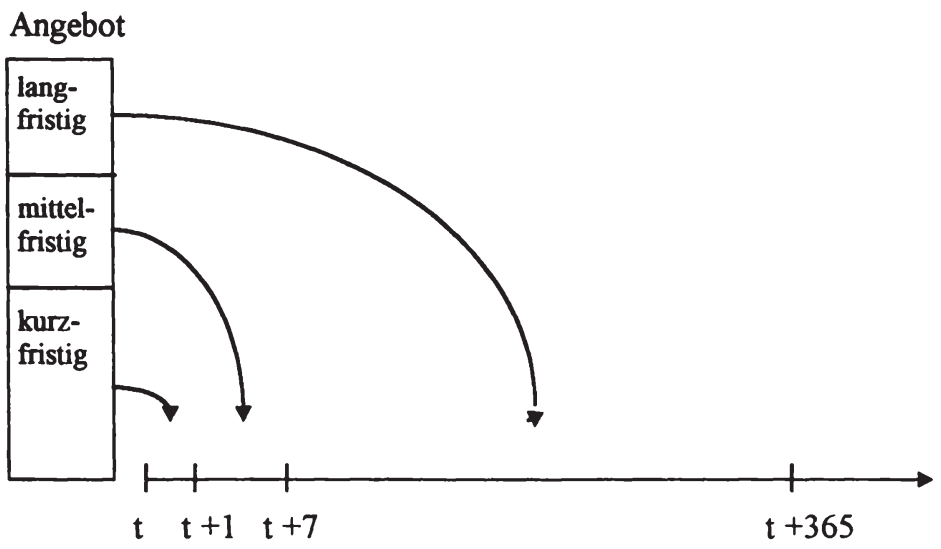

Am Tag nach der Angebotsabgabe ruft die Netzgesellschaft gemäß dem tatsächlich anfallenden Strombedarf der Verteilerunternehmen die Kraftwerke ab und übernimmt das Lastmanagement. Hierzu ist es erforderlich, daß die Erzeuger die

426 So kann z.B. in Frankreich, wo elektrische Raumheizungen weit verbreitet sind, ein strenger Winter berücksichtigt werden. Vgl. ZASK, J. : Exportkapazităt der französischen Stromwirtschaft, in: HARMS, W. (Hrsg.): Atomstrom aus Frankreich, Koln, Berlin, Bonn, München 1987, S. 6

427 Die Preisbildung auf dem Erzeugermarkt wirdim ndohsten Untorkapitel behandelo 
Steuerung ihrer Kraftwerke hinsichtlich Frequenz, Spannung und Kapazitätseinsatz der Netzgesellschaft überlassen. Sind prognostizierter und tatsächlicher Bedarf deckungsgleich, werden alle Kraftwerke eingesetzt. Bei einer höheren Nachfrage greift die Netzgesellschaft auf die Reservekraftwerke zurück. Unterschreitet die Nachfrage die Prognose, werden nicht alle Kraftwerke eingesetzt oder sie werden mit einem geringeren Auslastungsgrad betrieben werden. Bei Unterschreiten der tatsächlichen Abnahmemenge unterhalb der von der Netzgesellschaft georderten erhält der Erzeuger einen um die vermiedenen Brennstoffkosten reduzierten Preis ${ }^{428}$.

Im Bereich der Investitionsplanung ist es erforderlich, daß die Erzeugerunternehmen ihre beabsichtigten oder im Bau befindlichen Kraftwerksinvestitionen der Netzgesellschaft melden. Hierdurch ist die Netzgesellschaft in der Lage, ihre Leitungsinvestitionen an den Kraftwerksneubauten auszurichten oder mit diesen abzustimmen und zu erkennen, ob langfristig die Kapazität zur Deckung der prognostizierten Stromnachfrage ausreicht ${ }^{429}$. Bei einer erkennbaren Unterdeckung kann die Netzgesellschaft die fehlenden Erzeugungskapazitäten in einem ersten Schritt bekannt machen und, wenn kein Unternehmen neue Kraftwerke baut, als Notfallmaßnahme selbst den Bau von Kraftwerken in Auftrag geben. Sie sollte jedoch in diesem Fall die Auflage erhalten, die Kraftwerke nach Fertigstellung und Inbetriebnahme zu verkaufen.

Bei der Ausgestaltung der Stromhändler ist die Frage der Betriebsgröße und der Eigentumsverhältnisse für die Zielerfüllung von Bedeutung. In bezug auf die $\mathrm{Be}-$ triebsgröße der Netzgesellschaft als Stromhändler können die gleichen Überlegungen wie beim Common-Carrier-Konzept berücksichtigt werden. Denkbar ist ein einziges europäisches Unternehmen, welches außer dem Stromtransport die aufgezeigten weiteren Aufgaben übernimmt. Als Vorteile können die einheitliche Betriebführung, gleiche Anschlußbedingungen ${ }^{430}$ und die raumordnungspolitisch gewünschte Angleichung der Lebensbedingungen im Elektrizitätssektor angeführt werden. Ein weiterer Vorteil wäre ein einheitliches Tarif- und Preissystem. Ein einziges Unternehmen würde jedoch eine Marktmacht erreichen, die die Gefahr der Ausbeutung nahe legt. Gleichzeitig wäre damit das Entstehen einer "Mammut-Organisation" verbunden, deren erforderliche Kontrolle durch die Größe erschwert würde. Aus diesen Gründen schlägt der Verfasser eine mittlere Betriebsgröße vor. Sie kann bei der Netzgesellschaft als Stromhändler ein geographisch kleineres Gebiet umfassen als beim Common-Carrier-Konzept, weil das stromhandelnde Netzunternehmen neben dem Stromtransport die aufgezeigten Aufgaben über-

$428 \mathrm{Zu}$ dessen Festlegung sind weitere Richtlinien und Vereinbarungen erforderlich.

429 Ein derartiges Koordinationsinstrument wird in der niederländischen Elektrizitătswirtschaft verwandt. Vgl. hierzu: EISS, H. et al. : Die Ordnung ...., a.a.O.. S. 424

430 Diese könnten durch eine europäische Norraiunggerzidla wwerden. 978-3-631-75129-9 
nehmen muß. Hierdurch benötigt die Netzgesellschaft zusätzliche Unternehmensteile, wodurch die Betriebsgröße im Verhältnis zum Netzgebiet vergrößert wird. Als Eigentïmer der Netzgesellschaft kommen die bereits bei den anderen Modellen betrachteten Gruppen in Frage. GRÖNER spricht sich allgemein für eine private Transportgesellschaft aus ${ }^{431}$. Eine private Netzgesellschaft müßte wegen ihrer Marktmacht durch effiziente Regulierungsmechanismen und -instrumente überwacht werden. Geringer könnte die Überwachung bei einer paritätischen Beteiligung der Verteiler, Großabnehmer und Erzeugerunternehmen ausfallen. Für eine Beteiligung der europäischen öffentlichen Gebietskörperschaften in der Elektrizitätswirtschaft wird das Argument angeführt, hierdurch könne das "öffentliche Interesse" besser berücksichtigt werden.

Der Verfasser schlägt den paritätischen Besitz der Erzeuger, Verteiler und Großabnehmer an einer privaten Netzgesellschaft vor, nicht zuletzt deshalb, weil der Vergleich des amerikanischen Elektrizitätsversorgungssystems mit den nationalen europäischen Systemen keine Hinweise darauf zuläßt, daß bei privaten Gesellschaften das "öffentliche Interesse" weniger berücksichtigt wird. Ähnlich wie für das Common-Carrier-Konzept vorgeschlagen ist auch eine Beteiligung der europäischen Gebietskörperschaften an der stromhandelnden Netzgesellschaft denkbar.

\subsubsection{Die Mittel des Stromhändlerkonzeptes}

Im Gegensatz zu den anderen Modellen, bei denen als Mittel ein Instrument, z.B. der Pool oder eine gesetzliche Vorschrift (TPA), eingesetzt wurde, ist die Netzgesellschaft mit Teilen ihrer Tätigkeitsfelder als Mittel des Konzeptes zur Zielerreichung anzusehen. In diesem Zusammenhang wirkt die Preisgestaltung gegenüber den Erzeugerunternehmen und den Verteilern durch die Netzgesellschaft.

\subsubsection{Die Preisgestaltung gegenüber den Erzeugerunternehmen}

Auf dem Stromhändlerabsatzmarkt sind einheitliche Preise für alle Erzeuger oder je nach Angebotsabgabe differierende Preise denkbar. Die Wahl der Preistypen hat Auswirkungen auf die Zielerfüllung. 


\subsection{Einheitliche Strombezugspreise}

Gleiche Abnahmepreise der Netzgesellschaft von den Erzeugern können entweder durch eine vorab festgelegte einheitliche Bezahlung für gelieferte elektrische Energie entstehen oder durch eine einheitliche Preisfestlegung nach Angebotsabgabe seitens der Erzeuger.

Ersteres würde der in vielen europäischen Ländern praktizierten Lösung bei Kleineinspeisern in das öffentliche Netz entsprechen: Die Netzgesellschaft berechnet im voraus, bei welchem Preis welche Kapazitätsmengen angeboten werden und wählt den Preis, bei dem sie glaubt, daß er ein zur Nachfrage auf dem Stromhändlerabsatzmarkt passendes Angebot schaff. Dieses Verfahren hat als Vorteile die scheinbar einfache Durchführbarkeit und die niedrigen Transaktionskosten, nachteilig wirkt die Tatsache, daß die Netzgesellschaft zwar Erfahrungen über die Angebotskurven der Erzeuger hat, jedoch nie genau voraussagen kann, ob die durch den Preis erwirkte Angebotsmenge zur Bedarfsdeckung ausreicht. Bei Unterdeckung müßte sie dann zu wesentlich höheren Preisen Elektrizität von den Erzeugern abkaufen.

Die zweite Möglichkeit der einheitlichen Preisgestaltung ist die Anwendung der Grenzanbieter-Preisregel ${ }^{432}$. Als Vorteil wirken bei diesem Preisbildungsverfahren die großen Anreize zu Effizienzsteigerungen, da jede Kostenreduzierung den Gewinn des Erzeugers erhöhen kann. Gleichzeitig wird durch das Verfahren ein Selektionsprozeß implementiert, der die kostengünstigsten Anbieter zur Stromerzeugung heranzieht. Langfristig wirkt der Selektionsprozeß auf die Primärenergieträger, die zur Verstromung eingesetzt werden.

\subsection{Uneinheitliche Strombezugspreise}

\section{Reine Angebotspreise}

Uneinheitliche Abnahmepreise können dann entstehen, wenn die Netzgesellschaft Angebote von den Erzeugern erhält und diese gemäß ihrem Bedarf abnimmt und bezahlt. Der jeweilige Erzeuger muß vorab seine Gewinnerwartungen festlegen und darauf hoffen, seine Kapazitäten absetzen zu können. Anreize zu Effizienzsteigerungen sind vorhanden, da die Unternehmen mit günstigeren Kostenstrukturen durch ihre Erfahrungen im Bietwettbewerb langfristige Marktvorteile haben und einen zusätzlichen Gewinn erwirtschaften können. Die Gewinne sind jedoch niedriger als bei dem Grenzanbieterpreissystem, da die Erzeuger es nicht schaffen,

432 Zur Erklărung der Grenzanbieter-Preisregel vergleiche die Ausführungen zur Preisbildung im Common-Carrier-Konzept in Kapitel 7.2.2. diger Hermann - 978-3-631-75129-9 
ihre Angebote genau in Höhe des Grenzanbieterpreises festzulegen. Erzeuger mit kostengünstiger Struktur werden ihren Angebotspreis wegen der Gefahr, durch ein unwesentlich zu hohes Angebot nicht zum Einsatz zu kommen, unter den von ihnen erwarteten Grenzanbieterpreis setzen.

Nach Preisregeln (Split the Savings)

Preisbildungsregeln sind in einigen Staaten Amerikas elektrizitätswirtschaftliche Praxis ${ }^{433}$. Hieraus lassen sich Preisbildungsregeln für den Stromhändlerbeschaffungsmarkt ableiten:

Die Anwendung der „Split the Savings“-Regel für das Konzept bedeutet: Der Stromhändler bestimmt den Grenzanbieterpreis. Die einzelnen Angebote werden von der Stromhandelsgesellschaft abgerufen. Der Grenzanbieter erhält den Grenzanbieterpreis. Die anderen Erzeugerunternehmen werden mit einem Preis zwischen ihrem Angebot und dem Grenzanbieterpreis entlohnt. So kann die Differenz zwischen Angebots- und Grenzanbieterpreis paritätisch zwischen der Erzeugergesellschaft und dem Stromhändler geteilt werden. Die Anreize zu Effizienzsteigerungen sinken hierdurch, ohne jedoch zu verschwinden. Vorteilhaft ist, daß die dadurch eingesparten Beträge an die Verteiler und Letztabnehmer weitergegeben werden können. Außer der paritätischen Aufteilung sind alle anderen Aufteilungskombinationen denkbar. Dabei gilt: Je niedriger der Prozentsatz, die der Erzeuger erhält, desto größer die mögliche Einsparung für den Endverbraucher, desto geringer sind die Effizienzsteigerungsanreize.

\subsubsection{Die Preisgestaltung gegenüber den Verteilern und Großabnehmern}

Ähnlich wie bei der Preisgestaltung gegenüber den Erzeugerunternehmen hat die Stromhändlergesellschaft auf ihrem Absatzmarkt die Möglichkeit, einheitliche oder uneinheitliche Abgabepreise aufzustellen.

Unterschiedliche, individuelle Abgabepreise der Netzgesellschaft müssen auf $B e-$ zugsgrößen beruhen. Eine erste denkbare Bezugsgröße stellen die Anschlußkosten des Abnehmers an das Hochspannungsnetz des Stromhändlers dar. Problematisch ist hierbei die Festlegung der unterschiedlichen Anschlußkostenkriterien. Bis auf geringe Unterschiede durch Kostendegression oder -progressionseffekte bei höheren Anschlußleistungen sind die Kosten für den Anschluß aller Verteiler an ein bestehendes Leitungsnetz pro Leistungseinheit gleich. Erst wenn neue Verteilerunternehmen, Großabnehmer oder bestehende Verteiler einen höheren mit Lei- 
tungskapazitätszubauten verbundenen Netzzugang benötigen, können unterschiedliche Anschlußkosten entstehen. Die auf den Anschlußkosten beruhenden unterschiedlichen Stromabgabepreise entsprechen jedoch nicht der Konzeption eines mit einem öffentlichen Versorgungsauftrag ausgestatteten Unternehmens mit Dienstleistungscharakter. Aus diesen Gründen erscheint es dem Verfasser sinnvoll, die unterschiedlichen Anschlußkosten durch eine einmalige Gebühr zu erheben und den Abgabepreis unabhängig von den Anschlußkosten zu berechnen.

Als zweite Alternative können unterschiedliche Abgabepreise durch Staffelıng der Preise nach der Höhe der Abnahmemenge entstehen. In Kombination mit gleichen Einkaufspreisen der Netzgesellschaft ${ }^{43.4}$ wäre die Staffelung für die Verteiler und Großabnehmer nicht begründbar. Erst in Kombination mit differierenden Einkaufspreisen der Netzgesellschaft könnte mit viel Mühe über simulierte marktliche Preisbildungsprozesse die differierende Preisstaffelung nach der Abnahmemenge begründet werden.

Ein einheitlicher Stromhändlerabgabepreis kann dadurch entstehen, daß die Stromhandelsgesellschaft die Ankaufspreise bündelt und von ihren Abnehmern einen einheitlichen Preis verlangt. Der Preis kann im Lastverlauf variieren. Er setzt voraus, daß alle Abnehmer über ein Stromentnahmemeßgerät verfügen, welches die zeitliche Komponente des Stromverbrauchs festhält und an das Rechnung stellende Unternehmen weiterleitet. Dies ist bei den potentiellen Vertragspartnern der Netzgesellschaft bereits heute der Fall.

Ein einheitlicher Stromhändlerabgabepreis betont den Infrastrukturcharakter der Stromversorgung. Zudem entspricht er bei Anwendung der Grenzanbieterpreisregel den Bezugskosten des Stromhändlers; er ist daher für die Zielsetzung besser geeignet als unterschiedliche Abgabepreise.

\subsubsection{Die Preisgestaltung für die Koordinationsaufgaben und den Stromtransport}

Offen ist nun noch als letzter Aspekt der Preis für die Koordinationsaufgaben, die die stromhandelnde Netzgesellschaft erbringt. In der britischen Elektrizitätswirtschaft werden die Aufgaben durch eine Ankaufs- und Abgabepreisdifferenz finanziert $^{435}$. Die Vorteile des Verfahrens sind niedrige Transaktions- und Erhebungskosten. Neben den Kosten für die Reservehaltung, für die Bereitstellung der Versorgungssicherheit und für das Lastmanagement könnten so auch die Kosten für 
den Stromtransport erhoben werden. Nachteilig bei der pauschalisierten Erhebung und Berechnung ist die mangelnde Verursachergerechtigkeit. Als andere Variante kann die Netzgesellschaft, wenn die Grenzanbieterpreisformel auf dem Beschaffungsmarkt angewandt wird, einen Teil der Preisdifferenz zur Deckung der Koordinations- und Stromtransportkosten verwenden. Bei der "Split the Savings-Regel“ wäre denkbar, daß z.B. 50\% der Differenz an den Erzeuger bezahlt würden, und der verbleibende Teil zwischen Netzgesellschaft und Abnehmer aufgeteilt wird. Die Netzgesellschaft müßte dann von diesem Teil ihre Kosten decken. Dieses Verfahren läßt jedoch zusätzliche Transaktionskosten für die Abrechnung entstehen.

Wägt man abschließend die Preisbildungsalternativen ab, so erscheint dem Verfasser in der Anfangsphase, nach der Implementierung des Konzeptes, eine Preisbildung auf dem Beschaffungsmarkt nach der Grenzanbieterpreisregel geeignet. Von dieser Preisbildungsregel gehen die größten Anreize zur Steigerung der Effizienz beim Betrieb der bestehenden Kapazitäten und zum Bau neuer Kapazitäten aus.

Auf dem Stromhändlerabsatzmarkt sollten einheitliche Abgabepreise für die Stromleistungen den Infrastrukturcharakter der Netzgesellschaft unterstreichen ${ }^{436}$. Die Koordinations- und Stromtransportaufgaben der Netzgesellschaft sollten aus den aufgezeigten Gründen durch eine Ankaufs-/Abgabepreisdifferenz finanziert werden.

In einer späteren Phase nach der Einführung des Konzeptes können dann je nach Marktsituation andere Preisbildungsregeln wie z.B. die „Split-the-Savings“-Regel zur Anwendung kommen.

\subsubsection{Die Entwicklungsmöglichkeiten}

Bei der Betrachtung der Entwicklungsmöglichkeiten und -richtungen des Konzeptes kann von dem Verteilermarkt abgesehen werden, da er ebenso wie die Netzgesellschaft vollständig monopolisiert wird und die Preisgestaltung festen, gesetzlichen Auflagen unterliegen wird.

Die Entwicklungen kurz nach der Implementierung des Konzeptes hängen entscheidend von dem Ausmaß der Überkapazitäten auf dem Stromhändlerbeschaffungsmarkt ab. Drei unterschiedliche Entwicklungsszenarien können in bezug auf die Kapazitätssituation auf dem Stromhändlerbeschaffungsmarkt skizziert werden:

$\begin{array}{lll}\text { Szenario I } & : & \text { Keine Überkapazitäten } \\ \text { Szenario II } & : & \text { Niedrige Überkapazitäten } \\ \text { Szenario III } & : & \text { Hohe Überkapazitäten }\end{array}$

436 Beachtet werden sollte, daß hierdurch nicht die Abnahmepreisstruktur auf dem Endverbrauchermarkt festgelegt wird. 


\section{Abbildung 40: Übersicht Szenarien Stromhändlerkonzept}

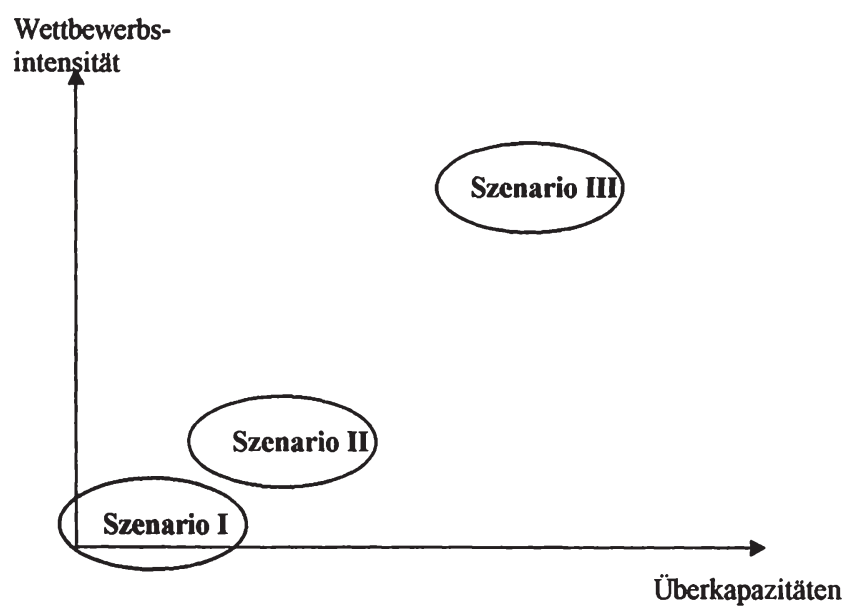

Szenario I : Keine Überkapazitäten und kein Wettbewerb

Das erste Szenario geht von fehlenden Überkapazitäten bei den Erzeugern aus. Ein Wettbewerb zwischen den Erzeugern ist daher solange ausgeschlossen, bis neue Anbieter auf den Markt drängen oder die bestehenden ihre Kapazitäten ausdehnen. Bis zu diesem Zeitpunkt können die Erzeuger den Preis für die gelieferte Elektrizität anheben. Die Preisobergrenze wird dabei durch die Nachfrager auf dem Endabnehmer- und Stromhändlerabsatzmarkt gebildet: Bei steigenden Strompreisen werden diese mittelfristig ihre Stromnachfrage reduzieren. Je schneller und je höher der Preis steigt, desto schneller reduzieren die Nachfrager ihren Stromkonsum. Durch die Nachfrageabsenkung werden Kapazitäten bei den Erzeugern frei, die in den Wettbewerb um die Versorgung eintreten. Gleichzeitig werden neue Anbieter um so schneller auf den Markt drängen je höher die erwartete Rendite für die Investitionen ist. Mit dem Eintreten der Neuanbieter erhöht sich die Erzeugungskapazität über den Strombedarf hinaus und es setzen Wettbewerbsprozesse ein. Faktoren die das Eintreten des Szenarios begünstigen sind:

- Stromnachfragesteigerungen z.B. durch starkes wirtschaftliches Wachstum,

- Stillegung von Kraftwerken,

- mangelnder Zubau von Kraftwerken, 
- lange Bau- und Genehmigungszeiten bei Investitionen in der Elektrizitätswirtschaft,

- Widerstand der Bevölkerung gegen Kraftwerksneubauten.

Szenario II: Niedrige Überkapazitäten und eine geringe Wettbewerbsintensität

Bei niedrigen Überkapazitäten müssen Anbieter mit hohen Elektrizitätspreisen damit rechnen, ihre Kapazität nicht absetzen zu können. Damit werden die Anbieter gezwungen, in einem begrenzten Preiswettbewerb um die Belieferung der Netzgesellschaft einzutreten. Wenn jedoch die Überkapazität auf dem Markt so gering ist, daß sie unterhalb der Produktion eines Erzeugungsunternehmens liegt, haben die Erzeuger, ohne ein Kartell bilden zu müssen, bis zum Eintritt von Neuanbietern Preismanipulationsmöglichkeiten. Der jeweilige Grenzanbieter kann seinen Preis und damit auch den Marktpreis selbst bestimmen ${ }^{437}$.

Bei Eintritt neuer Anbieter in den Markt wird die Erzeugungskapazität erhöht und es setzt ein Wettbewerb ein. Bis zur Stillegung von Kraftwerken oder bis zur Nachfrageausdehnung ist dann das Preisbeeinflussungpotential einzelner großer Erzeuger nicht vorhanden. Begünstigende Faktoren des Szenarios sind:

- der begrenzte Marktzutritt neuer Anbieter,

- die Stagnation des Strombedarfs,

- der verhaltene Ausbau der Kapazitäten bei Anbietern mit niedrigem Kostenniveau.

Szenario III : Hohe Überkapazitäten und eine hohe Wettbewerbsintensität

Anders ist die Situation bei hohen Überkapazitäten. Um Strom absetzen zu können, muß der einzelne Erzeuger andere unterbieten, d.h. er wird bestrebt sein, sein Angebot etwas unter dem erwarteten Grenzanbieterpreis zu plazieren. Hierdurch sinken die Beschaffungspreise und es steigt die Wettbewerbsintensität.

Die langfristige Wettbewerbsintensität hängt von dem Marktzugang neuer Anbieter und dem Verhalten der „etablierten“ Anbieter ab. Der Marktzugang neuer Anbieter erhöht die Kapazitäten auf dem Erzeugermarkt und initiiert bei konstanter Nachfrage einen Wettbewerb um den Stromabsatz. Verlassen Anbieter den Markt, ohne daß neue Anbieter auf den Markt eintreten, sinkt die Wettbewerbsintensität. Ebenso können Anbieter mit niedrigeren Preisen versuchen, ihre Kapazitäten aus-

437 Bei der britischen Umstrukturierung versuchten dies die beiden größten aus der Aufspaltung hervorgegangenen Erzeugerunternehmen Rüdiger Hermann - 978-3-631-75129-9 
zudehnen und den Marktanteil anderer Unternehmen zu übernehmen, wodurch die Wettbewerbsintensität steigt. Fördernde Faktoren sind:

- Strombedarfsstagnation oder -reduzierungen,

- massiver Markteintritt neuer Anbieter,

- der Ausbau bestehender Kraftwerke mit niedrigen Kostenstrukturen,

- Einführung einer kostengünstigen Technologie in der Stromerzeugung, z.B. in der Kraft-Wärme-Kopplung.

Die Überlegungen zu den Szenarien zeigen, daß für die langfristige Entwicklung auf dem Stromhändlerbeschaffungsmarkt die Marktzutrittsbarrieren für Neuanbieter eine entscheidende Einflußgröße sind. Ohne Marktzutrittsbarrieren entsteht unabhängig von der Ausgangssituation Wettbewerb um den Stromabsatz in wiederkehrenden Zyklen. Ohne den Marktzutritt potentieller Neuanbieter werden Wettbewerbsprozesse langfristig eingestellt. Für die Anbieter im Markt bildet sich die Preisobergrenze wie im Szenario I beschrieben. Überkapazitäten zu Beginn der Entwicklungsprozesses bestimmen lediglich die Gewinnerzielungspotentiale und die Preisentwicklungen am Anfang eines Entwicklungsprozesses.

\subsubsection{Die Akzeptanz des Stromhändlerkonzeptes}

Aussagen der an der Diskussion beteiligten Gruppen, die Rückschlüsse über die Akzeptanz des Stromhändlerkonzeptes zulassen, sind bisher wenig erfolgt. Einige Rückschlüsse lassen sich aus den Äußerungen und Ausführungen zum britischen System schließen, ohne jedoch generell übertragbar zu sein.

Die Kommission der Europäischen Union hat sich bisher sehr pauschal für ein Desintegrationskonzept ausgesprochen ${ }^{438}$, ohne eine genaue Ausgestaltung im Blickfeld zu haben. Deshalb kann angenommen werden, daß die Kommission grundsätzlich das Stromhändlerkonzept ähnlich wie das Common-Carrier-Konzept bewerten wird ${ }^{439}$. Aus den Veröffentlichungen zu den Umgestaltungen im Elektrizitätsmarkt kann man darüber hinaus schließen, daß die Kommission das CommonCarrier-Konzept dem Stromhändlerkonzept vorziehen würde, da die Marktmacht der Netzgesellschaft von ihr als potentielle Bedrohung für den Wettbewerb angesehen wird.

438 Vgl. o.V. : EG- und Internationales, in: ET 42. Jg. (1992), Heft 8, S. 558

439 Dieser Schluß wurde aus KOMMISSION DER EUROPÄISCHEN GEMEINSCHAFT Generaldirektion Energic : Energie in Europa - Der Binnenmarkt für Energic, a.a.O. gezogen. 
Die nationalen Regierungen haben sich bisher in der Mehrzahl gegen eine, nicht näher ausgestaltete, Desintegration ausgesprochen ${ }^{440}$. Der Widerstand gegen die einzelnen Konzepte wird jedoch je nach der heutigen Organisation der Elektrizitätswirtschaft unterschiedlich sein. Die Regierungen der Länder mit kontrollierten staatlichen Unternehmen werden das Stromhändlerkonzept weniger als das Common-Carrier-Konzept ablehnen. Eine Stromhandelsgesellschaft mit einer marktkontrollierenden Stellung in wenigstens teilweise öffentlichem Besitz wird diesen Ländern vertrauter vorkommen als eine Situation, bei der keine direkte Einflußnahme einer staatlichen Institution über die gesetzlichen Auflagen hinaus möglich ist. Weiterer Widerstand von den Regierungen ist gegen die geplanten Privatisierungen zu erwarten.

Die stromintensive Industrie, die Wettbewerb im Erzeugerbereich und direkten Zugang zu den Beschaffungsmärkten fordert ${ }^{441}$, wird das Konzept mehrheitlich befürworten. Nachteilig im Vergleich zum Common-Carrier-Konzept wirkt für sie die fehlende Flexibilität in der Lieferantenwahl und in der Ausgestaltung der Lieferverträge. Des weiteren haben die Unternehmen keine Möglichkeiten, die Wettbewerbsintensität auf dem Erzeugermarkt aktiv zu beeinflusssen. Vorteilhaft werden die Unternehmen der stromintensiven Industriezweige die Zuordnung der Versorgungsverantwortung auf die zentrale Netzgesellschaft einstufen, gleichzeitig jedoch die Marktmacht der Netzgesellschaft kritisieren.

Die bestehenden integrierten EVU werden das Konzept massiv ablehnen. Sie sprechen sich gegen jegliche Art der Desintegration aus und werden somit auch das Stromhändlerkonzept, als nicht den Besonderheiten der Branche Rechnung tragend, ablehnen.

Die Klein- und Haushaltsabnehmer werden in ihrer Haltung weitgehend von der Informationspolitik der EVU geprägt werden. Eine sachliche Aufklärung über die Zusammenhänge durch Verbraucherverbände und die Medien könnte ein Ausgleich zu den Äußerungen der EVU in der öffentlichen Diskussion bilden. Dabei ist es besonders wichtig, den Endverbrauchern aufzuzeigen, daß das Stromhändlerkonzept keine Einschränkung der Versorgungssicherheit bedeutet. Bei ausreichender Kenntnis der Zusammenhänge, insbesondere der klaren Versorgungsverantwortungszuweisung, werden sie dem Konzept weitgehend neutral gegenüberstehen. Es verbleibt jedoch ein Rest an Abnehmern, die sich gegen jede Veränderung der Struktur aussprechen.

440 Vgl. MATHIS, C. : a.a.O., S. 55 ff.

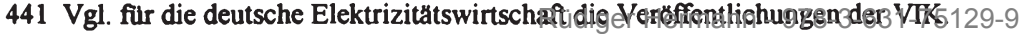




\subsubsection{Der Regulierungsbedarf des Konzeptes}

Der Regulierungsbedarf des Stromhändlerkonzeptes unterscheidet sich für den monopolisierten Verteilerbereich und für die Endversorgerpreise sowie für die Erzeuger nicht von dem des Common-Carrier-Konzeptes. Der Unterschied besteht in der Regulierung der Netzgesellschaft. Als Monopsonist auf dem Stromhändlerbeschaffungsmarkt und als Monopolist auf dem Stromhändlerabsatzmarkt verfügt sie über eine marktbeherrschende Stellung im Elektrizitätsversorgungssystem. Zusammen mit der Betriebsgröße der Netzgesellschaften ergibt sich folgender Regulierungsbedarf:

\section{a) Kontrolle der Koordinationsfunktion}

Hierbei ist die Koordinationsfunktion nicht vollständig zu überprüfen. Kontrolliert werden muß die strategische Koordinierungsfunktion der Netzgesellschaft. Werden von ihr „seriöse“ Elektrizitätsbedarfsprognosen erstellt? Schreibt sie fehlende Kapazitäten aus? Stimmt sie ihre Leitungsinvestitionen mit den Erzeugerunternehmen $\mathrm{ab}$ ? Diese langfristigen Koordinationsaufgaben sind für die Versorgungssicherheit einer Volkswirtschaft von entscheidender Bedeutung. Fehlentwicklungen sind schwer und zumeist nur mit hohen Kosten zu revidieren. Aus diesen Gründen muß die Koordinationsfunktion überwacht werden.

b) Kontrolle der Trennung zwischen Erzeugern, Verteilern und Netzgesellschaft Die Trennung der aus der Desintegration hervorgegangenen Versorgungsbereiche ist erforderlich, um eine organisatorische oder wirtschaftliche Reintegration zu verhindern, die letztlich mit geänderten Rechtsformen den alten Zustand wieder herstellt.

c) Geschäftsführungs- und Betriebskontrolle hinsichtlich deren Wirtschaftlichkeit Monopolunternehmen unterliegen keinem Wettbewerbsdruck, der im Zeitverlauf den Zwang zu Innovationen und Effizienzsteigerungen beinhaltet. Daher müssen diese Unternehmen hinsichtliche ihrer Geschäftsführung überwacht werden und eine Verbesserung der Wirtschaftlichkeit kontinuierlich gefordert werden. In der bisherigen Regulierungspraxis ist dieser Anspruch zumeist nicht erfüllt worden.

d) Preisaufsicht für die Stromtransport- und Koordinierungsleistungen

Die Preisaufsicht in bezug auf die erbrachten Leistungen der stromhandelnden Netzgesellschaft ist erforderlich, um die Ausnutzung ihrer durch das verliehende Monopol entstandenen Marktmacht zu begrenzen.

Als Regulierungsinstitution, die diesen Regulierungsanforderungen entspricht, kommt ausschließlich eine unabhängige Expertenkommission in Betracht. Eine Beteiligung der Erzeuger, Verteiler und Großabnehmer an der Netzgesellschaft würde den Regulierungsbedarf begrenzen $\mathrm{R}_{\mathrm{R}}$ Pie Aufgahe der Regulierungsingtitution 
wäre es dann in erster Linie, die Belange der Klein- und Haushaltsabnehmer zu berücksichtigten. Eingesetzt werden sollte die Regulierungskommission vom europäischen Parlament.

Die Finanzierung der Regulierungsinstitutionen kann durch einen Aufschlag auf die Leistungen der Netzgesellschaft erreicht werden. Der Verfasser schlägt aus den bereits beim Common-Carrier-Konzept ausgeführten Gründen eine Finanzierung über die Stromerzeugungslizenzen oder über die allgemeinen Steuern in der EU vor.

\subsubsection{Die Implementierung des Stromhändlerkonzeptes}

Die Implementierung des Stromhändlerkonzeptes beinhaltet eine Umstrukturierung des gesamten europäischen Elektrizitätsmarktes. Bis auf die Länder, in denen bereits Verteilerunternehmen bestehen, müssen die integrierten Unternehmen in $\mathrm{Er}$ zeugungsunternehmen, Netzgesellschaften und Verteilerunternehmen getrennt werden.

Ausgangspunkt ist die bereits bekannte Struktur ${ }^{442}$ :

\section{Abbildung 41: Ausgangspunkt: Vertikal integriete Unternehmen im Stromhändlerkonzept}

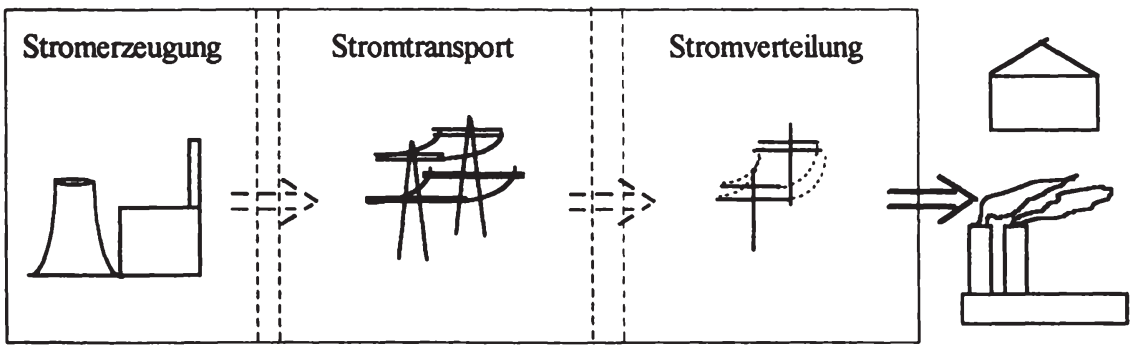

In einem ersten Implementierungsschritt sollte die Desintegration organisatorisch in den integrierten Unternehmen eingeführt werden ${ }^{443}$.

442 Die Legende zu den folgenden Graphiken befindet sich auf Seite 172

443 Dieser Implementierungsschritt ist identisch mit dem ersten des Common-Carrier-Konzeptes. Siehe hierzu Kapitel 7.2.6. 
Abbildung 42: 1. Implementierungsschritt: „unbundling“

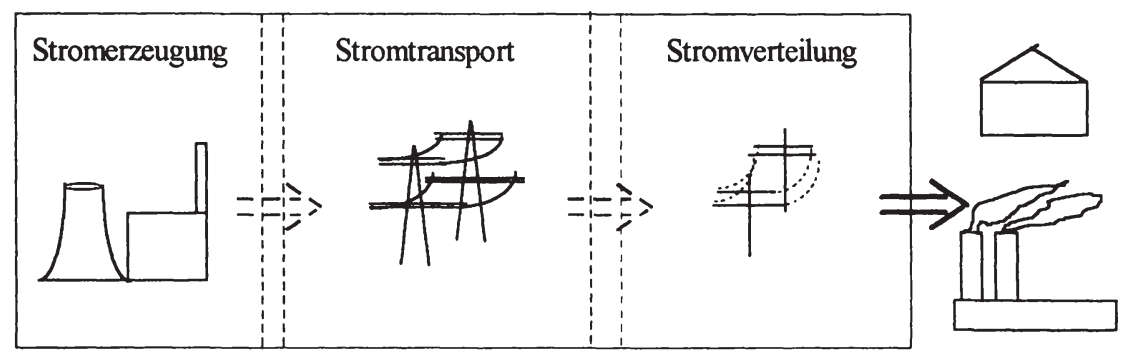

Anschließend werden der Erzeugungsbereich und der Verteilungsbereich von den integrierten Unternehmen abgetrennt und organisatorisch in die späteren Betriebsgrößen untergliedert (zweiter Implementierungsschritt). Hierbei ist eine Betriebsgrößenanpassung der bestehenden Verteilerunternehmen nicht zwingend erforderlich, da zwischen dem Unternehmen und der Netzgesellschaft kein Wettbewerb besteht. Lediglich der vergleichende Wettbewerb würde durch unterschiedliche Verteilerbetriebsgrößen ebenso wie die Regulierung erschwert. Ferner sollte im zweiten Implementierungsschritt der Netzbetrieb mit dem Stromtransportbereich vom Lastmanagement organisatorisch getrennt werden. Die Bezahlung der gelieferten Elektrizitätsmengen oder der bereitgestellten Leistungen erfolgt zunächst zu Verrechnungspreisen.

Im dritten Implementierungsschritt werden die Verteiler- und Erzeugungsunternehmen geschaffen und in die angestrebte Eigentumsform überführt. Die Verrechnungspreise werden durch Marktpreise ersetzt, die sich durch die bereits dargestellten Preisregeln bilden sollen. Gleichzeitig sollte der Marktzugang neuer Anbieter rechtlich ermöglicht werden. Zwischen den Unternehmenssparten des Stromhändlers werden Verrechnungspreise eingeführt. Der Lastmanagementbereich nimmt seine Stromhandelsaktivitäten auf dem eingerichteten Stromhändlerbeschaffungsmarkt auf.

Für die Durchfürung der Implementierungsschritte erscheint dem Verfasser ein Zeitraum von vier bis sechs Jahren angemessen. In diesem Zeitraum sollten feste Abnahmebeziehungen zwischen Erzeuger und Netzgesellschaft die Versorgungssicherheit des Gesamtsystems garantieren. Nach Abschluß der dritten Implementierungsphase können die Bindungen aufgelöst und durch Marktprozesse ersetzt werden. Damit treten die Erzeuger in den beabsichtigten Wettbewerb ein. 
Abbildung 43: 2. Implementierungsschritt: Abtrennung des Erzeuger- und Verteilerbereiches

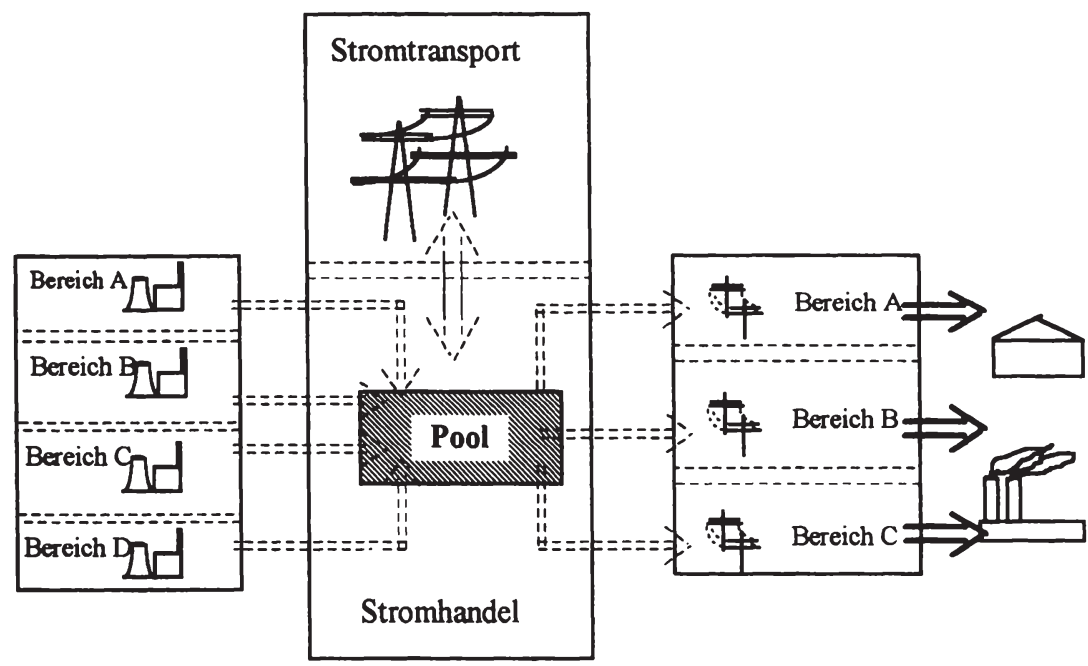

Abbildung 44: 3. Implementierungsschritt: Schaffung der Erzeuger- und der Verteilerunternehmen

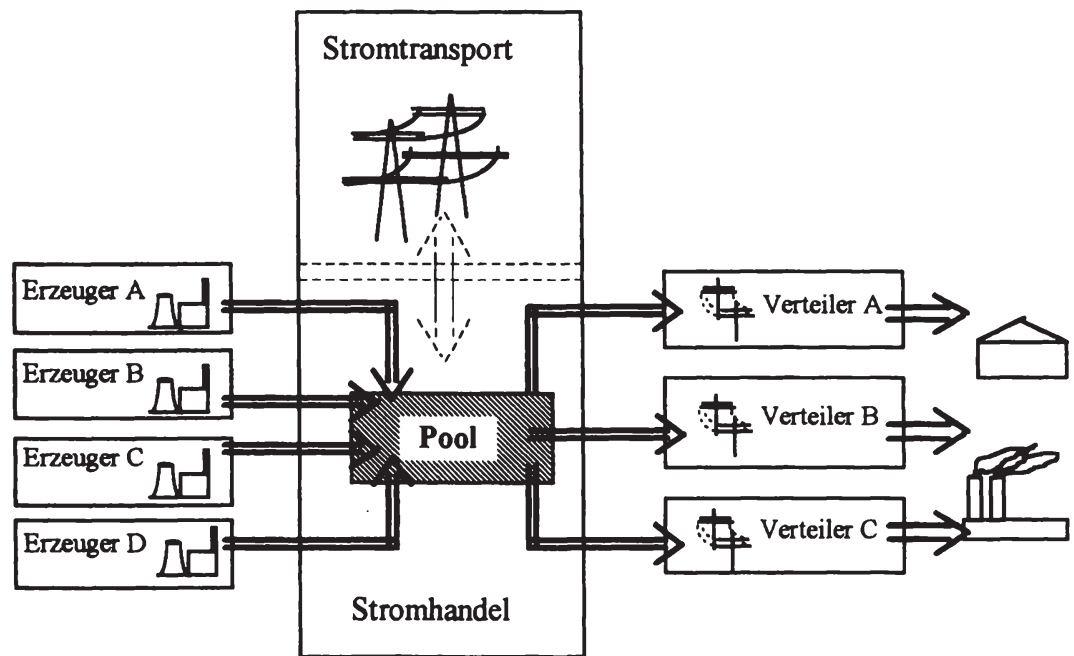


Durch die festen Abnahmebeziehungen kann es in keiner Implementierungsphase zu einer Gefährdung der Versorgungssicherheit kommen. Nach Abschluß der Implementierung geht die Versorgungsverantwortung auf die Netzgesellschaft und die Verteilerunternehmen über. Diese Aussage wird durch die begrenzten empirischen Erfahrungen mit derartigen Umstrukturierungen für die niederländische und britische Elektrizitätswirtschaft bestätigt.

Kosten für die Implementierung werden durch die Aufspaltung und die administrative sowie organisatorische Überführung der bestehenden Unternehmen in die neue Struktur entstehen. Weitere Implementierungskosten resultieren aus der begleitenden Marktaufsicht.

\subsection{Beurteilung der Desintegrationskonzepte}

\subsubsection{Beurteilung der Marktkonzeptionen}

Im Gegensatz zu den Integrationskonzepten ist die Auflösung der geschlossenen Versorgungsgebiete und die Aufspaltung der bestehenden integrierten Unternehmen ein wesentlicher Bestandteil der Desintegrationskonzepte. Unterschiede bestehen bei den beiden Konzepten hinsichtlich des Integrationsgrades. Im CommonCarrier-Konzept besteht für die Nachfrager des Elektrizitätsgroßhandelsmarktes die Möglichkeit, ihren Lieferanten frei zu wählen und durch aktives Handeln Preisvorteile zu erzielen. Ohne die Einführung von Endversorgungsunternehmen können Kleinabnehmer nicht ihren Stromlieferanten frei wählen. Das für die Kleinabnehmer auch bei Umsetzung des Common-Carrier-Konzeptes zuständige Verteilerunternehmen besitzt jedoch diese Option und bestimmt damit letztlich die Preise ihrer Kleinabnehmer.

Im Stromhändlerkonzept bestehen die Wahlmöglichkeiten der Großabnehmer und Verteilerunternehmen nicht; das Stromhändlerkonzept weist damit einen höheren Integrationsgrad als das Common-Carrier-Konzept auf. Deshalb besteht die Gefahr, daß durch den Wettbewerb auf dem Stromhändlerbeschaffungsmarkt bewirkte Erzeugungspreissenkungen über die monopolisierten Zwischenstufen des Stromtransportes und der Stromverteilung nicht den Endabnehmer erreichen.

Stärkere Unterschiede als beim Integrationsgrad weisen die Konzepte bei der Schaffung einer Stromhandelsinfrastruktur auf. Im Conmon-Carrier-Konzept kann bei geeigneter Umsetzung der Konzeption in die elektrizitätswirtschaftliche Realität durch den Pool und die Netzgesellschaften eine funktionsfähige Stromhandelsstruktur aufgebaut werden. Jedem Zugangsberechtigten ${ }_{7}$ steht $_{3}$ dänn dieg Infra- 
struktur zur Verfügung. Exklusive Verfügungsrechte über Teile der Stromhandelsinfrastruktur haben auf dem Großhandelsmarkt und dem Markt fü Aushilfslieferungen weder Nachfrager noch Anbieter, so daß hierdurch keine Wettbewerbsverzerrungen auftreten können. Anders die Situation im Stromhändlerkonzept; eine Stromhandelsinfrastruktur wird bei Verwirklichung des Konzeptes nicht geschaffen. Für die Marktsubjekte auf dem europäischen Elektrizitätsmarkt sind die Stromaustauschbeziehungen festgelegt. Die derartige Koordination des Gesamtmarktes durch die Netzgesellschaft verhindert ein Handeln im kaufmännischen Sinne mit freien Wahlmöglichkeiten.

Durch die Desintegration im Common-Carrier-Konzept entstehen vier Teilmärkte. Auf zweien dieser Teilmärkte, dem Elektrizitätsgroßhandel und dem Markt für Aushilfslieferungen können Wettbewerbsprozesse stattfinden, die Ausgangspunkt für Kosteneinsparungen und Preissenkungen sein können ${ }^{445}$. Dabei vereinfacht die Aufteilung der integrierten Unternehmen die Regulierung: In den verbleibenden Monopolbereichen können Fehlentwicklungen besser erkannt werden als bei den bestehenden integrierten EVU. Dies wirkt kosten- und preisreduzierend. Ein anderer Anreiz zu Preissenkungen kann durch die mit der Desintegration verbundene Änderung der Eigentumsverhältnisse im Common-Carrier-Konzept einhergehen: In zahlreichen EU-Mitgliedsländern werden staatliche Monopole aufgelöst und die entstehenden Unternehmen auf eine genossenschaftliche Basis gestellt oder privatisiert. Die so entstandenen Unternehmen werden weniger als die bisherig staatlichen kostensteigernden Einflußnahmen der jeweiligen nationalen Regierungen ausgesetzt sein. So sind z.B. privatisierte Unternehmen in ihrer Beschäftigungspolitik und in ihren Investitionsmaßnahmen freier. Hierdurch können Kosten gesenkt werden, die dann Auswirkungen auf die Preise haben können.

Im Stromhändlerkonzept entstehen drei Teilmärkte: Der Stromhändlerbeschaffungsmarkt und der Markt für langfristige Stromlieferungen können durch Wettbewerbsprozesse bei den Erzeugerunternehmen Kosten- und Preissenkungen erreichen. Der Stromhändlerabsatzmarkt ist wegen seiner Konstruktion nicht dazu geeignet.

Das Common-Carrier-Konzept baut Marktzutrittsbarrieren ab. Es muß jedoch zwischen den einzelnen Elektrizitätsversorgungsbereichen differenziert werden. Auf den beiden Teilmärkten, in denen Wettbewerbsprozesse stattfinden sollen dem Elektrizitätsgroßhandelsmarkt und dem Markt für Aushilfslieferungen - werden die Marktzutrittsbarrieren deutlich reduziert. Lediglich die bestehenden, zumeist abgeschriebenen Kraftwerke stellen für Neuanbieter eine Marktzutrittsbar-

445 Dies bestătigen die Australischen Erfahrungen. Vgl. SCHOLL, R. : Reform der Elektrizitătsindustrie in Australien..., a.a.O., S. 386. So ist die Arbeitsproduktivităt um $80 \%$ im FünfJahresvergleich gestiegen. Gleichzeitig konnte die Zahl der Beschäftigten um über $\mathbf{4 5 \%}$ reduziert werden. 
riere dar, die sich jedoch im Zeitverlauf relativiert: Ab einem kraftwerksspezifisch festgelegten Nutzungszeitraum steigen die Durchschnittskosten der bestehenden Kraftwerke wieder an, da Ersatz- oder Modernisierungsinvestitionen erforderlich werden ( $\mathrm{ab}$ dem Zeitpunkt t2 in der Abbildung 45). Bei weiterem Betrieb überschreiten die Durchschnittskosten der bestehenden Kraftwerke die Durchschnittskosten eines neuen Kraftwerken ( $\mathrm{t} 3$ ).

\section{Abbildung 45: Durchschnittskostenverlauf eines bestehenden und eines zu er- richtenden Kraftwerks}

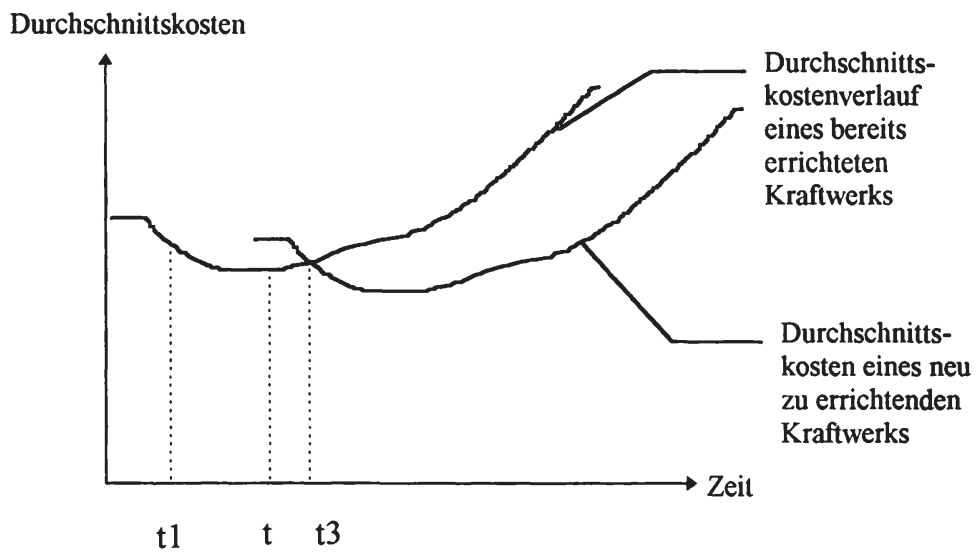

Die in vielen Mitgliedsländern bestehenden Marktzutrittsbarrieren für dezentrale Erzeugungsanlagen werden durch die Aufhebung der Auschließlichkeitsrechte im Common-Carrier-Konzept verschwinden. Neue Anbieter können Strom verkaufen, ohne an eine bestimmte Kraftwerksgröße gebunden zu sein. Dezentrale Anlagen konkurrieren direkt mit zentralen. Zudem können potentielle Neuanbieter durch die vorgesehenen regelmäßigen Veröffentlichungen der Preise auf dem Spotmarkt sowie der Lastflüsse im Stromtransportnetz ihr Absatzpotential abschätzen. Ferner ist der Zugang zu Beschaffungsmärkten für alle bestehenden und neuen Anbieter im Common-Carrier-Konzept gleich, so daß auch hieraus keine Marktzutrittsbarrieren resultieren.

Auf den weiterhin monopolisierten Teilbereichen der Elektrizitätsversorgung ist ein Marktzutritt konzeptionell ausgeschlossen. Lediglich der bereits angesprochene Ausschreibungswettbewerb um den Betrieb eines Verteilungsgebietes könnte die bestehenden Marktzutrittsbarrieren abbauen. 
Bei Umsetzung des Stromhändlerkonzeptes bestehen im Verteiler- und Stromtransportbereich nach der Einführung des Konzeptes weiterhin von Marktexternen nicht zu überwindende Markteintrittsbarrieren. Lediglich auf dem Erzeugermarkt können Neuanbieter auftreten und Wettbewerbsprozesse initiieren, die zu Preissenkungen bei den Verbrauchern führen können. Für diese Neuanbieter ist neben den staatlichen Auflagen zur Errichtung und zum Betrieb eines Kraftwerks die Anschlußkapazität der stromhandelnden Netzgesellschaft langfristig eine Marktzutrittsbarriere. Weitere Marktzutrittsbarrieren entstehen wie beim Common-Carrier-Konzept durch bereits abgeschriebene Kraftwerke. Marktzutrittsbarrieren aus asymmetrischen Informationen entstehen im Stromhändler-Konzept nicht.

Wettbewerb kann im Common-Carrier-Konzept auf dem Großhandelsmarkt und dem Markt für Aushilfslieferungen entstehen. Die Einflußfaktoren der Wettbewerbsintensität auf diesen Märkten sind:

\section{a) Die Anzahl der Anbieter und Nachfrager}

Die Anzahl der Anbieter auf dem Großhandelsmarkt direkt nach der Implementierung des Konzeptes wird bestimmt durch die Anzahl der aus der Desintegration hervorgegangenen Unternehmen. Die Anzahl der Konkurrenten auf dem Markt ergibt sich aus den Erzeugerabgabepreisen und den erforderlichen Stromtransportkosten. Deshalb wird an jedem Abnahmeort in Europa die Anzahl der Wettbewerber unterschiedlich sein. Jede Erzeugungspreisänderung hat gleichzeitig Auswirkungen auf die Anzahl der Wettbewerber. Ferner können neue Anbieter durch die bereits angesprochenen niedrigen Marktzutrittsbarrieren in den Wettbewerb eintreten, wodurch sich die Zahl der Wettbewerber erhöht. Die Zahl der Nachfrager ist durch die Zugangsberechtigung zum Großhandelsmarkt bestimmt. Neue Nachfrager können nur dann entstehen, wenn die Mindestabnahmenmengen auf dem Elektrizitätsgroßmarkt reduziert werden oder die Abnahmemenge einzelner Verbraucher über die Mindestabnahmemenge des Großmarktes angestiegen ist.

\section{b) Das Verhältnis aus Angebot und Nachfrage}

Überschreitet das Angebot die Nachfrage, sinken die Erzeugerpreise. Entspricht die Nachfrage der Verteiler und Großabnehmer dem Angebot, bleibt der Preis konstant. Merken die Erzeuger, daß die bestehenden Reservekraftwerke immer häufiger zum Einsatz kommen, können sie die Preise anheben. Als Folge sinkt tendenziell die Nachfrage. Ferner werden durch die niedrigen Marktzutrittsbarrieren und die steigenden Preise entweder neue Anbieter aktiv oder bestehende Anbieter dehnen ihre Kapazitäten aus, die, wenn auch zeitversetzt, das Angebot steigern und damit wieder Preissenkungen initiieren. Damit steuert der Marktmechanismus Angebot und Nachfrage und erfüllt eine seiner wesentlichsten Funktionen. 
c) Die Stromtransportgebühren und das Funktionieren der

Stromhandelsinfrastruktur

Die für die Inanspruchnahme der Infrastruktur von den Nutzern zu zahlenden Entgelte sind für die Wettbewerbsintensität bedeutend. Nur wenn die Stromtransportleistungen innerhalb des Binnenmarktes zu Preisen bereitgestellt werden, die Stromlieferungen von anderen Erzeugern ermöglichen, können Stromerzeugungspreisunterschiede von den Abnehmern genutzt werden.

Ein Problem ist die langfristige Wettbewerbsintensität. Verhalten sich die Erzeuger risikoavers und gehen zu abgestimmten Verhaltensweisen über, so können sie die Preise im Common-Carrier-Konzept beeinflussen und die Wettbewerbsintensität reduzieren. Langfristige Verträge zwischen Erzeugern und Abnehmern können diesen Trend unterstützen, insbesondere, wenn der Abnehmer nach Auslaufen des Vertrages keinen anderen Anbieter findet, der die gewünschte Kapazität bereitstellen kann. Aus diesem Grund erscheint dem Verfasser ein Anteil der langfristigen Lieferverträge am Großhandelsmarktvolumen von $70 \%$ und die Abwicklung der verbleibenden $30 \%$ über den Spotmarkt als maximales Verhältnis, um intensive Wettbewerbsaktivitäten auf der Erzeugerseite zu sichern. Der Spotmarktanteil kann bei einem hohen Sicherheitsbedürfnis der Abnehmer durch Optionsverträge abgesichert werden. Steigt der Anteil der langfristigen Verträge über den Wert hinaus, geht die Wettbewerbsintensität deutlich zurück und die Zielerreichung wird gefährdet. Gleichzeitig sollte die Laufzeit der langfristigen Lieferverträge auf acht bis zehn Jahren begrenzt werden, um keine, den deutschen System ähnliche, Demarkationsverträge zustande kommen zu lassen. Trotz dieser Gefahren für die langfristige Wettbewerbsintensität muß beim Common-Carrier-Konzept festgehalten werden, daß es bei geeigenter Aufteilung der bestehenden integrierten Unternehmen inhärent zu Wettbewerbsprozessen kommt, die Ausgangspunkt für Effizienzsteigerungen sein können. Das natürliche Bestreben der Wettbewerber nach Ausschluß der Konkurrenten muß durch eine Kartellaufsicht begrenzt werden. Dieses ist jedoch europäische Normalität.

Das Stromhändlerkonzept bietet eine gute Ausgangsposition für eine hohe Wettbewerbsintensität auf dem Stromhändlerbeschaffungsmarkt ${ }^{446}$. Neuanbieter können jederzeit versuchen, Kraftwerke zu errichten und ihren Strom an die Netzgesellschaft zu verkaufen. Alle Erzeugungsunternehmen, die an die gleiche Netzgesellschaft angeschlossen sind, stehen in direkter Konkurrenz zueinander. Bei Überkapazitäten ist ein Absatz der eigenen Kraftwerke nur durch eine Verdrängung eines anderen Anbieters realisierbar. Bei steigender Nachfrage können die Anbieter temporär höhere Preise erzielen, was neue Anbieter auf den Markt drängt und somit

446 Dies bestătigt das britische Vorbild. Die Akteure haben heftig um die Marktanteile gerungen. Vgl. hierzu KLOPFER, Th. : Die Elektrizitätswirtschaft in England und Wales Zwischenbilanz, in: Zeitschrift für Energiewirtsgaft, Heft tan 1993.78.26031-75129-9 
die Preise wieder sinken läßt. Je nach Wahl des Erzeugerabgabepreismodells wird der Nutzen der Umstrukturierung zwischen Erzeuger und Abnehmer aufgeteilt. Der größte Anreiz zu Effizienzsteigerungen und einer aktiven Teilnahme am wettbewerblichen Marktgeschehen geht für die Erzeugerunternehmen von der Anwendung der Grenzanbieterpreisformel aus.

Negative Auswirkungen auf die Wettbewerbsintensität im Stromhändlerkonzept haben die Bauzeiten von Kraftwerken. Bei knappen Angebotskapazitäten steigt die Wettbewerbsintensität erst an, wenn die errichteten Kapazitäten von Neuanbietern oder die erweiterten Kapazitäten von bestehenden Anbietern auf dem Markt verfügbar sind. In der Anfangsphase ist die Ausgangslage bei den Erzeugungskapazitäten für die Wettbewerbsintensität entscheidend; werden durch die Aufteilung der Erzeugungskapazitäten auf neue Erzeuger einige dieser Unternehmen mit einer Betriebsgröße ausgestattet, die bei ihnen die Möglichkeit zur Preismanipulation auf dem Spotmarkt entstehen läßt, sinkt die Wettbewerbsintensität. Die Verteilerund etwaige Versorgungsunternehmen sowie die Netzgesellschaft können den Preismanipulationen nicht mit marktwirtschaftlichen Mitteln entgegenwirken ${ }^{447}$. Langfristig steigt die Wettbewerbsintensität durch Marktzutritte neuer Erzeuger wieder an. Aus diesem Grund sind die negativen Auswirkungen fehlender Kapazitäten und fehlender Anbieter zeitlich begrenzt. Der Endverbraucher- und Verteilermarkt sowie die Bereitstellung der Stromtransport- und Stromhandelsdienstleistungen verbleiben in den Händen von Monopolunternehmen. Wettbewerb ist somit auf diesen Teilmärkten ausgeschlossen.

Die Unternehmen und Nachfrager auf Märkten versuchen durch die Ausdehnung ihrer Marktmacht Preisbeeinflussungspotentiale zu erlangen, die sie für ihre Ziele einsetzen können. Für den Großhandelsmarkt und den Markt für Aushilfslieferungen des Common-Carrier-Konzeptes gilt dies in gleichem Maße: So stellt das Ausdehnungsbestreben der geschaffenen Erzeugerunternehmen eine erste Gefahr dar. Dieses Bestreben ist ein integraler Bestandteil eines Wettbewerbsmarktes und daher nicht negativ zu werten, solange keine oder nur niedrige Marktzutrittsbarrieren auf dem Markt existieren. Konzeptionell kann immer dann ein neuer Erzeuger auf den Markt zu drängen, wenn ein im Markt befindlicher Erzeuger versucht, seine Marktmacht zu Preisanhebungen zu benutzen. Der seine Marktmacht ausnutzende Erzeuger hat jedoch den Vorteil, daß neue Anbieter erst Kraftwerke errichten müssen. Bis dahin werden die Preisanhebungen durch entfernte Lieferanten begrenzt, die vor den Preisanhebungen wegen der Stromtransportkosten, die die Anbieter der Netzgesellschaft im Common-Carrier-Konzept zahlen müssen, keinen Zugang zu dem regionalen Markt finden konnten.

447 Eine Aufnahme der Eigenerzeugung als Konkurrenz zu dem Fremdbezug ist den Verteilerund Erzeugungsunternehmen durch die Forderung der vollstăndigen vertikalen Trennung untersagt. 
Noch günstiger für eine hohe Wettbewerbsintensität und eine geringe Marktmacht des einzelnen Anbieters ist die Situation auf dem Markt für Aushilfslieferungen. Potentielle Anbieter auf diesem Markt können alle Erzeuger sein, deren Kapazitäten nicht ausgelastet sind. Marktzutrittsbarrieren für die bestehenden Erzeuger gibt es auf diesem Markt nicht, so daß eine hohe Marktmacht der einzelnen Anbieter und Nachfrager nicht entstehen kann. Langfristig können einzelne Erzeuger ihre Marktmacht durch Spezialisierung ausdehnen, die begrenzt wird durch die jederzeitige Marktzutrittsoption der Erzeuger aus dem Großhandelsmarkt.

Die Marktmacht der Verteilerunternehmen auf dem Großhandelsmarkt wird durch die bei der Implementierung des Common-Carrier-Konzeptes zugewiesenen Nachfrage eines Versorgungsgebietes bestimmt. Langfristig könnte jedoch ein extremes Wachstum der Endabnehmernachfrage die Marktmacht eines Verteilers steigern. Die Begrenzung der daraus resultierenden Effekte obliegt der auch in anderen Wirtschaftszweigen üblichen Kartellaufsicht.

Zusammenfassend kann somit festgehalten werden, daß im Vergleich zur gegenwärtigen Situation die Marktmacht jedes Untermehmens im Common-Carrier-Konzept gering ist. Kein Unternehmen verfügt über ein vollständiges Monopol in der Elektrizitätsversorgung. Die entstehenden Erzeuger initiieren einen Marktmacht begrenzenden Wettbewerb ${ }^{448}$.

Die Marktmacht ${ }^{449}$ des einzelnen Anbieters auf dem Beschaffungsmarkt des Stromhändlerkonzeptes hängt von seinem relativen Anteil an der Gesamtstromerzeugungskapazität eines Netzgesellschaftsgebietes und der Angebotssituation auf dem dazugehörigen Stromhändlerbeschaffungsmarkt ab. Gelingt es einem Erzeuger durch seine Angebotsabgabe gezielt zum Grenzanbieter ${ }^{450}$ zu werden, bestehen furr ihn Strompreismanipulationsmöglichkeiten. Diese werden kurzfristig nicht durch den Marktzugang neuer Anbieter oder die Ausdehnung der Kapazitäten bestehender Anbieter begrenzt. Versuchen nun Neuanbieter auf den Markt zu gelangen, wird das etablierte Unternehmen versuchen, Neuanbieter durch Absenkung des Preises wieder aus dem Markt drängen. Ob sich nun die etablierten oder neue Anbieter durchsetzen, hängt von der Summe der Unternehmensressourcen und der langfristigen Kostenstrukturen ab: Der Neuanbieter gelangt nur zum Absatz seiner Kapazitäten im Stromhändlerkonzept, wenn seine langfristigen Durchschnittskosten niedriger sind als die langfristigen Grenzkosten der etablierten Unternehmen

448 Und erfüllen hierdurch die zweite Komponente des Wettbewerbsbegriffes von STREIT in Kapitel 3.

449 Auf die Marktmacht der Netzgesellschaft oder der Verteilerunternehmen soll an dieser Stelle nicht eingegangen werden, da sie durch ihre Monopolstellung über die dominierende Marktmacht verfügen. Diese wird ausschlicßlich durch die Konzeption und die Regulierungsinstitutionen begrenzt.

450 Hierbei muß nicht zwingend die Grenzanbieterpreisregel eingeführt werden. Der Grenzanbieter ist auch bei anderen Preisfindungsprinzipien der letzte Anbieter, dessen Kapazităt zur Bedarfsdeckung herangezogen wird. Rüdiger Hermann - 978-3-631-75129-9 
mit weitgehend abgeschriebenen Anlagen. Das Unternehmen, welches im Wettbewerb andere Unternehmen verdrängt hat, kann nach Marktaustritt der Konkurrenten den Preis anheben. Dieser Prozeß wiederholt sich ständig. Somit kann über eine längere Zeitperiode betrachtet Marktmacht eines Erzeugungsunternehmens entstehen, die unter Wettbewerbsgesichtspunkten nicht negativ bewertet werden muß. Die Relevanz des Problems der Preismanipulationen zeigte sich auf dem britischen Strommarkt: Da nur drei Unternehmen aus der Aufteilung des britischen Netz- und Erzeugungsunternehmens (CEGB ${ }^{451}$ ) hervorgegangen und dabei die Erzeugungskapazitäten nicht gleichmäßig verteilt worden sind, konnten die beiden größten Erzeugungsgesellschaften auf dem britischen Strommarkt die Strompreise in begrenztem Umfang manipulieren ${ }^{452}$. Durch das Konzept vorgesehen ist jedoch die Schaffung einer Vielzahl von Erzeugerunternehmen innerhalb eines Netzgebietes. Des weiteren bestehen in den Mitgliedsländern Überkapazitäten in der Stromerzeugung. Deshalb wird es keinem Erzeuger nach der Implementierung des Stromhändlerkonzeptes gelingen, den Grenzanbieterpreis zu beeinflussen. Somit ist die Marktmacht zumindest in der Anfangsphase der Unternehmen gering. Langfristig wird die Marktmacht der Unternehmen durch die Marktzutritte neuer Anbieter begrenzt ${ }^{453}$.

Die Marktmacht der Netzgesellschaft im Stromhändlerkonzept wird durch die Preisbildungsregel vorgegeben. Bei einer konsequenten Anwendung der Grenzanbieterpreisregel mit vollständig automatisierter Angebotsabgabe durch die Erzeuger besitzt die Netzgesellschaft als Monopsonist keine Marktmacht in bezug auf den Strompreis.

451 Vergleiche zur Bedeutung des Marktzutritts von Neuanbietern für dic Preisgestaltung der bestehenden Anbieter Kapitel 3.

452 National Power und PowerGen. Vgl. KLOPFER, T. : Die Elektrizitătswirtschaft ..., a.a.O., S. 264. Zum Marktanteil der beiden Unternehmen: COLLIER, J.G. : 1994 Starting A New Era for Nuclear Power (überarbeitete Fassung eines Vortrages auf der Jahrestagung des Deutschen Atomforums e.V. am 26.01.94 in Bonn) abgedruckt in: atomivirtschaft, Mărz 94. S. $183 \mathrm{ff}$. und o.V. : Any Offers?, in: Energy Economist, 3/94. S. $20 \mathrm{ff}$.

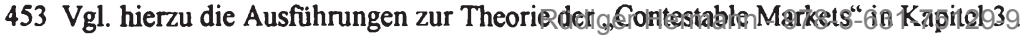




\subsubsection{Der Beitrag der beiden Desintegrationskonzepte zur Zielerfüllung}

\subsubsection{Reduzierung der Kosten der Stromversorgung}

\subsection{Kostenentwicklung bei Umsetzung des Common-Carrier- Konzeptes}

Gegen das Common-Carrier-Konzept wurde zumeist von seiten der bestehenden EVU angeführt, es steigere die Kosten der Elektrizitätsversorgung. Einen ersten Anhaltspunkt für die Stichhaltigkeit dieser Aussage gibt die Argumentation der deutschen EVU zur Begründung der realen Preisdifferenzen zur französischen $\mathrm{EdF}^{454}$. Die Vertreter der EVU führen eine ganze Reihe von Ursachen auf, geben als Ursache aber nicht die technisch und organisatorisch desintegrierte Struktur in einigen Teilen Deutschlands $a^{455}$. Wenn jedoch der technische und organisatorische Desintegrationsgrad kein kostenverursachender Faktor ist, kann von ihm keine Verteuerung bei Einfuhrung des Common-Carrier-Konzeptes ausgehen.

Näher betrachtet ergibt sich folgendes Bild: In der Stromerzeugung erreicht das Common-Carrier-Konzept erstmalig in der Elektrizitätswirtschaftsgeschichte die Stillegung nicht ausgelasteter Kraftwerke durch die Einführung eines Preiswettbewerbes $^{456}$. Bisher hatten die Kraftwerks-betreibenden EVU die Möglichkeit, die durch die nicht ausgelasteten Kraftwerke entstehenden Fixkosten dem Verbraucher aufzuerlegen. Dies läßt die Konzeption des Common-Carrier-Konzeptes nicht zu: Jeder Erzeuger oder potentielle Neuanbieter muß beim Kraftwerksbau die Rentabilität des Kraftwerks berücksichtigen und trägt das unternehmerische Investitionsrisiko. Hierdurch kommt es bereits kurzfristig zu Kosteneinsparungen. Langfristig werden diese Einsparungen noch verstärkt: Bestehende Kraftwerke werden in ihrer Preisobergrenze durch die Durchschnittskosten neuer Kraftwerke anderer Anbieter begrenzt. In der Anfangsphase des Konzeptes besitzen die bestehenden Erzeuger den Vorteil bereits abgeschriebener Anlagen. Dieser Vorteil relativiert sich mit zunehmender Dauer.

Die Stromtransportkosten werden durch den erhöhten Stromtransportbedarf der Anbieter und Nachfrager absolut steigen. Der erhöhte Stromtransportbedarf entsteht, weil Verbraucher und Verteiler ihren Strom bei ausreichenden Preisvorteilen von weiter entfernten Kraftwerksstandorten beziehen können. Die bestehende Be-

454 Vgl. SCHMIDT, D. : Im Grundlastbereich könnte Importkohle mit Atomkraft schon fast konkurrieren, in: Handelsblatt, 19.5.1987

455 Die Integration wird in diesen Teilen der Bundesrepublik über Demarkations- und Konzessionsvertrăge erreicht.

456 Hagenmeyer sicht den Preiswettbewerb als einzige Form des Wettbeiverb auf Elektrizitătsmärkten an. Vgl. HAGENMEYER. E. : Elektrizitätswirtschaft an der Jahrhundertwende ..., a.a.O. , S. 1268 
grenzung auf Standorte eines festgelegten Versorgungsgebietes entfällt. Zur Berechnung der Kostenentwicklung durch die Implementierung des Common-CarrierKonzeptes müssen nach der Einführung von den Stromtransportkosten die vorher vorhandenen abgezogen werden ${ }^{457}$.

Für den Verteilerbereich hat das Common-Carrier-Konzept keine Auswirkungen auf die Kosten. Die weiterhin mit einem Verteilungsmonopol in einem Gebiet ausgestatteten Unternehmen müssen auch im Common-Carrier-Konzept die zugewiesenen Aufgaben in der flächendeckenden Stromversorgung erbringen. Ohne Einführung weiterer Wettbewerbskomponenten bestehen im Common-Carrier-Konzept keine Anreize zu Kosteneinsparungen oder Ausdehnungen. Wird dagegen ein Ausschreibungswettbewerb um den Betrieb eines Verteilerunternehmens eingeführt, so verändert dies die Situation. Die Betreiberunternehmen konkurrieren über die Betriebskosten miteinander, wodurch positive Auswirkungen auf die Verteilungskosten erwartet werden können. Wird dieser Effekt wegen seiner mangelnden Quantifizierbarkeit ausgeklammert, können die Kosteneinsparungen in der Stromversorgung durch Einführung des Common-Carrier-Konzeptes wie folgt formal dargestellt werden:

$$
\begin{aligned}
& \Delta \mathrm{K}_{\mathrm{GM}}=\sum \mathrm{Kv}_{\mathrm{A}}-\left(\sum \mathrm{Kv}_{\mathrm{N}}+\sum \mathrm{K}_{\mathrm{T}}+\Delta \sum \mathrm{K}_{\mathrm{T}}\right)+\Delta \mathrm{K}_{\mathrm{FGM}} \\
& \text { mit : } \mathrm{K}_{\mathrm{GM}}=\text { Gesamtkosten der Elektrizitäts - } \\
& \text { versorgung in der EU } \\
& \mathrm{Kv}_{\wedge} \text { = variable Stromerzeugungskosten der "alten" } \\
& \text { merit order } \\
& \mathrm{Kv}_{\mathrm{N}}=\text { variable Stromerzeugungskosten der "neuen" } \\
& \text { merit order } \\
& \mathrm{K}_{\mathrm{T}}=\text { variable Stromtransportkostensalden } \\
& \mathrm{K}_{\mathrm{FT}}=\text { Fixkosten des Stromtransports } \\
& \mathrm{K}_{\mathrm{Fom}}=\text { Gesamtfixkosten }
\end{aligned}
$$

Bisher bestimmen staatliche Auflagen und das EVU das Versorgungssicherheitsniveau jedes Klein- und Mittelabnehmers. Lediglich Großabnehmer haben die Möglichkeit, ein individuelles Versorgungssicherheitsniveau mit ihrem EVU zu vereinbaren; eine Überprüfung des Versorgungssicherheitsniveaus für die Klein- und Mittelabnehmer wurde bisher in keinem Mitgliedsstaat vorgenommen. Das Postulat einer vollkommenen Versorgungssicherheit zu jedem Preis wird im Common-Car- 
rier-Konzept zugunsten eines Systems aufgegeben, das dem einzelnen Teilnehmer die individuelle Wahl seines Versorgungssicherheitsniveaus ${ }^{458}$ ermöglicht. In erster Linie werden Mittel- und Großabnehmer diese Option nutzen, um durch eine gezielte Reduzierung ihres Versorgungssicherheitsniveaus, ihre Strombezugspreise senken zu können. Hierdurch können Kosten für ein nicht nachfragekonformes Versorgungssicherheitsniveau eingespart werden:

$$
\begin{aligned}
& \Delta \mathrm{K}_{\mathrm{GM}}=\sum \mathrm{Kv}_{\mathrm{A}}-\left(\sum \mathrm{Kv}_{\mathrm{N}}+\sum \mathrm{K}_{\mathrm{T}}+\Delta \sum \mathrm{K}_{\mathrm{FT}}\right)+\Delta \mathrm{K}_{\mathrm{FGM}}+\Delta \mathrm{KvS}_{\mathrm{R}} \\
& \text { mit : } \mathrm{K}_{\mathrm{GM}}= \text { Gesamtkosten der Elektrizitäts - } \\
& \text { versorgung in der EU } \\
& \mathrm{Kv}_{\mathrm{A}}= \text { variable Stromerzeugungskosten der "alten" } \\
& \text { merit order } \\
& \mathrm{Kv}_{\mathrm{N}}= \text { variable Stromerzeugungskosten der "neuen" } \\
& \text { merit order } \\
& \mathrm{K}_{\mathrm{T}}= \text { variable Stromtransportkostensalden } \\
& \mathrm{K}_{\mathrm{FT}}= \text { Fixkosten des Stromtransports } \\
& \mathrm{K}_{\mathrm{FoM}}= \text { Gesamtfixkosten } \\
& \mathrm{KvS}_{\mathrm{R}}= \text { Gesamtfixkosten der Versorgungssicherheitsbereitstellung }
\end{aligned}
$$

Durch die Auflösung der geschlossenen Versorgungsgebiete sinkt der Grad der Integration; es entstehen Teilmärkte, die die vormals unternehmensinterne Koordinationsfunktion übernehmen müssen. Durch die marktliche Koordination fallen für kurzfristige und langfristige Stromlieferverträge Transaktionskosten an.

Auf dem Markt für kurzfristige Stromlieferungen (Spotmarkt) sind in erster Linie die Kosten für die Angebotsabgabe der Erzeuger und der Nachfragemengenabgabe der Verteiler und Großverbraucher beim Pool zu nennen. Hinzu kommen die Poolkosten zur Abwicklung des Spotmarktes und zur Abstimmung der Stromlieferungen mit den Netzgesellschaften. Informations- und Suchkosten für die Anbieter und Nachfrager entstehen nicht. Die Anbieter und Abnehmer kennen jedoch nicht den tatsächlichen Preis im vorhinein. Eine Möglichkeit, die Auswirkungen der Informationsdefizite in bezug auf den Spotmarktpreis zu begrenzen, ist der Abschluß von Optionsverträgen. Die Optionsentgelte der Unternehmen sind monetarisierte Transaktionskosten der Marktteilnehmer.

458 Dies ist nicht gleichzusetzen mit einem Verzicht auf Versorgungssicherheit. Ein Unternehmen kann z.B. mit seinem EVU vereinbaren, eigene Stromerzeugungsaggregate nach einer Mitteilung des EVUs einzusetzen oder kurzzeitig stromintensive Anwendungen zeitlich zu verlegen. 
Informations- und Suchkosten sind bei dem Markt für langfristige Stromlieferungen systemimmanent. Alle Marktteilnehmer müssen zur Bedarfsdeckung Informationen über Preise, Mengen, Versorgungssicherheitsniveaus etc. einholen und damit ihre Entscheidungen hinsichtlich der Strombedarfsdeckung treffen. Für Abnehmer und Nachfrager besteht das Problem der unvollständigen Information. Bei ihrer Entscheidung kennen sie den genauen Preis des Spotmarktes für den angestrebten Zeitraum nicht; der Preis furr langfristige Stromlieferungen ist dagegen ebenso gewiß wie die Durchführbarkeit der Stromlieferung durch die Netzgesellschaft, was zu einem Optimierungsproblem unter Ungewißheit führt. Die Optionsund langfristigen Lieferverträge garantieren nur einen Preis und kein bestimmtes Versorgungssicherheitsniveau. Die Versorgungssicherheitsdienstleistung muß auf einem weiteren Markt mit den daraus resultierenden Transaktionskosten eingekauft werden. Für den Markt für Versorgungssicherheitsleistungen gilt folgender Zusammenhang: Je individualisierter das gewünschte Versorgungssicherheitsniveau des jeweiligen Abnehmers ist, desto höher sind die damit verbundenen Transaktionskosten auf diesem Teilmarkt.

Die Höhe der auf allen Märkten anfallenden Transaktionskosten kann durch technische und organisatorische Instrumente reduziert werden ${ }^{459}$. So könnten z.B. Makler auf dem Markt für langfristige Stromlieferverträge die Transaktionskosten der Marktteilnehmer reduzieren ${ }^{460}$. Den Einsparungen muß jedoch die Maklerprovision entgegengestellt werden. Die gleiche Wirkung haben technische Installationen wie eine automatisierte Angebots- und Nachfrageangabe an den Pool und eine elektronische Informationsbörse über Optionsvertragsangebote.

Zusammenfassend kann festgehalten werden, daß Transaktionskosten zur Koordination des Gesamtversorgungssystems auf allen Märkten anfallen; ein Vergleich mit den bestehenden Transaktionskosten der unternehmensinternen Koordination kann wegen mangelnder Informationen über die derzeitigen Kosten nicht erbracht werden. Tendenziell können jedoch steigende Transaktionskosten angenommen werden.

Die Betrachtung der Regulierungsbedarfe im Common-Carrier-Konzept deutet auf hohe Regulierungskosten hin. Vergleicht man den erforderlichen Regulierungsaufwand jedoch mit dem der bestehenden integrierten Strukturen, so stellt man dessen Reduzierung fest:

459 Eine Maßnahme ist dic Optimierung der Wirk- und Blindleistung in Netzes. Vgl. HEINZ. D. : Kostenminimaler Wirk- und Blindleistungshaushalt in Hoch- und Höchstspannungsnetzen in: EW Jg. 93 (1994), Heft 7, S. 313 ff. und die Dissertation von BÜTTGENBACH, S. B. : Erweiterter Einsatz der Spannungs-Blindleistungs-Optimierung im Verbundsystem. RWTH Aachen 1992

460 Hagenmeyer geht nicht so weit. Er prognostiziert die Maklertătigkeit nur auf den Erzeugermărkten. Vgl. hierzu HAGENMEYER, E. : Elektrizitătswirtschaft an der Jahrhundertwenden ..., a.a.O., S. 1268 
Während bisher die vollständige Regulierung der Elektrizitätsversorgung erforderlich ist, entfällt im Common-Carrier-Konzept die Preisregulierung auf dem Erzeugungsmarkt und dem Markt für Aushilfslieferungen. Gleichzeitig sinkt durch die Desintegration die Möglichkeit für die verbleibenden Monopolbereiche, über interne Verrechnungspreise Gewinne zu verschleiern oder von einem Geschäftsbereich auf einen anderen zu transferieren ${ }^{461}$. Darüber hinaus wird die Regulierung durch die Aufteilung der Gesamtversorgung in abgegrenzte Teilbereiche erleichtert, wodurch die Kosten für die Regulierung sinken. Somit kann zusammengefaßt werden, daß die Kosten der Regulierung durch Einführung des Konzeptes, unabhängig von der absoluten Höhe und den Verlusten durch eine nicht funktionierende Regulierung, deutlich reduziert werden.

Die abschließende und zusammenführende Betrachtung der Gesamtkostenentwicklung durch die Einführung des Common-Carrier-Konzeptes läßt deutliche Kosteneinsparungspotentiale erkennen. Zwingende Voraussetzung für reale Kosteneinsparungen im Erzeugerbereich ist die Minimierung der Transaktionskosten durch geeignete Mittel und Instrumente sowie eine funktionierende Netzgesellschaft, die jederzeit ausreichende Kapazitäten zur Verfügung stellt. Ferner dürfen die Stromtransportpreise nicht eine Höhe annehmen, bei der sie jegliche Stromerzeugungspreisdifferenzen ausgleichen oder gar übersteigen. Des weiteren muß der Pool die ihm übertragenen Aufgaben übernehmen. Ist eine der Bedingungen nicht erfüllt, kehrt sich das Ergebnis der Gesamtkostenbeurteilung um.

\subsection{Kostenentwicklung bei Umsetzung des Stromhändlerkonzeptes}

Bei fehlenden Marktzutrittsbarrieren wird von den Erzeugern die Kraftwerkstechnologie mit den niedrigsten Durchschnittskosten langfristig eingesetzt werden müssen. Nationale Auflagen, z.B. im Umweltschutz oder für den Primärenergieträgereinsatz, werden von den Unternehmen im europäischen Wettbewerb als kostenverursachende Standortfaktoren angesehen, die sie bei ihren Standortentscheidungen berücksichtigen werden ${ }^{462}$. Durch dieses Verhalten der Erzeuger sinken die Stromerzeugungskosten ${ }^{463}$. Gleichzeitig steigen durch die Kraftwerks- oder Be-

461 Dies ist ein Nachteil des in dem EU-Richtlinienentwurf vorgeschlagenen "unbundling", d.h., der unternehmensinternen Auftrennung des Stromtransportes . der Stromerzeugung und der -verteilung in Unternehmenssparten.

462 Falls keine Harmonisierung der Auflagen auf EU-Ebene erreicht wird.

463 Vgl. LOTTERMANN, Th. : Die Preisentwicklung im englischen Elektrizitätssystem, a.a.O., S. 97 
zugsquellenverlagerungen ${ }^{464}$ zwangsläufig die Stromiransportkosten $n^{465}$, insbesondere für den bedarfsgerechten Ausbau des Leitungsnetzes und für die Abwicklung der Stromtransporte. Auch wenn in einzelnen Netzgesellschaftsgebieten ausreichende Leitungskapazitäten bestehen, müssen die Regelungs- und Netzsteuerungseinrichtungen im Stromtransport an die neue Netzsituation angepaßt werden, wodurch zusätzliche Kosten anfallen.

Der Stromverteilungshereich wird durch das Stromhändlerkonzept kostenmäßig nicht berührt.

Eine Gesamtkostenminimierung wird im Stromhändlerkonzept nicht erreicht. Die Erzeugungsunternehmen berücksichtigen für ihre Standortauswahl nicht die Auswirkungen der gestiegenen Stromtransportkosten. Für sie ist der Stromabgabepreis an den Stromhändler an jedem Ort innerhalb des Netzgebietes identisch ${ }^{466}$. Die durch eine Verlagerung der Kraftwerksstandorte hervorgegangenen Stromtransportkosten werden vom Stromhändler gleichmäßig an alle Abnehmer weitergegeben. Die Auswahl des Erzeugers beeinflussen sie nicht. Die folgenden beiden Graphiken verdeutlichen die Zusammenhänge.

Im Stromhändlerkonzept werden wesentliche Koordinationsaufgaben, die im Common-Carrier-Konzept vom Markt erbracht werden müssen, von der Netzgesellschaft wahrgenommen. Für die unternehmensinterne Koordination entstehen der Netzgesellschaft Kosten, die prinzipiell mit den gegenwärtigen in den integrierten Unternehmen identisch sind. Lediglich durch die Angebotsabgabe der Erzeuger entstehen zusätzliche Transaktionskosten, die wegen der möglichen Automatisierung nicht ins Gewicht fallen werden. Für die Erzeugerunternehmen entstehen ebensowenig Kosten für die Kundensuche wie für die Verteiler Informations- und Suchkosten auf dem Stromhändlerabsatzmarkt anfallen.

Im wesentlichen müssen von den Regulierungsinstitutionen die gleichen Regulierungsfelder wie bisher abgedeckt werden. Nur der Erzeugerbereich kann von der vollständigen Betriebs- und Preiskontrolle ausgenommen werden. Damit reduzieren sich die erforderlichen Kosten für die Regulierung. Die Reduktion wird jedoch, anders als beim Common-Carrier-Konzept, gering ausfallen.

Vergleichsmöglichkeiten zwischen den Netzgesellschaften, die kostenreduzierend bei der Regulierung wirken, sind von den aus der Desintegration hervorgehenden Betriebsgrößen abhängig.

464 Vgl. z.B. den Einfluß der Standortkosten auf die Kosten der Kernenergie in: HENSING. I. : Die Perspektiven von Kernenergie in wettbewerblich geöffneten Elektrizitătsmärkten, in: Zeitschrift für Energiewirtschaft, Heft 1 (1996), S. 53-63, hier S. 55 ff.

$465 \mathrm{Vgl}$. hierzu auch die Prognosen der Fachtagung der VDI-Gesellschaft Energietechnik. zusammengefaßt dargestellt von KETTERER, H. : a.a.O., S. 194

466 Vgl. hierzu die Ausführungen zur Preisbilłung im-Stromahăndlerkonzept in Kapitel 7.3.1 . 
Abbildung 46: Kraftwerksverteilung vor Einführung des Stromhändlerkonzeptes

$$
\mathbb{Q}=\text { Kraftwerke } \mathbb{Q}=\text { Verbrauchsschwerpunkte }
$$

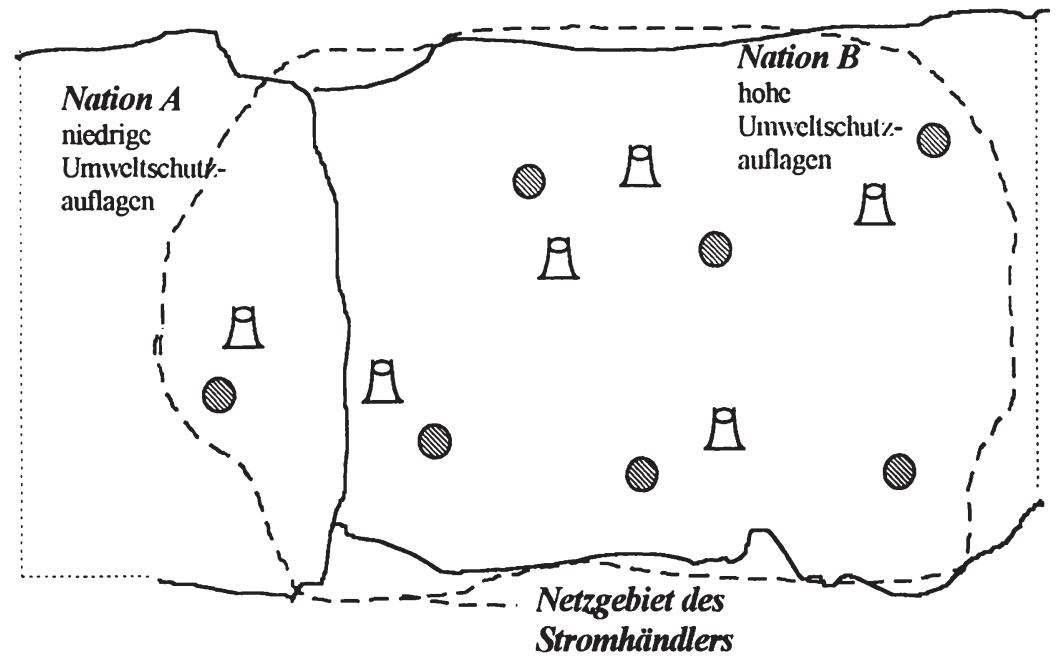

Abbildung 47: Kraftwerksverteilung nach Einführung des Stromhändlerkonzeptes

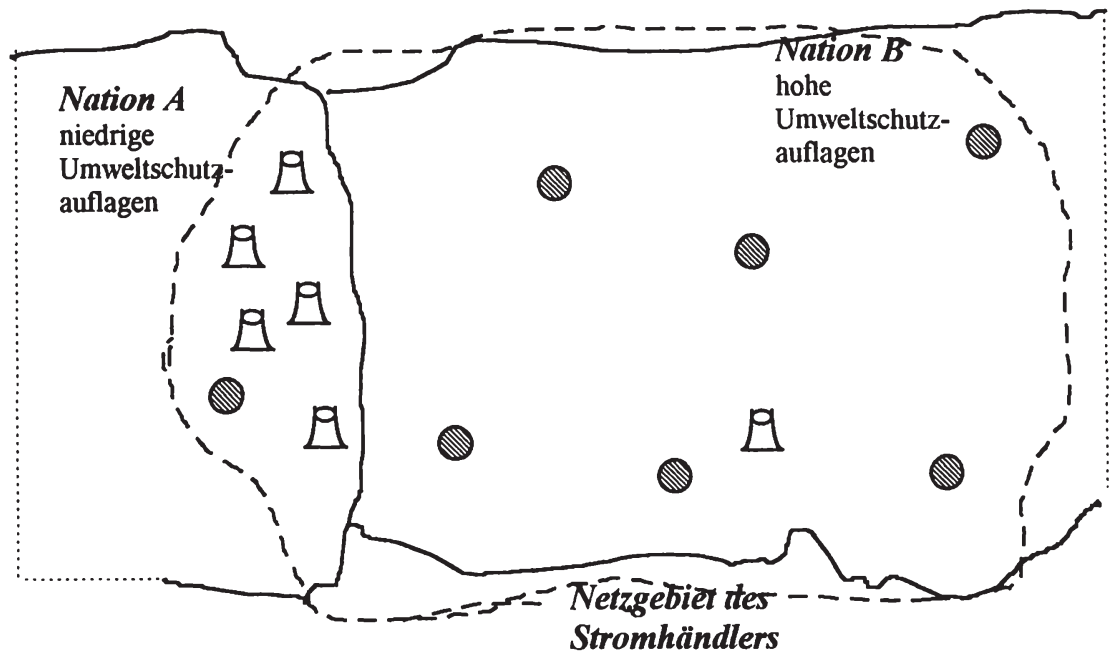


Zusammenfassend kann zur Kostenentwicklung bei Verwirklichung des Stromhändlerkonzeptes festgehalten werden, daß bei fehlenden Marktzutrittsbarrieren durch das Stromhändler-Konzept mit reduzierten Stromerzeugungskosten zu rechnen ist ${ }^{467}$. Bei unveränderten Verteilungskosten und nahezu unveränderten Regulierungskosten werden die Stromtransportkosten ansteigen. Die Saldogröße hängt entscheidend von der Wettbewerbsintensität auf dem Erzeugermarkt ab.

\subsubsection{Beurteilung der Versorgungssicherheit}

Die Verantwortung für die Versorgungssicherheit auf dem Elektrizitätsgroßhandelsmarkt des Common-Carrier-Konzeptes trägt die Poolleitung. Sie muß auf dem Markt für Aushilfslieferungen die nötigen Leistungen beschaffen. Daher hat sie imVergleich zu den Integrationskonzepten ständig das Problem, ohne zusätzliche Aufwendungen weder den genauen Reserveleistungsbedarf zu kennen, noch Einfluß auf die Stromproduzenten nehmen zu können. Sie muß also ein hohes Maß an Koordinationsleistungen erbringen, um die Versorgungssicherheit bereitzustellen. Hierdurch entstehen zusätzliche Aufwendungen, die nicht zuletzt auch von den Koordinationsmitteln abhängen. Doch es bestehen keine Hinweise dafür, daß das Common-Carrier-Konzept nicht das bisherige oder ein anderes definiertes Versorgungssicherheitsniveau gewährleisten kann ${ }^{488}$.

Ähnliches gilt auch für das Stromhändlerkonzept: Der Stromhändler ist jederzeit in der Lage, basierend auf seinen georderten Stromleistungen, ein entsprechendes Reservehaltungspotential zu beschaffen. Im Gegensatz zum Common-Carrier-Konzept kann der Stromhändler sogar Einfluß auf die Sicherheit der Stromlieferungen nehmen, in dem er z.B. Erzeuger, deren Einspeisung nicht den Sicherheits- und Qualitätsstandards genügen, von der Poolteilnahme ganz oder befristet ausschließt. Denkbar ist auch, daß der Ausfall von zugesagten Stromlieferungen ebenso mit einem befristenen Ausschluß vom Stromhandel sanktioniert wird. Mit diesen Mitteln kann der Stromhändler die Versorgungssicherheit gewährleisten.

Bei einem Vergleich der beiden Konzepte hinsichtlich des Zieles der Steigerung der Versorgungssicherheit ist das Stromhändlerkonzept besser zu bewerten.

467 So wurden in Großbritannien kostengünstige Gasturbinen-Kraftwerke mit der Liberalisierung des Strommarktes gebaut. Vgl. hierzu LOTTERMANN. T. : a.a.O., S. $94 \mathrm{f}$.

468 Groner führte 1989 an, daß die Aufhebung des Gebietsschutzes die Versorgungssicherheit eher steigen den sinken ließe, da durch die hohere Anzahl der Lieferanten bei Ausfall einzelner die Wirkungen geringer sind. Vgl. GRÖNER, H. : Überlegungen zum Fortbestand geschlossener Versorgungsgebiete in einem Binnenmarkt für Elektrizität, in: Zeitschrift für Energiewirtschaft . Heft 1 (19989). S. 22-25i,dhier \$125 Snann - 978-3-631-75129-9 


\subsubsection{Ausblick : Preisentwicklung bei Umsetzung der Desintegrationskonzepte}

Die Auswirkungen des Common-Carrier-Konzeptes auf die Strompreise für die verschiedenen Abnehmergruppen sind schwer abzuschätzen. Sie hängen von einer Vielzahl von Faktoren ab, die sich wechselseitig beeinflussen. Für Großabnehmer werden flexiblere Preise und Tarife durch den direkten Zugang zu den Erzeugerunternehmen zustande kommen. Ferner wird der Abbau der Quersubventionsmöglichkeiten in Europa Auswirkungen auf die Preisentwicklung der Großabnehmern haben. In Ländern, in denen Haushaltsabnehmer und kleinere Gewerbeabnehmer stromintensive (staatliche) Industrien über einen erhöhten Strompreis subventionieren, wird es zu einer Preisanhebung kommen ${ }^{469}$. In Ländern mit überzogenen Industriestrompreisen und/oder ineffizienten EVU können Großabnehmer durch geeigente Wahl ihrer Strombezugsquellen Preissenkungen realisieren.

Die Preise für die Klein- und Haushaltsabnehmer sind von den Beschaffungspreisen der Verteiler und einer erfolgreichen Verteilerregulierung abhängig. Je stärker die Verteilerunternehmen Erzeugungspreisdifferenzen zum günstigen Strombezug nutzen, desto stärker können sie die Preise für die Endverbraucher absenken. Die Aufgabe der Regulierungsinstitution ist es hierbei, die Weitergabe niedriger Erzeugerpreise an die Endverbraucher zu kontrollieren und die Verteilerunternehmen zu Effizienzsteigerungen anzuhalten ${ }^{470}$.

Allgemein werden die Preise stärker die Knappheitsverhältnisse und die AngebotsNachfrage-Konstellation auf dem Großhandelsmarkt des Common-Carrier-Konzeptes widerspiegeln. Weiteren Einfluß auf die Preisentwicklung wird die Wettbewerbsintensität haben: Je stärker sich der Wettbewerb auf dem Erzeugermarkt entwickeln wird, desto besser wird der Selektionsprozeß wirken und reale Preissenkungen bei den Endabnehmern verursachen. Weitere Kostenreduzierungen, die an den Verbraucher weitergegeben werden können, werden durch die erhöhte Regulierungseffizienz und die verminderten Eingriffsmöglichkeiten der nationalen Regierungen entstehen.

$\mathrm{Zu}$ einer vollständigen Angleichung der Preise auf dem europäischen Strommarkt wird es jedoch nicht kommen. In einem Marktgebiet von der Größe der EU existieren unterschiedliche Standortvorteile für die Elektrizitätsversorgung, die zwangsläufig unterschiedliche lokale Strompreise hervorrufen ${ }^{47}$. In Ländern mit für die Elektrizitätserzeugung verteuernd wirkenden Rahmenbedingungen wird es zu vorzeitigen Schließungen von Kraftwerken kommen, wenn Strom aus anderen EUStaaten billiger bezogen werden kann. Ist dies wegen hoher Stromtransportkosten

469 Dies bestătigt der empirische Trend in Großbritannien . Siehe Kapitel 4.1.3 .

470 In Großbritannien ist dies bisher nicht gelungen. Vgl. Kapitel 4.1.3 .

471 Gleiches schlagen auch die Vertreter der deutschen EVU für die Strompreisbildung bei einem verstärkten Wettbewerb vor. Vgl. BITTERER, R. : VDEW-Symposium „Strompreise in einem veränderten Markt", in: Elektrizitătswirtschaft, Jg. 95 (1996), Heft 4, S. 154-156, hier S, 155 
nicht möglich, werden die Rahmenbedingungen für die Endverbraucher zu höheren Preisen führen, auf die sie mit Duldung oder Abwanderung reagieren können ${ }^{47}$. Für die Unternehmen im Wettbewerb werden die politischen und rechtlichen Rahmenbedingungen Teile der Standortausstattung eines potentiellen Produktionsortes sein, die bei der Standortauswahl oder Verlagerung berücksichtigt werden müssen. Im Gegensatz zur bisherigen Kontinuität werden die Strompreise und die Strompreisentwicklung durch die Preisentwicklung auf dem Spotmarkt schwanken ${ }^{4 / 3}$. Je höher der Anteil des Spotmarktes ist, desto stärker werden die Elektrizitätspreise die kurzfristige Angebots- und Nachfragesituation wiedergeben und sich damit im Tages- und Jahresverlauf verändern. Die Spotmarktpreise werden anders als bei einem integrierten Elektrizitätssystem Anreize zum Konsumverhalten, zumindest bei den Großverbrauchern, geben. Die Wirkung auf die Kleinabnehmer ist von der Preisweitergabe und den eingesetzten Meßgeräten der Verteilerunternehmen abhängig ${ }^{474}$. Die bereits vorhandenen technischen Stromentnahmemeßgeräte lassen eine zeitliche Erfassung des Stromverbrauchs zu; bisher scheiterte ihr Einsatz bei Kleinverbrauchern an den hohen Anschaffungskosten. Diese können jedoch durch die Massenproduktion bei ausreichender Nachfrage reduziert werden ${ }^{4 \imath \varsigma}$.

Zusammenfassend kann man festhalten, daß eine genaue Preisentwicklungsprognose für jede Abnehmergruppe, an jedem Ort in Europa, bei Umsetzung des Common-Carrier-Konzeptes nicht möglich ist. Durch die Einführung von Wettbewerb auf zwei Teilmärkten unterliegen die Erzeuger aber erstmalig einem Wettbewerbsdruck, der zu Effizienzsteigerungen zwingt und ineffizient arbeitende Anbieter ausschließt. Hierdurch werden tendenziell die Elektrizitätspreise im Vergleich zur Situation vor Einführung des Konzeptes sinken.

Für das Stromhändlerkonzept haben die Ausführungen zur Wettbewerbsintensität und zur Marktmacht gezeigt, daß ein Preiswettbewerb auf der Erzeugerseite stattfinden wird. Die Geschwindigkeit, mit der dieser Preiswettbewerb nach dem Abschluß der Implementierung einsetzt, hängt von der Ausgangssituation bei den Stromerzeugungskapazitäten ab. Diese ist zur Zeit wegen den bestehenden Überkapazitäten und geringer Bedarfsanstiege für den Wettbewerb positiv einzuschätzen. Deshalb werden die Erzeugerabgabepreise und damit die Preise für Großab-

472 Abwanderung ist bei Haushaltsabnehmern wegen der Strompreise nicht zu erwarten. Sie können über ihr Wahlverhalten ihre Haltung zu den Rahmenbedingungen ausdrücken. Industrieunternehmen haben dagegen die langfristige Möglichkeit der Abwanderung und werden die Strompreise im Rahmen ihrer Standortwahl berücksichtigen.

473 Dies bestătigen die bisherigen britischen Erfahrungen. Vgl. BOULDING. P.G. : Die Preisentwicklung in der britischen Elektrizitătsıvirtschaf, in: Zcitschrift für Energiewirtschaft, Heft 4 (1995), S. 295-304

474 Siehe hierzu die Vorschlăge zur Einführung eines $96 \mathrm{~h}$-Tarifes in: BRANDMAIR, R. . Erfahrungen mit der 96-Stunden-Leistungsmessung, in: EW Jg. 94 (1995), Hcft 5, S. 233 ff.

475 O.V. : Überprüfung der Strompreisstrukturen - Beschluß der Wirtschaftsministerkonferenz

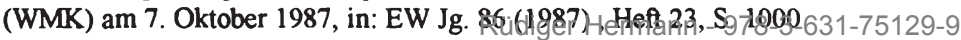


nehmer und Verteiler im Zyklus der Wettbewerbsintensität schwanken. Langfristig werden hohe Abweichungen von den durchschnittlichen Elektrizitätspreisen auf dem Stromhändlerbeschaffungsmarkt durch den Wettbewerb ausgeschlossen.

Die Auswirkungen des Stromhändlerkonzeptes auf die Endabnehmerpreise hängen von zahlreichen Faktoren ab. Ein erster bedeutender Faktor ist die Effizienz der Netzgesellschaft und der Verteilerunternehmen. Wegen ihres Monopols im Transport- und im Verteilungsbereich sowie den Kostenüberwälzungsmöglichkeiten bedeutet jeder Effizienzverlust bei der Netzgesellschaft höhere Preise für den Endverbraucher. Für die Verteilerunternehmen trifft dies in abgeschwächter Form zu, weil sie aufgrund der geringeren Betriebsgröße einfacher zu kontrollieren sind. Ein Vergleich zwischen den Gesellschaften kann erste Hinweise auf Effizienzverluste ergeben. Ein weiterer Einflußfaktor auf den Endabnehmerpreis ist das Funktionieren der Regulierungsmechanismen. Mangelnde Überwachung der Monopole führt zu Ineffizienzen und damit zu erhöhten Strompreisen ${ }^{476}$.

Die Netzgesellschaft gibt die Preise des Stromhändlerbeschaffungsmarktes mit einem Aufschlag für die Koordinierungs- und Stromtransportleistungen weiter. Deshalb werden die Preise auf dem Endabnehmermarkt den langfristigen Preisschwankungen des Stromhändlerbeschaffungsmarktes folgen. Der Trend der Schwankungen wird durch die Effizienzsteigerungen bestimmt. Sieht man von anderen Einflußfaktoren ${ }^{477}$ ab, wird er wegen niedriger Marktzutrittsbarrieren auf dem Stromhändlerbeschaffungsmarkt fallend $\operatorname{sein}^{478}$.

\section{Abbildung 48: Entwicklung der Endabnehmerpreise im Stromhändlerkonzept}

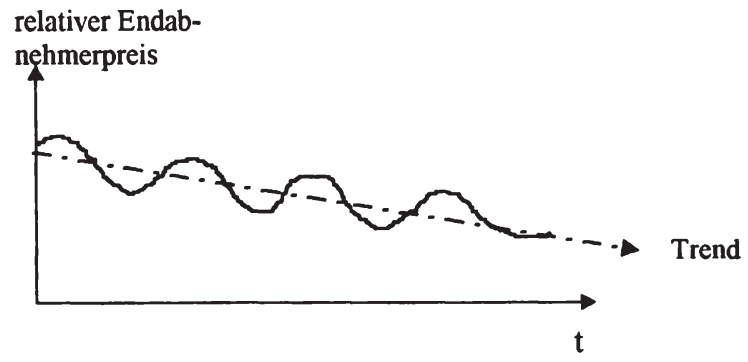

476 Dies bestätigen die britischen Erfahrungen. Vgl. hierzu LOTTERMANN. T. : Preisentwicklung im englischen Elcktrizitätssystem, a.a.O., S. $93 \mathrm{ff}$.

477 Z.B. der Primärenergicpreisentwicklungen oder der Steuern.

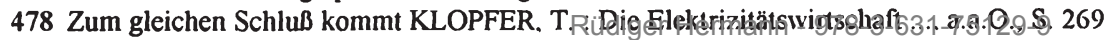


Tabelle 15: Übersicht Beurteilung Common-Carrier- und Stromhändlerkonzept

\begin{tabular}{|c|c|c|}
\hline Kriterien zur Beurteilung der Ordnungsmodelle & $\begin{array}{r}\text { erf } \\
\text { CC- Konzept }\end{array}$ & $\begin{array}{c}\text { IIf } \\
\text { Stromnändler- } \\
\text { konzept }\end{array}$ \\
\hline Vertikale Integration aufgelöst? & Ja & Ja \\
\hline Stromhandelsinfrastruktur geschaffen? & Ja & Nein \\
\hline Tellmärkte geschaffen ? & Ja & Ja \\
\hline Marktzuntittsbartieren reduzlent? & Ja & Ja \\
\hline Wettbewerbsintensität gestelgert? & Ja & Ja \\
\hline Marktmacht elnzelner Anbleter u.Nachfrager begrenzt ? & Ja & Ja \\
\hline Reduzlerung der Gesamtkosten der Elekttizlï̈tsversorgung ? & Ja & Ja \\
\hline Versorgungssicherhelt gewöhrlelstet? & Ja & Ja \\
\hline Ausbllck Prelsentwicklung: & $\begin{array}{l}\text { Sinkende } \\
\text { Strompreise } \\
\text { in Europa }\end{array}$ & $\begin{array}{c}\text { Sinkende } \\
\text { Strompreise } \\
\text { in Europa }\end{array}$ \\
\hline
\end{tabular}


Rüdiger Hermann - 978-3-631-75129-9

Downloaded from PubFactory at 01/11/2019 07:57:52AM

via free access 


\section{Die Bedeutung der Stromtransportkapazităten für den europăischen Binnenmarkt für Elektrizităt}

Die nähere Betrachtung der vier für den EU-Strommarkt vorgeschlagenen Ordnungsmodelle hat folgendes gezeigt:

- Der Preis für Stromtransporte ist bisher in keinem Ordnungsmodell näher betrachtet worden. Eventuelle Auswirkungen auf die Elektrizitätsversorgung wurden ausgeschlossen.

- Langfristig kann der europäische Strommarkt nur verwirklicht werden, wenn ausreichende Stromtransportkapazitäten vorhanden sind oder errichtet werden.

- Ohne eine funktionsfähige Stromhandelsinfrastruktur kann die Elektrizitätsversorgung nicht zu niedrigeren Kosten durch die Verwirklichung des Binnenmarktes bereitgestellt werden. Dies zeigt, daß eine abschliessende Bewertung der Ordnungsmodelle ohne eine weitere Betrachtung der Stromtransportkapazitäten nicht möglich ist.

- In allen Ordnungskonzepten bleibt das Stromtransportnetz im Besitz von Monopolunternehmen. Marktpreise oder eine Kontrolle der Preise für Stromtransportleistungen über den Markt ist ausgeschlossen.

Deshalb soll im folgenden Kapitel dargestellt werden, welche Anforderungen an Stromtransportpreise gestellt werden müssen, aus welchen Bestandteilen sie bestehen sollten und wie sie gebildet werden können. Anschließend soll herausgearbeitet werden, welcher Stromtransportpreis für welches Ordnungsmodell geeignet ist und die Stromtransportkapazitätsentwicklung in den Ordnungsmodellen prognostiziert werden. Dann wird festgestellt, welchen Einfluß die Kapazitätsentwicklung auf die Verwirklichung des europäischen Strommarktes im allgemeinen und speziell auf die Ziele der kostengünstigen und sicheren Versorgung hat.

\subsection{Stromtransportpreise}

\subsubsection{Anforderungen an Stromtransportpreise}

Zunächst einmal eine Definition, was unter Stromtransport zu verstehen ist:

Stromtransport ist die regionale und überregionale Übertragung elektrischer Energie von einem Hochspanmingseinspeisepunkt zu einem Hochspanmungsent- 
nahmepunkt. Der Stromtransport wird von einer oder mehreren Gesellschaften technisch und organisatorisch durchgeführt. Die dabei von den Leistungsinanspruchnehmenden zu zahlenden Entgelte sollen als Stromtransportpreise bezeichnet werden ${ }^{479}$.

Eine erste allgemeine Anforderung an Stromtransportpreise wurde von Pick aufgestellt. "Für Netzbetreiber muß die Gewährung von Durchleitungsrechten und die Abwicklung der Durchleitung eine reguläre wirtschaftliche Tätigkeit sein" ${ }^{180}$. Die Förderung dieser von Pick für das TPA-Konzept aufgestellten Forderungen sollte die Leitlinie für die aufzustellenden Grundsätze der Transportgebühren sein.

Es lassen sich fünf speziellere Anforderungen an die Stromtransportpreise im europäischen Binnenmarkt ableiten ${ }^{481}$. Sie sollten

- transparent,

- kostenorientiert ${ }^{482}$

- einfach zu erheben,

- planbar und

- verursachergerecht sein sowie

- die Knappheitsverhältnisse wiedergeben.

Die Transparen $z^{483}$ kann durch eine Publikation der Stromtransportpreise und ihrer Berechnungsgrundlagen erreicht werden.

Die Kostenbasis ist eine Frage der Kostenorientierung. In den europäischen Elektrizitätswirtschaften werden bisher, mit Ausnahme Frankreichs, die Vollkosten als Grundlage für die Preis- und Tarifberechnung herangezogen. In Frankreich entwikkelte sich das Grenzkostenpreisbildungsprinzip als Grundlage der Berechnung nach dem zweiten Weltkrieg ${ }^{484}$. Ein weiterer Grund für die geforderte Kostenorientie-

479 Der Stromtransport unterscheidet sich hierbei vollständig von dem leitungsgebundenen Gastransport. Ansätze für die Preisbildung bei Gastransporten sind daher nicht übertragbar. Vgl. hierzu : IEA: Natural Gas Transportation and Regulation. Paris 1994

480 PICK, H. : Ordnungsmodelle .... , a.a.O., S. 94

481 Swoboda stellt allgemein für die Elektrizitătswirtschaft andere Kriterien auf. Siehe SWOBODA, P. : Kostenermittlung und Tarifbildung bei Elektrizitătsversorgungsunternehmen insbesondere hinsichtlich kalkulatorischer Kosten, in: Journal für Betriebswirtschaft, Heft 2, 1990, S. $66 \mathrm{ff}$.

482 Traditionell orientierte sich die Preisaufsicht regulierter Unternehmen an der Kostensituation. Vgl. RIECHMANN, C. : Price-Cap Regulierung in: Zeitschrift für Energiewirtschaft, Heft 2, 1995, S. 157

483 Vgl. MEIER, H. : Unipede Tarifkonferenz ..., a.a.O., S. 1687. Dies ist bereits durch die Richtlinie Transitrichtlinie erreicht worden. Vgl. hierzu LANG, G. : Die EG-Richtlinie zur Strompreistransparenz ..., a.a.O., S. 1357

484 Vgl. QUOILLIN, J. : Der Verkauf zu Grenzkosten bei der EdF, in: Analen der Gemeinwirtschaft, 45. Jg.. Lüttich 1976, S. 129-155, S. 133 und weiter MEIER, K. : Spitzenlasttarifie-

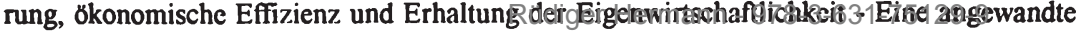


rung der Stromtransportkosten ist die Ausstattung der Netzgesellschaften oder der Netze betreibenden EVU mit einem Monopol ${ }^{485}$.

Die Forderung nach einer einfachen Erhebungs- und Verrechnungsmöglichkeit ist notwendig, um die Kosten für die Infrastrukturbereitstellung zu minimieren ${ }^{486}$.

Die Forderung nach der Planbarkeit der Stromtransportpreise resultiert aus dem Bedürfnis der potentiellen Vertragspartner bei den Vertragsverhandlungen, die tatsächlichen Kosten des Vertragsgegenstandes zu kennen. Nicht planbare, d.h. zum Zeitpunkt der Liefer- und Bezugsvertragsverhandlungen in ihrer Höhe nicht bekannte Stromtransportpreise steigern das Risiko von Stromhandelsgeschäften und erschweren die Auswahl des kostengünstigsten Lieferanten für die Abnehmer ${ }^{487}$. Gleichzeitig steigern sie die Transaktionskosten. Die Folge wird ein reduziertes Stromhandelsvolumen sein.

Eine traditionell in der Elektrizitätswirtschaft an Preise und Gebühren gestellte Forderung ist die der Ausrichtung an dem Verursacherprinzip ${ }^{488}$. Das Verursacherprinzip sieht vor, daß die tatsächlich durch eine Inanspruchnahme verursachten Kosten durch den Inanspruchnehmenden getragen werden. Hieraus resultieren idealtypisch individuelle Stromtransportpreise.

Darüber hinaus sollten die Tarife für die Übertragungsleistungen Anreize zur kostenminimierenden Ausnutzung der Kapazitäten geben.

Studie der elektrizitătswirtschaftlichen Preisbildung. Bern. Stuttgart 1983. S. $18 \mathrm{ff}$. und BOITEUX, M. : La tarification des demandes en pointe: application de la theorie de la vente au cout marginal, in: Reveue General de l'Electricité , Bd. 58, 1963. S. 321-340 und weiter LANG. G. : EG Strompreise 1992 im Vergleich, in: ET 41. Jg. (1992), Heft 10, S. $693 \mathrm{ff}$.

$485 \mathrm{Vgl}$. hierzu KLOPFER, Th. : Die Elektrizitătswirtschaft ..., a.a.O., S. 274 zur Kostenorientierung der britischen Netzgebühren.

$486 \mathrm{Vgl}$. hierzu die Ausführungen zu den Zielen des europäischen Strommarktes in Kapitel 1.

487 Trotzdem hat man sich bei der Neustrukturierung im australischen Victoria für expost festzulegende Stromtransportpreise entschieden. Vgl. SCHOLL. R. : Reform der Elektrizitătsindustrie in Australien ..., a.a.O., S. 390

488 Vgl. MEYER, H. : Unipede Tarifkonferenz in Lausanne, in: EW Jg. 91(1992), Heft 26, S. 1687. Bisher wurde die Orientierung an dem Verursacherprinzip allgemein für die Strom-

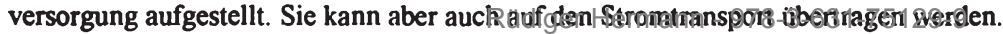




\subsubsection{Pauschalisierte Stromtransportpreise}

Auch hier zunächst die Definition:

Als pauschalisierter Stromtransportpreis soll das Entgelt fïr die Leistungen einer Netzgesellschaft bezeichnet werden, welches auf einer oder wenigen standardisierten, vorher festgelegten und veröffentlichten physikalischen Größe beruht ${ }^{49}$.

Wie könnte ein pauschalisierter Stromtransportpreis berechnet werden?

Zunächst einmal kann er ebenso wie die Mehrzahl der zur Zeit in den Mitgliedsstaaten verwandten Stromtarife einen festen Anteil pro Stromtransport enthalten. Dieser Betrag dient dem Stromtransporteur zur Deckung seiner Kosten, die unabhängig von der transportierten Stromleistung entstehen ${ }^{490}$. Der zweite Strompreisbestandteil entspricht dem Arbeitspreis der derzeitigen Stromtarife. Eine einfache Form eines Stromtransportarbeitspreises berücksichtigt ausschließlich die übertragene Energie (in $\mathrm{kWh}$ ). Nachteilig wirkt bei dieser Transportpreisberechnung die mangelnde Berücksichtigung von Leistungselementen. Eine in einem längeren Zeitabschnitt übertragene Stromleistung verursacht geringere Kosten als die gleiche übertragene Leistung innerhalb eines kürzeren Zeitabschnitts. Die physikalischen Zusammenhänge in elektrischen Leitungen bewirken, daß der Widerstand einer Leitung mit zunehmender Stromstärke im Quadrat ansteigt. Deshalb sollte in einem pauschalisierten Stromtransportpreis die angeschlossene, vorher vertraglich zwischen den Stromhandelspartnern und der Transportgesellschaft bestellte Leistung eine Berücksichtigung finden. Denkbar wäre auch eine Berücksichtigung des Auslastungsgrades bei der pauschalisierten Preisberechnung, da die gleiche transportierte Stromleistung mit gleichem Leistungsabnahmeprofil bei niedrigem Auslastungsgrad der Leitung geringere Netzverluste verursacht als die gleiche Stromleistung bei fast ausgelasteter Leitung. In der Praxis besteht das Problem in der Messung des Auslastungsgrades während einer Stromlieferung, denn die einzelnen Leitungen, Kupplungen, Transformatoren und sonstigen technischen Einrichtungen sind zu jedem Zeitpunkt anders ausgelastet. Die Veränderung des Auslastungsgrades durch eine spezifische Lieferung ist durch die technischen Besonderheiten der Elektrizitätsbranche nur mit einem hohen Meßaufwand feststellbar. Da die Auslastungssituation für die Stromerzeuger und Abnehmer insbesondere bei langfristigen Stromvertragsverhandlungen im vorhinein nicht vollständig planbar und damit auch ein Stromtransportpreis nicht exakt bekannt ist, entstehen durch die Berücksichtigung des Auslastungsgrades bei der pauschalisierten Stromtransportpreisbe-

489 Die Bezeichnung „pauschal“ wurde von Hedtkamp übernommen, der unter Pauschgebühren offentliche Gebühren für cin Leistungsbündel versteht. Vgl. HEDTKAMP, G.: Lehrbuch der Finanzwissenschaft, 2. Auflage, Neuwied 1977, S. 286

490 Dies sind z.B. Verwaltungskosten für die Rechnungserstellung und das Inkasso sowie die Anschlußgebühren. Vgl. LOTTERMANN, Th. : Die Preisentwicklung im englischen Elektrizitătssystem. a.a.O., S. 95 
rechnung Kosten zur Begrenzung dieser Unsicherheit für die Unternehmen. Damit ist die Berücksichtigung des Auslastungsgrades bei einem pauschalisierten Entgelt nicht zieladäquat. Deshalb muß eine andere Kenngröße herangezogen werden, die als Indikator für die Leistungsinanspruchnahme und damit auch für die Kostenverursachung durch die Kunden der Netzgesellschaft genommen werden kann. Der Verfasser schlägt hierfür die zu überwindende Entfernung vor: Sie berücksichtigt zwar weder den Auslastungsgrad des Netzes noch die tatsächlichen Stromflüsse, ist aber einfach zu berechnen und für die Kunden der Netzgesellschaft planbar ${ }^{491}$. Folgende Größen können also zur Berechnung des variablen Anteil eines pauschalisierten Stromtransportpreises herangezogen werden:

a) die zu übertragende absolute Spitzenleistung,

b) die zu übertragende kWh-Menge,

c) die zu überwindende Entfernung.

Das Ergebnis eines derartigen Stromtransportpreises ist ein Tarif, der zu sinkenden Durchschnittspreisen bei steigender Leistungsinanspruchnahme führt ${ }^{492}$.

\section{Abbildung 49: Durchschnittspreisentwicklung bei zweigeteiltem Stromtransporttarif}

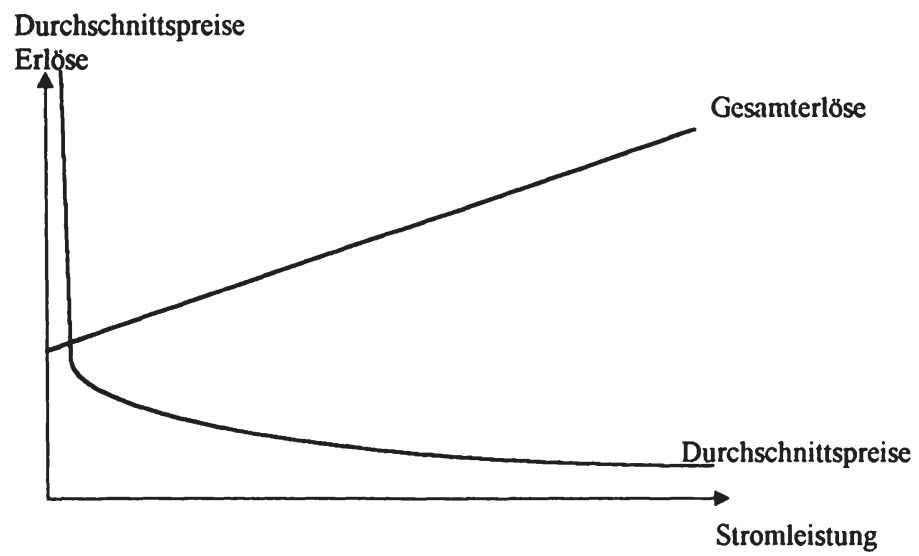

491 Hierdurch werden die erforderlichen Transaktionskosten auf einem niedrigen Niveau gehalten.

492 Siehe auch VDEW Arbeitskreis „Tarifgestaltung“: Tariflicher Bercitstellungspreis ..., a.a.O., S. 1055 
Die Vorteile von pauschalisierten Stromtransportpreisen bestehen in der guten Planungsgrundlage für Stromgeschäfte und den damit verbundenen niedrigen Transaktionskosten. Weitere Vorteile sind die hohe Transparenz und die einfache Berechnungs- und Erhebungsmöglichkeit.

Ein besonderes Problem bei einem pauschalisierten Preis ohne Berücksichtigung des Netzzustandes liegt in der erforderlichen Kapazitätszuweisung bei Transportübernachfrage. Bei einer Übernachfrage nach Transportdienstleistungen kann es dazu kommen, daß das Stromhandelsgeschäft mit dem größten volkswirtschaftlichen Nutzen nicht zustande kommt, weil die Kapazitäten anderen Unternehmen zugewiesen worden sind. Des weiteren sind pauschalisierte Durchleitungspreise für den Einzellfall nicht verursachungsgerecht.

Empirisches Beispiel für einen pauschalisierten Stromtransportpreis ist der im britischen Energy Act von 1983 vorgesehene Berechnungsmodus: Der Energy Act sollte die Möglichkeiten einer privaten Stromerzeugung in Großbritannien verbessern. Deshalb war es nötig, den EVU eine Durchleitungsverpflichtung aufzuerlegen und Stromtransportpreise vorzuschreiben ${ }^{493}$. Sie umfaßten 1984 eine Grundgebühr von 24 Pence pro Monat und Kilowattstunde je in Anspruch genommener Transformationsstufe kleiner oder gleich $132 \mathrm{kV}$ und eine Gebühr pro übertragenem Kilometer. Darüber hinaus mußten die Lieferanten ihre Einspeisung 2,5\% höher ausrichten als die zu erwartende Entnahme des Durchleitungsempfängers, um die Netzverluste auszugleichen.

Ein weiteres empirisches Beispiel ist seit dem 01.01.1991 das nicht zur EU gehörende Norwegen ${ }^{494}$. Verteilerunternehmen sind in Norwegen verpflichtet, ihre Leitungen Kunden zu öffnen und dafür einen einheitlichen, zu veröffentlichenden Preis zu verlangen. Der Preis wird an den Gesamtkosten ausgerichtet und beinhaltet eine Verzinsung des eingesetzten Kapitals.

In den folgenden Ausführungen sollen die Vor- und Nachteile eines pauschalisierten Stromtransportpreises fur jedes der vier vorgeschlagenen Ordnungsmodelle aufgezeigt werden; auch dann, wenn aus theoretischen Überlegungen ein pauschalisiertes Entgelt nicht sinnvoll erscheint. Die europäische Realität zeigt jedoch insbesondere für die leitungsgebundenen Energien, daß in allen Staaten Durchschnittspreise, teilweise staatlich vorgegeben, bestehen. Man muß daher davon ausgehen, daß unabhängig von dem in der Zukunft gewählten Ordnungsmodell bei den politischen Entscheidungsträgern der traditionelle Drang zu einer derartigen Preisbildung und -setzung vorhanden sein wird.

493 Vgl. COTTERELL, J.W. ; HEINRICH, D.O. : Stromwirtschaftliche Zusammenarbeit in England, in: Recht der Elcktrizitätswirtschaft, Heft 9, 1985, S. $170 \mathrm{ff}$.

494 Vgl. MEYER. H. : Unipedc Tarifkonferenz R.,̣i.g.@.HSrn69lh - 978-3-631-75129-9 


\subsubsection{Einzelfallorientierte Stromtransportpreise}

Die Definition lautet:

Als einzelfallorientierte Stromtransportpreise sollen solche bezeichnet werden, die an der tatsächlichen Leistungsinanspruchnahme ${ }^{495}$ oder der Marktsituation orientiert werden ${ }^{496}$.

Einzelfallorientierte Stromtransportpreise können in kosten- und markıorientierte unterteilt werden.

Einzelfallorientierte kostenbezogene Stromtransportpreise erfordern eine geeignete Meßtechnik, um die tatsächlich vom einzelnen Kunden in Anspuch genommene Leistung des Stromtransporteurs zu erfassen und die Kosten zuordnen zu können. Faktisch bedeuten einzelfall- und kostenorientierte Stromtransportgebühren nichts anderes als eine wesentlich höhere Anzahl von Kennziffern, die zur Berechnung des Preises herangezogen werden. Neben den Leistungs- und Entfernungsgrößen werden als Einflußfaktoren der Auslastungsgrad des Netzes (durch den elektrischen Widerstand jeder Teilstrecke) gemessen, sowie die Inanspuchnahme von Netzregelungseinrichtungen bei schwankender Einspeisung und die Entnahme- und die Abrechnungskosten berücksichtigt. Ein weiterer zu berücksichtigender Kostenfaktor sind die Netzverluste eines Stromtransportes.

Einzelfall- und kostenorientierte Transitpreise besitzen die Möglichkeit der Grenzkosten- und Grenznutzenberücksichtigung. Sie können verursacher- und marktgerecht sein und damit die richtigen Anreize zur Allokation geben. Die Nachteile von kostenorientierten Preisen sind die hohen Meß- und Erhebungskosten sowie die mangelnde Planbarkeit. Eine genaue Übersicht über die Kosten hat das durchführende EVU erst nach einem Stromtransport.

Marktlichen Stromtransportpreise liegen Nachfragesituationen zugrunde. Bei steigender Leitungskapazitätsnachfrage steigt und bei fallender sinkt der Preis. Hierdurch werden den Marktteilnehmern Anreize gegeben, die Stromtransportkapazitäten kontinuierlich zu benutzen und die Knappheit der Infrastrukturkapazitäten bei ihren Entscheidungen zu berücksichtigen. Praktisch ist die Umsetzung mittels einer Kapazitätsorder durchführbar. Zu einem festgelegten Zeitpunkt, z.B. einen Tag vor Inanspruchnahme, wird die tatsächliche Nachfragesituation mit der durchschnittlichen oder der bereits abgeforderten Kapazitätsmenge verglichen. Bei Über-

495 Die Berechnung der Gebühr nach der tatsächlichen Leistungsinanspruchnahme unterscheidet sich von der Berechnung nach physikalischen Größen durch die Menge der Einfluß nehmenden Faktoren und dem Abrechnungszeitraum. Die genaueste Berechung der Gebühren nach den Kosten ist erst im nachhinein, also nach der Stromtransportleistung, möglich.

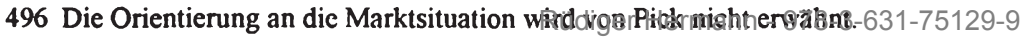


schreiten einer festgelegten Auslastung des Netzes steigt der Preis für die noch freien Übertragungskapazitäten an. Hierdurch werden die Knappheitsverhältnisse der Stromtransportkapazitäten von den Marktteilnehmern berücksichtigt werden müssen. Im Zeitverlauf gewinnen die Kapazitätsnachfrager Erfahrungen über die Netzsituationen im Jahres- und Tagesverlauf, so daß sie den Preis schätzen können und ihre Geschäfte daran orientieren. Hierdurch könnten teure Spitzenlastkapazitäten im Stromtransportbereich reduziert werden und gleichzeitig die richtigen Marktsignale an die Abnehmer von Stromtransportkapazitäten gegeben werden. Die Zusammenhänge verdeutlicht die Abbildung 50.

Nachteilig wirken die hohen Transaktionskosten und die Restunsicherheit über den Marktpreis der Übertragungskapazitäten bei Verhandlungsaufnahme potentieller Stromhandelspartner.

Als dritte Möglichkeit können einzelfallorientierte Stromtransportpreise aus einer Mischung zwischen kosten- und marktorientierten Preisen bestehen. $\mathrm{Zu}$ bedenken ist jedoch, daß jede zusätzlich erforderliche Messung und Erhebung kosten- und damit preissteigernd für die Abnehmer der Stromtransportleistung wirkt. Deshalb sollte auf eine Mischung der beiden einzelfallorientierten Stromtransportpreisarten verzichtet werden.

\subsubsection{Die Stromtransportpreisarten für die Ordnungskonzepte}

\subsubsection{Stromtransportpreise im Weiterentwicklungskonzept}

Bei Stromgeschäften zwischen benachbarten EVU ist eine Stromtransportkostenbezahlung in der Vergangenheit weder üblich noch erforderlich gewesen. Im europäischen Strommarkt des Weiterentwicklungskonzeptes verändert sich diese Situation nicht; durch das niedrige Stromtransitaufkommen besteht für die beteiligten Unternehmen kein großer Druck, durch Standardisierung die anfallenden Transaktionskosten zu mindern. Ihre Ertragssituation ändert sich durch die Stromtransportdienstleistung nicht, da sie weiterhin mit einem Versorgungsmonopol in Europa ausgestattet sind, welches ihnen die vollständige Gesamtkostenüberwälzung an den Letztabnehmer ermöglicht. Daraus folgt, daß eine Tendenz der EVU zu partnerschaftlichen Lösungen im Stromhandel und -transport sowie in der Preisberechnung vorhanden ist. Sollen nun, aus welchen Gründen ${ }^{497}$ auch immer, die Stromtransite monetär entgolten werden, stellt sich die Frage, ob einzelfall orien-

497 Ein denkbarer Grund wäre z.B.. daß die Verbund-EVU ihren Stromtransporten im Binnenmarkt einen marktwirtschaftlichen „Anstrich“ geben wollen, ein anderer wäre die deutlich höhere Nutzung der Infrastruktur eines EVUSidutgeh eimanderes. 978-3-631-75129-9 
Abbildung 50: Marktliche einzelfallorientierte Stromtransportpreise Stromtransport-

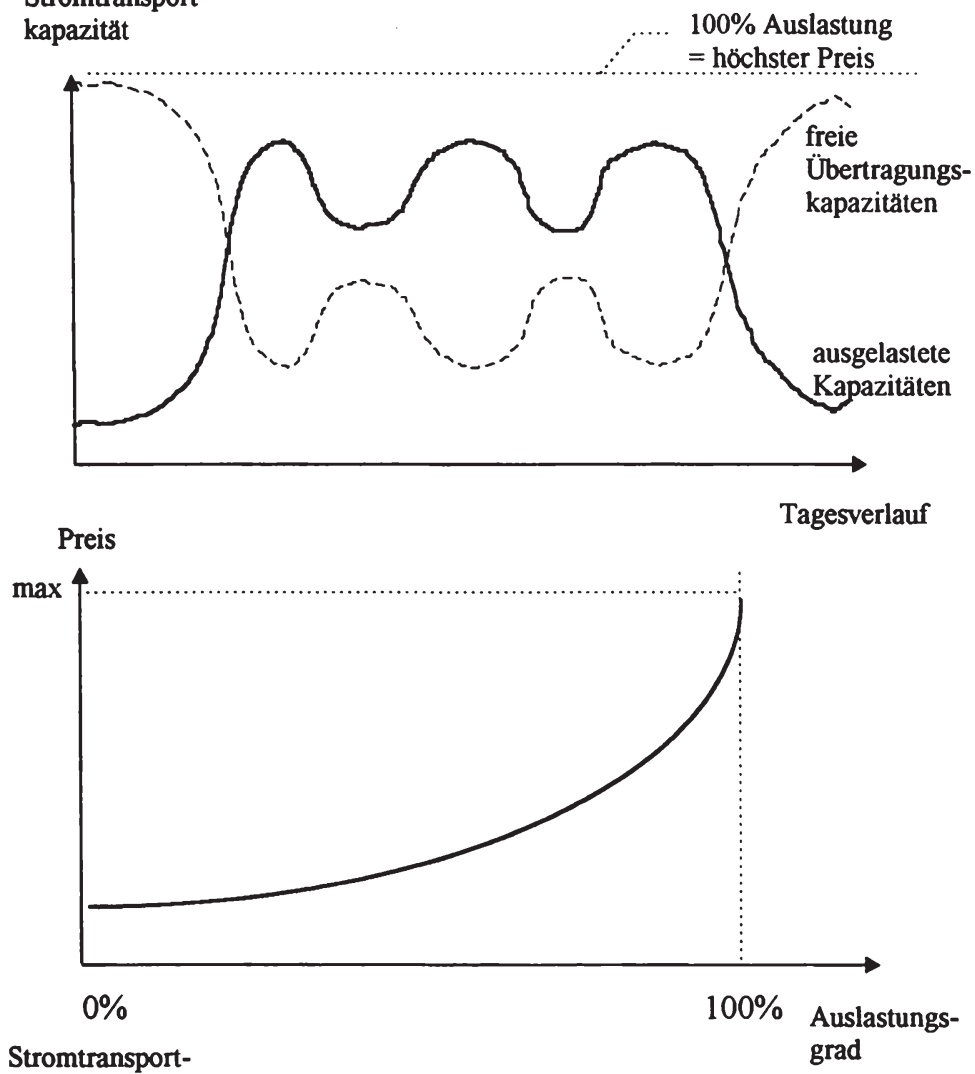
preis

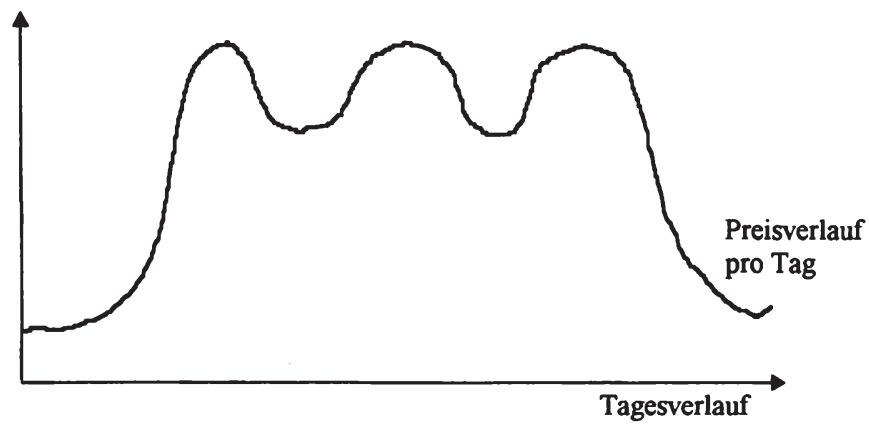


tierte oder pauschale Stromtransportpreise für das Weiterentwicklungskonzept geeigneter sind. Einzelfallorientierte Stromtransportpreise kommen dem Ziel der verbesserten Aushilfs- und Reserveleistung entgegen. Je nach Inanspruchnahme und Netzsituation kann der Preis berechnet werden. Die Bezahlung kann an den Grenzkosten orientiert werden, weil das transportierende EVU seine Kapazitätsplanung nicht an der potentiellen Stromtransportnachfrage anderer Unternehmen ausrichtet. In Schwachlastzeiten werden lediglich die nicht benötigten Kapazitäten zur Verfügung gestellt. Die Preisuntergrenze liegt bei dem Preis, bei dem das transportierende EVU gerade noch einen positiven Deckungsbeitrag erzielt. Die Preisobergrenze wird durch die maximale Zahlungsbereitschaft des Transitbeantragenden festgelegt. Der Preis und damit auch die Aufteilung der Kosten und Gewinne kommt durch freie Verhandlung der EVU zustande. Der einzelfallorientierte, verhandelte Preis hat jedoch den Nachteil, daß er nicht vollständig auf den Kosten beruht; das Transitunternehmen wird die hohen Kosten für die Erhebung der für die Grenzkostenberechnung erforderlichen Größen scheuen und deshalb nur eine Schätzung der Kosten vornehmen. Dies bedeutet jedoch, daß letztlich die Abnehmer des Transit-EVUs entweder die Stromlieferungen anderer EVU subventionieren müssen oder ihre Stromversorgungskosten durch andere Abnehmer bezahlt werden ${ }^{498}$. Ferner weist der Stromtransportpreis fur die beantragenden EVU keine Transparenz auf. Überschreitet die Nachfrage die verfügbaren Stromtransportkapazitäten, wird keine Abwägung zwischen Kosten und Nutzen einer Transportleistung getroffen, sondern lediglich die über das Angebot hinausgehenden Stromtransportanträge werden abgelehnt. Denkbar ist eine Situation, in der der Nutzen aus dem Stromtransport deutlich höher ist als der Nutzen aus der uneingeschränkten Versorgung in dem Versorgungsgebiet des Verbund-EVUs. Trotzdem wird das den Stromtransit ablehnende EVU, unabhängig vom Preis, nicht bereit sein, seine eigenen Abnehmer zu verprellen. Dies führt zu keiner wohlfahrtsoptimalen Verwendung der Stromtransportkapazitäten.

Eine an der Marktsituation orientierte Berechmung der Stromtransportleistung ist für das Weiterentwicklungskonzept nicht sinnvoll, weil die Anzahl der Nachfrager für die Stromtransportüberkapazitäten eines EVU zu gering sind.

Für einen pauschalisierten Stromtransportpreis bei Transitleistungen als denkbare Alternative zwischen den EVU der Verbundebene spricht die hohe Planungssicherheit für die den Transit in Anspruch nehmenden EVU; hierdurch werden die Transaktionskosten reduziert. Es muß jedoch die Netzauslastıngssituation des transportierenden EVU mit berücksichtigt werden. Zur Verdeutlichung der Zusammen-

498 Diese Effekte werden jedoch wegen des geringen Umfanges der Stromtransite keine große Bedeutung erlangen. 
hänge soll im folgenden die Situation eines EVUs in einer Hochlastphase und in einer Schwachlastphase dargestellt werden:

Situation eines EVUs in einer Hochlastphase

An ein EVU wird in einer Hochlastphase (z.B. Winter) von einem Verbund-EVU I und einem Verbund-EVU II die Anfrage gerichtet, ob es 500 MW über einen Zeitraum von zwei Monaten übertragen kann (Stromtransit). Hierfür erhielte es den pauschalisierten Durchleitungspreis, der die Lastsituation des EVUs nicht berücksichtigt. Der Preis kann z.B. aus der Entfernung und der Leistung berechnet werden (vgl. Abb. 51 und 52).

Das Transit-EVU lehnt dies ab, da seine Leitungen nahe der wirtschaftlichen Kapazitätsgrenze ausgelastet sind und durch die zusätzliche Übertragungsleistung Verluste bei dem EVU entstehen können.

Bei der Ablehnung des Stromtransportantrages berücksichtigt das EVU nicht, welchen Grenznutzen diese Durchleitung bei den beiden beantragenden Unternehmen stiftet. Es könnte möglich sein, daß bei EVU I mehrere Kraftwerke ausgefallen sind und die Versorgung ohne den Stromimport eingeschränkt werden müßte. In dieser Situation sind die Kosten des zusätzlichen Stromtransportes niedriger als die Nutzenverluste bei den Abnehmern des EVUs I ${ }^{49}$. Bei einer freien Verhandlung des Strompreises zwischen den EVU wäre dies kein Problem; das EVU I mit dem Kraftwerksausfall wäre bereit, jeden Preis für den Stromtransport zu bezahlen, um die Versorgung seiner Kunden gewährleisten zu können.

Situation eines EVUs in einer Schwachlastperiode

EVU I und II beantragen nun in einer Schwachlastperiode beim gleichen Unternehmen einen Stromtransit (vgl. Abb. 53).

Sie sehen jedoch davon ab, weil die Transitpreise die Einsparungen durch die Lieferung überschreiten, der Nutzen von Stromtransporten niedriger ist als der Preis (in der graphischen Darstellung nicht erkennbar). Das Transit-EVU wäre jedoch wegen der geringen Auslastung des eigenen Netzes und seiner Grenzkostensituation bereit, den Stromtransit zu einem Preis unter dem pauschalisierten Transportpreis durchzuführen.

Die beiden Situationen zeigen, daß in beiden Fällen Wohlfahrtsverluste durch den pauschalisierten Durchleitungspreis entstehen, wenn die Netzauslastungssituation nicht berücksichtigt wird.

499 Grund hierfür ist , daß der pauschalisierte Stromtransportpreis nicht identisch ist mit dem Grenznutzen des Nachfragers. 


\section{Abbildung 51: Lastsituation, Stromtransportkapazitäten eines EVU}

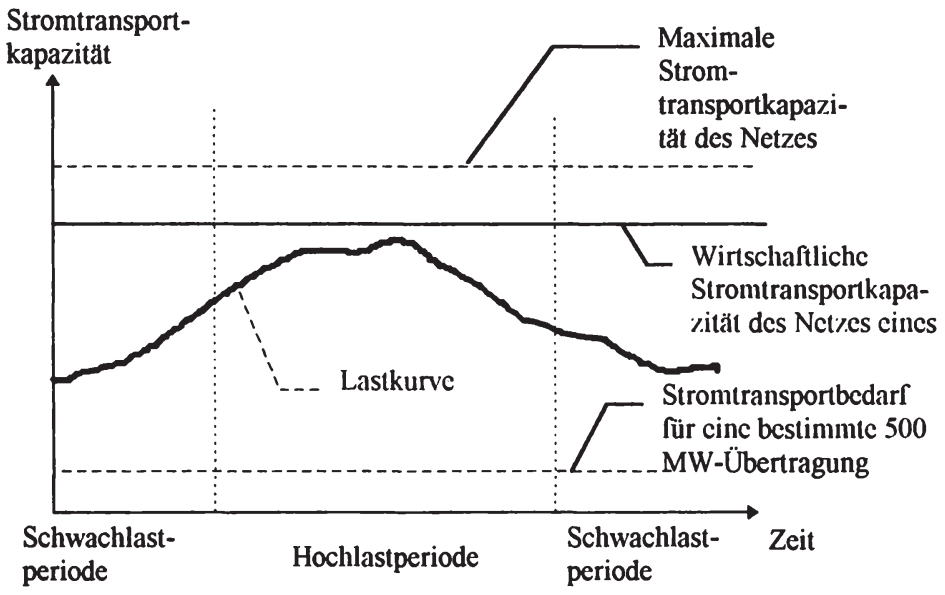

\section{Abbildung 52: Kostensituation für das EVU in einer Hochlastphase}

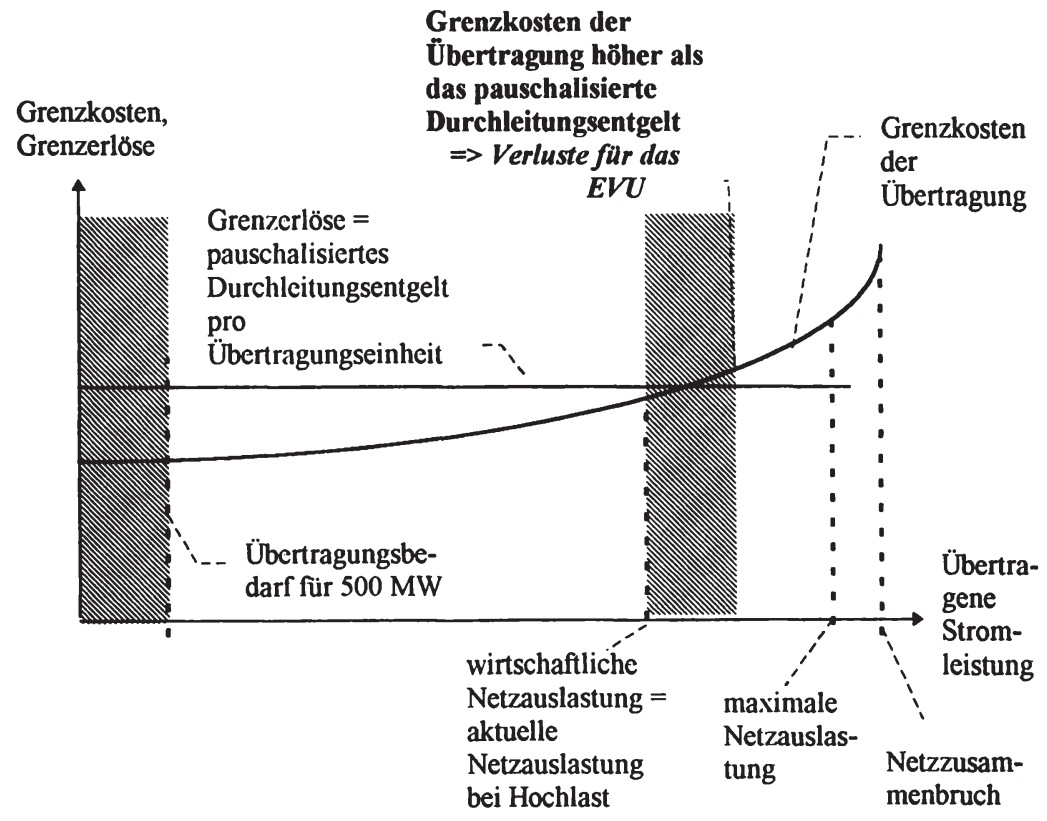




\section{Abbildung 53: Kostensituation eines EVUs in einer Schwachlastphase}

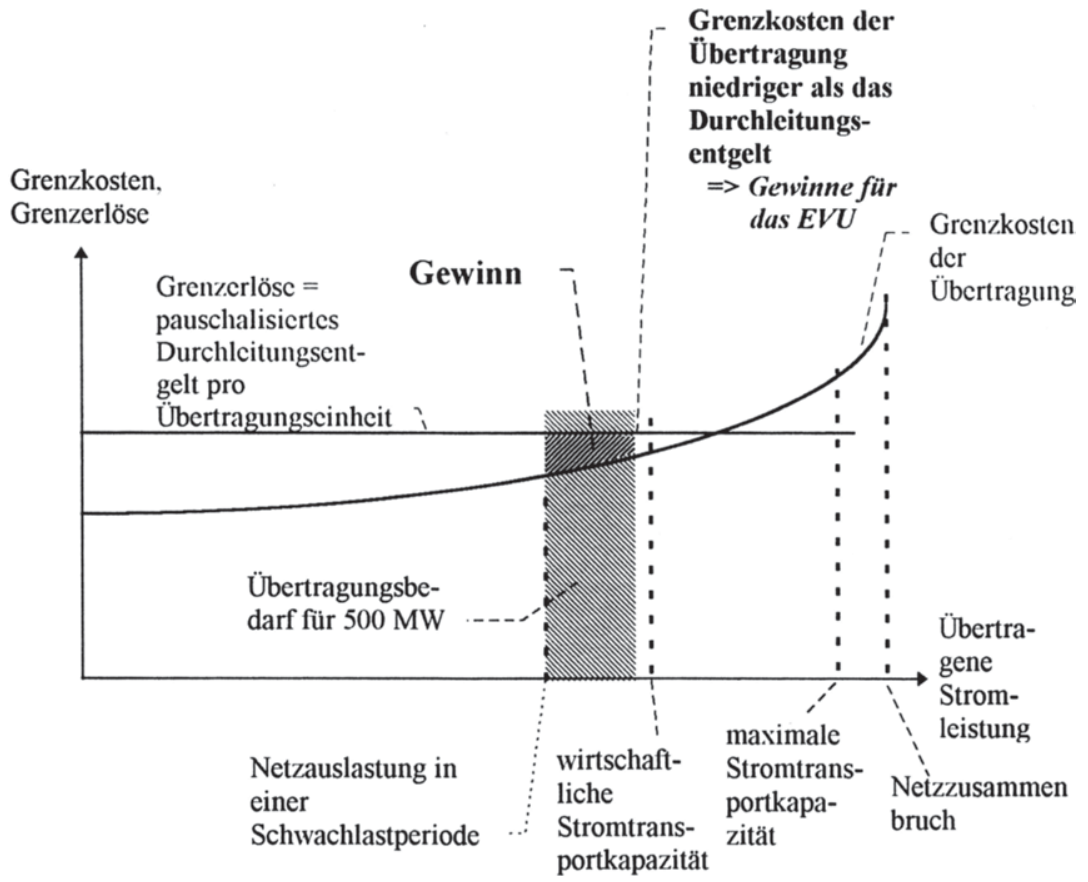

Bei einer abschätzenden Bewertung der Vor- und Nachteile der beiden Stromtransportpreisarten für das Weiterentwicklungskonzept erscheint dem Verfasser die einzelfallorientierte Lösung besser geeignet. Bei einer freien Verhandlung der potentiellen Vertragspartner können die individuellen Grenzkosten und Grenznutzen leichter berücksichtigt werden als bei einem pauschalisierten Preis. Ferner werden die EVU die einzelfallorientierte Preisbildung der pauschalisierten vorziehen, weil sie ihnen größere Handlungsspielräume gibt. Unterstützt werden sollte die einzelfallorientierte Verhandlungslösung durch Richtlinien zur Transportpreisberechnung. Die Richtlinien sollten als Berechnungsgrundlage für den Transportpreis ohne Zusatzkosten zu gewinnende Kostenindikatoren aufweisen, die eine einfache Schätzung der Grenzkosten erlauben. Darüber hinaus sollten sie durch Vereinbarung zwischen den EVU zustande kommen. Daß dabei die tatsächlichen Grenzkosten von den geschätzten abweichen, ist anzunehmen. Der dadurch entstehende Schaden wird durch die geringen Erhebungskosten ausgeglichen. Die Vergangenheit hat gezeigt, daß sich die Verbund-EVU an solche durch Konsens getragenen Richtlinien gehalten haben. Des weiterendbesteht für die-EVIJ Kein 1 Anngeiz, sich 
nicht an die Richtlinien zu halten, da die eventuell nicht durch den Preis abgedeckten Kosten in den meisten europäischen Staaten über die Gesamtkostendeckung den Endverbrauchern auferlegt werden können. Die mit der einzelfallorientierten Preiserhebung zusammenhängenden Transaktionskosten werden durch die geringe Anzahl der Stromtransporte im Weiterentwicklungskonzept begrenzt.

\subsubsection{Im TPA-Konzept}

Das Hauptmittel des TPA-Konzeptes, um die gesteckten Ziele zu erreichen, ist die Einführung einer Zwangsdurchleitung. Die Durchleitung soll gegen ein "angemessenes Entgelt" durchgeführt werden. Hieraus resultiert die Erforderlichkeit eines Durchleitungspreissystems. Aus dem Wesen der Zwangsdurchleitung ergibt sich, daß das Preissystem von der EU reguliert werden muß.

Anhaltspunkte für die Preisbildung geben die für das TPA-Konzept aufgestellten Forderungen: ${ }^{500}$

\section{a) Durchleitungen sind ausschließlich bei freien Übertragııngskapazitäten erzwingbar.}

Dies bedeutet, daß von dem Konzept kein Zwang zu Kapazitätserweiterungen ausgeht, aber auch keine Kapazitätsfinanzierung durch den Durchleitungspreis erfolgen soll; die Fixkosten des Stromtransportes werden nicht von dem Preis gedeckt.

\section{b) Durchleitungen sollen für das Gebiets-EVU zı einer gewöhnlichen Geschäfts- aktivität werden}

Dies können sie nur dann werden, wenn das Gebiets-EVU auch seine Fixkosten langfristig durch die Erlöse aus dem Stromtransport gedeckt bekommen.

Diese beiden Forderungen, auf die Preisbildung umgesetzt, ergeben einen inhärenten Widerspruch des Konzeptes, den kein Preissystem optimal lösen kann. Deshalb soll nun geprüft werden, welche Preise für die Zielerfüllung des Konzeptes annähernd geeignet sind.

Einzelfall- und kostenorientierte Preise für Durchleitungsleistungen können eher verursachungsgerecht sein als pauschalisierte; zu deren Berechnung ist jedoch ein hoher Aufwand an Messungen und ein hoher Kontrollbedarf der Regulierungsinstitution erforderlich. Trotz der Kontrolle hat das EVU die Möglichkeit, die eigenen Kosten zu verschleiern und die Durchleitungen zu diskriminieren. Für die Durchleitungsbegehrenden führt eine einzelfall- und kostenorientierte Berechnung des Durchleitungspreises zu einer höheren Planungsunsicherheit als die pauschali- 
sierte Preisbildung. Vor Geschäftsabschluß müßten sie zuerst Informationen über den zu erwarteten Durchleitungspreis einholen, der sich bis zum Abschluß des Geschäftes verändern kann.

Einzelfall- und marktorientierte Durchleitungspreise hätten den Nachteil, daß wegen der derzeitigen Versorgungsgebietsgrößen in Europa nur in wenigen Regionen mehrere Duchleitungsbegehren bei einem Gebiets-EVU vorliegen würden, insbesondere in der ersten Konzeptphase. Deshalb wird sich eine Marktsituation für Durchleitungen nur in wenigen Versorgungsgebieten bilden (market thinness). In den anderen Versorgungsgebieten werden entweder die Bedingungen für Durchleitungen nicht vorliegen, oder es wird kein oder nur ein geringes Interesse an Durchleitungen bestehen. In diesem Fall wird ein Durchleitungspreis, welcher auf der Nachfragesituation aufbaut, nicht zu einer Kostendeckung für das durchleitende Unternehmen führen. Dies bedeutet aber, daß die Mehrzahl der Abnehmer, die weiter ihren Strom von dem durchleitenden EVU beziehen, die wenigen Abnehmer, die Duchleitungen in Anspruch nehmen, subventionieren müßten. Damit wird jedoch gegen eine der beiden mit dem Konzept verbundenen Forderungen verstoßen.

Ein pauschalisierter Durchleitungspreis hätte für das TPA-Konzept mehrere Vorteile. Die integrierten EVU brauchen nach Festlegung eines Durchleitungspreises ihre Kostenstrukturen im Transportbereich nicht offenzulegen, es sein denn, sie wollen eine Anhebung des Durchleitungspreises erzielen. Eine Kontrolle der EVU durch die Regulierungsinstitution kann sich auf die Ablehnung wegen fehlender Kapazitäten beschränken. Hierdurch wird der Kontroll- und Regulierungsaufwand auf einem niedrigen Niveau gehalten. Gleichzeitig wird für die in Anspruch nehmenden Planungssicherheit hinsichtlich des Durchleitungspreises geschaffen.

Nachteilig fur das TPA-Konzept wirkt bei einem pauschalisierten Durchleitungspreis die fehlende Verursachergerechtigkeit. Unabhängig von den tatsächlich durch eine Durchleitung anfallenden Kosten werden alle, die Durchleitungen in Anspruch nehmen, mit dem gleichen Preis belastet. Andererseits könnte hiervon ein Anreiz auf die Durchleiter zu Effizienzsteigerungen ausgehen, wenn sie versuchen, ihre Kosten unter den festgelegten Preis zu senken, um dadurch höhere Gewinne zu erzielen.

Quersubventionierung ist eine weitere Gefahr, die von einem pauschalisiertem Durchleitungspreis ausgeht. Des weiteren spiegelt der pauschalisierte Durchleitungspreis keine Angebotssituation wider. Bei zahlreichen Durchleitungsbegehren und dafür nicht ausreichenden Kapazitäten muß das EVU eine Auswahl treffen, 
ohne über den Durchleitungspreis einen Ausgleich zwischen Angebot und Nachfrage herbeiführen zu können ${ }^{501}$.

Für einen pauschalisierten Durchleitungspreis kommen die gleichen Bemessungsgrundlagen wie für das Weiterentwicklungskonzept in Frage.

Der Verfasser schlägt aus den aufgezeigten Vor- und Nachteilen ein gemischtes Preissystem vor:

Überschreitet die Nachfrage an Durchleitungskapazitäten in einem Versorgungsgebiet das Angebot, so wird ein Bietwettbewerb durchgeführt. Die Anbieter mit den höchsten Geboten können ihre gewünschte Durchleitung erhalten.

Zur Verdeutlichung folgendes Beispiel: Zwei EVU (EVU1 und EVU2) wünschen eine Durchleitung von dem Gebiets-EVU. EVU1 möchte 500 MW und EVU2 300 MW in der gleichen Zeitperiode durchleiten lassen. Da sie mit ihren Kunden einen konstanten Preis ausgehandelt haben, ist ihr Grenznutzen pro MW konstant. Die Durchleitungskapazität des Gebiets-EVU beträgt $700 \mathrm{MW}$ bei dem gegebenen pauschalisierten Stromtransportpreis.

\section{Abbildung 54: Angebotsabgabe bei knappen Stromtransportkapazitäten}

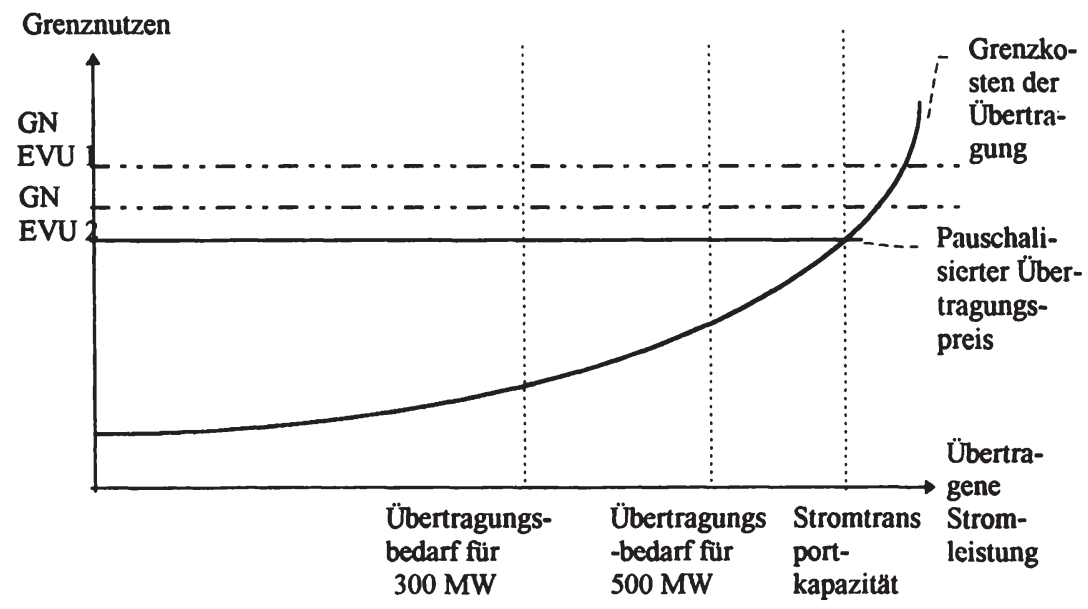

501 Dies wăre z.B. bei der Nutzung der skandinavischen Wărmekraftpotentiale und deren Absatz über eine HGÜ-Verbindung denkbar. Siehe hierzu auch die Ausfihrungen zu den möglichen Zuordnungsregeln im TPA-Konzep̈tinđKapiteh6.29.1-.978-3-631-75129-9 
In dieser Situation wird jedes EVU einen Preis bieten, der seinem Grenznutzen entspricht. EVU 1 wird daraufhin den Zuschlag erhalten und $500 \mathrm{MW}$ vom Gebiets-EVU durchleiten lassen können. EVU 2 kann dann noch $200 \mathrm{MW}$ durchleiten lassen. Da der von EVU 2 gebotene Preis auch bei Überschreiten der Durchleitungskapazität des pauschalisierten Preises noch über den Grenzkosten des Gebiets-EVU liegt, könnte dieses theoretisch mehr als $200 \mathrm{MW}$ übertragen. Davon wird es im TPA-Konzept aber Abstand nehmen, wenn das EVU 2 Abnehmer im Versorgungsgebiet des Gebiets-EVU beliefern will ${ }^{52}$. Handelt es sich jedoch um einen reinen Stromtransit, wird das EVU zu einer Ausdehnung des Stromtransportvolumens für das EVU 2 bereit sein.

Unterschreitet die Stromtransportnachfrage das bereitgestellte Angebot eines Gebiets-EVU, so sollte der Durchleiter einen pauschalisierten Durchleitungspreis erhalten. Bemessungsgrundlage sollte die übertragene Leistung, die Zeit und die Entfernung sein.

\subsubsection{Im Common-Carrier-Konzept}

Im ersten Desintegrationskonzept entsteht eine Netzgesellschaft, die sich ausschließlich auf den Stromtransport als Geschäftsaktivität beschränkt. Sie hat damit keinen direkten Einfluß auf die Stromtransportnachfrage; die von den Erzeugern und Verteilern bestimmt wird. Damit übernimmt die Netzgesellschaft Infrastrukturaufgaben in der Stromversorgung. Für die Netzgesellschaft besteht kein Anreiz, ihre Kosten zu verbergen oder bestimmte Abnehmer zu bevorzugen. Einige Bereiche ihrer Dienstleistung haben den Charakter eines öffentlichen Gutes, so z.B. die Versorgungssicherheitsbereitstellung und die Spannungs- und Frequenzhaltung. Die Kosten für den Netzbetrieb und die Ausgaben für Netzinvestitionen muß die jeweilige Netzgesellschaft aus den Erlösen des Transportes finanzieren ${ }^{503}$. Aus diesen Gründen muß eine Gesamtkostendeckung erzielt werden.

Ein pauschalisierter Stromtransportpreis kommt dem Dienstleistungscharakter der Transportgesellschaft entgegen. Die Vertragsgestaltung und -abwicklung beim Pool wird durch einen pauschalisierten Preis vereinfacht. Gleichzeitig wird eine gute Planungsbasis für die an der Stromversorgung beteiligten Unternehmen geschaffen. Nachteilig wirkt die fehlende Berücksichtigung der Auslastungssituation der Netzgesellschaft. Eine pauschalisierte Preisberechnung kann zwar auch die

502 Annahme: Der Grenznutzen aus dem Stromtransport ist niedriger als der Grenznutzen aus der Versorgung des Abnehmers.

503 Die Kosten für die Versorgungssicherheit sind gesondert z.u erheben, da sie von der Stromtransportpreisbemessungsgrundlage unabhaimgirgeist:.ermann - 978-3-631-75129-9 
Netzauslastungssituation berücksichtigen, ist dabei aber jeweils auf Annahmen und Prognosen angewiesen. Die tatsächliche Auslastungssituation ist erst bei Durchführung der Stromtransporte bekannt. Bei nicht ausreichenden Transportkapazitäten müssen Aufteilungs- und Vergabekriterien geschaffen werden. Des weiteren ist ein pauschalisierter, auf den Durchschnittskosten beruhender Stromtransportpreis nicht verursachergerecht.

Ein einzelfallorientierler Transportpreis im Common-Carrier-Konzept kann erst ex post berechnet werden, wenn die Lastflüsse im Netz und die tatsächlichen Auslastungsgrade sowie der erforderliche Regelungs- und Steuerungsaufwand bekannt sind. Als Nachteile sind bei einer einzelfallorientierten Berechnung der Transportkosten die hohen Transaktionskosten zu bedenken. Vor jeder Verhandlung über langfristige Stromhandelsverträge müßten die Vertragspartner erst Prognosen zu den Stromtransportpreisen für ihre beabsichtigte Stromleistung bei der Netzgesellschaft einholen. Die angenommenen Preise können jedoch bereits während der Vertragsverhandlungen Änderungen unterliegen; dies hat Auswirkungen auf die Rentabilität der angestrebten Lieferung für die Vertragspartner. Aus diesen Gründen spricht sich der Verfasser für einen pauschalisierten, an den Durchschnittskosten orientierten Stromtransportpreis aus. Um Kapazitätsengpässe zu vermeiden, kann die Netzgesellschaft ein Datensystem für die Verteiler- und Erzeugungsunternehmen bereitstellen, das potentiellen Stromhandelspartnern mittels einer Datenverbindung Auskunft über die Netzsituation und die von ihnen für das Geschäft zu entrichtenden Stromtransportpreise erteilt. Hierzu geben die Geschäftspartner den Entnahmeort und den Einspeisungsort in das System sowie die beabsichtigte Übertragungsmenge, Übertragungsleistung und Zeit ein und erhalten die erforderlichen Informationen. Darüber hinaus ist eine Bestellung der Transportdienstleistung der Netzgesellschaft über dieses Datensystem denkbar ${ }^{504}$.

\subsubsection{Im Stromhändlerkonzept}

Das Stromhändlerkonzept weist im Gegensatz zu dem ersten Desintegrationskonzept einen höheren Integrationsgrad mit einem umfangreicheren Wertschöpfungsprozeß der Netzgesellschaft auf.

Die Gesamtkosten der stromhandelnden Netzgesellschaft unterteilen sich in die Kosten des Stromtransportes und die des Stromhandels. Eine Abgrenzung ist ohne

504 Die Praktikabilităt eines solchen Systems kann vielfăltig im Dienstleistungssektor beobachtet werden. Erschwerend kommen für die Elektrizitătsıvirtschaft die Besonderheiten der Stromausbreitung und des Verhaltens von elektrischen Netzen hinzu. Die Probleme können jedoch über interne Lastsimulations- und Streckenauslastungsprogramme gelöst werden. 
grőßere Probleme mit den üblichen Instrumentarien des betrieblichen Rechnungswesens möglich ${ }^{505}$.

Die Stromhandelskosten sind unabhängig von der gehandelten Stromleistung, der Dauer und der Leistung. Des weiteren ist die vom Stromhändler zu erbringende Dienstleistung für jeden Handelsvorgang annähernd gleich. Abweichungen können in bezug auf das gewünschte Versorgungssicherheitsniveau auftreten. Daher ist es sinnvoll, die Preise für den Stromhandel an den Durchschnittskosten zu orientieren und über ein festgelegtes Entgelt pro Handelsvorgang abzudecken.

Bei einer ausreichenden Trennung zwischen Stromhandel und Stromtransport gelten für die Stromtransportleistıngsberechmıng die gleichen Aussagen wie für das erste Desintegrationskonzept.

In der Praxis würde dies zu einem zweigeteilten Preis führen, welcher aus einem festen Stromhandelspreis und einem leistungsabhängigen Stromtransportpreis besteht. Der Stromtransportpreis sollte wie im Common-Carrier-Konzept pauschalisiert berechnet werden (vgl. Tabelle 16).

\subsection{Die Stromtransportkapazitătsentwicklung}

\subsubsection{Die Stromtransportkapazitätsentwicklung im Weiterentwicklungskonzept}

Die Konzeption des Weiterentwicklungskonzeptes hat keine Auswirkungen auf den Betrieb und die Organisation der nationalen Netze sowie auf die Teilnahme am bestehenden Stromverbund, dessen Voraussetzung die Eigenverantwortlichkeit und Freiwilligkeit ist. Des weiteren verbleibt die Versorgungsverantwortung und damit die Versorgungssicherheitsvorsorge bei den bestehenden EVU. Die Netzinvestitionen richten die EVU in einem europäischen Strommarkt des Weiterentwicklungskonzeptes an ihrem erwarteten Strombedarf und dessen geographischer Verteilung aus. Die Verantwortung für die Versorgungssicherheit des Netzes läßt die EVU die prognostizierten Kapazitätsbedarfe um einen versorgungssicherheitsspezifischen Prozentsatz erhöhen ${ }^{506}$ (vgl. Abb. 55).

505 Wobei trotzdem zahlreiche Gemeinkostenzurechnungsalternativen verbleiben.

506 Vgl. als Beispiel die Stromtransportkapazitătsbedarfsentwicklung in DVG: Jahresbericht 1993, Heidelberg 1994, S. 15 
Tabelle 16: Übersicht: Stromtransportpreisarten

\begin{tabular}{|l|l|l|}
\hline \multicolumn{1}{|c|}{ Ordnungsmodell } & \multicolumn{1}{|c|}{ Stromtransportpreisart } & Bemessungsgä̈Be \\
\hline \hline Weiterentwicklungskonzept & einzelfallorientierte Preise & Grenzkosten \\
\hline $\begin{array}{c}\text { TPAKonzept bei Nachfrage > Angebot } \\
\text { bei Nachfrage < Angebot }\end{array}$ & einzelfallorientierte Preise & Bietwettbewerb \\
\cline { 2 - 4 } & pauschalisierte Preise & Durchschnittskosten \\
\hline Cormmon-Carrier-Konzept & pauschalisierte Preise & Durchschnittskosten \\
\hline Stromhändlerkonzept & pauschalisierte Preise & Durchschnittskosten \\
\hline
\end{tabular}

\section{Abbildung 55: Stromtransportkapazitätsentwicklung im \\ Weiterentwicklungskonzept ohne Stromhandelsaktivitäten}

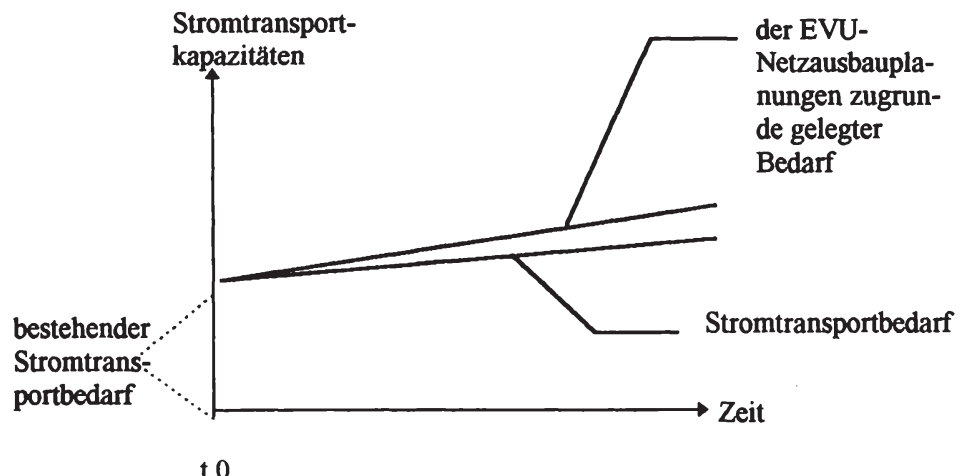

Der Anreiz zu Netzinvestitionen wird durch das Weiterentwicklungsmodell nicht beeinträchtigt.

Zu prüfen ist, ob die EVU im Weiterentwicklungskonzept Stromtransportkapazitäten zum Stromhandel errichten werden. Dies hängt im wesentlichen von dem Nutzen ab, den das jeweilige EVU aus den Stromtransportinvestitionen erwartet. Ein Nutzen aus der Stromtransportkapazitätserweiterung kann bei den EVU durch

a) die substituierende Funktion des Netzes in bezug auf Reservehaltungskapazitäten,

b) zusätzliche Deckungsbeiträge und

c) die Regulierungspraxis resultieren. 
$\mathrm{Zu}$ a):

Leitungskapazitäten und Netzinvestitionen sowie Kupplungen zu anderen Stromerzeugern können die Reservehaltung ersetzen ${ }^{507}$. Je höher die Kosten der Reservehaltung werden, um so stärker wird der Anreiz sein, Leitungskapazitäten zu deren Ersatz zu verwenden und auszubauen. Ein hoher Anreiz zur Substitution kann entstehen, wenn sich die Kraftwerksstruktur in einem Land verändert hat: Meist werden alte fossile Kraftwerke, z.B. auf Ölbasis, zur Reservehaltung eingesetzt. Steigt nun die Nutzungsdauer von Grund- und Mittellastkraftwerken wegen einer höheren Lebensdauer in der jeweiligen Lastart an, kommt es zu folgender Kostensituation: Die Kosten zum Betrieb der "Altanlage" für die Reservehaltung steigen mit zunehmender Lebensdauer an; die Gründe für die Kostensteigerung sind bei zunehmenden Alter steigende Ersatzinvestitionen und ein erhöhter Reparaturaufwand. Gleichzeitig sind die eingesetzten Anlagen in der Grund- und Mittellast nicht erneuerungsbedürttig. Ein EVU wird dann keine Anlagen aus dem Grund- und Mittellastbereich in den Spitzenlast- und Reservehaltungsbereich überführen. In dieser Situation wägt das EVU die Kosten zwischen der Errichtung von Spitzenlastkraftwerken und dem Ausbau des Netzes ab. Gegen diese Überlegungen spricht der zu Punkt drei beschriebene Sachverhalt.

Zu b) :

Zusätzliche Deckungsbeiträge aus dem Stromtransport können die EVU dann erwirtschaften, wenn durch das Stromtransportentgelt nicht nur die variablen Kosten gedeckt werden. Dies war bisher in Europa nicht der Fall. Sie entstehen im Weiterentwicklungsmodell, wenn die Grenzkosten je Mengeneinheit mit zunehmender Inanspruchnahme steigen, also ein progressiver Grenzkostenverlauf vorliegt. Dies ist nach Überschreiten der durchschnittskostenminimalen Stromtransportmenge im Stromtransport der Fall.

Zu c) :

Solange die EVU die Kostendeckung von seiten der nationalen Parlamente zugebilligt bekommen, entsteht kein Anreiz, die Eigenerstellung von elektrischer Energie durch fremdbezogenen Strom zu ersetzen. Die konsequente Folge sind Kraftwerksneubauten, auch wenn die substituierenden Netzinvestitionen geringere Kosten verursachen. Verstärkt wird dieser Effekt dadurch, daß die EVU nur begrenzt Gewinne im Verhältnis zum eingesetzten Eigenkapital oder gar keine Gewinne erzielen dürfen ${ }^{508}$, so daß sie die Option von Gewinnsteigerungen durch reduzierte Kosten nicht nutzen können. Je geringer der Anteil ist, der von den Einsparungen

507 Vgl. LICHTENBERG, H. : Der europäische Stromverbund, in: LUKES, R.(Hrsg.) : Ein EWG-Binnenmarkt für Elektrizităt: Realităt oder Utopie; Vortrăge und Diskussionsberichte anlåBlich e. wiss. Tagung in Münster/W. A, 29./30. Oktober 1987, Köln, Berlin, Bonn, München 1988, S.87 ff.

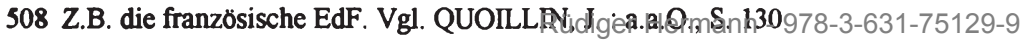


den Unternehmensgewinnen zugute kommt, desto geringer ist der Anreiz der EVU an einem Ausbau des Verbundes und damit des Leitungsnetzes über den Eigenbedarf hinaus ${ }^{509}$.

Anders stellt sich die Situation bei einer Höchstpreisregulierung ${ }^{510}$ dar. Rechnet ein EVU mit konstanten Elektrizitätspreisen in den nächsten Jahren, d.h. fehlenden Durchsetzungsmöglichkeiten von Strompreissteigerungen bei der Regulierungsbehörde, so kann es versuchen, über Einsparungen durch den Stromhandel Verluste zu vermeiden oder Gewinne zu erzielen. Die bei konventionellen Kraftwerken und Stromtransportkapazitäten bestehenden Planungs- und Bauzeiträume beschränken jedoch die diesbezüglichen Handlungspotentiale der EVU.

Zusammenfassend kann zur Kapazitätsentwicklung im Weiterentwicklungskonzept folgendes festgehalten werden: Für den Stromhandel werden die europäischen Gebiets-EVU nur dann Kapazitäten und eine Infrastruktur errichten, wenn sie positive Deckungsbeiträge durch den Stromhandel erwirtschaften können. Die bestehenden Versorgungsgebietsgrößen und die derzeitigen Erzeugungskostendisparitäten lassen jedoch, wie die Entwicklung der Szenarien gezeigt hat ${ }^{511}$, kein hohes Stromhandelsvolumen erwarten, bei dem ein Stromtransport durch das Gebiet eines EVUs erforderlich wird. Aus diesem Grund werden die Gebiets-EVU bei Verwirklichung des Weiterentwicklungskonzeptes keine Stromtransportkapazitäten für den Stromhandel errichten.

\subsubsection{Stromtransportkapazitätsentwicklung im TPA-Konzept}

Prinzipiell unterscheidet sich die Motivation der EVU zum Bau von Stromtransportkapazitäten im TPA-Konzept nicht von der des Weiterentwicklungskonzeptes. Die EVU müssen jedoch bei ihrer Investitionsentscheidung berücksichtigen, daß nicht selbstbenötigte Kapazitäten von Wettbewerbern zum Stromexport genutzt werden können; die EVU werden daher neben Rentabilitätsgesichtspunkten auch ihre strategische Wettbewerbsposition einschätzen und bei der Entscheidung berücksichtigen müssen.

509 Der Einwand, daß bei staatlich regulierten Monopolunternehmen oder im staatlichen Besitz befindliche Unternehmen erwirtschaftete Gewinne keine Anreize darstellen, vergißt, daß hăufig die Gewinne von Staatsunternehmen dem jeweiligen Staatshaushalt zufließen und somit als Erfolgsgröße angesehen werden. Ferner orientieren sich Manager traditionell an Erfolgsgrößen insbesondere dem Unternehmensgewinn.

510 Ein Beispiel für eine Höchstpreisregulierung ist die Bundesrepublik Deutschland. Vgl. hierzu WEISHEIMER, M. : Preise und Preisaufsicht im Egebnis der Systemtransformation Erfahrungen der neuen Bundesländer, in: ET 45. Jg. (1995), Heft 5, S. 286

511 Siehe hierzu Kapitel 6.2.3.1. 


\subsubsection{Die Auswirkungen der Regulierung auf die Stromtransportkapazitätsentwicklung}

In Ländern, in denen die zumeist staatlichen Unternehmen keine Gewinne erzielen dürfen, müßten die Unternehmen bei ihren Investitionsentscheidungen im Stromtransportbereich ausschließlich Kostengesichtspunkte berücksichtigen. Sofern die Unternehmen im europäischen Vergleich eine gute Erzeugungskostensituation aufweisen, wird dies auch der Fall sein. Für die anderen Unternehmen besteht jedoch die Gefahr, daß ihre Stromtransportkapazitäten bei einer schlechteren oder sich verschlechternden Kostensituation zum Stromexport durch Konkurrenten in ihr Versorgungsgebiet verwendet werden. Mit dem dann erzwingbaren Stromimport werden Arbeitsplätze in den Staaten abgebaut und die Bedeutung der Unternehmen geht ebenso wie deren Akzeptanz in der Bevölkerung zurück. Daran haben weder die Manager der Unternehmen noch die sie beeinflussenden Politiker ein Interesse. Deshalb werden diese EVU keine Stromtransportkapazitäten bauen, die zum Stromexport von anderen EVU genutzt werden könnten ${ }^{512}$.

EVU, die einer Höchstpreisregulierung unterliegen, können Stromtransportkapazitäten nutzen, um zusätzliche Deckungsbeiträge zu erwirtschaften, die ihnen unternehmenspolitische Freiräume schaffen. Sie müssen jedoch stets die Chancen und die Gefahren abwägen, weshalb ihre Marktsituation für die Investitionsentscheidung über Stromtransportkapazitäten zum Stromhandel ausschlaggebend sein wird $^{\text {s13. }}$.

In Ländern, in denen den EVU ein fester Prozentsatz des eingesetzten Kapitals als Verzinsung oder als Gewinn zugestanden werden ${ }^{514}$, wird es zu einer Abwägung kommen. Durch den Ausbau des Verbundes und des Stromhandels konkurrieren eigene Erzeugungsanlagen und Stromtransportkapazitäten um das einzusetzende Kapital. Entgegen den ökonomischen Grundsätzen, in denen das Kapital seiner produktivsten Verwendung zugeführt werden soll, wird die Entwicklung der Kapitalintensität für das Verhalten der EVU entscheidend sein:

a)

Übersteigt das einzusetzende Kapital zum Ausbau des Stromhandels das Kapital der dadurch ersetzten Erzeugungskapazitäten in einem Versorgungsgebiet, so werden die Stromtransportkapazitäten ausgebaut und das EVU beteiligt sich aktiv an

512 Die Auswirkungen der jeweiligen Wettbewerbsfăhigkeit auf dem europăischen Strommarkt werden im năchsten Unterkapitel ausführlich dargestellt.

513 Vgl. RIECHMANN, Ch.: Price-Cap Regulierung, in: ZfE 2/95, S. 158 ff.

514 In Spanien wird, als ein europăisches Beispiel, diese Regulierungsform gewăhlt. Vgl. hierzu EISS et al. : Die Ordnung ..., a.a.O., S. 612 . Zur Berücksichtigung der Kapitalkosten bei der Preisregulierung siehe SOWODA, P. : a.a.O., S. 66 ff. und LIPSEY, R.G. : Einführung

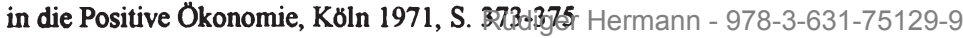


der Strombörse. Es gilt folgender Zusammenhang, wobei andere Fixkosten aus der Betrachtung ausgeschlossen sind (Verwaltung, Personal etc.):

$$
\begin{aligned}
& \Delta \text { KaStrTr }+\left(\mathrm{kv}_{\mathrm{v}} \text { StrTr }+ \text { peFremd }\right) * \mathrm{x}<\Delta \text { KaStrE }+\mathrm{kv}, \operatorname{StrE} * \mathrm{x} \\
& \text { Nebenbedingung: } \Delta \text { KasurTr }>\Delta \text { KastrE } \\
& \text { mit: } \\
& \text { KastrTr }=\text { Kapitalkosten Stromtransport des Gebiets - EVUs } \\
& \mathrm{kv}_{\mathrm{v}, \mathrm{StrTr}}=\text { Variable Stromtransportkosten des Gebiets - EVUs } \\
& \text { peFremd }=\text { Preis des Exporteurs } \\
& \mathrm{x}=\text { Strommenge } \\
& \text { KastrE = Kapitalkosten der Stromerzeugung des Gebiets - EVUs } \\
& \mathrm{kv}, \text { SurE = Variable Stromerzeugungskosten des Gebiets - EVUs }
\end{aligned}
$$

Als Folge dieses Zusammenhanges findet ein Fremdbezug statt. Effizienzsteigerungen werden durch die Konkurrenz bei den EVU eingeleitet und das Konzept ist zieladäquat.

b)

Ist die Kapitalintensität des Stromtransportausbaus geringer als die dadurch substituierte Kapitalintensität der Erzeugungsanlagen, wird die Substitution unterbleiben und das EVU Maßnahmen tätigen, um den Stromhandel zu unterbinden.

$$
\Delta \text { KaStrTr }<\Delta \text { KaStrE }
$$

Die erforderlichen Stromtransportkapazitäten zur erfolgreichen Implementierung der Strombörse unterbleiben. Daraus erwachsen Wohlfahrtsverluste in Höhe der Gesamtkostendifferenz.

Der in bezug auf den europäischen Strommarkt positiv erscheinende erste Fall (a) bedeutet jedoch, daß die Durchschnittskosten der Elektrizitätsversorgung bei Fremdbezug und gestiegener Kapitalintensität niedriger sein müssen als bei der Eigenerzeugung, also große Erzeugungspreisdisparitäten vorliegen müssen. 
$\mathbf{k}_{\mathbf{v}, \text { SurTr }}+$ peFrumd $<\mathbf{k v}_{\text {. StrE }}$

Nebenbedingung: $(\Delta \mathrm{kv}$.StrE $-\Delta \mathrm{kv}, \operatorname{StrTr}-$ peFremu $) * \mathrm{x}>\Delta$ KaStritr $-\Delta$ KasurL

mit:

KastrTr = Kapitalkosten Stromtransport des Gebiets - I:VUs

$\mathbf{k v}$, StrTr $=$ Variable Stromtransportkosten des Gebiets - EVUs

peFrend $=$ Preis des Exporteurs

$\mathrm{x} \quad=$ Strommenge

KasurE = Kapitalkosten der Stromerzeugung des Gebiets - EVUs

$\mathbf{k v}_{\mathbf{v}}$ StrE = Variable Stromerzeugungskosten des Gebiets - EVUs

Die folgenden Graphiken verdeutlichen diesen Zusammenhang: Ausgangspunkt ist die Situation eines Gebiets-EVUs, welches seinen benötigten Strom ausschließlich selbst erzeugt. Bei einer nachgefragten Stromleistung von X0 entstehen Kosten in Höhe von K0 (vgl. Abb. 56).

\section{Abbildung 56: Ausgangssituation eines Gebiets-EVU im TPA-Konzept}

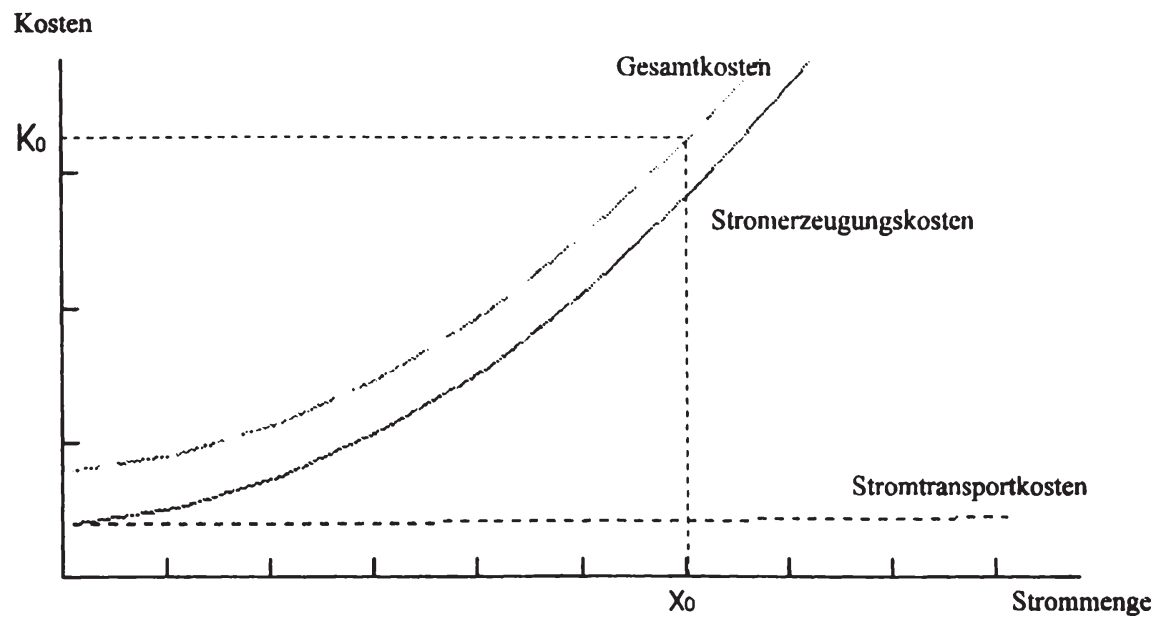


Die Kosten des Gebiets-EVUs gliedern sich, wie in Abbildung 56 dargestellt, auf. Von den Verteilungskosten wird dabei zur Vereinfachung abgesehen, da sie durch die Substitution der Erzeugungskapazitäten durch den Stromimport nicht betroffen werden. Ferner wird im folgenden ausschnittsweise nur die Kapazität mit ihren Kostenstrukturen betrachtet, die substituiert werden kann. Die beim Gebiets-EVU aus technischen oder kapazitativen Gründen verbleibenden Erzeugungs- und Übertragungskapazitäten werden nicht mit einbezogen, da sie auf die Unternehmensentscheidung für oder gegen einen Bezug der substituierbaren Kapazität keinen Einfluß haben.

Die Abbildung 57 zeigt die Kostenstrukturen eines potentiellen Anbieters, also eines anderen EVUs oder eines Erzeugers, außerhalb des Versorgungsgebietes des zu betrachtenden EVUs. Dieses Unternehmen führt noch keinen Stromexport durch. $\mathrm{Da}$ er wegen seiner Kostenstrukturen jedoch als potentieller Stromlieferant für Gebiets-EVU (Importeure) mit einer schlechteren Kostensituation angesehen werden kann, soll er im folgenden als Exporteur bezeichnet werden.

\section{Abbildung 57: Kostensituation eines potentiellen Exporteurs}

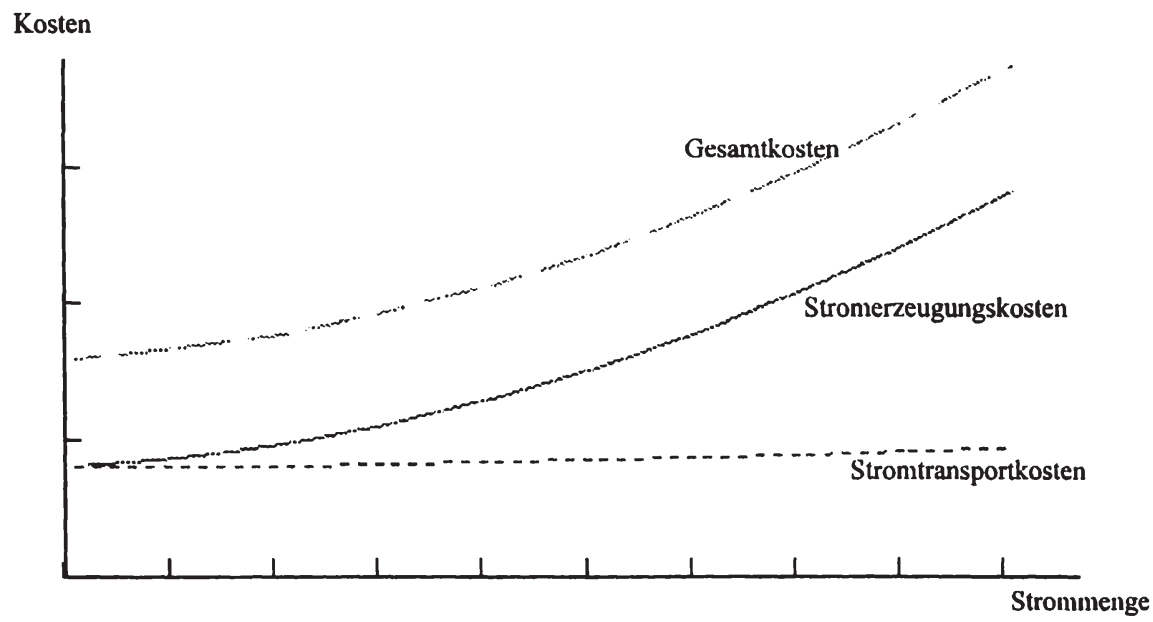

Strom wird dieses Unternehmen in andere Versorgungsgebiete zu seinen Grenzkosten oder einem Preis der über seinen Grenzkosten liegt, liefern. Durch den Stromexport fallen jedoch zusätzliche Stromtransportkosten an. Auch für diese wird das exportierende Unternehmen zumingest 
seinen Grenzkosten entspricht. Hieraus ergibt sich eine Preisuntergrenze im Versorgungsgebiet eines Importeurs, die in Abbildung 58 dargestellt ist. Abbildung 59 stellt die Gesamtkosten eines Importeurs dar.

Damit die Regulierungsinstutionen die Substitution des eigenerzeugten Stroms durch importierten bei einem Gebiets-EVU zustimmen und hierdurch auch das Ziel der sinkenden Kosten durch den europäischen Strommarkt erreicht wird, müssen die Gesamtkosten durch den Stromimport sinken (vgl. Abbildung 60).

Aus diesen Betrachtungen kann folgendes geschlossen werden:

Damit das Gebiets-EVU Strom importieren wird, muß die Kapitalintensität steigen. Gleichzeitig müssen die Gesamtkosten der Stromversorgung jedoch sinken; das bedeutet, die Grenzkosten des Stromimports müssen deutlich niedriger sein als die Grenzkosten bei Eigenerzeugung. Dies ist wegen des geringen Anteils der Stromtransportgrenzkosten an den Gesamtgrenzkosten bei Stromimport nur dann möglich, wenn große Erzeugungskostenunterschiede vorliegen. Da diese Konstellationen der Strompreisdisparitäten mittelfristig unrealistisch $\operatorname{sind}^{515}$, kann aus dem Modell geschlossen werden, daß ein Stromhandel unterbleibt. Stromtransportkapazitäten werden deshalb bei dieser Regulierungspraxis nicht errichtet werden. Würde aus wohlfahrtstheoretischen Gründen von den europäischen Regierungen dennoch der Bau der erforderlichen Kapazitäten gewünscht werden ${ }^{516}$, so müßte der Bau gegen den Willen der EVU durchgesetzt werden, wodurch die Aufwendungen steigen und der Nutzen aus der Maßnahme sinkt.

Außer über die Preisregulierung nimmt die Regulierungsinstitution durch die Ausgestaltung der beiden Durchleitungsverhinderungsgrïnde und deren Kontrolle Einfluß auf die Stromtransportkapazitätsentwicklung der EVU im europäischen Strommarkt des TPA-Konzeptes:

a) Ablehnung wegen fehlender freier Durchleitungskapazitäten

Das TPA-Konzept sieht in der bisherigen Fassung die Ablehnung eines Durchleitungsantrages bei fehlenden Übertragungskapazitäten vor. Nicht ausgeführt werden dabei die Bemessungsgrundlagen, wobei zwei Fragen von Bedeutung sind:

515 Vgl. hierzu die Übersichten zu den Strompreisen in der EU in Kapitel 4.2.2.2 .

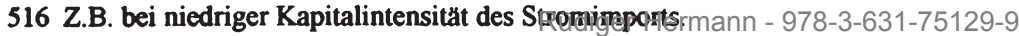


Abbildung 58: Preis eines Exporteurs für ein importierendes Unternehmen

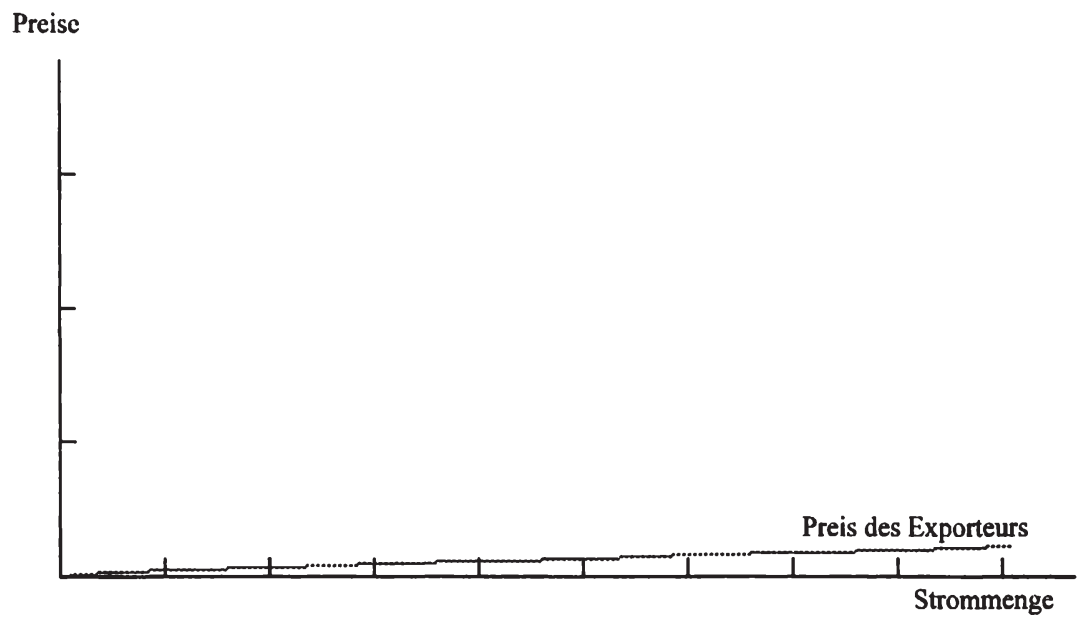

\section{Abbildung 59: Gesamtkosten eines Importeurs ohne Eigenerzeugung}

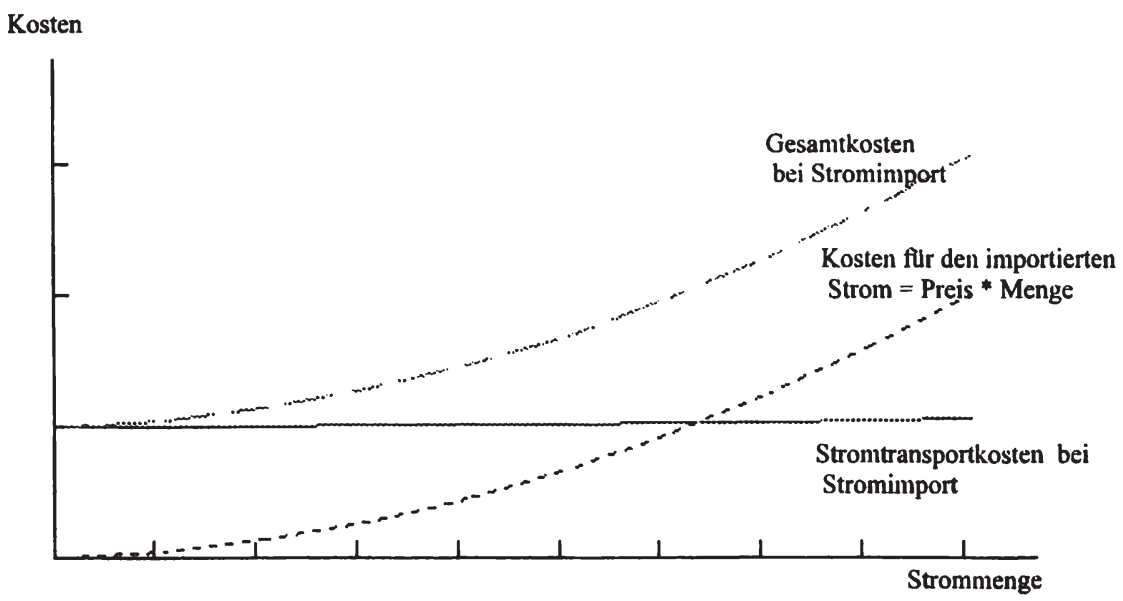




\section{Abbildung 60: Vergleich der Gesamtkosten bei vollständiger Eigenerzeugung und bei vollständigem Stromimport}

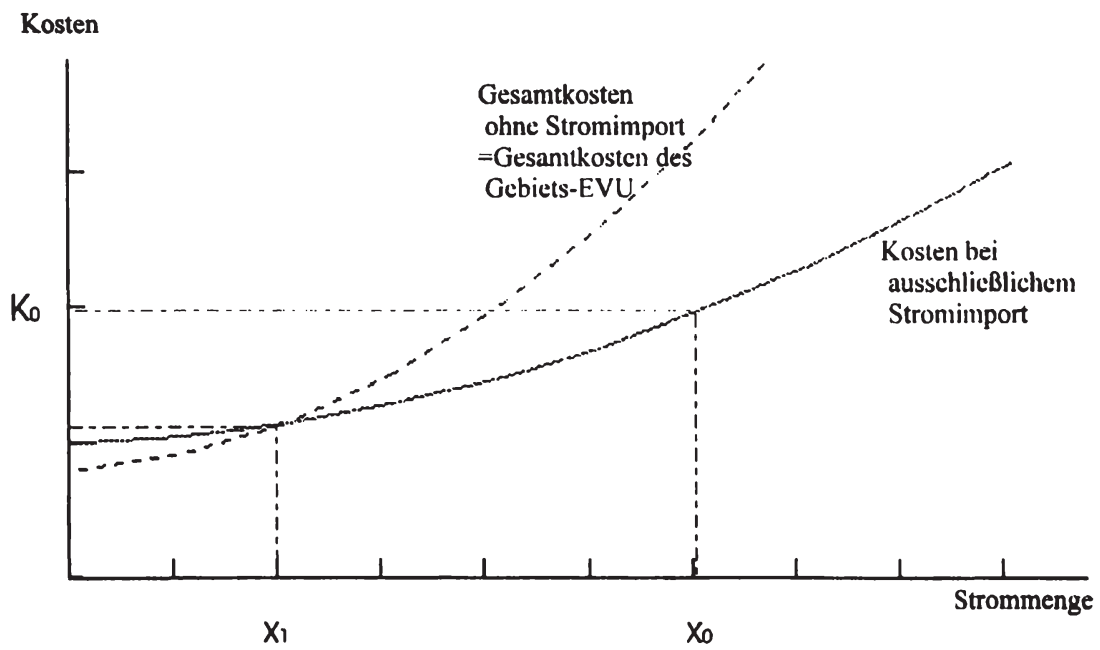

aa) Welche Kapazitätsgrenze soll herangezogen werden?

Zur Verfügung stehen die technische und die wirtschaftliche Kapazitätsgrenze.

Bei der Festlegung der wirtschaftlichen Kapazitätsgrenze bleibt jedoch ein Spielraum, den die EVU, die Durchleitungen verhindern wollen, nutzen werden. Deshalb gilt folgender Zusammenhang: Je präziser die Kriterien zur Festlegung der wirtschaftlichen Kapazitätsgrenze aufgestellt werden und je transparenter und kontrollierbarer sie sind, desto geringer sind die Möglichkeiten für EVU, über eine Reduzierung der wirtschaftlichen Kapazitätsgrenze Überkapazitäten scheinbar abzubauen.

ab) Werden Lastspitzen bei der Festlegung der freien Übertragungskapazitäten mit berücksichtigt?

Wird als Überkapazität in einem Hochspannungsnetz die Differenz zwischen wirtschaftlicher Kapazitätsgrenze und Stromtransportbedarf eines Gebiets-EVUs bei der Jahreshöchstlast definiert, werden nirgendwo in Europa Überkapazitäten existieren, die es einem Durchleitungsbeantragenden erlauben, eine Durchleitung zu erzwingen. 
b) Ablehnung wegen der Beeinträchtigung des Netzbetriebes

Ähnliches gilt für den zweiten Ablehnungsgrund. Je einfacher ein EVU, welches negativ durch eine Durchleitung (Stromimporte / -exporte) betroffen wird, diese mit dem Hinweis, es werde in seinem Netzbetrieb durch die Durchleitung gestört, ablehnen kann, desto weniger Durchleitungen werden durchgeführt werden.

Gelingt es den Regulierungsinstitutionen jedoch, durch klare Begriffs- und Sachverhaltsbestimmungen sowie eine kontinuierliche Kontrolle der netzbetreibenden EVU, Durchleitungen für die TPA-Berechtigten zu ermöglichen, bleibt den EVU mittel- und langfristig ausschließlich die Transportkapazitätsreduzierung durch verringerten Zubau oder verminderten Einsatz von Altanlagen.

\subsubsection{Die Abhängigkeit von der Marktsituation}

Bisher orientierten die integrierten EVU ihre Stromtransportkapazitätsentwicklung ausschließlich an ihrem Eigenbedarf. Zu dem prognostizierten Bedarf wurde von ihnen eine meist großzügig berechnete Versorgungssicherheitsmarge hinzugefügt, so daß die Stromtransportkapazitäten zu jedem Zeitpunkt den Anforderungen der Versorgung entsprachen. Bedarfsanstiege konnten ohne Probleme und Eingriffe in den Netzbetrieb sowie das Abnahmeverhalten von Verbrauchern bedient werden. Als Folge entstanden in fast allen europäischen Ländern Überkapazitäten im Stromtransportsektor, die durch die Versorgungssicherheit gerechtfertigt wer$\operatorname{den}^{517}$. Mit der Einführung des TPA-Konzeptes wird sich dieses Verhalten der Gebiets-EVU ändern: EVU, die durch Durchleitungen Abnehmer für ihre Erzeugungskapazitäten verlieren können, werden versuchen, über die Stromtransportkapazitätsentwicklung die erste der beiden zulässigen Bedingungen zur Ablehnung einer beantragten Durchleitung zu erfüllen ${ }^{518}$. Die Strategie, die ein Gebiets-EVU wählt, ist abhängig von seinem Stromerzeugungspreisniveau und seinen derzeitigen Stromtransportkapazitäten. Hinsichtlich des Erzeugungspreisniveaus soll furr die weitere Analyse in Unternehmen mit niedrigem, mittlerem und hohem Erzeugungspreisniveau unterschieden werden. Für die Stromtransportkapazitätssituation werden folgende drei Typen unterschieden:

- Ausgangssituation 1: Ausgelastete Stromtransportkapazitäten (Abb. 61)

- Ausgangssituation 2: Stromtransportkapazitätsknappheit (Abb. 62)

- Ausgangssituation 3: Stromtransportkapazitätsüberkapazitäten (Abb. 63)

517 PAFFENBERGER, W. ; SCHEELE, U. : Kosten und Nutzen von Stromhandel in Europa, Diskussionsbeitrag Nr. V-61-90 der Universităt Oldenburg, August 1990

518 Vergleiche hierzu Kapitel 6.2.1 . 
Abbildung 61: Ausgangssituation 1 - Ausgelastete Stromtransportkapazitäten

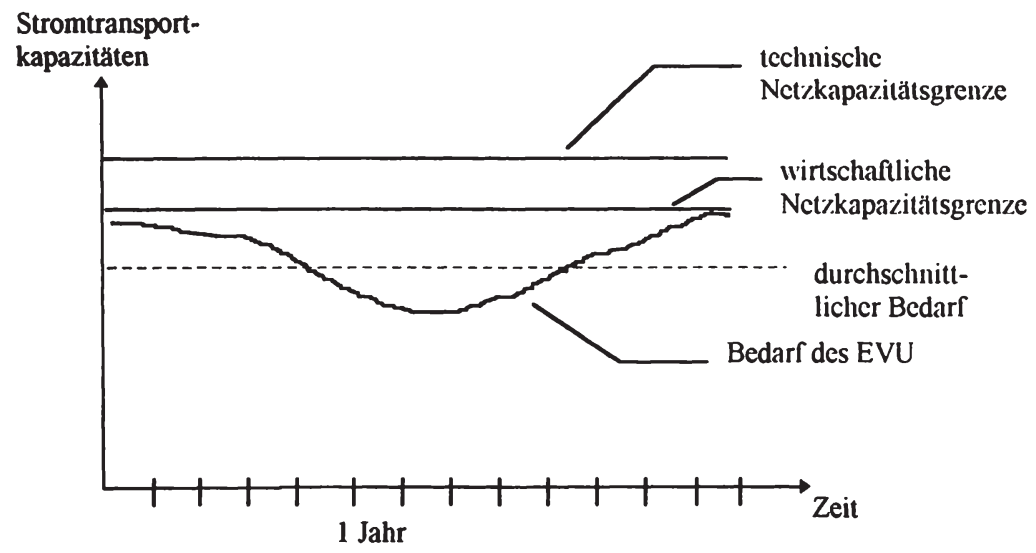

Abbildung 62: Ausgangssituation 2 - Stromtransportkapazitätsknappheit

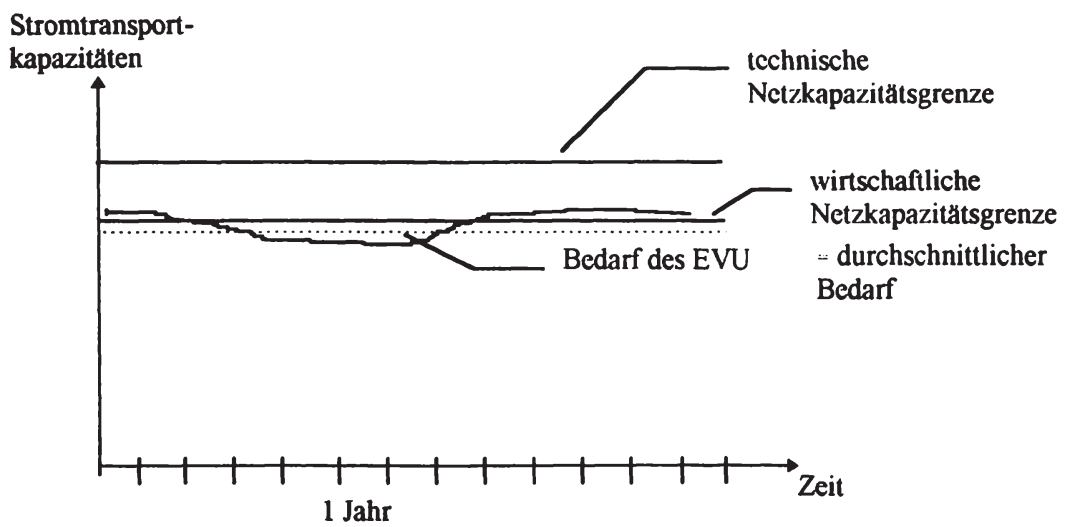

Die technische Kapazitätsgrenze ist die Übertragungsleistung, bei deren weiteren Ausdehnung die Spannung im gesamten Netz zusammenbricht. Die wirtschaftliche Übertragungskapazitätsgrenze ist abhängig vom Widerstandsverhalten einer Leitung. Sie kann dort gezogen werden, wo der Widerstand einer Übertragungsleitung expotentiell ansteigt. In der Praxis wird die wirtschaftliche Kapazitätsgrenze in den Widerstandbereich verlegt, der einem exponentiellen Anstieg unterliegt (Vgl. hier- 
zu die Abbildung 64). Der Anstieg des Widerstandes ist mit steigenden Leitungsverlusten gleichzusetzen ${ }^{519}$.

\section{Abbildung 63: Ausgangssituation 3 - Stromtransportüberkapazitäten}

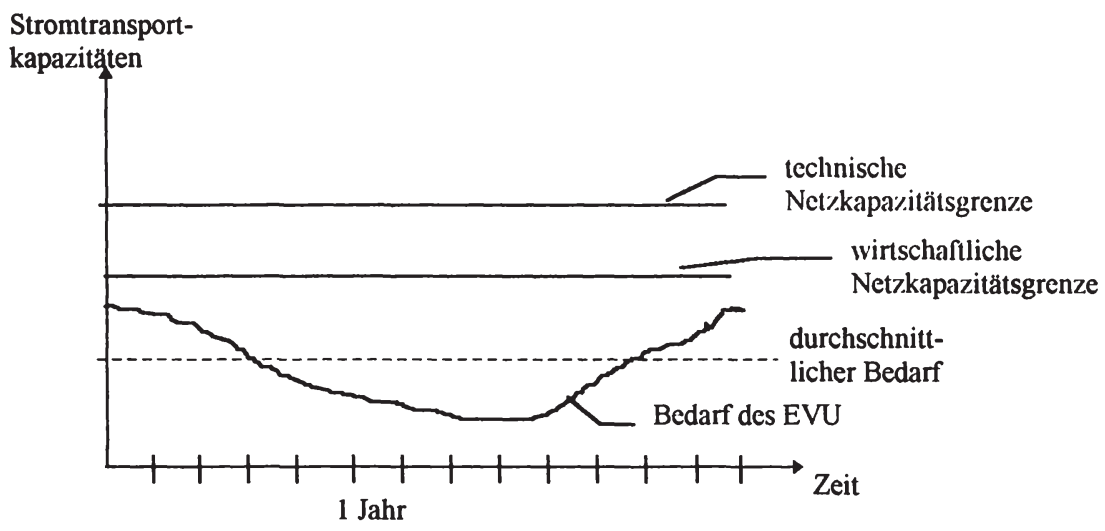

\section{Abbildung 64: Zusammenhang zwischen Widerstand und Übertragungsmenge einer elektrischen Leitung}

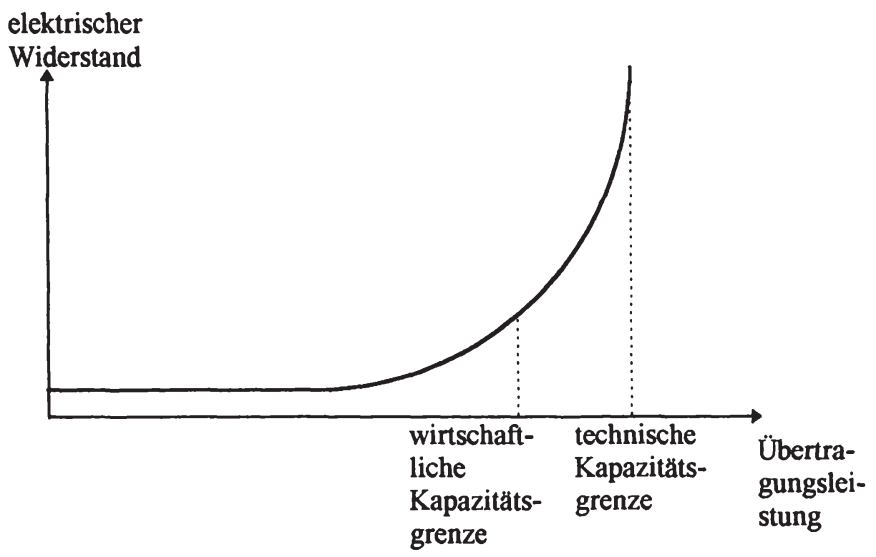

519 Trotzdem ist die wirtschaftliche Kapazitătsgrenze keine feststchende Größe. Durch technische Regelungs- und Steuereinrichtungen kann sie verändert werden. Siehe hierzu ESSL, H. FELDMANN, W. ; RENZ, K. : ASC- der Schlüssel für bessere Nutzung elektrischer Netze, in: EW Jg. 93 (1994), Heti8,g3ri396;399nn - 978-3-631-75129-9 
Durch das Konzept vorgegeben ist eine Durchleitung oder ein Stromtransit ausschließlich in der dritten Ausgangssituation (bei Stromtransportüberkapazitäten) erzwingbar.

Hieraus ergibt sich folgende Matrix, die die unterschiedlichen Marktsituationen von Gebiets-EVU in Abhängigkeit von ihren Stromtransportkapazitätsauslastungen und ihren Erzeugungspreisniveaus typisiert.

\section{Tabelle 17: Marktsituationen für EVU im TPA-Konzept}

\begin{tabular}{|l|c|c|c|}
\hline $\begin{array}{l}\text { Mono- Preisniveau } \\
\text { polkapazităt }\end{array}$ & $\begin{array}{c}\text { Erzeugungspreis } \\
\text {-niveau } \\
\text { niedrig }\end{array}$ & $\begin{array}{c}\text { Erzeugungspreis } \\
\text {-niveau } \\
\text { mittel }\end{array}$ & $\begin{array}{c}\text { Erzcugungspreis } \\
\text {-niveau } \\
\text { hoch }\end{array}$ \\
\hline $\begin{array}{l}\text { Ausgangssituation 1 } \\
\text { (Ausgelastete Kapa.) }\end{array}$ & I & II & III \\
\hline $\begin{array}{l}\text { Ausgangssituation 2 } \\
\text { (Kapazitătsknappheit) }\end{array}$ & IV & V & VI \\
\hline $\begin{array}{l}\text { Ausgangssituation 3 } \\
\text { (Überkapazităten) }\end{array}$ & VII & VIII & IX \\
\hline
\end{tabular}

\section{I und IV}

Die Gebiets-EVUs mit den Marktsituationen I und IV müssen keine Durchleitungen in den Konzeptphasen fürchten. Ihre Erzeugungspreissituation läßt sie zu potentiellen Lieferanten für Abnehmer in anderen Versorgungsgebieten werden. Die EVU, die wegen ihrer Erzeugungspreise Absatzchancen auf dem europäischen Strommarkt haben, werden ihre Stromtransportkapazitäten ausdehnen, um die Voraussetzungen zum Stromabsatz in anderen Versorgungsgebieten zu schaffen.

Die Differenz aus Kapazitätsentwicklung ohne TPA und dem prognostizierten Kapazitätsbedarf ist die Stromtransportkapazitätsreserve, die das EVU einplant. Die Höhe des Kapazitätszuwachses wird bestimmt durch die erwarteten Absatzchancen der EVU auf dem europäischen Strommarkt sowie die dazu zur Verfügung stehenden Stromerzeugungspotentiale. Ohne Absatzchancen oder ausreichendes Stromtransportpotential auf dem europäischen Strommarkt werden diese Gebiets- 
EVU ihre Kapazitätsentwicklung an dem erwarteten Eigenbedarf orientieren. Dann ändert sich ihre Kapazitätsentwicklung durch die Einführung des TPA-Konzeptes nicht.

\section{Abbildung 65: Stromtransportkapazitätsentwicklung bei einem EVU mit niedrigen Erzeugungskosten und Absatzchancen}

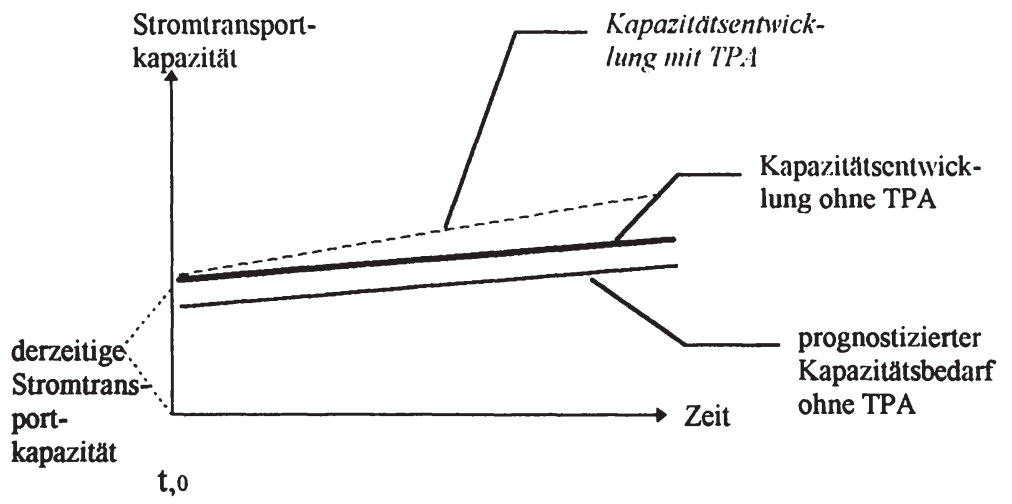

II

Gebiets-EVU mit mittlerem Erzeugungspreisniveau und ausgelasteten Stromtransportkapazitäten müssen kurz- und mittelfristig nicht mit einer erzwungenen Durchleitung rechnen. Deshalb werden sie ihr Verhalten beim Ausbau der Stromtransportkapazität nicht ändern, jedoch bei ihren Planungen in bezug auf den Eigenbedarf tendenziell niedrigere Werte zugrunde legen und die Marge fur die Versorgungssicherheit knapper auslegen, um den Auswirkungen einer zukünftigen verschlechterten Marktposition vorzubeugen.

\section{III}

EVU mit dieser Marktsituation werden ausschließlich durch ihre ausgelasteten Übertragungskapazitäten vor Durchleitungen ab der zweiten Konzeptstufe geschützt. Deshalb müssen diese EVU bestrebt sein, ihre Kapazitäten möglichst präzise am Eigenbedarf auszurichten und tendenziell die Übertragungskapazitäten weiter verknappen. Dann können sie bei Nachfragerückgängen nicht plötzlich zu Durchleitungen gezwungen werden, weil Stromtransportüberkapazitäten entstanden sind.

$V$

Ein Gebiets-EVU mit mittlerem Erzeugungspreisniveau und knappen Stromtransportkapazitäten unterliegt kurz- und mittelfristig nicht der-Gefahn s seine a 
durch die Implementierung des TPA-Konzeptes zu verlieren. Deshalb wird es seine Übertragungskapazitäten ausbauen, um die Knappheit zu beseitigen, jedoch die Errichtung von Überkapazitäten vermeiden.

\section{$V I$}

Die EVU mit hohem Preisniveau und knappen Übertragungskapazitäten werden ihre Kapazitäten äußerst restriktiv an den Eigenbedarf anpassen. Sie werden versuchen, jede Situation zu vermeiden, in denen Abnehmer eine Durchleitung mit Hinweis auf ausreichende Übertragungskapazitäten erzwingen können.

\section{VII}

Die Höhe des Kapazitätszuwachses bei einem EVU mit niedrigem Preisniveau und Überkapazitäten wird durch die erwarteten Absatzchancen der EVU auf dem europäischen Strommarkt sowie der dazu zur Verfugung stehenden Stromerzeugungspotentiale bestimmt.

\section{VIII}

EVU mit dieser Marktsituation werden ihre Übertragungskapazitäten abhängig von ihren Absatzpotentialen auf dem europäischen Strommarkt entwickeln. Müssen sie eine Verschlechterung ihrer relativen Erzeugungspreissituation, z.B. durch bereits beschlossene staatliche Auflagen oder Kraftwerkserneuerungen, befurchten, werden sie ihre Kapazitäten zu reduzieren versuchen. Bei Absatzchancen und erwarteten relativen Erzeugungspreisverbesserungen werden sie die vorhandenen Überkapazitäten zum Stromexport nutzen und ihre Kapazitätsentwicklung an den langfristigen Absatzchancen orientieren.

\section{IX}

EVU mit hohem Erzeugungspreisniveau und Überkapazitäten im Stromtransportbereich werden zu Beginn der zweiten Konzeptstufe Abnehmer an externe Lieferanten verlieren, sofern ein ausreichendes Angebot vorhanden ist. Sie müssen daher versuchen, durch eine gezielte Stromtransportkapazitätsentwicklung eine der beiden Bedingungen zur Ablehnung eines Durchleitungsantrages zu erfüllen.

Wie werden die Unternehmen in der Marktsituation IX ihre Kapazitäten abhängig von der Implementierungsphase verändern, um ihre durch das Konzept verschlechterte Absatzchancen wieder zu verbessern?

In der ersten Konzeptstufe kann lediglich der Stromtransit durch das Netz eines Gebiets-EVUs erzwungen werden. Die Durchleitung für andere Gebiets-EVUs beinhaltet somit für das stromtransportierende EVU keine direkten negativen Auswirkungen. Durch das Durchleitungsentgelt erhält es je nach Stromtransportpreis einen zusätzlichen Deckungsbeitrag. Das Interesse für einen Stromtransport 
kapazitätszubau ist damit isoliert betrachtet von den Deckungsbeiträgen abhängig. Berücksichtigt werden müssen jedoch die indirekten Auswirkungen für das Gebiets-EVU in der Situation IX: Durch die Abwicklung von Stromtransporten in der ersten Konzeptstufe offenbart es den Regulierungsinstitutionen und potentiellen Durchleitungsbegehrenden der zweiten Implementierungstufe seine vorhandenen Überkapazitäten. Das Gebiets-EVU hat es dann in der zweiten Stufe schwerer, Durchleitungen mit Hinweis auf fehlende Stromtransportkapazitäten abzulehnen. Aus diesem Grund werden die EVU versuchen, ihre Überkapazitäten zu reduzieren und technisch durchführbare Stromtransite zu verhindern. Begleitend können sie offensichtliche Überkapazitäten im Stromtransport durch langfristige Stromtransitverträge mit anderen Gebiets-EVU auslasten, bis die Maßnahmen zur Kapazitätsreduktion greifen.

Tabelle 18: Veränderung der Absatzchancen der EVU in Abhängigkeit von der Marktsituation

\begin{tabular}{|c|c|c|c|c|}
\hline & \multicolumn{3}{|c|}{ Unternehmen mit } \\
\hline & & $\begin{array}{c}\text { nledrigem } \\
\text { Erzeugungspreis- } \\
\text { nlveau }\end{array}$ & $\begin{array}{l}\text { milttlerem } \\
\text { Erzeugungspreis- } \\
\text { niveau }\end{array}$ & $\begin{array}{c}\text { hohem } \\
\text { Erzeugungspreis- } \\
\text { nlveau }\end{array}$ \\
\hline \multirow{3}{*}{ 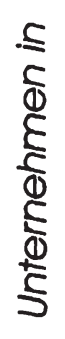 } & $\begin{array}{l}\text { Ausgangssituation I } \\
\text { (Ausgelastete Kapa.) }\end{array}$ & $\begin{array}{c}1 \\
\text { unveränderte } \\
\text { Absatzchancen }\end{array}$ & $\begin{array}{c}\text { II } \\
\text { unveränderte } \\
\text { Absatzchancen }\end{array}$ & $\begin{array}{c}\text { III } \\
\text { unveränderte } \\
\text { Absatzchancen }\end{array}$ \\
\hline & $\begin{array}{l}\text { Ausgangssituation } 2 \\
\text { (KapazitöIsknappheit) }\end{array}$ & $\begin{array}{c}N \\
\text { unveränderte } \\
\text { Absatzchancen }\end{array}$ & $\begin{array}{c}\text { V } \\
\text { unveränderte } \\
\text { Absatzchancen }\end{array}$ & $\begin{array}{c}\mathrm{V} \\
\text { unveränderte } \\
\text { Absatzchancen }\end{array}$ \\
\hline & $\begin{array}{l}\text { Ausgangssituation } 3 \\
\text { (Überkapazitäten) }\end{array}$ & $\begin{array}{c}\text { VII } \\
\text { verbesserte } \\
\text { Absatzchancen }\end{array}$ & $\begin{array}{c}\text { XX } \\
\text { verbesserte } \\
\text { Absatzchancen }\end{array}$ & $\begin{array}{c}1 \\
\text { verschlechterte } \\
\text { Absatzchancen }\end{array}$ \\
\hline
\end{tabular}

In der zweiten Stufe des Konzeptes ändert sich die Situation grundlegend: Für Gebiets-EVU in der Marktsituation IX wird die Stromtransportüberkapazität zu einer das Ergebnis negativ beeinflussenden Größe. Deshalb werden die EVU stärker als in der ersten Konzeptstufe versuchen, eine der beiden Bedingungen zu erfüllen, die von den Regulierungsinstitutionen als gerechtfertigtigter Ablehnungsgrund für eine beantragte Durchleitung akzeptiert werden. Dies kann durch zwei Maßnahmen erfolgen: 
a)

Das EVU versucht, seine Stromtransportkapazitäten an der VersorgungsgebietsPeripherie und den Kupplungsstellen zu anderen Netzen zu verringern oder zumindest konstant zu halten. Bei einem Anstieg des Eigenbedarfes reduzieren sich die Stromtransportüberkapazitäten und ein Durchleitungsbegehren kann abgelehnt werden.

\section{Abbildung 66: Überkapazitätsreduktion}

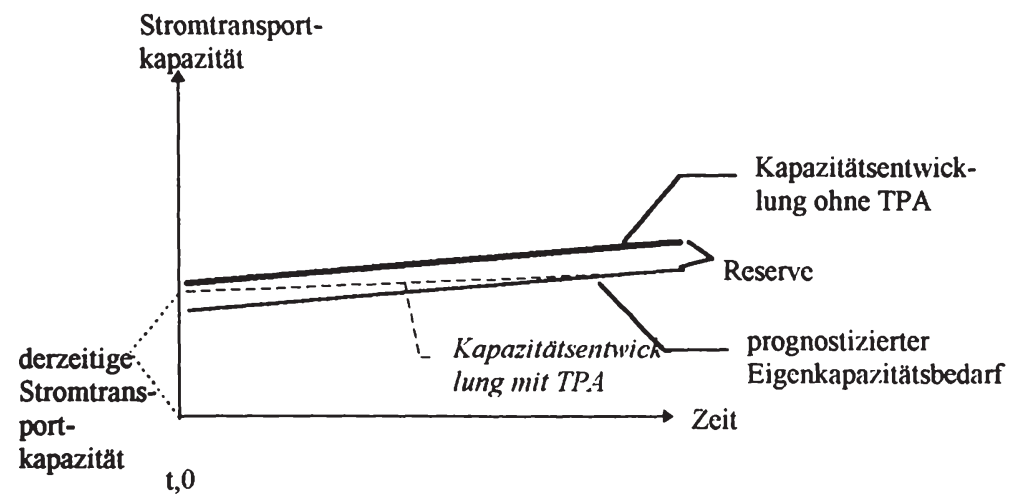

b)

Durch Beeinflussung seiner Lastkurve versucht das Gebiets-EVU ein Netzbenutzungsprofil des Typs 2 der Abbildung 67 herzustellen, um damit die zweite Durchleitungsablehnungsbedingung zu erfüllen. Eine derartige Lastkurvenveränderung kann das EVU durch folgende Maßnahmen erreichen:

- Einstellen aller stromverbrauchsreduzierenden oder -ausgleichenden Maßnahmen (Demand-Side-Management ${ }^{520}$ ),

- Abbau von Preisnachlässen für eine kontinuierliche Stromentnahme bei Großabnehmern ${ }^{521}$,

520 Vgl. SCHULZ, E. : Vom regulierungsorientierten zum kundenorientierten DSM. in: EW. Jg. 94 (1995), Heft 1/2, S. 65 ff. und DÜWALL. P. ; LANGE-HÜLSKEN. M. ; ZYBELL. G. : Lastganglinien der Haushalte - Ergebnisse und Schlußfolgerungen der Studie „Ermittlung der Lastganglinien bei der Benutzung elektrischer Energie durch die bundesdeutschen Haushalte während eines Jahres, in: EW Jg. 84 (1985) . Heft 25, S. 1059 ff. und zum aktuellen Stand der Entwicklungen zum Demand-Side-Management in den USA: DRILLISCH, J. : Der Einfluß wettbewerblicher Strukturen in der US-Elektrizitătstvirtschaft auf das Demand-Side Management, in: Zeitschrift für Energiewirtschaft, Heft 1 (1996), S. 37-52, hier S. $37 \mathrm{f}$.

521 Hierdurch unterlassen die Großabnehmer Anstrengungen, ihren Strom kontinuierlich aus

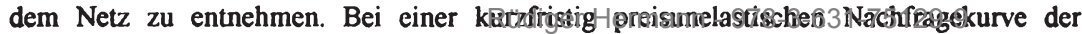


- Werbemaßnahmen für Strom bei Haushaltskunden, um Bedarfsspitzen auszudehnen.

\section{Abbildung 67: Netznutzungsprofiländerung}

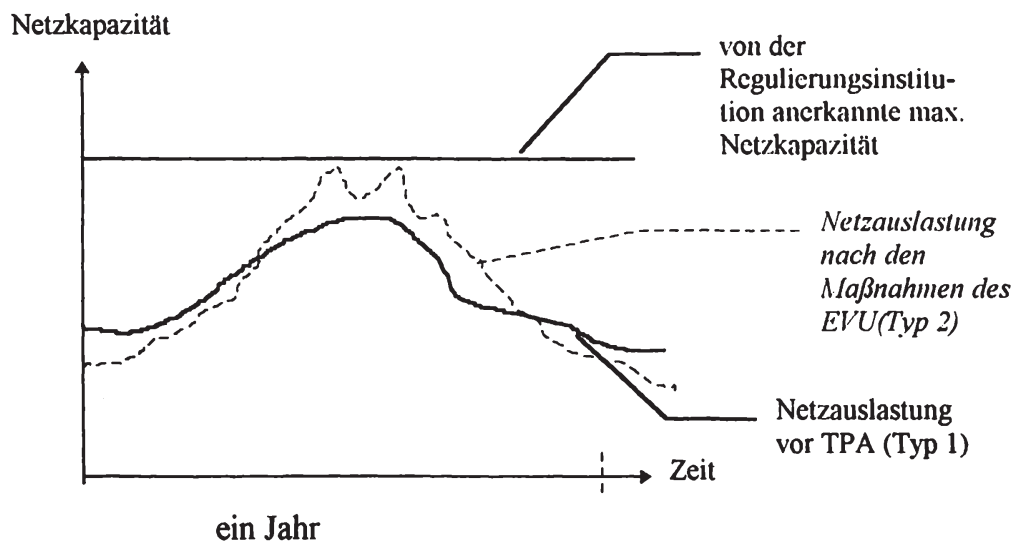

In der dritte Konzeptstufe werden die Gebiets-EVU in der Situation IX die gleichen Maßnahmen wie in der zweiten Konzeptstufe zur Abwehr von Durchleitungen nutzen oder die bereits begonnen Enwicklungen weiterführen.

\subsubsection{Der Einfluß von Bedarfsprognosen}

Bisher sind die EVU bei der Schätzung des zukünftigen Elektrizitätsbedarfes ${ }^{522}$ und des daraus resultierenden Erzeugungs- und Transportkapazitätsbedarfes von tendenziell höheren Werten ausgegangen, um bei Eintreffen der Prognosen, welche eine überdurchschnittliche Verbrauchssteigerung voraussagen, die Versorgungssicherheit gewährleisten zu können. Bei Einführung der Durchleitungsverpflichtung müssen die EVU in den Marktsituationen II, III, V, VI, VIII und IX bestrebt sein,

Großabnehmer sinkt die Nachfragemenge dieser Kundengruppe 7.war langfristig, das EVU kann aber mit diesem Mittel seine Durchleitungskapazität kurzfristrig reduzieren.

522 Zur Planung von Kraftwerken, Kapazităten und Leitungen siehe z.B. : BELKHOFER, H. ; JAKOB, K. ; SACHS, U. : Planung elektrischer Netze mit Sincal, in: Elektrizitătswirtschaft. Jg. 91 (1992), Heft 22, S. 1444-1455 und PIEHL, E. : CIRED-Sitzung 6: Entwurf und Planung offentlicher Verteilungsnetze, in: Elektrizitătswirtschaft, Jg. 90 (1991), Heft 16/17, S. 939-943 und die dort angegebene Literatur. Rüdiger Hermann - 978-3-631-75129-9 
keine Überkapazitäten entstehen zu lassen, um Abnahmeausfälle durch Abwanderung der Versorgungskunden zu vermeiden.

Ein EVU, welches aufgrund der Eigeneinschätzung seiner zukünftigen Marktsituation die Durchleitungsanträge anderer EVU oder von Verbrauchern auch vor einer Aufsichtsbehörde ablehnen will, wird seine Transportnetzausbauentscheidung an den ersten beiden Prognosen ausrichten. Hierdurch verringert sich das dem Stromhandel zur Verfügung stehende Stromtransportkapazitätspotential. Bei einem darin nicht enthaltenen Anstieg des Strombedarfes und dem daraus resultierenden Stromtransportbedarf innerhalb eines Versorgungsgebietes werden die fehlenden Kapazitäten durch eine höhere Auslastung einzelner Kraftwerke und, wenn dies nicht mehr möglich ist, durch eine Reduzierung der Reservehaltungkapazitäten aufgefangen. Ferner werden teilweise Hochspannungsleitungen über die wirtschaftliche Auslastungsgrenze hinaus genutzt. Die Leitungsverluste steigen hierdurch an, die Versorgung bricht jedoch nicht zusammen. Die Folgen sind eine geringere Versorgungssicherheit und höhere Kosten für die Stromversorgung. Die Gebiets-EVU ziehen diese Situation der Durchleitungssituation vor, da sie weiterhin ihre Kostendeckung zugesichert bekommen und potentiellen Konkurrenten den Zugriff auf ihre Abnehmer verwehren. Gleichzeitig gewinnen die EVU bei den Regulierungsinstitionen durch den Hinweis auf die negativen Auswirkungen des TPA-Konzeptes eine stärkere Verhandlungsposition. Eine weitere negative Auswirkung dieses Verhaltens ist die Verringerung der EU-weiten Reservehaltungspotentiale, die bei Kraftwerksausfällen oder trockenen Sommern wenigstens teilweise benötigt wer$\operatorname{den}^{523}$.

\section{Abbildung 68: Kapazitätsbedarfsentwicklungsprognosen und deren Auswahl für die Ausbauentscheidungen bei den EVU}

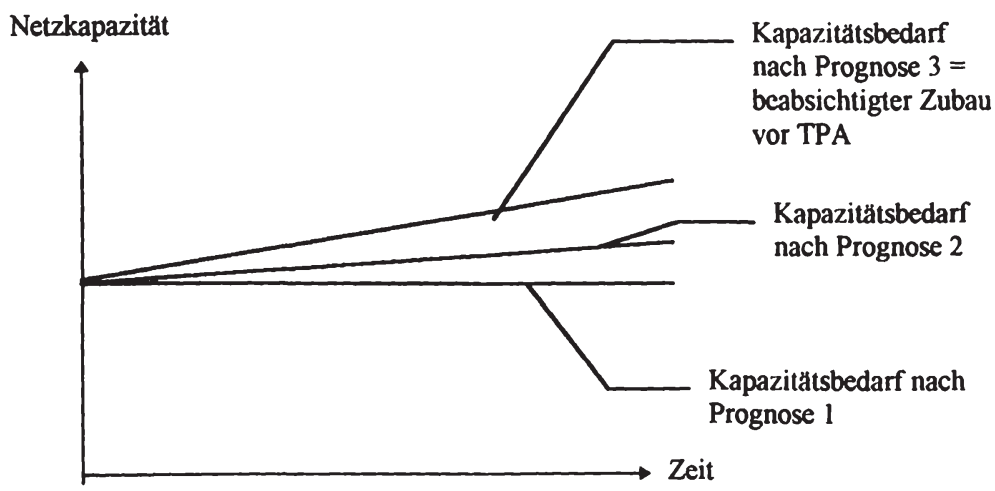

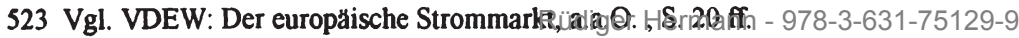


Aus den Überlegungen zur Kapazitätsentwicklung im TPA-Konzept folgt: Die langfristig maximale für die Durchleitungen zur Verfügung stehende Transportkapazität sinkt in dem Maße, wie die Maßnahmen der Gebiets-EVU in der Situation IX greifen, vorausgesetzt in der Marktsituation IX befinden sich Unternehmen. $\mathrm{Zu}$ einem Zeitpunkt tx kann jedoch jedes EVU in der Marktsituation IX nach Durchführung der Anpassungsmaßnahmen eine Durchleitung mit Hinweis auf fehlende Übertragungskapazitäten ablehnen. Die vorhandenen Überkapazitäten der GebietsEVU in der Situation VII bleiben bestehen ${ }^{524}$. Die Unternehmen in der Marktsituation VIII finden keine Abnehmer für ihre Überkapazitäten. Sie werden daher keine Kapazitäten zum Stromhandel errichten, gegebenenfalls ihre Überkapazitäten reduzieren, um Kosten zu sparen.

\section{Abbildung 69: Stromtransportpotential des Gesamtmarktes für Durchleitungen}

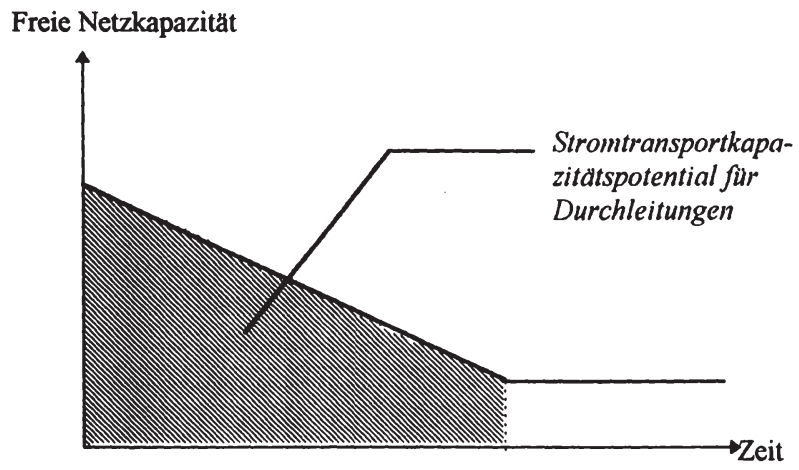

$\mathrm{tx}$

Es stellt sich die Frage nach der Stromtransportkapazitätsentwicklung für Durchleitungen nach dem Zeitpunkt tx. Der tatsächlich eintretende Bedarf an Stromtransportkapazitäten ist von den EVU ja nur annähernd planbar, da letztendlich die Abnehmer den Bedarf bestimmen ${ }^{525}$. Aus diesem Grund wird es nach tx zu einem, um den geplanten Wert schwankenden Stromtransportbedarf kommen (vgl. Abbildung 70).

524 Abgesehen wird bei den Ausführungen von den Stromtransportkapazitătseinsparungen durch die Durchleitungen beim Gebiets-EVU. Die Einbeziehung dieses Sachverhaltes würde die Darstellung verkomplizieren, ohne jedoch die Ergebnisse zu beeinflussen.

525 Dieser unterliegt nicht zuletzt auch konjunktürellear Schwankungeñ.-3-631-75129-9 
Dies bedeutet, daß kurzfristig begrenzte Stromtransportkapazitäten für Durchleitungen entstehen (b). Eine Nutzung dieser Kapazitäten kann sich der Verfasser durch kurzfristig gestiegene temporäre Strombedarfe einzelner Großabnehmer vorstellen. In der Realität wird diese Konstellation selten vorkommen, da häufig Rezessionszeiten die Ursache für eine Stromnachfrage, die niedriger ist als die erwartete, sind. In solch einer Situation wird selten ein Großabnehmer unvorhergesehene Bedarfszuwächse haben. ${ }^{526}$. Zur erneuten Einführung eines wettbewerblichen Verhalten oder zu Anreizen für Effizienzsteigerungen werden die nur kurzfristig nutzbaren Kapazitäten nicht ausreichen.

\section{Abbildung 70: Stromtransportkapazitätsabweichungen in TPA-Konzept}

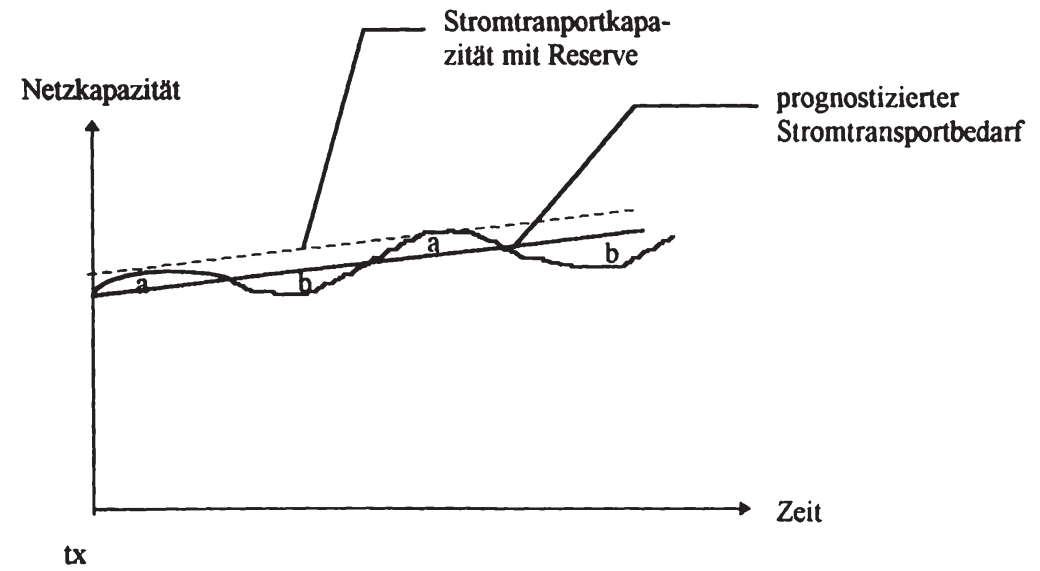

\subsubsection{Stromtransportkapazitätsentwicklung im Common-Carrier-Konzept}

Durch die Desintegration der EVU bei Einführung des Common-Carrier-Konzeptes entstehen eine oder mehrere eigenständige Netzgesellschaften, deren Aufgabe es ist, den Stromtransportbedarf des europäischen Binnenmarktes zu decken. Da-

526 Vgl. hierzu die Ergebnisse der Expertengruppe für die Analyse des Industric- und Tertiărverbrauchs (STATIND) des XXII . Kongresses der UNIPEDE vom 10.-14.06.1991 in Kopenhagen, abgedruckt in: UNIPEDE: Fachberichte Synthesen, XXII. Kopenhagen. MărZ 1991 
durch wird die Abwicklung des Stromtransportgeschäftes zur einzigen Geschäftsaktivität der Netzgesellschaft, auf die ihr alleiniges Augenmerk gerichtet sein muß. Für den Binnenmarkt übernehmen die Netzgesellschaften eine Dienstleistung mit Infrastrukturcharakter. Durch die Trennung der Leistungserstellung in der Elektrizitätsversorgung kann die jeweilige Netzgesellschaft das Stromtransportgeschäft nicht zu Lasten anderer Geschäftsbereiche diskriminieren; im Gegenteil: der Erfolg des Unternehmens wird von der Regulierungsinstitution, die über die Unternehmensgewinne und Spielräume mit entscheidet, an der Infrastrukturbereitstellung gemessen. Den Netzgesellschaften wird innerhalb der EU die Gesamtkostendekkung zugebilligt, um zu verhindern, daß eine mangelnde Investitionstätigkeit im Transportsektor die Versorgungssicherheit gefährdet. Daraus folgt für die Netzgesellschaft, daß sie keinerlei Anreize hat, Netzinvestitionen zu unterlassen.

Ein Problem für die Netzgesellschaft bei der Stromtransportkapazitätsentwicklung ist die fehlende Beeinflußbarkeit der Kraftwerksstandorte und -verteilungen innerhalb ihres geograhischen Gebietes. Ohne eine Anzeige eines Kraftwerksbauvorhabens bereits in der Planungsphase würde die Netzgesellschaft bei ihrer Kapazitätsentwicklung den Kraftwerksbauten ,hinterher hinken“. Dies kann jedoch nicht im Interesse der Netzgesellschaft und des Kraftwerksbetreibers sein, der durch einen unzureichenden oder fehlenden Hochspannungsnetzanschluß im Absatz seiner Erzeugungskapazität behindert wird. Jedoch nutzt die Anzeige von Kraftwerksneubauten oder Ausbauten nur dann, wenn die Bauzeiten für Kraftwerke mindestens so lang sind wie die Bauzeiten für die erforderlichen Stromleitungen. Für die heute überwiegend zur Stromversorgung eingesetzten Großkraftwerke ist diese Bedingung erfüllt. Bei Einführung des Common-Carrier-Konzeptes muß jedoch verstärkt mit dem Bau dezentraler Erzeugungsanlagen in schnell zu errichtender Modularbauweise gerechnet werden ${ }^{527}$. Bei diesen dezentralen Anlagen ist ein Anschluß an das Niederspannungsnetz unproblematisch, so daß die Netzgesellschaft von dem Kraftwerksbau nicht betroffen ist ${ }^{528}$. Andere Auswirkungen haben dagegen Erzeugungsanlagen, die einen Anschluß an das Mittelspannungsnetz eines Verteilungsunternehmens, das selber keine Erzeugungsanlagen besitzt, erfordern. Das Verteilerunternehmen muß für die zusätzliche Erzeugungsanlage eine Regelungstechnik installieren, die ohne den Anschluß nicht erforderlich wäre. Entschärft wird dieses Problem dadurch, daß neue Kraftwerksstandorte in den Mitgliedsstaaten politisch zumeist nicht mehr durchsetzbar sind ${ }^{529}$. Deshalb werden Kraftwerksneubauten zumeist auf dem Gelände bestehender oder ehemaliger Kraftwerke

527 Z.B. auf Gasbasis . Vgl. hierzu z.B. BETLING, T. : Blockheizkraftwerke, in: Fachinformationszentrum Karlsruhe, Karlsruhe 1991

528 Zur technischen Realisierbarkeit vgl. JANSSEN, R. : Niederspannungsnetze, in: EW 92. Jg. (1993), Heft 21, S. 1283 ff. . Auf die daraus resultierenden Probleme mit der Vertragsgestaltung und der Laststeuerung zwischen Verteilungsunternehmen und dezentralem Erzeuger soll im Rahmen dieser Arbeit nicht eingegangen werden.

529 So besteht z.B. in Frankreich starker Widerstand gegen neue Wasserkraftanlagen und in Italien generell gegen neue Kraftwerksstandotteiger Hermann - 978-3-631-75129-9 
durchgeführt, zu denen die entsprechenden Hochspannungstrassen bereits bestehen oder erweitert werden können.

In bezug auf die Kupplungen zu anderen Netzen hat die Netzgesellschaft kein Interesse, diese bei ihrer Kapazitätsplanung zu benachteiligen. Insofern wird sie bei ausreichendem Bedarf jederzeit bereit sein, Stromleitungen, die über das zur Reservehaltung und zur Versorgungssicherheit erforderliche Maß hinausgehen, zu anderen Netzen zu errichten. Hierdurch werden die Voraussetzungen für einen europäischen Stromhandel geschaffen.

Somit kann zusammenfassend festgehalten werden, daß die Netzgesellschaften des Common-Carrier-Konzeptes die Stromtransportkapazitäten an dem erwarteten Bedarf orientieren werden. Die Bedarfsschätzung wird durch die Anzeigepflicht von Kraftwerkszubauten erleichtert. Es besteht jedoch folgende Gefahr: Wird der Netzgesellschaft eine angemessene Kapitalverzinsung zugesprochen, so neigen Unternehmen dazu, ihre Leitungsinvestitionen über den zur Bedarfsdeckung erforderlichen Umfang aufzubauen. Hierdurch wollen sie zusätzliche absolute Gewinne erzielen. Es wird daher die Aufgabe der Regulierungsinstitution sein, die Leitungsinvestitionen auf ihre Notwendigkeit hin zu überprüfen.

\subsubsection{Stromtransportkapazitätsentwicklung im Stromhändlerkonzept}

Grundsätzlich unterscheidet sich das Stromhändlerkonzept hinsichtlich der Stromtransportkapazitäten nicht von dem Common-Carrier-Konzept; unterschiedlich ist, $\mathrm{da} ß$ der Stromtransport innerhalb der Stromhandelsgesellschaft eine Unternehmenssparte, also kein eigenständiges Unternehmen ist. Aus der Stellung als Unternehmenssparte resultiert die Ausrichtung der Aktivitäten an dem Gesamtunternehmensinteresse. Dies bedeutet konkret: Die Koordination zwischen Stromtransportbereich und Stromhandel sowie dem Lastmanagement wird unternehmensintern zu lösen sein.

Für die Erzeuger- und Verteilerunternehmen, die als Stromhandelspartner des Stromhändlers auftreten, verliert der Stromtransport seinen infrastrukturellen Dienstleistungscharakter; von ihnen wird der Stromtransport als Vertriebsweg eines Zwischenhändlers angesehen.

In bezug auf die Kapazitätsentwicklung hat die Stromhandelsgesellschaft kein Interesse, die Stromtransportkapazitäten zu verknappen. Durch eine Verknappung würden die Stromhandelsspielräume reduziert und die Gesamtaufgabenerfüllung in Frage gestellt. Ferner wird der Stromhändler als direkter Vertragspartner der Erzeuger und Verteiler verstärkt auf die Sicherheit der Übertragungsleitungen und -einrichtungen sowie einen angemessenen Kapazitätsausbau achten. Tendenziell 
wird die Stromhandelsgesellschaft, ähnlich wie die Netzgesellschaft des CommonCarrier-Konzeptes, dazu neigen, Überkapazitäten im Stromtransport zu errichten, um ein hohes $\mathrm{Ma} \beta$ an Versorgungssicherheit in ihrem Versorgungsbereich zu gewährleisten. Dadurch wächst der Spielraum für Stromhandelsaktivitäten im Netzgebiet. Andere Stromhandelsgesellschaften wird die Netzgesellschaft als zusätzlichen Anbieter betrachten. Ein Interesse, Stromlieferungen anderer Stromhandelsgesellschaften gegenüber den Anbietern in ihrem Gebiet zu benachteiligen, hat sie nicht. Sie wird deshalb auch bereit sein, die Kupplungskapazitäten bedarfsgerecht auszubauen.

Die erforderliche Überkapazitätsbegrenzung durch die Regulierungsinstitution wird jedoch durch den Integrationsgrad erschwert. Der Stromhändler hat, im Gegensatz zum Common-Carrier-Konzept, bessere Möglichkeiten, den tatsächlichen Bedarf zu verschleiern. 


\section{Die Folgen der Stromtransportkapazitätsentwicklungen für die Ordnungskonzepte}

\subsection{Für das Weiterentwicklungskonzept}

Stromtransportkapazitäten werden im Weiterentwicklungskonzept ebensowenig wie eine Infrastruktur für den Stromhandel errichtet. Die bisher existierenden Anlagen sind auf dem bestehenden UCPTE-Verbund und dem NORDEL-Verbund ausgerichtet. Dies wirkt auf die geplante Strombörse des Weiterentwicklungskonzeptes: Sie benötigt zum Funktionieren, oder grundsätzlicher, zur Abwicklung von Stromhandelsgeschäften, ein ausgebautes Stromtransportnetz und eine Meß- und Regelungsinftrastruktur. Daraus folgt, daß die Strombörse kein hohes Stromhandelsvolumen aufweisen wird. Langfrisitig werden von ihr ausschließlich die bereits in der Vergangenheit durchgeführten Stromhandelsgeschäfte in einer anderen institutionellen Form abgewickelt werden. Die in Schwachlastphasen bestehenden freien Stromtransportkapazitäten werden wegen mangelnder Nachfrage nicht genutzt werden. Die Stromlieferungen und Bezugsmengen werden überwiegend für Aushilfen bei außergewöhnlichen Betriebssituationen verwendet werden.

Somit verhindert die Stromtransporıkapazitätsentwicklung im Weiterentwicklıngskonzept einen funktionierenden Stromhandel in Europa und schließt damit gleichzeitig aus, daß die Stromversorgung in Europa zu niedrigeren Kosten durchgefuihrt wird.

\subsection{Für das TPA-Konzept}

Die Stromtransportkapazitätsentwicklung bei Implementierung des TPA-Konzeptes fürt dazu, daß alle Gebiets-EVU, die durch eine Durchleitung in der zweiten und dritten Konzeptstufe negativ betroffen werden könnten, versuchen, ihr Monopol in der Versorgung von Endabnehmern und Verteilungsunternehmen zurückzugewinnen (vgl. Abb. 71).

Die Durchleitungen werden jedoch nicht erst dann verhindert, wenn die Stromtransportkapazität identisch ist mit dem Stromtransportbedarf des Gebiets-EVU (tx), sondern bereits vorher, zum Zeitpunkt tx-1, wenn die zur Verfügung stehenden Stromtransportkapazitäten den Mindestumfang für wirtschaftliche Stromübertragungen unterschreiten. Der Mindestumfang ergibt sich aus der verkauften 


\section{Abbildung 71: Rückgewinnung des Monopols durch ein Gebiets-EVU in der Situation IX}

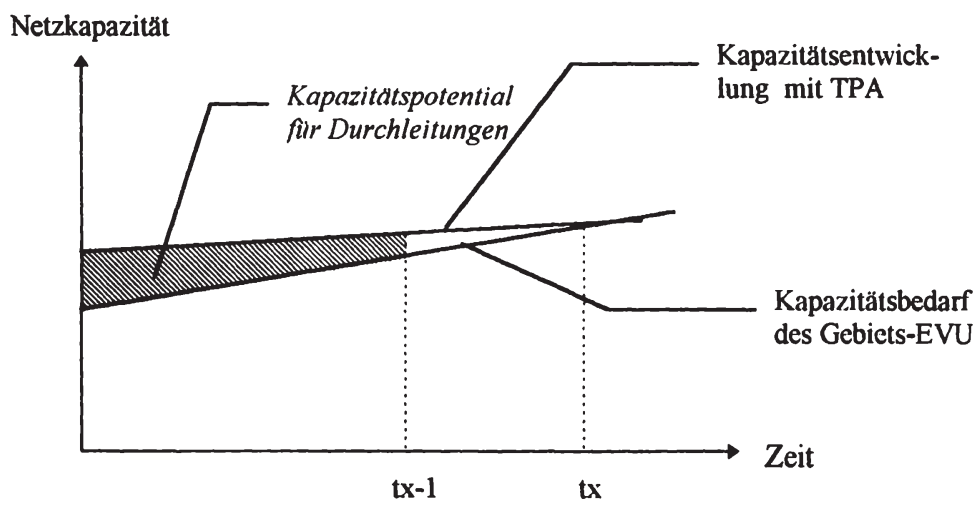

Stromleistung, die gerade soviele Deckungsbeiträge erwirtschaftet, wie die Fixkosten des Stromverkaufs inklusive der erforderlichen Durchleitung betragen.

Für die Wettbewerber bedeutet dies:

- Zum Zeitpunkt tx-1 hat das Gebiets-EVU trotz Durchleitungsverpflichtung auf allen Teilmärkten das vollständige Monopol wiedererlangt und gleichzeitig seine Wettbewerbsposition gegenüber der Ausgangssituation verbessert, da die Drohung der Durchleitungsverpflichtung keine Wirkung mehr erzielen kann. Wichtig ist das besonders für die Unternehmen in der Situation $\mathrm{IX}^{530}$.

- Externe Unternehmen ${ }^{531}$, die an einem Stromabsatz in einem Versorgungsgebiet interessiert sind, werden ihre eigenen Stromtranportkapazitäten nicht nutzen können, da die erforderlichen Leitungen zur Belieferung eines Abnehmers in dem Versorgungsgebiet langfristig nicht zur Verfügung stehen. ${ }^{532}$ Jedem potentiell stromliefernden EVU steht ein Gebiets-EVU entgegen, daß die Durchleitung verhindern will.

$530 \mathrm{Vgl}$. hierzu die Ausführungen zur Stromtransportkapazitätsentwicklung im TPA-Konzept in Kapitel 8.2.2 .

531 Beipiele sind ein EVU in der Situation XII aber auch Erzeuger ohne Versorgungsgebiet.

532 Ausnahmen bilden Versorgungsgebiete, in denen das Gebiets-EVU nicht in der Lage ist, die benötigte Erzeugungskapazităt bereitzustellęü digine soiches Beispiel \&sB dỉe ENEi Zin Italien. 
Abbildung 72: Grund für nicht genutzte Durchleitungskapazitäten

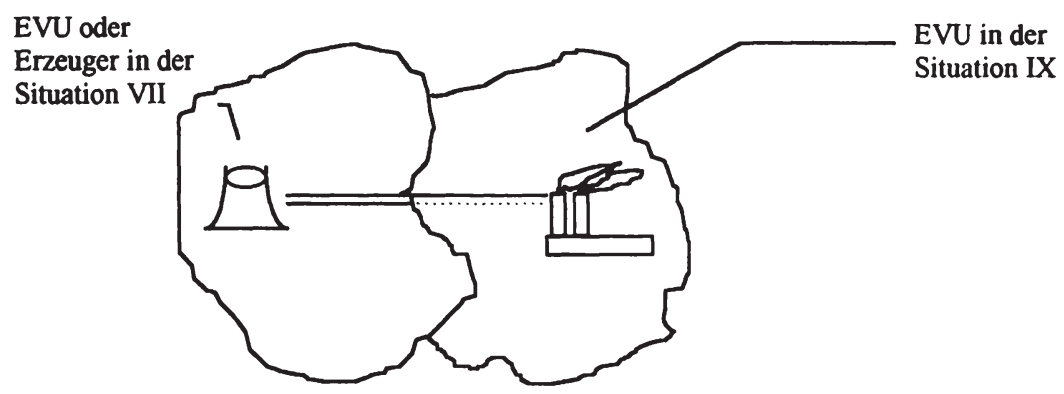

Ab dem Zeitpunkt an dem die Gebiets-EVU, die von einer Durchleitung negativ betroffen werden könnten, ihr Monopol wiedererlangt haben, ist eine Erreichung der Ziele des europäischen Strommarktes durch das TPA-Konzept ausgeschlossen. Die Situation nach diesem Zeitpunkt tx-1 ist identisch mit der Situation vor Implementierung des TPA-Konzeptes. Die noch durch die Unternehmen in der Situation VII bereitgestellten Durchleitungskapazitäten können nicht für Durchleitungen genutzt werden.

Die Durchleitungspreise nehmen auf die Wiedererlangung des Monopols durch die Gebiets-EVU keinen Einfluß. Der Durchleitungspreis bestimmt lediglich die Erträge der durchleitenden Gebiets-EVU bis zur Einstellung der Stromhandelsaktivitäten.

Offen bleibt die Frage, wie die für Durchleitungen zur Verfügung stehenden Kapazitäten bis zum Zeitpunkt tx-1 genutzt werden (vg. Abb. 73). Zunächst muß von dieser verfügbaren Leistung der Teil abgezogen werden, der wegen zu geringen Erzeugungspreisdifferenzen in der elektrizitätswirtschaftlich-geographischen Nähe nicht genutzt wird (vgl. Abb. 74).

Für die Nutzung der Durchleitungskapazitäten sind verschiedene Kombinationen aus Zeit, Stromtransportkapazitätsinanspruchnahme und Unternehmensanzahl denkbar. Zur Verdeutlichung der Zusammenhänge wird zunächst die Nutzung der Durchleitungskapazität eines Gebiets-EVUs durch ein Unternehmen dargestellt. Im Anschluß wird die Zahl der Durchleitungsbeantragenden auf drei erhöht. 


\section{Abbildung 73: Stromtransportpotential für Durchleitungen}

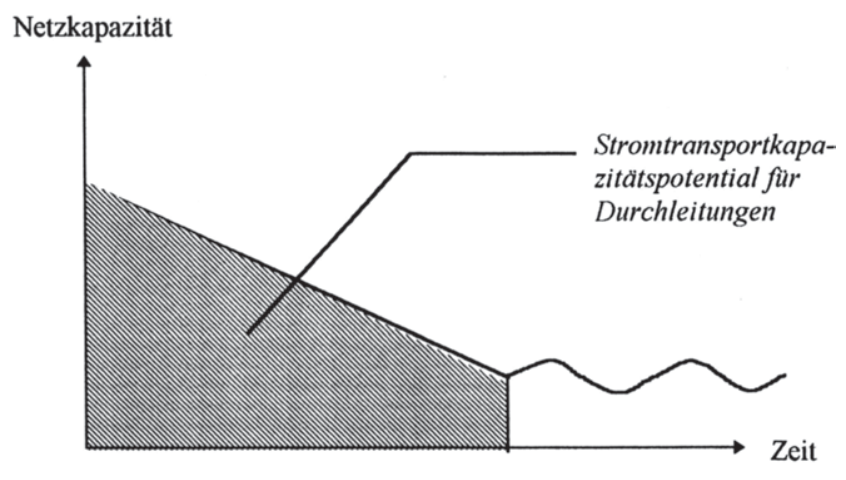

tx-1

Abbildung 74: Stromtransportpotential nach Abzug der nicht nutzbaren Leistung

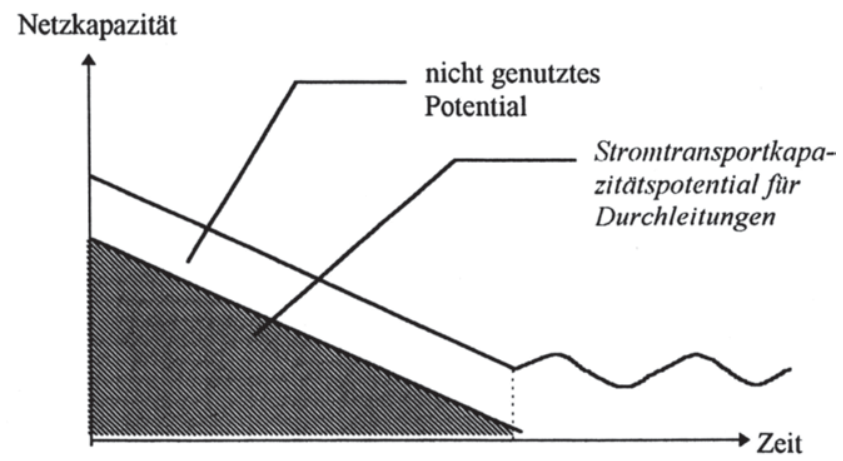

tx-1

$\mathrm{tx}$

a) Ein externes Unternehmen beantragt eine Durchleitung

Ein Erzeuger oder EVU beantragt eine Durchleitung, die identisch ist mit der maximalen Durchleitungskapazität des Gebiets-EVUs. 
Abbildung 75: Max. Durchleitungskapazität = beantragte

Durchleitungskapazität

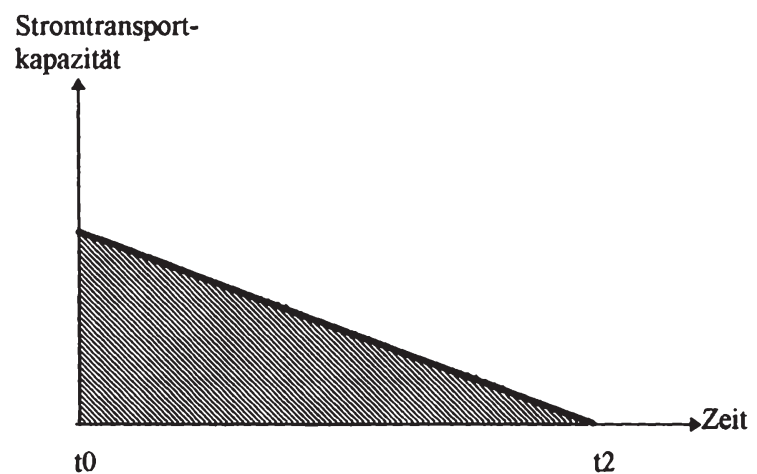

Dieser Fall wird in der Praxis keine Bedeutung haben, da kein EVU oder Erzeuger einen Abnehmer zu einer im Zeitverlauf kontinuierlichen reduzierten Abnahme wie in vorherigen Abbildung gewinnen kann.

Das gleiche Unternehmen kann auch alternativ eine hohe kontinuierliche Übertragungskapazität $\mathrm{K} 1$ beantragen. Wegen der schrumpfenden maximalen Durchleitungskapazität kann das Unternehmen die Durchleitungskapazität nur für einen kurzen Zeitraum, bis $\mathrm{t} l$, nutzen.

\section{Abbildung 76: Kurzer Durchleitungszeitraum, hohe Durchleitungskapazität}

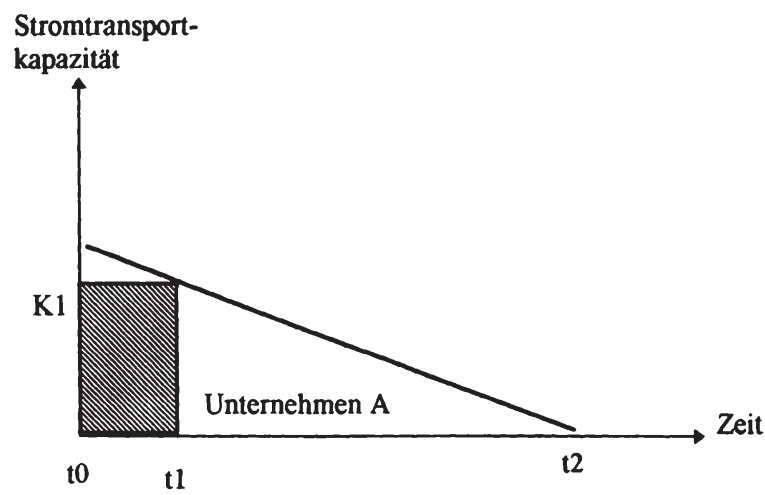


Das Unternehmen kann als dritte Option eine Durchleitung mit niedriger, konstanter Übertragungskapazitätsinanspruchnahme beantragen und kann diese dann länger nutzen.

\section{Abbildung 77: Überkapazitäten > Durchleitungskapazität ; langer Durchlei- tungszeitraum, geringe Durchleitungskapazität}

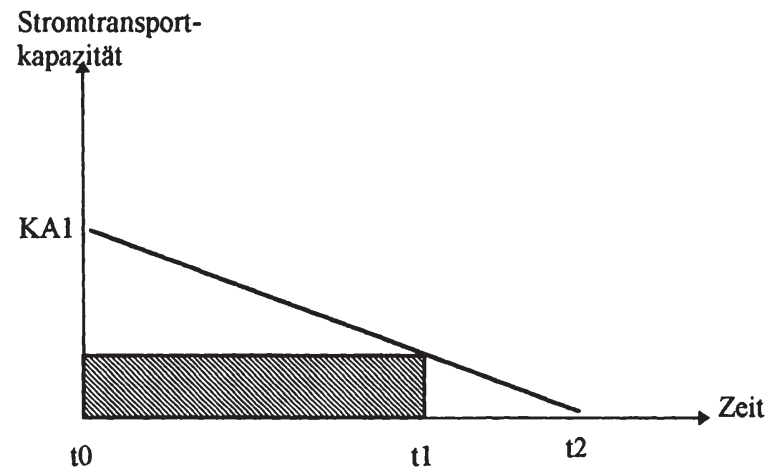

Diese Situation kann dann eintreten, wenn gezielt einzelne Abnehmer von externen Anbietern umworben werden und einen Stromliefervertrag abschließen.

Als dritte Möglichkeit kann das Unternehmen versuchen, seine beantragten Durchleitungen sukzessive zu reduzieren, um dadurch möglichst die vom GebietsEVU in Anspruch genommene Durchleitungskapazität zu maximieren (vgl. Abb. 78). Bis zum Zeitpunkt tl beantragt es die hohe Kapazität KA1. Anschließend reduziert es seine durchgeleiteten Stromleistungen, so daß die Durchleitungskapazität KA2 bis zum Zeitpunkt $\mathrm{t} 2$ beansprucht. In der dritten Zeitspanne kann das Unternehmen nur noch die verbleibende Durchleitungskapazität nutzen. Denkbar ist diese stufenweise Inanspruchnahme bei einem externen Erzeugungsunternehmen, daß über niedrige Erzeugungskosten, z.B. durch alte abgeschriebene Erzeugungskapazitäten verfügt und für die Anlagen zusätzliche Deckungsbeiträge erzielen will, bevor es die Kraftwerke stilllegt. Der potentielle Abnehmerkreis für ein derartiges Angebot ist jedoch gering. Theoretisch denkbar sind Abnehmer, die gerade ihre Eigenerzeugung stufenweise ausbauen. Deshalb wird eine derartige Inanspruchnahme von abnehmenden Stromtransportkapazitäten keine praktische Relevanz haben. 


\section{Abbildung 78: Mittlere Durchleitungszeiträume, mittlere Durchleitungskapazitäten}

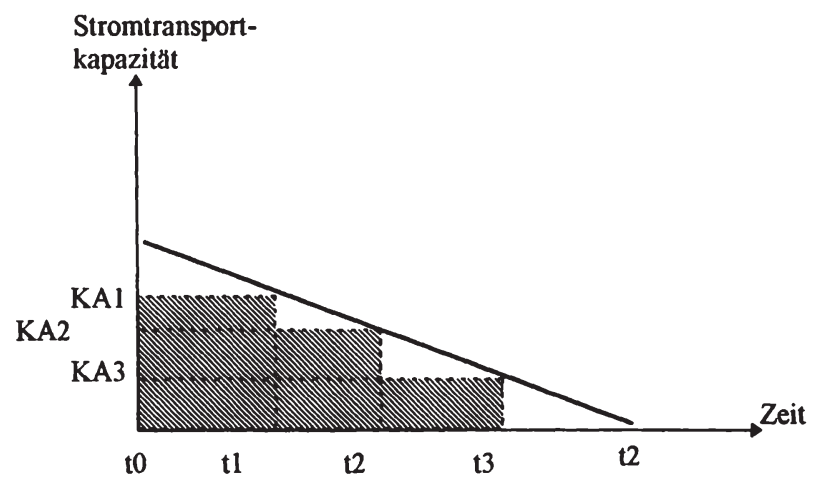

b) Drei Unternehmen beantragen eine Durchleitung bei einem Gebiets-EVU

Die externen Unternehmen sind bestrebt, die Durchleitungen über einen möglichst langen Zeitraum zu nutzen, um ihre Erzeugungskapazitäten mit konstanter Höhe auszulasten. Unternehmen A beantragt als erstes eine Durchleitung und kann das Stromtransportnetz des Gebiets-EVU gegen Zahlung eines Preises bis zum Zeitpunkt tA nutzen ${ }^{533}$. Die Zeitspanne, über die das Unternehmen B eine Durchleitungskapazität (KB-KA) nutzen kann, ist geringer - von t0 bis tB. Für das Unternehmen $C$ verbleibt dann nur noch eine kurzzeitig nutzbare Durchleitungskapazität (KC-KB), die zum Aufbau langfristiger Lieferbeziehungen nicht geeignet ist. Denkbar ist der Stromabsatz von Unternehmen $C$ an Industrieunternehmen, die einen kurzfristig begrenzten zusätzlichen Strombedarf haben ${ }^{534}$ (vgl. Abb. 79).

Theoretisch denkbar ist auch ein identischer Nutzungszeitraum der Durchleitungskapazität des Gebiets-EVU für alle drei Unternehmen. Dies würde jedoch bedeuten, daß bei allen drei externen Anbietern die Abnahmemenge kontinuierlich reduziert werden müßte; diese theoretische Möglichkeit besitzt aber keine Relevanz $z^{535}$ (vgl. Abb. 80).

533 Zur Vereinfachung wurde hier die Zuordnung nach der zeitlichen Beantragung der Durchleitungskapazităten gewăhlt. Bei den anderen Zuordnungsmethoden ăndern sich die Ergebnisse nicht.

534 In der Praxis kőnnte dieser Bedarf bei Unternehmen anfallen, deren Eigenerzeugungsanlagen einen Defekt aufweisen. Ein kontinuierlicher Bedarf in Europa. von dem ein Wettbewerb um die Belieferung ausgehen kann, ist dies jedoch nicht.

535 Theoretisch moglich ist auch die Kombinatiotigderlbeiden fäHe278-3-631-75129-9 
Abbildung 79: Unterschiedliche Laufzeiten der Verträge und unterschiedliche Durchleitungskapazitäten

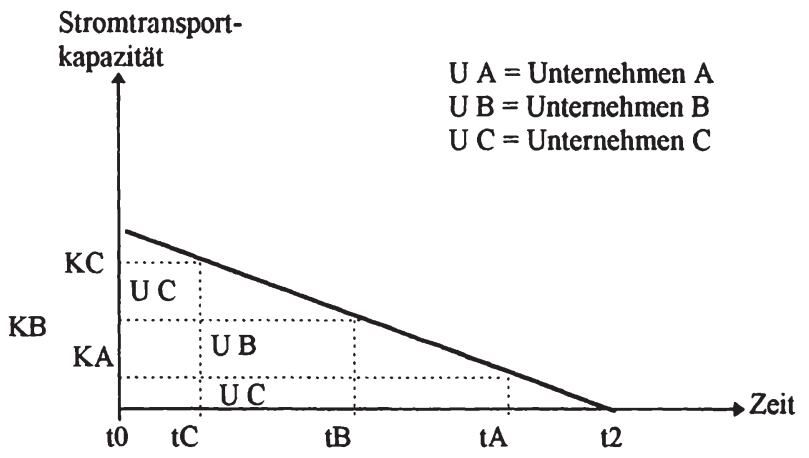

Abbildung 80: Mehrere Unternehmen mit gleicher Laufzeit der Verträge und abnehmenden Durchleitungskapazitäten

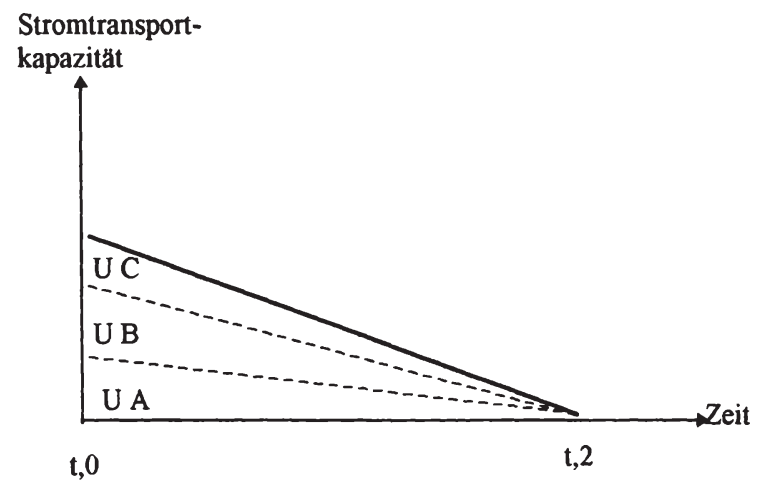

Die zusammenfassende Beurteilung des TPA-Konzeptes unter Berücksichtigung der Stromtransportproblematik ist in Tabelle 19 dargestellt. 


\subsection{Für das Common-Carrier-Konzept und das Stromhändler- Konzept}

Die Konzeptionen der beiden Ordnungsmodelle beinhalten keine Anreize für die Netzgesellschaften, die Stromtransportkapazitäten zu verknappen; im Gegenteil: das Funktionieren des Stromhandels und im Stromhändlerkonzept der gesamten Großhandelsstufe ist für die Netzbetreiber zwingend erforderlich, da ihnen bei einem Scheitern des jeweiligen Konzeptes die Reintegration und damit die Aufgabe der unternehmerischen Unabhängigkeit droht. Aus diesem Grund bestehen im Gegensatz zum TPA- und Weiterentwicklungskonzept im Common-Carrier- und Stromhändlerkonzept inhärente Anreize, bestehende Kapazitätsengpässe abzubauen und langfristig für ausreichende Stromtransportkapazitäten zu sorgen. Der Ausbau der Transportnetze wird von den Unternehmen an der von ihnen erwarteten Nachfrage nach Transportdienstleistungen orientiert. Darüber hinaus wird von den mit einem Monopol ausgestatteten Netzunternehmen wie bei den bestehenden integrierten EVU die Nachfrage eher höher eingeschätzt und ausreichende Sicherheitsmargen einkalkuliert. Eine Regulierungsinstitution muß tendenziell eher Überkapazitäten begrenzen als die Errichtung von notwendigen Kapazitäten überwachen.

Durch die Reduzierung der Kapazitätsengpässe im Stromtransportbereich wird der infrastrukturelle Charakter der Elektrizitätsversorgung im europäischen Binnenmarkt betont und ein Beitrag zur Integration der anderen Wirtschaftszweige durch die Elektrizitätswirtschaft geleistet.

Anders als die Stromtransportkapazität nehmen die Stromtransportpreise, die die Nutzer der Infrastruktur zu zahlen haben, einen Einfluß auf die Zielerfullung des jeweiligen Konzeptes: Je höher die Stromtransportpreise sind, desto höher müssen die Strompreisunterschiede der Erzeuger oder Anbieter von Elektrizität sein, damit der beabsichtigte Wettbewerb um Abnehmer stattfindet und die bestehenden Preisunterschiede nicht eingeebnet werden. Die Netzbenutzungspreise legen somit wesentlich die Anzahl der Konkurrenten um einen einzelnen Abnehmer fest. Daher resultiert aus diesen Zusammenhängen die Erfordernis einer Stromtransportpreisregulierung. Die Beurteilung der Konzepte muß durch die Berücksichtigung der Stromtransportproblematik nicht korrigiert oder ergänzt werden ${ }^{536}$.

536 Vergleiche hierzu die zusammenfassende Bewertung der beiden Desintegrationskonzepte in Kapitel 7.4.3 
Tabelle 19: Zusammenfassende Beurteilung der Integrationskonzepte

\begin{tabular}{|c|c|c|}
\hline Kriterlen zur Beurtellung der Ordnungsmodelle & $\begin{array}{c}\text { erfü } \\
\text { Welterentwickl.- } \\
\text { konzept }\end{array}$ & TPA-Konzept \\
\hline Vertlkale Integratlon aufgelöst? & Nein & Nein \\
\hline Stromhandelsinfrastruktur geschaffen? & Nein & Nein \\
\hline Tellmärkte geschaffen? & Nein & Nein \\
\hline Marktzutrittsbarrieren reduziert? & Nein & Ja \\
\hline Wettbewerbsintensität gestelgert? & Nein & Nein \\
\hline Marktmacht elnzelner Anbleter u.Nachfrager begrenzt? & Nein & Nein \\
\hline Reduzlerung der Gesamtkosten der Elektriztütsversorgung? & Nein & Nein \\
\hline Versorgungssicherhelt gewährleisteł? & Ja & Ja \\
\hline Ausblick Prelsentwicklung: & $\begin{array}{c}\text { Unveränderte } \\
\text { Strompreise } \\
\text { in Europa }\end{array}$ & $\begin{array}{l}\text { Lgfr. kelne } \\
\text { Stromprels- } \\
\text { senkungen }\end{array}$ \\
\hline
\end{tabular}




\section{Schlußbetrachtungen}

Politisches Ziel der EU-Mitgliedsstaaten ist die Schaffung einer politischen Union. Für den Elektrizitätssektor wird ein gemeinsamer Markt ohne nationale Grenzen, der die Kosten sinken läßt und die Sicherheit der Elektrizitätsversorgung gewährleistet, angestrebt. Obwohl die Ziele somit klar umrissen waren, fehlten bisher konkrete Konzepte, wie diese Ziele in absehbaren Zeiträumen erfüllt werden können. Von der EU-Kommission und den Interessenverbänden der Stromversorgungsunternehmen wurden in der Vergangenheit ausschließlich Ansätze zur Schaffung eines europäischen Strommarktes eingebracht: Die bestehenden EVU schlugen eine Intensivierung des bestehenden zwischenstaatlichen Stromaustausches insbesondere für Aushilfs- und Reservestromlieferungen vor, um durch eine verminderte nationale Reservehaltung Kosten einzusparen. Zusätzlich soll eine Strombörse zu einem vermehrten bilateralen Stromhandel beitragen (Weiterentwicklungsansatz).

Der zweite, von der EU-Kommission eingebrachte Ansatz - Third-Party-Access geht weiter. Er sieht die stufenweise Einführung einer Stromtransportverpflichtung für die EVU vor, die ein Stromnetz unterhalten. Abnehmer und Anbieter aus anderen Versorgungsgebieten sollen in die Lage versetzt werden, die Stromtransportinfrastruktur auch gegen den Willen des EVUs benutzen zu können.

Der dritte, ebenfalls von der EU-Kommission bereits Mitte der achtziger Jahre angedachte Ansatz - Common-Carrier - sieht die Errichtung von Unternehmen vor, die die Stromnetze betreiben und den Stromtransport zwischen Abnehmern und Anbietern übernehmen. Die Erzeugung und Verteilung von Strom soll durch rechtlich und wirtschaftlich selbständige Unternehmen vorgenommen werden.

Die Übertragung der englisch-walisischen Elektrizitätsstruktur auf den europäischen Strommarkt ist der vierte und letzte Ansatz, der in die Diskussion eingebracht worden ist. Nach diesem Ansatz sollen ein oder mehrere Unternehmen in Europa als Stromhändler zwischen Erzeugern und Abnehmern fungieren und gleichzeitig den erforderlichen Stromtransport übernehmen.

Alle Ansätze haben eines gemeinsam: Sie sind bisher derart gering operationalisiert, daß ihre zügige Umsetzung derzeitig nicht möglich ist. Deshalb war es das Ziel dieser Arbeit:

- die bestehenden Ansätze zu Konzepten auszubauen,

- zu zeigen, wie die Konzepte mit welchem Aufwand und in welcher Zeit realisiert werden können,

- zu überprüfen, welches Konzept die wirtschafts- und gesellschaftspolitischen Ziele am besten gewährleisten kann,

- aufzuzeigen, welche Auswirkungen die nicht geeigneten Konzepte auf die Ziele haben. 
Hierzu wurden zunächst die politischen Ziele herausgearbeitet und konkretisiert sowie in einem zweiten Schritt die von der EU-Kommission für den europäischen Strommarkt identifizierten Hemmnisse dargestellt. Diese sind:

- die nationalen rechtlichen Rahmenbedingungen,

- die Stromerzeugung durch öffentliche Unternehmen,

- die unterschiedliche steuerliche Behandlung und der Zugang zu den Finanzmärkten,

- die Verfahren für die Genehmigung von Kraftwerksneubauten,

- der Zugang der Stromerzeuger zur Primärenergie und

- die Sicherheitsauflagen und Umweltschutznormen.

Begründet wurden diese Hemmnisse in den Mitgliedsstaaten traditionell mit den Erfordernissen des Elektrizitätssektors, weshalb der Verfasser im Anschluß diese grundlegende Aussage überprüfte. In einem ersten Schritt wurden die Besonderheiten des Elektrizitätssektors dargestellt, typisiert und hinterfragt, ob und inwieweit sie bei der Entwicklung von Konzepten für den europäischen Strommarkt berücksichtigt werden müssen und ob sie eine wettbewerbliche Ausnahmebehandlung des Elektrizitätssektors im europäischen Wettbewerbsrecht rechtfertigen. Die Betrachtungen ergaben, daß die Besonderheiten entgegen den zahlreichen Pro-Statements keine generelle Ausnahmeregelung im Wettbewerbsrecht und in der Wettbewerbspolitik begründen. Im Bereich des Stromtransportes und der Stromverteilung erfordern jedoch die technischen Besonderheiten Versorgungsgebiete, in denen eine Netz- oder Verteilungsgesellschaft zumindest zeitlich begrenzt den Stromtransport oder die Stromverteilung allein durchführt.

In einem zweiten Schritt wurde dann gepüft, ob in der ökonomischen Theorie Anhaltspunkte für den Ausschluß von Marktstrukturen mit Wettbewerb im Elektrizitätssektor zu finden seien. Diese ließen sich weder in der ,klassischen“ noch in der „neueren“ Preistheorie finden. Die Übertragung der Überlegungen des Transaktionskostenansatzes auf dem Elektrizitätssektor zeigte ferner, daß die marktliche Koordinationsform für den Elektrizitätssektor effizient ist. Dies bedeutet für die zu entwickelnden Konzepte: Es gibt keinen Grund, warum die bestehenden Hindernisse nicht abgebaut und in der Stromerzeugung sowie der Endabnehmerversorgung Wettbewerb nicht eingeführt werden kann. Des weiteren konnte aus den theoretischen Überlegungen geschlossen werden, daß folgende Bedingungen gegeben sein sollten, damit Wettbewerb im zukünftigen europäischen Strommarkt kostensenkend wirken kann:

- Die vertikale Integration der Elektrizitätsstrukturen muß aufgehoben werden.

- Eine Stromhandelsinfrastruktur muß geschaffen werden.

- Es müssen Teilmärkte entstehen.

- Es müssen Mitbewerber oder potentielleKonkursentencexistierang 
- Es sollten Innovationen vorhanden sein, die Effizienzsteigerungen für den Gesamtmarkt bewirken, weil sie von den Wettbewerbern übernommen werden müssen.

- Es dürfen keine oder nur geringe Marktzutritts- oder Austrittsbarrieren vorhanden sein.

- Informationen über Angebots- und Nachfragepreismengenkombinationen müssen von den Marktsubjekten beschaffbar sein.

- Die einzelnen Marktsubjekte müssen eine begrenzte Marktmacht aufweisen.

- Die Transaktionen müssen eine niedrige Spezifität bei begrenzter Unsicherheit aufweisen.

Aus diesen Bedingungen entwickelte der Verfasser Kriterien, mit denen die Konzepte an späterer Stelle beurteilt wurden.

Das nächste Kapitel stellt die bestehenden Strukturen der nationalen Elektrizitätssektoren dar. Die Darstellung sollte die derzeitige Ausgangssituation verdeutlichen und den hieraus entstehenden Handlungsbedarf sowie den erforderlichen Umfang der Umstrukturierungen aufzeigen. Hierzu wurden die bestehenden nationalen Elektrizitätssektoren der Mitgliedsstaaten in drei Gruppen eingeteilt und ausgewählte Staaten jeder Gruppe exemplarisch näher dargestellt. Im Anschluß daran wurden die bestehenden Organisationen zum Stromaustausch in Europa betrachtet, da sie ein weiterer Ausgangspunkt fur jede Umgestaltung sind. Das an dieser Stelle aufgezeigte niedrige Stromaustauschvolumen wurde als Indikator für den niedrigen Integrationsgrad der europäischen Elektrizitätssektoren herangezogen. Das Ergebnis: Von einem gemeinsamen Markt für Elektrizität kann in der EU nicht gesprochen werden. Die Freiheit der Lieferantenwahl ist, außer für Großabnehmer und Stromversorger in England und Wales, nirgendwo innerhalb der EU verwirklicht. Eine Preisfestsetzung im freien Wettbewerb findet für alle Abnehmergruppen nicht statt. Eine Durchleitungsmöglichkeit für Erzeuger besteht bisher nur in den Niederlanden und in Großbritannien. Die staatlichen Beihilfen für die Elektrizitätswirtschaft sind weder transparent noch einheitlich und die von der EU-Kommission identifizierten Austausch- und Handelshemmnisse bestehen weiter. Daraus zog der Verfasser den Schluß, daß die bestehenden Strukturen zu keinem europäischen Binnenmarkt für Elektrizität führen können und die von der EU-Kommission identifizierten Hemmnisse nicht abgebaut werden. Der wirtschaftliche Integrationsgrad des Stromsektors wird ohne gezielte Veränderungen hinter dem anderer Wirtschaftszweige auch in Zukunft hinterherhinken. Es zeigte sich ferner, daß die Vielfalt der nationalen Strukturen hohe Anforderungen an die zu entwickelnden Konzepte stellt.

Das nächste Kapitel beschreibt die unterschiedlichen Ansätze, die einzelne Institutionen und Interessengruppen zur Veränderung der derzeitigen 5 eurgpäischen 
Strukturen in die energiepolitische Diskussion eingebracht haben. Hierbei zeigten sich auch die hinter den Ansätzen stehenden Absichten der einzelnen an der Willensbildung beteiligten Gruppen: Die $E V U$ sind am Erhalt ihrer bisherigen weitgehend durch Monopole gekenntzeichneten Strukturen interessiert. Sie sichern ihnen ihre bisherige Wirtschaftsmacht und schaffen wegen der erzielbaren Monopolrenten unternehmenspolitische Freiräume. Die einzelnen nationalen Regierungen geben ungern Kompetenzen und Einflußmöglichkeiten an EU-Institutionen ab, insbesondere in den letzten Jahren, in denen die Betonung der nationalen Eigenständigkeiten in der EU wieder „modern" geworden ist. Ferner lassen die in allen europäischen Mitgliedsstaaten vorliegenden Verbindungen zwischen Politik und EVU den Schluß zu, daß einige Regierungen bei ihren Überlegungen von den Wünschen ihrer EVU beeinflußt wurden. Die EU-Kommission will endlich einen weiteren bedeutenden Schritt in Richtung Öffnung der nationalen Märkte erreichen. Der starke Widerstand der EVU und des Europäischen Parlamentes, in dem die französischen Vertreter meinungsbildend wirkten, sowie einzelner nationaler Regierungen zwang sie jedoch zu Kompromissen.

Nachdem nun eine Bestandsaufnahme der derzeitigen Elektrizitätsstrukturen in den einzelnen Mitgliedsstaaten durchgeführt und der Handlungsbedarf aufgezeigt worden war, konnte mit der Operationalisierung der in die Diskussion eingebrachten Ansätze zur Schaffung eines liberalisierten europäischen Elektrizitätsmarktes begonnen werden. Hierbei wurden zuerst die beiden Ansätze aufgegriffen, die vollständig integrierte EVU (Integrationskonzepte) vorsehen. Der Weiterentwicklungsansatz, der bisher von allen vier Ansätzen der am weitesten operationalisierte ist, beinhaltet, daß ausschließlich zwischen den europäischen Verbund-EVU Stromlieferungen stattfinden. Endverbraucher erhalten keine Möglichkeit, ihren Stromlieferanten zu wählen. Es bliebe bei den vollständig integrierten Versorsorgungsstrukturen in Europa, wobei die bestehenden nationalen Unterschiede nicht beseitigt werden würden.

Beim TPA-Konzept soll eine Stromtransportverpflichtung, die den EVU mit einem Leitungsnetz auferlegt wird, Wettbewerb zwischen Elektrizitätserzeugern um die Belieferung von Abnehmern mit elektrischer Energie bewirken. Diese Verpflichtung soll schrittweise eingeführt werden, wodurch der Kreis der Abnehmer, die ihren Lieferanten frei wählen können, gleichfalls schrittweise ansteigt. Beim TPAKonzept wurden Lösungsmöglichkeiten zu folgenden offenen Punkten vom Verfasser entwickelt:

- zu der Vertragsgestaltung,

- zu der Rückkehr der Verbraucher zum Gebiets-EVU,

- zu der Versorgungspflicht und

- zu der Auswahl der Durchleitungsanträge. 
Nach dem Entwurf der beiden Konzepte wurden Entwicklungsszenarien gebildet und die Faktoren herausgearbeitet, die das Eintreten der einzelnen Szenarien fördern. Hierdurch sollte aufgezeigt werden, welche Auswirkungen einzelne Details der Konzeption auf den europäischen Strommarkt haben. Es zeigte sich, daß ein intensiver Stromhandel bei der Umsetzung der beiden Integrationskonzepte nicht zu erwarten ist, weil die Bedingungen, durch die ein entsprechendes Szenario eintreten könnte, nicht erfüllt werden.

Des weiteren wurde bei jedem Integrationskonzept diskutiert, auf welche Akzep$\operatorname{tanz}$ es bei welchen Gruppen stoßen wird oder bereits gestoßen ist. Diese Diskussion ist erforderlich, weil die Vollendung eines gemeinsamen Strommarktes ein Bestandteil der Bildung einer politischen Union ist. Dieses Ziel und der Weg dorthin ist in den Mitgliedsstaaten nicht unumstritten. Der Trend, die einzelstaatliche Identität zu betonen, zeigt die Unsicherheit in der Bevölkerung und den verbreiteten Wunsch, gewohnte Strukturen zu erhalten. Eine fehlende Akzeptanz der Umgestaltungen in den Elektrizitätssektoren würde die Verwirklichung des europäischen Strommarktes gefährden und Rückwirkungen auf den allgemeinen Einigungsprozeß haben. Hierbei ergab sich das folgende Bild:

\section{Tabelle 20: Akzeptanz der Integrationskonzepte}

\begin{tabular}{|c|c|c|}
\hline Gruppe/Institution & $\begin{array}{c}\text { Weiterentwicklungs- } \\
\text { konzept }\end{array}$ & $\begin{array}{c}\text { TPA- } \\
\text { Konzept }\end{array}$ \\
\hline Bevölkerung & hoch & mäßig \\
\hline EU-Kommission & niedrig & hoch \\
\hline Regierungen & uneinheitlich & uneinheitlich \\
\hline Evv & hoch & niedrig \\
\hline Industrie & niedrig & hoch \\
\hline
\end{tabular}

Im Anschluß daran wurde der Frage nachgegangen, welche Veränderungen die Konzepte bei den bestehenden Regulierungsmechanismen erfordern. Hierbei zeigte es sich, daß das Weiterentwicklungskonzept prinzipiell keine_andere, Regulierung 
als die bestehende benötigt, die jedoch um eine Stromhandelsteilnahmekontrolle ergänzt werden muß. Bei Einführung des TPA-Konzeptes muß zusätzlich eine umfassende Netzbetreiberkontrolle eingeführt und die bestehende Preisregulierung ausgedehnt werden. Der Regulierungsbedarf steigt deutlich an.

Bei beiden Konzepten wurde ein möglicher Implementierungsprozeß dargestellt. Hinsichtlich der Implementierungszeiträume und des jeweiligen Implementierungsaufwandes bei Umsetzung der Konzepte zeigten sich folgende Ergebnisse:

Tabelle 21: Übersicht: Implementierung der Integrationskonzepte

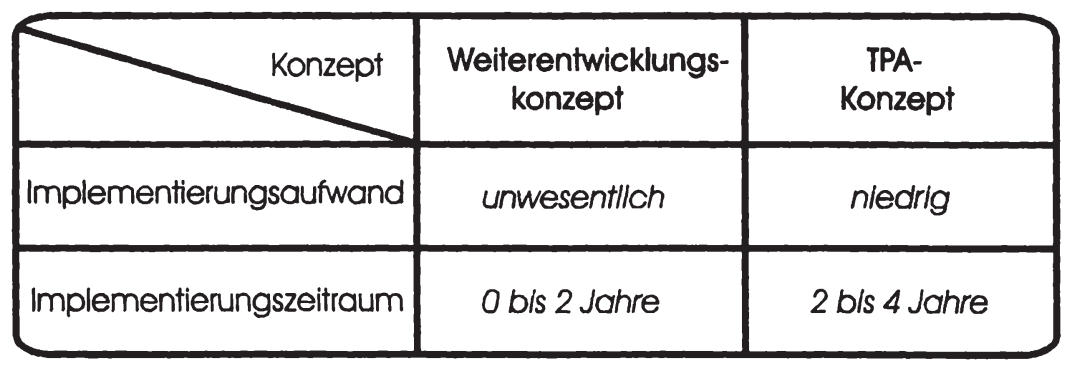

Die beiden Desintegrationskonzepte beinhalten grundlegende Veränderungen der europäischen Elektrizitätssektoren. Beide sehen die Teilung bestehender integrierter Unternehmen sowie das Verbot von Ausschließlichkeitsverträgen vor. Im Common-Carrier-Konzept entstehen vier und im Stromhändlerkonzept zwei Teilmärkte, in denen Wettbewerbsprozesse stattfinden sollen. Während im Stromhändlerkonzept ein zentrales Unternehmen den gesamten erforderlichen Strom aufkauft, wieder verkauft und die Koordination übernimmt, treffen auf den Märkten des Common-Carrier-Konzeptes eine Vielzahl von Anbieter auf die Abnehmer. Die aus den Besonderheiten des Sektors resultierende erforderliche Gesamtkoordination übernimmt eine spezielle Organisation - der Pool. Offen waren in beiden Konzepten folgende Fragen:

- Wieviele Unternehmen sollen aus den integrierten Unternehmen entstehen?

- Welche Betriebsgröße sollen die Unternehmen haben?

- Wer soll der Eigentümer der Unternehmen sein, welche Eigentumsform soll gewählt werden?

- Wer ist für die Sicherheit der Stromversorgung zuständig?

- Welche Organisationen sind erforderlich, damit der Gesamtmarkt funktioniert? 
Diese und weitere Fragen wurden vom Verfasser eingehend diskutiert. Es entstanden zwei Konzepte, zu denen wie bei den Integrationsmodellen Entwicklungsszenarien entworfen und ein Bild von der weiteren Entwicklung des europäischen Strommarktes und seiner Einflußvariablen gezeichnet wurden. Im Gegensatz zu den Integrationskonzepten sind intensive Wettbewerbsprozesse, die darüber hinaus zu sinkenden Strompreisen führen, in beiden Konzepten wahrscheinlich, sofern die Betriebsgrößen der zu schaffenden Unternehmen eine marktbeherrschende Stellung einzelner Unternehmen in der Anfangsphase nach der Einführung des Konzeptes ausschließen. Das Stromhändlerkonzept besitzt diesbezüglich eine höhere Toleranz als das Common-Carrier-Konzept. Im Stromhändlerkonzept muß jedoch ausgeschlossen werden, daß ein Erzeugungsunternehmen gezielt zum Grenzanbieter wird und damit den Preis fur den produzierten Strom festsetzen kann. Zur Akzeptanz der beiden Desintegrationsmodelle kann folgendes festgehalten werden:

\section{Tabelle 22: Akzeptanz des Common-Carrier-Konzeptes und des Stromhändlerkonzeptes}

\begin{tabular}{|c|c|c|}
\hline Gruppe/lnstitution & $\begin{array}{c}\text { Common-Carrier- } \\
\text { Konzept }\end{array}$ & $\begin{array}{c}\text { Stromhändler- } \\
\text { konzept }\end{array}$ \\
\hline Bevölkerung & nledrig & nledrlg \\
\hline EU-Kommission & hoch & hoch \\
\hline Reglerungen & uneinheitlich & uneinheitlich \\
\hline EvU & niedrig & niedrig \\
\hline Industrie & hoch & hoch \\
\hline
\end{tabular}

Der Regulierungsbedarf geht bei der Umsetzung der beiden Desintegrationsmodelle zurück: Der gesamte Erzeugungsbereich der Elektrizitätsversorgung muß nicht mehr der Preiskontrolle unterzogen werden. Eine besondere Regulierung für die Elektrizitätsversorgung bei Einführung des Common-Carrier-Konzeptes ist ausschließich furr die Netzgesellschaften und Verteilerunternehmen notwendig. Im Stromhändlerkonzept muß die Netzgesellschaft weiterhin reguliert werden, damit 
Effizienzgewinne aus den anderen Versorgungsbereichen nicht durch eine geringere Effizienz im Stromhandels- und Stromtransportbereich kompensiert werden. Im Anschluß mußte ein möglicher Umgestaltungsprozeß entwickelt werden, der die Versorgungssicherheit nicht gefährdet und möglichst niedrige Kosten verursacht. Für beide Desintegrationskonzepte sieht der Verfasser eine schrittweise Veränderung vor, die diesen Anforderungen gerecht wird, jedoch auch einen langen Zeitraum in Anspruch nimmt.

\section{Tabelle 23: Implementierungsaufwand und -zeitraum der Desintegrationskonzepte}

\begin{tabular}{|l|c|c|}
\hline Konzept & $\begin{array}{c}\text { Common-Carrier- } \\
\text { Konzept }\end{array}$ & $\begin{array}{c}\text { Stromhändler- } \\
\text { konzept }\end{array}$ \\
\hline Implementlerungsaufwand & hoch & hoch \\
\hline Implementierungszeitraum & $6 \mathrm{~b} / \mathrm{s} 8 \mathrm{Jahre}$ & $4 \mathrm{~b} / \mathrm{s} 6 \mathrm{Jahre}$ \\
\hline
\end{tabular}

Der Versuch einer ersten Beurteilung der Integrationskonzepte ergab, daß beide Konzepte ohne die Analyse der Zusammenhänge im Stromtransport nicht abschließend bewertet werden können. Eine erste Beurteilung der Desintegrationskonzepte war jedoch im Gegensatz zu den Integrationskonzepten möglich und zu allen Kriterien konnten Aussagen getroffen werden. Trotzdem blieb auch bei den beiden Desintegrationskonzepten die Frage offen, wie die Preisbildung für den Stromtransportbereich durchgeführt werden sollte und wie sich die Kapazitäten entwickeln würden. Aus diesen Gründen folgte anschließend eine vertiefende Analyse desjenigen Elektrizitätsversorgungsbereiches, der bisher mit seinen Auswirkungen auf den zukünttigen europäischen Strommarkt in der Literatur vollständig vernachlässigt worden ist - des Stromtransportbereichs. Als erstes wurde der Frage nachgegangen, wie Preise für die Stromtransportleistungen gebildet werden können. Reine Marktpreise schieden aus, da die Unternehmen auch bei den Desintegrationskonzepten über ein staatlich gesichertes vollständiges Monopol verfügen sollen, welches es zu regulieren gilt. Die Stromtransportpreise sollten transparent, kostenorientiert und einfach zu erheben sein. Ferner sollten sie zur Minimierung der Transaktionskosten planbar sein. Von politischer Seite wurde die Verursachergerechtigkeit gefordert. Traditionell vorherrschend sind im Elektrizitätssektor an die Kosten der Leistungserstellung gebundene Preise. Der Verfasser demonstrierte jedoch, daß auch Stromtransportpreise denkbar sind die Nachfrage_/Angebotskon- 
stellationen berücksichtigen und damit Marktpreise simulieren. Für die weitere Analyse wurden die denkbaren Preismodelle wie folgt typisiert:

Abbildung 81: Übersicht: Stromtransportpreisarten

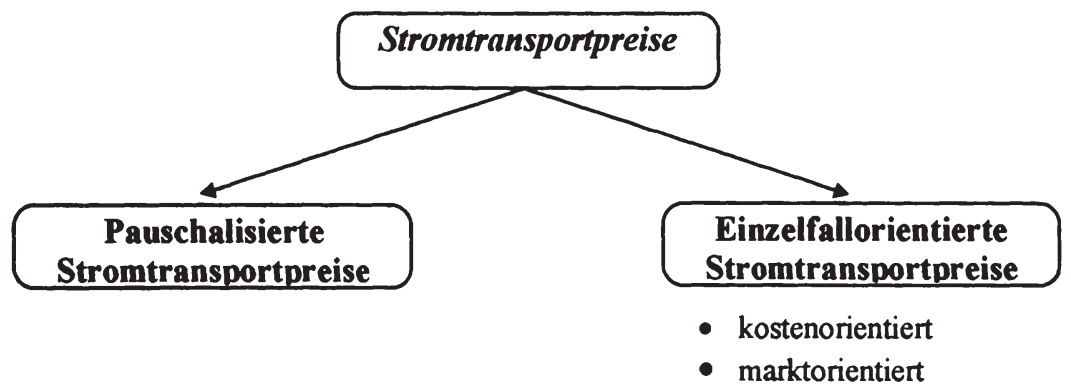

Dabei zeigte sich, daß die jeweiligen Preistypen den Anforderungen in unterschiedlichem Maße genügten:

Tabelle 24: Anforderungen an Stromtransportpreise

\begin{tabular}{|c|c|c|c|}
\hline Preisart & $\begin{array}{l}\text { Pauschalisierte } \\
\text { Stromtransport- } \\
\text { preise }\end{array}$ & $\begin{array}{c}\text { Kostenorientierte } \\
\text { Stromtransport- } \\
\text { preise }\end{array}$ & $\begin{array}{l}\text { Marktorientiente } \\
\text { Stromtransport- } \\
\text { preise }\end{array}$ \\
\hline Transparent & ja & nein & ja \\
\hline Kostenorientiert & bedingt & ja & neln \\
\hline Einfach zu erheben & ja & nein & nein \\
\hline Planbar & ja & nein & nein \\
\hline Verursachergerecht & nein & ja & nein \\
\hline $\begin{array}{l}\text { Knappheltsverhältnisse } \\
\text { wiedergebend }\end{array}$ & nein & ja & ja \\
\hline
\end{tabular}


Hinzu kommt, daß die speziellen konzeptionellen Anforderungen einzelner Ordnungsmodelle von den politischen Anforderungen an die Stromtransportpreise abweichen. Die nähere Betrachtung verdeutlichte, daß für das Weiterentwicklungskonzept eine einzelfallorientierte Stromtransportpreisbildung, die auf den Kosten der jeweiligen Stromtransportleistung aufbaut, geeignet ist. Das TPA-Konzept mit der Zwangsdurchleitung als zentrales Mittel zur Zielerfullung schließt eine vollständig einzelfallorientierte Preissetzung aus. Der Verfasser sieht für das TPAKonzept folgende Preissetzung vor: Ist die Nachfrage von anderen Erzeugern und EVU geringer als das Angebot an verfügbaren Stromtransportkapazitäten eines Gebiets-EVU, so wird ein pauschalisiertes Stromtransportentgelt erhoben. Bemessungsgrundlage sollte die übertragene Leistung, die Zeit und die Entfernung sein. Überschreitet die Nachfrage an Durchleitungskapazitäten in einem Versorgungsgebiet das Angebot, so wird ein Bietwettbewerb durchgeführt. Die Anbieter mit den höchsten Geboten können ihre gewünschte Durchleitung erhalten.

Bei Umsetzung des Common-Carrier-Konzeptes ergibt sich zwangsläufig eine hohe Stromtransportinfrastrukturnutzung durch eine Vielzahl von Anbietern und Abnehmern. Hierdurch ist es zwingend erforderlich, daß die Beteiligten bei Verhandlungen über Stromliefergeschäfte und beim Abschluß von Verträgen die Preise für den erforderlichen Stromtransport kennen. Ansonsten sind sie nicht in der Lage, die Rentabilität eines Geschäftes zu beurteilen. Deshalb wird für das Common-Carrier-Konzept die pauschalisierte, an den Durchschnittskosten des Stromtransportes orientierte Preissetzung vorgesehen. Auch bei Implementierung des Stromhändlerkonzeptes ist die Inanspruchnahme der Stromtransportinfrastruktur und die dafür im Konzept vorgesehene Bezahlung tägliche Routine. Dadurch, daß der Stromhändler jedoch als alleiniger Ankäufer von elektrischer Energie auf dem Stromhändlerbeschaffungsmarkt und als einziger Anbieter auf dem Stromhändlerabsatzmarkt wirkt, findet die Stromtransportkostenverrechnung zunächst unternehmensintern statt. Die vom Verfasser entwickelte Regulierung sieht jedoch die vollständige Trennung der beiden Unternehmensbereiche - Stromtransport und Stromhandel - vor. Deshalb werden auch im Stromhändlerkonzept die gleichen Anforderungen an die Preisbildung gestellt wie im Common-Carrier-Konzept. Folglich ist auch im Stromhändlerkonzept eine pauschalisierte Preisbildung, die die Transaktionskosten begrenzt und an den Durchschnittskosten des Stromtransportes orientiert ist, geeignet. Die Kosten für den Stromhandel als zweiten Unternehmensbereich des Stromhändlers können durch ein ebenfalls an den Durchschnittskosten orientiertes, festgelegtes Entgelt pro Stromhandelsvorgang beglichen werden.

Nachdem die Konzeptionalisierung der Ansätze mit der Preisbildung im Stromtransportbereich abgeschlossen war, wurde die Stromtransportkapazitätsentwicklung mit ihren Rückwirkungen auf die Zielerfüllung durch die Konzepte analysiert. Hierbei stellte sich folgendes heraus: Im Weiterentwicklungskonzept wird der Anreiz zu Netzinvestitionen für ein Gebiets-Fy/Jezu dessen Ejigenbedarf night verän- 
dert. Ein Gebiets-EVU wird Stromtransportkapazitäten für den Stromhandel nur dann errichten, wenn ein zusätzlicher Nutzen aus der Stromtransportkapazität erzielt wird. Dies ist jedoch wegen der bestehenden Versorgungsgebietsgrößen, den daraus resultierenden Stromtransportkosten und der Erzeugungskostensituation in Europa nur in wenigen Konstellationen denkbar; die Infrastrukturbereitstellung und der Ausbau furr den Stromhandel unterbleiben.

Im TPA-Konzept hängt die Stromtransportkapazitätsentwicklung des jeweiligen Gebiets-EVUs entscheidend von der Regulierung und der Marktsituation der EVU ab. Ausschließlich in Staaten, die ihren EVU frei erzielbare Gewinne erlauben oder die eine Höchstpreisregulierung durchführen, werden die EVU in wenigen Fällen bereit sein, Stromtransportkapazitäten für den Stromhandel zu errichten. Zur Verdeutlichung der Einflüsse, die die jeweilige Marktsituation auf die Investitionsentscheidung der EVU im Stromtransportbereich haben, bildete der Verfasser neun Marktsituationstypen in Abhängigkeit vom Erzeugungspreisniveau und der Stromtransportkapazitätssituation: Die potentiellen Stromlieferanten wären bereit, Stromtransportkapazitäten für den Stromhandel zu errichten, doch die EVU, in deren Gebiete der Strom geliefert werden soll, werden ihre Kapazitäten nicht ausbauen, um keine Abnehmer zu verlieren. Falls in ihren Versorgungsgebieten die Stromtransportkapazitäten nicht ausgelastet sind, werden sie versuchen, diese zu reduzieren, um somit die erzwingbaren Durchleitungen zu verhindern. Die Mittel für diese gezielte Kapazitätsreduktion sind:

- die Beeinflussung der Lastkurve,

- unterlassene Ersatzinvestitionen,

- reduzierte Neuinvestitionen bei Stromtransportbedarfsanstiegen,

- die Auswahl restriktiver Bedarfsentwicklungsszenarien für die Kapazitätsplanung.

Durch diese Maßnahmen werden die Gebiets-EVU erreichen, daß ausschließlich zeitlich begrenzte Stromtransportkapazitäten entstehen, die Abnehmer und Konkurrenten zu langfristigen Stromlieferungen oder -bezügen nicht nutzen können.

Gänzlich anders stellt sich die Situation im Common-Carrier- und im Stromhändlerkonzept dar. In beiden Konzepten haben die Unternehmen, die die Stromtransportinfrastruktur betreiben, ein starkes Interesse, zu jedem Zeitpunkt ausreichende Kapazitäten bereitzuhalten; ihr Umsatz ist direkt abhängig von der Infrastrukturnutzung. Wegen der garantierten Vollkostendeckung besteht bei den Stromtransporteuren der Desintegrationskonzepte die Gefahr, daß Kapazitäten über das erforderliche Ausmaß hinaus errichtet werden. Hier muß die Regulierung dieser Monopole ansetzen.

Die Betrachtung der Stromtransportkapazitätsentwicklungen führen zu folgenden Ergebnissen: Im Weiterentwicklungskonzept werden keine zusätzlichen Kapazitäten für den Stromhandel errichtet. Eine Intensivierung des Stromhandels ist damit

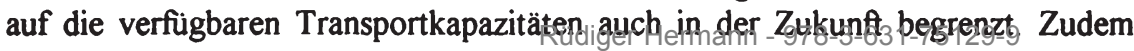


muß langfristig mit einer Zunahme des Stromverbrauchs gerechnet werden, so daß die derzeit für den Stromhandel zur Verfügung stehenden Kapazitäten reduziert werden. Im TPA-Konzept können die EVU über eine gezielte Reduzierung der Stromtransportkapazitäten ihre bestehenden Monopole zurückgewinnen und jeden Stromhandel verhindern. Im Common-Carrier- und Stromhändlerkonzept besteht ein starker Anreiz für Unternehmen, die Stromnetze betreiben, jederzeit ausreichende Stromtransportkapazitäten zum Stromhandel zur Verfügung zu stellen.

Für die Kosten und die Versorgungssicherheit der Elektrizitätsversorgung in Europa bedeutet die Umsetzung der einzelnen Ordnungskonzepte: Durch die Einführung des Weiterentwicklungskonzeptes kann kein Stromhandel in Europa initiiert werden, der zu Kostensenkungen führen könnte. Die bestehenden Monopolrenten werden nicht abgebaut und die ineffizienten, vollständig integrierten Strukturen in den europäischen Elektrizitätssektoren bleiben erhalten. Das TPA-Konzept bewirkt nur kurzfristig begrenzte Wettbewerbsprozesse. Die Reaktion der Gebiets-EVU, die den Verlust ihrer Abnehmer befürchten müssen, führt dazu, daß Stromtransportkapazitäten reduziert werden, um die Durchleitungsbegehren von anderen Stromanbietern und Abnehmern ablehnen zu können. Nachdem die Unternehmen ihre alte Marktposition als Monopolist wiedererlangt haben, hat die Androhung einer Zwangsdurchleitung durch die EU-Institutionen ihre Wirkung verloren. Die Umsetzung des TPA-Konzeptes kann daher nicht die Kosten der Stromversorgung langfristig reduzieren und die Effizienz der Unternehmen steigern.

Anders ist die Situation im Common-Carrier- und Stromhändlerkonzept: Der Verfasser konnte keine Hinweise darauf finden, daß die durch die Umsetzung der Konzepte beabsichtigten Wettbewerbsprozesse langfristig versiegen werden. Die niedrigen Marktzutrittsbarrieren ermöglichen jederzeit den Eintritt neuer Anbieter auf der Großhandelsstufe des Common-Carrier-Konzeptes und des Stromhändlerbeschaffungsmarktes, wodurch die Prozesse stets neu belebt und wettbewerbsbedrohende abgestimmte Verhaltensweisen der Unternehmen im Markt begrenzt werden. Unterschiede zwischen den beiden Konzepten bestehen hinsichtlich der Anzahl der Märkte, auf denen Wettbewerbsprozesse kostenmindernd wirken können, der Transaktionskosten und der Regulierungskosten. Hier weist das Common-CarrierKonzept Vorteile gegenüber dem Stromhändlerkonzept auf. Ferner existieren die freien Wahlmöglichkeiten der Nachfrager auf der Großhandelsstufe im Stromhändlerkonzept nicht. Das Stromhändlerkonzept ist jedoch einfacher zu implementieren und wird in Europa auf eine höhere Akzeptanz stoßen. Nach Meinung des Verfassers gleichen diese Vorteile jedoch nicht die Vorzüge des Common-Carrier-Konzeptes hinsichtlich der dynamischen Effizienz aus (vgl. Tabelle 25).

Die vorliegende Arbeit kommt somit zu folgendem Ergebnis: Ausschließlich die beiden Desintegrationskonzepte können die Ziele - sinkende Kosten in der Stromversorgung und ein ausreichendes $\mathrm{Maß}$ an Versorgungssicherheit - gewährlei- 
$\operatorname{sten}^{537}$. Die Einfuihrung eines der beiden Integrationskonzepte bedeutet das Scheitern eines gemeinsamen europäischen Strommarktes und keine Effizienzsteigerung

Tabelle 25: Vergleichende Beurteilung der Ordnungskonzepte

\begin{tabular}{|c|c|c|c|c|}
\hline Kriterien zur Beurtellung der Ordnungsmodelle & $\begin{array}{c}\text { Welterentuicld. } \\
\text { konzept }\end{array}$ & TPA-Konzept & $\begin{array}{c}\text { CC.- } \\
\text { Konzept }\end{array}$ & $\begin{array}{l}\text { Stromhöndles. } \\
\text { konzept }\end{array}$ \\
\hline Vertikale Integration aufgelöst? & Nein & Nein & Ja & Ja \\
\hline Stromhandelsinfrostuktur geschatien? & $\mathrm{Ne}$ & Nein & $\mathrm{Ja}$ & Nein \\
\hline Tellmärite geschaffen ? & Neil & Nein & Ja & Ja \\
\hline Marktzutititsbarileren reduzert? & & Ja & Ja & Ja \\
\hline Wettbewerbsintenstiöt gestelgert? & & Nein & Ja & Ja \\
\hline Marktmacht einzeliner Anbleter u. Nochfrager begrenzt? & $\mathrm{Ne}$ & Nein & Ja & Ja \\
\hline Reduzienung der Gesamtkosten der Elektriztlätsversorgung? & in & Nein & Ja & Ja \\
\hline Versorgungssicherhelt gewährleistet? & $\mathrm{Ja}$ & Ja & $\mathrm{Ja}$ & $\mathrm{Ja}$ \\
\hline Ausblick Presentwicklung: & $\begin{array}{l}\text { Unveränderte } \\
\text { Strompreise } \\
\text { in Europo }\end{array}$ & $\begin{array}{l}\text { Lgtr. keine } \\
\text { Strompreis- } \\
\text { senkungen }\end{array}$ & $\begin{array}{l}\text { Sinkende } \\
\text { Strompreise } \\
\text { in Europo }\end{array}$ & $\begin{array}{l}\text { Sinkende } \\
\text { Strompretse } \\
\text { in Europo }\end{array}$ \\
\hline
\end{tabular}

in der Stromversorgung. Unter den Gesichtspunkten der politischen Ökonomie ist die vollständige Trennung von Elektrizitätsversorgung und Politik zu empfehlen. Die in Europa übliche Vergabe gut bezahlter Posten an Politiker hat dazu geführt, daß weder Unternehmen noch Politiker unabhängig in ihren Entscheidungen und deren Findung waren und sind. Hierdurch wurden in der Vergangenheit oftmals progressive Ansätze und Entwicklungen verhindert und damit auch deren Chancen zu Effizienzsteigerungen. Ferner sollte die Aufsicht über die Elektrizitätssektoren einer europäischen Institution übertragen werden. Dies würde zwar nicht dem Subsidiaritätsgedanken entsprechen, jedoch eine Harmonisierung der Rahmenbe-

537 In Großbritannien sind z.B. durch Einführung der neuen Struktur und die Implementierung von Wettbewerbsprozessen 22.000 Arbeitsplătze wegrationalisiert worden, ohne daß negative Auswirkungen auf die Sicherheit der Stromversorgung spürbar wurden. Vgl. COLLIER, J.G. : a.a.O., S. 184 
dingungen für Produzenten und Abnehmer sowie eine Entkopplung der nationalen Elektrizitätswirtschaften von den nationalen Wirtschaftspolitiken bewirken. In Anbetracht der aktuellen Diskussion über den Weg zu einem europäischen Strommarkt sollten alle politischen Entscheidungsträger einsehen, daß Märkte mit Wettbewerb bei geeigneten Rahmenbedingungen zu besseren Ergebnissen führen als unzureichend regulierte staatlich geschützte Monopole. Mit Hilfe der bei den Desintegrationskonzepten aufgezeigten Mitteln sollte es möglich sein, die Einflußnahmen von Interessengruppen oder einzelnen Regierungen auf die Elektrizitätsversorgung in Zukunft zu begrenzen. Diese Empfehlungen münden in die folgenden Forderungen an die politischen Entscheidungsträger:

- Die EU-Kommission, verschiedene nationale Regierungen und das Europäische Parlament sollten davon Abstand nehmen, Modifikationen des TPA-Konzeptes als Mittel zur Verwirklichung des europäischen Strommarktes anzusehen. Ferner sollten die Gegner der Desintegrationskonzepte ihren Widerstand aufgeben.

- Es sollte ein klares Bekenntnis zu einer wettbewerblichen Elektrizitätsstruktur in Europa abgegeben werden, um deutlich zu machen, daß ein gemeinsamer Markt das ökonomische Ziel der EU auch auf dem Elektrizitätssektor ist.

- Ein genauer Zeitplan sollte für die einzelnen Phasen des Implementierungsprozesses des Common-Carrier-Konzeptes erstellt werden.

- Ein entsprechendes Gesetzeswerk sollte von den europäischen Institutionen zügig verabschiedet werden.

Parallel dazu sollte die europäische Öffentlichkeit über die Ziele und Erfordernisse der Umstrukturierung informiert werden, damit eine Akzeptanz der Umstrukturierungen erreicht wird.

Die Hauptgefahren drohen dem vorgeschlagenen Common-Carrier-Konzept durch zahlreiche politische Kompromisse und einzelstaatliche Rücksichtnahmen. Sie können bewirken, daß die Maßnahmen nur zögerlich und zeitlich verspätet durchgeführt werden und hierdurch Probleme bei der technischen Realisierung entstehen. Jeder Bereich, in dem ein möglicher Wettbewerb politisch gewollt verhindert wird, läßt den Erfolg des Konzeptes sinken und gefährdet somit auch langfristig dessen Akzeptanz.

Die derzeitigen Entwicklungen deuten darauf hin, daß ein gemeinsamer europäischer Strommarkt in die ferne Zukunft entschwindet. Die schrittweise Abkehr der EU-Kommission von ihren ursprünglichen Gedanken zur Einführung eines Strommarktes mit Wettbewerbsprozessen zeigt, daß sie nicht über das politische Gewicht verfügt, die grundlegenden ökonomischen Zielsetzungen der EU durchzusetzen. Einzelnen nationalen Regierunger und Parteien sowiederen -Vertizetern im 
Europäischen Parlament gelang es bisher, jede durchgreifende Veränderung zu verhindern. Das Ende dieser Entwicklung sind zwei Modifikationen des Weiterentwicklungskonzeptes - Negotiated Access und Single-Buyer. Ob und wieweit die Gebiets-EVU Stromdurchleitungen durchführen müssen, ist derzeit offen. Fest steht jedoch bereits heute, daß es zu keiner umfassenden von Abnehmern oder Produzenten erzwingbaren Durchleitungsverpflichtung kommen wird. Somit wird langfristig jeder Wettbewerb im europäischen Elektrizitätssektor ausgeschlossen. Hierdurch verzichten die politischen Entscheidungsträger auf einen gemeinsamen europäischen Strommarkt, der durch Effizienzsteigerungen relativ sinkende Elektrizitätspreise bewirken kann. Es wird politisch eine Chance vertan, die internationale Wettbewerbsfähigkeit des Wirtschaftsstandortes Europa zu verbessern. Anstatt ein elektrizitätswirtschaftliches Konzept für das nächste Jahrtausend umzusetzen, werden die zu Beginn unseres ausklingenden Jahrhunderts geschaffenen einzelstaatlichen Strukturen fortgeschrieben.

Doch noch sind die abschließenden Entscheidungen nicht getroffen. Die elektrizitätswirtschaftliche Kleinstaaterei muß überwunden werden. Veränderungsprozesse sind mit Chancen und Risiken verbunden. Es gilt, die Risiken durch eine geeignete Konzeption zu begrenzen und die Chancen eines marktwirtschaftlichen europäischen Strommarktes zu nutzen.

Seit Abschluß der Dissertation im April 1996 kam es auf der europäischen Ebene zu einer politischen Entscheidung über den Weg und die Mittel mit denen ein europäischer Binnenmarkt für Elektrizität geschaffen werden soll. Zu Beginn des Jahres 96 verstärkte sich der Druck auf die Mitgliedsstaaten und die EU-Kommission nach nunmehr zehnjährigem Ringen zu einem Ergebnis auf dem Stromsektor zu kommen. Die Mitgliedsstaaten teilten sich weiterhin in die bereits angesprochenen politischen Lager auf: Auf der einen Seite Staaten, die jede Auflösung ihrer öffentlichen integrierten Unternehmen und Strukturen ablehnten und auf der anderen Seite Staaten, die wie die Niederlande und die Bundesrepublik durchgreifende Veränderungen forderten. Das Ergebnis dieses poltischen Prozesses ist die Richtlinie 96/92 EG vom 19.12.96 ${ }^{538}$, die bereits im Juni des gleichen Jahres durch einen gemeinsamen Standpunkt der Mitgliedsstaaten vorgegeben wurde.

In dieser Richtlinie werden in den Vorbemerkungen zunächst noch einmal die Gründe dargestellt, warum und wie es zu der Richtlinie gekommen ist. Ebenso werden zu Beginn unter Ziffer 1 die Ziele für den Elektrizitätsbinnenmarkt wieder-

538 Vgl. KOMMISSION DER EUROPÄISCHEN GEMEINSCHAFT: Richtlinie 96/92/EG des europasischen Parlaments und des Rates vom 19. Dezember 1996 betreffend gemeinsame Vorschriften für den Elektrizitätsbinnenmarkt, in: Amtsbaltt der Europäischen Gemeinschaften Nr. L 27/20 vom 30.01.97. Auf diese Richtlinisavird Bezug gemommen-9 
holt. Die Schaffung des angestrebten Binnenmarktes soll schrittweise erfolgen wobei die Richtlinie für den Transit von Elektrizitätslieferungen über große Netze ${ }^{539}$ und die Richtlinie zur Einführung eines gemeinschaftlichen Verfahrens zur Gewährleistung der Transparenz der von industriellen Endverbrauchern zu zahlenden Gasund Strompreise ${ }^{540}$ als der erste Schritt angesehen wird. Die weiteren Maßnahmen sollen durch die Richtlinie vom 06.12.96 festgelegt werden. Zum Ausgangspunkt der Richtlinie wird in den Vorbemerkungen festgehalten, daß ,in den einzelnen Mitgliedsstaaten strukturelle Unterschiede und dementsprechend unterschiedliche Regelungen für den Elektrizitätssektor ${ }^{\text {“ } 541}$ bestehen. Ferner werden in den Vorbemerkungen bereits die im folgenden konkret ausgeführten Alternativen für den Netzzugang zur Gestaltung der Elektrizitätsstrukturen in den Mitgliedsstaaten umrissen $^{542}$.

Nachdem im Kapitel I der Richtlinie die erforderlichen Begriffe definiert worden sind $^{543}$, weist Artikel 3 Absatz 1 des zweiten Kapitels den Mitgliedsstaaten die Verantwortung dafür zu, daß die Elektrizitätsversorgungsunternehmen unabhängig von der Wahl des Netzzugangskonzeptes nach den Grundsätzen der Richtlinie und im Hinblick auf die Erreichung eines wettbewerbsorientierten Elektrizitätsmarktes betrieben werden ${ }^{544}$. Ferner schreibt Artikel 3 Absatz 1 ausdrücklich die Gleichbehandlung der Unternehmen vor. Die beiden dargelegten alternativen Netzzugangskonzepte müssen zu einem vergleichbaren Marktzugang führen. Absatz 2 des dritten Artikels gibt den Mitgliedsstaaten die Möglichkeit, den Versorgungsunternehmen gemeinwirtschaftliche Verpflichtungen aufzuerlegen und wenn dies geschieht, diese Unternehmen von den Artikeln 5, 6, 17 und 18 auszunehmen, sofern die Anwendung der Artikel die Erfüllung der gemeinwirtschaftlichen Aufgaben durch diese Unternehmen verhindern würde. In den Artikeln 5, 6, 17 und 18 werden die Vorschriften zur Umsetzung eines Genehmigungsverfahrens für Kraftwerke geregelt. Gleichzeitig sieht die Richtlinie jedoch vor, daß die Nichtanwendung der genannten Artikel nicht den Interessen der Gemeinschaft bei der Entwicklung des Handelsverkehrs zuwider laufen darf.

539 Vgl. KOMMISSION DER EUROPÄISCHEN GEMEINSCHAFT : Richtlinie des Rates vom 29.10.1990 über den Transit von Elektrizitătslieferungen über große Netzc (90/547/EWG)

$540 \mathrm{Vgl}$. KOMMISSION DER EUROPÄISCHEN GEMEINSCHAFT : Richtlinie des Rates vom 29. Juni 1990 betreffend der Einführung eines gemeinschaftlichen Verfahrens zur Gewährleistung der Transparenz der vom industrellen Endverbraucher zu zahlenden Gas- und Strompreise, in: Amtsblatt der Europäischen Gemeinschaften, Nr. L. 185 vom 17.7.1990

$541 \mathrm{Vgl}$. KOMMISSION DER EUROPÄISCHEN GEMEINSCHAFT: Richtlinie 96/92/EG, a.a.O.

542 Vgl. KOMMISSION DER EUROPÄISCHEN GEMEINSCHAFT: Richtlinie 96/92/EG, a.a.O. Ziffer $23 \mathrm{fr}$. der Vorbemerkungen

$543 \mathrm{Vgl}$. KOMMISSION DER EUROPÄISCHEN GEMEINSCHAFT: Richtlinie 96/92/EG, a.a.O. Artikel 1 Absatz 2

$544 \mathrm{Vgl}$. KOMMISSION DER EUROPÄISCHEN GEMEINSCHAFT: Richtlinie 96/92/EG, a.a.O. Artikel 3 Absatz 1 
Das Kapitel III ${ }^{\text {s.t5 }}$ legt die Maßnahmen im Erzeugungsbereich fest. Die Richtlinie gibt den Mitgliedsstaaten die Wahl beim Bau neuer Erzeugungsanlagen zwischen einem Genehmigungs- und einem Ausschreibungsverfahren zu wählen ${ }^{546}$. Im Falle des Genehmigungsverfahrens werden die Kriterien für die Erteilung einer Genehmigung durch die jeweiligen Mitgliedsstaaten festgelegt ${ }^{547}$. Die Kriterien und die Verfahren müssen veröffentlicht werden und bei einer Verweigerung einer Genehmigung müssen die Gründe dargelegt, Rechtsmittel zur Verfügung stehen und die Kommission unterrichtet werden ${ }^{548}$. Beim Ausschreibungsverfahren berechnen die Mitgliedsstaaten regelmäßig einen Plan über die erforderlichen Ersatzkapazitäten, die dann nach festgelegten Modalitäten ausgeschrieben werden müssen. Hierbei müssen auch unabhängige Erzeuger eine Genehmigung zum Kraftwerksbau erhalten können ${ }^{\text {s.19. }}$.

Hinsichtlich des Übertragungsnetzes sieht die Richtlinie in Kapitel IV $V^{550}$ vor, daß die Mitgliedsstaaten die Eigentümer der Übertragungsnetze benennen ${ }^{551}$, denen die Verantwortung für den Netzausbau und die Versorgungssicherheit auferlegt wird. Im wesentlichen werden dies die bereits in der Richtlinie über den Transit von Elektrizität in Europa angesprochenen Unternehmen sein. Ferner sieht die Richtlinie Vorschriften fur die Zusammenarbeit der Netzbetreiber in der EU vor ${ }^{52}$. Zusätzlich zu den Aufgaben im Stromtransport ist der Betreiber eines Netzes gemäß Artikel 8 auch für die „Inanspruchnahme der Erzeugungsanlagen in seinem Gebiet und für die Nutzung der Verbindungsleitungen mit anderen Netzen ${ }^{\text {"s553 }}$ verantwortlich. Damit müssen die Netzbetreiber letztlich die wesentlichen Koordinationsaufgaben der Elektrizitätsversorgung erbringen ${ }^{554}$. Die Kriterien für die Lieferung an

545 Vgl. KOMMISSION DER EUROPÄISCHEN GEMEINSCHAFT: Richtlinie 96/92/EG, a.a.O. Artikel 4 bis 6

546 Vgl. KOMMISSION DER EUROPÄISCHEN GEMEINSCHAFT: Richtlinic 96/92/EG, a.a.O. Artikel 4

547 Vgl. KOMMISSION DER EUROPÄISCHEN GEMEINSCHAFT: Richtlinie 96/92/EG. a.a.O. Artikel 5 Absatz 1

548 Vgl. KOMMISSION DER EUROPÄISCHEN GEMEINSCHAFT: Richtlinie 96/92/EG. a.a.O. Artikel 5 Absatz 2 und 3

$549 \mathrm{Vgl}$. KOMMISSION DER EUROPÄISCHEN GEMEINSCHAFT: Richtlinie 96/92/EG, a.a.O. Artikel 6Absatz 1 bis 6

550 Vgl. KOMMISSION DER EUROPÄISCHEN GEMEINSCHAFT: Richtlinic 96/92/EG, a.a.O. Artikel 7 bis 9

551 Vgl. KOMMISSION DER EUROPÄISCHEN GEMEINSCHAFT: Richtlinie 96/92/EG, a.a.O. Artikel 7 Absatz 1

552 Vgl. KOMMISSION DER EUROPÄISCHEN GEMEINSCHAFT: Richtlinie 96/92/EG, a.a.O. Artikel 7 Absatz 3

553 Vgl. KOMMISSION DER EUROPÄISCHEN GEMEINSCHAFT: Richtlinie 96/92/EG, a.a.O. Artikel 8 Absatz 1

554 Vgl. hierzu die Ausführungen im siebten Kapitel zu den Aufgaben des Pools im CommonCarrier-Konzept 
Endverbraucher ${ }^{55}$ und somit auch für die Einspeisung in das Elektrizitätsnetz legen die Mitgliedsstaaten fest. Sie müssen objektiv begründet sein, veröffentlicht und in „nichtdiskriminierender Weise“ angewandt werden. In diesem Zusammenhang gibt die Richtlinie den Mitgliedsstaaten ausdrücklich die Möglichkeit, den Netzbetreibern die Stromeinspeisung aus bestimmten Energieträgern vorzuschreiben. Hierbei werden Stromeinspeisungen aus Erzeugungsanlagen von erneuerbaren Energieträgern oder Abfällen sowie der Kraft-Wärme-Kopplung genannt und den Staaten freigestellt, bis zu 15\% des gesamten Elektrizitätsverbrauchs eines Staates aus heimischen Energieträgern zu beziehen ${ }^{556}$.

Bezüglich des Verteilungsnetzes schreibt die Richtinie im fünften Kapitel ${ }^{557}$ vor, $\mathrm{da} ß$ den bestehenden Verteilerunternehmen die Versorgungspflicht für Kunden auferlegt werden kann.

Für alle Versorgungsstufen der Elektrizitätsversorgung sieht die Richtlinie in Kapitel VI vor, daß unabhängig von der Eigentumsform und des vertikalen Integrationsgrades der Unternehmen eine getrennte Buchführung eingerichtet werden mu ${ }^{558}$, in die die Kontrollorgane der Mitgliedsstaaten einsicht nehmen können ${ }^{559}$. Hiermit sollen „Diskriminierungen, Quersubventionen und Wettbewerbsverzerrungen ${ }^{\text {c560 }}$ verhindert werden.

Beim Netzzugang haben die Mitgliedsstaaten wie im Erzeugerbereich die Wahl zwischen zwei Modellen ${ }^{561}$ :

1. Der Netzzugang auf Vertragsbasis ${ }^{562}$

2. Das Alleinabnehmersystem ${ }^{53}$.

Beim Netzzugang auf Vertragsbasis müssen die Mitgliedsstaaten Maßnahmen treffen, „damit die Elektrizitätserzeuger und, soweit die Mitgliedsstaaten solche

555 Vgl. KOMMISSION DER EUROPÄISCHEN GEMEINSCHAFT: Richtlinie 96/92/EG, a.a.O. Artikel 8 Absatz 2

556 Vgl. KOMMISSION DER EUROPÄISCHEN GEMEINSCHAFT: Richtlinie 96/92/EG, a.a.O. Artikel 8 Absatz 3 und 4

557 Vgl. KOMMISSION DER EUROPÄISCHEN GEMEINSCHAFT: Richtlinie 96/92/EG, a.a.O. Artikel 13 bis 15

558 Vgl. KOMMISSION DER EUROPÄISCHEN GEMEINSCHAFT: Richtlinie 96/92/EG, a.a.O. Artikel 14

559 Vgl. KOMMISSION DER EUROPÄISCHEN GEMEINSCHAFT: Richtlinie 96/92/EG, a.a.O. Artikel 13

$560 \mathrm{Vgl}$. KOMMISSION DER EUROPÄISCHEN GEMEINSCHAFT: Richtlinie 96/92/EG, a.a.O. Artikel 14 Absatz 3

561 Vgl. Kapitel VII der Richtlinie 96/92/EG

562 Vgl. KOMMISSION DER EUROPÄISCHEN GEMEINSCHAFT: Richtlinie 96/92/EG, a.a.O. Artikel 17

563 Vgl. KOMMISSION DER EUROPÄISCHEN GEMEINSCHAFT: Richtlinie 96/92/EG, a.a.O. Artikel 18 
Unternehmen zulassen, die Elektrizitätsversorgungsunternehmen sowie die zugelassenen Kunden, die sich innerhalb und außerhalb des Netzgebietes befinden, einen Netzzugang aushandeln können, um untereinander Lieferverträge auf der Grundlage freiwilliger kommerzieller Vereinbarungen schließen zu können. “564 Der Netzbetreiber muß hierzu eine Preisspanne für die Übertragungsleistung veröffentlichen. Als Alternative wird den Mitgliedsstaaten ein geregeltes Netzzugangssystem mit veröffentlichten Tarifen für die Übertragungsleistung vorgeschlagen. Besonders wichtig furr die Bewertung der Richtlinie ist Absatz 5 des Artikels 17:

„Der Betreiber des betreffenden Verteiler- bzw. Übertragungsnetzes kann den $\mathrm{Zu}$ gang verweigern, wenn er nicht über die nötige Kapazität verfügt. Die Verweigerung ist insbesondere unter Berücksichtigung des Artikels 3 entsprechend zu begründen."

Anders lauten die Vorschriften der Richtlinie bei Wahl des Alleinabnehmersystems durch Mitgliedsstaaten der EU. In diesem Falle müssen die Mitgliedsstaaten dafür Sorge tragen, daß „ein nichtdiskriminierender Tarif für die Nutzung des Übertragungs- und Verteilersystems veröffentlicht wird; die zugelassenen Kunden zur Deckung ihres Eigenbedarfs Lieferverträge mit Elektrizitätserzeugern und soweit Mitgliedsstaaten solche Unternehmen zulassen, mit Elektrizitätsversorgungsunternehmen außerhalb des von dem System abgedeckten Gebietes schließen kön-

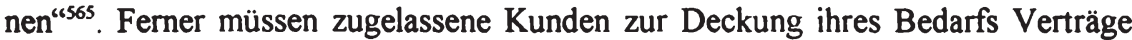
mit Erzeugern innerhalb des Gebietes des Alleinabnehmers und Verträge mit dem Netzbetreiber über die Nutzung des Übertragungsnetzes für Stromlieferungen von außerhalb des Netzgebietes schließen können. Für das Verständnis des Alleinabnehmermodells bedeutend sind die beiden Alternativen über das Zustandekommen der in Artikel 18 Absatz 1 vorgesehenen Verträge mit anderen Anbietern innerhalb und außerhalb des Netzgebietes des Alleinabnehmers, die in Artikel 18 Absatz 2 ausgeführt werden. Danach kann der Alleinabnehmer dazu verpflichtet werden, von einem Erzeuger Strom zu seinem Abgabepreis, abzüglich eines Übertragungsentgeltes, abzunehmen ${ }^{566}$. Voraussetzung hierfür ist, daß der Erzeuger einen Stromliefervertrag mit einem zugelassenen Kunden im Versorgungsgebiet des Alleinabnehmers geschlossenen hat. Der Alleinabnehmer berechnet dem zugelassenen Verbraucher seinen Abgabepreis. Die Vertragspartner müssen dann im Anschluß den tatsächlichen Strompreis für die Lieferung mit den Zahlungen an den Alleinabnehmer verrechnen. Warum ein derartiges Konstrukt gewählt wurde, ist in der Richtline nicht ausgeführt worden. Falls dem Alleinabnehmer diese Verpflichtung nicht auferlegt wird, müssen die Mitgliedsstaaten geeigente Maßnahmen treffen,

564 Vgl. KOMMISSION DER EUROPÄISCHEN GEMEINSCHAFT: Richtlinie 96/92/EG, a.a.O. Artikel 17 Absatz 1

565 Vgl. KOMMISSION DER EUROPÄISCHEN GEMEINSCHAFT: Richtlinie 96/92/EG, a.a.O. Artikel 18 Absatz 1 Ziffer I und II

566 Vgl. KOMMISSION DER EUROPÄISCHEN GEMEINSCHAFT: Richtlinie 96/92/EG, a.a.O. Artikel 18 Absatz 2 
damit ein Netzzugang und damit Lieferverträge zwischen Erzeugern und Verbrauchern auf Vertragsbasis zustande kommen können ${ }^{567}$.

Zum Zeitplan der Marktöffnung sieht die Richtlinie folgendes vor: Es wird eine europäische Marktquote gebildet, wobei alle Endverbraucher, die pro Betriebsstätte einschließlich der Eigenerzeugung mehr als $40 \mathrm{GWh}$ Strom verbrauchen, gewertet werden ${ }^{568}$. Deren Elektrizitätsverbrauch wird im Verhältnis zum gesamten Elektrizitätsverbrauch gesetzt. Die daraus gebildete Quote ist die Quote, mit der der jeweilige nationale Elektrizitätsmarkt in der zweiten Stufe geöffnet werden muß. Drei Jahre nach dem Inkrafttreten der Richtlinie, am 19.02.1999, wird dann die Quote auf der Grundlage von Endverbrauchern mit über $20 \mathrm{GWh}$ neu berechnet. Nach weiteren drei Jahren werden Verbraucher mit einer Abnahme von mehr als $9 \mathrm{GWh}$ zugrunde gelegt ${ }^{569}$. Verteilungsunternehmen können durch die Mitgliedsstaaten als zugelassene Kunden definiert werden. Anderenfalls haben sie zumindest über die Strommenge Marktzugang, die der Strommenge entspricht, die zugelassene Kunden der Verteiler von den Verteilerunternehmen beziehen ${ }^{570}$. Ungleichgewichte bei der Marktöffnung sollen dadurch verhindert werden, daß Lieferungen zwischen einem Erreuger und einem Kunden in einem anderem Netzgebiet dann nicht untersagt werden dürfen, wenn der Kunde in beiden Gebieten als Kunde zugelassen ist. Untersagt ein Unternehmen eine Übertragung, weil der Kunde nur in einem der Mitgliedsstaaten zugelassen ist, hat die Kommission das Recht, der verweigernden Netzgesellschaft die Ausführung der Übertragung aufzuerlegen $^{571}$.

Artikel 21 der Richtlinie bezieht sich auf Direktleitungen. Elektrizitätserzeuger und EVU, die in den Mitgliedsstaaten zugelassen sind, erhalten das Recht, Tochterunternehmen und zugelassene Kunden über eine Direktleitung zu versorgen ${ }^{572}$. Die Mitgliedsstaaten müssen Kriterien für den Bau von Direktleitungen festlegen und veröffentlichen. Ausnahmen regelt Artikel 21 Absatz 4: „Die Mitgliedsstaaten können die Genehmigung zur Errichtung einer Direktleitung entweder von der Ver-

567 Vgl. KOMMISSION DER EUROPÄISCHEN GEMEINSCHAFT: Richtlinie 96/92/EG, a.a.O. Artikel 18 Absatz 3

568 Vgl. KOMMISSION DER EUROPÄISCHEN GEMEINSCHAFT: Richtlinie 96/92/EG, a.a.O. Artikel 19 Absatz 1

569 Vgl. KOMMISSION DER EUROPÄISCHEN GEMEINSCHAFT: Richtlinie 96/92/EG, a.a.O. Artikel 19 Absatz 2

570 Vgl. KOMMISSION DER EUROPÄISCHEN GEMEINSCHAFT: Richtlinie 96/92/EG, a.a.O. Artikel 19 Absatz 3

571 Vgl. KOMMISSION DER EUROPÄISCHEN GEMEINSCHAFT: Richtlinie 96/92/EG, a.a.O. Artikel 19 Absatz 5

572 Vgl. KOMMISSION DER EUROPÄISCHEN GEMEINSCHAFT: Richtlinie 96/92/EG, a.a.O. Artikel 21 Absatz 1 
weigerung des Netzzugangs auf der Grundlage, soweit anwendbar, des Artikels 17 Absatz 5 oder des Artikel 18 Absatz 4 ( ...) abhängig machen. “573

Die Artikel 22 bis 29 regeln die weiteren Rahmenbedingungen und generelle Ausnahmen für Krisenzeiten. Ferner werden Übergangsregelungen für ausgewählte Mitgliedsstaaten und Berichtspflichten der Kommission festgelegt.

Die Modelle der Richtlinie und die vorgeschlagenen Wege zu ihrer Umsetzung weisen deutliche Parallelen zu den in der Dissertation vorgestellten Ordnungsmodellen auf:

Die Kombination aus Genehmigungsmodell und Netzzugang auf Vertragsbasis ist weitgehend identisch mit dem TPA-Konzept. Der Unterschied besteht darin, daß die Position des Netzbetreibers in der Konzeption der Richtlinie stärker ist. Der Netzzugang kann von Kunden oder Erzeugern nicht direkt zu festgelegten Preisen erzwungen werden, sondern muß mit dem Netzbetreiber als Monopolist verhandelt werden. Hierbei hat der Netzbetreiber die Möglichkeit, seine starke Verhandlungsposition für eigene Interessen zu nutzen. Neuanbieter muß der Netzbetreiber nicht fürchten, da der Bau von Direktleitungen beschränkt ist und er gegenüber einem Unternehmen, welches ein neues Netz errichten will, wesentliche Zeit- und Kostenvorteile besitzt. Der Netzbereiber als Monopolist wird einen höheren Preis für die Übertragungsleistung fordern können, als es bei mehreren Anbietern, die untereinander in Konkurrenz stehen, möglich wäre. Dies ist jedoch noch die am wenigsten negative Wirkung, die nur dann eintreten wird, wenn die bestehenden integrierten Unternehmen mit einer zukünttig getrennten Buchführung keine Gesamtunternehmensstrategie verfolgen. Durch mündliche, schwer nachweisbare Absprachen und die im achten und neunten Kapitel aufgezeigten Maßnahmen können die Übertragungsbereiche der EVU mittel- und langfristig die Marktpositionen ihrer Erzeugungsbereiche stärken, bis diese wieder das Monopol bei der Elektrizitätsproduktion erreichen. Die Ausfuihrungen zum TPA-Konzept haben gezeigt, welche wirksamen Mittel den Unternehmen zur Verfügung stehen ${ }^{574}$. Die Richtlinie schränkt diese Mittel nicht ein. Im Gegenteil, sie läßt ausdrücklich die Ablehnung einer Lieferung mit Hinweis auf mangelnde Kapazitäten oder einer Einschränkung des Versorgungsauftrages zu, ohne diese Tatbestände so zu präzisieren, daß deren Mißbrauch ausgeschlossen werden kann. Staatliche Kontrollorgane der Mitgliedsstaaten werden wegen ihrer eingeschränkten Informationslage entgegen den Auflagen der Richtlinie den Einsatz dieser Mittel nicht verhindern können. Aus diesen Gründen kann die Bewertung des TPA-Konzeptes vollständig für die Bewertung der Richtlinie hinsichtlich der im dritten Kapitel aufgestellten Kriterien übernommen werden. Damit werden durch die Richtlinie bezüglich der Kombination aus Genehmigungsverfahren und Netzzugang auf Vertragsbasis die Ziele, die mit der

573 Vgl. KOMMISSION DER EUROPÄISCHEN GEMEINSCHAFT: Richtlinie 96/92/EG, a.a.O. Artikel 21 Absatz 4

574 Vgl. hierzu die Ausführungen im achten Kajpitgl. Hermann - 978-3-631-75129-9 
Verwirklichung des Binnenmarktes verbunden werden, nicht erreicht werden können.

Das zweite in der Richtlinie vorgesehene Modell zur Organisation der nationalen Elektrizitätswirtschaften, die Kombination aus Ausschreibungsverfahren und Alleinabnehmer, ist weitgehend mit den wesentlichen Inhalten des dargestellten Weiterentwicklungskonzeptes identisch. Die Beibehaltung von staatlichen Unternehmen mit einem vollständigen Monopol in allen Bereichen der Elektrizitätsversorgung als Alleinabnehmer verändert die bisherigen Strukturen mit ihren negativen Auswirkungen auf dem Binnenmarkt nicht. Auch das zukünftige Ausschreiben von benötigten Erzeugungskapazitäten wird wenig daran ändern, weil durch die Ausschreibung zunächst nur ein geringer Anteil im Verhältnis zur Gesamtkapazität ausgeschrieben wird. Ferner verfuggt das staatliche Unternehmen, welches gleichzeitig auch im Besitz des Übertragungs- und Verteilungsnetzes ist, über eine wesentliche bessere Informationslage als neue Anbieter und kann diese nutzen, um Konkurrenten zu unterbieten. Ein weiterer Vorteil sind die bestehenden Kraftwerksstandorte des staatlichen Unternehmens. In den meisten Mitgliedsstaaten sind neue Kraftwerksstandorte schwer durchzusetzen, insbesondere dann, wenn keine regenerativen Energien genutzt werden. Ein bereits bestehender Anbieter hat den Vorteil, daß seine Standorte weiter ausgebaut werden können, wobei er von der Erzeugungstechnologie begünstigt wird, die immer höhere Kapazitäten auf gleichen Raum ermöglicht. Die Kombination aus Ausschreibungsverfahren und Alleinabnehmer weist jedoch auch Bestandteile des Stromhändler-Konzeptes auf. So ist die Funktion der Unternehmen als Alleinabnehmer für die Erzeuger zunächst identisch mit den Aufgaben der Netzgesellschaft des Stromhändler-Modells. Die Aufgaben des Pools im dargestellten Stromhändler-Konzept werden in der Richtlinie jedoch nicht vom Alleinabnehmer übernommen. Während im Stromhändler-Konzept der Pool eine Preisgestaltung durch direkten Wettbewerb der Erzeuger einleitet, nimmt der Alleinabnehmer den Strom von den Produzenten innerhalb und außerhalb seines Netzgebietes zu seinen Preisen ab und berechnet eine Übertragungsgebühr. Damit beseitigt die in der Richtlinie vorgesehende Kombination aus Ausschreibungs- und Alleinabnehmersystem nicht die Defizite, die bei der Beurteilung der Integrationskonzepte angesprochen worden $\operatorname{sind}^{575}$.

Eine Zusammenfassung der wesentlichen Regelungen der Richtlinie in Hinblick auf die aufgestellten Ziele des Binnenmarktes ergibt folgendes Bild:

1. Man konnte sich innerhalb der EU nicht auf einheitliche Strukturen in der Elektrizitätswirtschaft einigen. Deshalb sieht die Richtlinie zwei unterschiedliche Konzeptionen für die Organisation des Erzeugungsbereiches und den Netzzugang vor. 
2. Die vollständige Marktöffnung ist in der Richtlinie in absehbarer Zeit nicht geplant. Es soll schrittweise für definierte Großverbraucher eine Marktöffnung erreicht werden.

3. Die bisherigen, häufig vollständig integrierten und meist mit Monopolen ausgestatteten Unternehmen in der Elektrizitätsversorgung werden nicht aufgelöst. Sie müssen lediglich eine getrennte Buchführung einrichten.

4. Die bestehenden Netzbetreiber haben die Möglichkeit den Netzzugang zu verweigern, wenn ihre Kapazitäten nicht ausreichen.

5. Die Richtlinie gibt den Mitgliedsstaaten vielfältige Möglichkeiten, ihre energiepolitischen Vorstellungen in der Elektrizitätswirtschaft umzusetzen.

6. Das gewählte Verfahren der Marktöffnung kann dazu führen, das ähnliche Betriebe eines Unternehmens in dem einen Mitgliedsstaat marktzugangsberechtigt sind und in dem anderen nicht.

7. Die zu schaffenden Regulierungsstellen und Instrumenmte sind bei der erforderlichen Informationsbeschaffung vollständig auf die Elektrizitätsversorgungsunternehmen angewiesen.

8. Die Mitgliedsstaaten können den EVU gemeinwirtschaftliche Verpflichtungen im Allgemeininteresse auferlegen. Hierfür werden diese Unternehmen eine Kompensation fordern und auch erhalten.

Es wird somit durch die Richtlinie kein einheitlicher europäischer Binnenmarkt für Elektrizität geschaffen und die Rahmenbedingungen für die Volkswirtschaften nicht angeglichen. Unter dem Deckmantel der Subsidarität werden die bestehenden einzelstaatlichen Strukturen fortgeschrieben und ein grenzüberschreitender Handel mit Elektrizität weitgehend unterbunden. Damit ist eine Chance vertan worden, die nationale Kleinstaaterei im Energiebereich durch die Richtlinie zu überwinden. Es bleibt zu hoffen, daß die Elektrizitätswirtschaft unter diesen Bedingungen in der europäischen Zukunft weiterhin ein Ausnahmebereich bleiben und nicht zum Modell für andere Wirtschaftsbereiche wird. 
Rüdiger Hermann - 978-3-631-75129-9

Downloaded from PubFactory at 01/11/2019 07:57:52AM

via free access 


\section{Literaturverzeichnis}

ALCHIAN, A. A. : Information Costs, Pricing and Resource Unemployment, in: PHELPS, E.S. et al. : Microeconomic Foundations of Employment and Inflation Theory, London 1970

ARBEITSGEMEINSCHAFT REGIONALER ENERGIEVERSORGUNGSUNTERNEHMEN - ARE-e.V. : Tätigkeitsbericht 1986-1987, Hannover 1988

AVERCH, H. ; JOHNSON, L. : Behavior of the Firm under Regulatory Contraint, in: American Economic Review, Vol. 52, 1962, S. 1053-1069

BACKWINKEL, R. ; FREUND, H. ; SEELE, W. : Batteriespeicher zur Spannungsstützung in Niederspannungsnetzen, in: Elektrizitätswirtschaft, Jg. 91 (1992), Heft 15, S. $975-981$

BACKWINKEL, R. ; FREUND, H. ; GRETSCH, R. ; KAUFMANN, W. ;

SCHMIDT, G. ; SEELE, W. ; WEISSMÜLLER, G. : Entwicklung, Errichtung und Erprobung eines Niederspannungsnetzstützpunktes mit NaS-Batterien zur Spannungsstützung, Abschlußbericht zum BMFT-Forschungsvorhaben, Essen, Februar 1991

BAILEY, E.E. : Economic Theory of Regulatory Constraint, Lexington 1973

BAILEY, E.E. ; COLEMANN, R.D. : The Effect of Lagged Regulation in an AverchJohnson Model, in: Bell Journal of Economics, Vol. 2 (1971), S. 278-292

BARKER, J.M. Jr. : Electric Energy Brokering: An Explanation and Status Report, in: Public Utility Fortnightly, Februar 1982, S. 28-36

BARON, D. P. : Design of Regulatory Mechanisms and Institutions, in: SCHMALENSEE, R. ; WILLIG, R.D. : Handbook of Industrial Organisation, Vol. 2, Amsterdam 1989, S. 1346-1447

BARTELS, R. ; COHEN, R. ; HOEHN, T. : Das Elektrizitätssystem in Großbritannien: Erste Erfahrungen und Perspektiven, in: Zeitschrift furr Energiewirtschaft, Heft 1 (1991), S. 26-27

BASSELER, U. ; HEINRICH, J. ; KOCH, W. : Grundlagen und Probleme der Volkswirtschaft, 13. Auflage, Köln 1991

BAUER, J.F. : Die Europäische Gemeinschaft und das Recht der leitungsgebundenen Energie, Baden-Baden 1993

BAUMOL, W.J. : Contestable Markets: An Urprising in the Theory of the Industry Structure, in: American Economic Review, Vol. 72 (1982), S. 1-15

BAUMOL, W.J. ; PANZAR, J.C. ; WILLIG, R.D. : Contestable Markets and the Theory of Industry Structure, New York 1982

BAUMOL, W.J. ; PANZAR, J.C. ; WILLIG, R.D. : Contestable Markets and the Theory of Industry Structure, erweiterte Fassung, San Diego 1988

BAUMOL, W.J. : Determinants of Industry Structure and Contestable Markets Theory, in: GREENAWAY, D. ; BLEANEY, M.F. : Companion to Contemporary Economic Thought, London 1991, S. 507-522 
BELKHOFER, H. ; JAKOB, K. ; SACHS, U. : Planung clektrischer Netze mit Sincal, in: Elektrizitätswirtschaft, Jg. 91 (1992), Heft 22, S. 1444-1455

BERGBACKER, F. ; WESSEL, T. : Die Energiewirtschaft im gemeinsamen Markt, Baden-Baden 1963

BERGER, T. (Hrsg.): Finanzlexikon, München 1971

BETLING, T. : Blockhcizkraftwerke, Karlsruhe 1991

BIERHOFF, R. : Ausblick auf die künftige elektrizitätswirtschaftliche Zusammenarbeit, in: Elcktrizitätswirtschaft, Jg. 93 (1994), Heft 13, S. 744-748.

BITTERER, R. : VDEW-Symposium „Strompreise in einem veränderten Markt“, in: Elcktrizitätswirtschaft, Jg. 95 (1996), Heft 4, S. 154-156

BOADWAY, R. : The Role of Second-Best Theory in Public Economics, Kingston (Ontario) 1994

BÖRNER, B. : Frankreichstrom nach Deutschland?, Baden-Baden 1990

BÖRNER, B. (Hrsg.) : Reform des Energierechts und Natur der Sache, Düsseldorf 1971

BÖWING, H. ; DEHMER, R. ; DIENES, K. : EG und Internationales, in: Elektrizitätswirtschaftliche Tagesfragen, 42. Jg. (1992), Heft 7, S. 482

BOITEUX, M. : Die französischen Arbeiten über die Strompreisbildung nach Grenzkosten, in: BLANKART, B. ; FABER, M. (Hrsg.) : Regulierung öffentlicher Unternehmen, Königstein 1992, S. 78-90

BOITEUX, M. : La tarification des demandes en pointe: Application de la theorie de la vente au cout marginal, in: Reveue General de l'Electricité , Bd. 58, 1963, S. 321340

BOLLE, F. : Wettbewerb und Kooperation in der Elektrizitätswirtschaft, Schriftenreihe des Energiewirtschaftlichen Institutes der Universität Köln, Nr. 39, Köln 1990

BORCHERT, M. ; GROSSEKETTLER, H. : Preis- und Wettbewerbstheorie: Marktprozesse als analytisches Problem und ordnungspolitische Gestaltungsaufgabe, Stuttgart 1985

BOULDING, P.G. : Die Preisentwicklung in der britischen Elektrizitätswirtschaft, in: Zeitschrift für Energiewirtschaft, Heft 4 (1995), S. 295-304

BRAATZ, U. : Elektrizität ohne Grenzen - XXII. UNIPEDE Kongreß in Kopenhagen, in: Elektrizitätswirtschaft, Jg. 90 (1991), Heft 16 u. 17, S. 907-910

BRAATZ, U. : „Power to Progress“ - Die Zukunft meistern - XXIII. Unipede Kongreß 1994 in Birmingham, in: Elektrizitätswirtschaft, Jg. 93 (1994), Heft 20, S. $1182-$ 1184

BRANDMAIR, R. : Erfahrungen mit der 96-Stunden-Leistungsmessung, in: Elektrizitätswirtschaft, Jg. 94 (1995), Heft 5, S. 233-238

BRAUN, D. : Reform des Konzessionsabgabewesens, in: Elektrizitätswirtschaftliche Tagesfragen, 41. Jg. (1991), Heft 7, S. 456-467

BRINKMANN, K. : Einführung in die elektrische Energiewirtschaft, Braunschweig 1971

BRUCHE, G. : Elektrizitätsversorgung und Staatsfunktion, Frankfurt/Main 1977

BUDDE, H.-J. : „Wettbewerb überfällig“, in: ENERGIE SPEKTRUM, Mai 1994, S. 2433 
BUDERATH, J. : Dic Ordnungspolitik in der deutschen Elcktrizitätswirtschaft, Bad Homburg 1962

BÜTTGENBACH, S. B. : Erweiterter Einsatz der Spannungs-Blindleistungs-Optimierung im Verbundsystem, Aachen 1992

CARLTON, D.W. : The Theory and Facts of How Markets Clear: Is Industrial Organisation Valuable for Understanding Microeconomics?, in: SCHMALENSEE, R. : WILLIG, R.D. (Hrsg.) : Handbook of Industrial Organisation, Vol. 2, Amsterdam 1989, S. 909-947

COASE, R.H. : The Nature of Firm Economic, nachgedruckt von STIGLER, G.J. : BOULDING, K.E. (Hrsg.) : Readings in Pricc Theory, Homewood 1994

COLLIER, J.G. : 1994 Starting A New Era for Nuclear Power (überarbeitete Fassung eines Vortrages auf der Jahrestagung des Deutschen Atomforums e.V. am 26.01.94 in Bonn) abgedruckt in: atomwirtschaft, März 1994, S. 183-188

COPELAND, B.R. : Strategic Interaction Among Nations: Negotiable and Non-negotiable Trade Barriers, in: Canadian Journal of Economics, Vol. 23 (1990), Nr.1, S. 84108

COTTERELL, J.W. ; HEINRICH, D.O. : Stromwirtschaftliche Zusammenarbeit in England, in: Recht der Elektrizitätswirtschaft , Heft 9, 1985, S.170-178

CREW, M. ; KLEINDORFER, P. : The Economics of Public Utility Regulation, London 1986

CRONENBERG, M. : Reform der Konzessionsabgaben, in: Elektrizitätswirtschaftliche Tagesfragen, 41. Jg. (1991), Heft 7, S. 450-454

DEMOUGIN, D. ; SINN, H.-W. : Privatisation, Risk-taking and the Communist Firm, in: Journal of Public Economics, Jg. 55 (1995), S. 203-231

DEMSETZ, H. : Why Regulate Utilities? Journal of Law and Economies, Bd. 11, 1968, S. $55-66$

DEMSETZ, H. : Barriers of Entry, in: American Economic Review, Vol. 72 (1982), S. 47-57

DEPARTMENT of ENERGY : Electricity Privatisation, London 1988

DEREGULIERUNGSKOMMISSION : Markt und Wettbewerb in der Stromwirtschaft, Bericht der Deregulierungskommission, Sonderdruck, Stuttgart 1991

DRILLISCH, J. : Der Einfluß wettbewerblicher Strukturen in der US-Elektrizitätswirtschaft auf das Demand-Side Management, in: Zeitschrift für Energiewirtschaft, Heft 1 (1996), S. 37-52

DTV : Atlas zur Weltgeschichte, Bd. 2, 8. Auflage, München 1983

DÜWALL, P. ; LANGE-HÜLSKEN, M. ; ZYBELL, G. : Lastganglinien der Haushalte Ergebnisse und Schlußfolgerungen der Studie „Ermittlung der Lastganglinien bei der Benutzung elektrischer Energie durch die bundesdeutschen Haushalte während eines Jahres, in: Elektrizitätswirtschaft, Jg. 84 (1985) , Heft 25, S. 1051-1064

DVG : Daten aus der Verbundwirtschaft der Bundesrepublik Deutschland, Heidelberg 1993

DVG : Jahresbericht 1993, Heidelberg 1994

DVG : Jahresbericht 1992, Heidelberg 1993 
DVG : Jahresbericht 1990, Hcidelbcrg 1991

DVG : Stellungnahme der deutschen Elektrizitätswirtschaft zur Mitteilung der Kommission an Rat und Parlament: Die Ausweitung der innergemeinschaftlichen Stromlieferungen, ein grundlegender Beitrag zur Vollendung des Binnenmarktes für Energie, Heidelberg 06.08.1989

DVG : Die Stromversorgung in Deutschland, Heidelberg 1994

EDF : Entreprise nationale industrielle et comerciale, La documentation francaise, notes et études documentaires, Paris 1981

EISS, H. ; LUKES, R. : PICK, H. : SCHULZ, W. : Dic Ordnung des Elcktrizitätsmarktes in der Europäischen Gcmeinschaft, München 1990

ENEL : Jahresbericht 1985, Rom 1986

ENTE NAZIONALE PER L'ENERGIA ELETTRICA : I Prezzi del l'energia elettrica in Italia - in vista delmercato mico europeo, Rom 1991

ERNO, V.d' : Die italienische Energiewirtschaft - Entwicklung und Aussicht, in: Zeitschrift für Energiewirtschaft, Heft 1 (1990), S. 17-28

ESSER, C. : Umstrukturierung und Privatisierung der Elektrizitätswirtschaft in England und Wales, 2. korrigierte Auflage, Frankfurt 1991

ESSL, H. ; FELDMANN, W. ; RENZ, K. : ASC- der Schlüssel für bessere Nutzung elektrischer Netze, in: Elektrizitätswirtschaft, Jg. 93 (1994) , Heft 8, S. 396-399

FEDERAL ENERGY REGULATORY COMMISSION : The Transmission Task Forces Report to the Commission, Electricity Transmission: Realities, Theory and Policy Alternatives, London 1989

FEHL, U. ; SCHREITER, C. : Neuere Entwicklungen in der Preistheorie, in: Wirtschaftswissenschaftliches Studium, Heft 6, Juni 1993, S. 276-285

FIQUET, A. : Rechtlicher Rahmen der Elektrizitätswirtschaft in Frankreich, in: Elektrizitätswirtschaftliche Tagesfragen, 42. Jg. (1992), Heft 8, S. 554-557

FREUND, H. : Batteriespeicher in Netzen der Energieversorgung , Brigton 1989

FREUND, H. ; ROSENOW, V. ; WOLFF, H.-P. : Ein erweitertes (n-1) - Planungsprinzip für vermaschte Hochspannungsnetze, in: Elektrizitätswirtschaft, Jg. 90 (1991), Heft 13, S. 747-752

FRIEDMAN, D. ; OSTROY, J. : Competitivity in auction markets: An experimental and Theoretical Investigation, in: The Economic Journal, Vol. 95 (1995), S. 22-53

FUDENBERG, D. ; TIROLE, J. : Noncooperative game theory for industrial organisation: An introduction and overview, in: SCHMALENSEE, R. ; WILLIG, R.D. : Handbook of Industrial Organisation, Vol. 1, Amsterdam 1989, S. 260-327

GILBERT, R. J. : Mobility barriers and the value of incumbency, in: SCHMALENSEE, R. ; WILLIG, R.D. : Handbook of Industrial Organisation, Vol. 1, Amsterdam 1989 , S. $260-327$

GILSBACH, A. : Die Konzeption der französischen Energiepolitik, München 1965

GINOCCHIO, R. : Législation de l'éctricité, Paris 1977

GRAWE, J. : Der Kommissionsentwurf für eine EG-Richtlinie "Elektrizität" aus der Sicht der deutschen Elektrizitätswirtschaft, in: Elektrizitätswirtschaft, Jg.91 (1992), Heft 14 , S. 880-886 
GRAWE, J. : Encrgierechtliche Rahmenbedingungen für Energiedienstleistungsmärkte, in: Elektrizitätswirtschaft, Jg. 94 (1995), Heft 1 u. 2 , S. 9-10

GRAWE, J. : Ein gemeinsamer Strommarkt in der EG aus der Sicht der Energieversorgungsunternehmen, in : LUKES, R. (Hrsg.) : Ein EWG- Binnenmarkt für Elektrizität: Realität oder Utopie; Vorträge und Diskussionsberichte anläßlich einer wissenschaftlichen Tagung in Münster/W. am 29./30. Oktober 1987, Köln, Berlin, Bonn, München 1988, S. 165-175

GRAWE, J. : Die Rolle des Stroms zur Optimierung der Energiebedarfsdeckung, in: Elektrizitätswirtschaft, 91. Jg. (1992), Heft 18, S. 1139-1151

GREEN, R. ; NEWBERY, D.M. : Competition in the British Electricity Spot Market, Diskussionspapicr des Centre for Economic Policy Research, Nr. 557, London 1991

GREENAWAY, D. ; BLEANEY, M.F. : Companion to Contemporary Economic Thought, London 1992

GRONER, H. : Energieversorgungskonzepte als Problem der Wirtschaftspolitik, in: Zeitschrift für Energiewirtschaft, Heft 3 (1985), S. 171-175

GRONER, H. : Energy policy between competition and control, in: TIETZEL, M. (Hrsg.) : Oilshock - Five years later, Göttingen 1979, S. 189-211

GRONER, H. : Grundzüge einer Wettbewerbsordnung für dic Elektrizitätswirtschaft, in: ROPER, B. (Hrsg.): Wettbewerbsprobleme der Elektrizitätswirtschaft - Der Begriff Monopolgrad, Berlin 1972, S. 47-69

GRONER, H. : Energiepolitische Konzeptionen zwischen Wettbewerb und Lenkung, in: Energiewirtschaft, Heft 2 (1978), S. 91-98

GRONER, H. : Die Ordnung der deutschen Elektrizitätswirtschaft, Baden-Baden 1975

GRONER, H. : Property Rights und staatlich regulierte Industrien, in: SCHÜLLER, A.(Hrsg.) : Property Rights und ökonomische Theorien, München 1983, S. 219239

GRÖNER, H. : Überlegungen zum Fortbestand geschlosener Versorgungsgebiete in einem EG-Binnenmarkt für Elektrizität, in: Zeitschrift für Energiewirtschaft, Heft 1 (1989), S. 22-25

GRÖNER, H. : Die Vollendung des EG-Binnenmarktes und die Elcktrizitätsversorgung, in: DÜRR, E. ; SIEBER, H. (Hrsg.) : Weltwirtschaft im Wandel - Festgabe für Egon Tuchtfeldt zum 65. Geburtstag , Bern Stuttgart 1988, S. 359-376

GRÖNER, H. : Wettbewerb in der Elektrizitätsversorgung, in: Energiewirtschaft, Heft 2 (1979), S. 112-117

GRONNER, H. ; SCHÜLLER, A. (Hrsg.) : Die europäische Integration als ordnungspolitische Aufgabe, Stuttgart, Jena, New York 1993

GRÜNDLING, F-J. ; PICK, H. : Die europäische Energiewirtschaft vor dem Binnenmarkt, in: Zeitschrift furr Energiewirtschaft, Heft 1 (1990), S. 1-15

HAGENMEYER, E. : Elektrizitätswirtschaft an der Jahrhundertwende - europäischer Wettbewerb kontra lokale Partnerschaft?, in: Elektrizitätswirtschaft, Jg. 94 (1995), Heft 20, S. 1267-1271 
HAGENMEYER, E. : Stromversorgung in Europa, in: atomwirtschaft, Juli 1993, S. 508516

HANCHER, L. : Dic Almelo-Entscheidung des Europäischen Gerichtshofs - Hintergrund, Bewertung, mögliche Auswirkungen, in: Elektrizitätswirtschaft, 45. Jg. (1995), Heft 1 u.2, S. 68-76

HANSELMANN, M. ; ALBIGER, J. ; KALTSCHMITT, M. : Mittelfristige Kraftwerkseinsatzplanung - Problemstellung und Lösungsansätze, in: Elektrizitätswirtschaft, Jg. 95 (1996), Heft 4, S. 204-209

HARMS, W. : Wettbewerb in der leitungsgebundenen Energiewirtschaft, Zeitschrift für Energiewirtschaft, Heft 1 (1989), S. 13-21

HARPOLT, H. ; OEDING, G. : Elcktrische Kraftwerke und Netze, 5. Auflage, Berlin 1978

HARTMANN, U. : Anmerkungen zur Energiepolitik, in: Elektrizitätswirtschaft, Jg. 94 (1995), Heft 3, S. 89-90

HAUBRICH, H.-J. : Neue Ordnungsrahmen und Systemfuihrungskonzepte in der Elektrizitätswirtschaft, in: Elektrizitätswirtschaftliche Tagesfragen, 43. Jg. (1993), Heft 10, S. 662

HAVER, R. : Versunkene Kosten - Zur Funktionsweise der unsichtbaren Hand, Freiburg 1990

HAYEK, F.A. von : Der Wettbewerb als Entdeckungsverfahren, in ders. : Freiburger Studien, Tübingen 1969

HEDTKAMP, G. : Lehrbuch der Finanzwissenschaft, 2. Auflage, Neuwied 1977

HEINEMANN, W.-R. : Das englische Elektrizitätssystem - Eine Zwischenbilanz, in: Elektrizitätswirtschaft, Jg. 93 (1994), Heft 1 u. 2, S. 12-15

HEINZ, D. : Kostenminimaler Wirk- und Blindleistungshaushalt in Hoch- und Höchstspannungsnetzen in: Elektrizitätswirtschaft, Jg. 93 (1994), Heft 7, S. 313-318

HELFRISCH, F. : EU-Binnenmarkt für Strom aus Verbrauchersicht, in: Elektrizitäts-wirtschaftliche Tagesfragen, 44. Jg. (1994), Heft 1 u.2, S. 26-29

HELM, D.R. ; YARROW, G. : The Regulation of Utilities, Oxford 1988

HENNEY, A. : A Study of the Electricity Industry in England and Wales, London 1994

HENSING, I. : Die Perspektiven von Kernenergie in wettbewerblich geöffneten Elektrizitätsmärkten, in. Zeitschrift für Energiewirtschaft, Heft 1 (1996), S. 91-92

HERPPICH, W. : Least-Cost Planning in den USA: ein Vorbild für Europa?, in: Elektrizitätswirtschaft, Jg. 95 (1996), Heft 9, S. 542-544

HEUK, K. : Vorlesungsskript für den Studienzweig Elektrotechnik der Universität der Bundeswehr Hamburg

HILSCHER, U. ; LAUBSCHER, H.-D. : Finanzierungskosten, Frankfurt/M. 1976

HOEK, van G.A.L. : Europa im Stromverbund - Vortrag anläßlich des VDEW-Sym-posiums furr Wirtschaftsjournalisten "Perspektiven der Stromversorgung" vom 25. bis 27.11.1991, Veröffentlichung der VDEW, Frankfurt 1992

HOLMES, A. : Twisting by the Pool, in: Energy Economist, Juli 1991, Heft 17, S. 7-13 
HOLZER, J. : Energiewirtschaftliche Perspektiven in Mittel- und Osteuropa aus der Sicht der Elektrizitätsversorgung, Köln 1992

HÜFFER, U. ; TETTINGER, J. : Rechtsfragen beim Versorgenwechsel nach Ablauf von Konzessionsverträgen, Stuttgart, München, Hannover, Berlin 1992

IEA : Natural Gas Transportation and Regulation, Paris 1994

INFORMATIONSZENTRALE DER ELEKTRIZITÄTSWIRTSCHAFT c.V. (Hrsg.) : StromBASISWISSEN : Stromerzeugung in Wärmekraftwerken. Nr. 105, S. 1-4 Frankfurt/M. 1988

INSTITUT FÜR BILANZANALYSEN : Dic Elcktrizitätswirtschaft in der Bundes-republik Deutschland, Würzburg 1979

JANSSEN, R. : Niederspannungsnetze, in: Elcktrizitätswirtschaft, 92. Jg. (1993), Heft 21, S. $1283-1289$

JOSKOW, P.L. : Vertical integration and long-term contracts: The case of coal burning electric generating plants, in: Journal of Law, Economics and Organisation, Heft 1, 1985 , S. 33-80

JOSKOW, P.L. ; ROSE, N. : The Effects of Economic Regulation, in: SCHMALENSEE, R. ; WILLIG, R.D. : Handbook of Industrial Organisation, Vol. 2, Amsterdam 1989, S. 1450-1506

JÜNGST, H. : Umweltschutz und Leitungsbau - ein zwangsläufiger Konflikt?, in: Elektrizitätswirtschaft, Jg. 91(1992), Heft 23, S. 1514-1519

KAAS, K.P. : Marketing als Bewältigung von Informations- und Unsicherheitsproblemen im Markt, in: Die Betriebswirtschaft, 50. Jg. (1990), S. 539-548

KAAS, K.P. ; FISCHER, M. : Der Transaktionskostenansatz in: Wirtschaftswissenschaftliches Studium, Heft 8 u. 9, 1993, S. 686-693

KAHN, A.E. : The Economics of Regulation, Vol. 1, New York 1970

KETTERER, H. : Global-Link und Interkontinentaler Energieverbund, in: Elektrizitätswirtschaft, Jg. 94 (1995), S. 192-196

KETTERER, H. : Stromspeicher für die Energieversorgung, in: Elektrizitätswirtschaft, Jg. 94 (1995), Heft 14, S. 842-845

KLOPFER, T. : Die Elektrizitätswirtschaft in England und Wales - Zwischenbilanz, in: Zeitschrift für Energiewirtschaft, Heft 4 (1993), S. 259-290

KLOPFER, T. : Märkte für Strom - Internationale Erfahrungen und Übertragbarkeit auf Deutschland, München 1993

KLOPFER, T. ; HENSING, I. ; RIECHMANN, C. ; SCHULZ, W. ; WERNER, O. : Bewertung von Deregulierungsmodellen, in: Zeitschrift für Energiewirtschaft, Heft 1 (1996), S. 91-92

KOBARG, B. : Elektrizitätswirtschaft und Wettbewerb, Kiel 1973

KOEPPEL, P. F. : Typische Verträge der Energiewirtschaft in kartellrechtlicher Betrachtung, Köln 1959

KOMMISSION DER EUROPÄISCHEN GEMEINSCHAFT : Bulletin der EG 11, 1992

KOMMISSION DER EUROPÄISCHEN GEMEINSCHAFT : Bulletin der EG 1/2, 1992

KOMMISSION DER EUROPÄISCHEN GEMEINSCHAFT : Bulletin der EG, Beilage $2 / 86$, S. 5 
KOMMISSION DER EUROPÄISCHEN GEMEINSCHAFT : EG-Nachrichten - Berichte und Informationen, Nr.17 vom 29.04.1991, S.6

KOMMISSION DER EUROPÄISCHEN GEMEINSCHAFT - Generaldirektion Energie : Energie in Europa - Der Binnenmarkt für Energie, Luxemburg 1988

KOMMISSION DER EUROPÄISCHEN GEMEINSCHAFT : Richtlinie 90/547/EWG des Rates vom 29.10.1990 über den Transit von Elektrizitätslieferungen über große Netze in: Amtsblatt der Europäischen Gemeinsschaften Nr. L 313 vom 13.11.1990

KOMMISSION DER EUROPÄISCHEN GEMEINSCHAFT : Richtlinic 90/377/EWG des Rates vom 29. Juni 1990 zur Einführung eines gemeinschaftlichen Verfahrens zur Gewährleistung der Transparenz der vom industricllen Endvcrbraucher zu zahlenden Gas- und Strompreise, in : Amtsblatt der Europäischen Gemeinschaften Nr. L 185 vom 17.7.1990

KOMMISȘION DER EUROPÄISCHEN GEMEINSCHAFT : Richtlinie 96/92/EG des europäischen Parlaments und des Rates vom 19. Dezember 1996 betreffend gemeinsame Vorschriften für den Elektrizitätsbinnenmarkt, in: Amtsblatt der Europäischen Gemeinschaften Nr. L 27 vom 31.01.97

KOMMISSION DER EUROPÄISCHEN GEMEINSCHAFT - Generaldirektion Energie : Vollendung des Binnenmarktes für Gas und Elektrizität, Brüssel 15.07.1991

KOMMISSION DER EUROPÄISCHEN UNION : Energy in Europe, 1994 Annual Energy Review, Luxemburg 1995

KOOPMANN, T. : Three Essays of the State of Economic Science; New York 1957

KRAMER, H. : Nuklearpolitik in Westeuropa und die Forschungsaufgaben der Euratom, Köln 1975

KRISTOF, K. : Dezentralisierung in der Elektrizitätswirtschaft, Frankfurt/Main, New York 1992

LAMBRECHT, F. : Wettbewerb und Regulierung in der britischen Elektrizitätswirtschaft, in: Elektrizitätswirtschaftliche Tagesfragen, 42. Jg. (1992), Heft 11, S. 775-779

LAMBRECHT, F. : Transmission and Distribution in Liberalized Power Markets, in: Elektrizitätswirtschaftliche Tagesfragen, 45. Jg. (1995), Heft 1 u. 2, S. 105

LANE, D. : ENEL to market, in: Energy Economist, September 1993, S. 15-19

LANG, G. : Die EG-Richtlinie zur Strompreistransparenz, in: Elektrizitätswirtschaft, Jg. 89 (1990), Heft 24, S. 1357-1363

LANG, G. : EG Strompreise 1992 im Vergleich, in: Elektrizitätswirtschaftliche Tagesfragen, 41. Jg. (1992), Heft 10, S. 693-697

LANG, G. : Spielball der Wechselkurse? Strompreise im internationalen Vergleich, in: ENERGIE, Jg. 39, Nr. 4, April 1987, S. 47-50

LANG, G. : Die Strompreisentwicklung in England und Wales seit der Privatisierung, in: Elektrizitätswirtschaft, Jg. 91 (1992), Heft 21, S. 1385-1386

LANG, G. : Das Strompreisniveau in den EG-Ländern 1991, in : Elektrizitätswirtschaftliche Tagesfragen 41. Jg. (1991), Heft 10, S. 672-677 
LANGBEIN, G. : Stromimport und EG-Niederlassungsrecht - Aufriß der Problemstellungen, in: Elcktrizitätswirtschaftliche Tagesfragen, 37. Jg. (1987), Heft 11, S. 941944

LEDERER, P. ; BOUTTES, J.P. : Elektrizitätsmonopol kontra Wettbewcrb. in: Elcktrizitätswirtschaft, Jg. 90 (1991), Heft 6, S. 224-230

LEFTWICH, R.H. : The Price System and Ressource Allocation, 6. Auflage, Heimsdale (Illinos) 1976

LEWINGTON, I. ; WEISHEIMER, M. : Zur Regulicrung in der deutschen Elektrizitätswirtschaft - Einc Analyse anhand neuer ökonomischer Ansätze und praktischer Erfahrungen, in: Zeitschrift furr Energiewirtschaft, Heft 4 (1995), S. 277-304

LICHTENBERG, H. : Der europäische Stromverbund, in: LUKES, R. (Hrsg.) : Ein EWG-Binnenmarkt für Elektrizität: Realität oder Utopie; Vorträge und Diskussionsberichte anläßlich einer wissenschaftlichen Tagung in Münster/W. am 29./30. Oktober 1987, Köln, Berlin, Bonn, München 1988, S.86-108

LIPSEY, R.G. : Einführung in die Positive Ökonomie, Köln 1971, S. 373-375

LIPSEY, R.G. ; LANCASTER, K. : The General Theory of Second Best, in: Review of Economic Studies, Vol. 24 (1956), S. 11-32

LOTTERMANN, T. : Preisentwicklung im englischen Elektrizitätssystem, in: Elektrizitätswirtschaft, Jg. 94 (1995), Heft 3, S. 93-98

MAGERL, H. : Elektrizitätswirtschaftliche Tagesfragen, in: Elektrizitätswirtschaft, Jg. 92 (1993), Heft 14, S. 867-869

MANSFELD, L. : Gebietsmonopole für Elektrizitätsversorger?, in: technologie \& management, Heft 1 (1989), S. 29-37

MARTIN, J.M. : Die französische Energiewirtschaft - Entwicklung und Perspektiven, in: Zeitschrift für Energiewirtschaft, Heft 3 (1990), S. 224-237

MATHIS, C. : Vorschläge für eine Neuorientierung der Elektrizitätswirtschaft in Frankreich - Der „Rapport Mandil“, in: Elektrizitätswirtschaftliche Tagesfragen, 45. Jg. (1995), Heft 1 u.2 , S. 54-58

MEIER, K. : Spitzenlasttarifierung, ökonomische Effizienz und Erhaltung der Eigenwirtschaftlichkeit - Eine angewandte Studie der elektrizitätswirtschaftlichen Preisbildung, Bern, Stuttgart 1983

MEISTER, H. : Elektrotechnische Grundlagen, 8. Auflage, Würzburg 1986

MEYER, H. : Unipede Tarifkonferenz in Lausanne, in: Elektrizitätswirtschaft, Jg. 91 (1992), Heft 26, S. 1687-1692

MICHAELIS, H. : Gesamtwirtschaftliche Aspekte, Ein EWG-Binnenmarkt für Elektrizität, in: Elektrizitätswirtschaftliche Tagesfragen, 37. Jg. (1987), Heft 16 u. 17, S. $1029-1033$

MICHAELIS, H. : Der Weg zu einem europäischen Binnenmarkt für Energie, in: Energiewirtschaftliche Tagesfragen, 46. Jg. (1996), Heft 4, S. 214-217

MIKSCH, L. : Wettbewerb als Aufgabe, Bad Godesberg 1947

MONIG, W. ; SCHMITT, D. ; SCHNEIDER, H.K. ; SCHÜRMANN, J. : Konzentration und Wettbewerb in der Energiewirtschaft, München 1977 
MÜLLENDORF, R. : Dic französische Elektrizitätswirtschaft und der europäische Stromhandel, in: Glückauf 125 (1989), Nr. 21 u. 22, S. 1351-1355

MÜLLER, J. ; VOGELSANG, I. : Staatliche Regulierung, Baden-Baden 1978

MÜLLER, L. : Network Ost, in: ENERGIE SPEKTRUM, April 1993, S. 30-38

NATIONAL WESTMINSTER BANK : Privatising Electricity in the United Kingdom Problems in Storc, Quartcrly Review, November 1988

NIJKAMP, P. ; PERRELS, A. : Impacts of Electricity Rates on Industrial Location, in: Energy Economics, April 1988, S. 107-116

NOLL, R.G. : Economic Perspectives on the Politics of Regulation, in: SCHMALENSEE, R. ; WILLIG, R.D. : Handbook of Industrial Organisation, Vol. 2, Amsterdam 1989, S. 1252-1287

NORDEL : Jahresstatistik, Kopenhagen 1990

O.V. : Any Offers?, in:Energy Economist, März 1994, S. 20-21

O.V. : EG und Internationales, in: Elektrizitätswirtschaftliche Tagesfragen, 42. Jg. (1992), Heft 8, S. 558

O.V. : EG und Internationales, in: Elektrizitätswirtschaftliche Tagesfragen, 42. Jg.(1992), Heft 10, S. 712

O.V. : EG und Internationales - Anhörung zu Strom- und Gas-Richtlinienvorschlägen, in: Elektrizitätswirtschaftliche Tagesfragen, 42. Jg. (1992), Heft 12, S. 850

O.V. : EU und Internationales: Die Europäische Kommission zur Vereinbarkeit des französischen Single-Buyer-Vorschlages mit dem Kommissionsvorschlag des verhandelten Netzzuganges, in: Elektrizitätswirtschaftliche Tagesfragen, 45. Jg. (1995), Heft 5, S. 324

O.V. : Energiewirtschaft - BMWi für Liberalisierung des Ordnungsrahmens, in: Elektrizitätswirtschaftliche Tagesfragen, 42. Jg. (1992), Heft12, S. 859

O.V. : Energiewirtschaft und Umweltschutz im EG-Binnenmarkt - Interview mit Dr. Dietmar Kuhnt in: Elektrizitätswirtschaftliche Tagesfragen, 42. Jg. (1992), Heft 6, S. 346-350

O.V. : „Es sei denn höhere Gewalt" - EdF, Badenwerk und Cattenom, in: ENERGIE SPEKTRUM, November 1989, S. 65-68

O.V. : Jahrbuch der Atomwirtschaft, Düsseldorf 1993

O.V. : (Elektrizitätswirtschaftliche Tagesfragen-Redaktion) - Kompromißsuche hält an, in: Elektrizitätswirtschaftliche Tagesfragen, 42. Jg. (1992), Heft 8, S. 500

O.V. : Liberalisierung der Energiemärkte umstritten, in: Elektrizitätswirtschaftliche Tagesfragen, 42. Jg. (1992), Heft 7, S. 491

O.V. : „Nachrichten“, in: Elektrizitätswirtschaftliche Tagesfragen, 43.Jg. (1993), Heft 7, S. 486

O.V. : Finnland: Start der Strombörse im Juni, in: Energiewirtschaftliche Tagesfragen, 46. Jg. (1996), Heft 4, S. 261

O.V. : Für ungeteilten Strom-Wettbewerb, in: Elektrizitätswirtschaftliche Tagesfragen, 44. Jg. (1994), Heft 4, S. 256

O.V. : That curious UK electricity pool price, in: Energy Economist, Februar 1991, S. 9- 
O.V. : Überprüfung der Strompreisstrukturen - Beschluß der Wirtschaftsministerkonferenz (WMK) am 7. Oktober 1987, in: Elektrizitätswirtschaft, Jg. 86 (1987) , Heft 23, S. 994-1005

O.V. : Feilschen um mehr Wettbewerb, in: ENEGIE SPEKTRUM, Januar 1994, S. 8

PAFFENBERGER, W. ; SCHEELE, U. : Kosten und Nutzen von Stromhandel in Europa, Diskussionsbcitrag Nr. V-61-90 der Universität Oldenburg, Oldenburg August 1990

PERRIDON, L. ; STEINER, M. : Finanzwirtschaft der Unternchmung, 3. Auflage, München 1984

PERRY, M.K. : Vertical integration: The monopsony case, in: American Economic Review, Vol. 63 (1978), S. 561-570

PICK, H. : Ordnungsprobleme eines europäischen Binnenmarktes für Elcktrizität, Idstein 1991

PHELPS, E.S. et al. : Microeconomic Foundations of Employment and Inflation Theory, London 1970

PRIECKAERTS, J.M. : Regionale Energieversorgung der Niederlande - Entivicklung und Strukturwandel, in: Elektrizitätswirtschaftliche Tagesfragen, 41. Jg. (1991), S. 382-386

PIEHL, E. : CIRED-Sitzung 6: Entwurf und Planung öffentlicher Verteilungsnetze, in: Elektrizitätswirtschaft, Jg. 90 (1991), Heft 16/17, S. 939-943

PRITZSCHE, K. : EU-Liberalisierung - jüngste Entwicklungen, in: Energiewvirtschaftliche Tagesfragen, 46. Jg. (1996), Heft 4, S. 218

QUOILlIN, J. : Der Verkauf zu Grenzkosten bei der EdF, in: Analen der Gemeinwirtschaft, 45. Jg., Lüttich 1976, S. 129-155

RADZIO, H. : Die deutsche Stromwirtschaft blickt nach Osten, aber auch nach Westen, in: Elektrizitätswirtschaftliche Tagesfragen, 43. Jg. (1993), Heft 10, S. 654

RECKNAGEL, H. : Mehr Wettbewerb in der Energiewirtschaft?, in: Elcktrizitätswirtschaftliche Tagesfragen, 40. Jg. (1990), Heft 4, S. 220-225

RIECHMANN, C. : Price-Cap Regulierung, in: Zeitschrift für Energiewirtschaft, Heft 2 (1995), S. 157-167

RIEMER, H.W. : EG-Binnenmarkt - Technische Aspekte der Herausforderung der deutschen Elektrizitätswirtschaft, in: Elektrizitätswirtschaft, 91. Jg. (1992), Heft 17, S. 1075-1090

RIORDAN, M.H. : Uncertainty, asymmetric information and bilateral contracts, in: Review of Economic Studies, Vol. 51 (1984), S. 83-93

RUCHTI, H. : Die Bedeutung der Abschreibung für den Betrieb, Berlin 1942

SCHAVOIR, R. : Common Carriage: Geordneter Rückzug?, in: Elektrizitätswirt-schaftliche Tagesfragen 40. Jg. (1990), Heft 3, S. 109

SCHIFFER, H.-W. : Energiemarkt Bundesrepublik Deutschland, Köln 1988

SCHIFFER, H.-W. : Preisbildung in der Energiewirtschaft, in: Elektrizitätswirtschaftliche Tagesfragen, 41. Jg. (1991), Heft 12, S. 810-819

SCHIFFER, H.W. : Stabile Preise in Sicht, in: Elektrizitätswirtschaftliche Tagesfragen, Heft 6 (1996), S. 484- 494 
SCHMALENSEE, R. : A note on the theory of vertical integration, in: Journal of Political Economy, Vol. 81 (1973), S. 442-449

SCHMALENSEE, R. ; WILLIG, R.D. (Hrsg.) : Handbook of Industrial Organisation, Vol. 1, Amsterdam 1989

SCHMIDT, D. : Im Grundlastbereich könnte Importkohle mit Atomkraft schon fast konkurrieren, in: Handelsblatt, 19.05.1987

SCHMITT, F.J. : Europäische Stromversorgung auf neuen Wegen, in: Glückauf , Jg. 127 (1991), Nr. 5 u. 6, S. 222-225

SCHMITZ, E. : Dic Anwendung der Wettbewerbsregeln des EWG-Vertrages auf die Elektrizitätswirtschaft, in: Elektrizitätswirtschaft, Jg. 90 (1991), Heft 12, S. 650658

SCHNEIDER, R. : Elektrizität unter wettbewerbspolitischem Aspekt, Bamberg 1966

SCHOLL, R. : Reform der Elcktrizitätsindustric in Australien - Das Beispiel der Statc Elẹctricity Commission of Victoria, in: Elektrizitätswirtschaftliche Tagesfragen, 45. Jg. (1995), Heft 6, S. 386-392

SCHULZ, E. : Vom regulierungsorientierten zum kundenorientierten DSM, in: Elektrizitätswirtschaft, Jg. 94 (1995), Heft 1 u. 2, S. 65-66

SCHWEPPE, F.-C. : Spotpricing in Electricity, Boston 1988

SCHWEPPE, F.-C. : Economic Impediments to Power Transfer, in: Kelly, K. (Hrsg.): Non Technical Impediments to Power Transfer, Columbia, Ohio 1987, S.176-183

SCHWICKARDI, G. : Elektroenergietechnik, Bd. 2, Aarau 1979

SHAPIRO, C. : Theories of Oligopoly Behavior, in: SCHMALENSEE, R. ; WILLIG, R.D. : Handbook of Industrial Organisation, Vol. 1, Amsterdam 1989, S. 329-413

SIMON, H.A. : Administrative Behavior, New York 1961

SOLTWEDEL, H. et al : Deregulierungspotentiale in der Bundesrepublik, Tübingen 1986

SPANN, R.M. ; Rate-of-Return Regulation and Effiency in Production: An Empirical Test of the Averch-Johnson Thesis, in: Bell Journal of Economics and Management Science, Vol. 5 (1974), S. 38-54

STIGLER, G. : The Organization of Industry, Homewood 1968

STIGLER, G.J. ; BOULDING, K.E. (Hrsg.) : Readings in Price Theory, Homewood 1994

STOTZ, J. : Anbindung des Höchstspannungsnetzes der neuen Bundesländer an den westeuropäischen Verbund, in: Elektrizitätswirtschaft, Jg. 93 (1994), Heft 13, S. 715722

STRASSBURG, W. : Energie-, Umwelt- und Wettbewerbspolitik im Widerstreit, in: Elektrizitätswirtschaftliche Tagesfragen, 44. Jg. (1994), Heft 9, S. 592-595

STRASSBURG, W. : Stromverbund für Gesamteuropa, in: INFORMATIONSZENTRALE DER ELEKTRIZITÄTSWIRTSCHAFT e.V. (Hrsg.): StromThemen Extra, 8. Jg., Frankfurt 1991, S. 1-8

STRAUSS, L. : Osteuropa bereitet Anbindung ans Westnetz vor, in: INFORMATIONSZENTRALE DER ELEKTRIZITÄTSWIRTSCHAFT e.V. (Hrsg.): StromThemen 10. Jg. Nr. 5, Frankfurt 1993, S. 1-3 
STRAUSS, L. ; KARAS, P. : Dic Bedeutung der Gleichstromnetzkupplung EtzenrichtHradec für die Stromnetzc West- und Osteuropas, in: Elcktrizitätswirtschaft, Jg. 92 (1993), Hcft 12, S. 713-724

STREIT, M.E. : 13 Thesen zu einer marktprozeßoricntierten Wettbewcrbspolitik, in: Wirtschaftswissenschaftliches Studium, Heft 4, April 1993, S. 167-170

SWOBODA, P. : Kostcnermittlung und Tarifbildung bei Elcktrizitätsversorgungs-unternehmen insbesondere hinsichtlich kalkulatorischer Kosten, in: Journal für Betriebswirtschaft, Heft 2 (1990), S. 66-76

TOLKSDORF, M. : Dynamischer Wettbeiverb - Einführung in dic Grundlagen der deutschen und internationalen Wettbeiverbspolitik, Wicsbaden 1994

TUROWSKI, R. : Hohe Stromimportc in Däncmark, in: Elcktrizitätswirtschaft, Jg. 89 (1990), Heft 25, S. 1441-1442

UCPTE : Betrieb des westcuropäischen Verbundsystems, Arnhcim 1990

UCPTE : Jahresbericht 1993, Wien 1994

UNIPEDE : Fachberichte Synthesen, Kopenhagen 1991

VAHLENS Großes Wirtschaftslexikon, Bd. 1, A-K, 2. Auflage, München 1993

VDEW : Für europäische Kooperation - gegen Zwangsregulicrung durch Brüssel, Stellungsnahme vom 15.07.1991, Frankfurt/M. 1991

VDEW : Der europäische Strommarkt - Dokumentation der deutschen Elcktrizitäts-ivirtschaft, Frankfurt/M. 1988

VDEW-Arbeitskreis „Tarifgestaltung“: Tariflicher Bercitstellungsprcis - Möglichkeiten für eine Umstellung auf eine elektrische Bemessungsgröße bei Gewerbe und Landivirtschaft, in: Elektrizitätswirtschaft, Jg. 93 (1994), Heft 25, S. 1051-1073

VICKERS, J. ; YARROW, G. : The British clcctricity experiment, in: Economic policy A European Forum, Nr. 1, 1991, S. 187-232

VIK : „Durchleitung als Wettbewerbsinstrument in der Stromversorgung“, Essen 1990

VILIAN, M. : La politique de l'énergie en France, de la seconde guerre mondial a l'horizon 1985 , Paris 1969, S. 98-110

VOGELSANG, I. : Die staatliche Regulierung der amerikanischen Elektrizitätswirtschaft Eine kritische Bestandsaufnahme, in: Zeitschrift für Energicwirtschaft, Heft 1 (1979), S. 25-32 VOGT, H. : Probleme der Versorgungswirtschaft, München 1950

VORMBAUM, H. : Finanzierung der Betriebe, 7. Auflage, Wiesbaden 1986

WAGNER, G. : Abbau regionaler Strompreisdisparitäten, Studie der Bundesanstalt für Landeskunde und Raumordnung, Bonn 1985

WEISHEIMER, M. : Preise und Preisaufsicht im Ergebnis der Systemtransformation Erfahrungen der neuen Bundesländer, in: Elektrizitätswirtschaftliche Tagesfragen, 45. Jg. (1995), Heft 5, S. 284-289

WESSEL, T. : Wirtschaftliche und rechtliche Grundlagen der Energiewirtschaft, Tagungsberichte des Energiewirtschaftlichen Instituts der Universität Köln, Heft 1, Köln 1951

WEYMANN-JONES, T.G. : Electricity Privatisation, Aldershot-Brookfield, Hong Kong, Singapore, Sydney 1990 
WIEDSWANG, G. : Dic Liberalisierung des norwegischen Energiemarktes - Konse-quenzen für den internationalen Stromaustausch, in: Elcktrizitätswirtschaftliche Tagesfragen, 43. Jg. (1993), Heft 10, S. 678-687

WILLIAMSON, O.E. : Dic ökonomischen Institutionen des Kapitalismus, Tübingen 1990

WILLIAMSON, O.E. : Markets and Hicrachies. Analysis and Antitrust Implications, New York 1975

WILLIAMSON, O.E. : Transaction Cost Economics, in SCHMALENSEE, R. ; WILLIG, R.D. (Hrsg.): Handbook of Industrial Organisation, Vol. 1, Amsterdam 1989, S. 135-182

WÖHE, G. : Grundzügc der Unternehmensfinanzierung, 5. Auflage, München 1988

ZASK, J. : Exportkapazität der französischen Stromwirtschaft, in: HARMS, W. (Hrsg.) : Atomstrom aus Frankreich?, Beiträge zum Wirtschaftsrecht, Bd. 2, Köln, Berlin, Bonn, München 1987, S. 3-8

ZIJL, G. :.Geplanter Markt oder Monopol mit Wettbewerb? - Eine Bilanz derniederländischen Lösung, in: Elektrizitätswirtschaftliche Tagesfragen, 43. Jg. (1993), Heft 10, S. 674-677 


\section{SCHRIFTEN ZUR WIRTSCHAFTSTHEORIE UND WIRTSCHAFTSPOLITIK}

Herausgegeben von Rolf Hasse, Wolf Schäfer, Thomas Straubhaar, Klaus W. Zimmermann

Band 1 Lars Bünning: Die Konvergenzkriterien des Maastricht-Vertrages unter besonderer Berücksichtigung ihrer Konsistenz. 1997.

Band 2 Andreas Henning: Beveridge-Kurve, Lohnsetzung und Langzeitarbeitslosigkeit. Eine theoretische Untersuchung unter Benücksichtigung des Insider-Outsider-Ansatzes und der Entwertung des Humankapitals. 1997.

Band 3 Iris Henning: Die Reputation einer Zentralbank. Eine theoretische Untersuchung unter besonderer Berücksichtigung der Europäischen Zentralbank. 1997.

Band 4 Rüdiger Hermann: Ein gemeinsamer Markt für Elektrizität in Europa. Optionen einer Wettbewerbsordnung zwischen Anspruch und Wirklichkeit. 1997. 
Rüdiger Hermann - 978-3-631-75129-9

Downloaded from PubFactory at 01/11/2019 07:57:52AM

via free access 


\section{Prinzipien wirtschaftlichen Handelns und ihre Anwendung}

\section{Umriß einer Wirtschaftsphilosophie}

Frankfurt/M., Berlin, Bern, New York, Paris, Wien, 1996. 145 S., 1 Abb.

ISBN 3-631-30477-3 • br. DM 48.-*

Die enge Verbindung von Wirtschaft und Weltgeschehen verlangt nach einer exakten Untersuchung ihrer Notwendigkeit, Möglichkeiten und Grenzen. Die Ökonomie wird auf ihre erlernbaren theoretischen Prinzipien, die Möglichkeiten ihrer Anwendung und die Bedingungen ihrer praktischen Durchsetzbarkeit hin untersucht. Ergebnis ist eine gründliche Neubestimmung des Verhältnisses von Theorie, Anwendung und Praxis. Die historisch bekannten Wirtschaftsschulen und -richtungen werden auf ihren Geltungsanspruch hinterfragt. Politische und ideologische Rahmenbedingungen werden in ihrem Einfluß auf Privat- und Gemeinwirtschaft erläutert und Modelle zur Überprüfung von Wirtschaftspolitik und Wirtschaftsethik aufgezeigt.

Aus dem Inhalt: Wissenschaftsanspruch, Definition und Methoden der Ökonomie - Einfluß von politischen und ideologischen Rahmenbedingungen . Privat- und Gemeinwirtschaft - Wirtschaftsschulen und Wirtschaftsmodelle Wirtschaftsrecht und Wirtschaftsethik - Ökonomische Begriffsbildung und Wissenschaft · Ökonomie und Philosophie

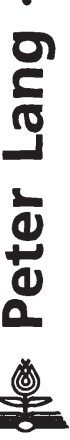

Frankfurt/M - Berlin - Bern - New York · Paris - Wien

Auslieferung: Verlag Peter Lang AG

Jupiterstr. 15, CH-3000 Bern 15

Telefax (004131) 9402131

*inklusive Mehrwertsteuer

Preisănderungen vorbehalten 
Rüdiger Hermann - 978-3-631-75129-9

Downloaded from PubFactory at 01/11/2019 07:57:52AM

via free access 